\title{
Health technology assessment of hyperphosphatemia management among hemodialysis patients in Lebanon
}

Citation for published version (APA):

Rizk, R. (2017). Health technology assessment of hyperphosphatemia management among hemodialysis patients in Lebanon. [Doctoral Thesis, Maastricht University]. Datawyse / Universitaire Pers Maastricht. https://doi.org/10.26481/dis.20170622rr

Document status and date:

Published: 01/01/2017

DOI:

10.26481/dis.20170622rr

Document Version:

Publisher's PDF, also known as Version of record

\section{Please check the document version of this publication:}

- A submitted manuscript is the version of the article upon submission and before peer-review. There can be important differences between the submitted version and the official published version of record.

People interested in the research are advised to contact the author for the final version of the publication, or visit the DOI to the publisher's website.

- The final author version and the galley proof are versions of the publication after peer review.

- The final published version features the final layout of the paper including the volume, issue and page numbers.

Link to publication

\footnotetext{
General rights rights.

- You may freely distribute the URL identifying the publication in the public portal. please follow below link for the End User Agreement:

www.umlib.nl/taverne-license

Take down policy

If you believe that this document breaches copyright please contact us at:

repository@maastrichtuniversity.nl

providing details and we will investigate your claim.
}

Copyright and moral rights for the publications made accessible in the public portal are retained by the authors and/or other copyright owners and it is a condition of accessing publications that users recognise and abide by the legal requirements associated with these

- Users may download and print one copy of any publication from the public portal for the purpose of private study or research.

- You may not further distribute the material or use it for any profit-making activity or commercial gain

If the publication is distributed under the terms of Article $25 \mathrm{fa}$ of the Dutch Copyright Act, indicated by the "Taverne" license above, 
Health technology assessment of hyperphosphatemia management among hemodialysis patients in Lebanon 
$\square$

$\square$

$\square$

$\square$

$\square$

$\square$

$\square$

$\square$

$\square$

$\square$

$\square$

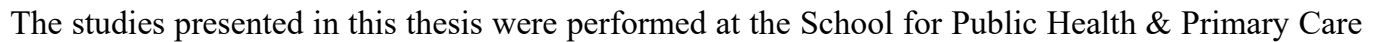

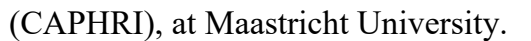

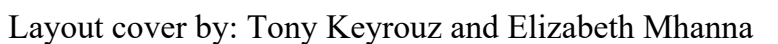

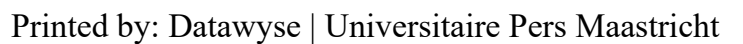

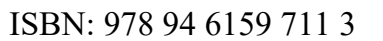

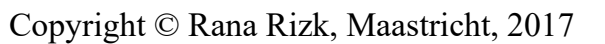

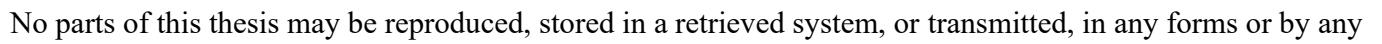

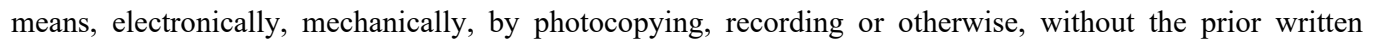

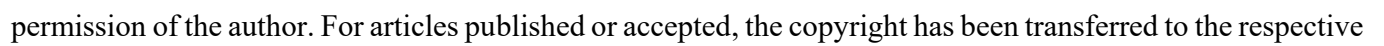

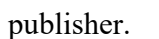


Health technology assessment of hyperphosphatemia management among hemodialysis patients in Lebanon

Proefschrift

ए 


\section{Promotores}

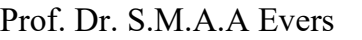

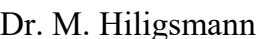

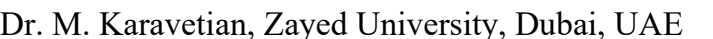

\section{BEOORDELINGSCOMMISSIE}

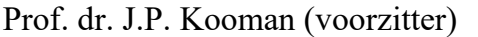

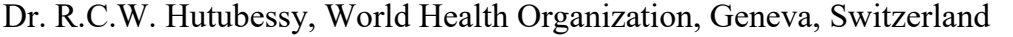

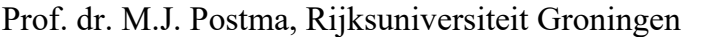

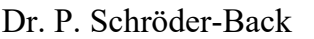

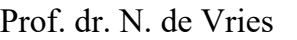

$\square$ 


\section{CONTENT}

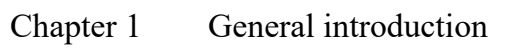

$\square$

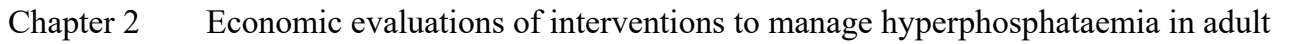

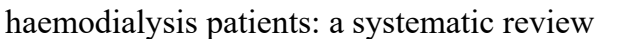

$\square$

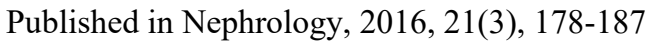

$\square$

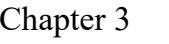

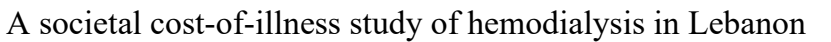

$\square$

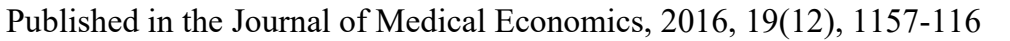

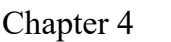

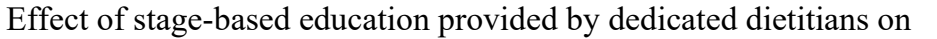

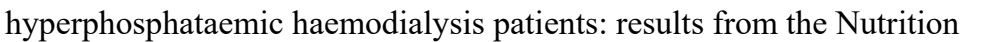

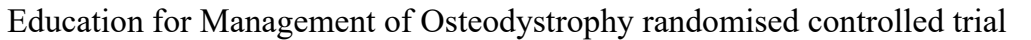

$\square$

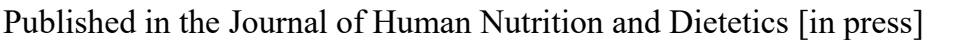

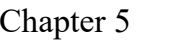

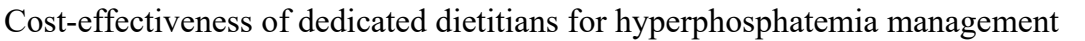

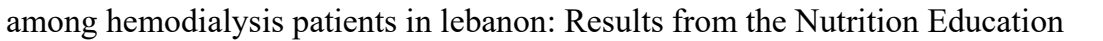

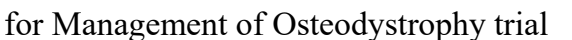

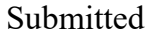

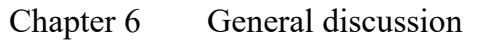

$\square$

$\square$

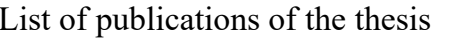

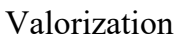

$\square \square$

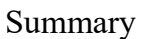

पाए

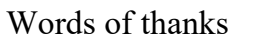

$\square \square$

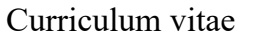

$\square 11$ 

$\square$
$\square$
$\square$
$\square$
$\square$
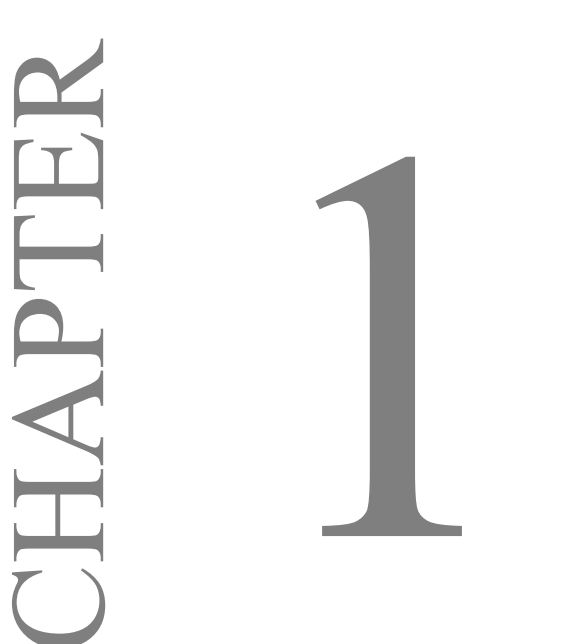

GENERAL INTRODUCTION $\square$ 


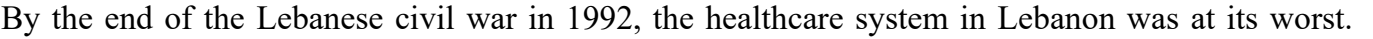

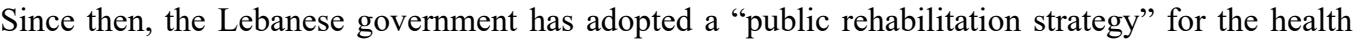

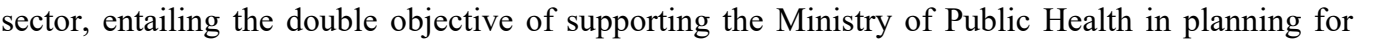

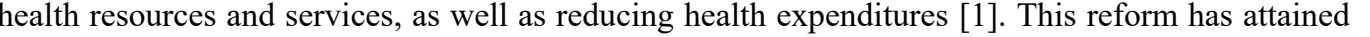

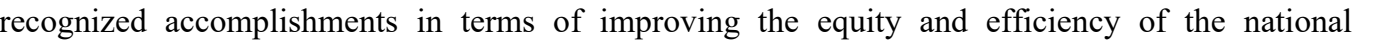

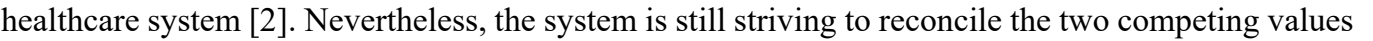

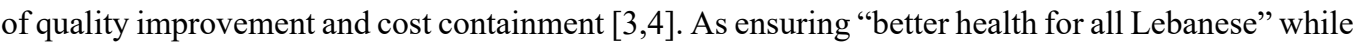
पा

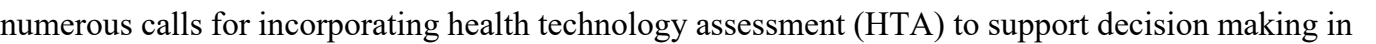

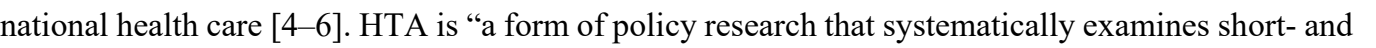

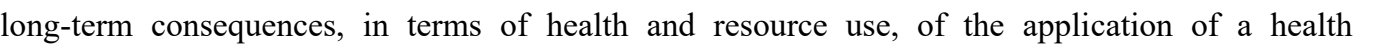

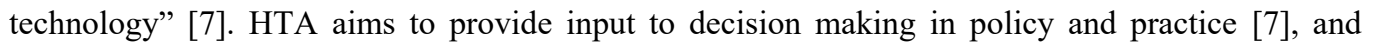

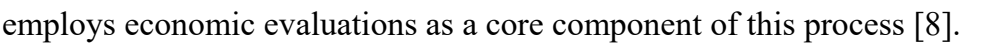

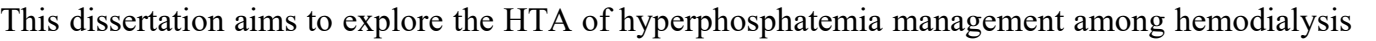

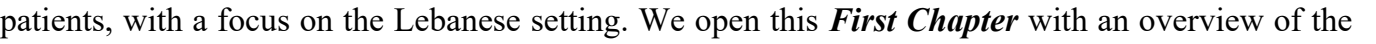

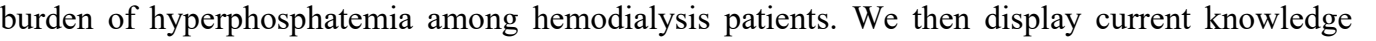

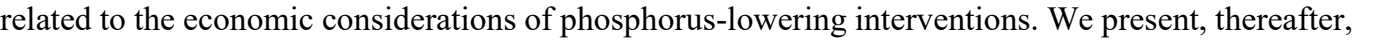

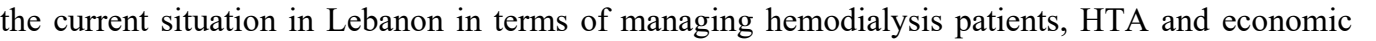

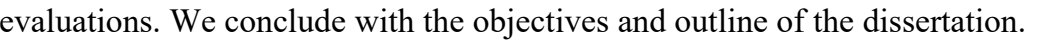

\section{HYPERPHOSPHATEMIA: A HEAVY CLINICAL AND ECONOMIC BURDEN}

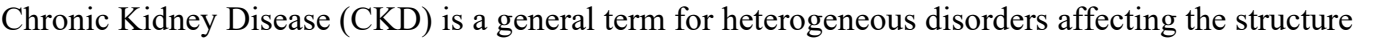

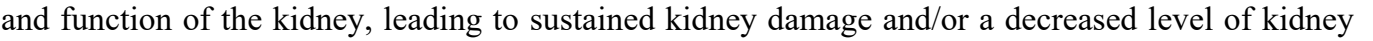

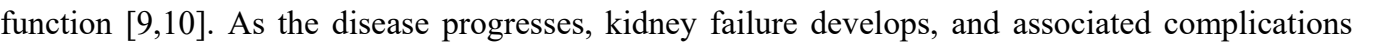

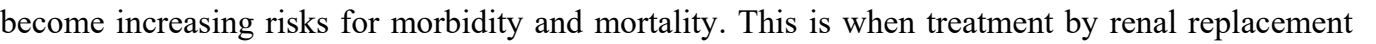

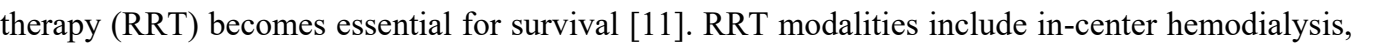

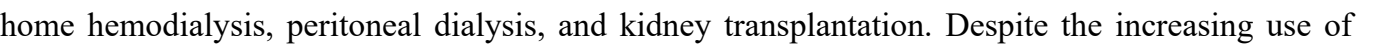

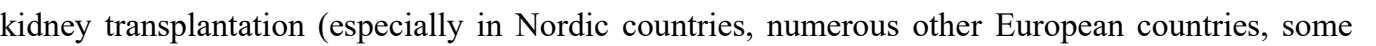

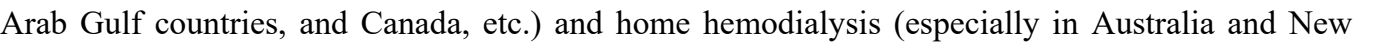

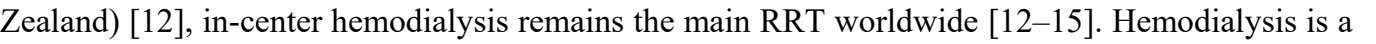

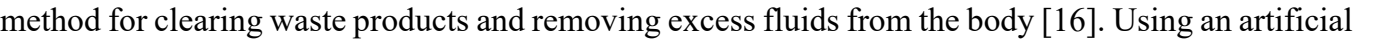

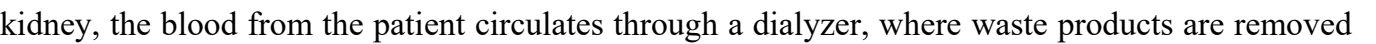

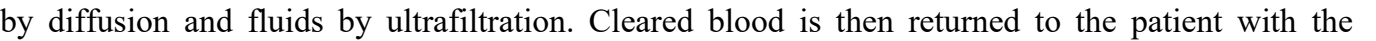

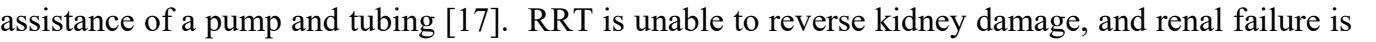

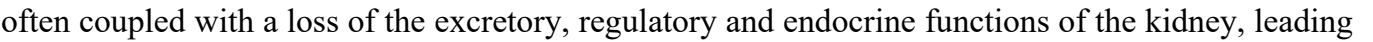




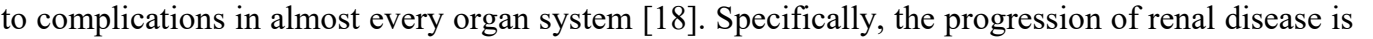

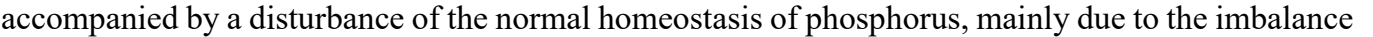

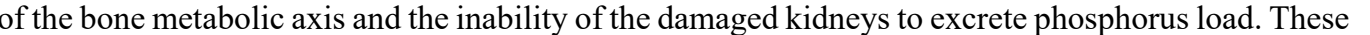

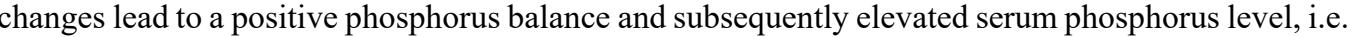

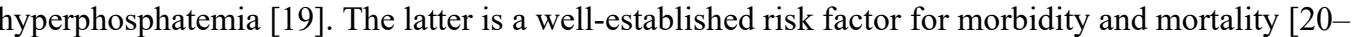

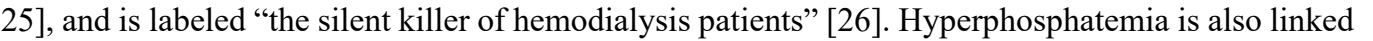

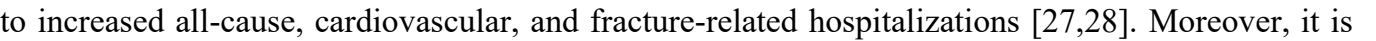

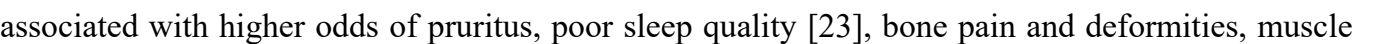

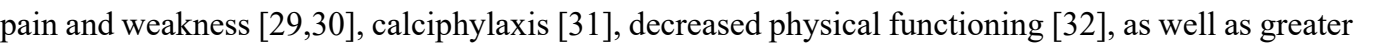

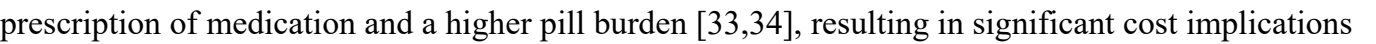

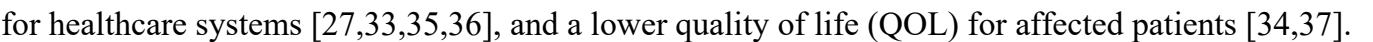

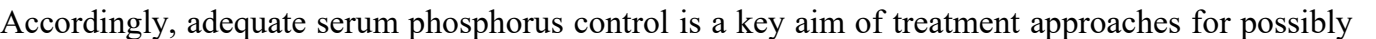

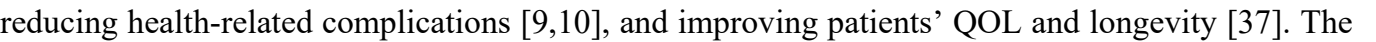

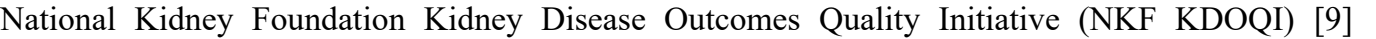

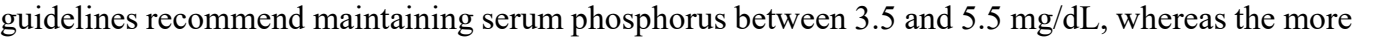

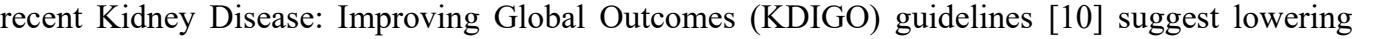

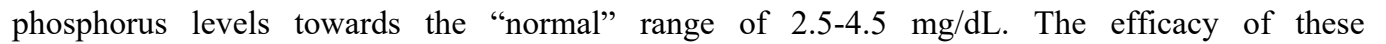

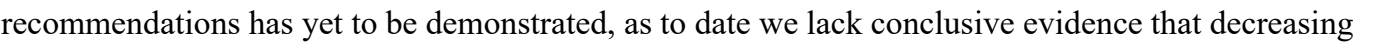

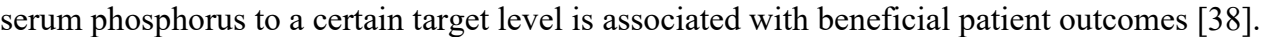

\section{HYPERPHOSPHATEMIA MANAGEMENT: THE EVERLASTING CHALLENGE}

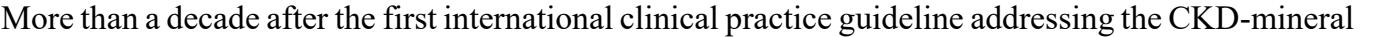

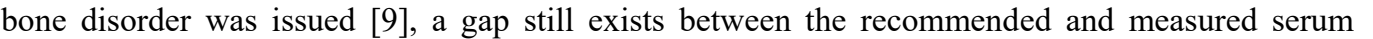

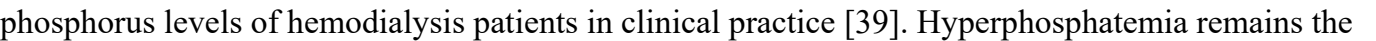

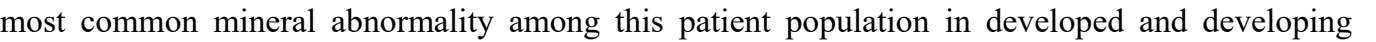

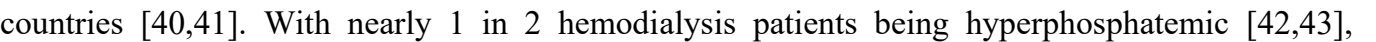

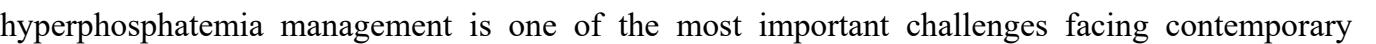

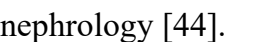

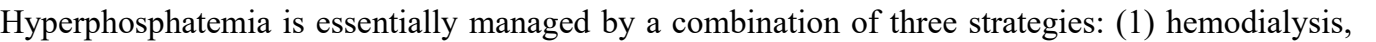

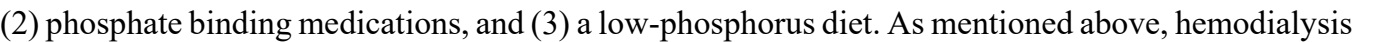
ए

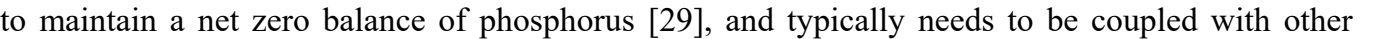

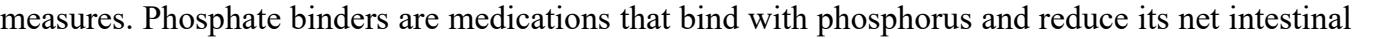

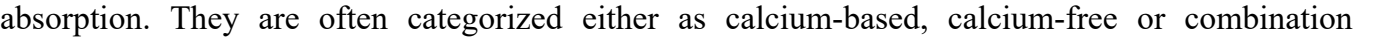

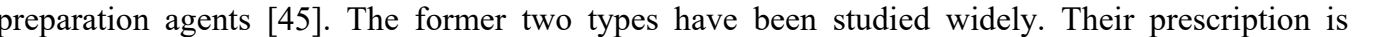




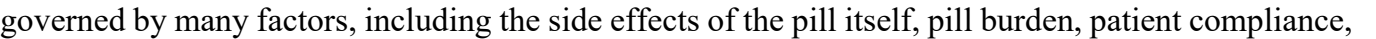

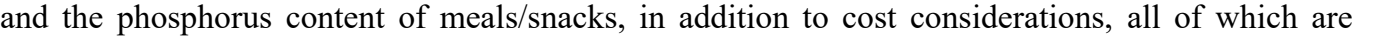

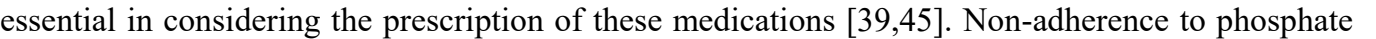

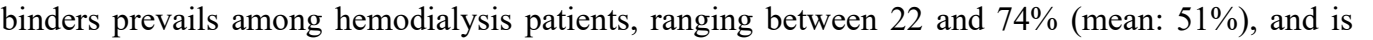

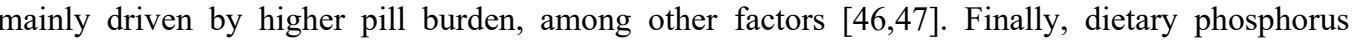

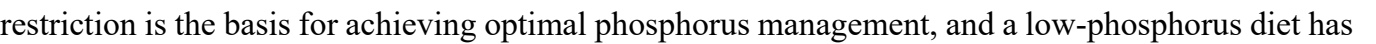

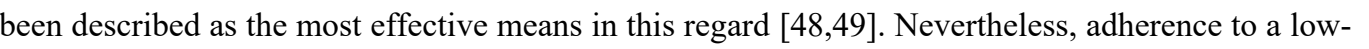

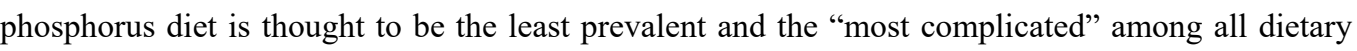

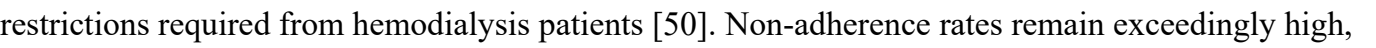

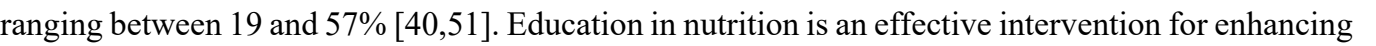

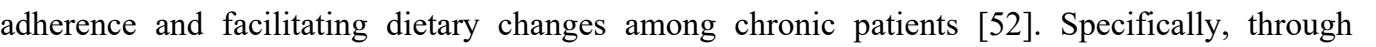

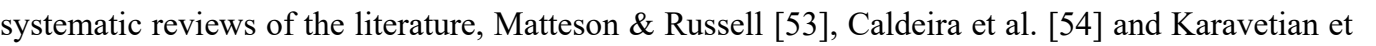

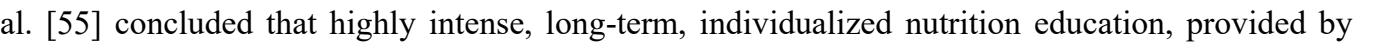

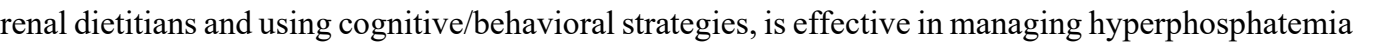

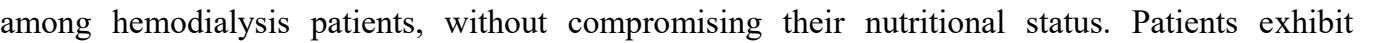

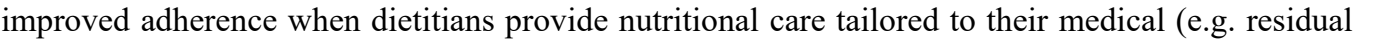

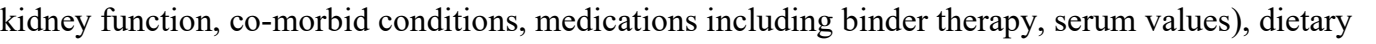

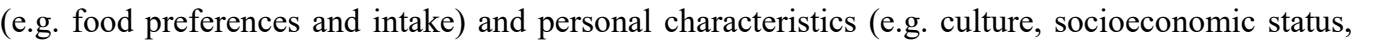

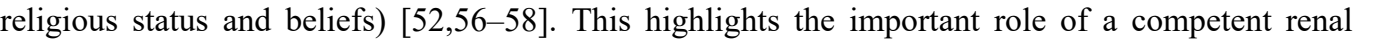

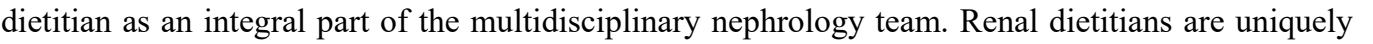

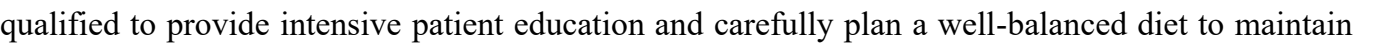

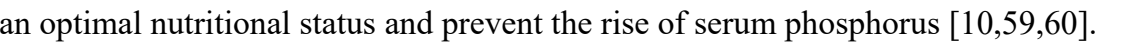

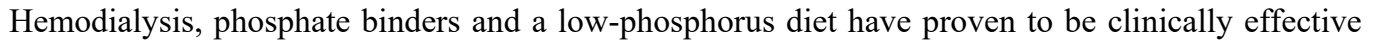

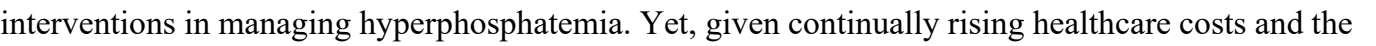

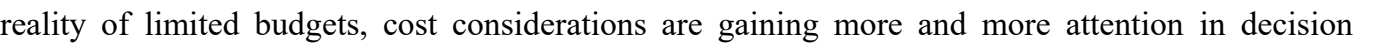

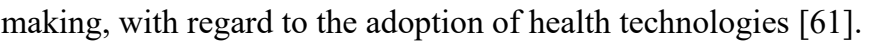

$\square$

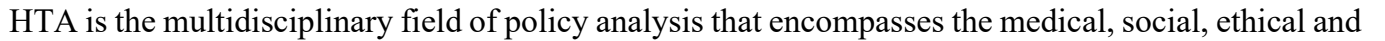

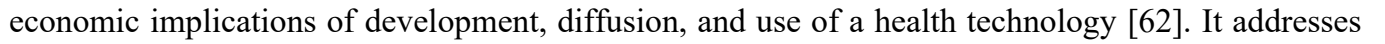

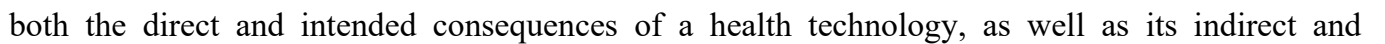

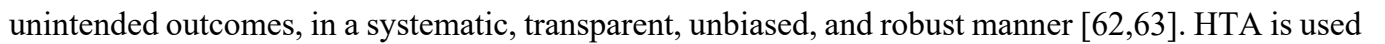

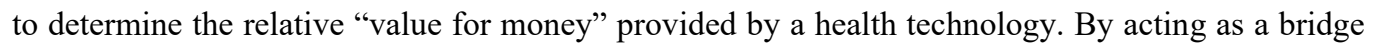

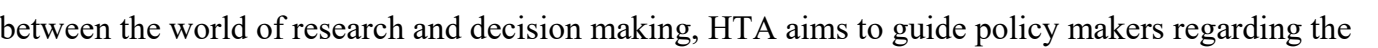

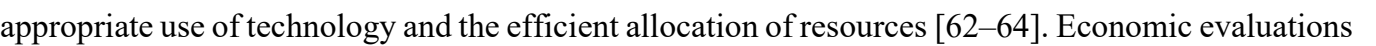

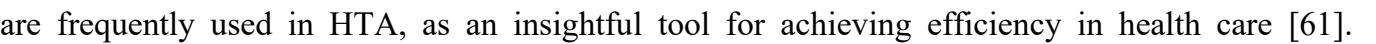




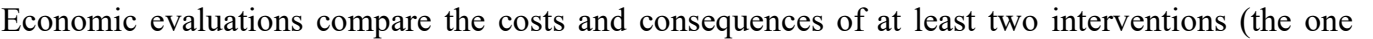

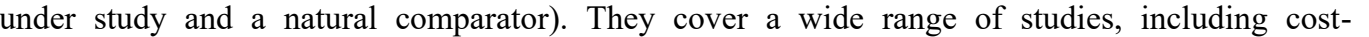

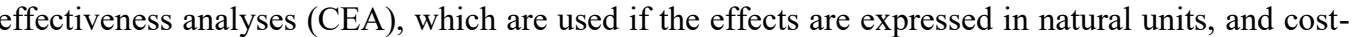

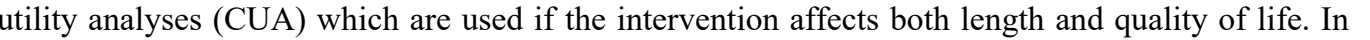

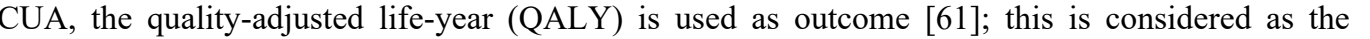

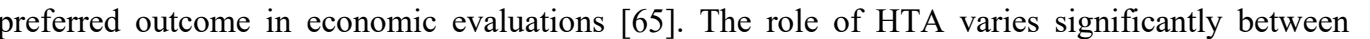

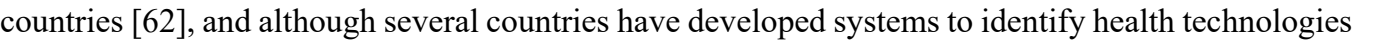

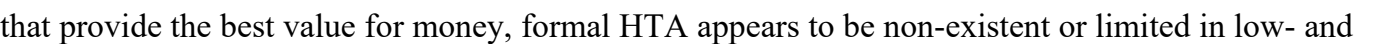

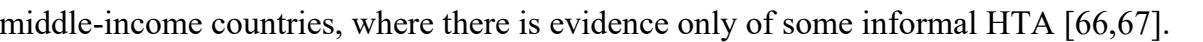

$\square$

\section{HYPERPHOSPHATEMIA MANAGEMENT: ECONOMIC CONSIDERATIONS}

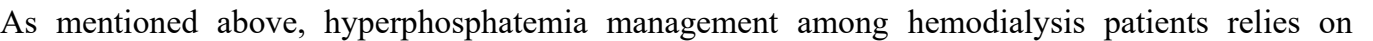

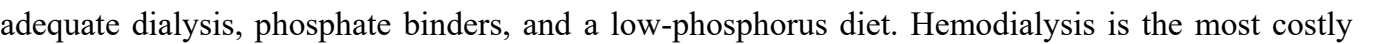

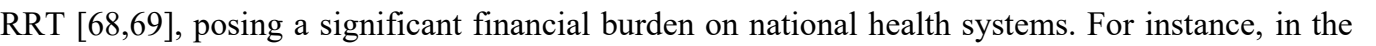

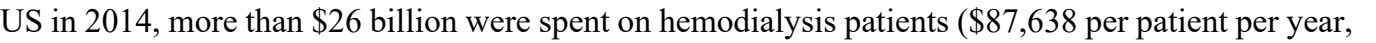

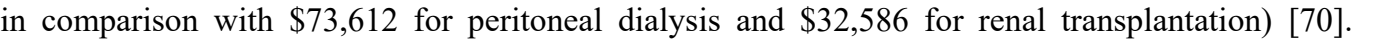

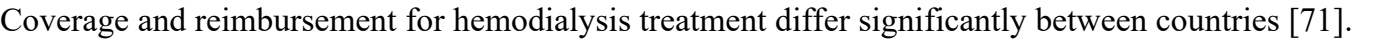

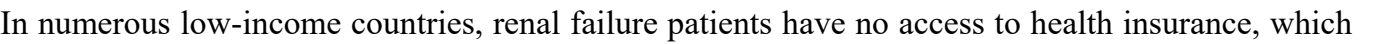

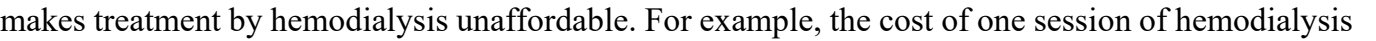

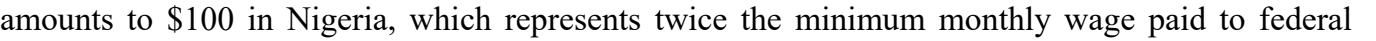

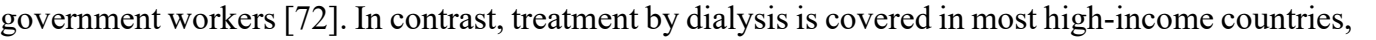

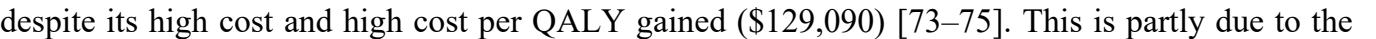
पा山ाWwithout dialysis, patients face certain death, and as a "rule of rescue," societies tend to adopt

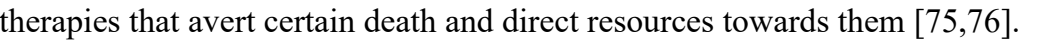

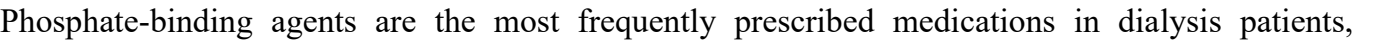

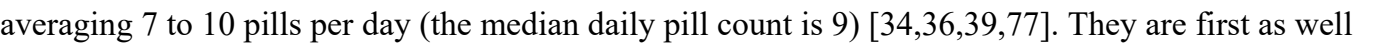

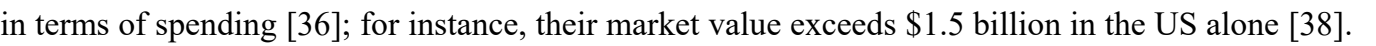

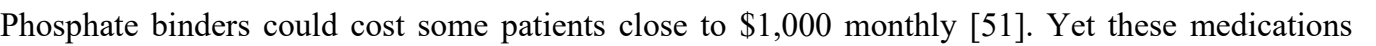

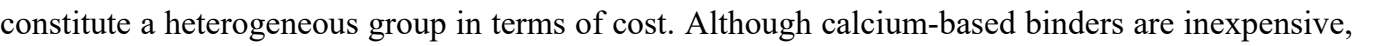

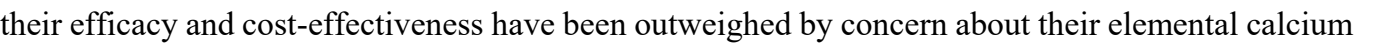

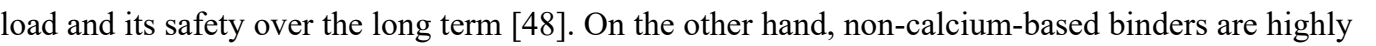

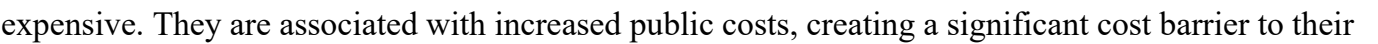

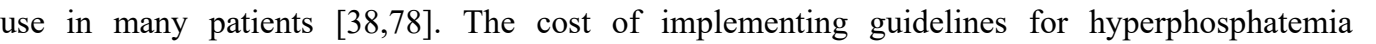

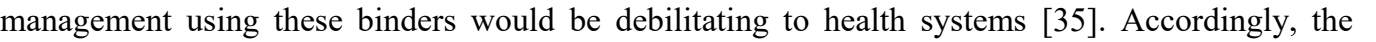

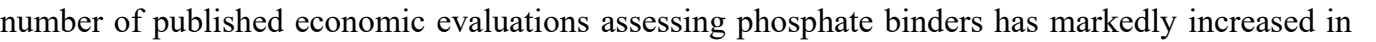




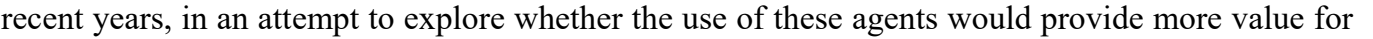

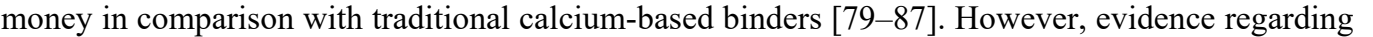

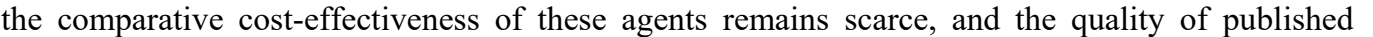

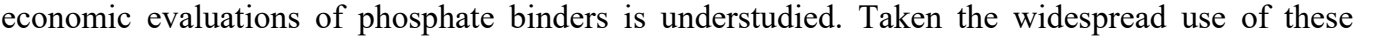

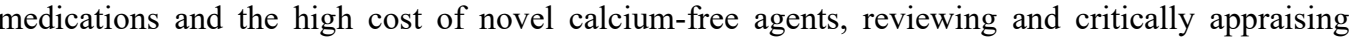

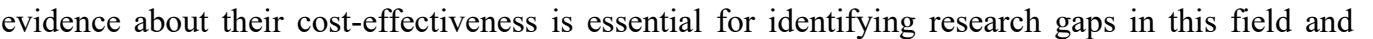

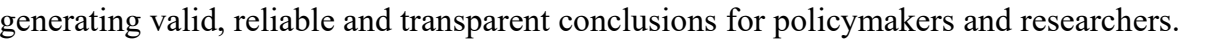

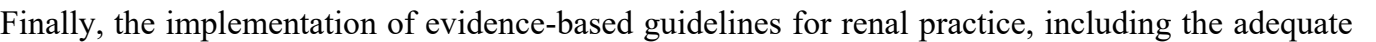

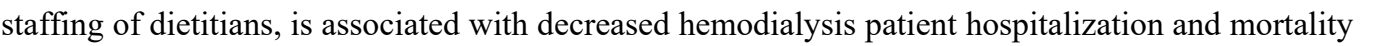

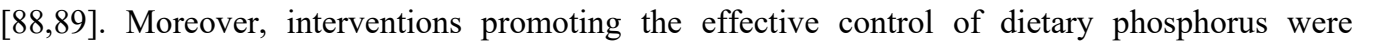

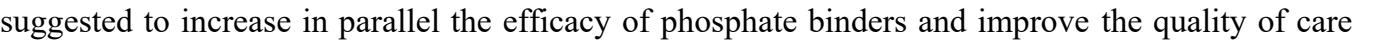

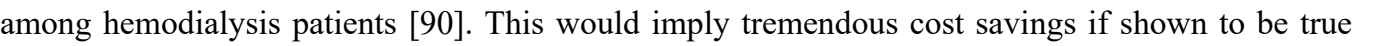

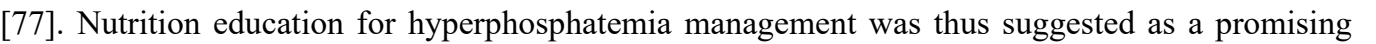

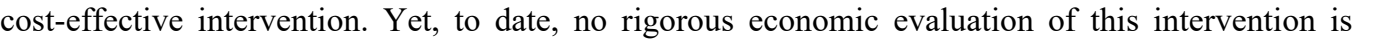

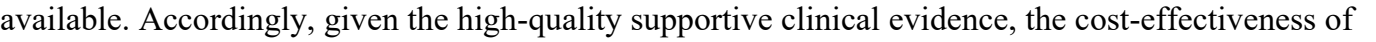

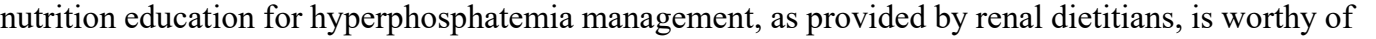

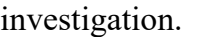

$\square$

\section{MANAGEMENT OF HEMODIALYSIS PATIENTS IN LEBANON}

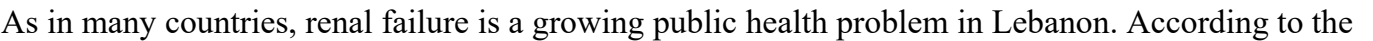

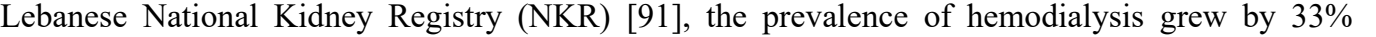

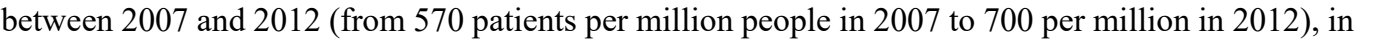

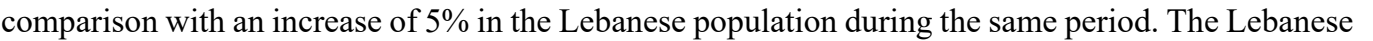

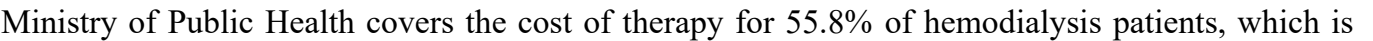

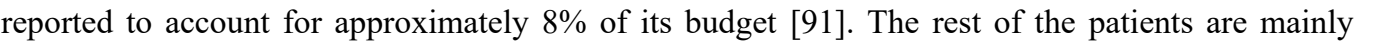

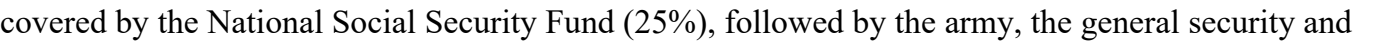
एवा

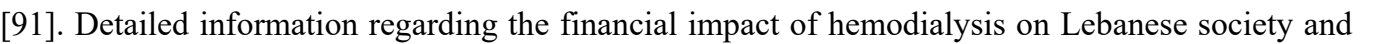

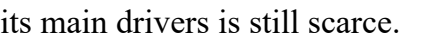

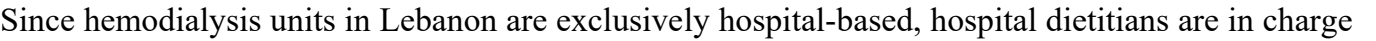

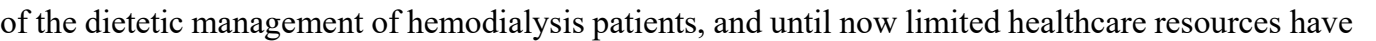
पा

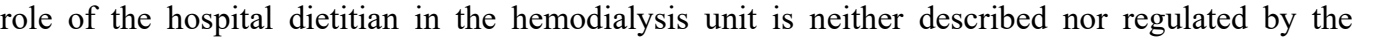

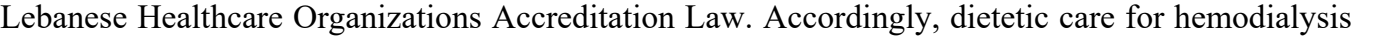

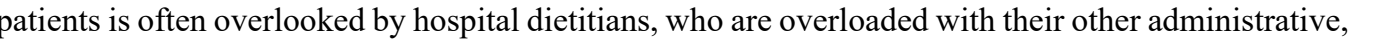




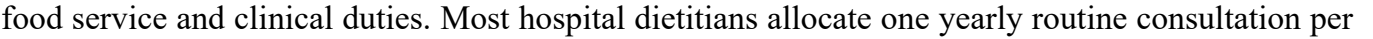

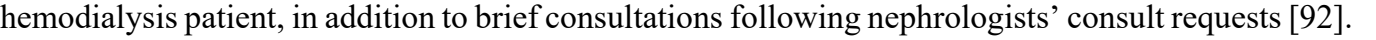

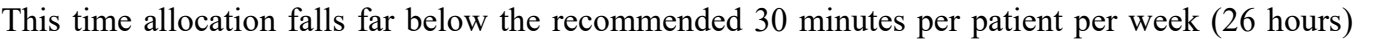
ए

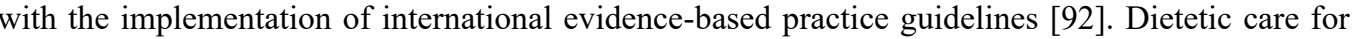

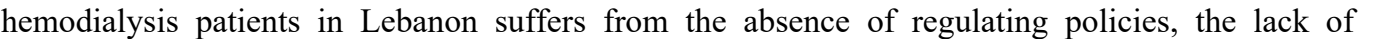

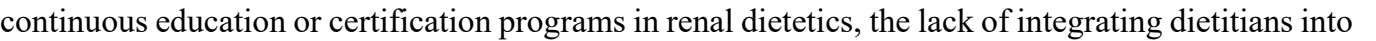
ए।

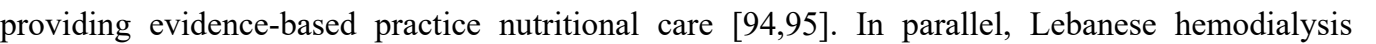

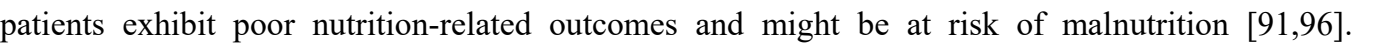

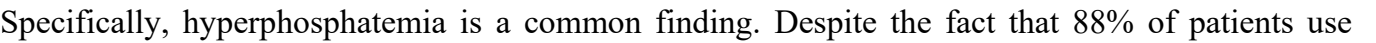

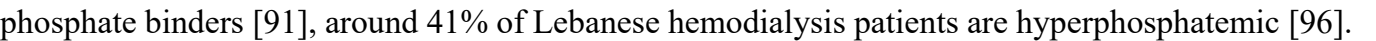

$\square$

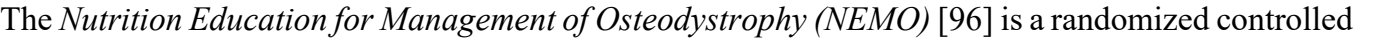

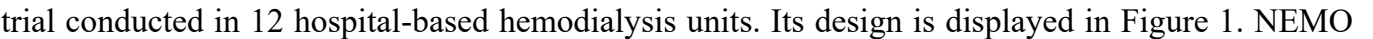

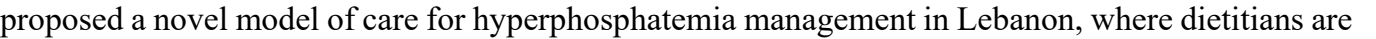

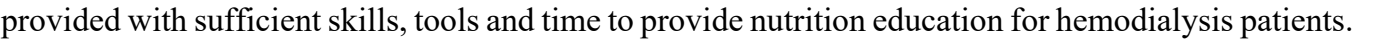

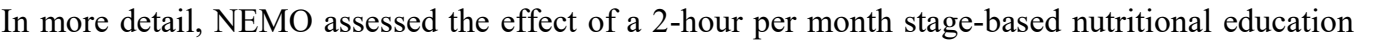

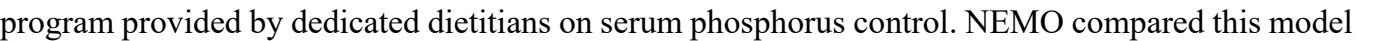

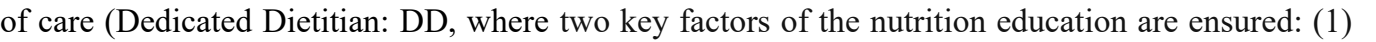

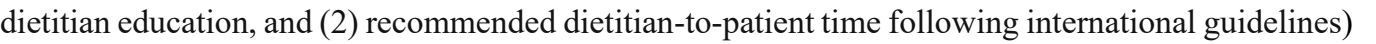

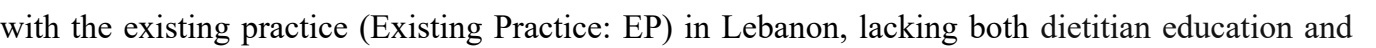

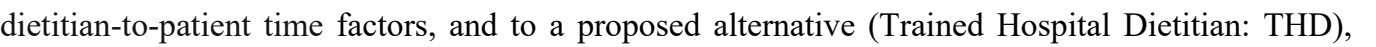

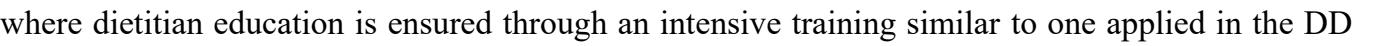

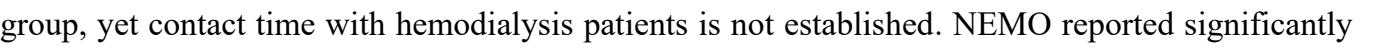

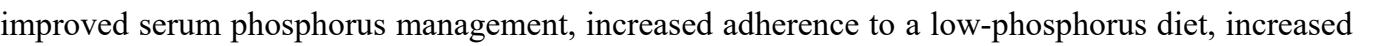

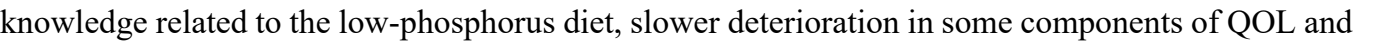

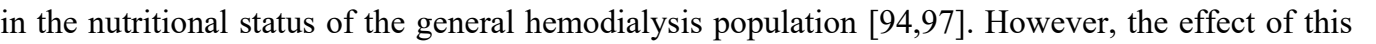

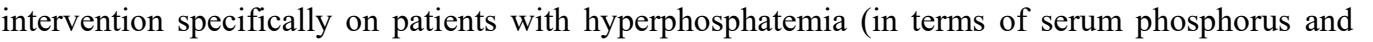

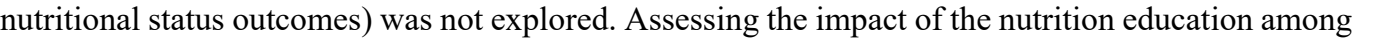

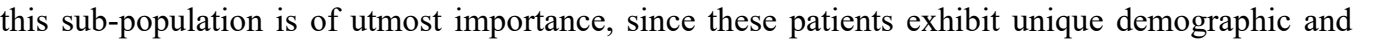

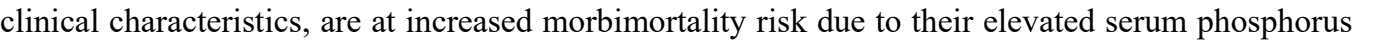

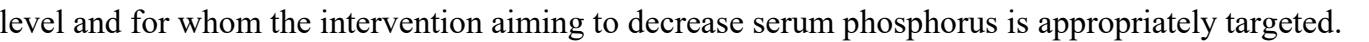

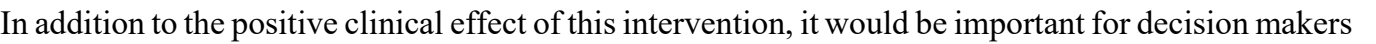

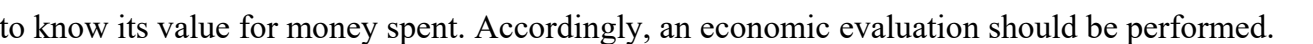




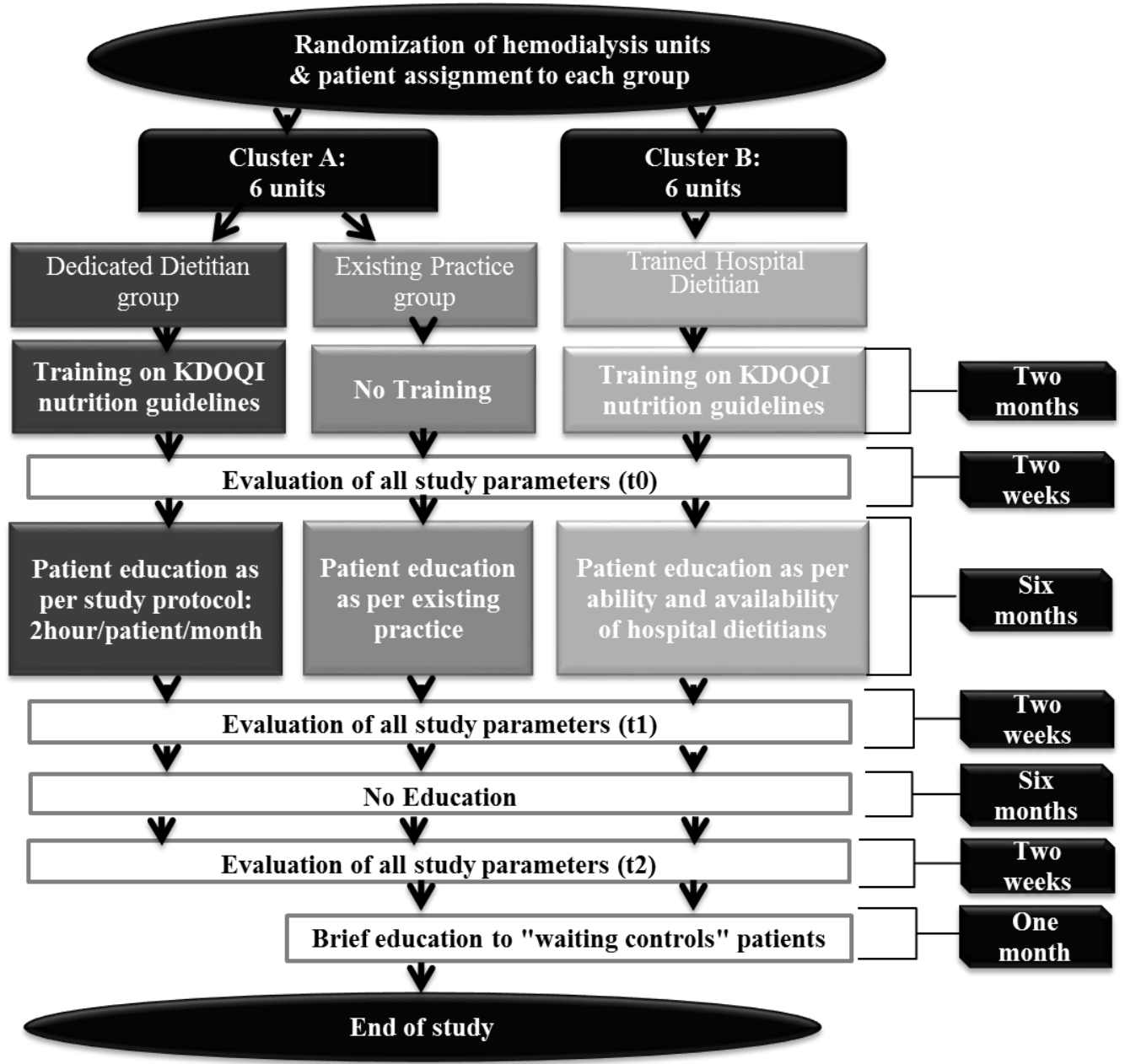

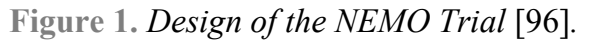

HEALTH TECHNOLOGY ASSESSMENT AND ECONOMIC EVALUATIONS IN LEBANON

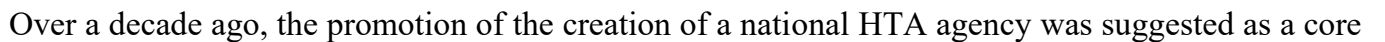

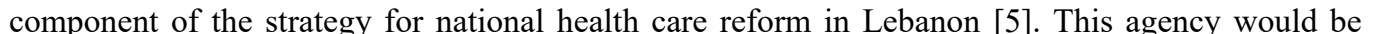

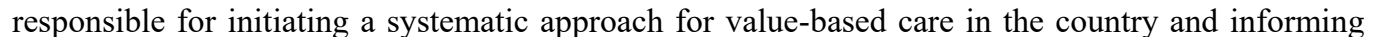

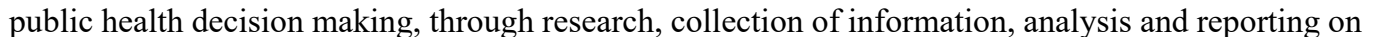
ए ए

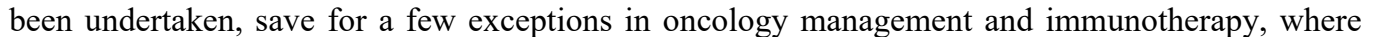

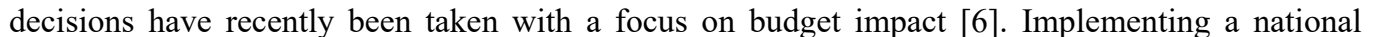

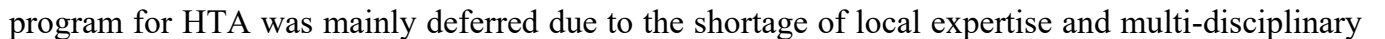




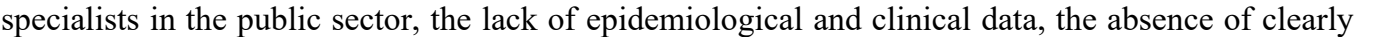

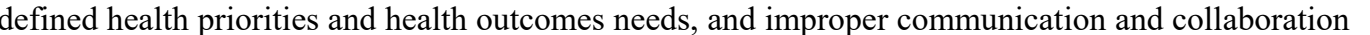

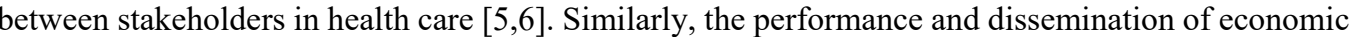

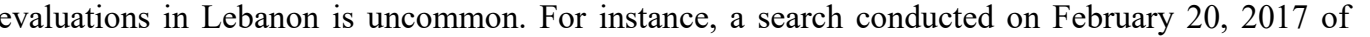

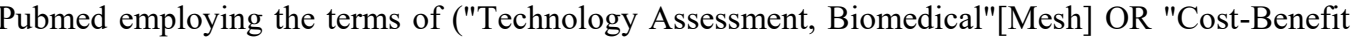

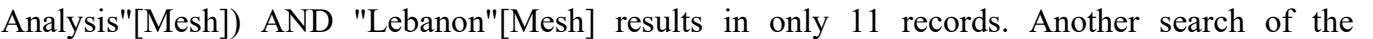
University of York's Centre for Reviews and Dissemination (CRD) on the NHS Economic Evaluation

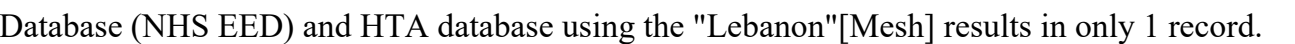

$\square$

\section{OBJECTIVES AND OUTLINE OF THE THESIS}

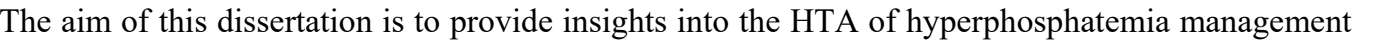

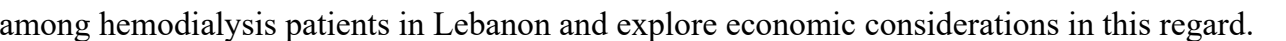

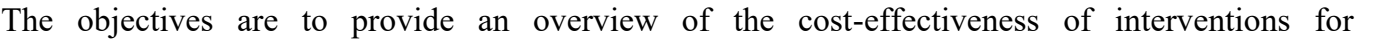

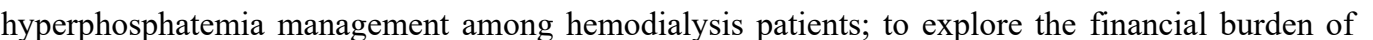

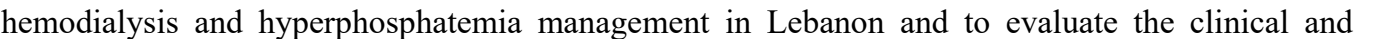

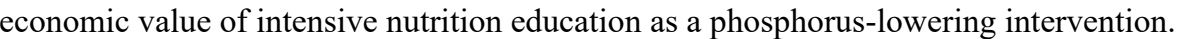

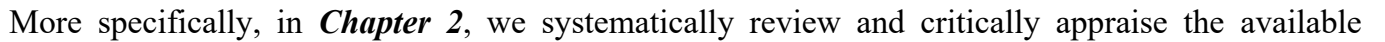

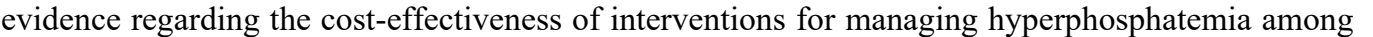

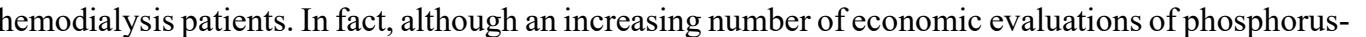

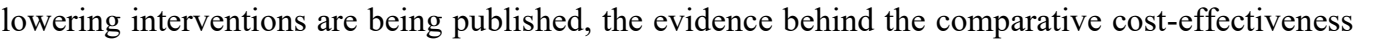

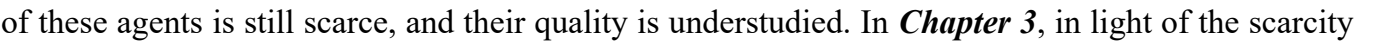

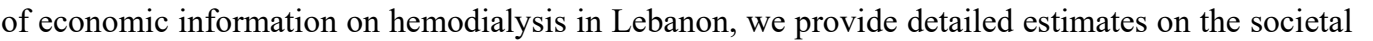

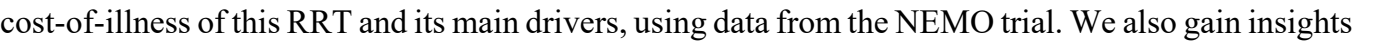

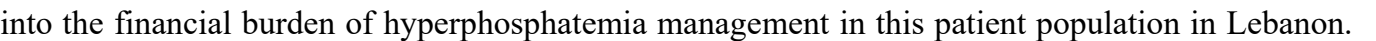

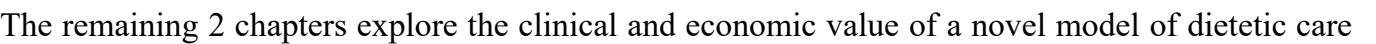

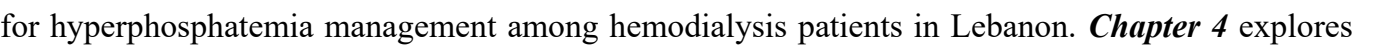

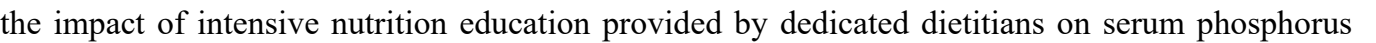

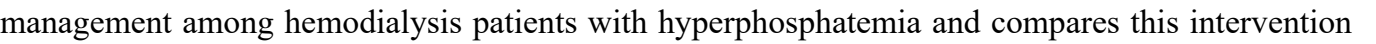

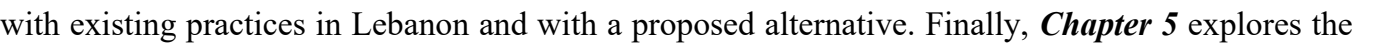

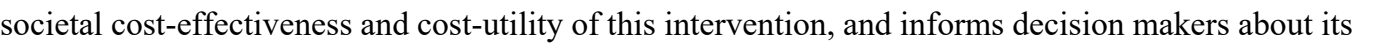

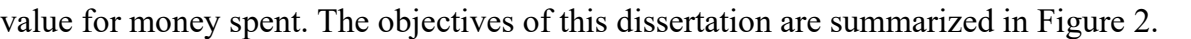




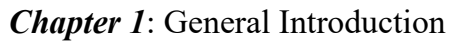

\section{$+1$}

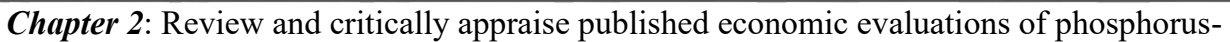

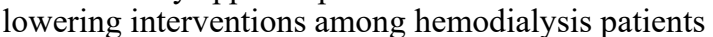

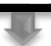

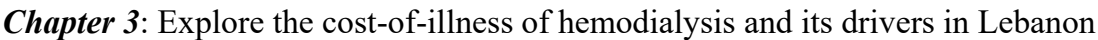

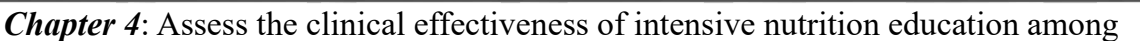

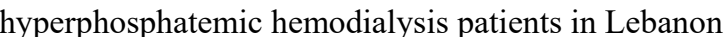

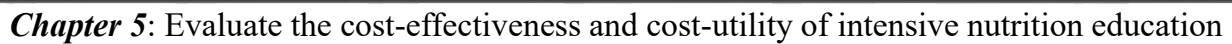

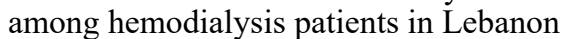

\section{$1-$}

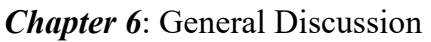

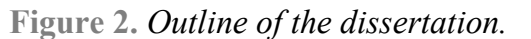

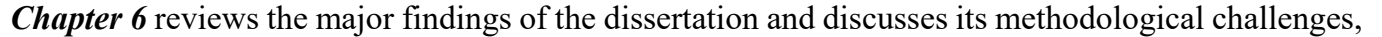

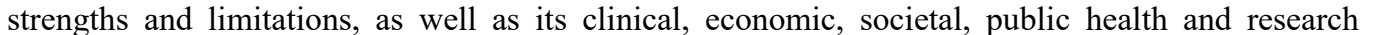

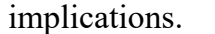




\section{REFERENCES}

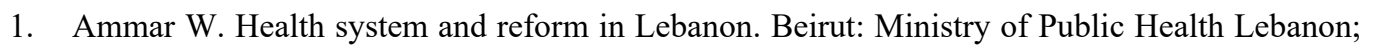

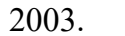

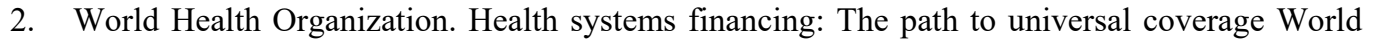

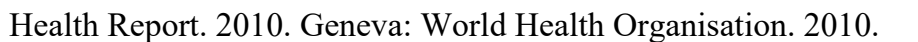

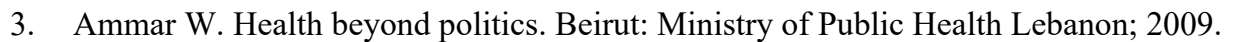

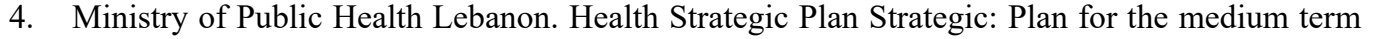

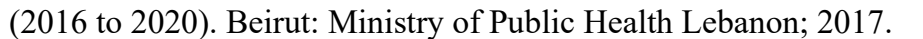

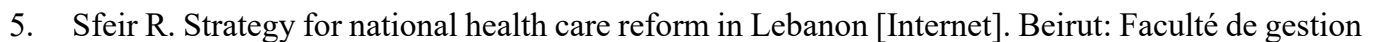

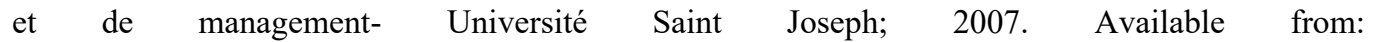

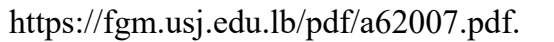

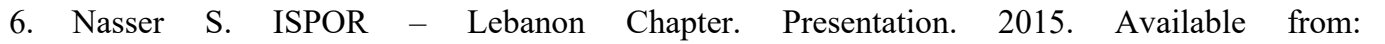

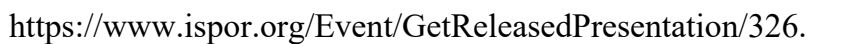

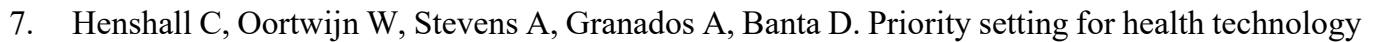

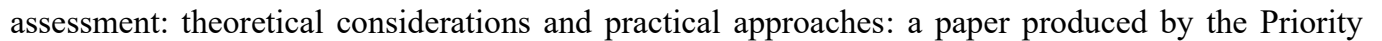

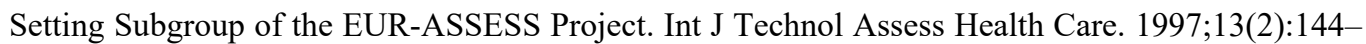
$\square \square$

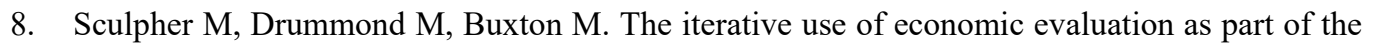

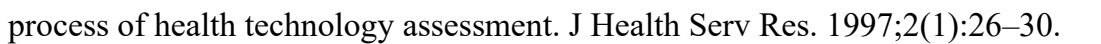

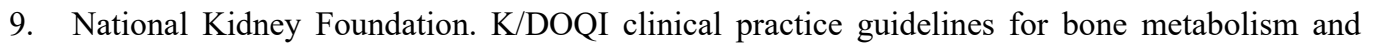

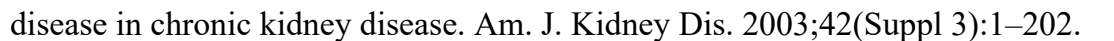

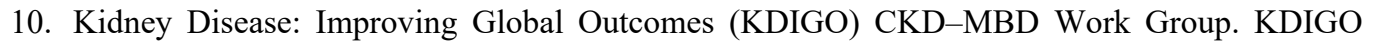

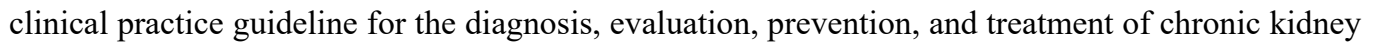

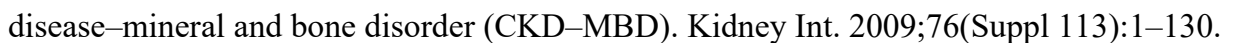

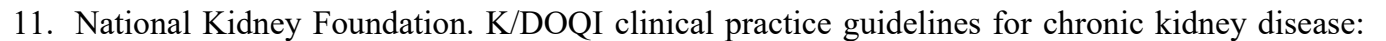

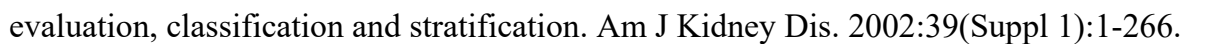

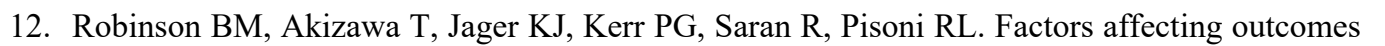

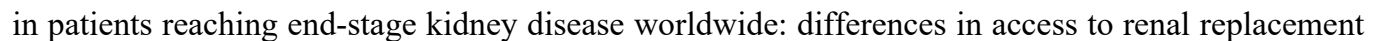

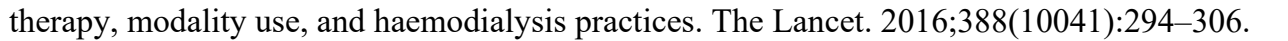

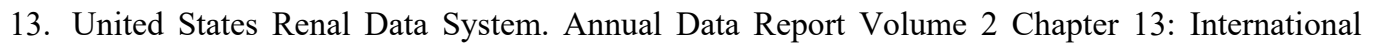

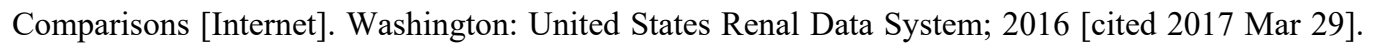

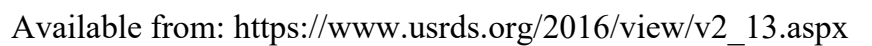

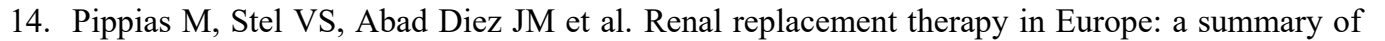

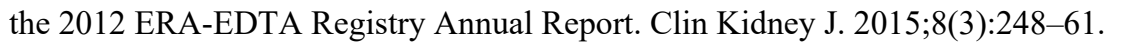

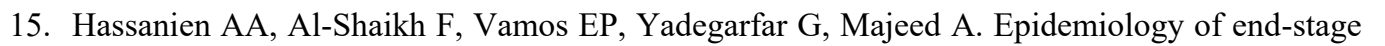

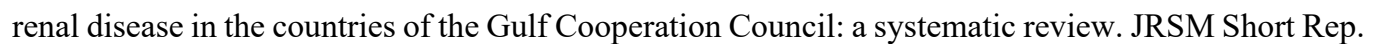

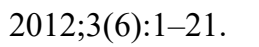

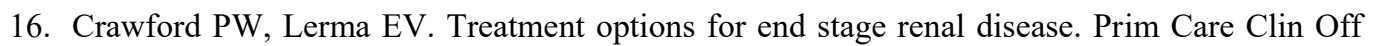

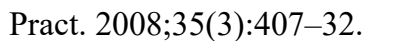




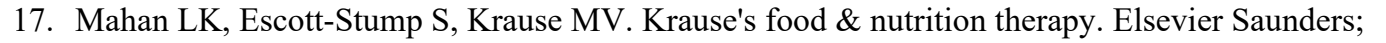
$\square 11$

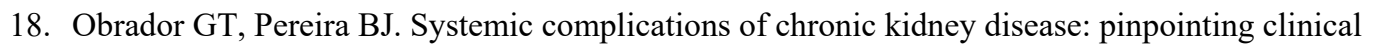

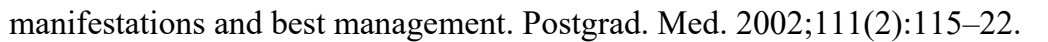

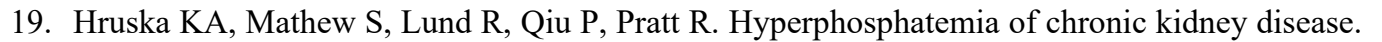

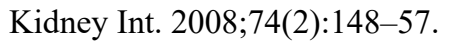

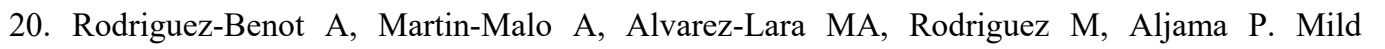

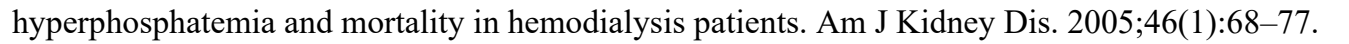

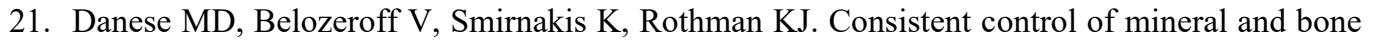

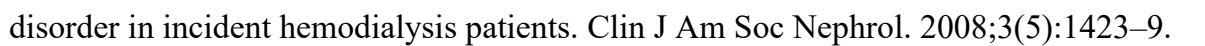

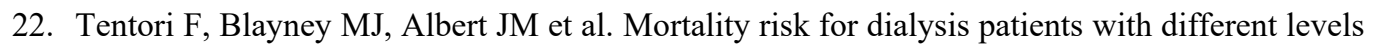

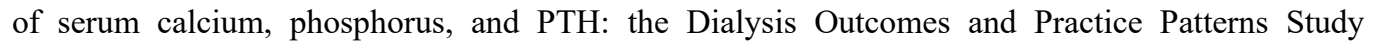

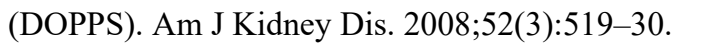

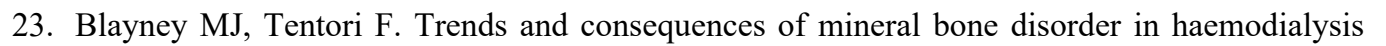

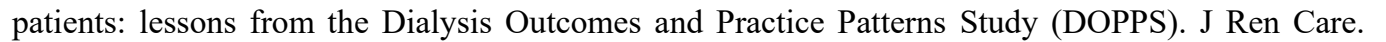

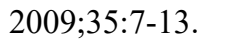

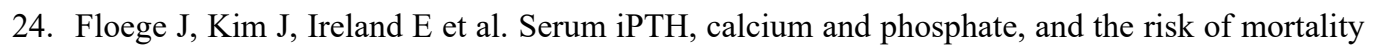

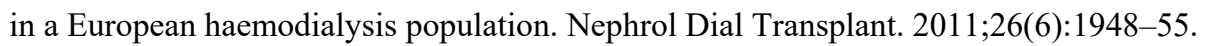

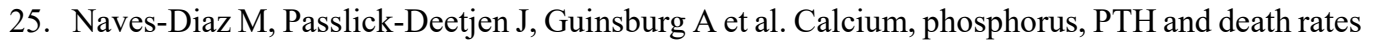

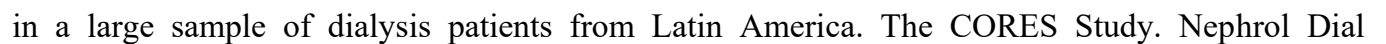

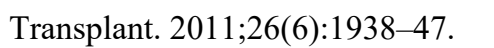

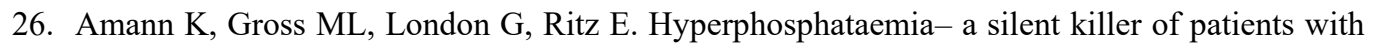

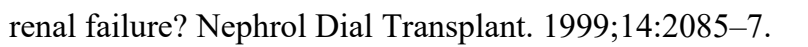

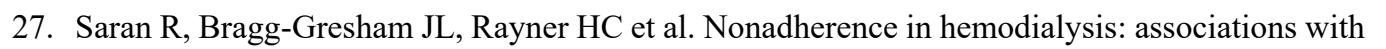

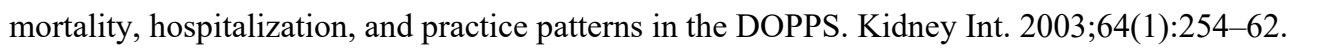

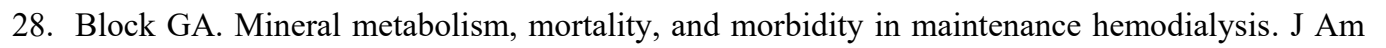

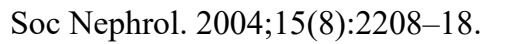

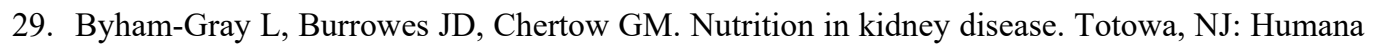

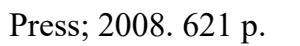

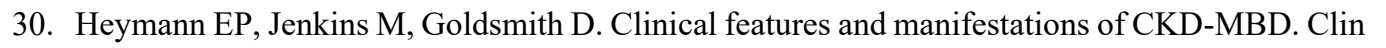

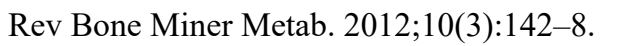

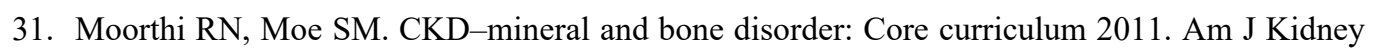

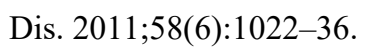

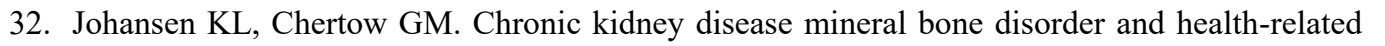

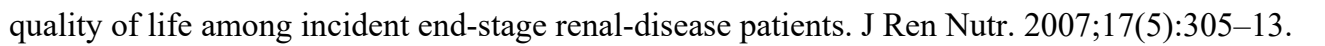

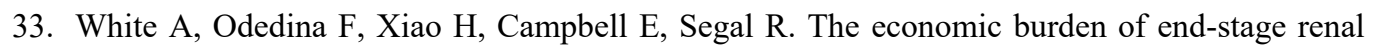

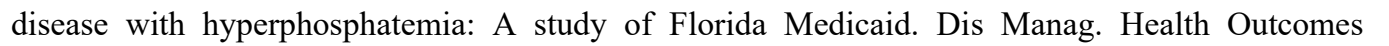
$\square|\Pi| \Pi-\square \mid \Pi$ 


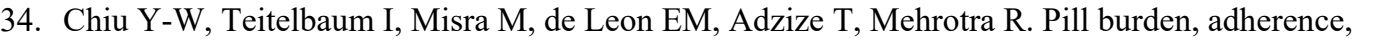

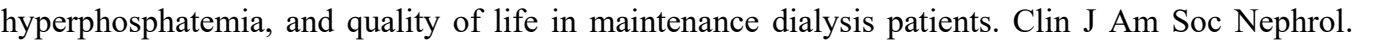

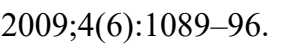

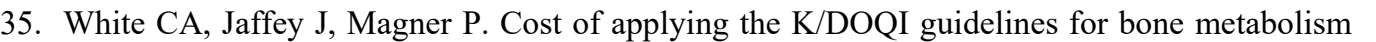

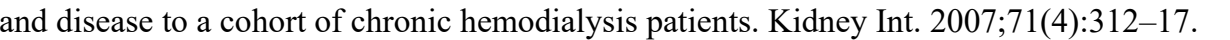

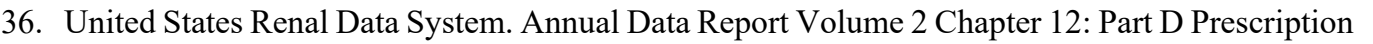

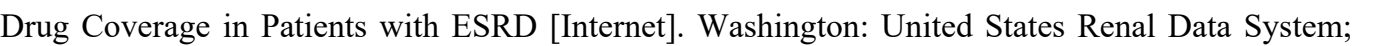

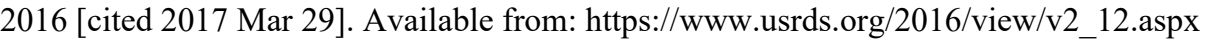

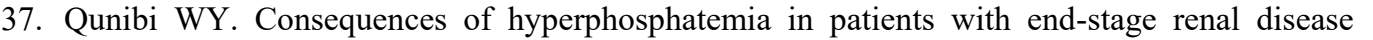

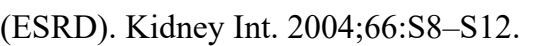

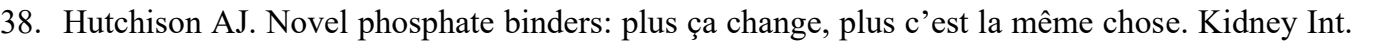

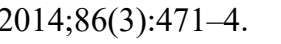

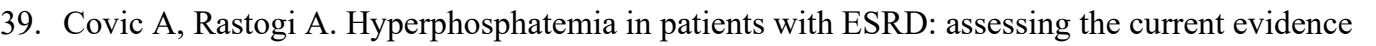

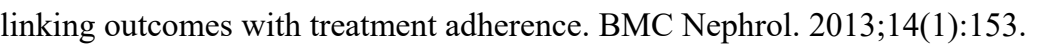

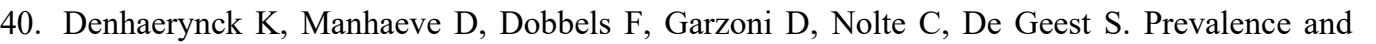

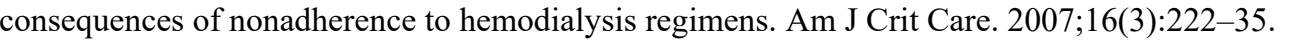

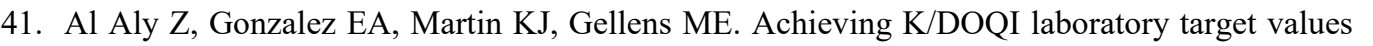

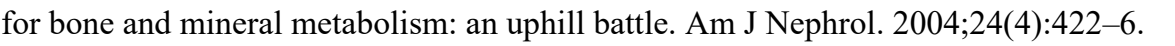

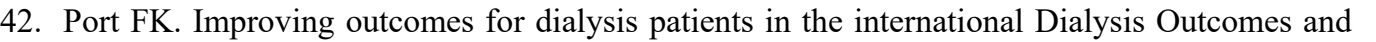

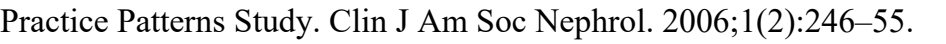

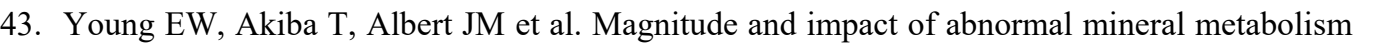

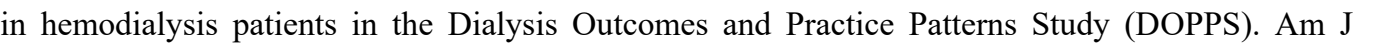

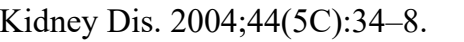

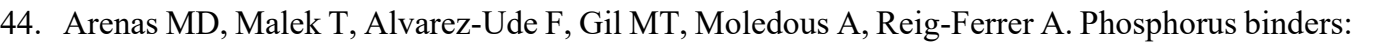

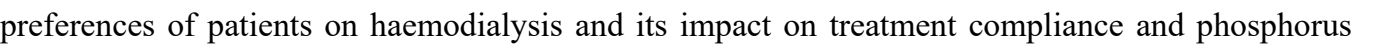

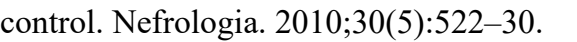

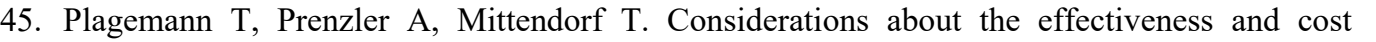

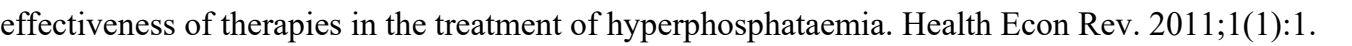

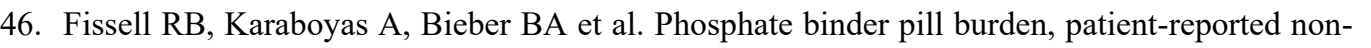

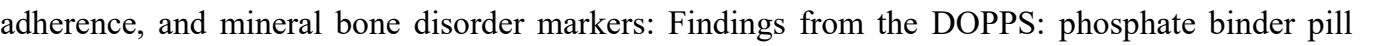

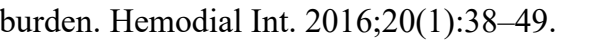

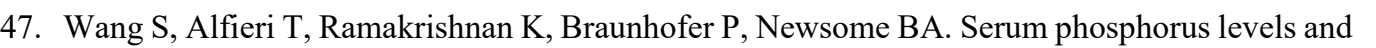

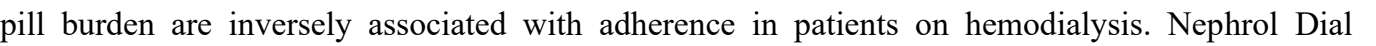

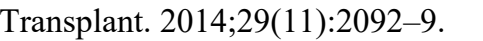

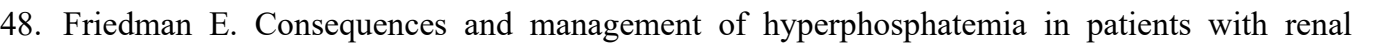

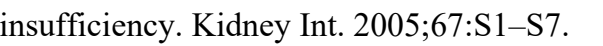

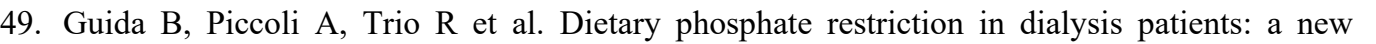

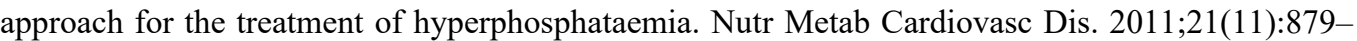
$\square \square$ 


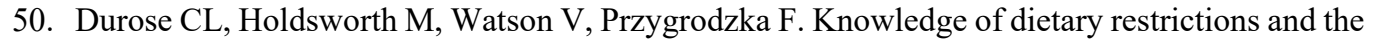

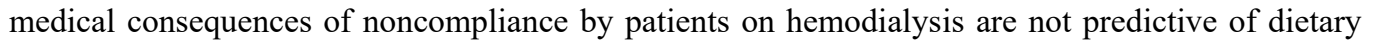

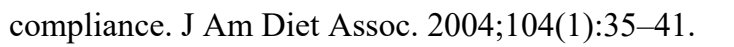

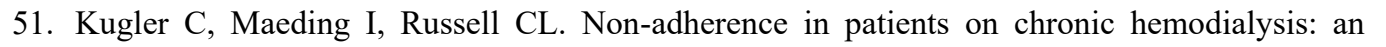

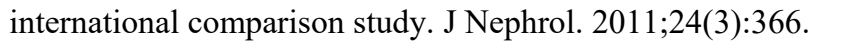

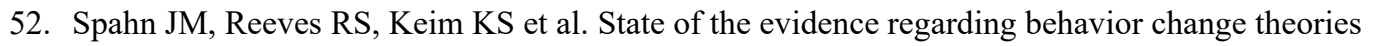

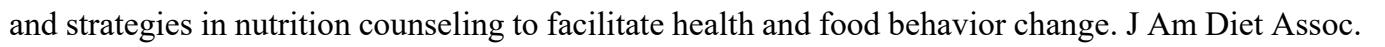

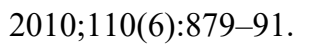

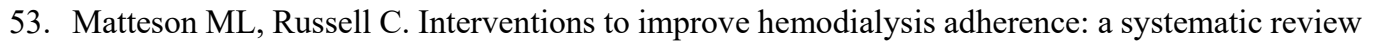

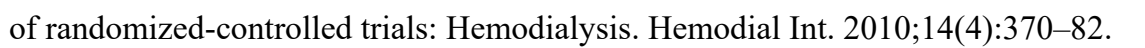

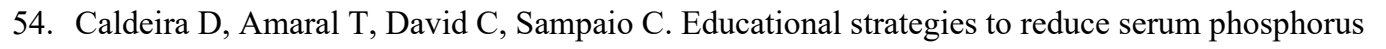

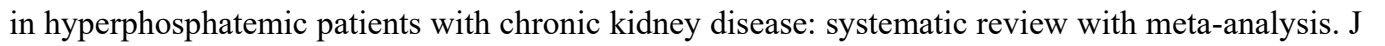

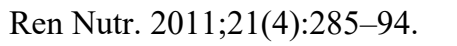

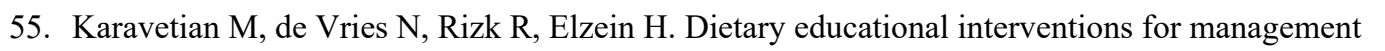

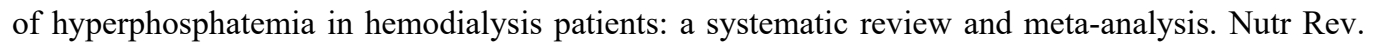
$\square||||||||||-\square \mid \square$

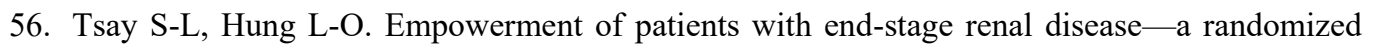

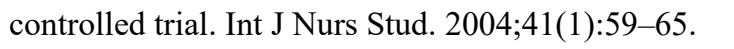

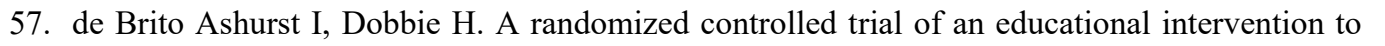

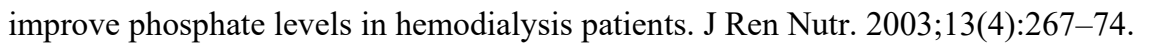

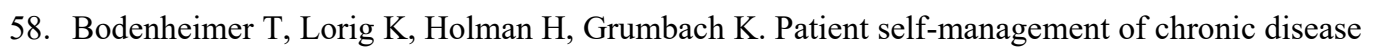

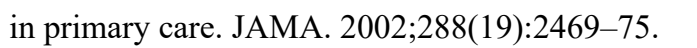

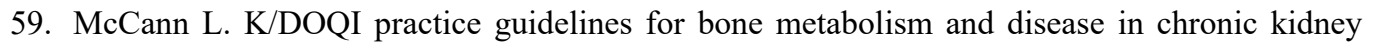

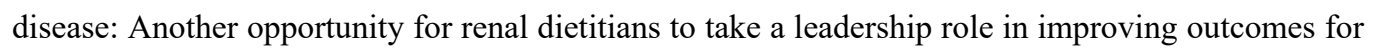

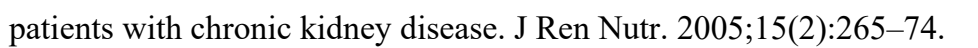

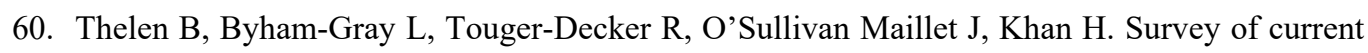

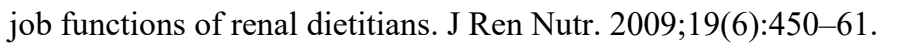

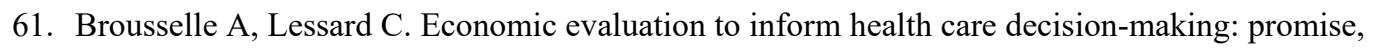

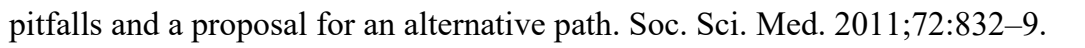

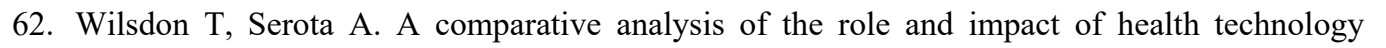

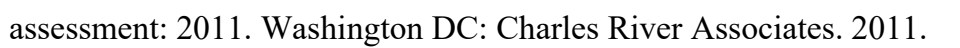

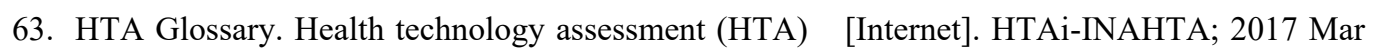

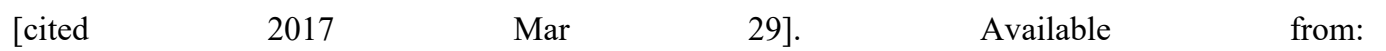

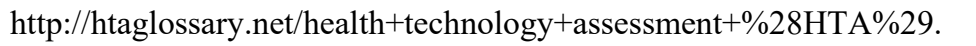

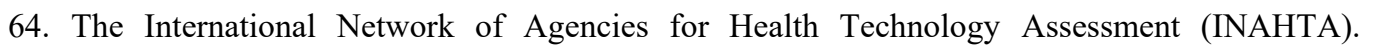

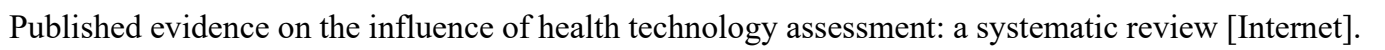

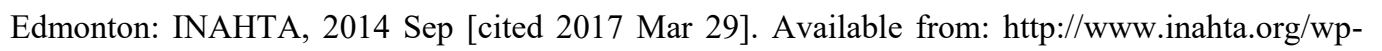

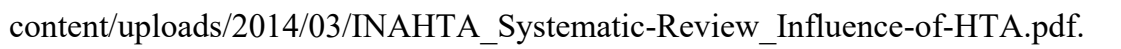

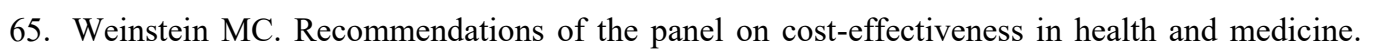

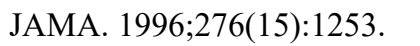




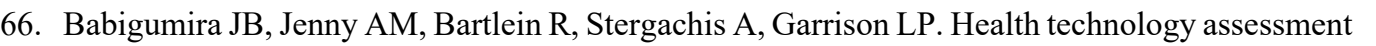

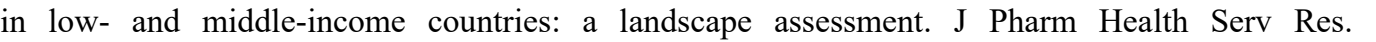

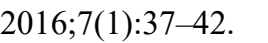

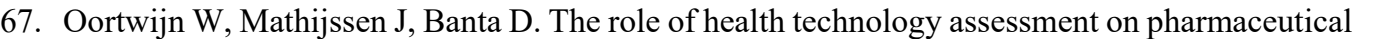

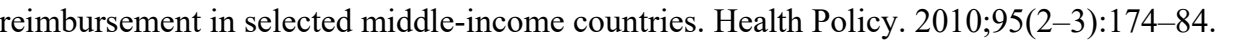

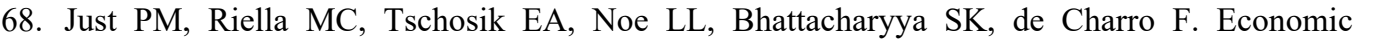

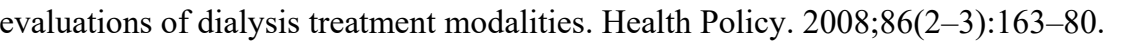

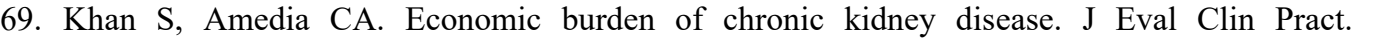

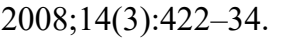

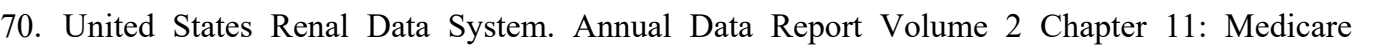

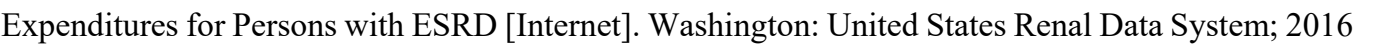

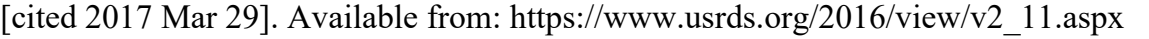

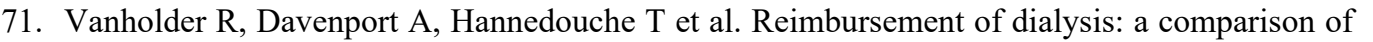

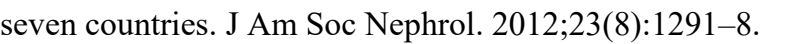

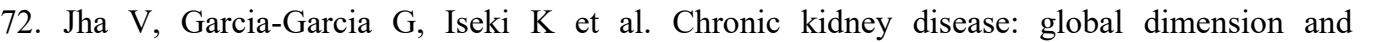

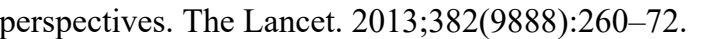

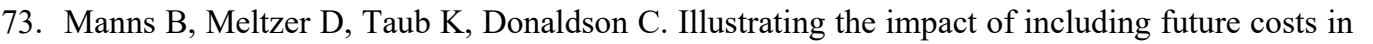

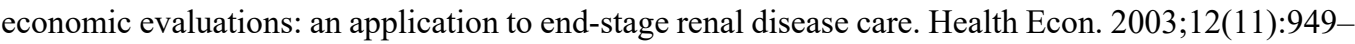
$\square 1 \mathrm{II}$

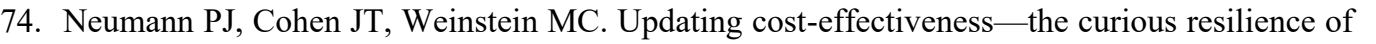

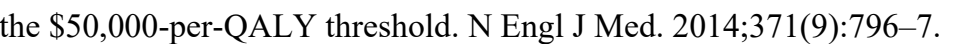

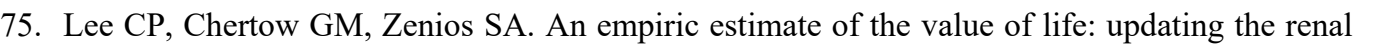

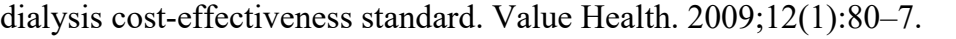

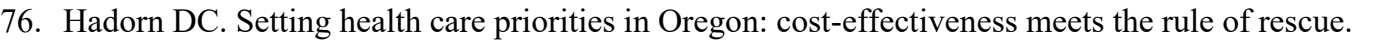

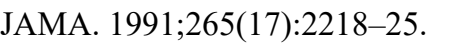

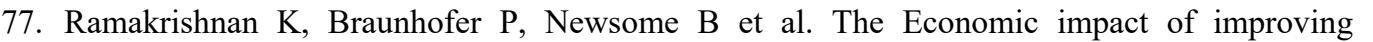

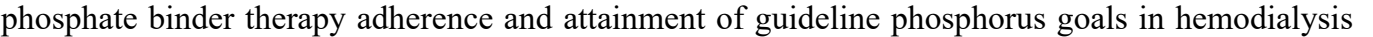

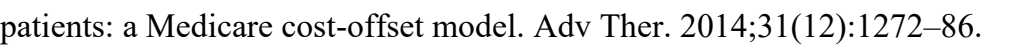

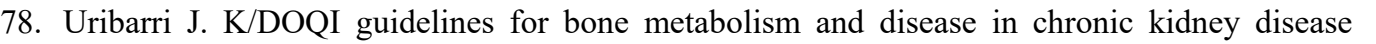

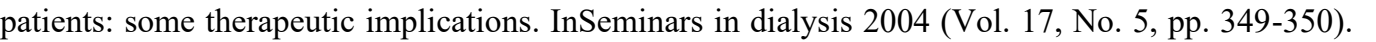

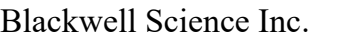

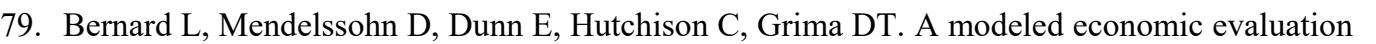

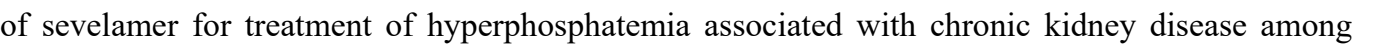

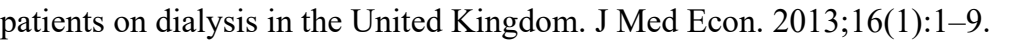

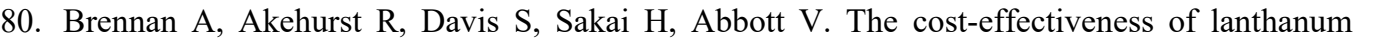

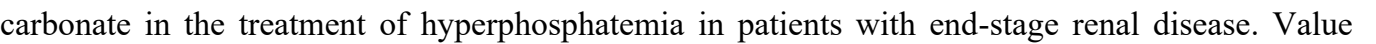

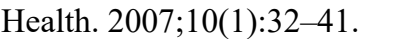

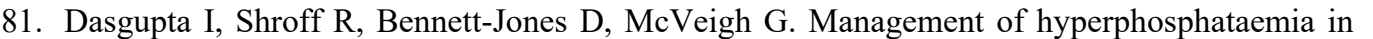

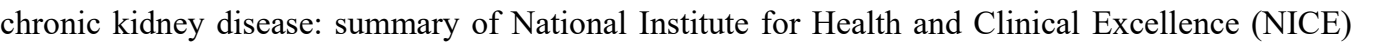

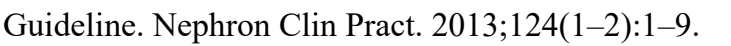




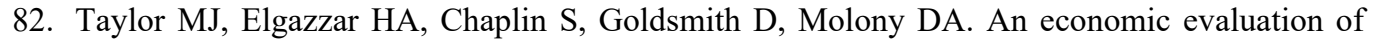

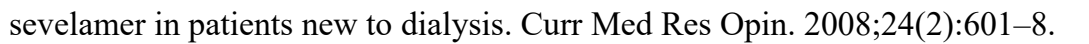

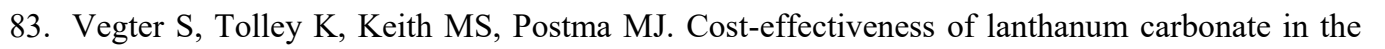

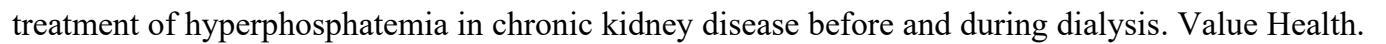

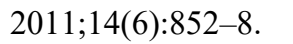

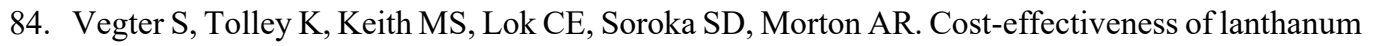

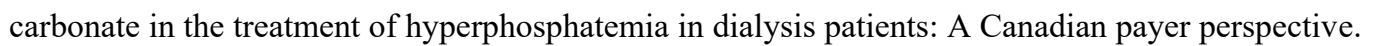

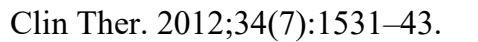

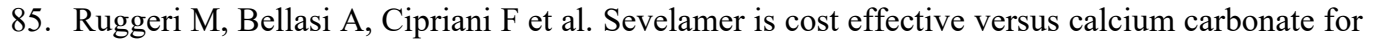

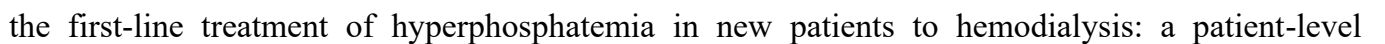

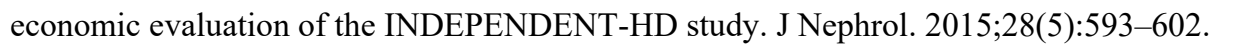

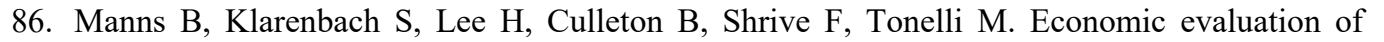

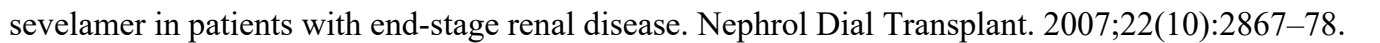

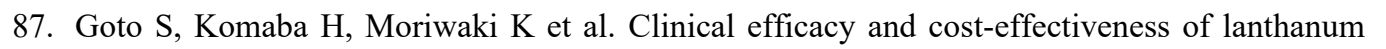

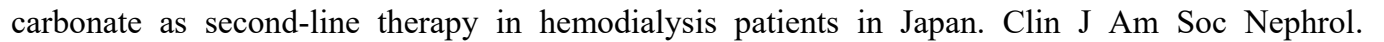

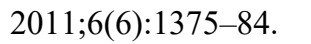

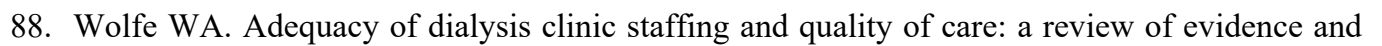

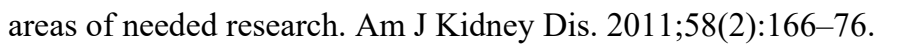

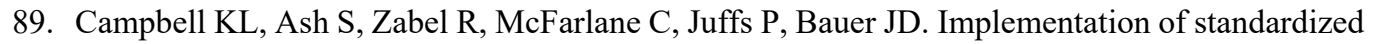

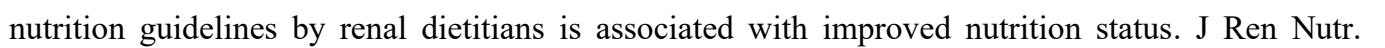
$\square|||||||||| \mid-\square+\square$

\section{$\square$ Cupisti A, D’Alessandro C, Baldi R, Barsotti G. Dietary habits and counseling focused on}

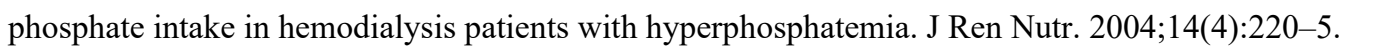

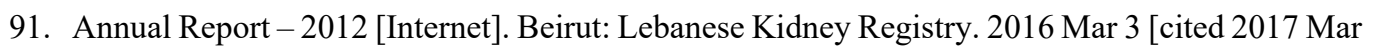

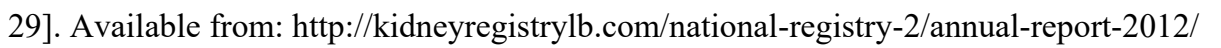

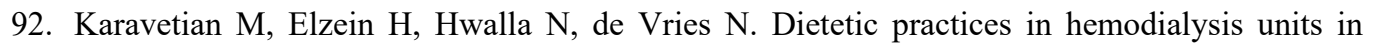
पा

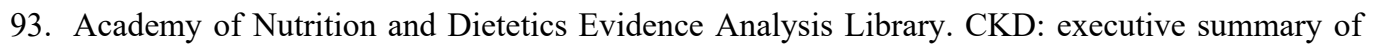

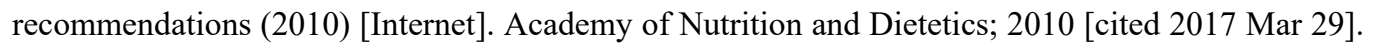

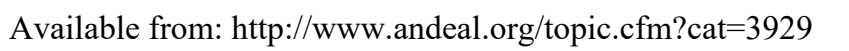

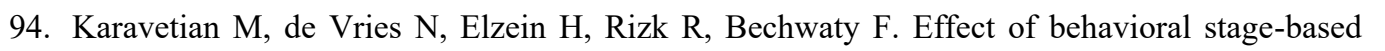

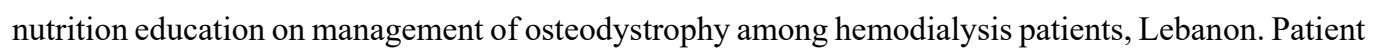

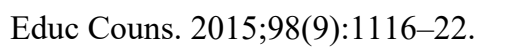

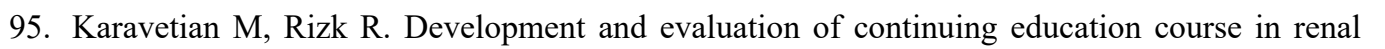

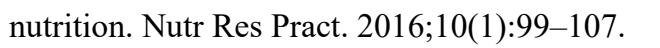

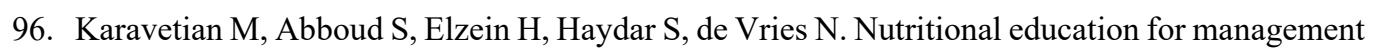

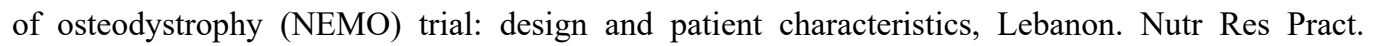

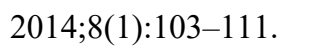




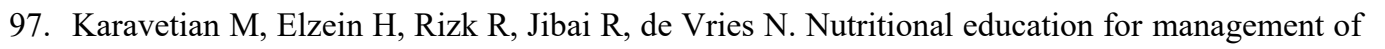

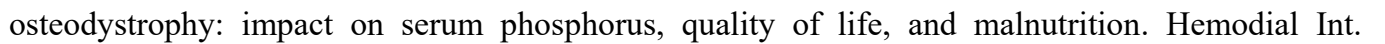

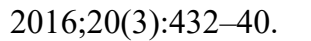



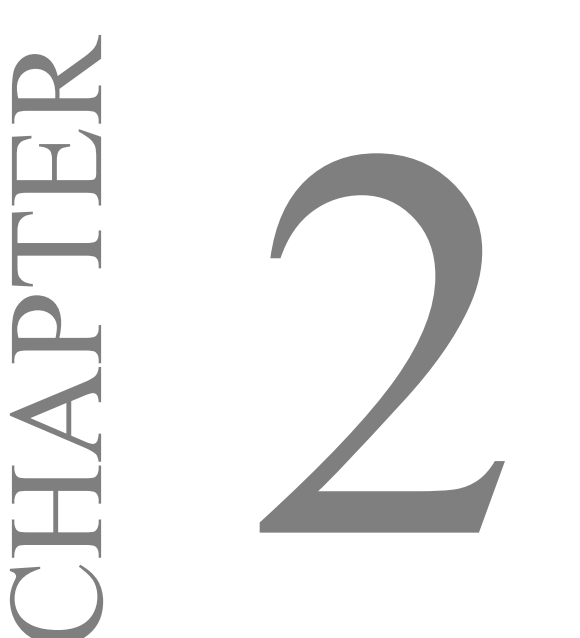

ECONOMIC EVALUATIONS OF INTERVENTIONS TO MANAGE HYPERPHOSPHATAEMIA IN ADULT HAEMODIALYSIS PATIENTS: A SYSTEMATIC REVIEW $\square$

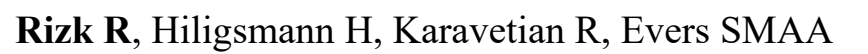




\section{Abstract}

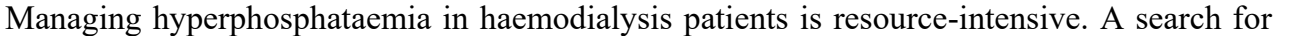

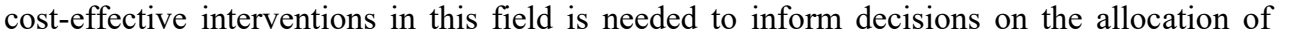

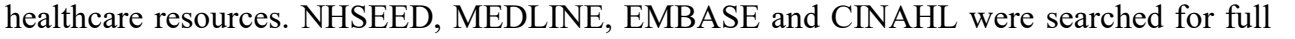

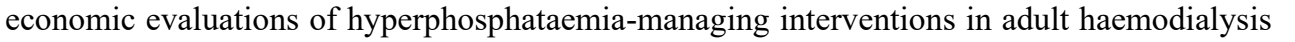

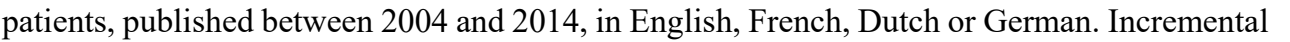

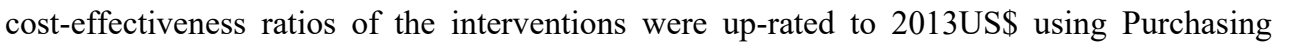

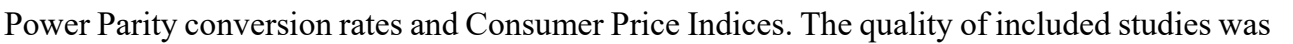

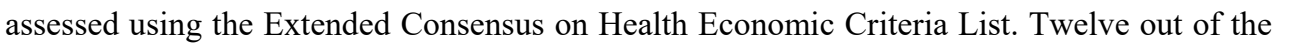

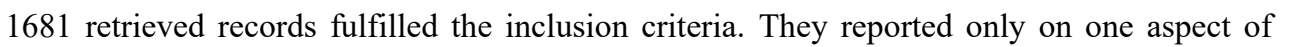

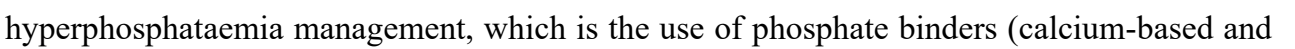

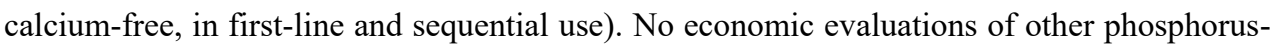

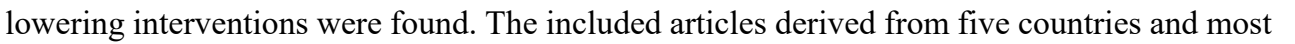

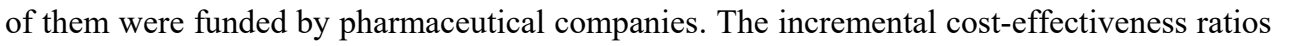

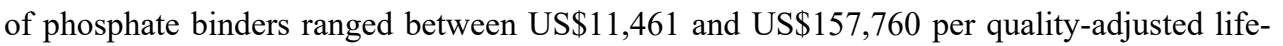

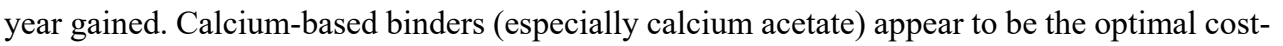

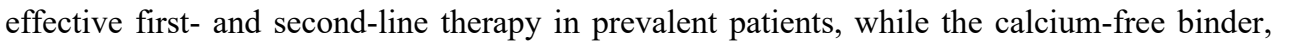

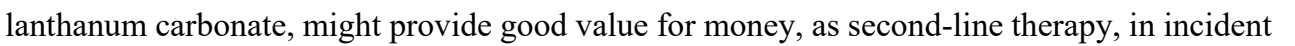

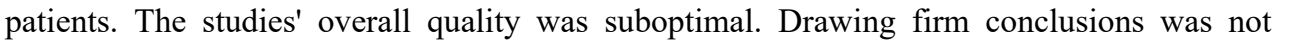

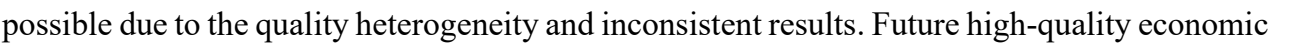

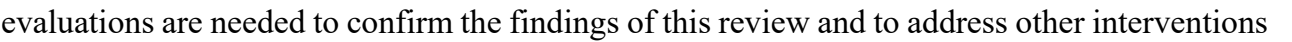

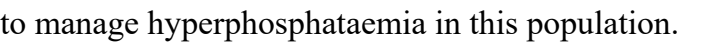

\section{KEYWORDS}

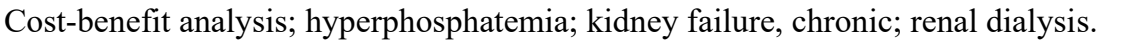
$\square$ 


\section{INTRODUCTION}

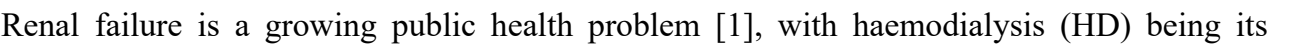

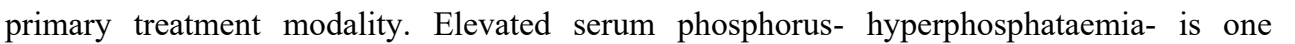

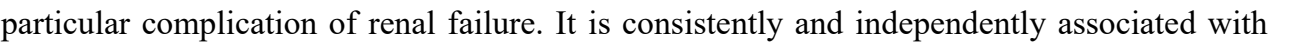

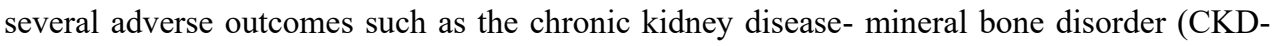

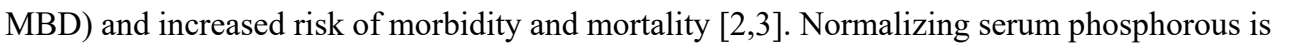

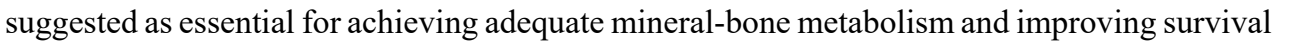

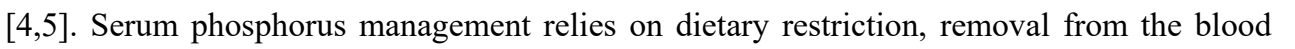

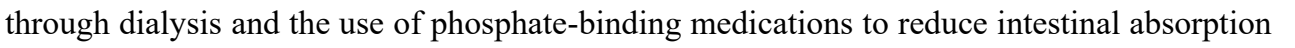

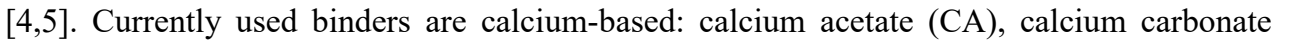

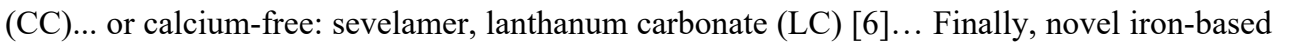

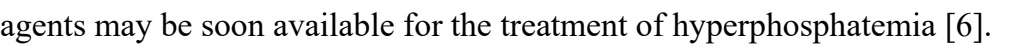

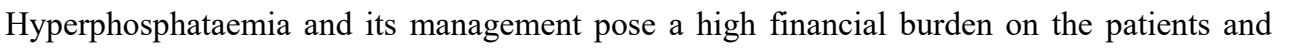

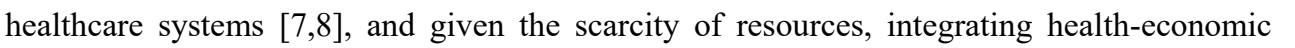

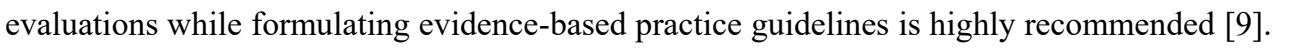

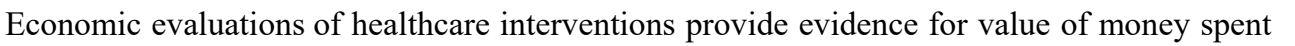

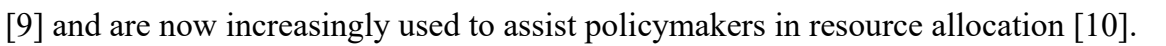

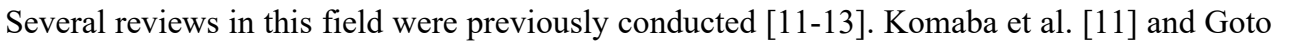

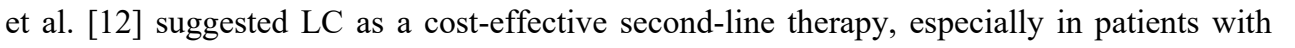

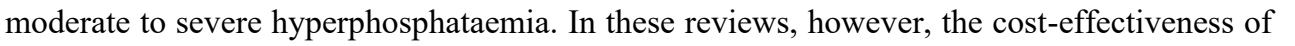

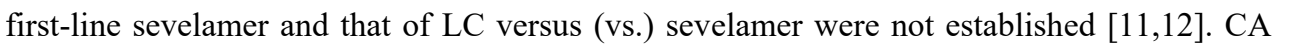

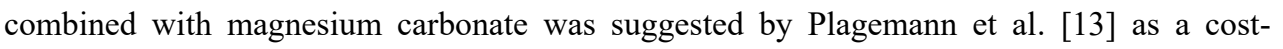

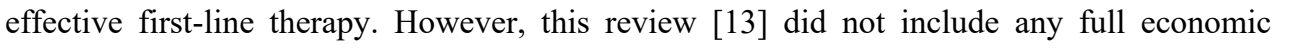

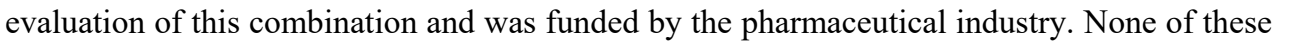

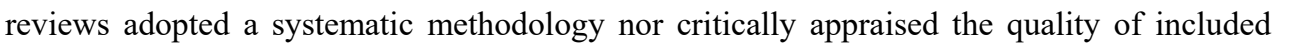

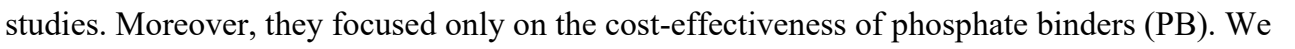

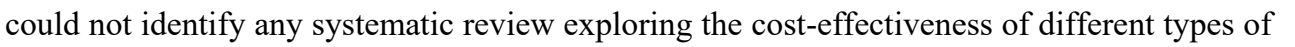

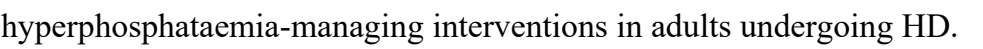

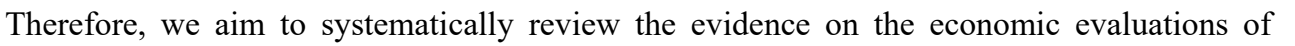

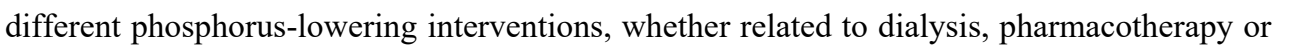

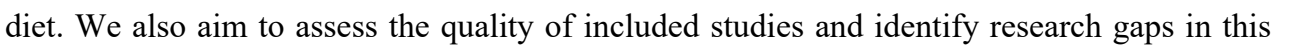

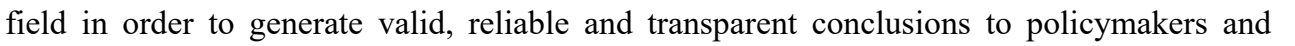

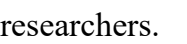

$\square$ 


\section{METHODS}

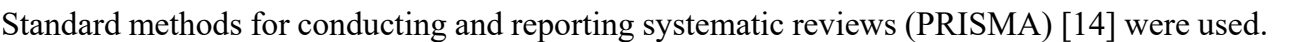

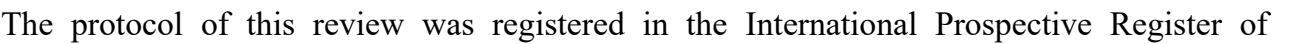

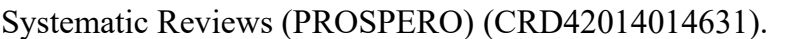

$\square$

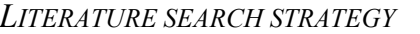

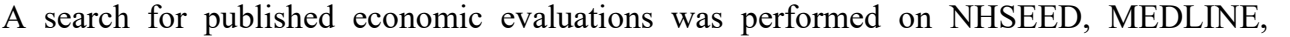

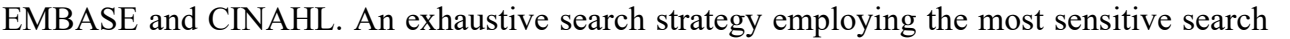

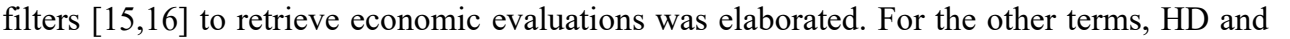

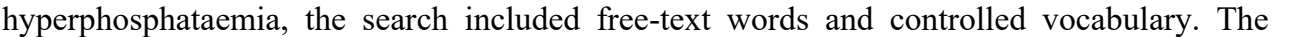

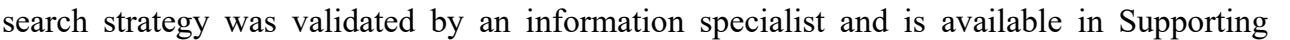
प

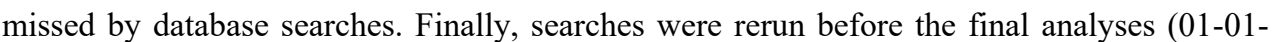
$\square|| \mid \|$

$\square$

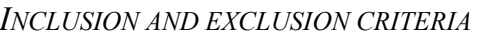

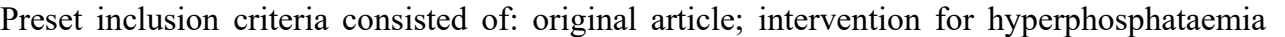

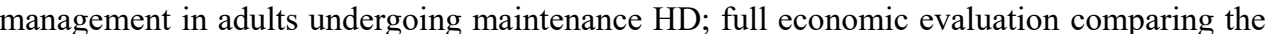

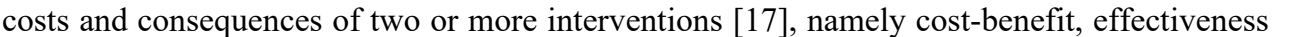

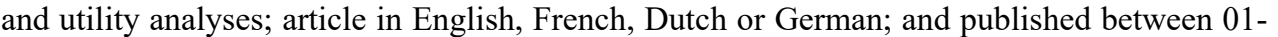

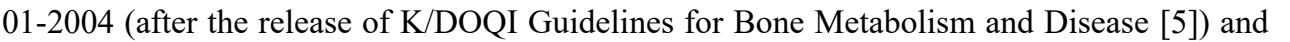

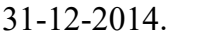

$\square$

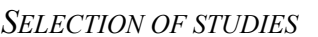

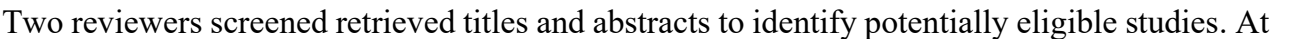

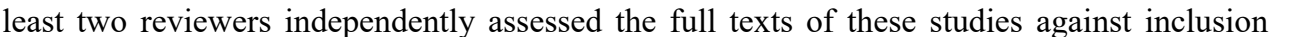

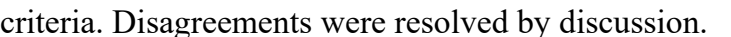

$\square$

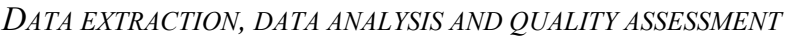

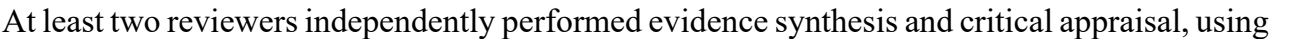

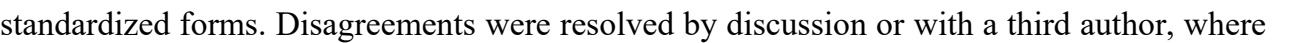

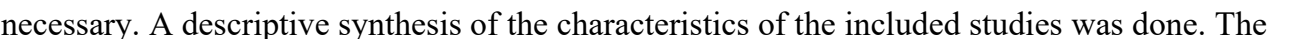




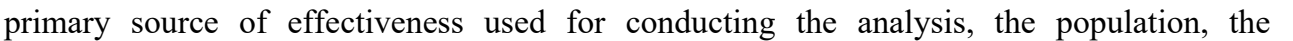

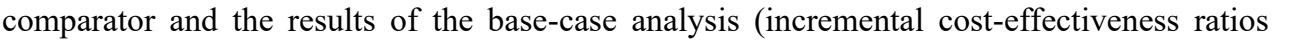

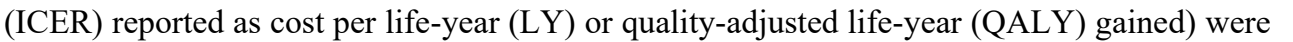

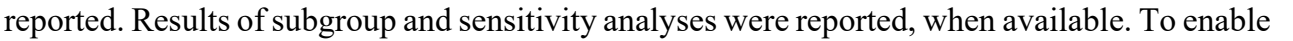

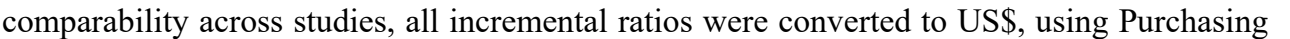

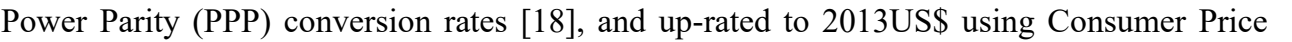

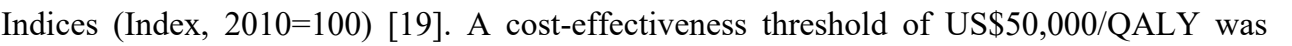

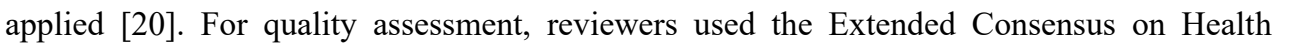

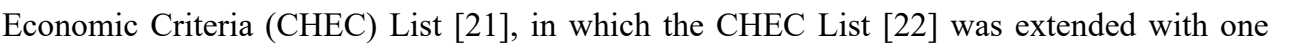

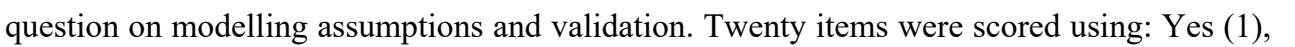

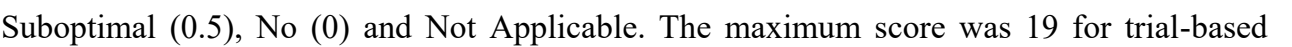

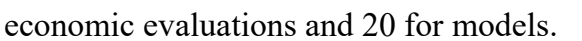

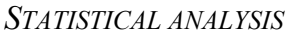

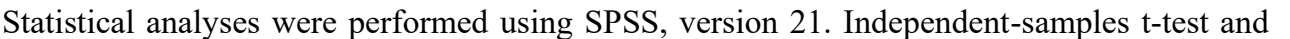
Fisher's exact test were employed to investigate the associations between the studies'

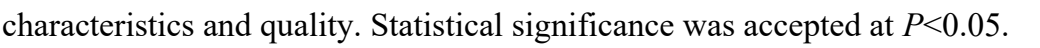

\section{RESULTS}

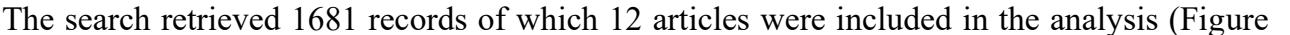
$\square \square$

\section{$\square$}




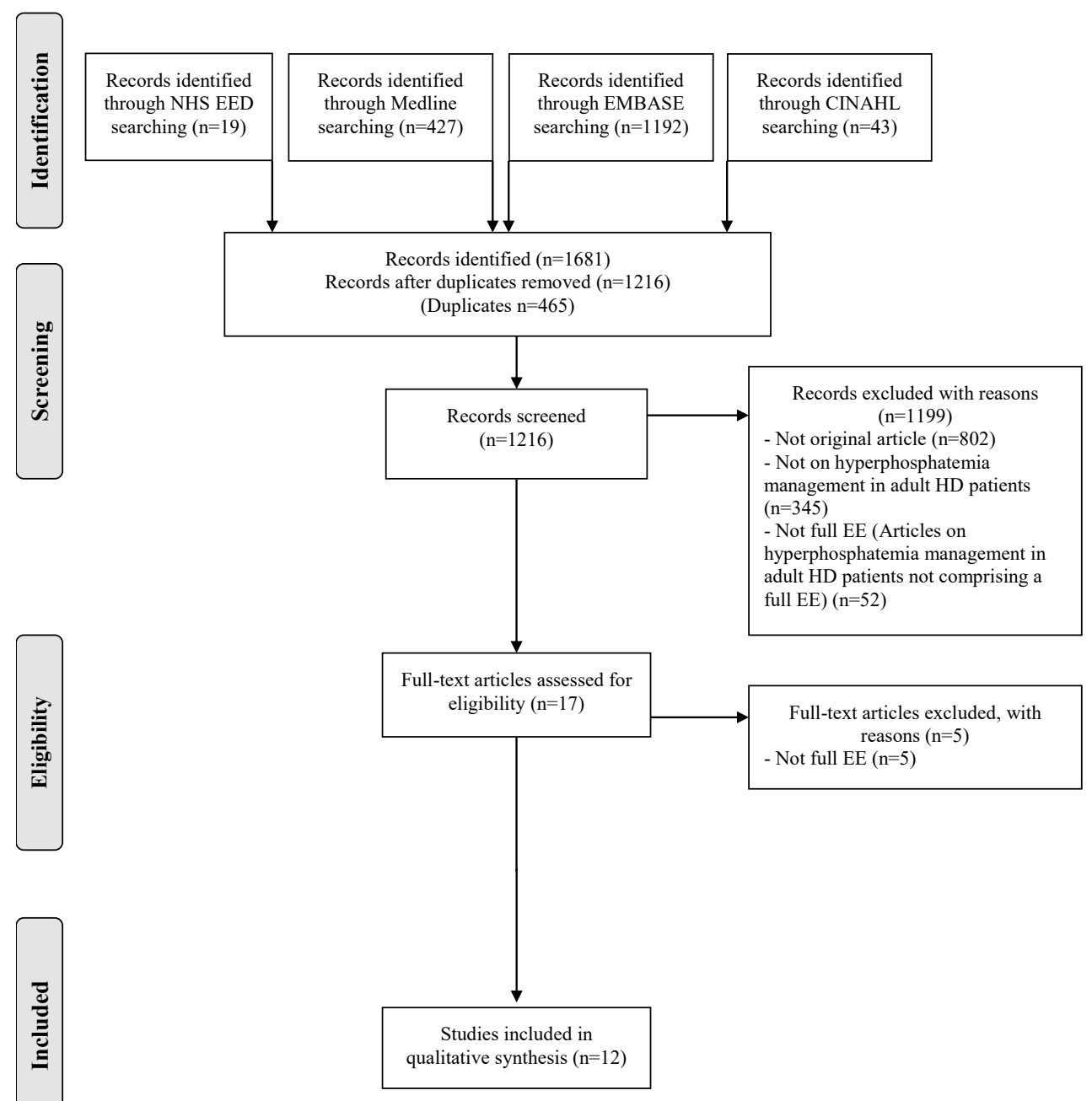

Figure 1.

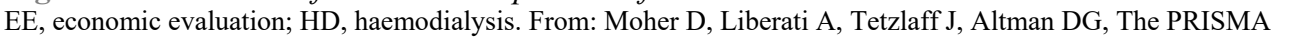

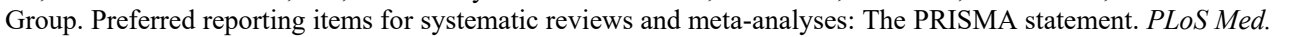

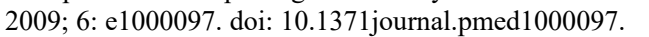

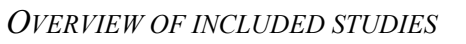

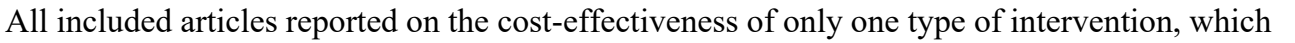

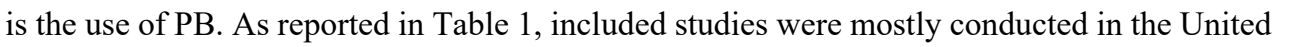

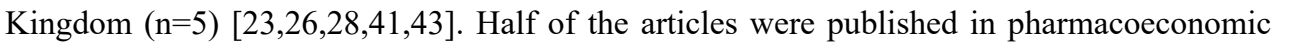

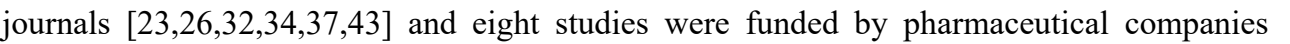

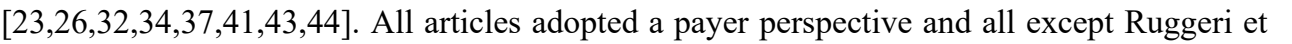




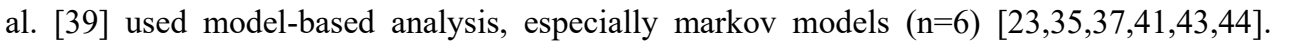

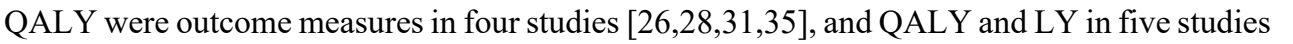

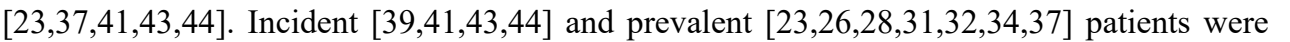

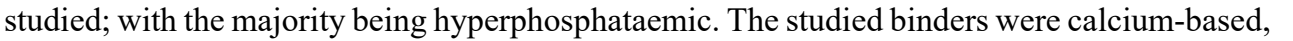

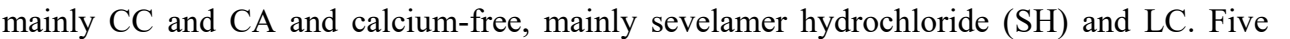

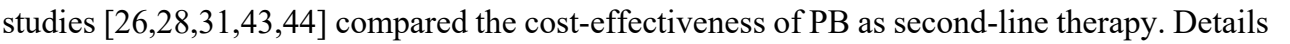

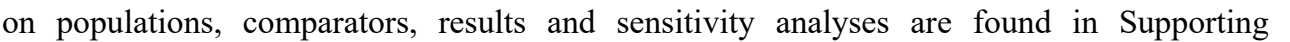

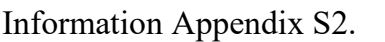

$\square$

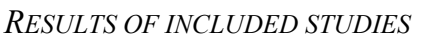

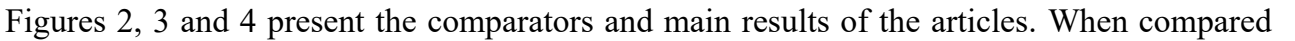

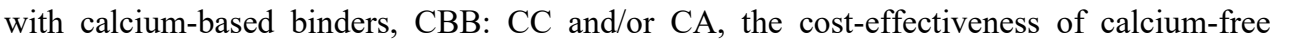

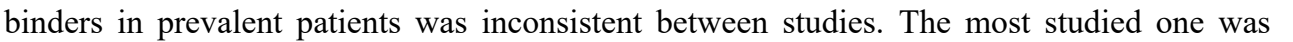

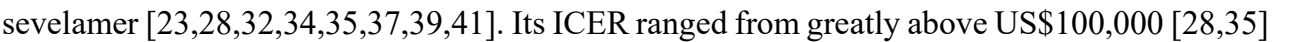

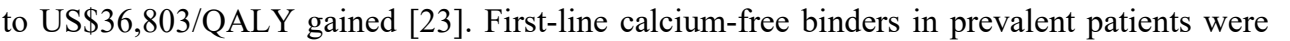

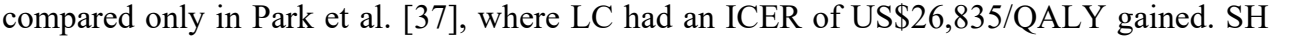

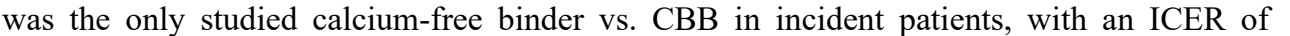

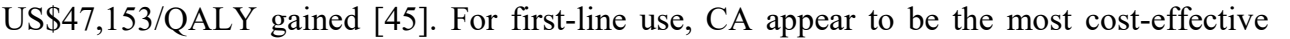
ए ए।

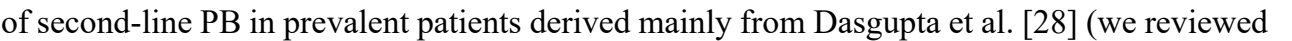

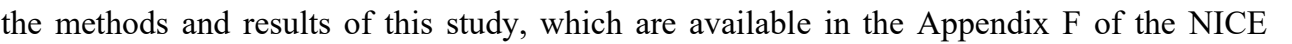

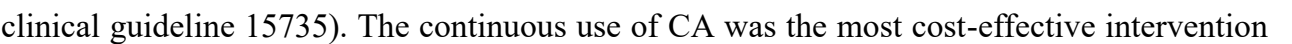

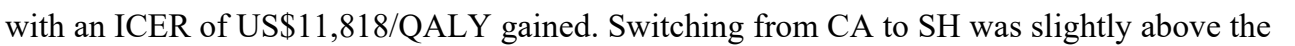

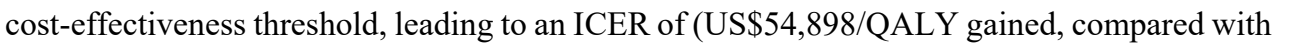

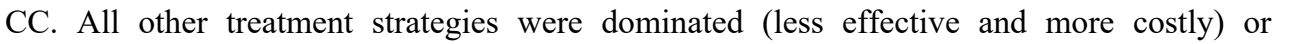

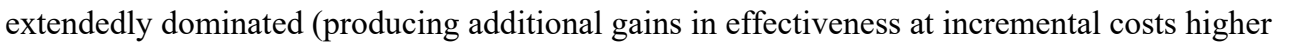
ए

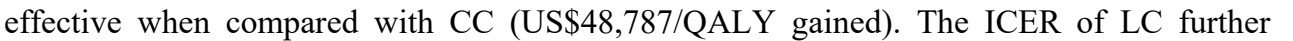

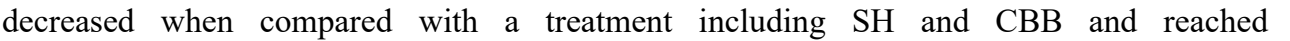

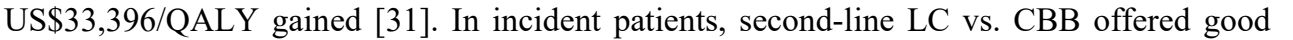

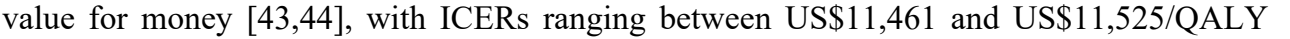
$\square[110$ 


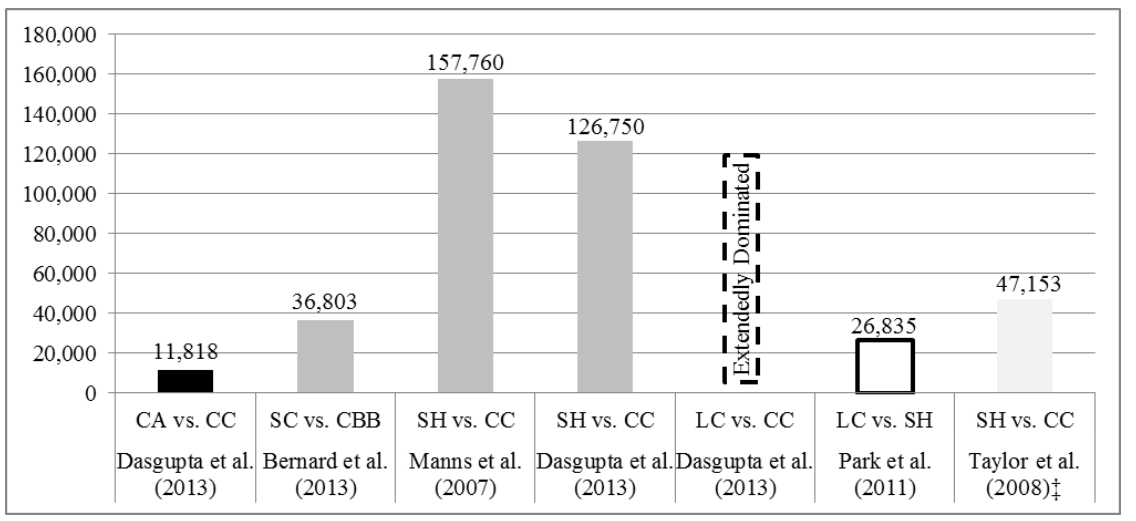

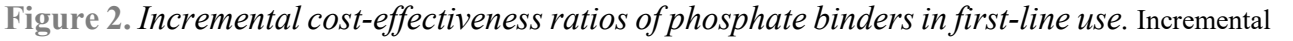

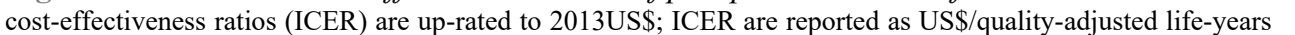

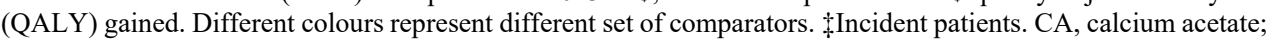

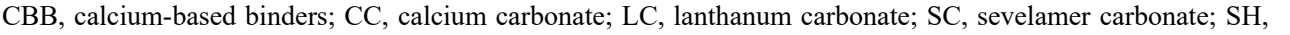

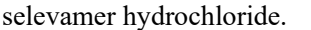

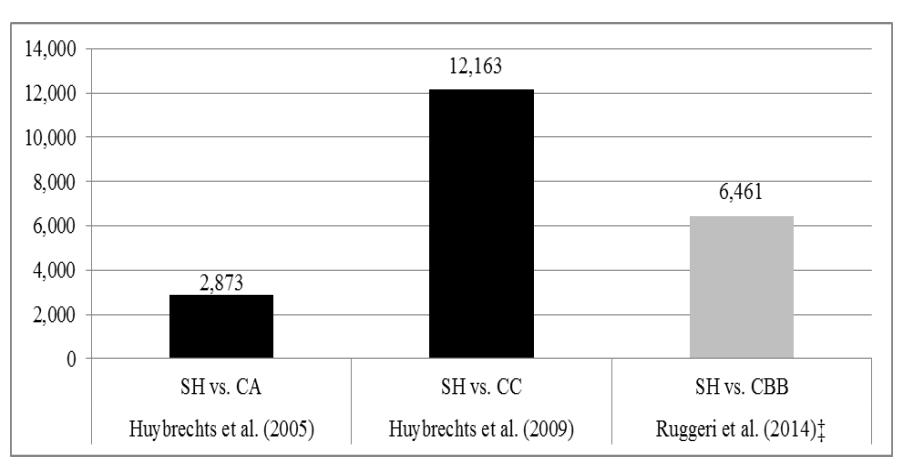

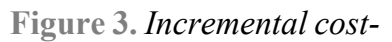

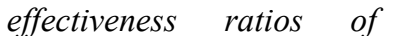

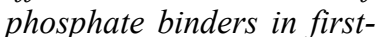
पाणाणाणाए

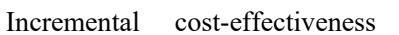

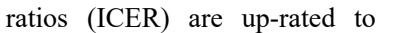

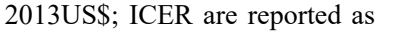

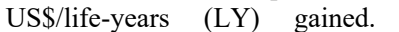

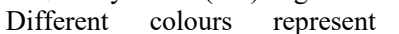

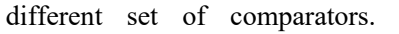
$\$$ Incident patients. CA, calcium

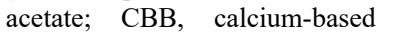

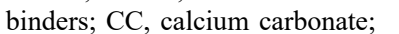

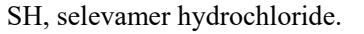

$\square$

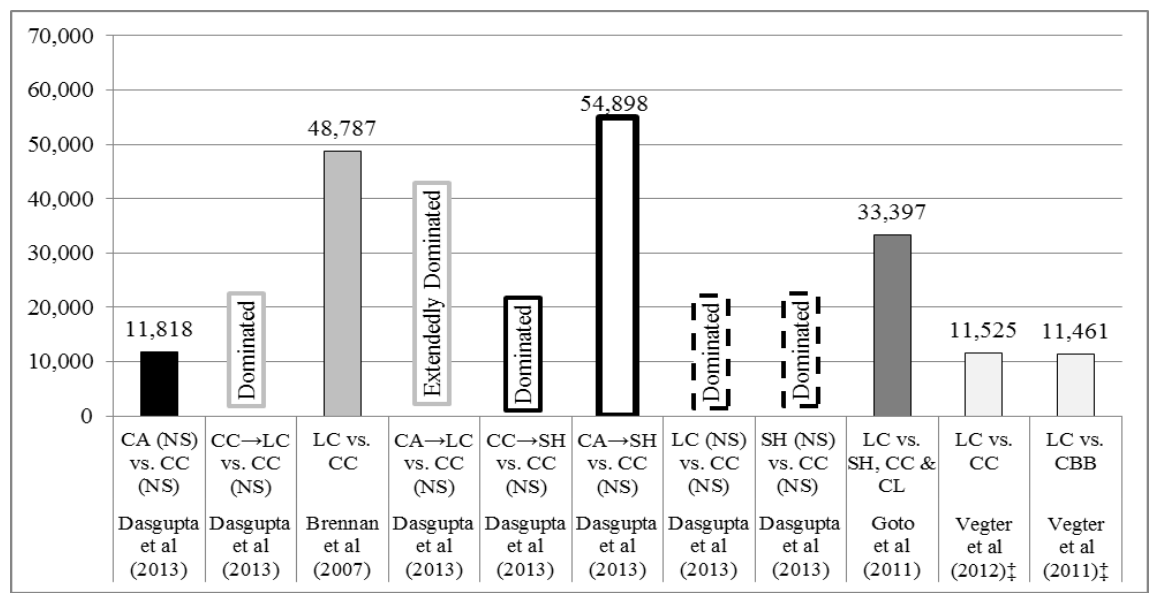

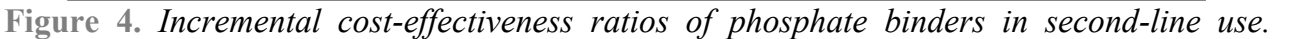

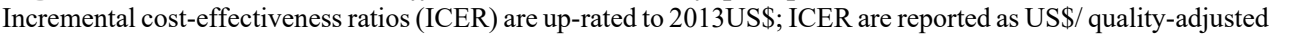

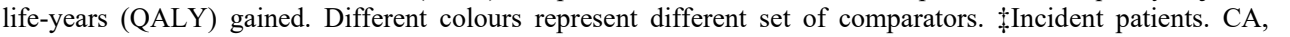

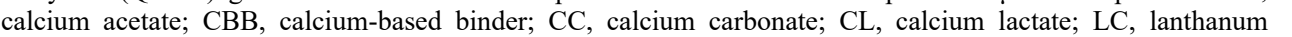

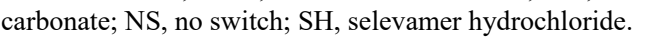




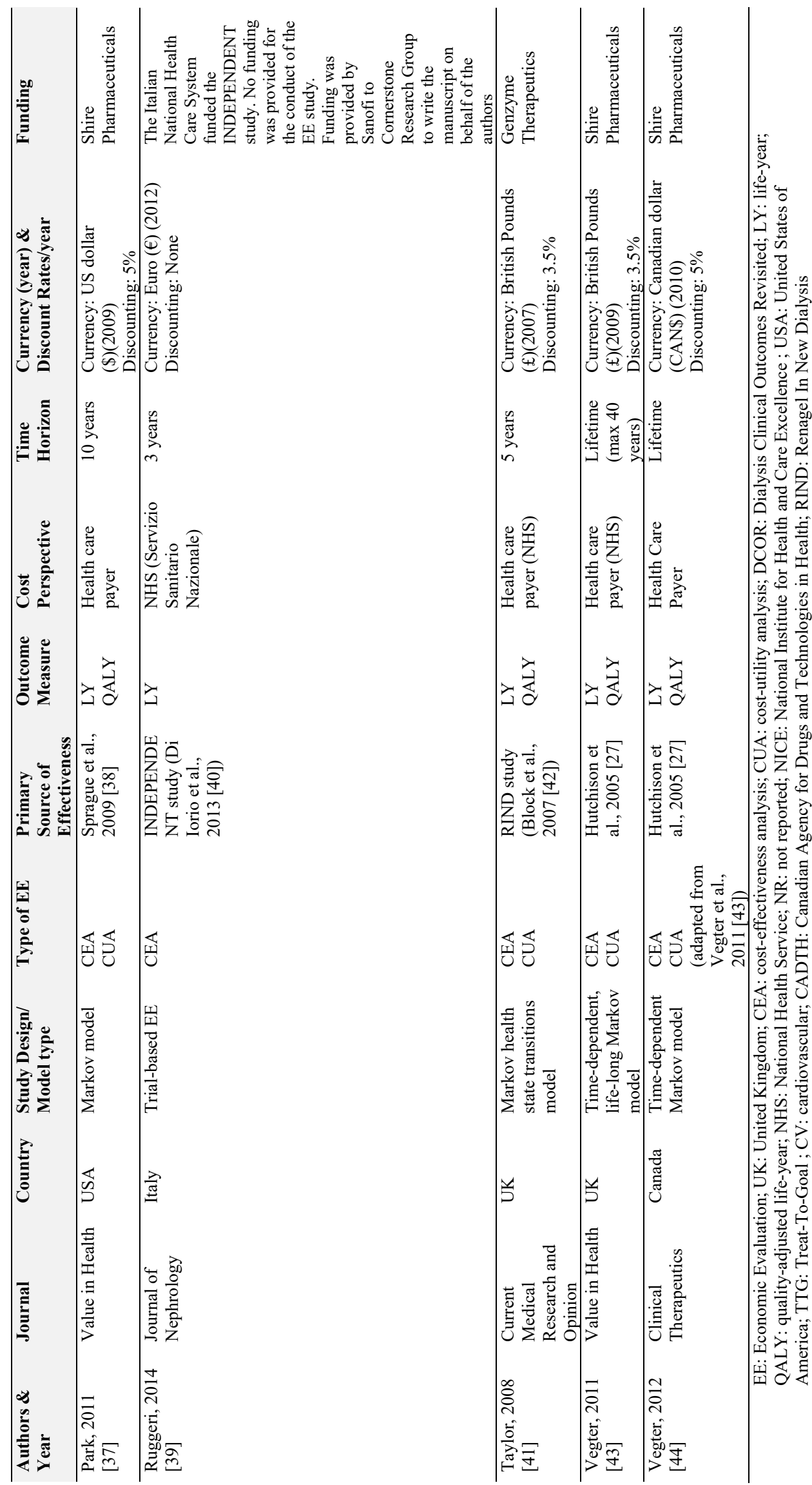




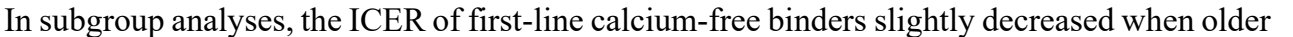

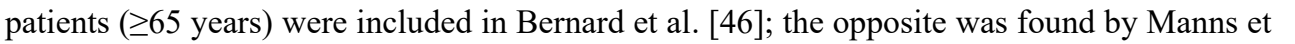

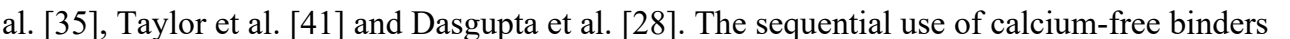

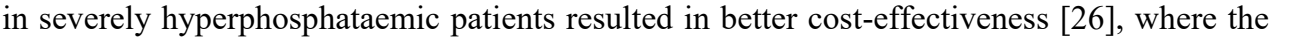

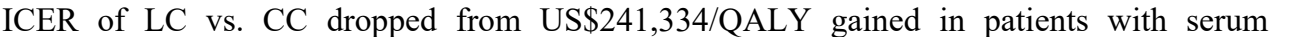

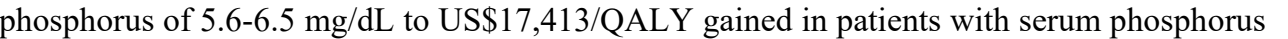
पामाणापणमाप

ए

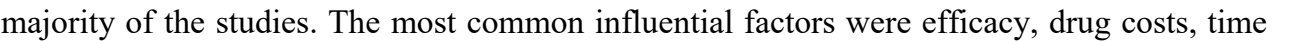

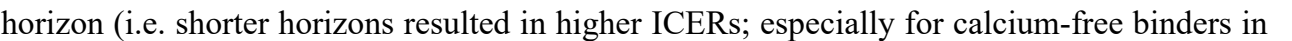

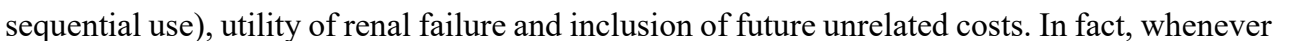
एणा

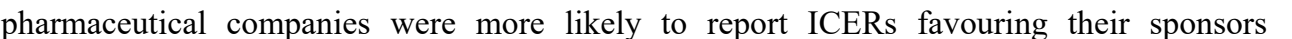

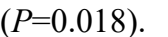

$\square$

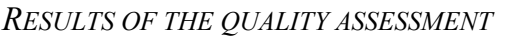

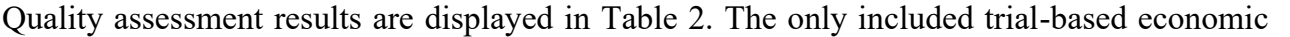

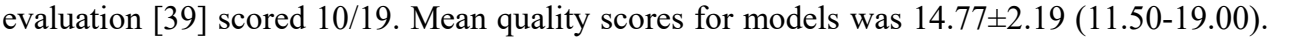

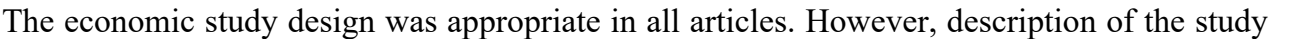

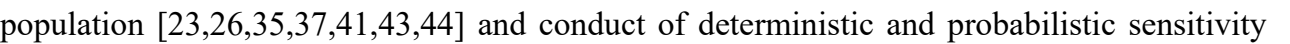

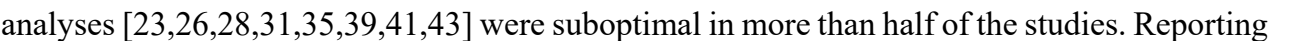

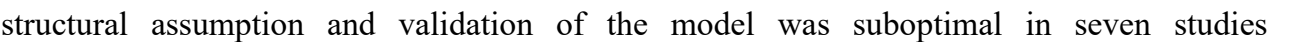

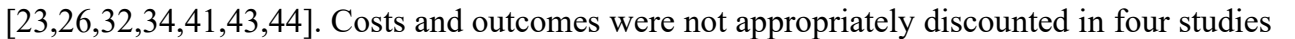

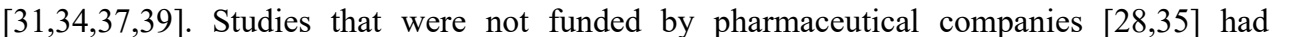

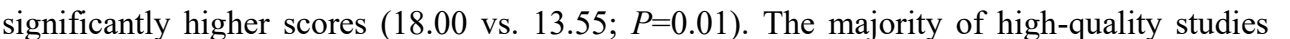

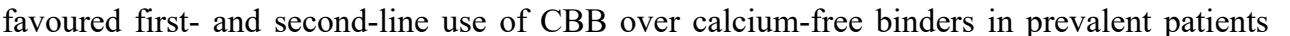

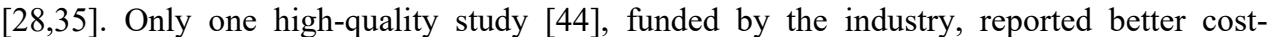

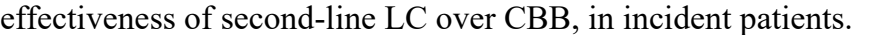

$\square$

$\square$

$\square$ 


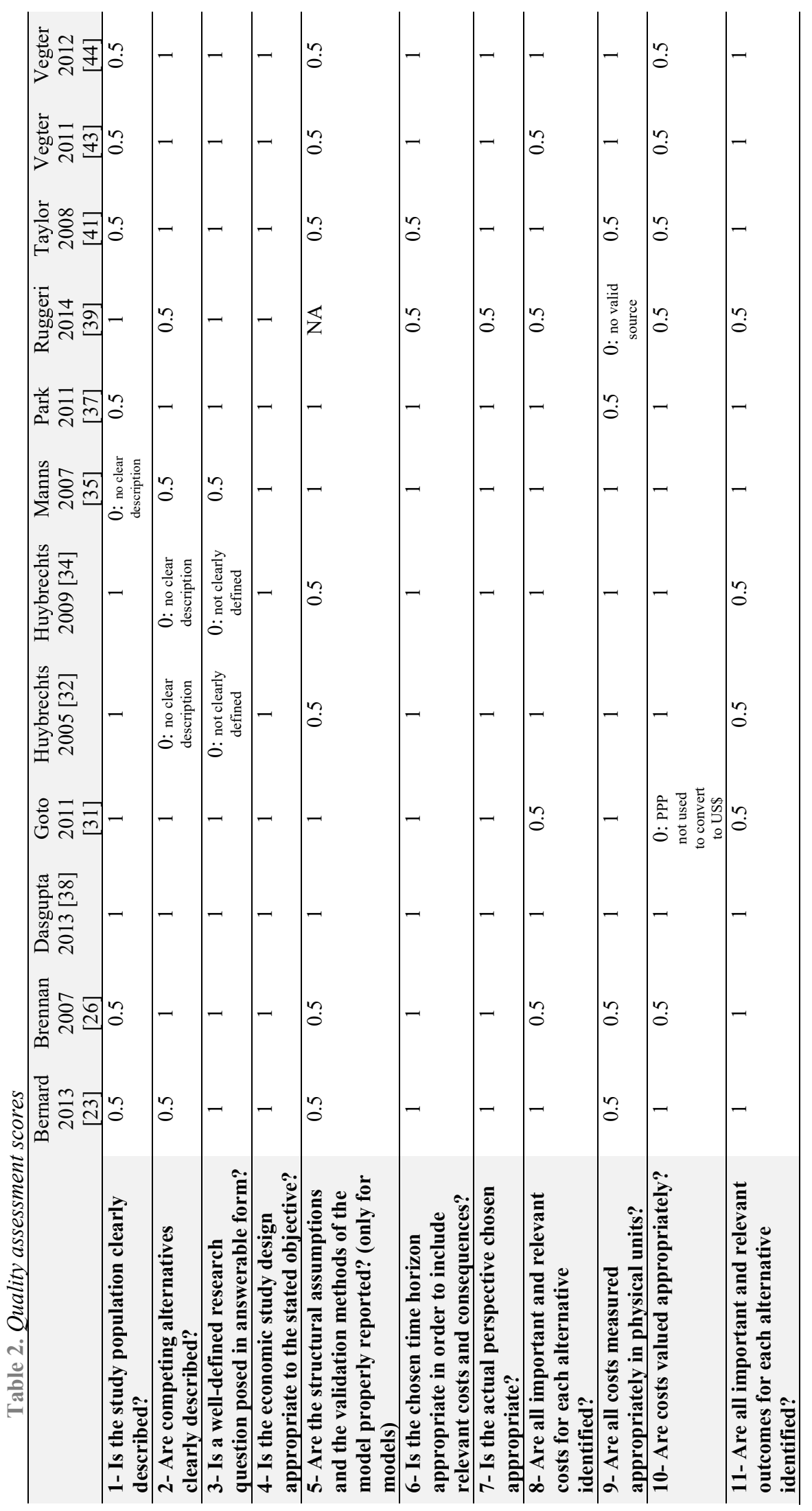




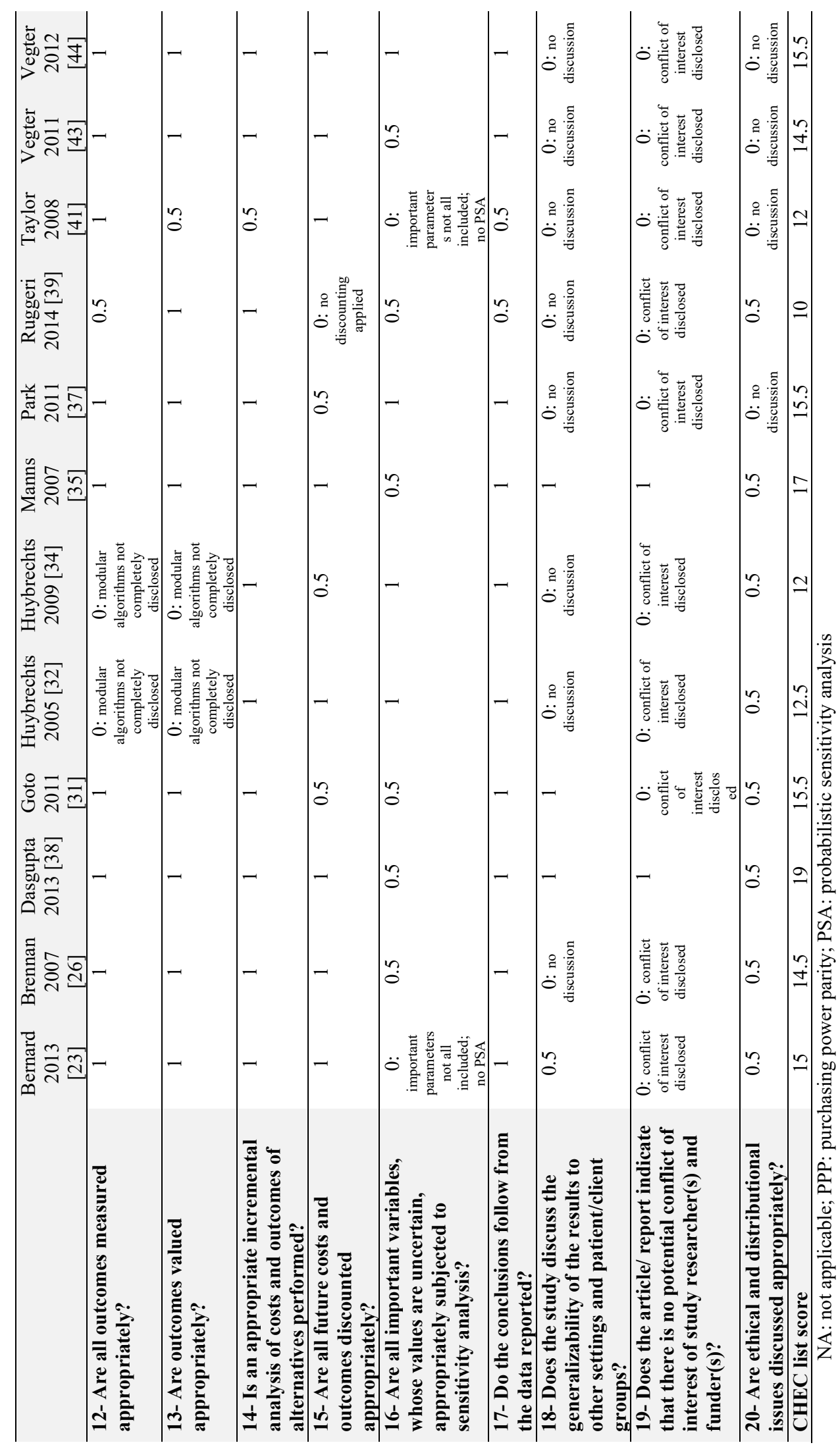




\section{DISCUSSION}

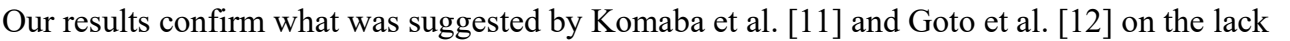

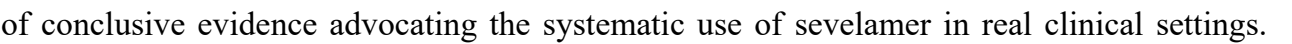

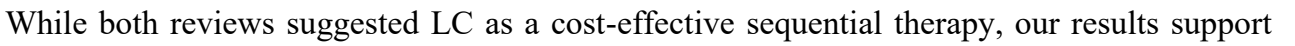

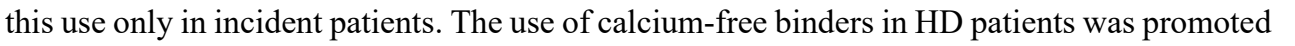

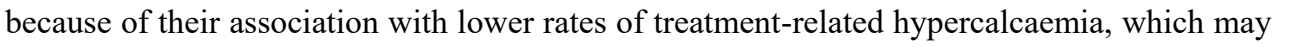

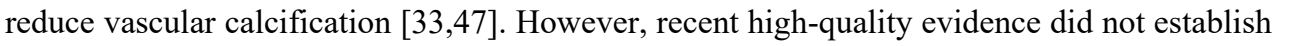

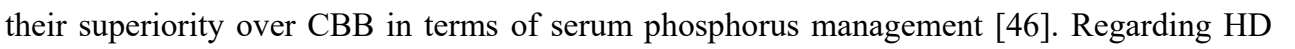

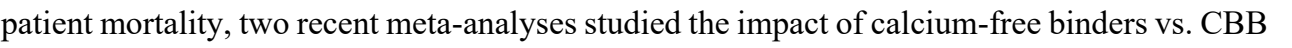

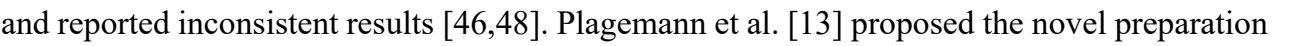

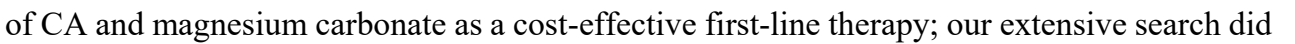

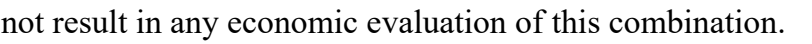

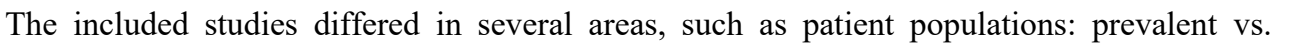

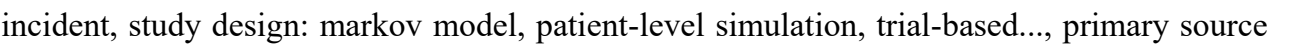

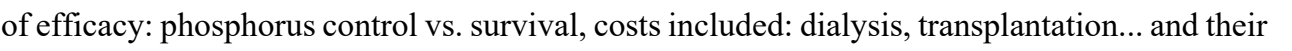

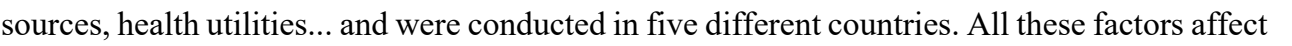

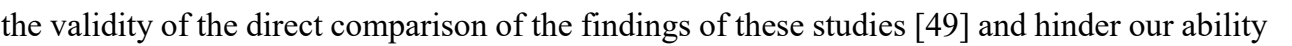

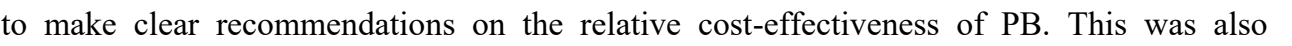

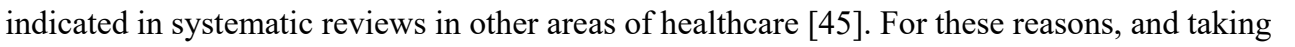

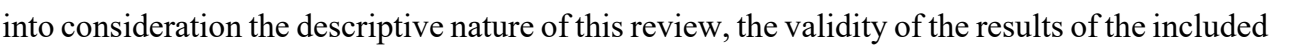

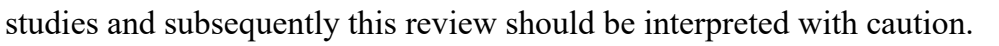

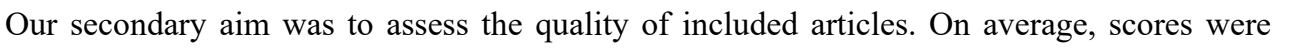

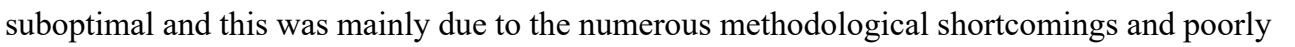

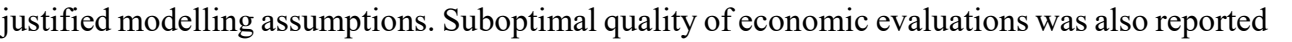

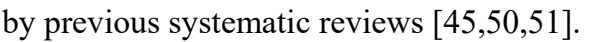

$\square$

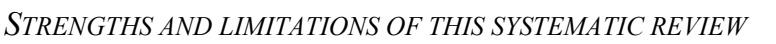

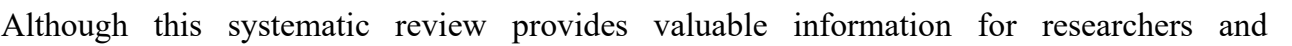

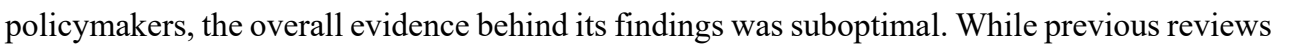

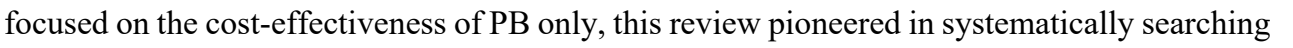

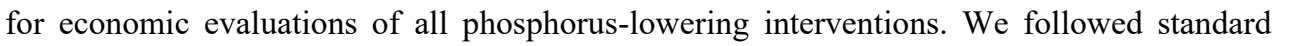

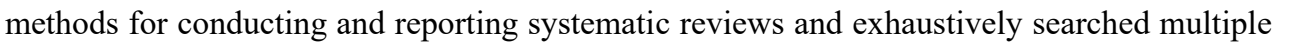

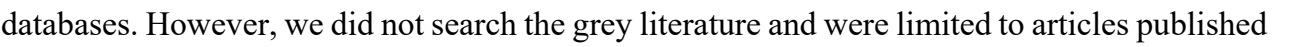




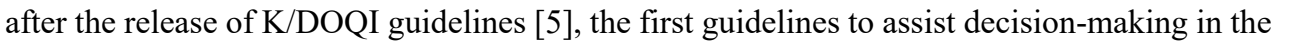

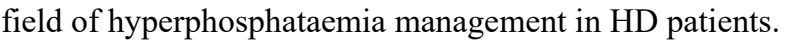

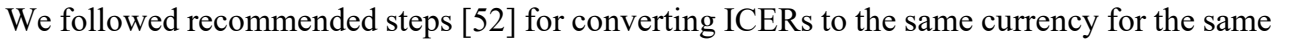

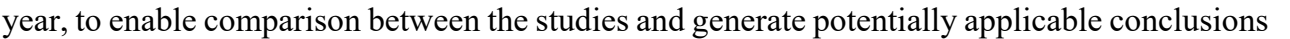

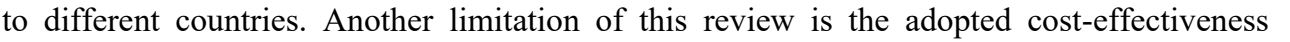

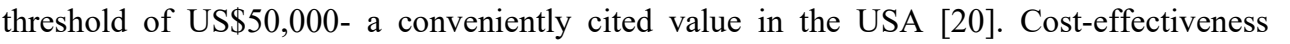

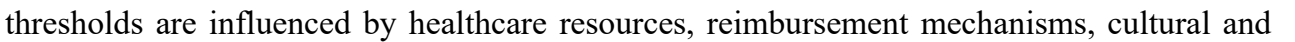

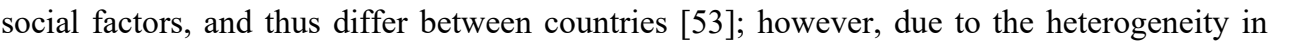

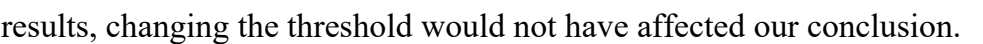

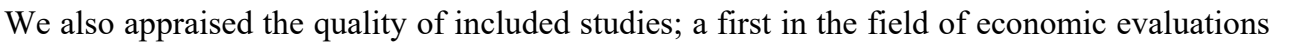

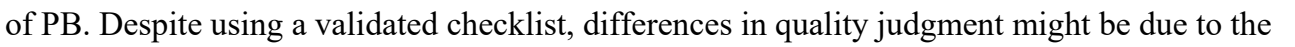

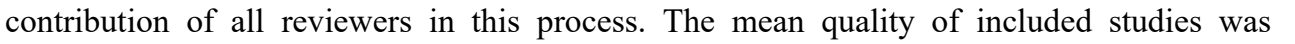

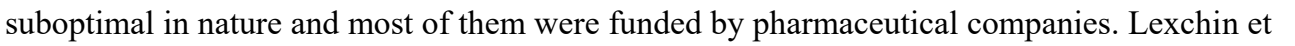

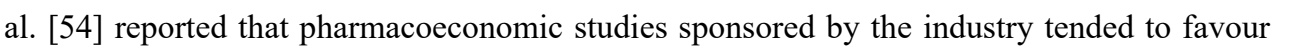

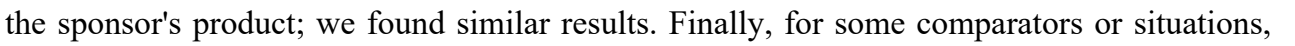

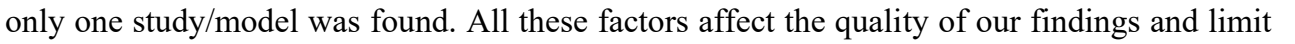

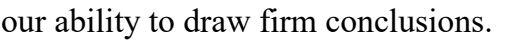

$\square$

पा

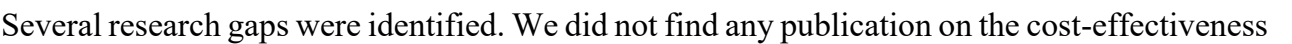

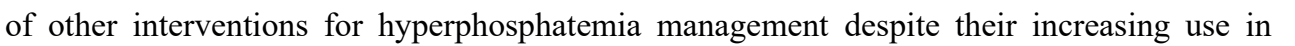

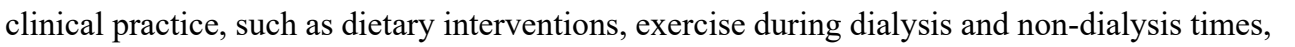

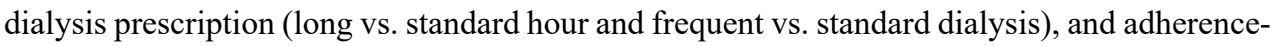

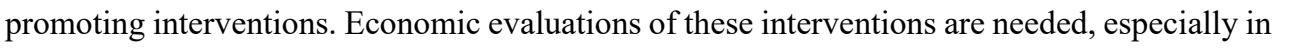

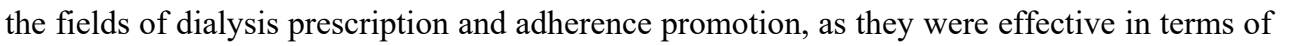

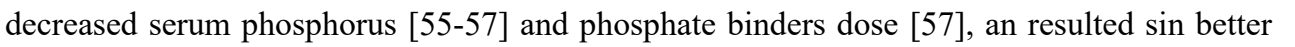

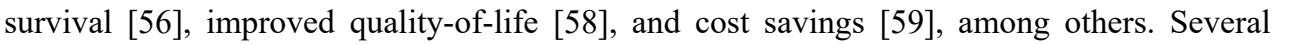

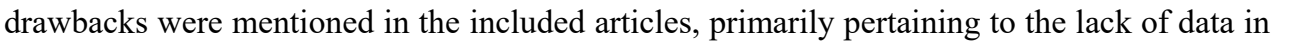
ए

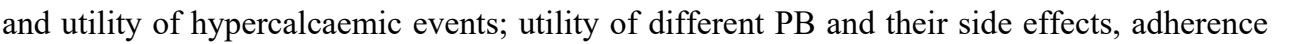
rates with different binders and their effects on outcomes and costs... Also, we could not

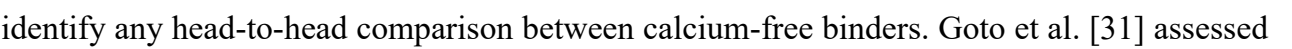

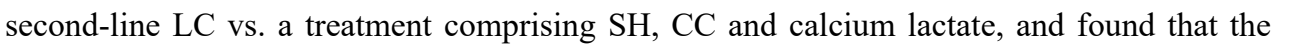

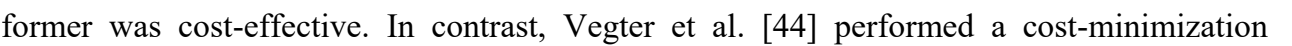




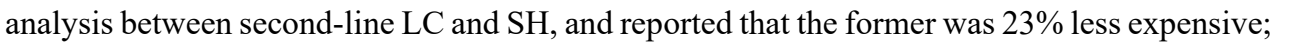

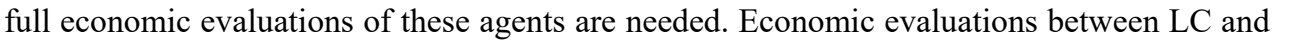

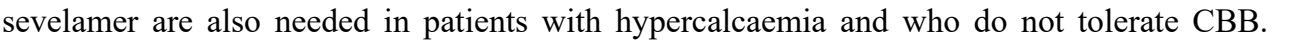

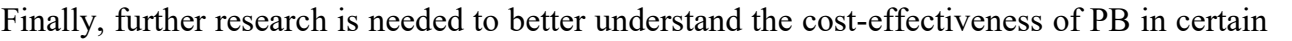

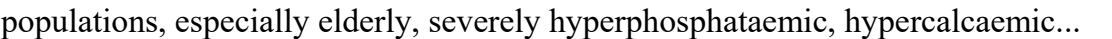

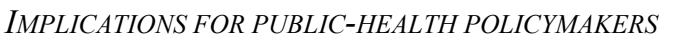

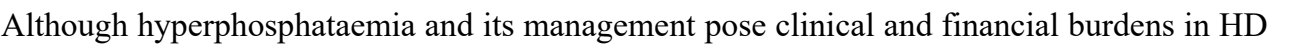

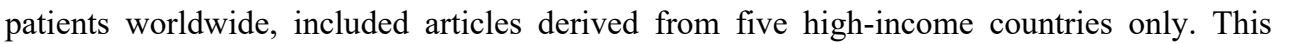

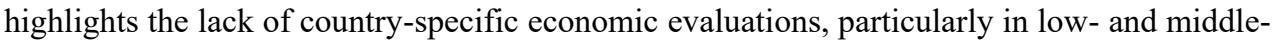

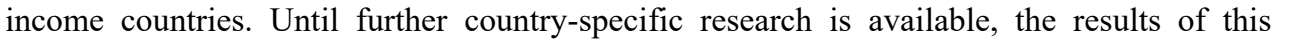

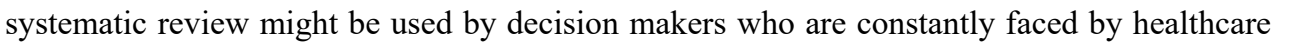

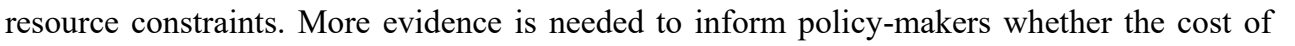
पाए

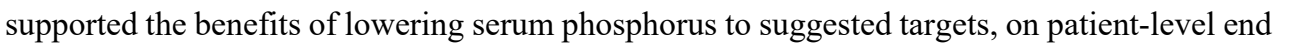

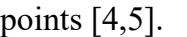

$\square$

\section{CONCLUSION}

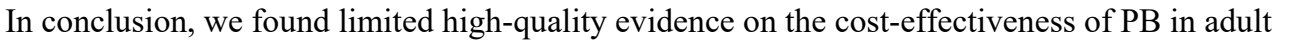

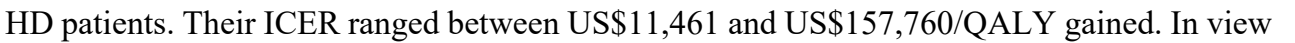

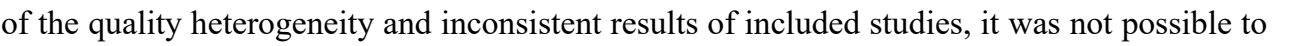

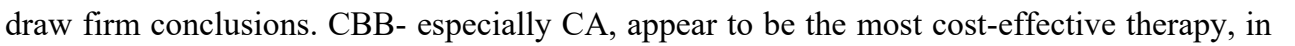

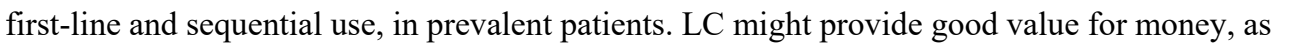

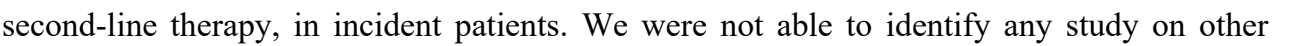

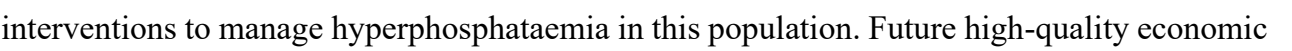

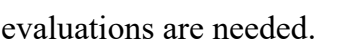

\section{Acknowledgements}

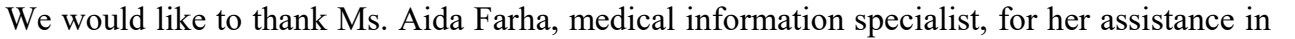

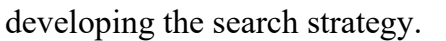

$\square$ 


\section{REFERENCES}

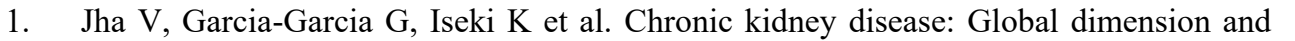

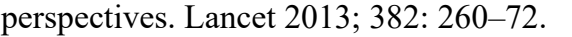

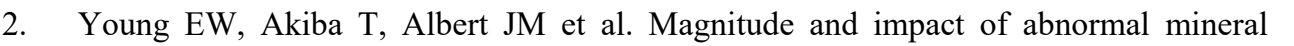

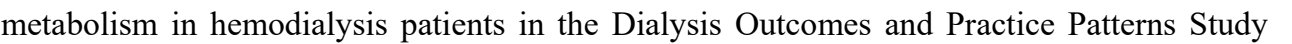

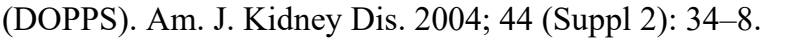

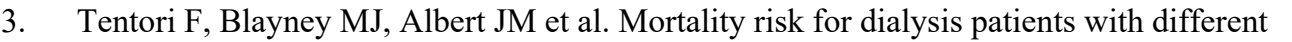

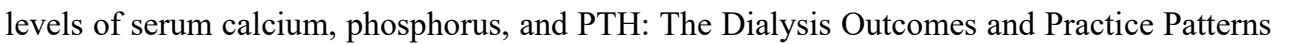

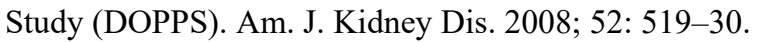

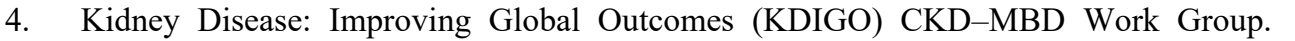

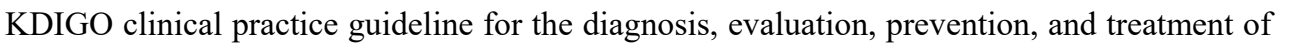

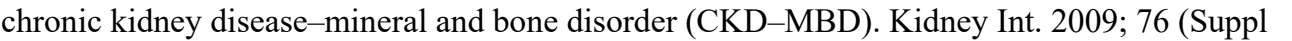
$\square 1 \| \square-\square 1 \square$

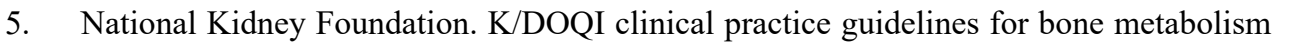

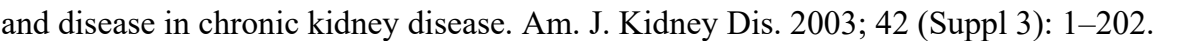

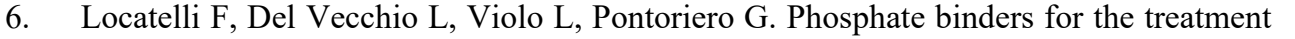

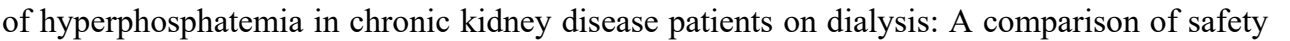

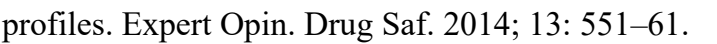

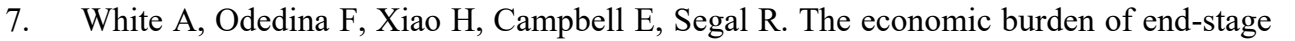

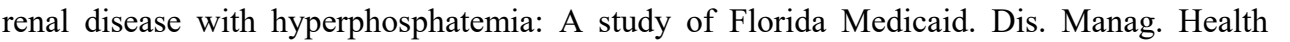

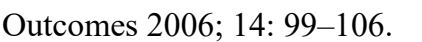

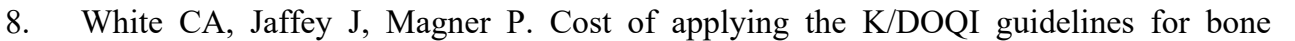

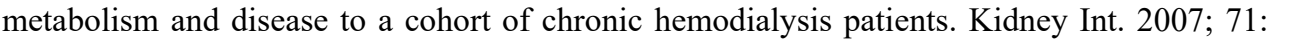
$\square+\square$

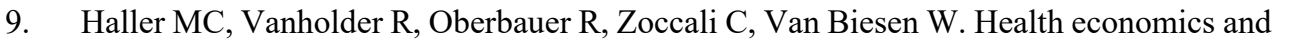

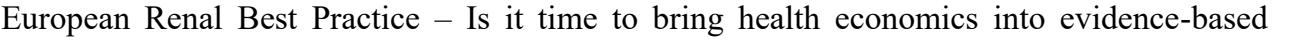

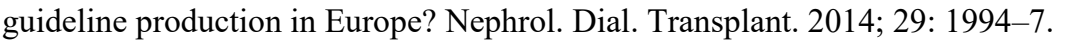

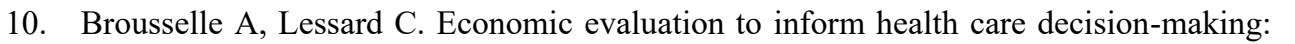

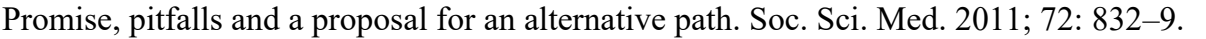

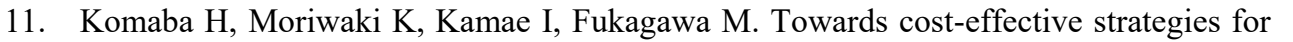

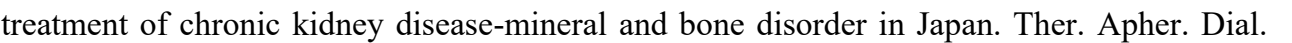

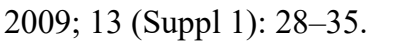

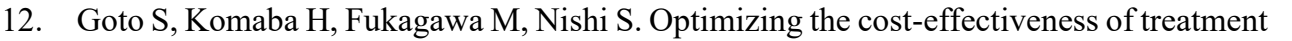

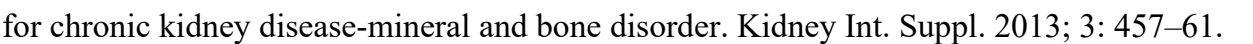




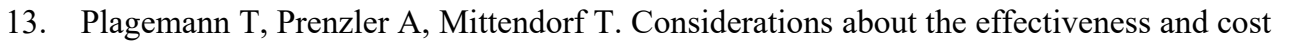

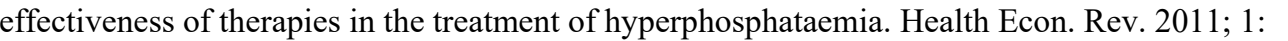
$\square \square$

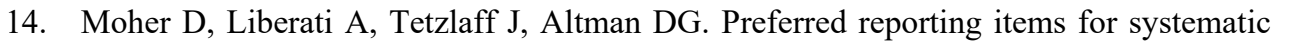

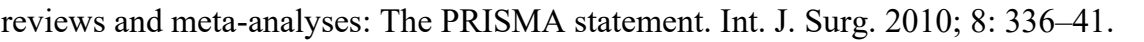

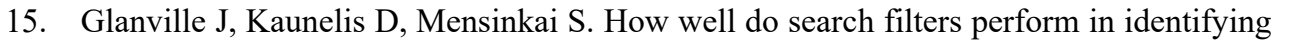

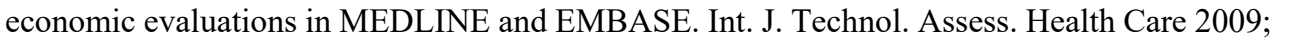
$\square \mid \Pi 1-\square \square$

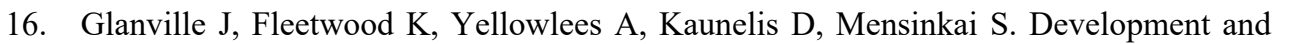

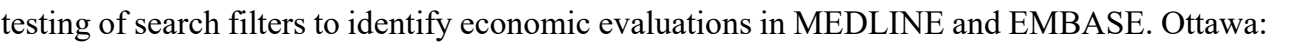

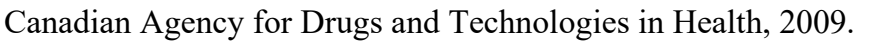

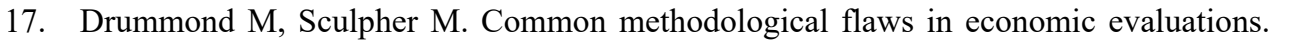

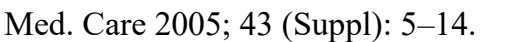

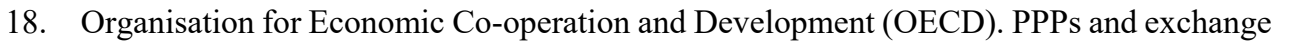

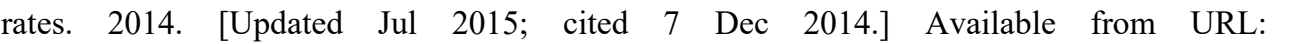

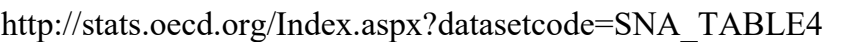

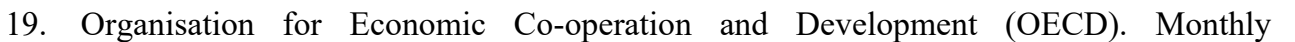

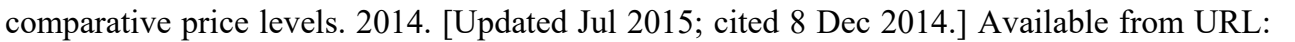

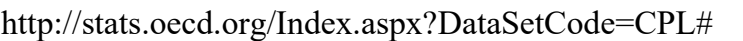

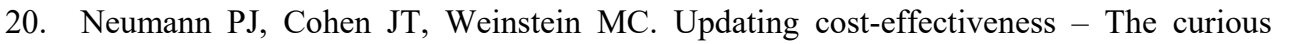

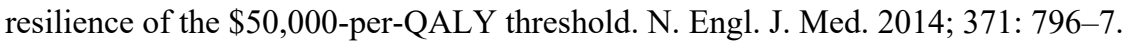

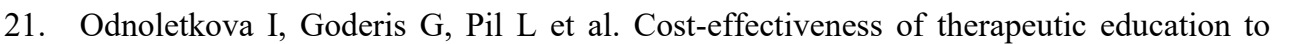

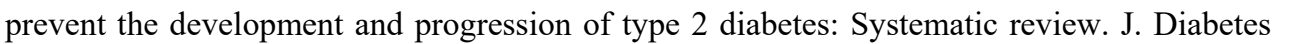

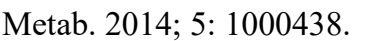

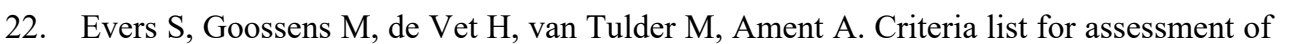

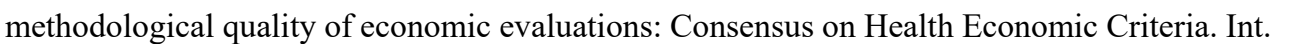

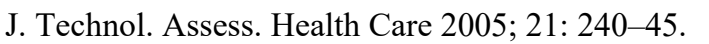

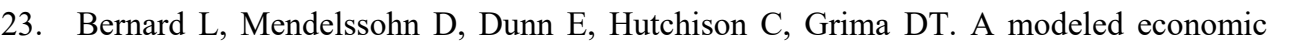

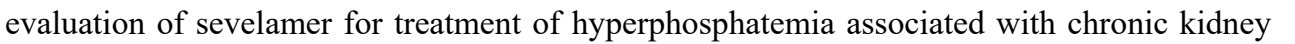

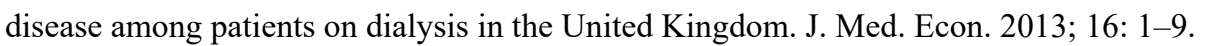

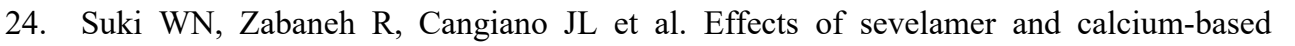

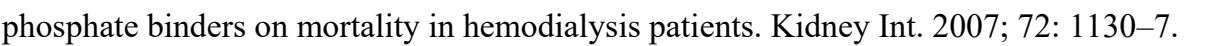

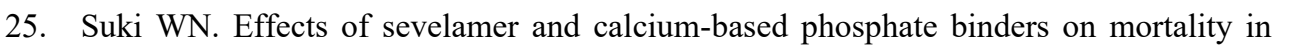

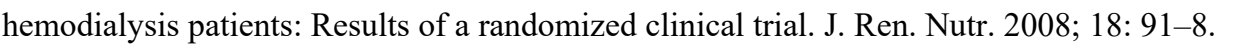




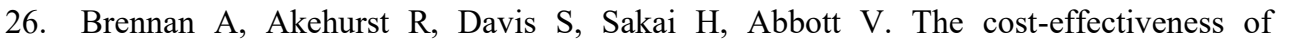

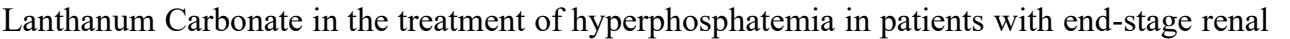

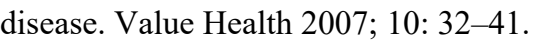

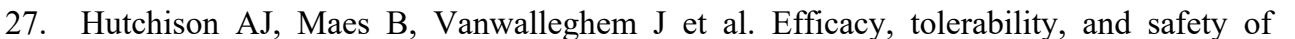

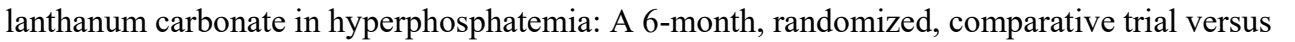

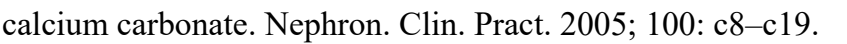

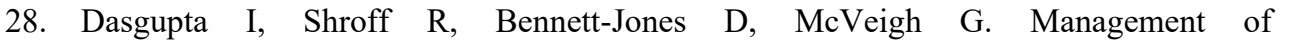

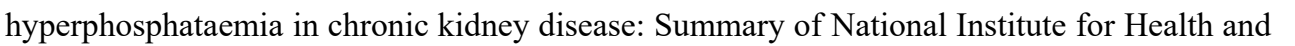

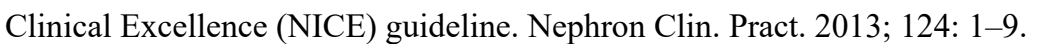

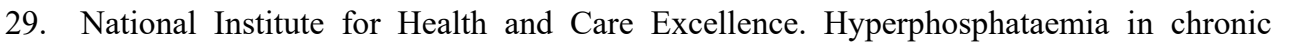

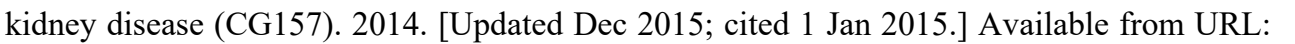

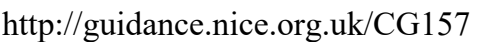

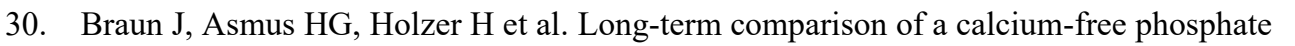

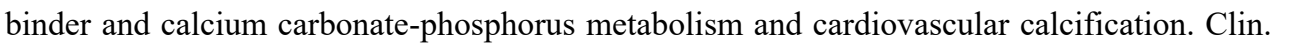

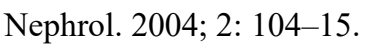

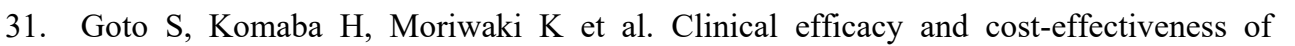

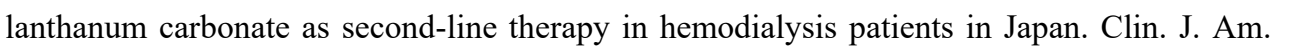

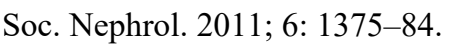

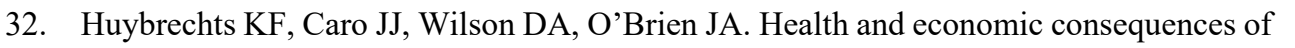

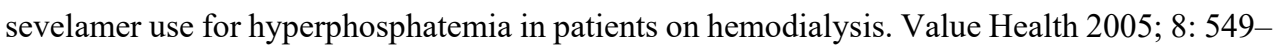
$\square \square$

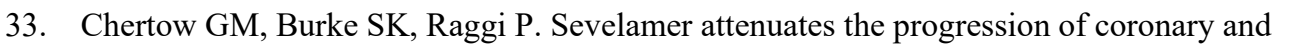

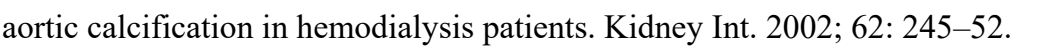

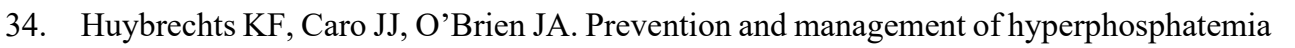

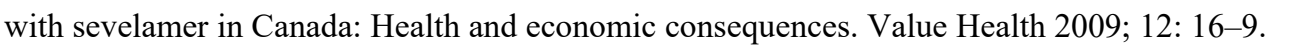

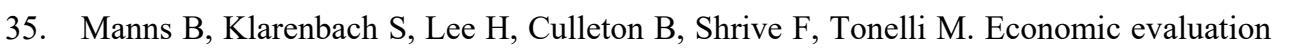

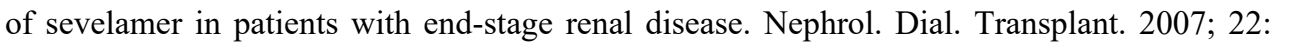
$\square 1+\square \square$

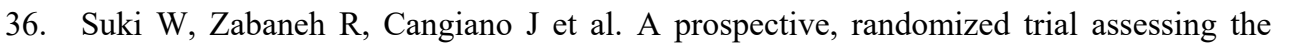

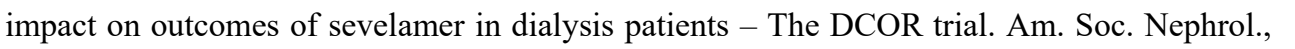

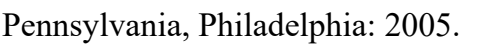

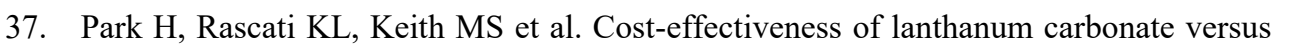

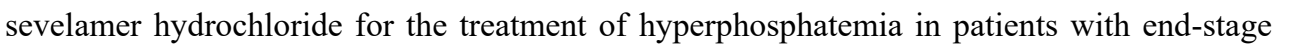

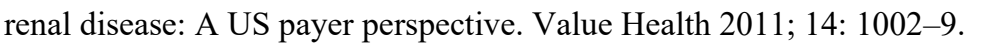




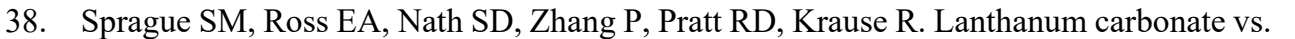

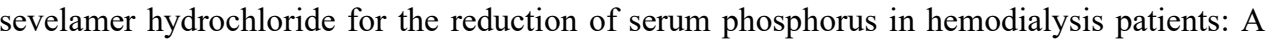

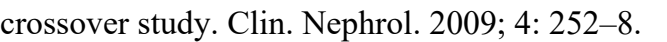

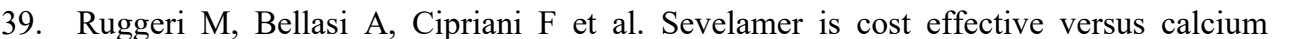

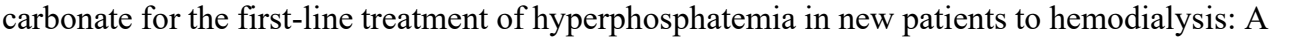

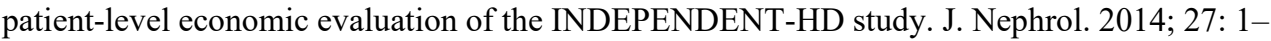
$\square \square$

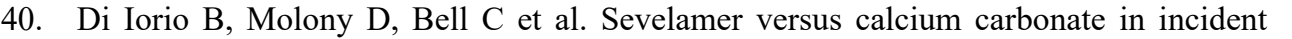

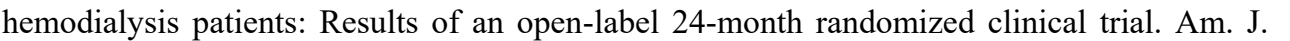

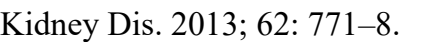

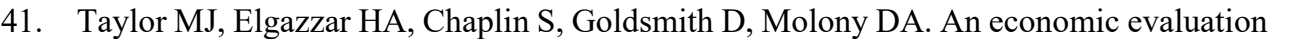

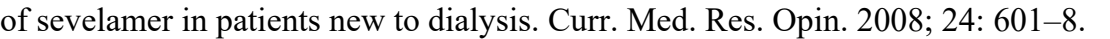

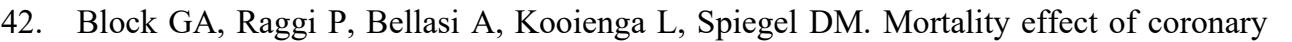

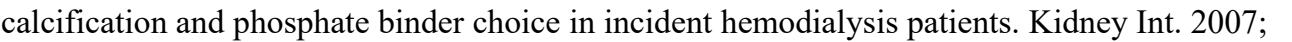
$\square|\nabla| 1-\square 10$

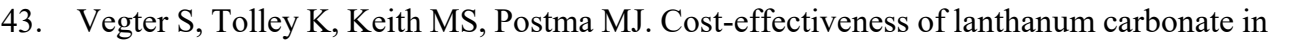

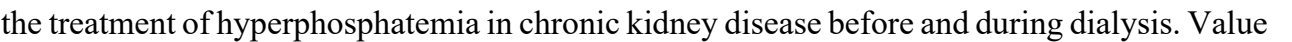

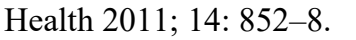

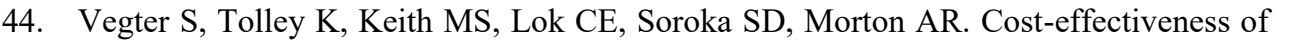

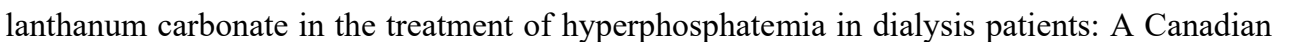

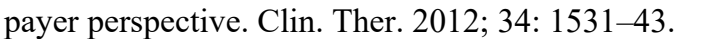

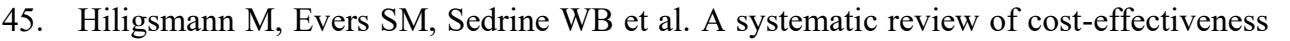

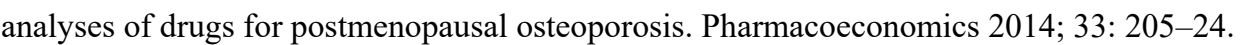

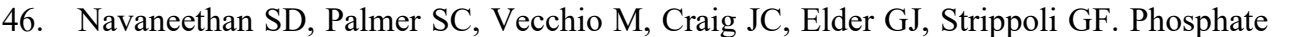

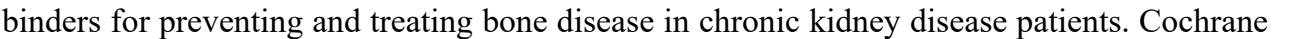

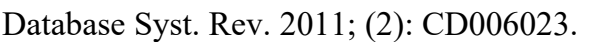

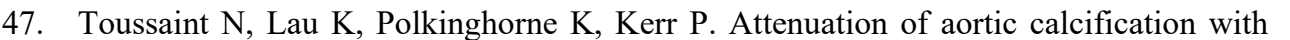

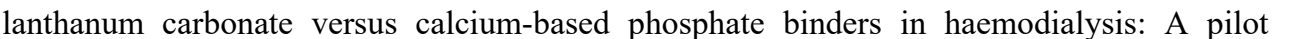

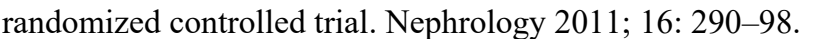

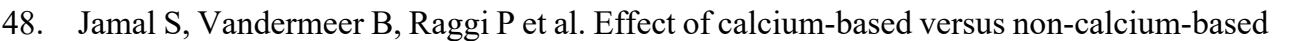

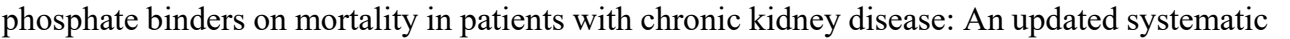

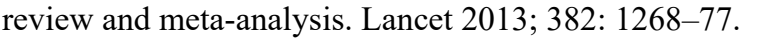

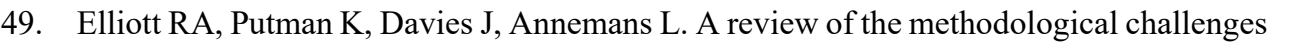

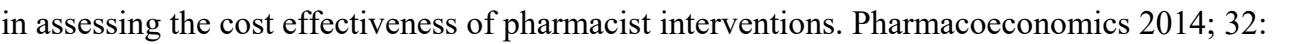
$\square 11-\square \square$ 


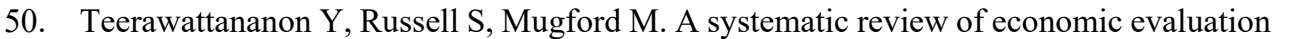

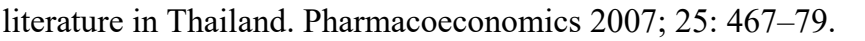

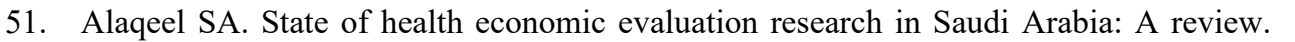

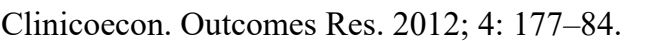

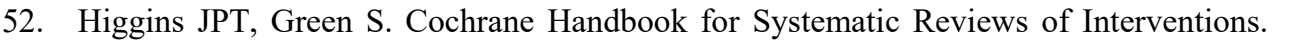

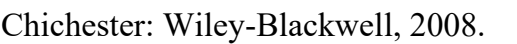

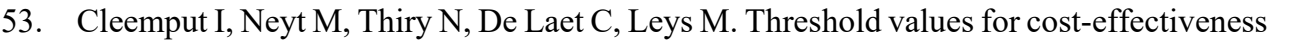

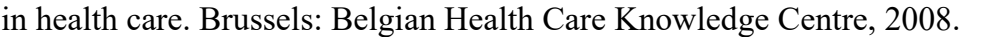

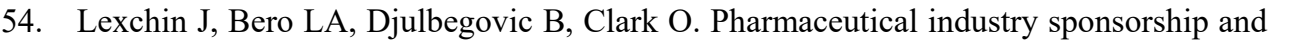

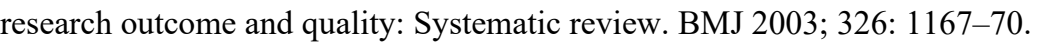

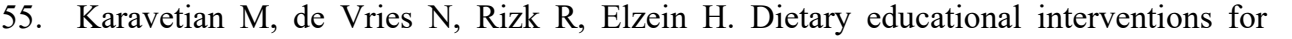

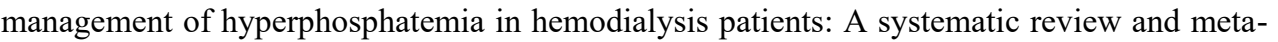

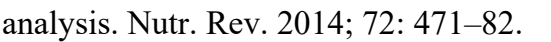

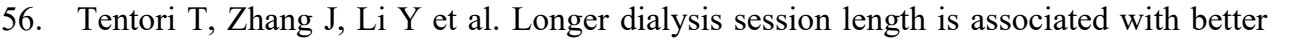

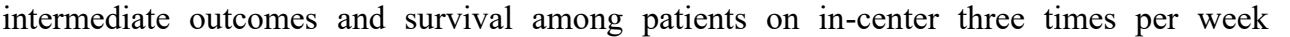

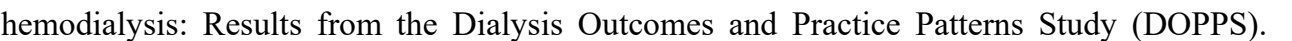

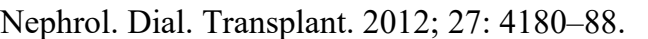

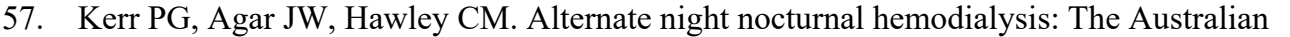

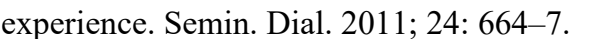

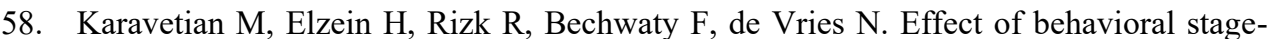

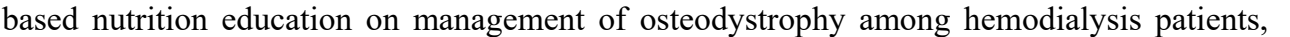

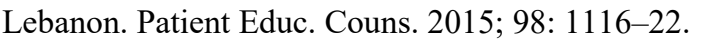

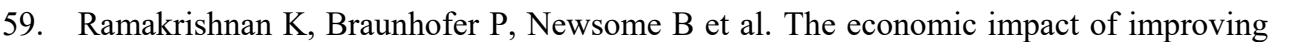

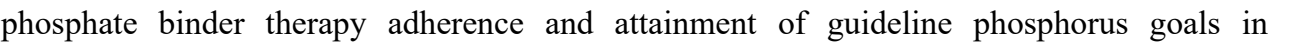

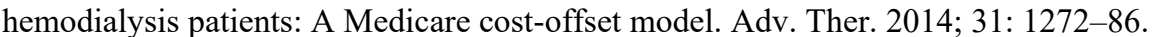




\section{Supporting Information Appendix S1: Search strategy}

\section{1) NHSEED via CRD}

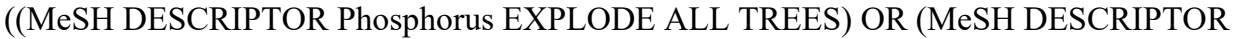

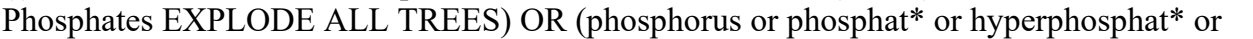

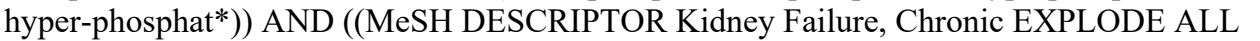

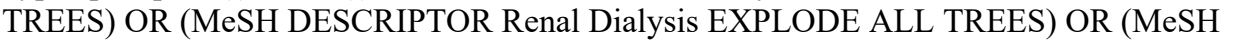

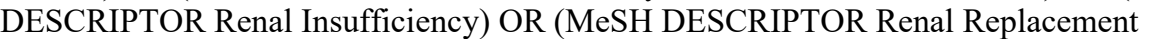

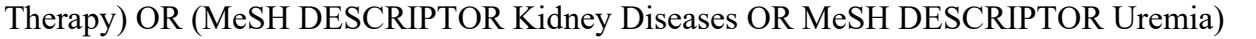
प

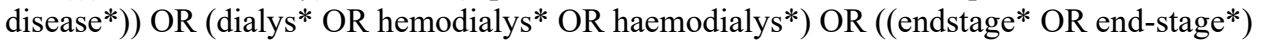

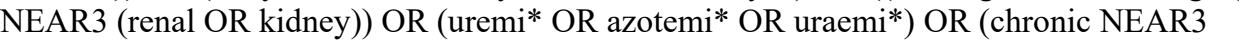

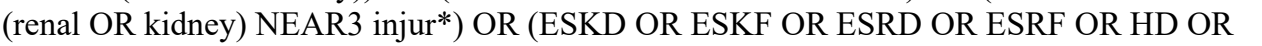

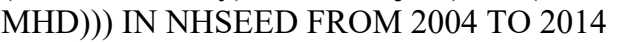

\section{2) MEDLINE using OVID}

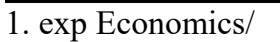

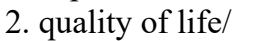

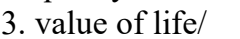

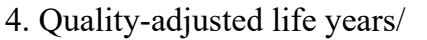

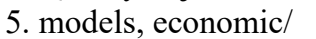

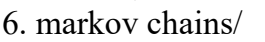

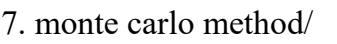

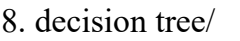

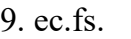

पाणापापापा

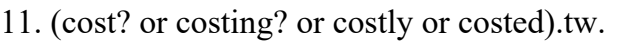

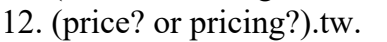

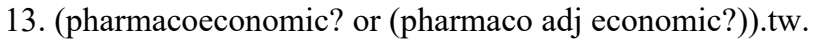

पाणाणापा

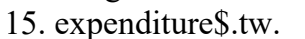

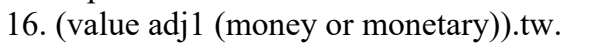

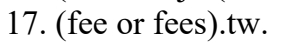

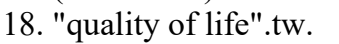

पाणाणाणापा

पाणाणाणा

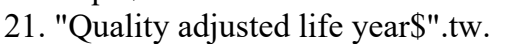

एणाणाणा

पाणाणाण

पाणापापा

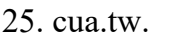

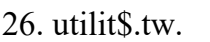

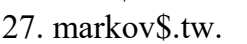

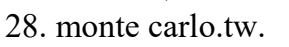

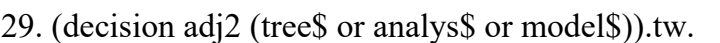

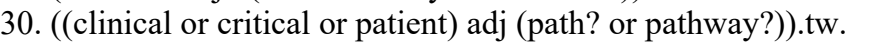

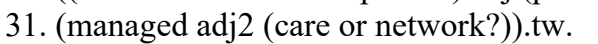

पाएापाणा

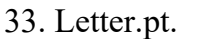




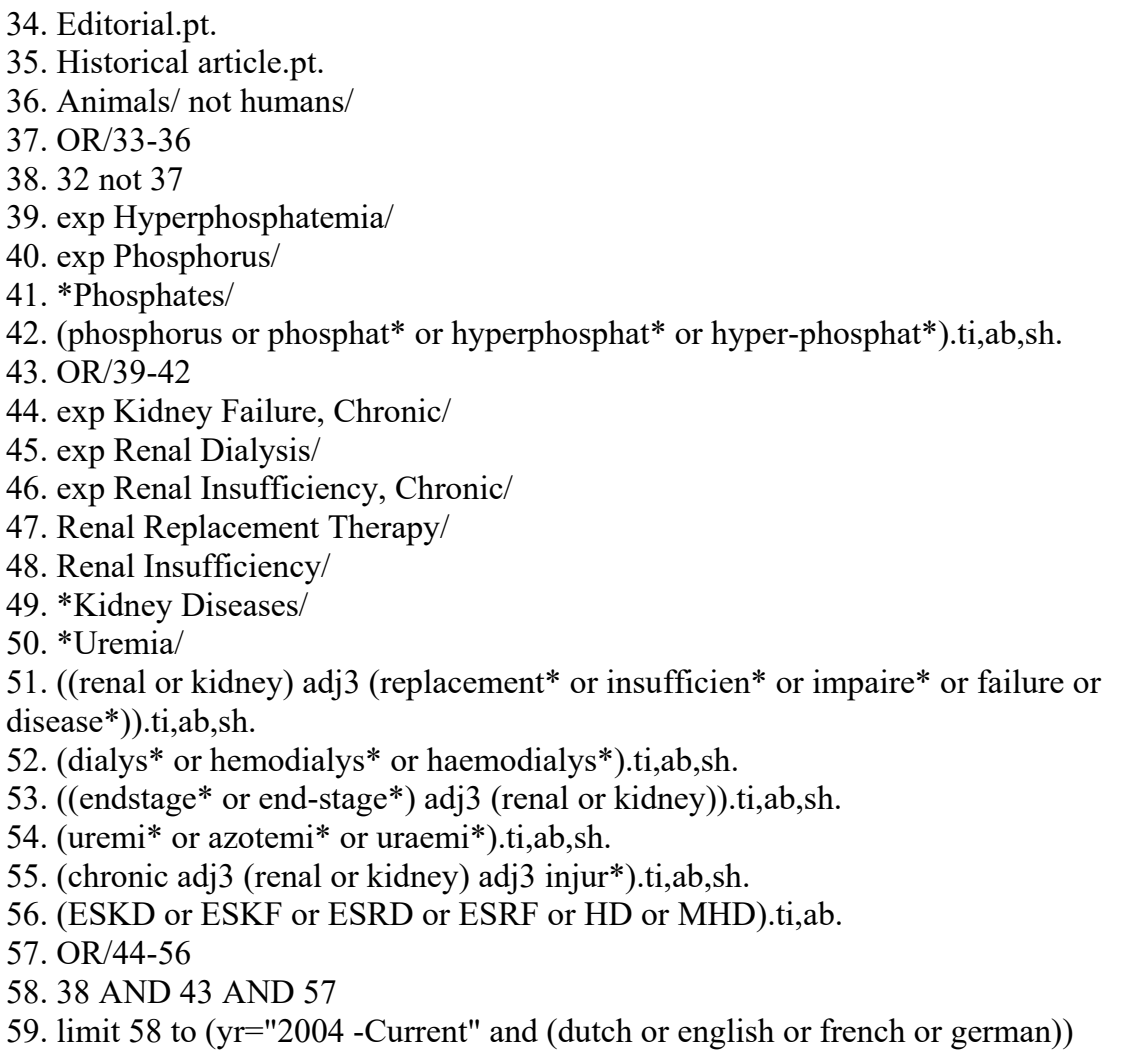




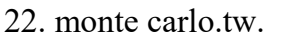

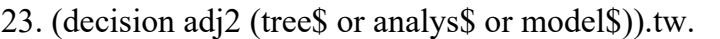

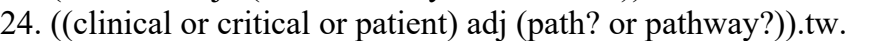

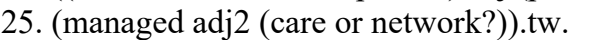

पाயमणाम

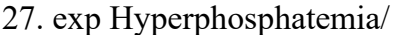

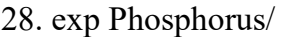

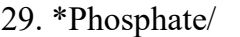

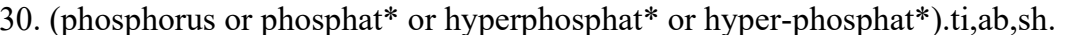

पाயमणाण

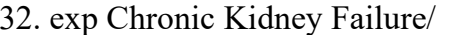

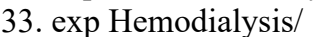

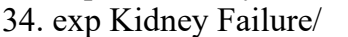

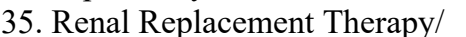

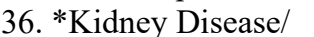

पणापणाण

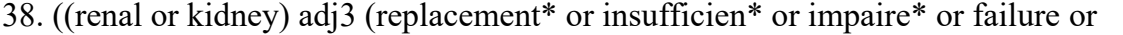

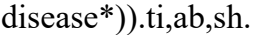

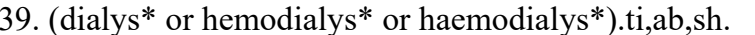

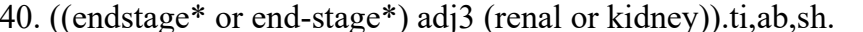

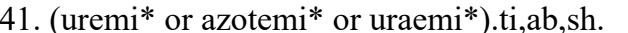

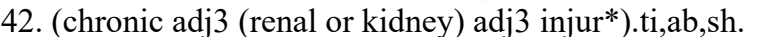

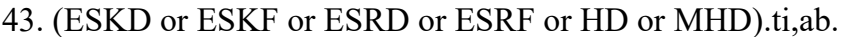

पाएवाणाम

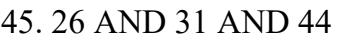

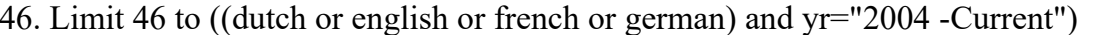

\section{4) CINAHL using EBSCO}

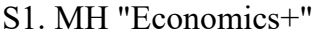

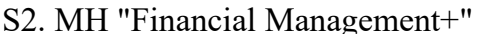

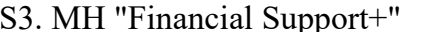

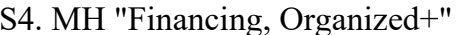

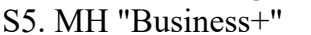

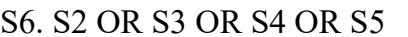

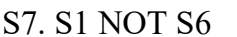

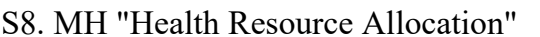

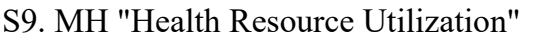

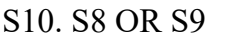

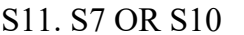

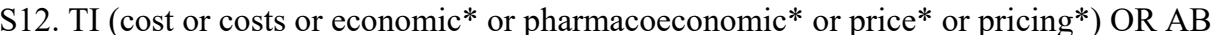

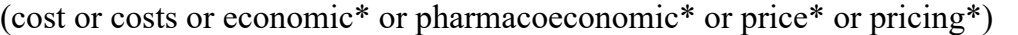

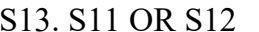

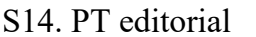

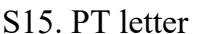

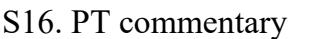

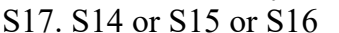

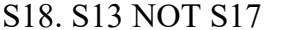

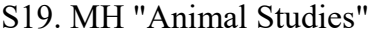

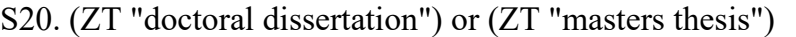

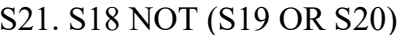




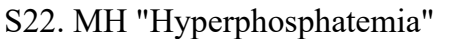

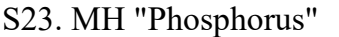

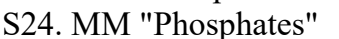

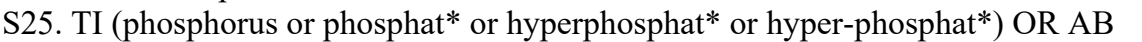

पा

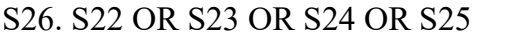

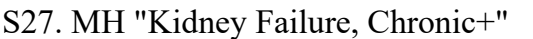

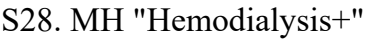

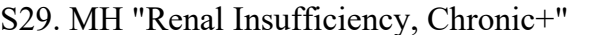

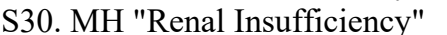

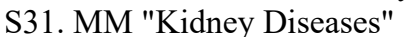

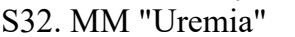

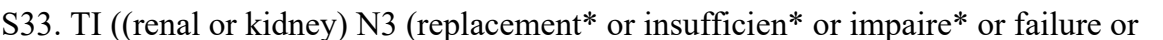

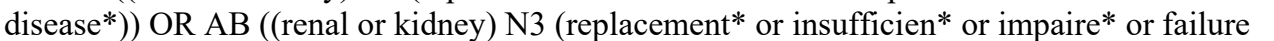

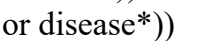

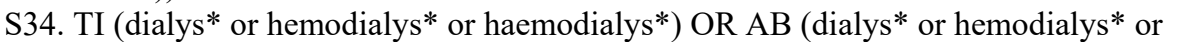

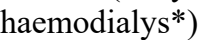

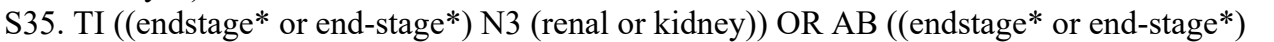

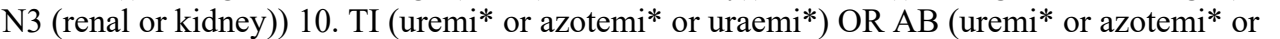

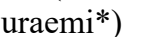

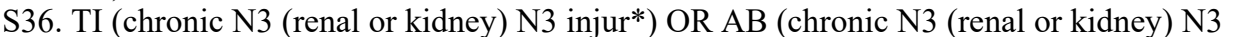
पाणाणा

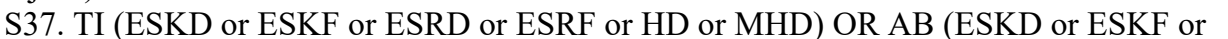

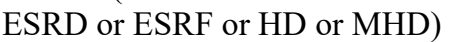

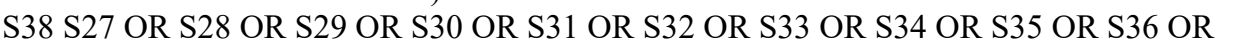

$\square \square \square$

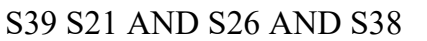

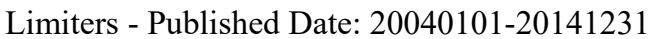

$\square$ 


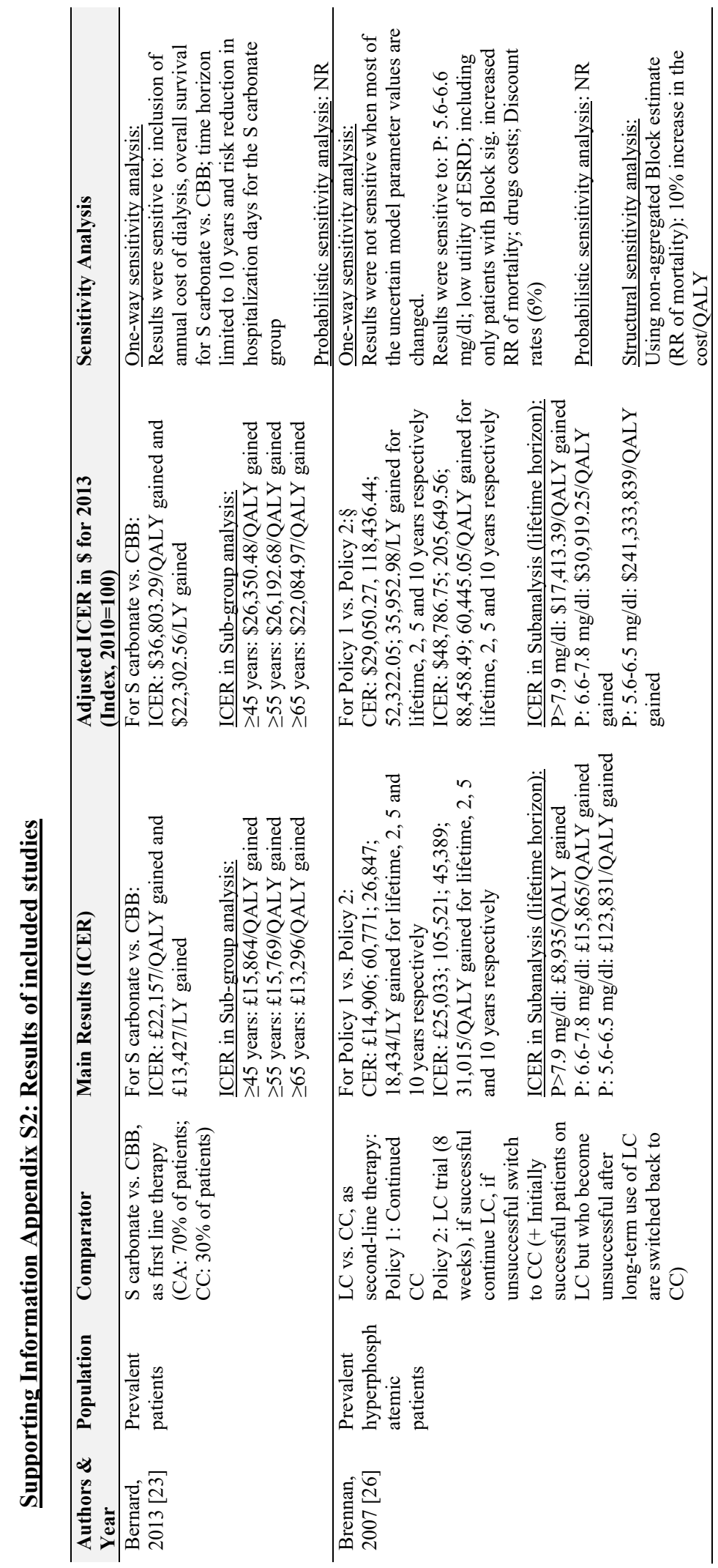




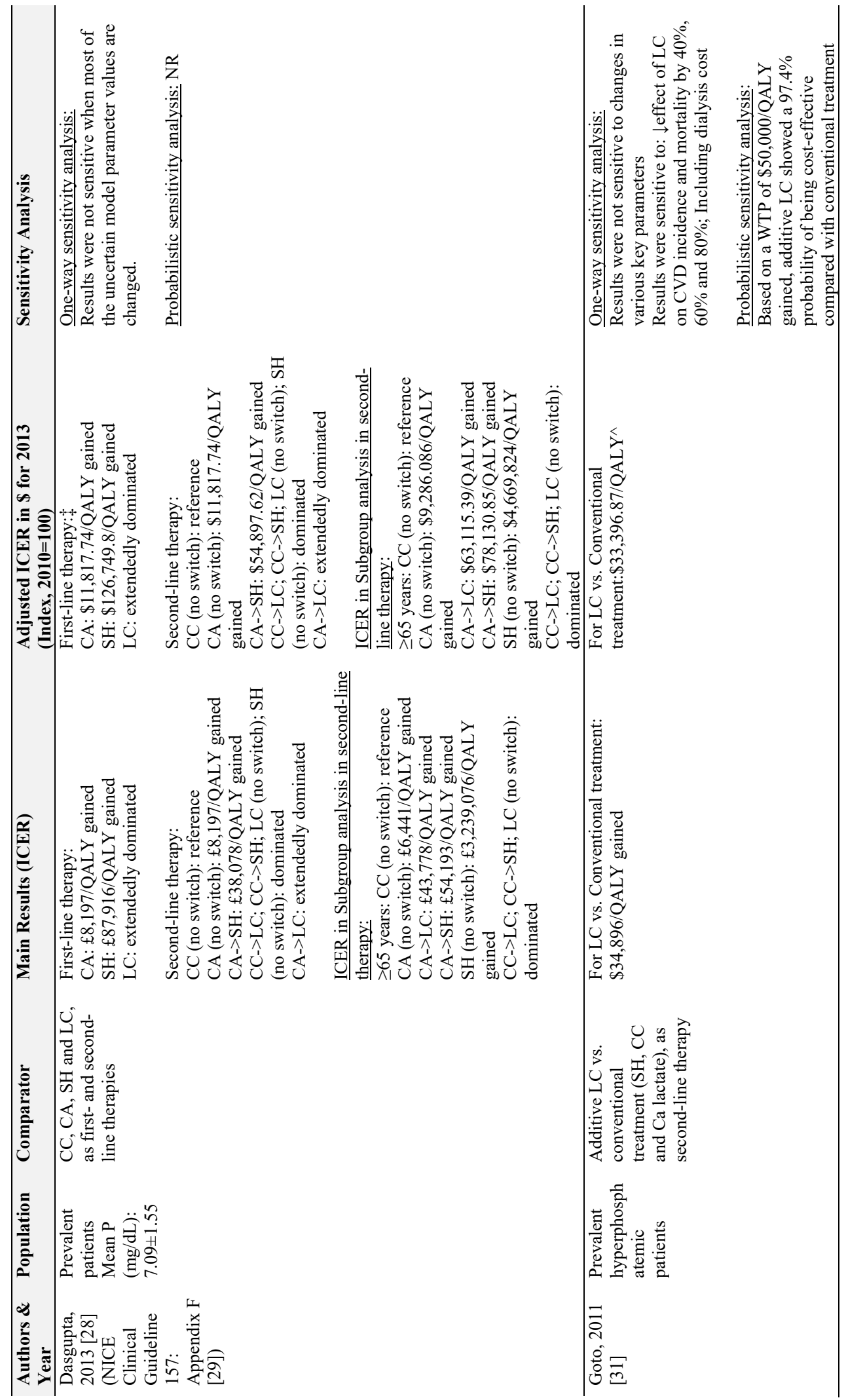




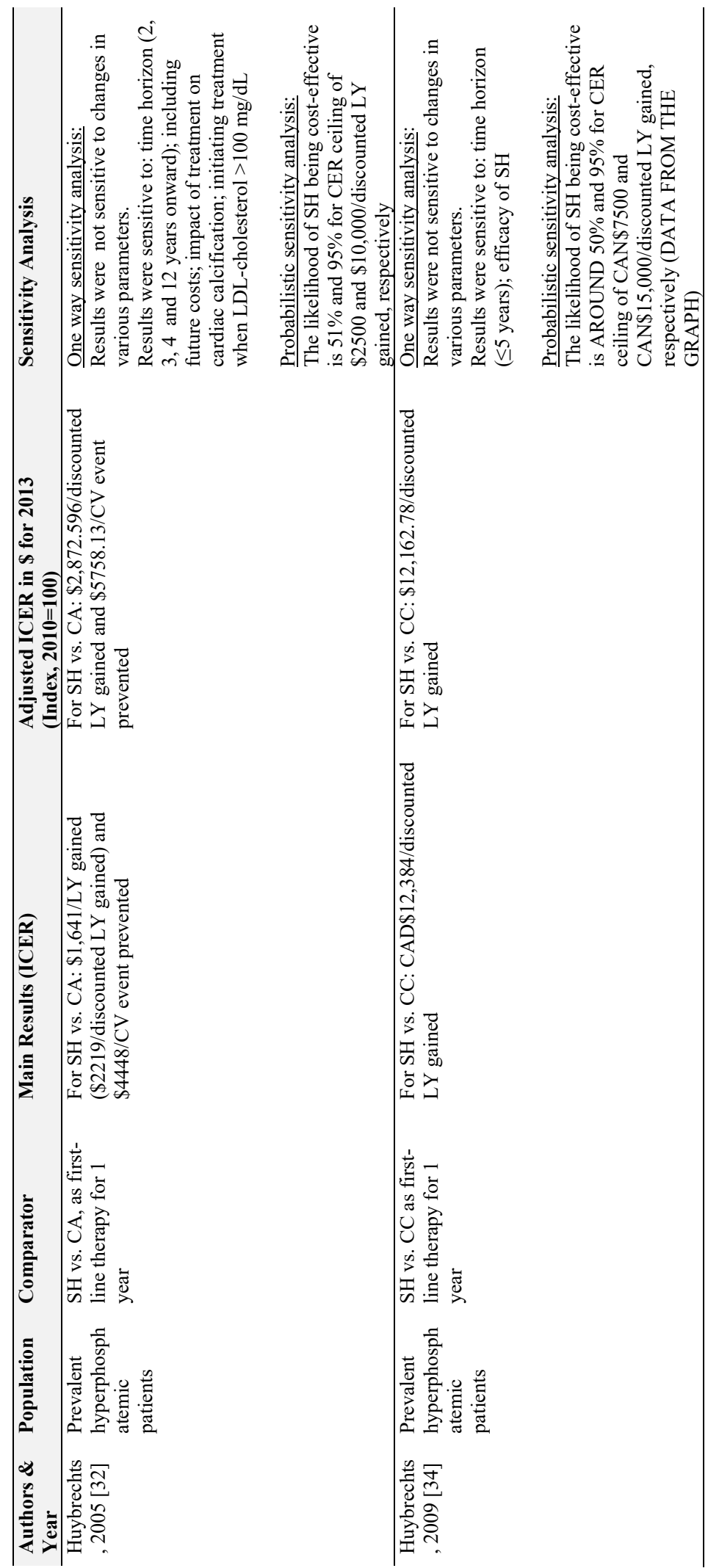




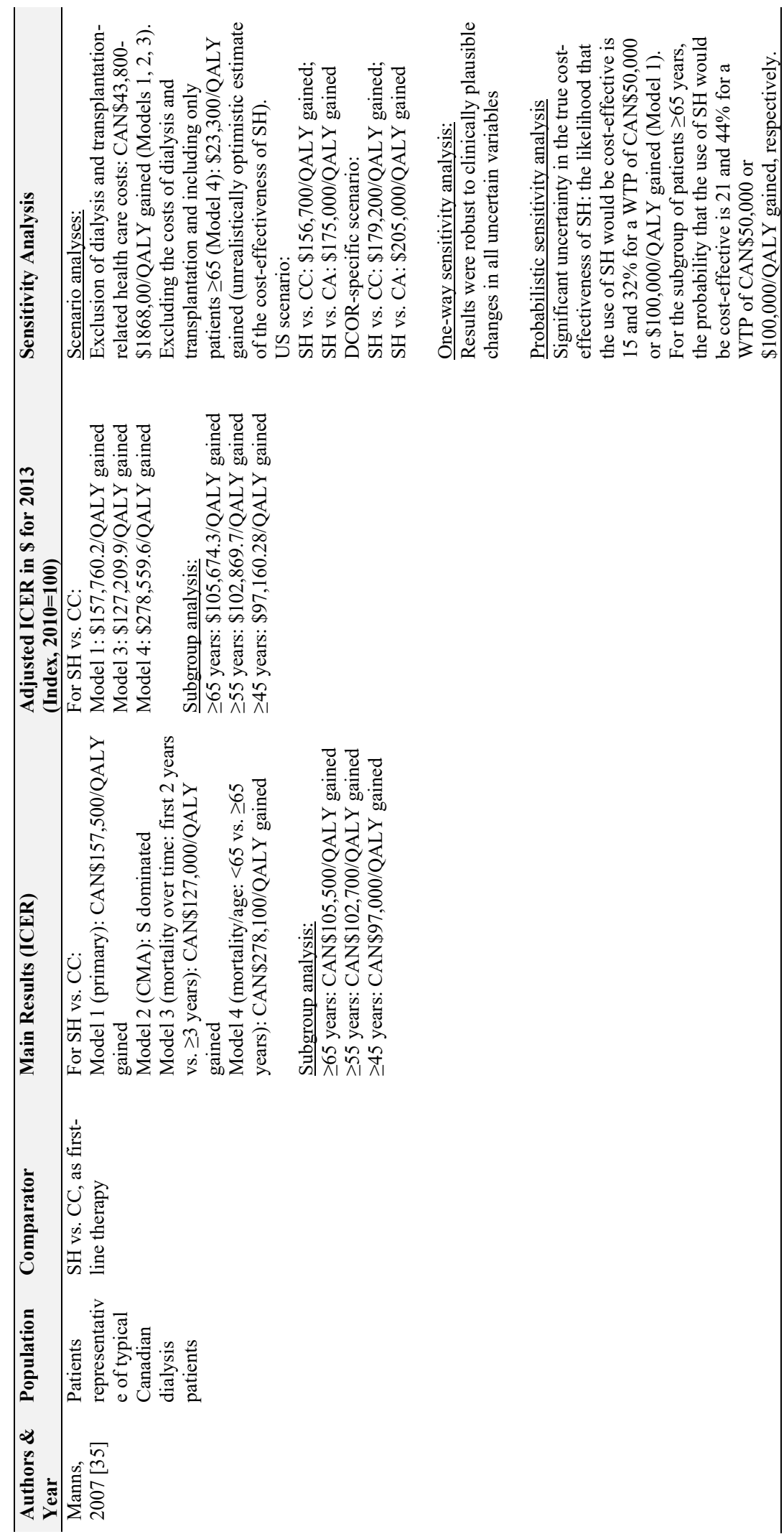




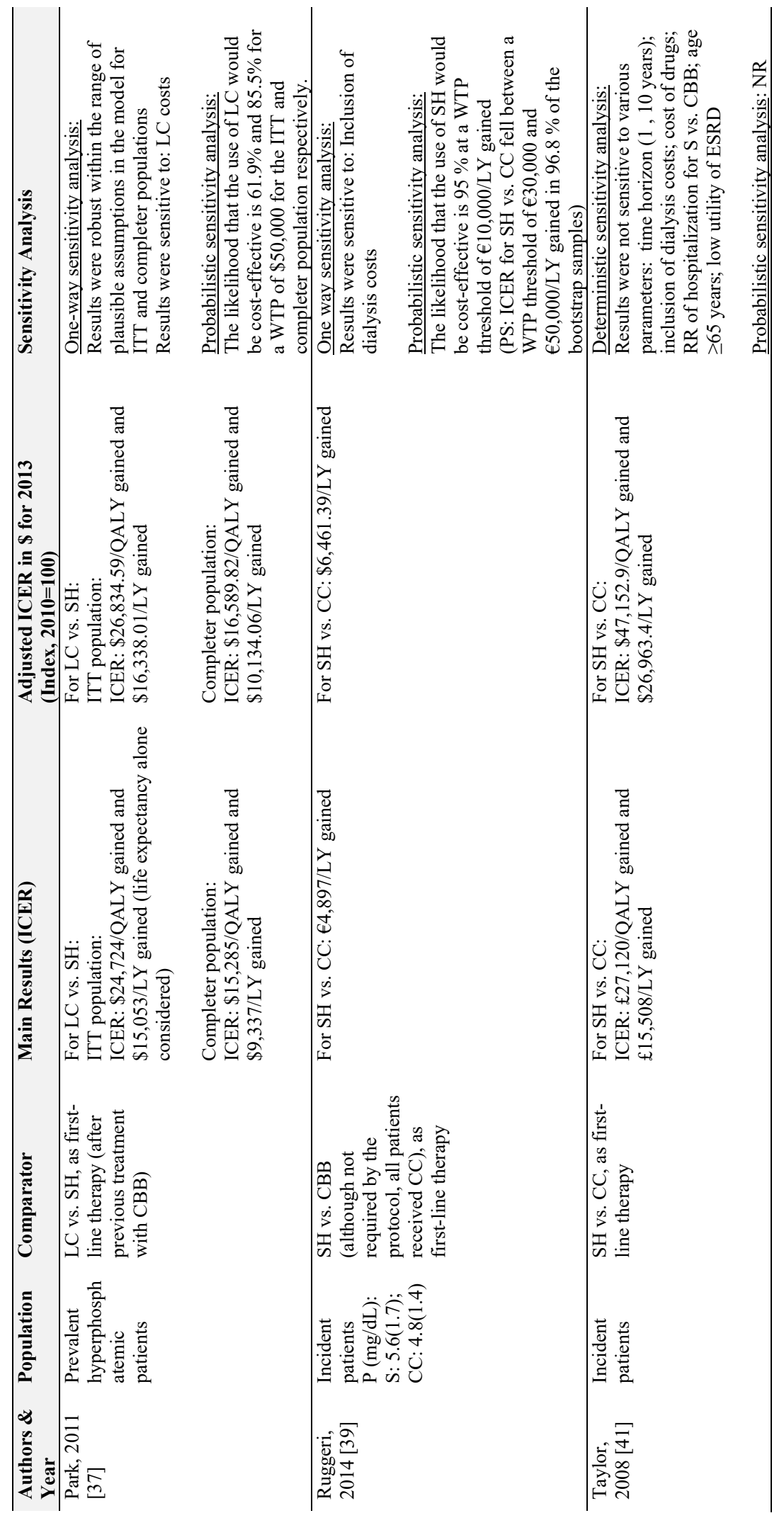




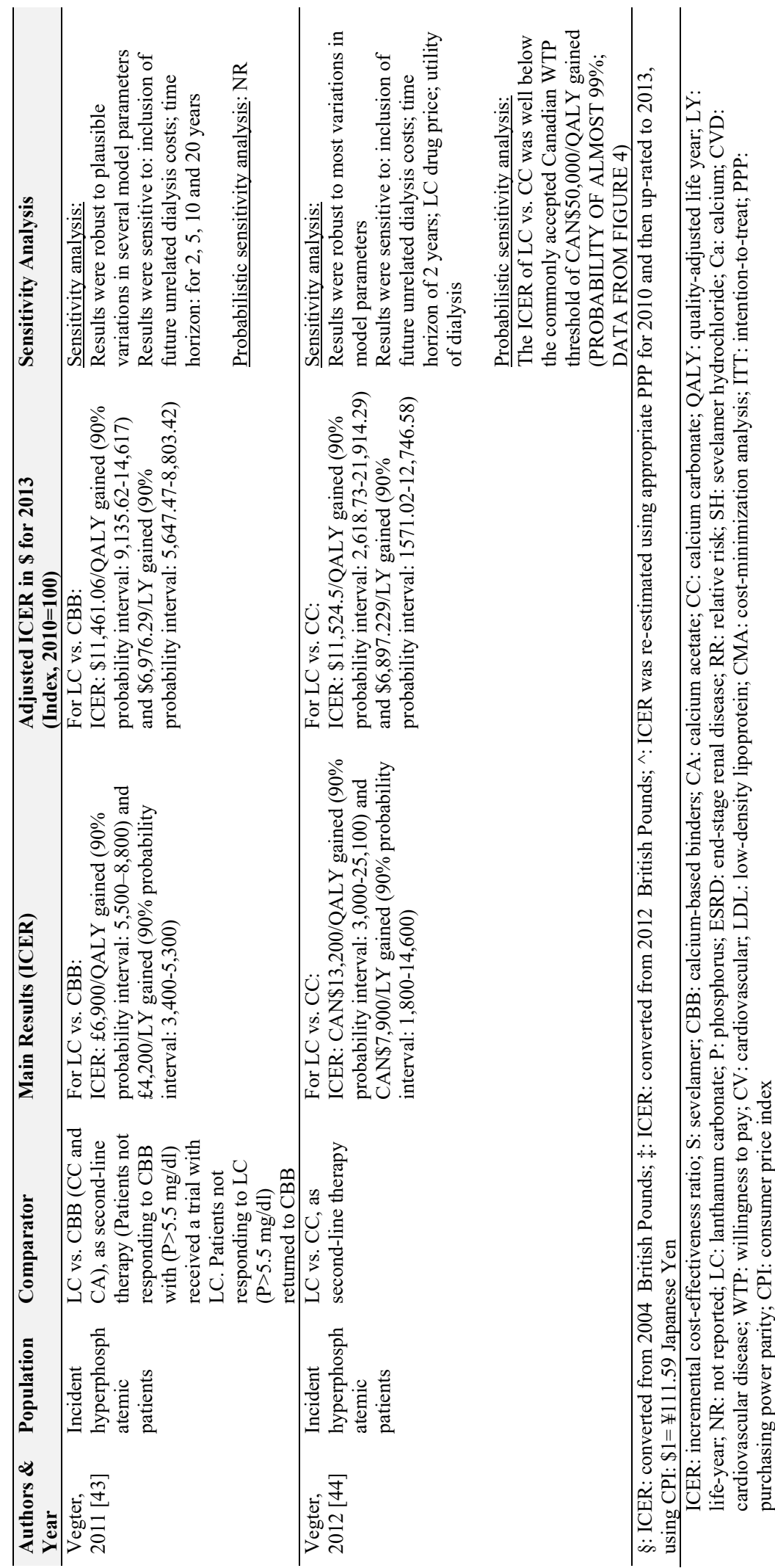



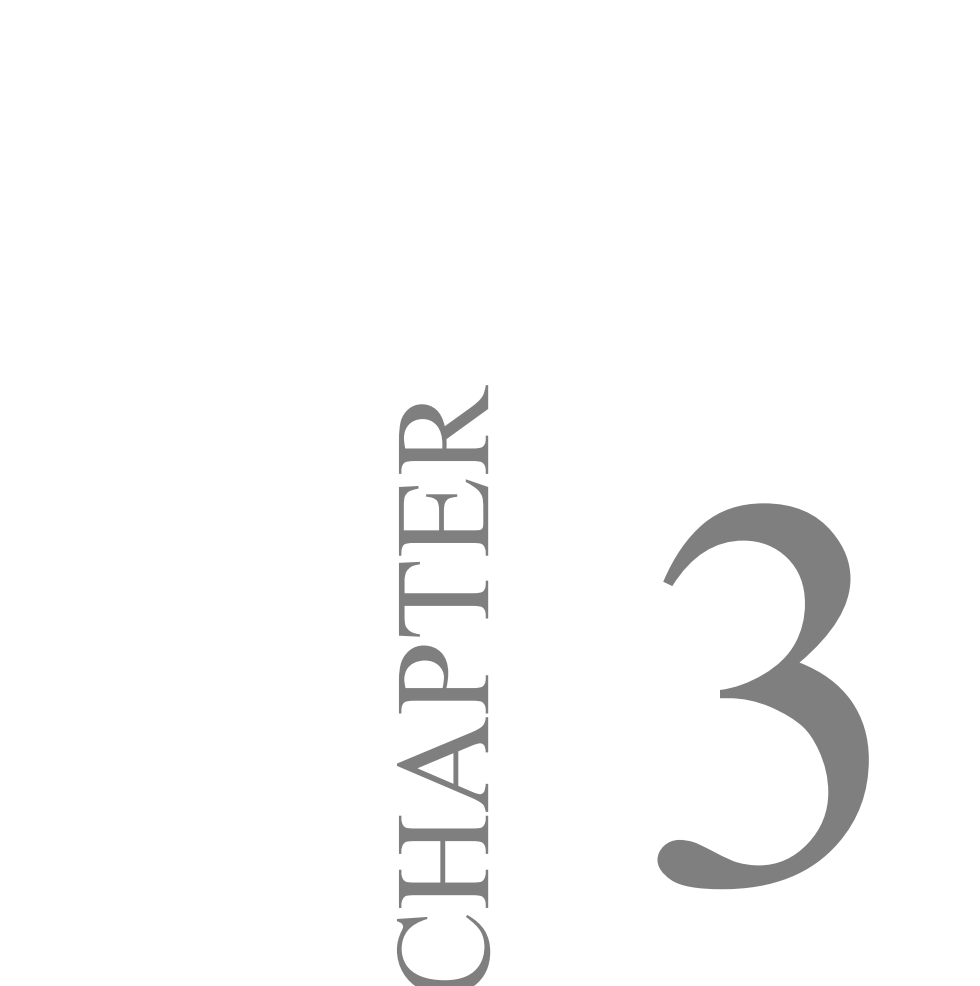

\section{A SOCIETAL COST-OF-ILLNESS STUDY OF HEMODIALYSIS IN LEBANON $\square$}

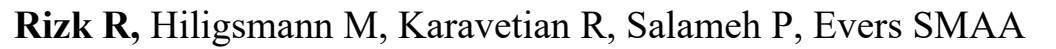




\section{Abstract}

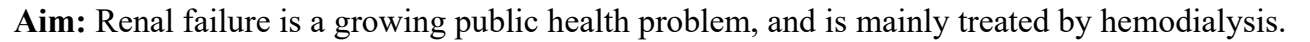

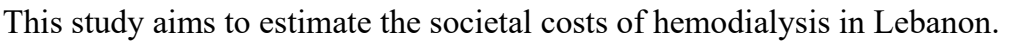

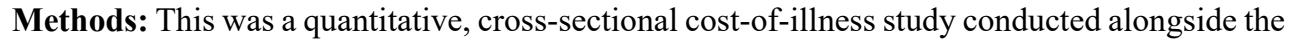

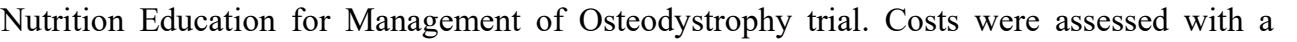

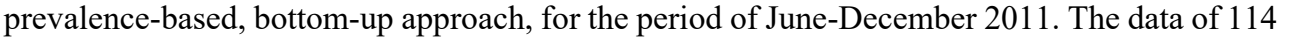

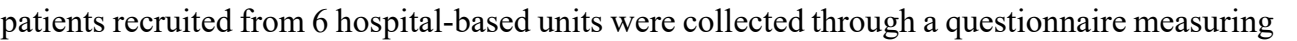

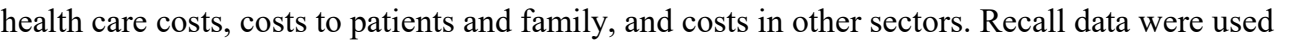

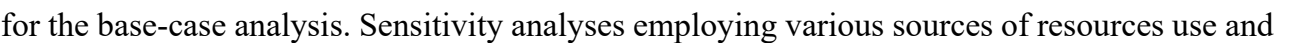

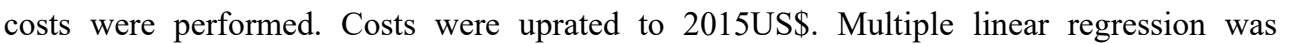

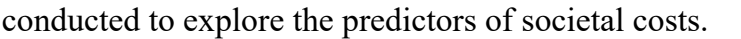

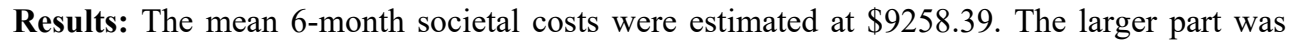

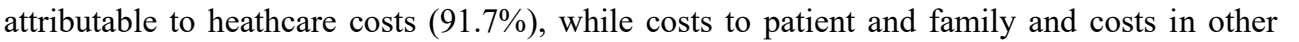

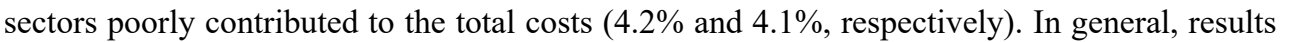

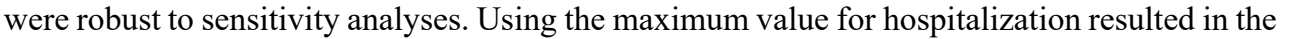

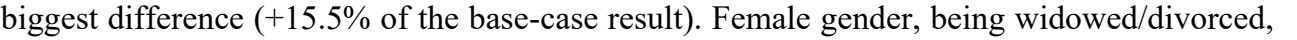

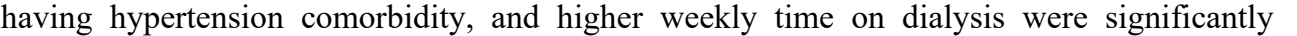

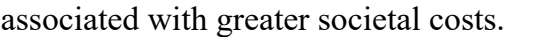

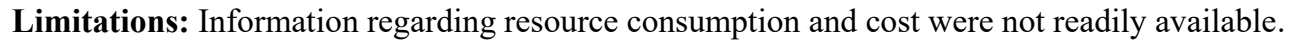

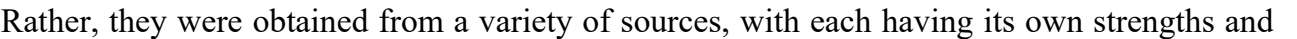

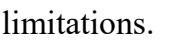

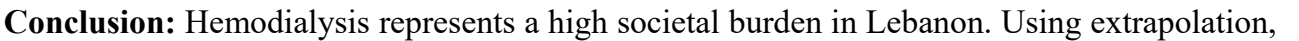

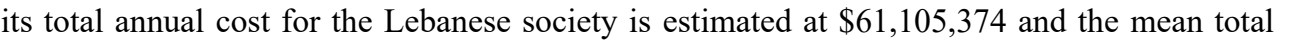

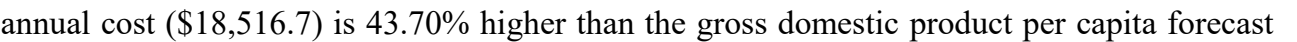

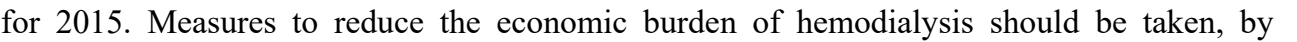

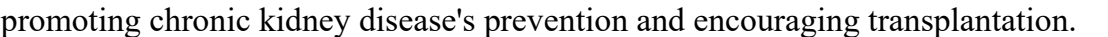

\section{KEYWORDS}

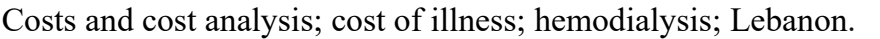

\section{INTRODUCTION}




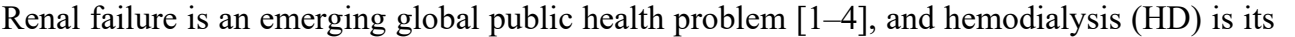

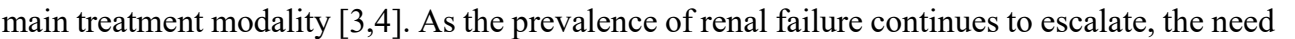

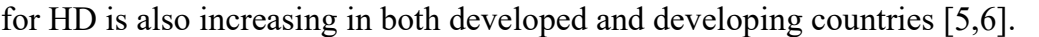

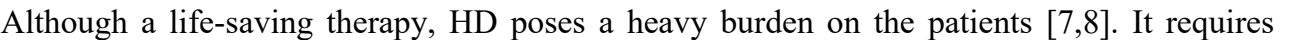

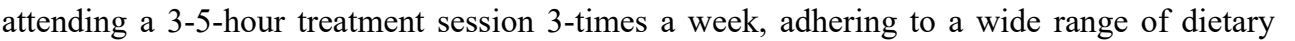

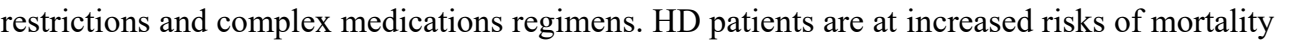

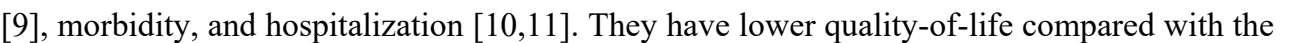

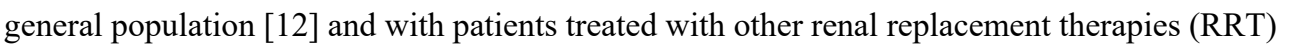

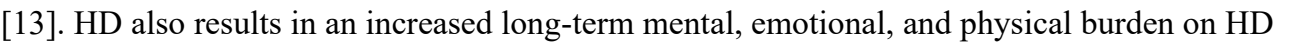

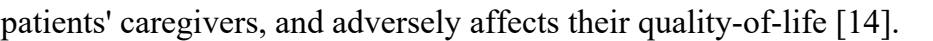

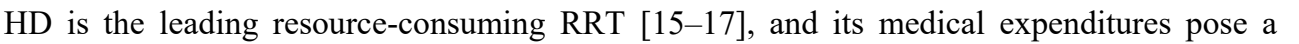

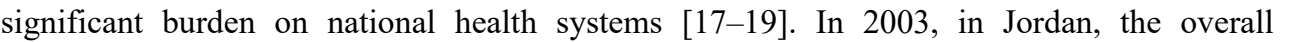

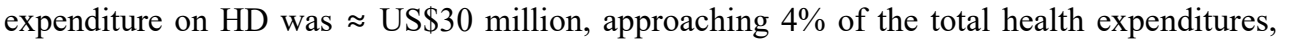

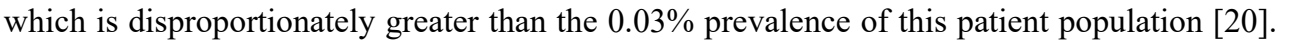

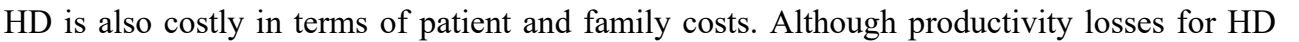

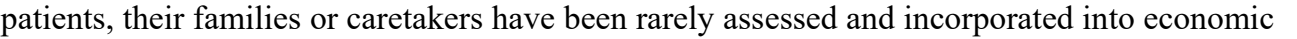

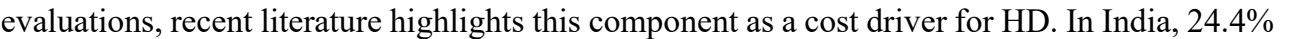

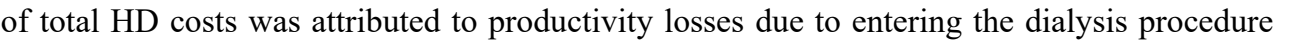
$\begin{array}{ll} & \end{array}$

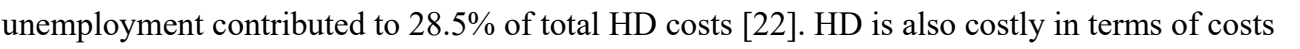

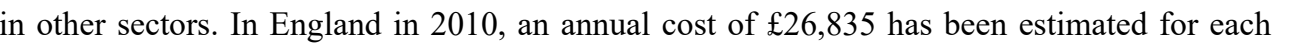
$\square \square \square$ 's journeys to HD centers $\square \square \square \square$

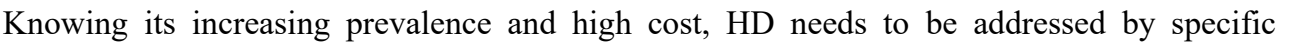

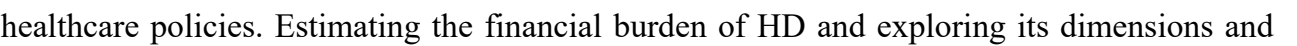

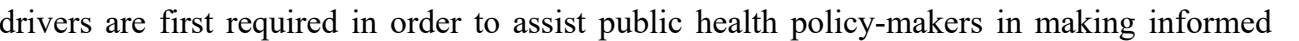

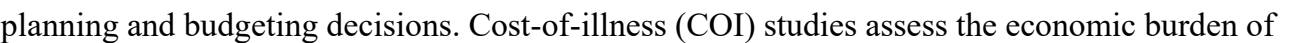

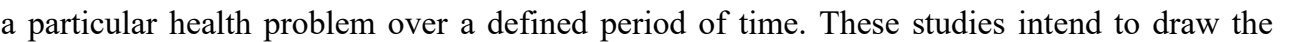

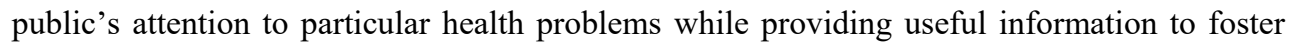

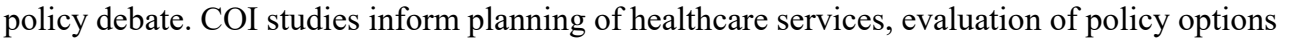

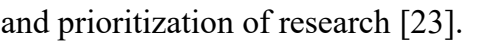

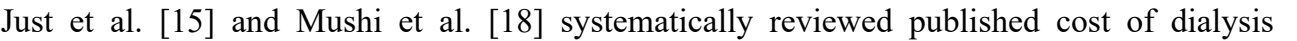

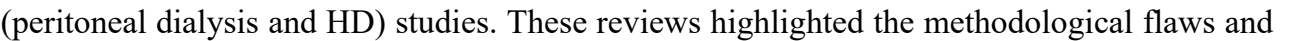

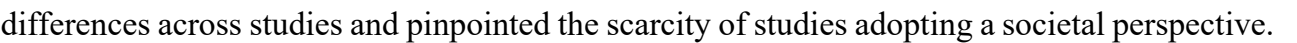

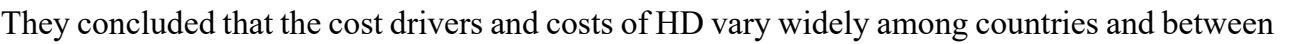

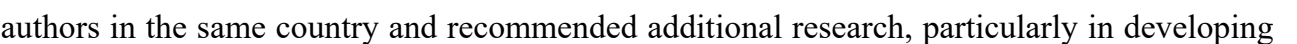




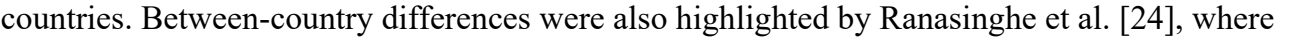

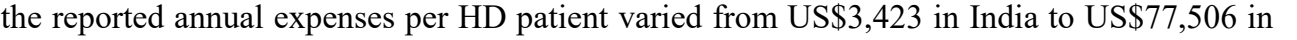

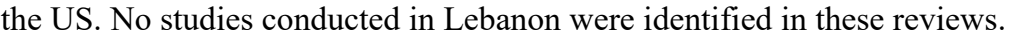

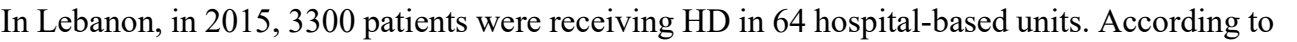
पणी

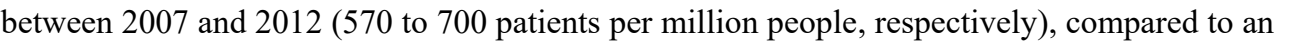

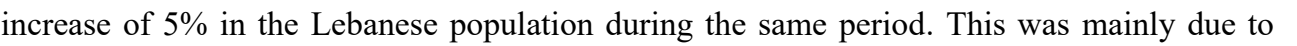

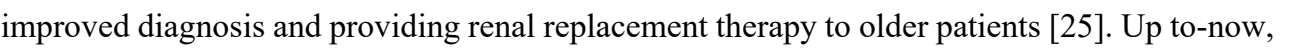

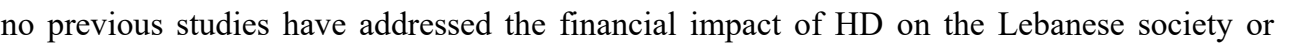

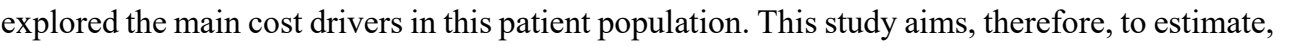

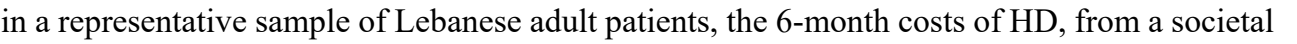
$\square \mid\|\|\|\| \| \square$

$\square$

\section{MeTHODS}

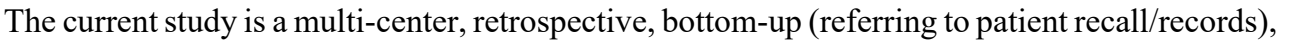

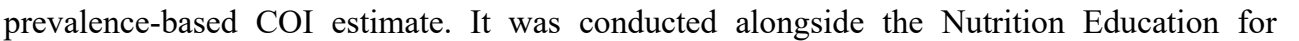

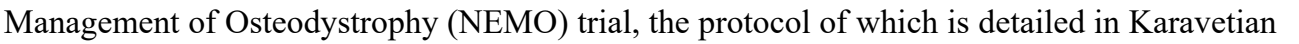

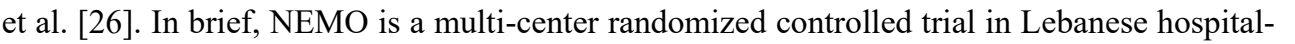

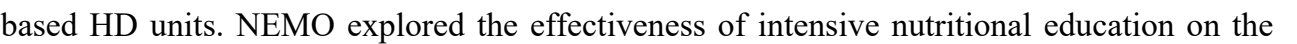

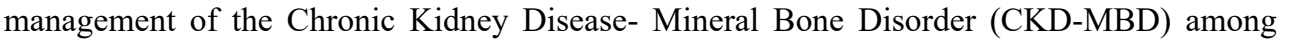

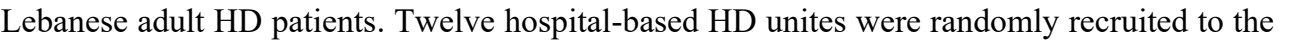

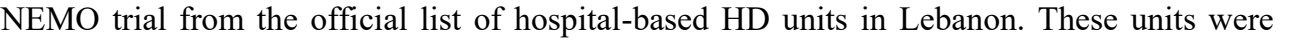

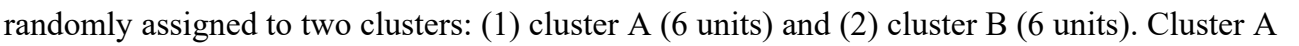

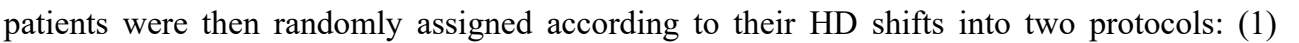

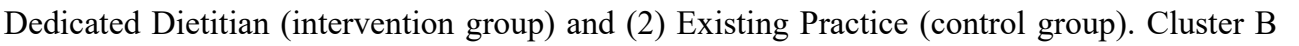

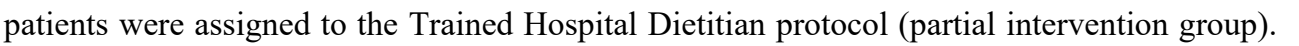

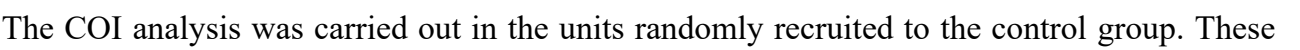

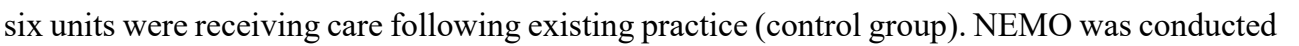

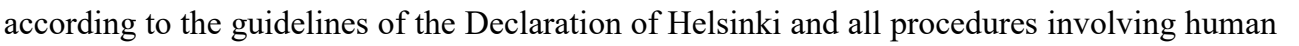

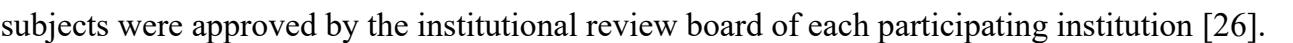

$\square$

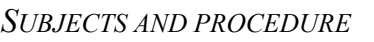

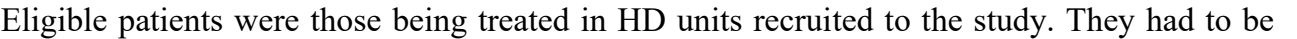

Lebanese, adults ( $\geq 18$ years), stable (free of the following diseases: cancer, infection with the 
Human Immunodeficiency Virus and hepatitis), and on HD for $\geq 6$ months. Eligible patients

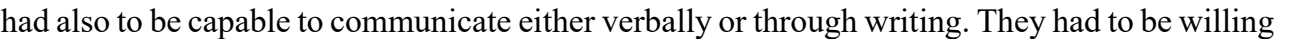

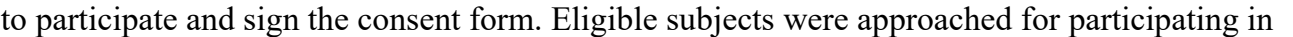

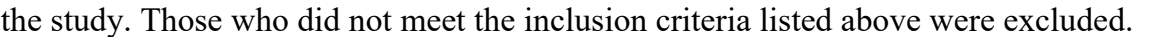

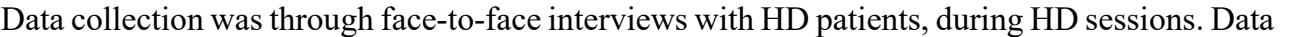

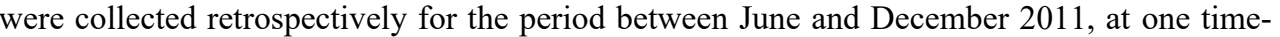

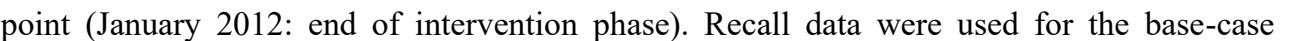

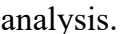

$\square$

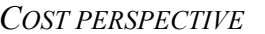

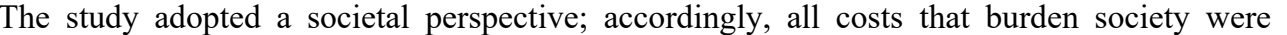

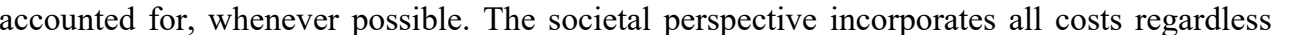

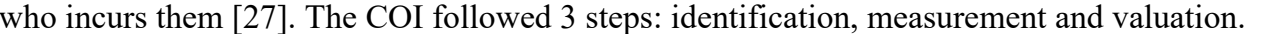

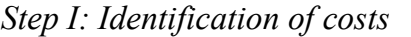

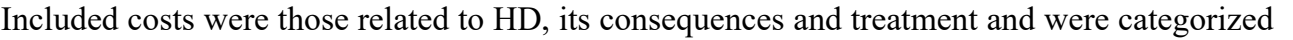

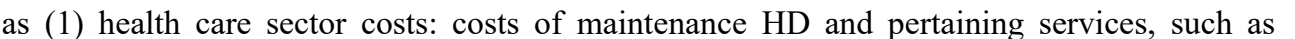

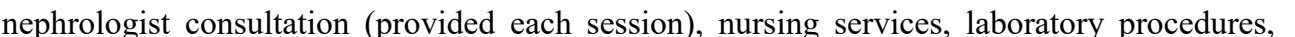

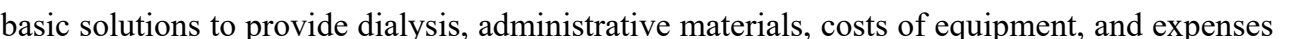

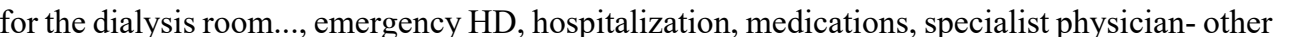
than nephrologist, i.e. cardiologist, neurologist, gastroenterologist...

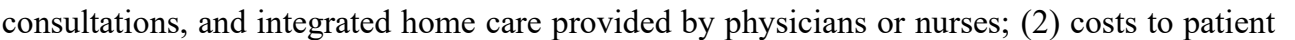

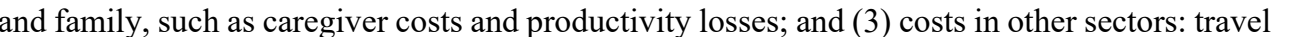

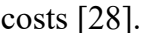

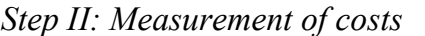

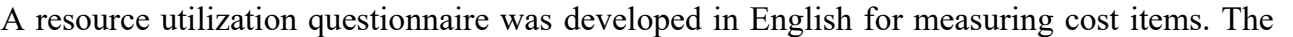

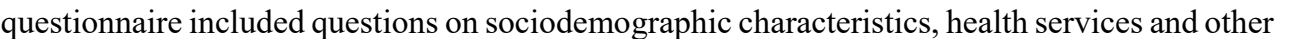

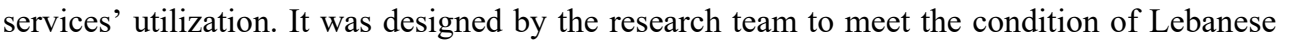

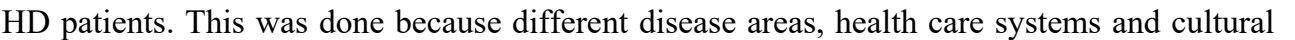

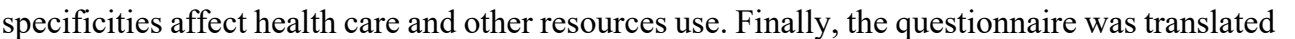

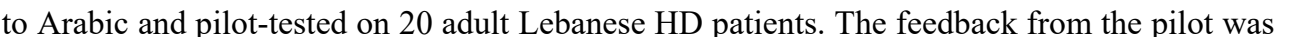

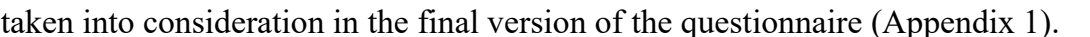

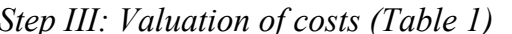




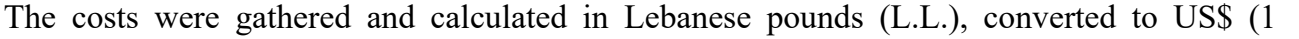

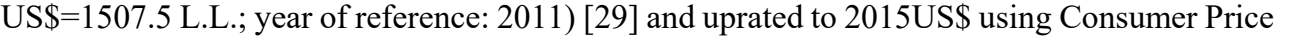

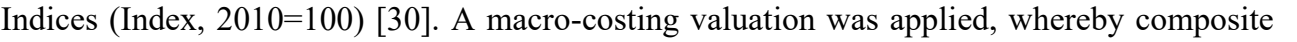

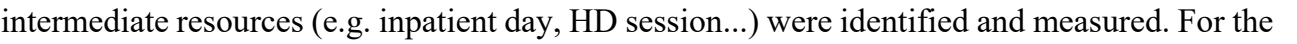

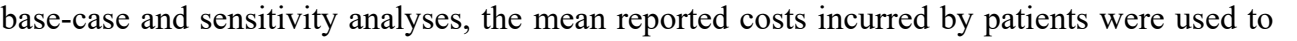

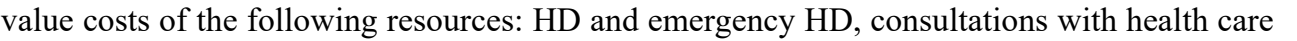

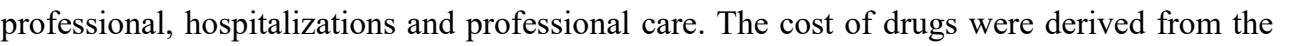

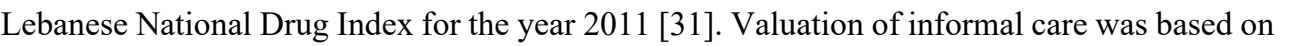

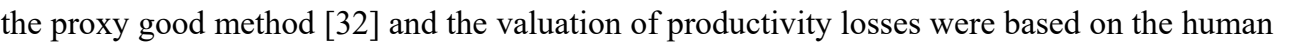

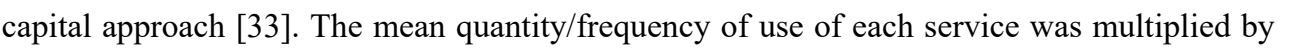

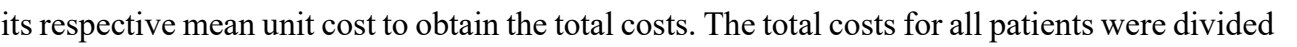

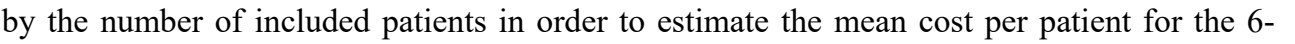

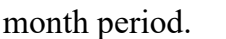

$\square$

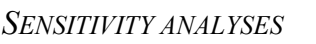

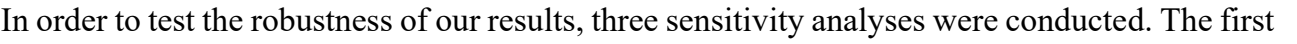

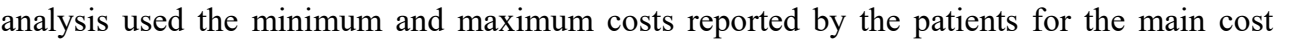

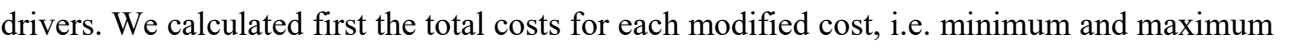

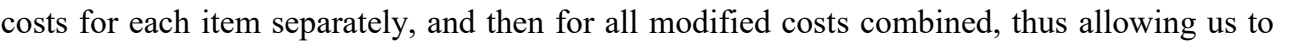

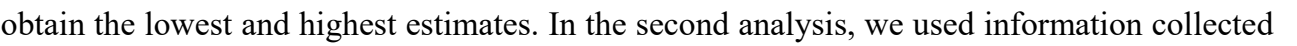

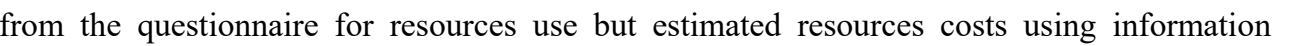

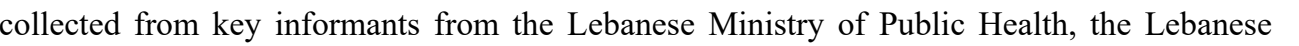

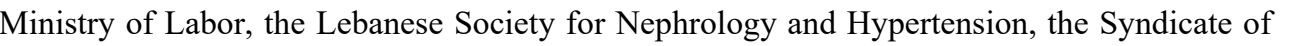

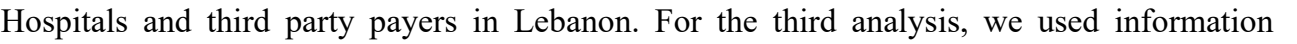

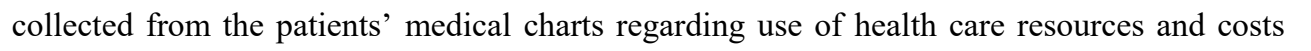

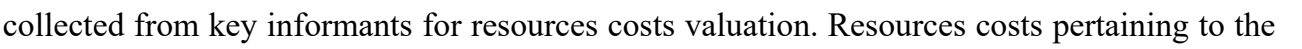

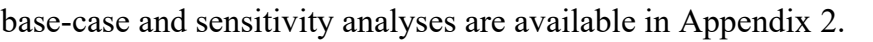

$\square$

$\square$

$\square$

$\square$

$\square$

$\square$ 


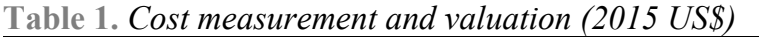

\begin{tabular}{|c|c|c|c|c|}
\hline \multirow[t]{3}{*}{$\square$} & \multirow[t]{3}{*}{ Unit } & \multicolumn{3}{|l|}{ Cost } \\
\hline & & \multirow[t]{2}{*}{ Base-case analysis } & \multicolumn{2}{|c|}{ Sensitivity analysis } \\
\hline & & & Minimum & Maximum \\
\hline \multicolumn{5}{|l|}{ Health care sector costs } \\
\hline \multicolumn{5}{|l|}{ HD } \\
\hline$\square \square \square$ & 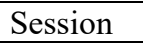 & \begin{tabular}{l|l|l|}
$\square \square \square$ \\
\end{tabular} & \begin{tabular}{l|l|l|}
$\square \square \square$ \\
\end{tabular} & $\square \square \| \square$ \\
\hline $\begin{array}{c}\square \square\|\|\|\| \square \square \square \\
\end{array}$ & 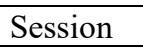 & \begin{tabular}{ll|l|}
$\square \square \square$ \\
\end{tabular} & 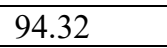 & 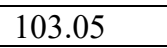 \\
\hline \multicolumn{5}{|l|}{ Health care professionals } \\
\hline 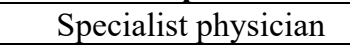 & $\square \square \square\|\|\|\|$ & \begin{tabular}{l|l|l|}
$\square$ \\
\end{tabular} & \begin{tabular}{l|l|l|}
$\square|\square|$ \\
\end{tabular} & $\square \square \| \square$ \\
\hline 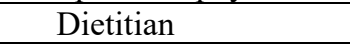 & 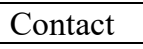 & $\square \square \square$ & $\square \square \square$ & \\
\hline 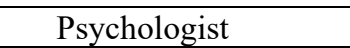 & 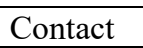 & $\square \mid \square \square$ & $\square \square \square$ & \\
\hline Medications & 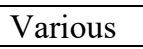 & 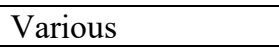 & $\square \square \square$ & \\
\hline Hospitalization & $\square \square \square$ & $\square \square \| \square$ & 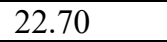 & 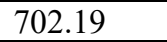 \\
\hline Professional home care & $\square \square \square \square$ & $\square \square \square$ & \begin{tabular}{ll|l|}
$\square \square \square$ \\
\end{tabular} & $\square|\Pi| \square$ \\
\hline \multicolumn{5}{|l|}{ Costs to patient and family } \\
\hline Informal care & 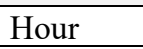 & 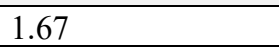 & $\square \square \square$ & 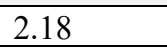 \\
\hline Productivity losses & 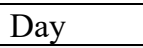 & 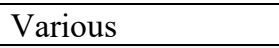 & $\square \square \square$ & \\
\hline \multicolumn{5}{|l|}{ Costs in other sectors } \\
\hline Travel & 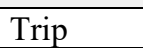 & $\square \square \square$ & $\square \square \square$ & \\
\hline
\end{tabular}

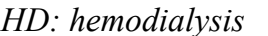

$\square$

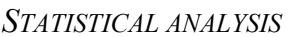

Descriptive analysis of the patients' characteristics, consumption of different resources and

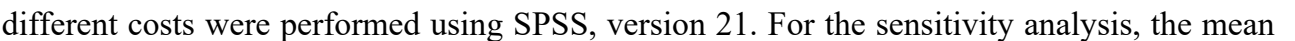

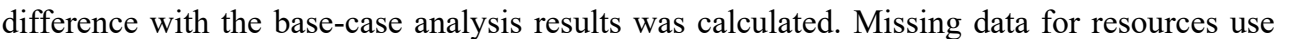
प

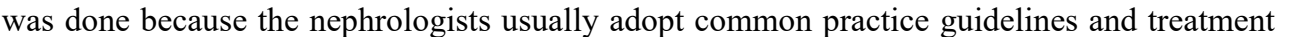

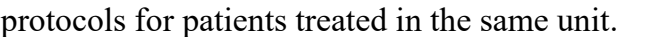

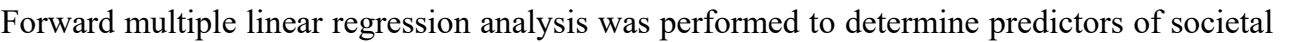

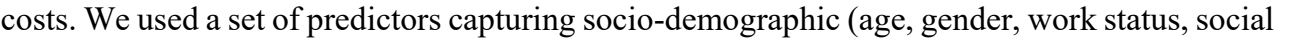

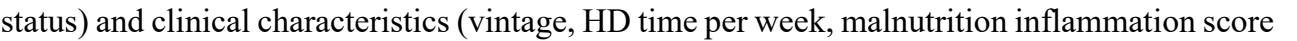

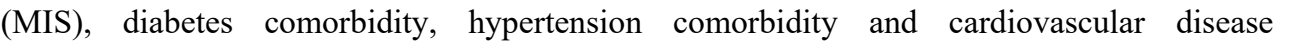

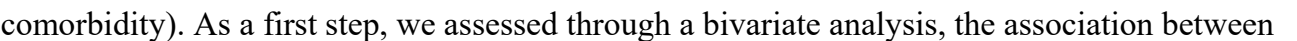

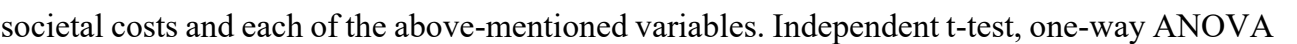

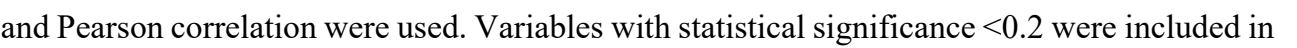

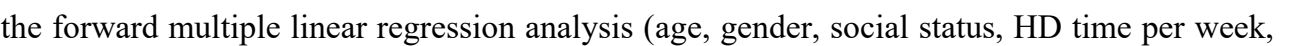

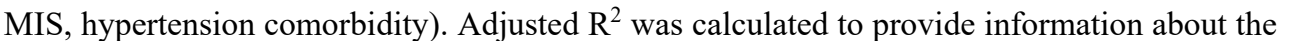

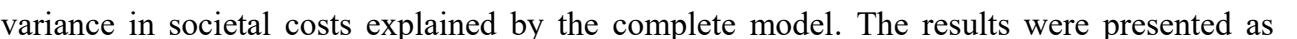

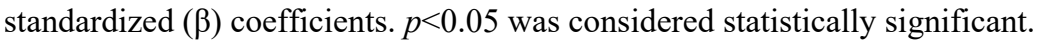




\section{RESULTS}

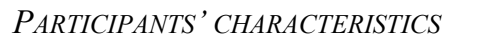

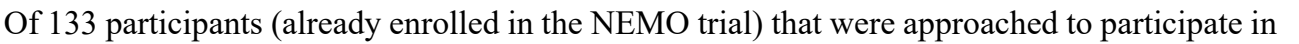

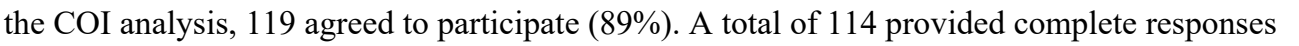

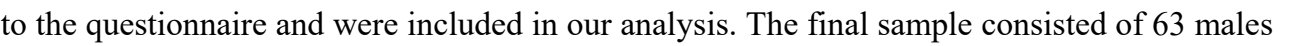

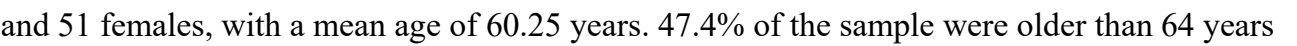

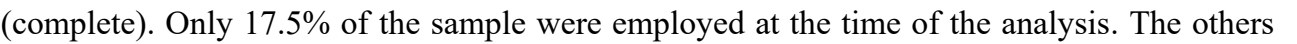

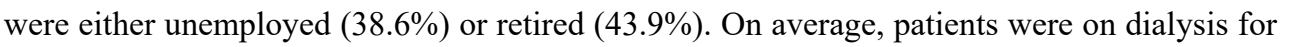

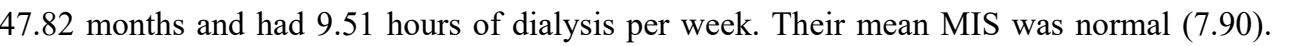

Patients' characteristics are further displayed in Table 2.

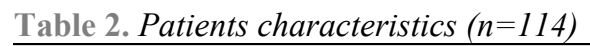

\begin{tabular}{|c|c|}
\hline Socio-demographic characteristics & $\mathbf{N}(\%)$ \\
\hline Gender, Male & 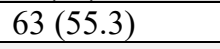 \\
\hline Social status & $\square$ \\
\hline \begin{tabular}{ll|l|} 
\\
\end{tabular} & 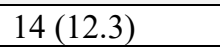 \\
\hline 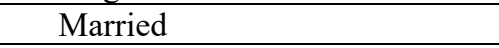 & 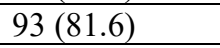 \\
\hline$\square \square \square \square \square$ & 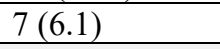 \\
\hline Educational level & $\square$ \\
\hline 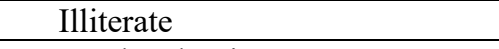 & 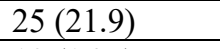 \\
\hline 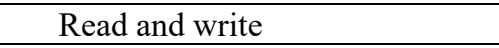 & 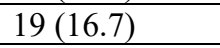 \\
\hline 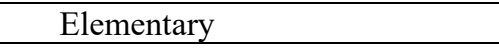 & 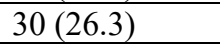 \\
\hline 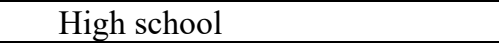 & 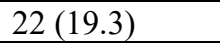 \\
\hline 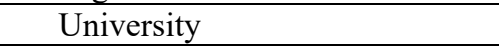 & 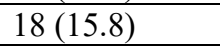 \\
\hline \multirow[t]{2}{*}{ Employment status, Employed } & 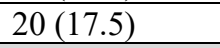 \\
\hline & Mean (SD) \\
\hline Age (years) & 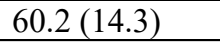 \\
\hline Clinical characteristics & $\mathbf{N}(\%)$ \\
\hline Primary cause of HD initiation & $\square$ \\
\hline$\square \square|||| \mid$ & 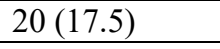 \\
\hline 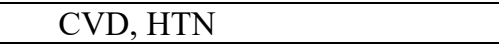 & 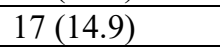 \\
\hline$\square \square\|\|\|\|\|\| \| \square$ & 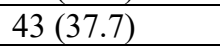 \\
\hline 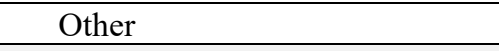 & 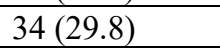 \\
\hline Co-morbidities & $\square$ \\
\hline 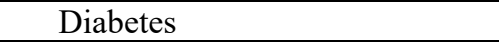 & 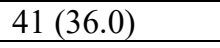 \\
\hline $\begin{array}{ll}\square \square \square \\
\end{array}$ & 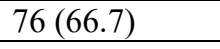 \\
\hline \multirow[t]{2}{*}{$\begin{array}{l}\square \square \square \\
\end{array}$} & 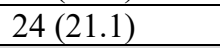 \\
\hline & Mean (SD) \\
\hline HD vintage (months) & 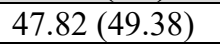 \\
\hline HD time/week (hours) & 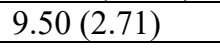 \\
\hline MIS & 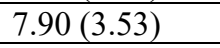 \\
\hline
\end{tabular}

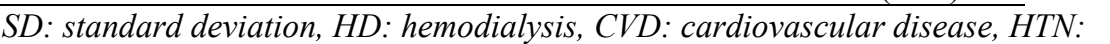

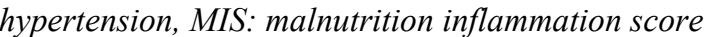




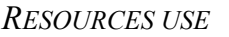

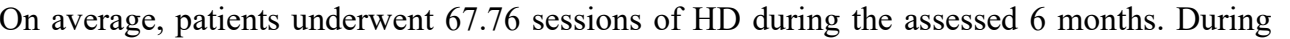

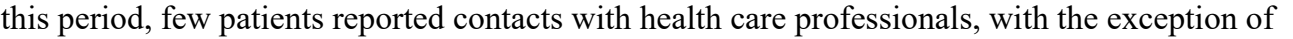

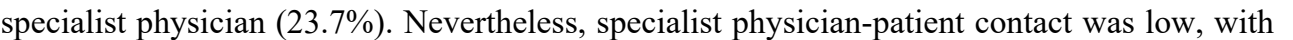

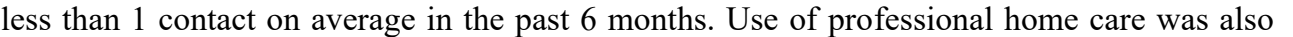

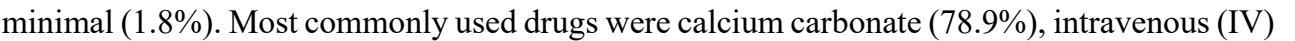

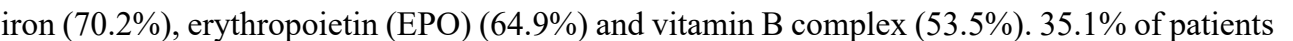

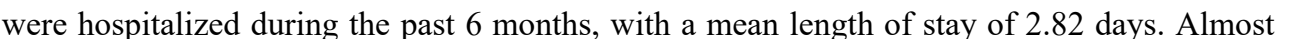

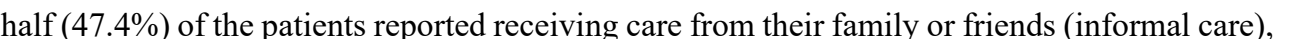

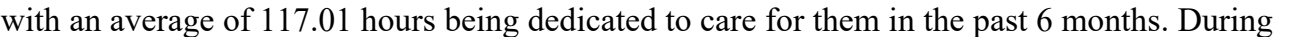

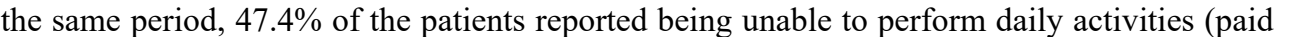

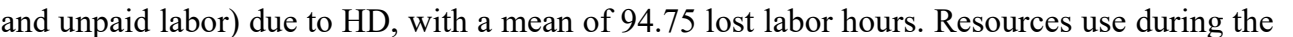

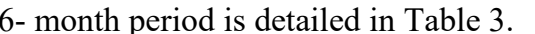

$\square$

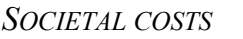

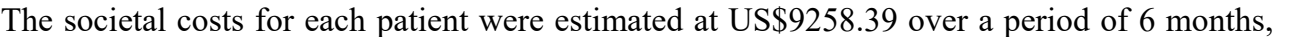

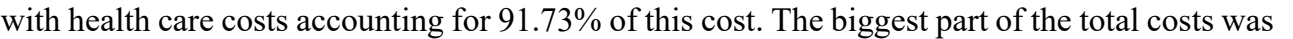

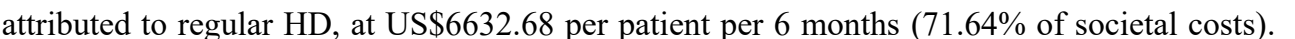

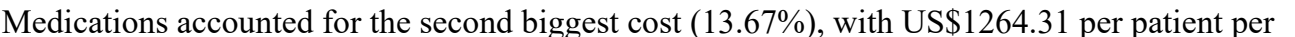

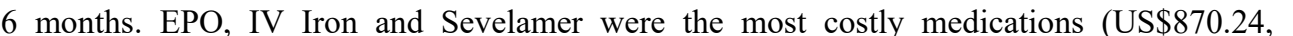

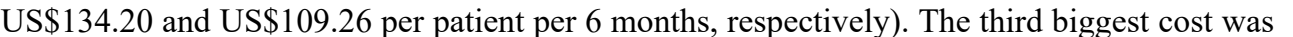

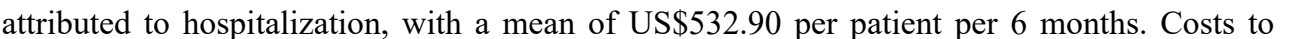

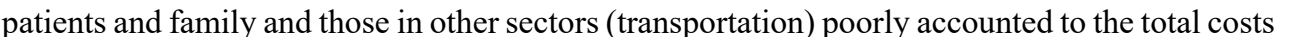

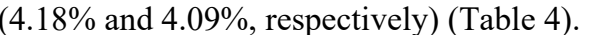

$\square$

$\square$

$\square$

$\square$

$\square$

$\square$

$\square$

$\square$ 


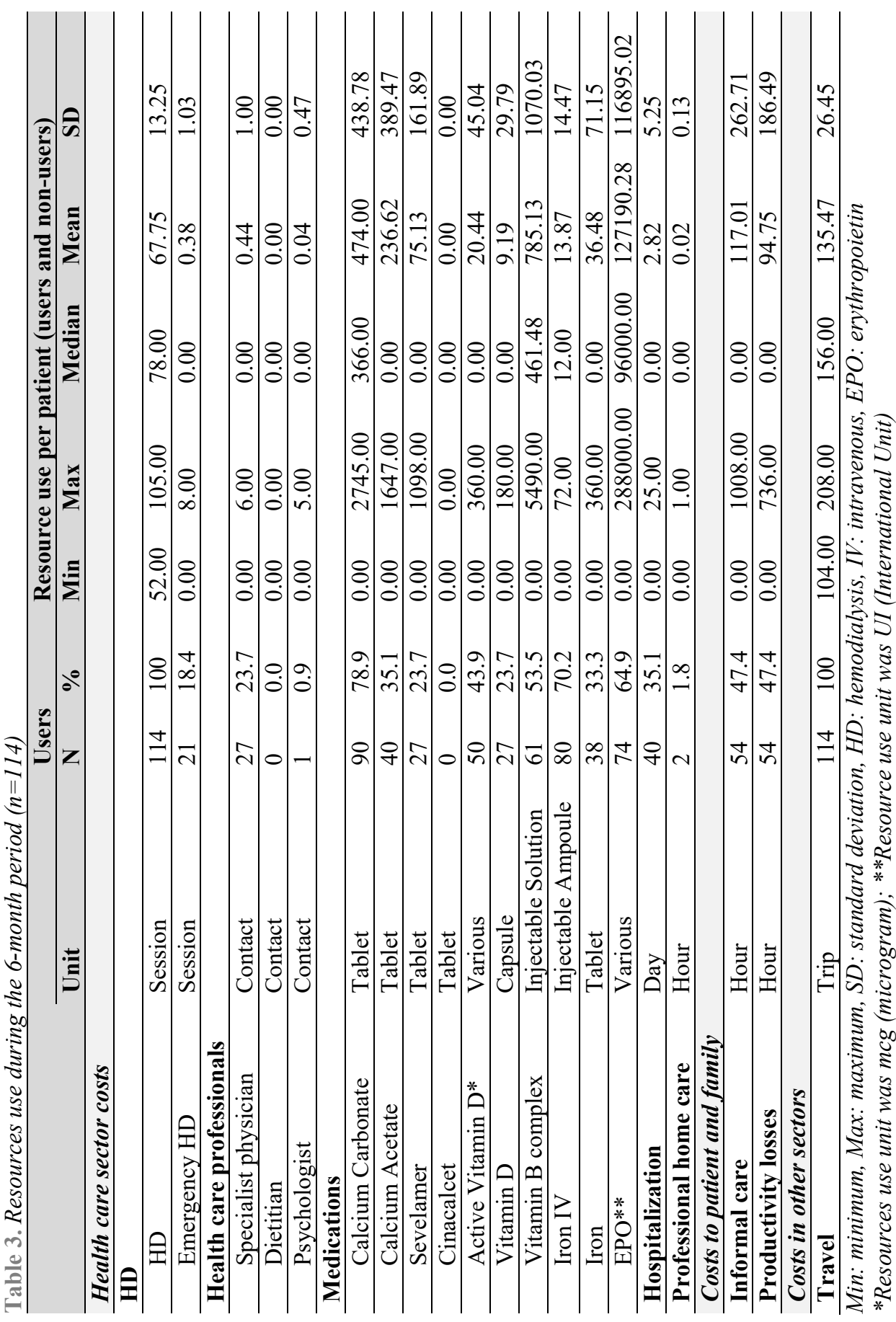




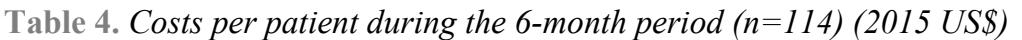

\begin{tabular}{|c|c|c|c|}
\hline$\square$ & Mean cost & $\%$ of total costs & Median cost \\
\hline \multicolumn{4}{|l|}{ Health care sector costs } \\
\hline \multicolumn{4}{|l|}{ HD } \\
\hline पणाए & पामाण & पाणाए & 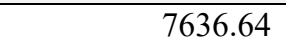 \\
\hline \multirow[t]{2}{*}{ 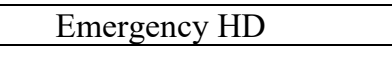 } & $\square \| \square$ & $\square \square$ & 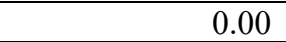 \\
\hline & 6670.27 & 72.05 & 7636.64 \\
\hline \multicolumn{4}{|l|}{ Health care professionals $\square$} \\
\hline 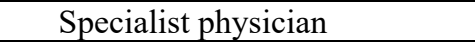 & $\square \square \square$ & $\square \square$ & 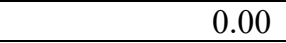 \\
\hline 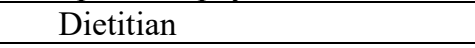 & \begin{tabular}{ll|l|}
$\square \square$ \\
\end{tabular} & 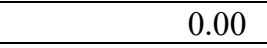 & 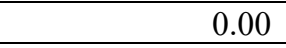 \\
\hline \multirow[t]{2}{*}{ 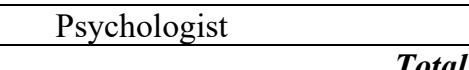 } & 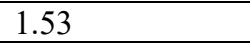 & 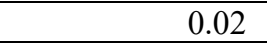 & 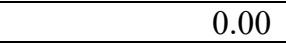 \\
\hline & 24.69 & 0.27 & 0.00 \\
\hline \multicolumn{4}{|l|}{ Medications $\square$} \\
\hline 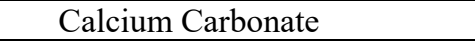 & $\square \| \square \square$ & $\square \amalg$ & $\square \| \square$ \\
\hline 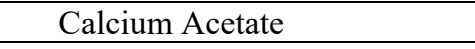 & 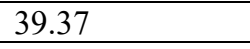 & 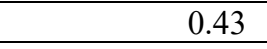 & $\square \|$ \\
\hline 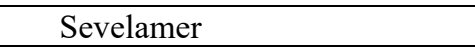 & $\square\|\| \square$ & $\square \square$ & 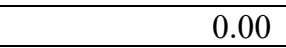 \\
\hline 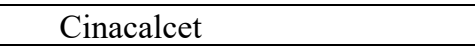 & $\begin{array}{lll}\square \\
\end{array}$ & $\square \square$ & 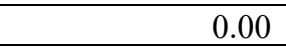 \\
\hline 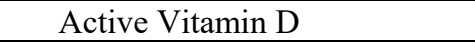 & 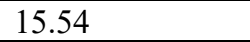 & $\square \square$ & $\square \square$ \\
\hline 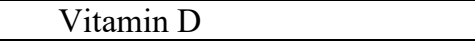 & 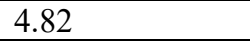 & $\square \amalg$ & $\square \|$ \\
\hline 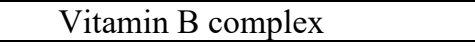 & $\square \square \square$ & $\square \amalg$ & $\square \square \square$ \\
\hline 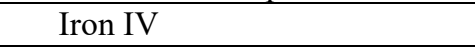 & $\square|\|| \square$ & $\square \square I$ & $\square \square\|\|$ \\
\hline 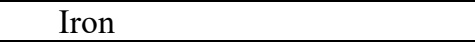 & 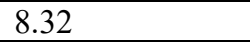 & 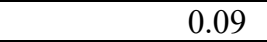 & 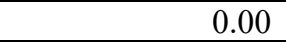 \\
\hline \multirow[t]{2}{*}{$\square \square \square \square$} & $\square|| \Pi \mid \square$ & $\square \square$ & 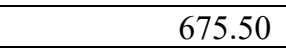 \\
\hline & 1264.31 & 13.67 & 1315.28 \\
\hline Hospitalization & $\square \| \Pi \square$ & $\mathrm{III}$ & 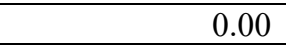 \\
\hline Professional home care & $\square \square \square$ & 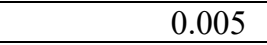 & $\square \Pi \|$ \\
\hline Total & 8492.61 & 91.73 & 8439.30 \\
\hline \multicolumn{4}{|l|}{ Costs to patient and family } \\
\hline Informal care & $\square \| \square$ & $\mathrm{IIII}$ & पाII \\
\hline \multirow[t]{2}{*}{ Productivity losses } & $\square\|\| \square$ & $\begin{array}{lll}\mathrm{III} \\
\end{array}$ & 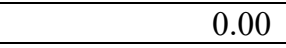 \\
\hline & 387.16 & 4.18 & 131.00 \\
\hline \multicolumn{4}{|l|}{ Costs in other sectors } \\
\hline Travel & $\square\|\| \square$ & $\square \square I$ & $\square \mid\|\| \|$ \\
\hline Total & 378.62 & 4.09 & 435.99 \\
\hline Total societal costs & 9258.39 & 100 & 9201.22 \\
\hline
\end{tabular}

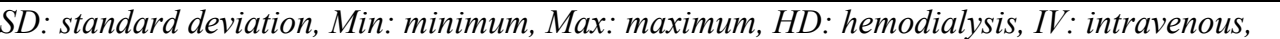

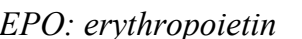

$\square$

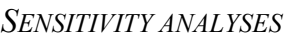

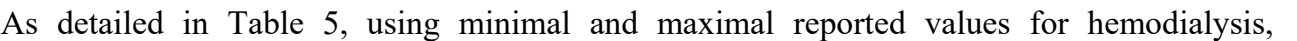

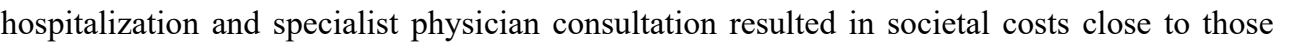

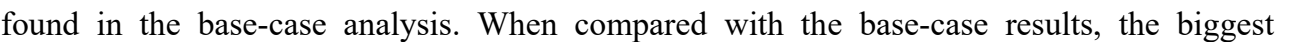

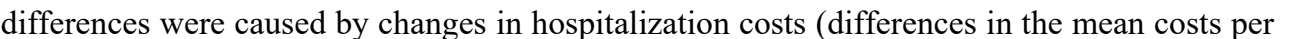

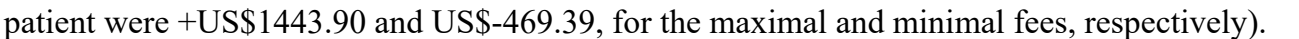




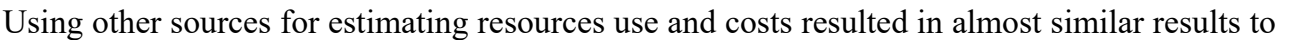

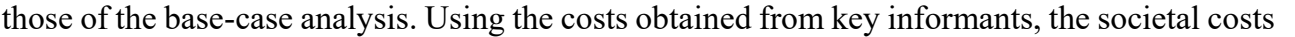

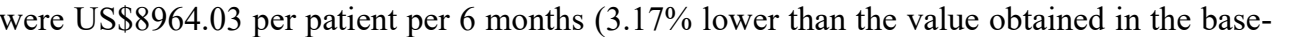

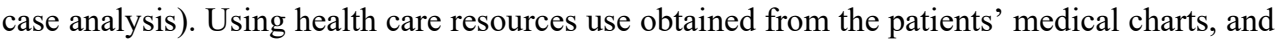

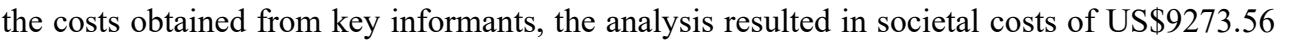

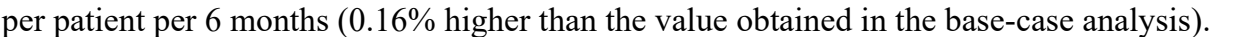

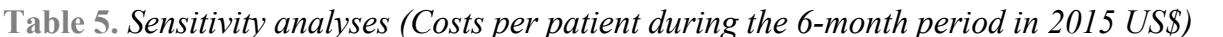

\begin{tabular}{|c|c|c|c|}
\hline \multirow[t]{2}{*}{$\square$} & \multirow{2}{*}{$\begin{array}{l}\text { Mean total } \\
\text { costs } \\
2015 \text { US\$ }\end{array}$} & \multicolumn{2}{|c|}{$\begin{array}{l}\text { Difference from the mean costs } \\
\text { obtained in the base-case analysis }\end{array}$} \\
\hline & & 2015 US\$ & $\%$ \\
\hline \multicolumn{4}{|l|}{ Analysis $1 \square$} \\
\hline \multicolumn{4}{|l|}{ Different costs $\square$} \\
\hline \multicolumn{4}{|l|}{ Hemodialysis $\square$} \\
\hline Minimum & पा\|णम & 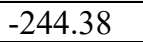 & 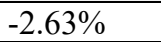 \\
\hline Maximum & 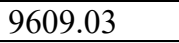 & 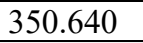 & 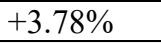 \\
\hline \multicolumn{4}{|l|}{ Hospitalization $\square$} \\
\hline Minimum & 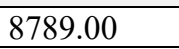 & \begin{tabular}{|l|l|l|l|l|}
$\square$ & \|\|$\|$ \\
\end{tabular} & 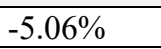 \\
\hline $\begin{array}{ll}\text { Maximum } \\
\end{array}$ & 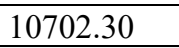 & 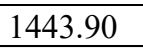 & 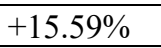 \\
\hline \multicolumn{4}{|l|}{ Specialist physician consultation $\square$} \\
\hline Minimum & $\square\|\| \square$ & \begin{tabular}{l|l|l|l|}
$\|$ & \\
\end{tabular} & 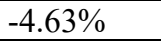 \\
\hline Maximum & 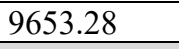 & $\square \square \square \square$ & 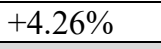 \\
\hline Highest estimate & $\square\|\|\|\|$ & $\square\|\Pi\| \square$ & 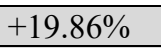 \\
\hline Lowest estimate & $\square 11 \| \Pi 1 \square$ & पा\|ा\|ा & 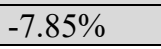 \\
\hline \multicolumn{4}{|l|}{ Analysis $2 \square$} \\
\hline Costs from key informants & 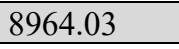 & 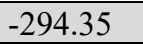 & $\square \| \square \square$ \\
\hline \multicolumn{4}{|l|}{ Analysis $3 \square$} \\
\hline $\begin{array}{l}\text { Resources use from medical charts } \\
\text { and costs from key informants }\end{array}$ & $\square\|\Pi\| \square$ & $\square\|\| \|$ & $\square \square|\Pi| \square \square$ \\
\hline
\end{tabular}

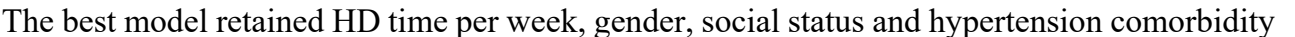

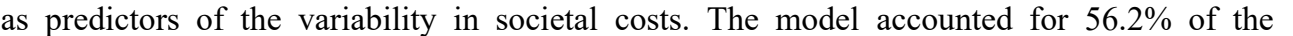

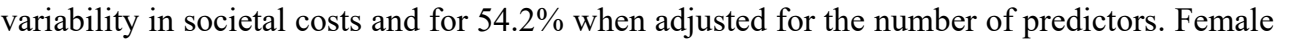

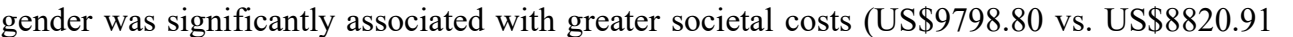

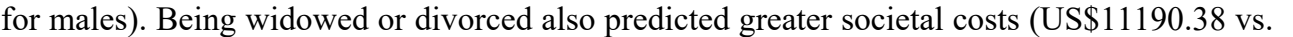

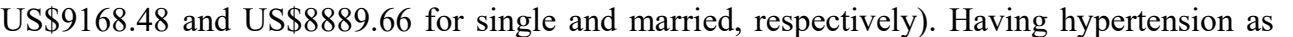

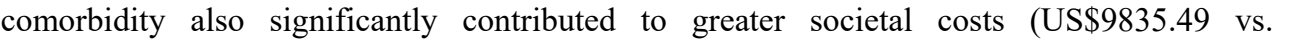

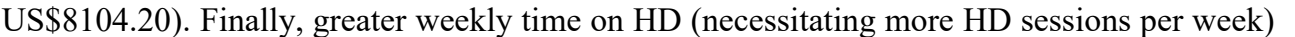

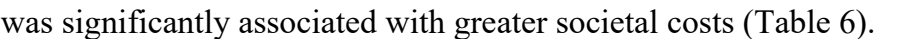




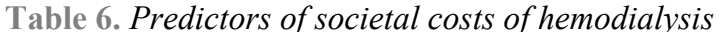

\begin{tabular}{|c|c|c|c|c|}
\hline \multirow[t]{2}{*}{ Predictor } & \multirow{2}{*}{$\begin{array}{l}\text { Standardized } \\
\text { Coefficients }\end{array}$} & \multirow[t]{2}{*}{$\mathbf{p}$} & \multicolumn{2}{|c|}{ 95.0\% Confidence Interval } \\
\hline & & & Lower Bound & Upper Bound \\
\hline 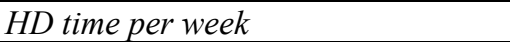 & 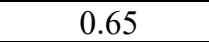 & $\square \square\|\|$ & $\square \square \square \square$ & $\square\|\| \square$ \\
\hline 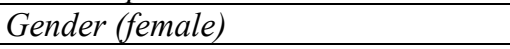 & 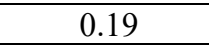 & $\square \square \| \square$ & 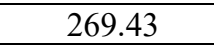 & $\square\|\| \|$ \\
\hline 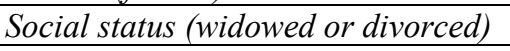 & 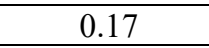 & पाण & $\square \square \mid \square \square$ & $\square\|\| \| \square$ \\
\hline 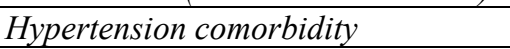 & 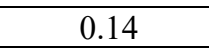 & $\square \square \square$ & $\square \square \square$ & $\square|||\Pi| \square$ \\
\hline
\end{tabular}

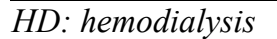

$\square$

\section{DISCUSSION}

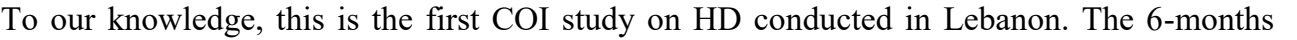

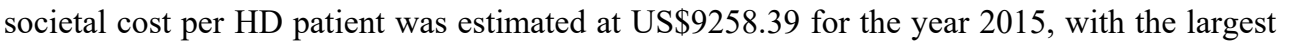

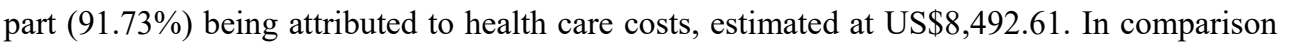

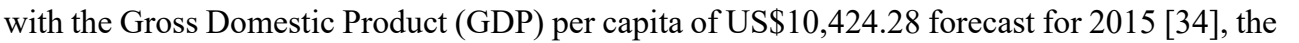

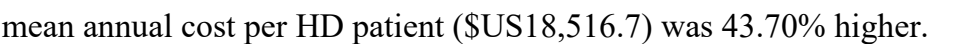

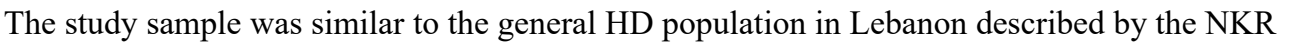

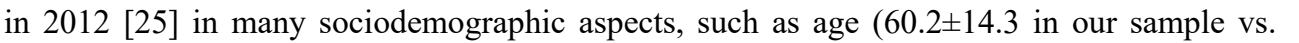

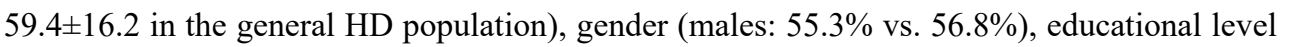

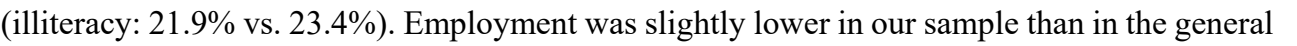

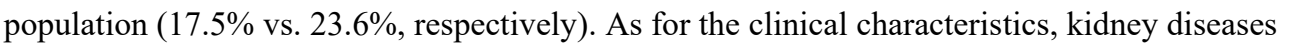

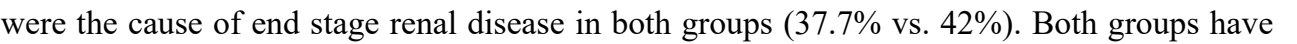

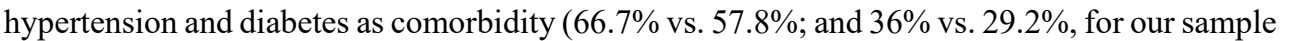

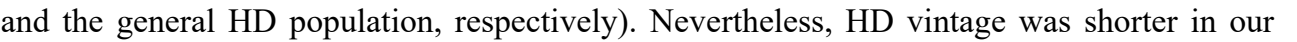

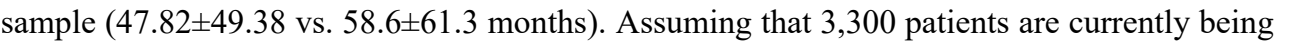

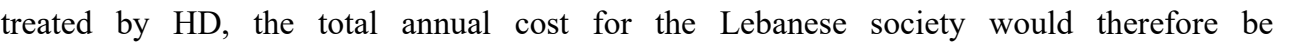

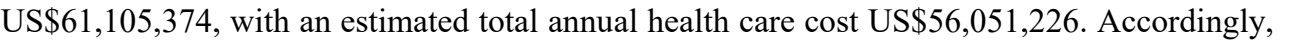

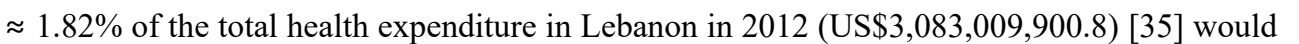

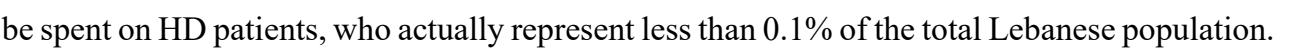

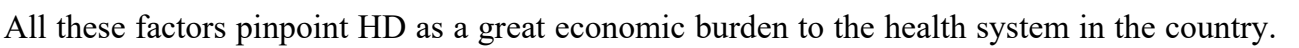

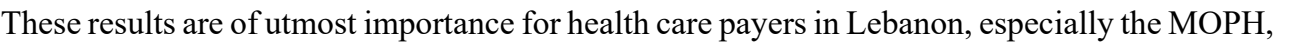

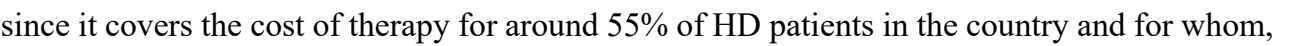
about $8 \%$ of the Ministry's budget is allocated $\square[\mid \| ा$

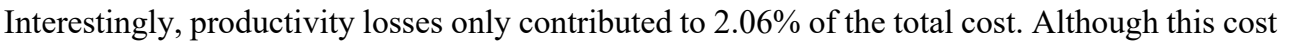

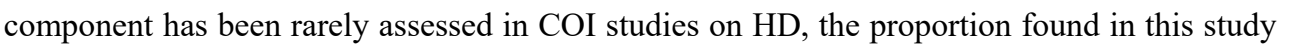
प

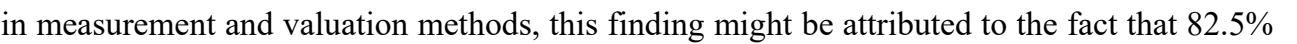




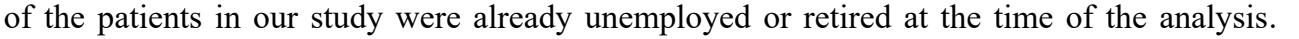

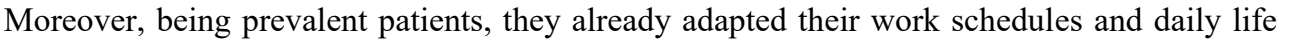

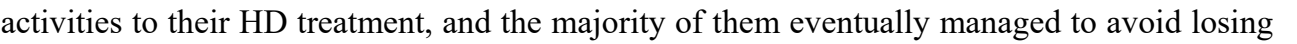

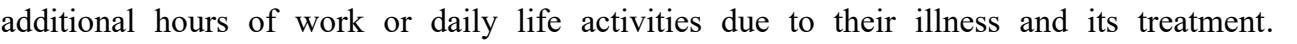

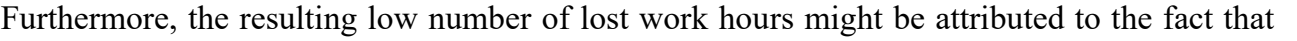

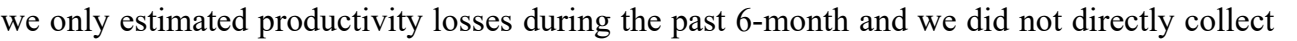

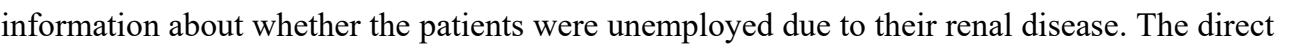

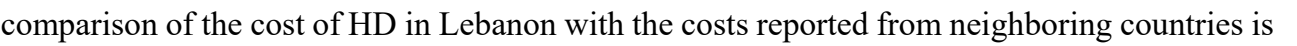

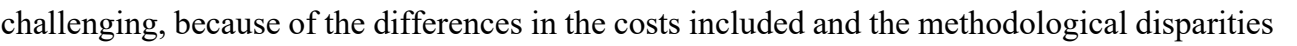

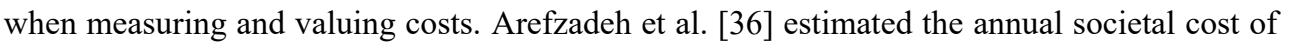

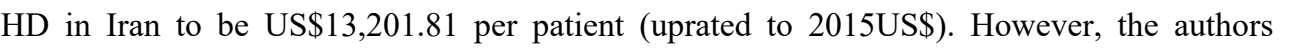

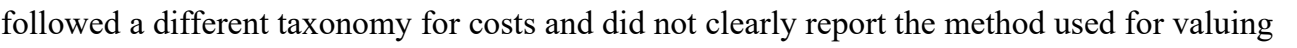

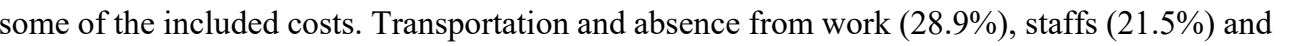
ए

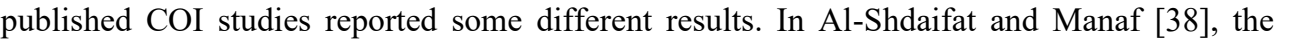

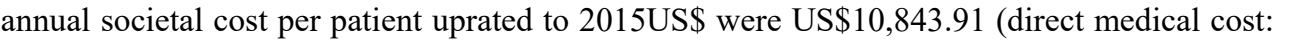

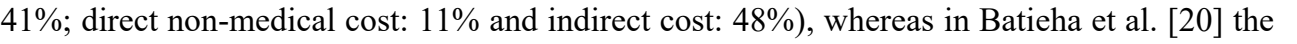

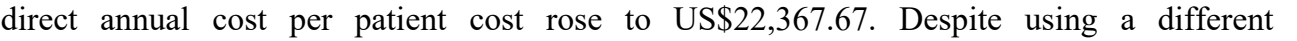

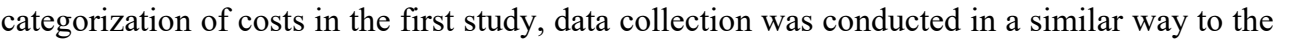

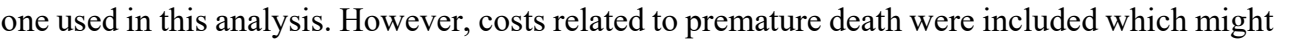

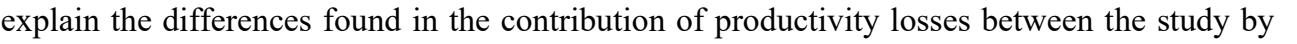

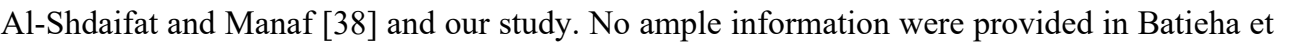

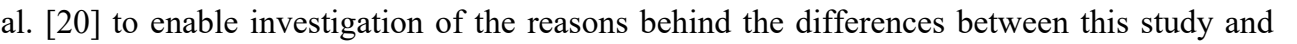
पाणाए

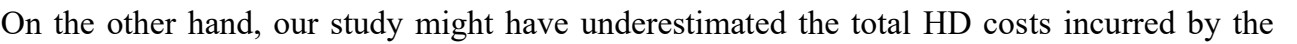

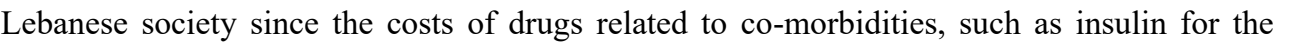

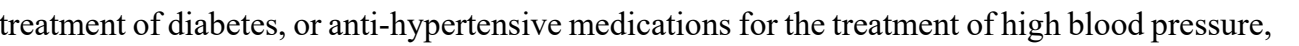

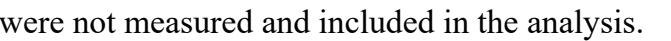

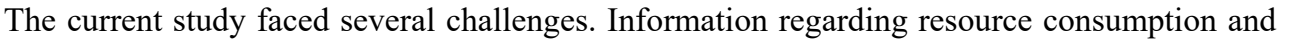

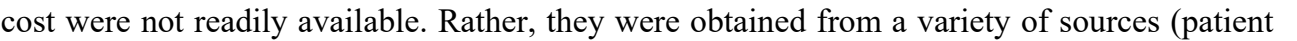

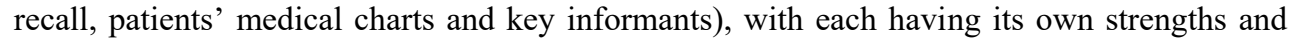

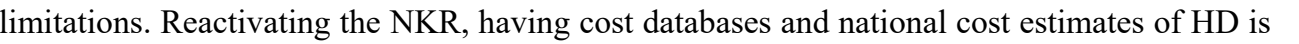

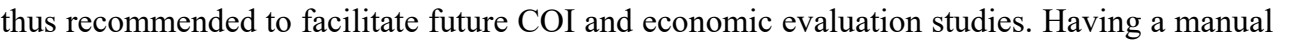

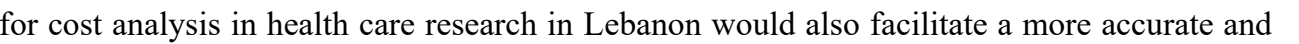

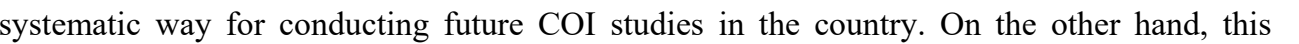




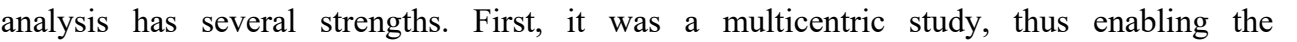

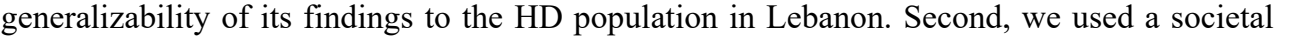

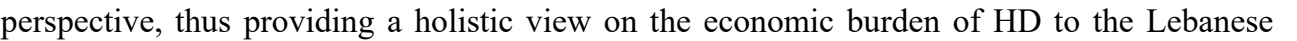

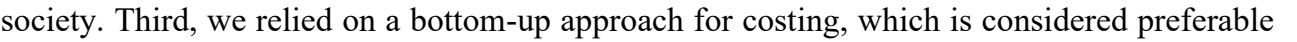

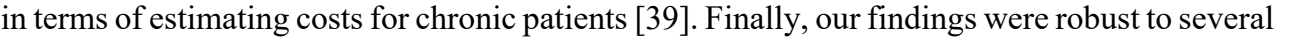

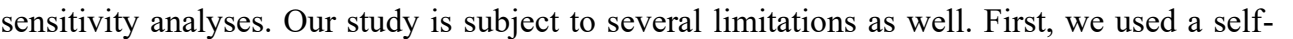

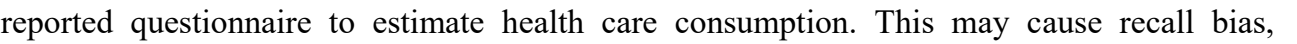

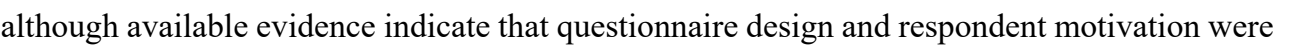

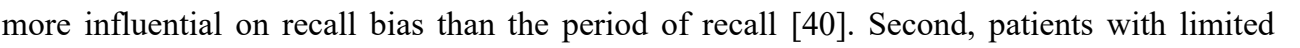

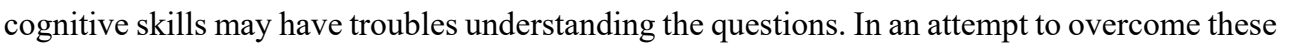

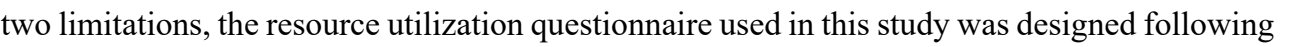

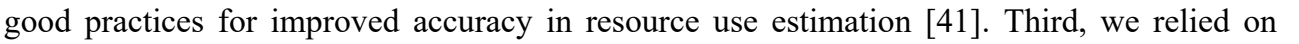

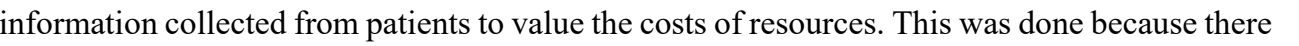

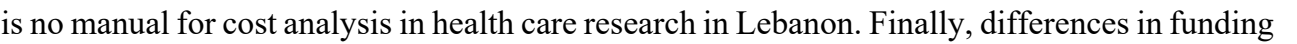

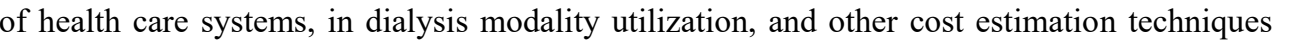

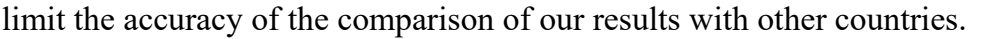

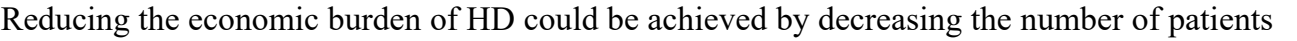

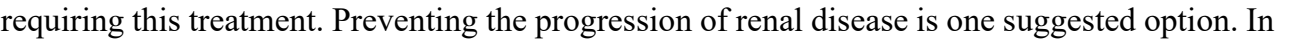

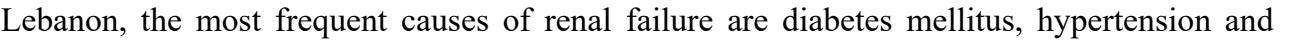

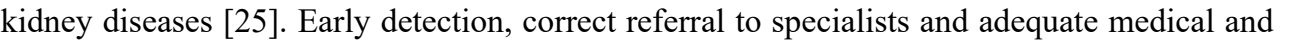

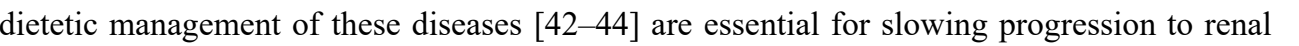

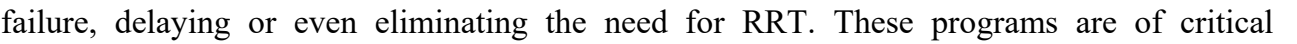

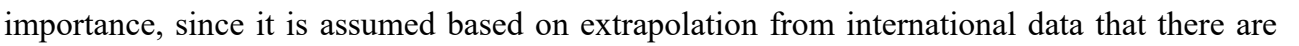

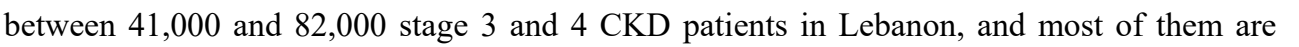

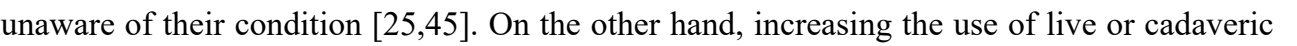

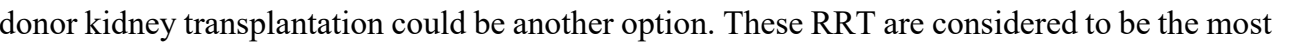

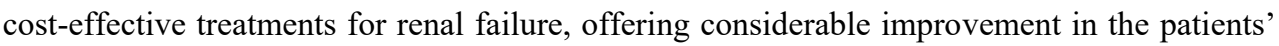
पा |

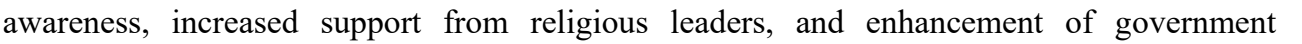

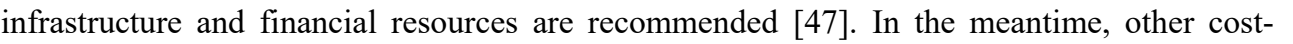

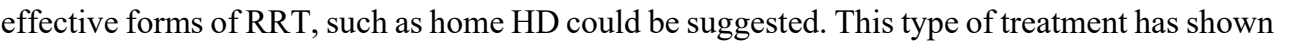

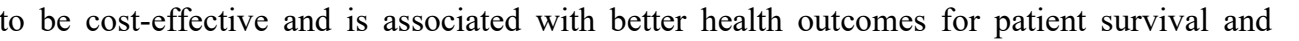

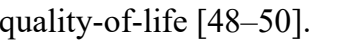

$\square$

$\square$ 


\section{CONCLUSION}

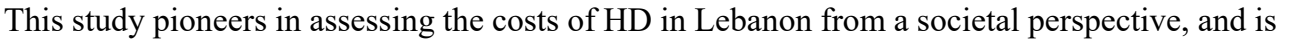

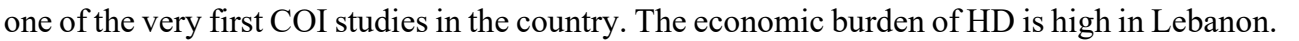

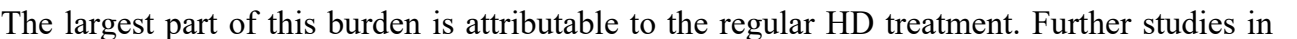

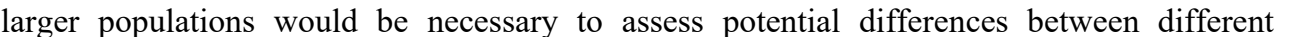

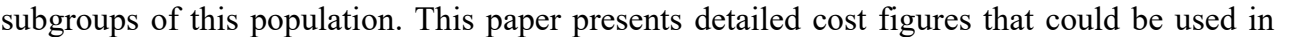

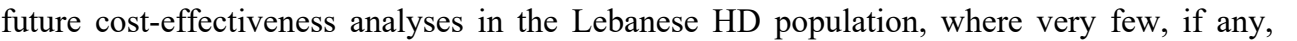

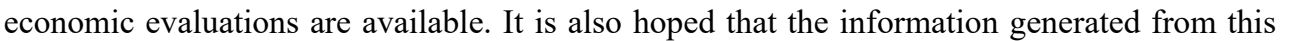

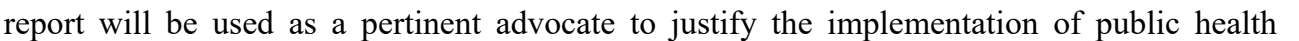

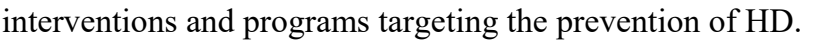

\section{Acknowledgments}

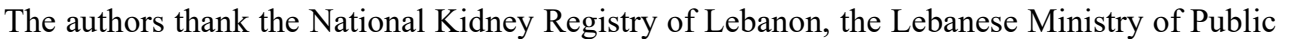

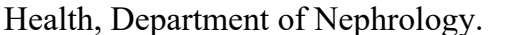




\section{REFERENCES}

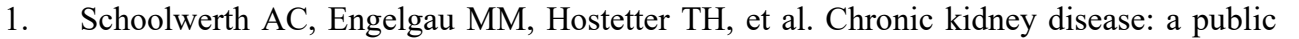

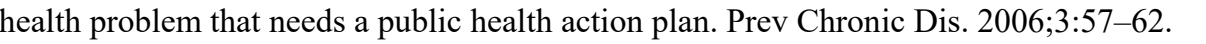

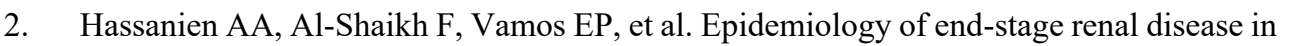

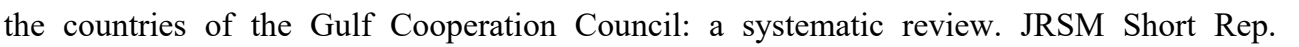
$\square[\Pi \Pi m-\square \Pi$

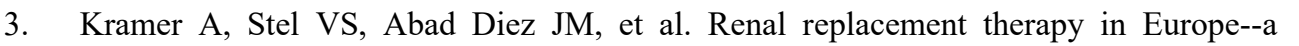

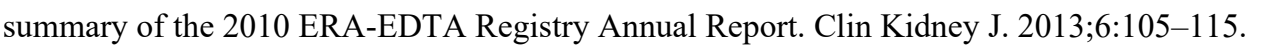

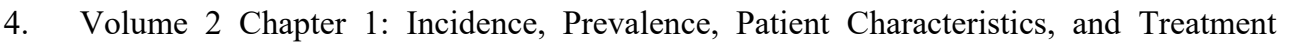

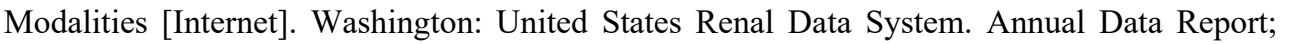

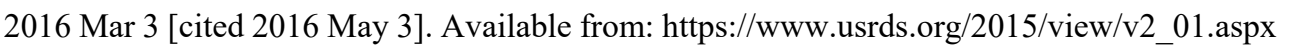

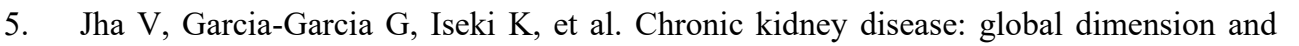

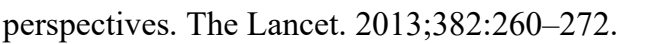

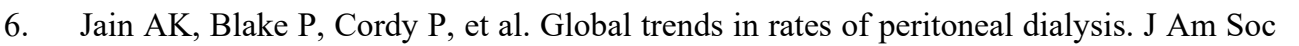

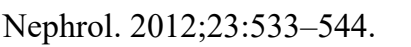

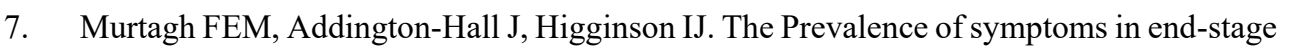

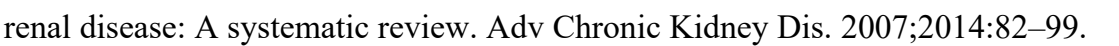

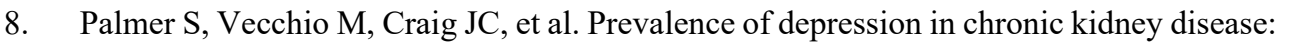

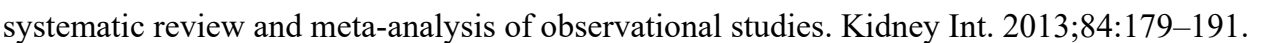

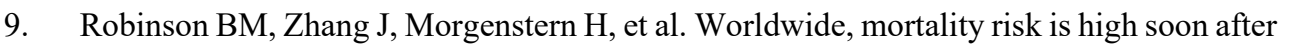

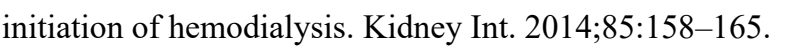

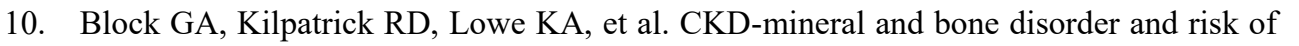

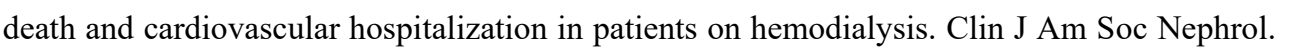

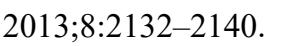

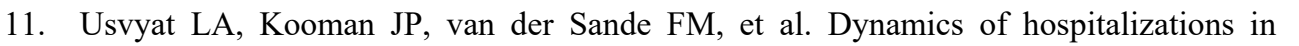

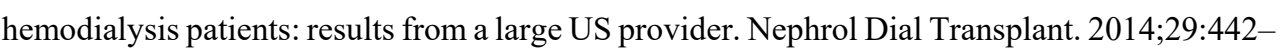
$\square 1$

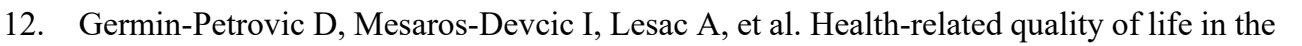

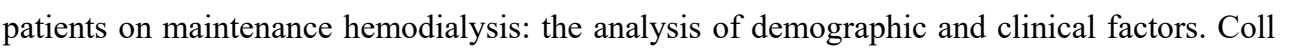

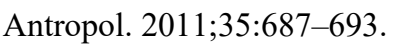

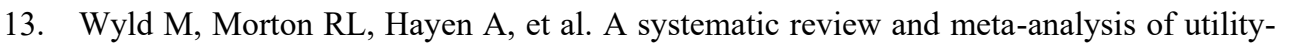

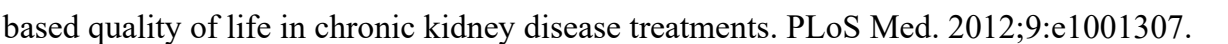

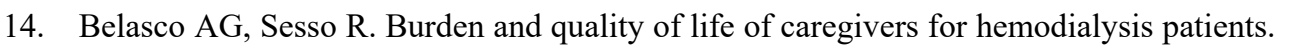

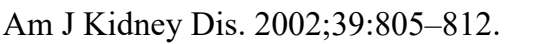




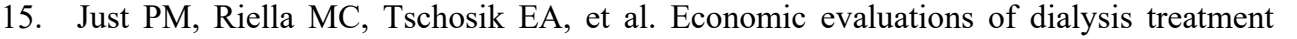

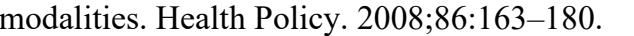

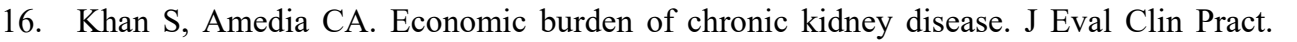
पामाणाम-पाएा

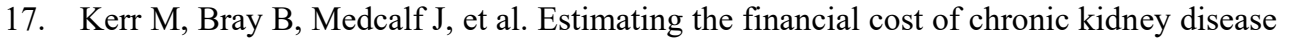

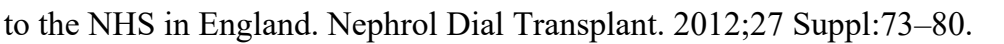

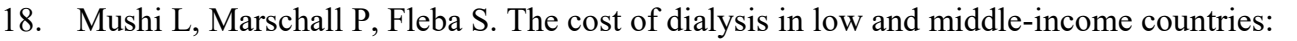

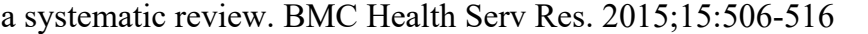

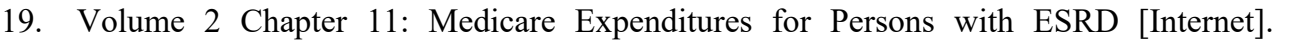

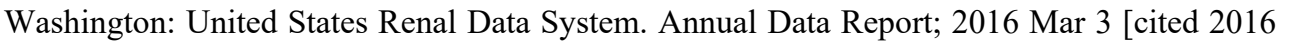

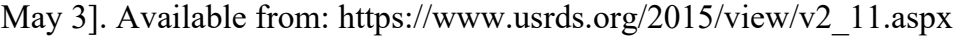

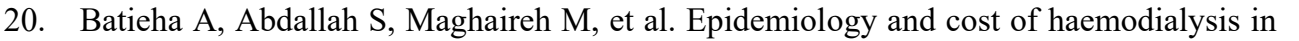

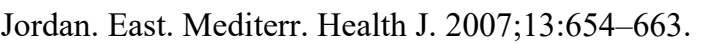

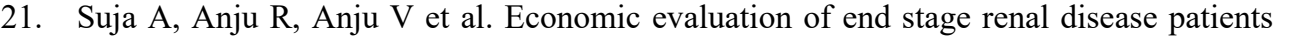

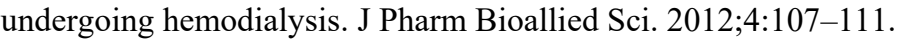

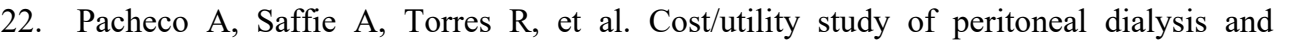

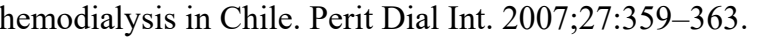

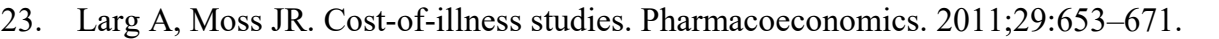

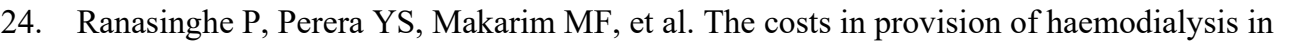

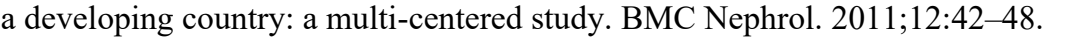

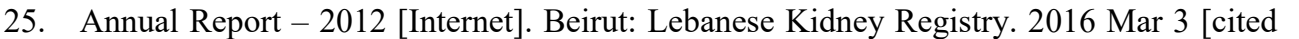

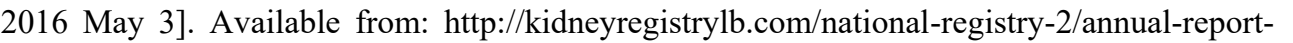
$\square 110$

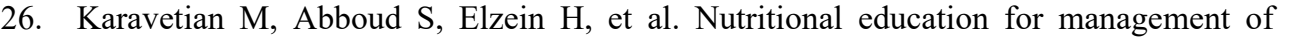

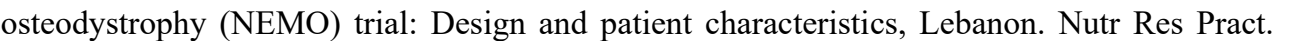

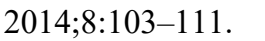

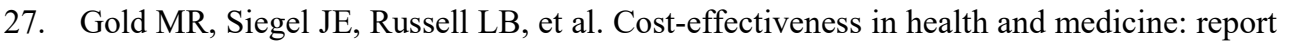

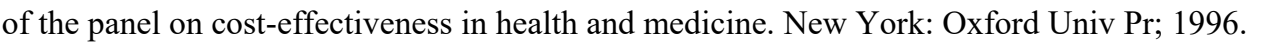

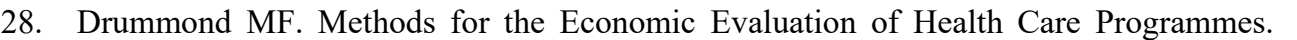

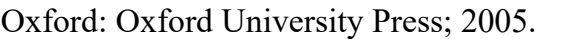

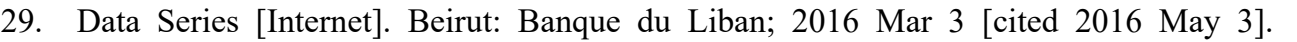

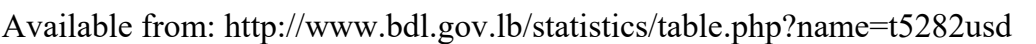




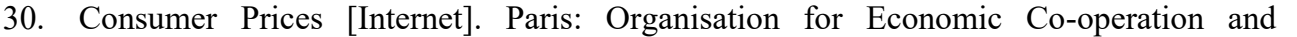

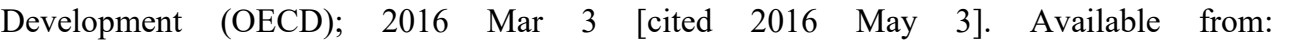

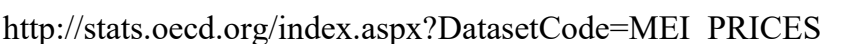

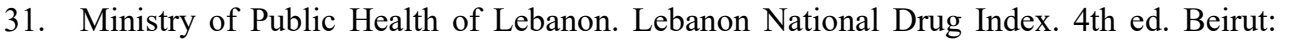

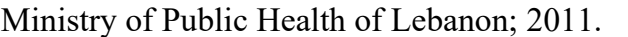

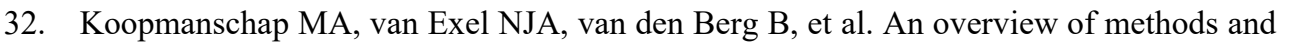

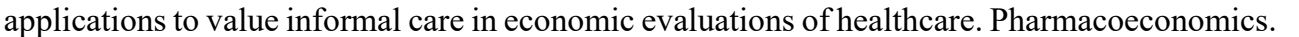
पापणाणम-पाएा

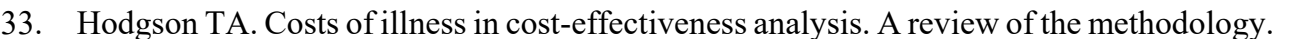

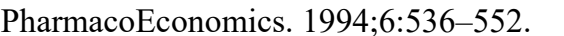

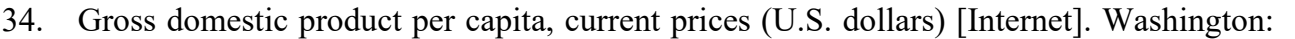

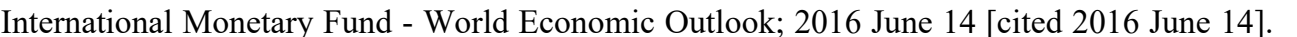

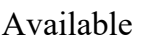
미밈

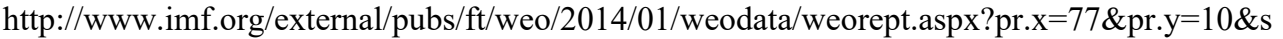

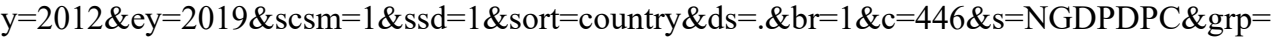
$\square \square \square$

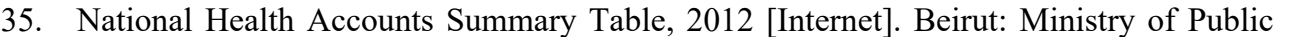

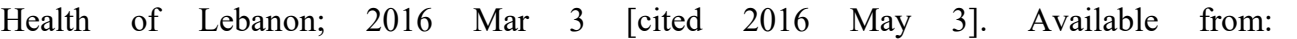

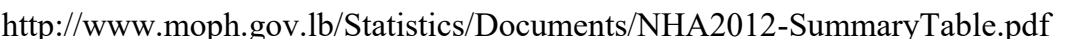

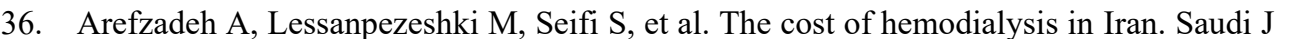

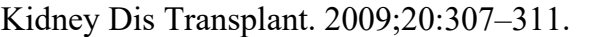

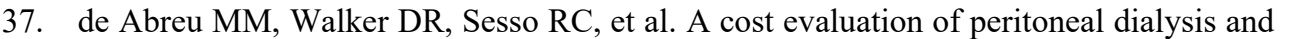

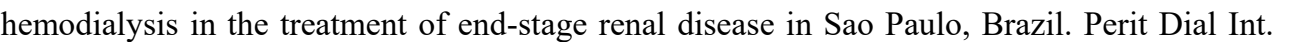

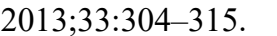

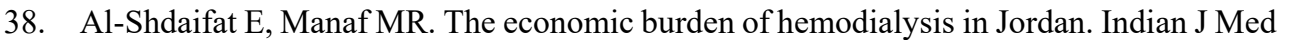

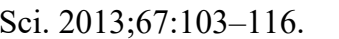

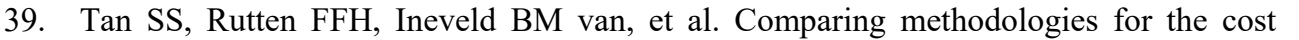

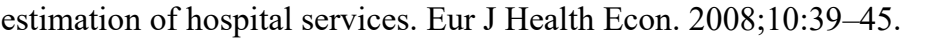

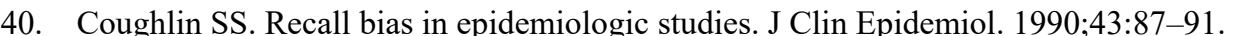

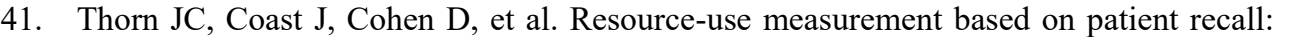

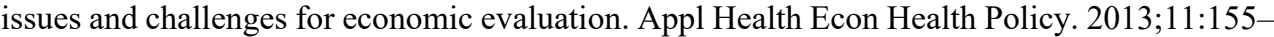
$\square \mathrm{II}$

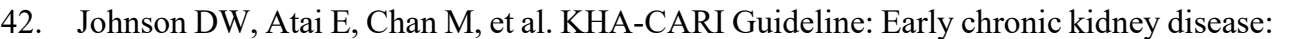

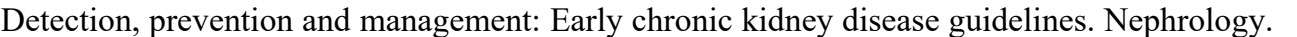

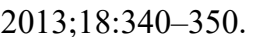




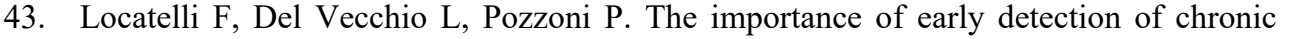

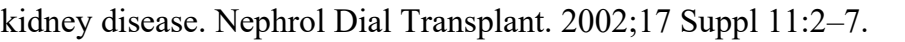

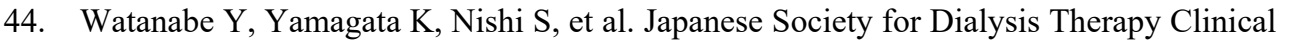

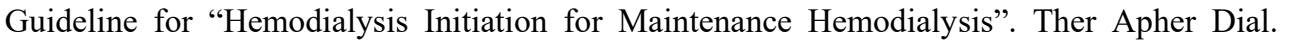
$\square|| \Pi|\Pi-\square| \Pi \mid \square$

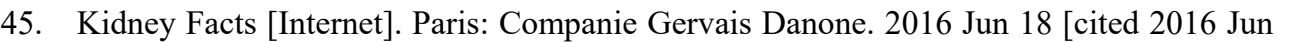

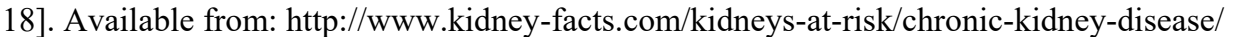

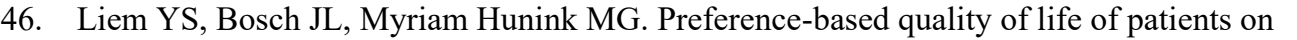

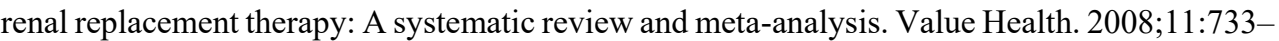
$\square 10$

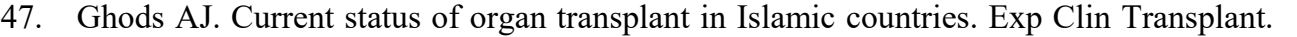

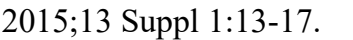

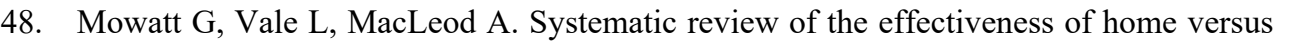

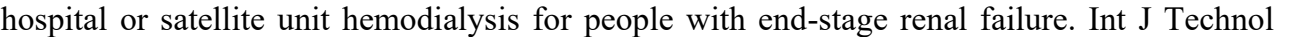

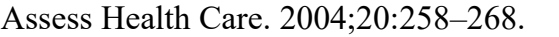

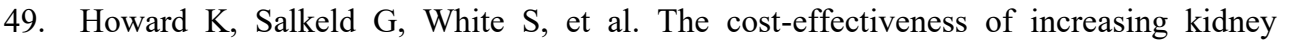

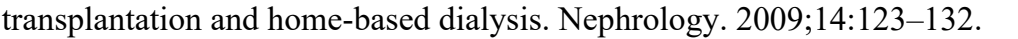

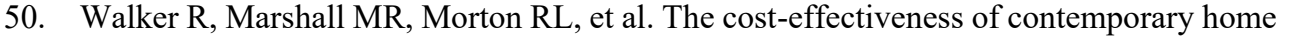

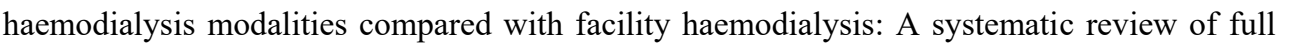

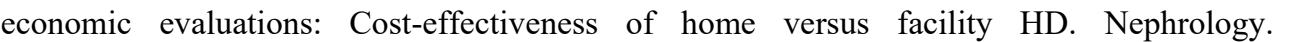

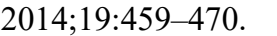


Appendix 1: Resource utilization questionnaire

\begin{tabular}{|c|c|c|c|c|c|c|}
\hline$\square$ & \multicolumn{6}{|c|}{ 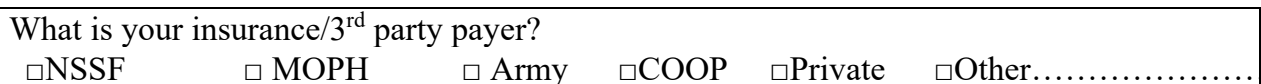 } \\
\hline$\square$ & \multicolumn{6}{|c|}{ 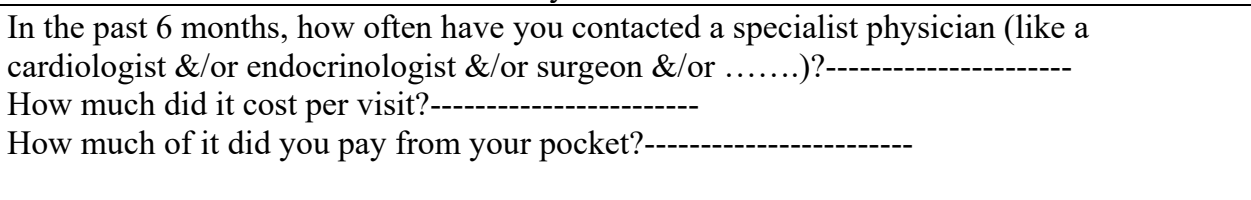 } \\
\hline$\square$ & \multicolumn{6}{|c|}{ 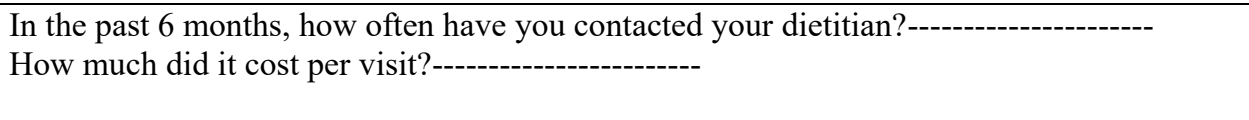 } \\
\hline$\square$ & \multicolumn{6}{|c|}{ 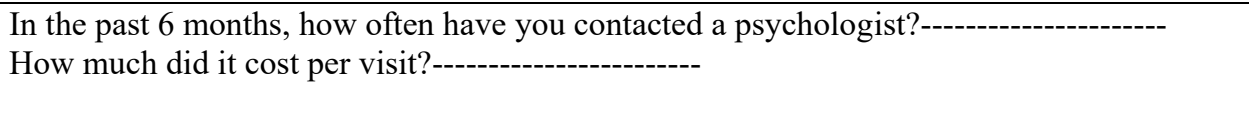 } \\
\hline$\square$ & \multicolumn{6}{|c|}{ 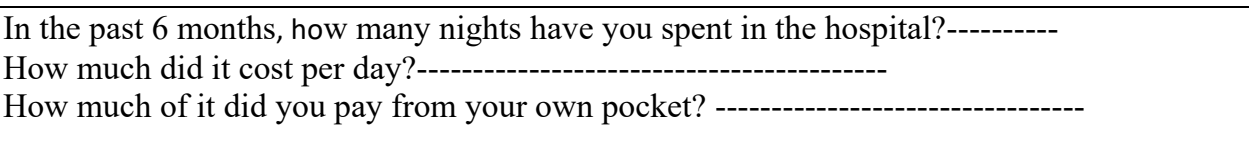 } \\
\hline$\square$ & \multicolumn{6}{|c|}{ 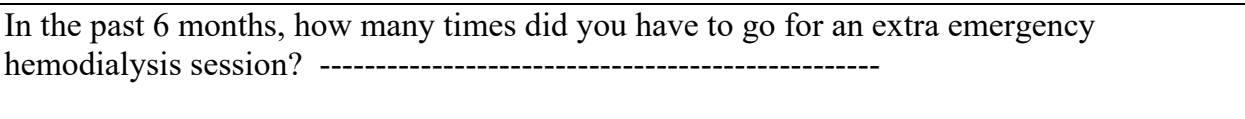 } \\
\hline$\square$ & \multicolumn{6}{|c|}{ 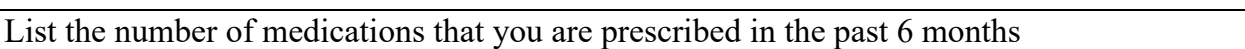 } \\
\hline & \begin{tabular}{|l|l}
$\square$ &
\end{tabular} & 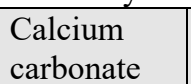 & 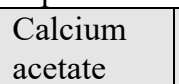 & 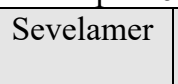 & 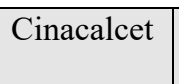 & पणापणा \\
\hline & 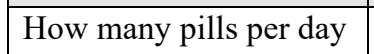 & $\square$ & $\square$ & $\square$ & $\square$ & $\square$ \\
\hline & $\square$ & 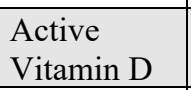 & 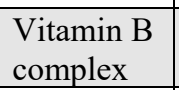 & 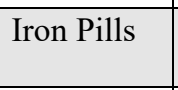 & 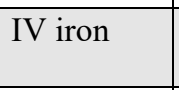 & \\
\hline & 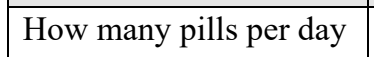 & $\square$ & $\square$ & $\square$ & $\square$ & $\square$ \\
\hline & \multicolumn{6}{|c|}{ 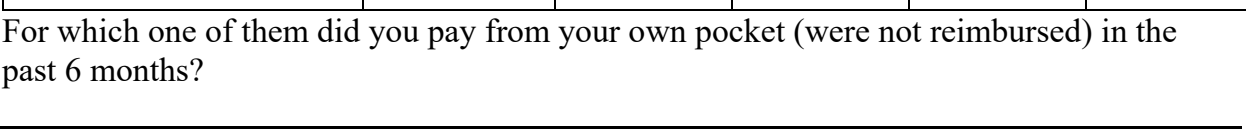 } \\
\hline & \multicolumn{6}{|l|}{$\square$} \\
\hline & \multicolumn{6}{|l|}{$\square$} \\
\hline$\square$ & \multicolumn{6}{|c|}{ 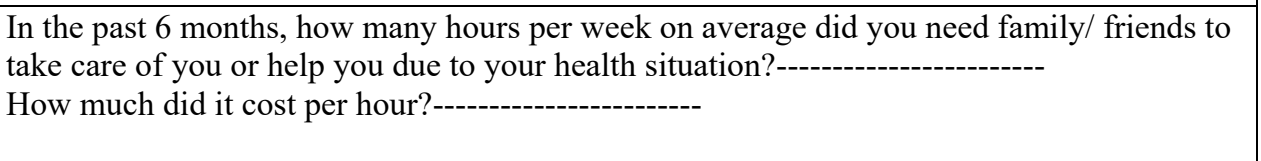 } \\
\hline$\square$ & \multicolumn{6}{|c|}{ 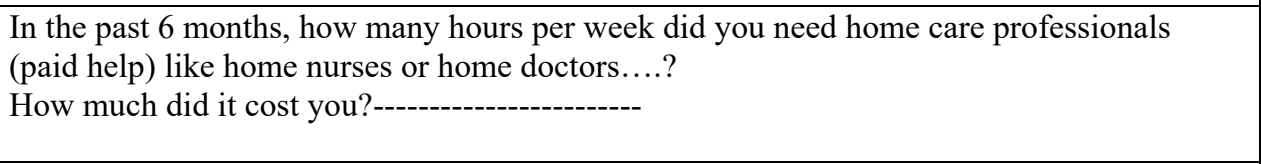 } \\
\hline$\square 1$ & \multicolumn{6}{|c|}{ 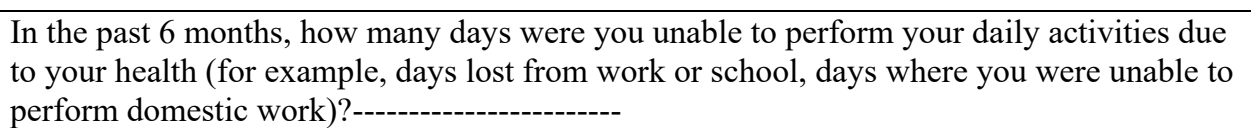 } \\
\hline
\end{tabular}




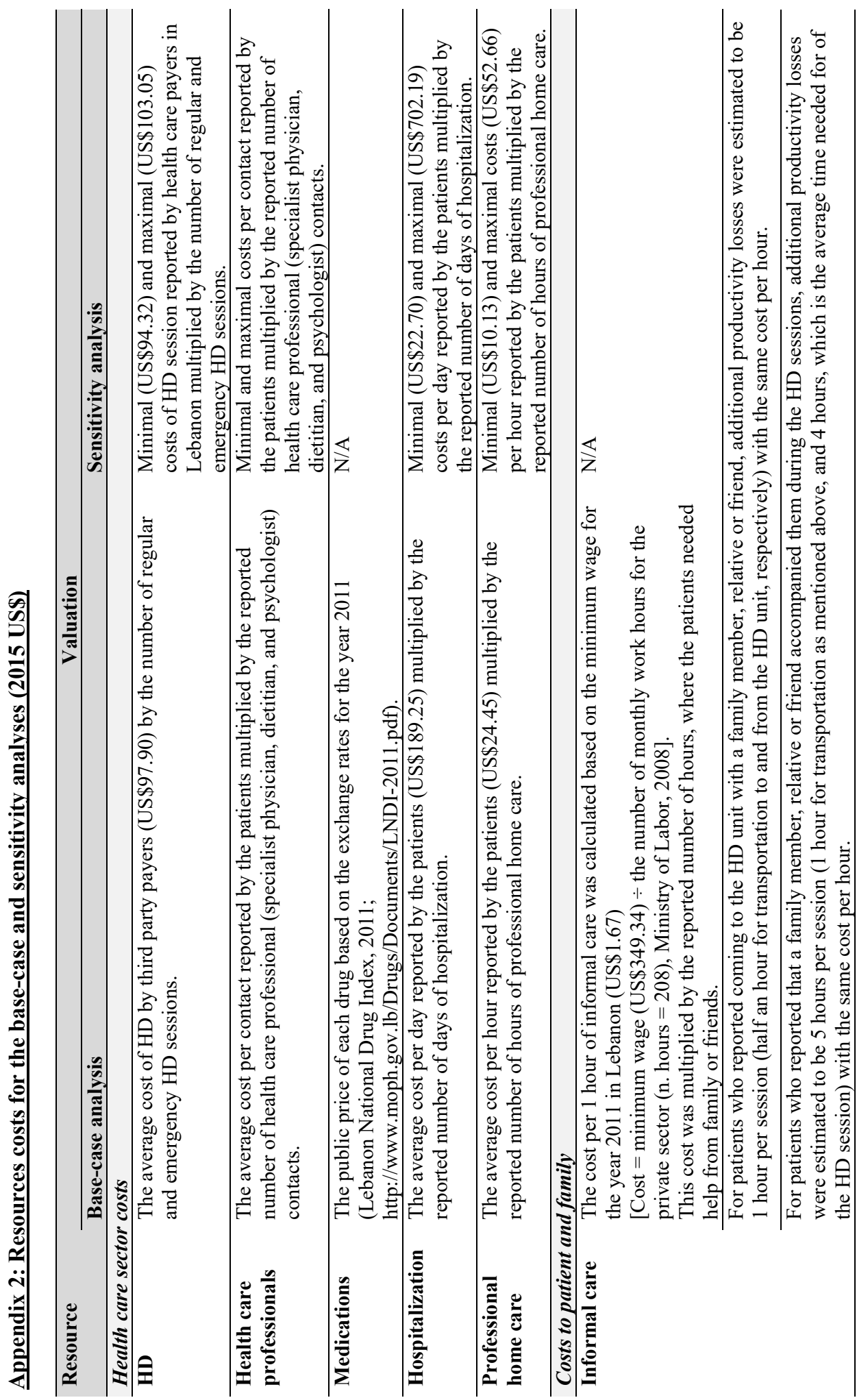




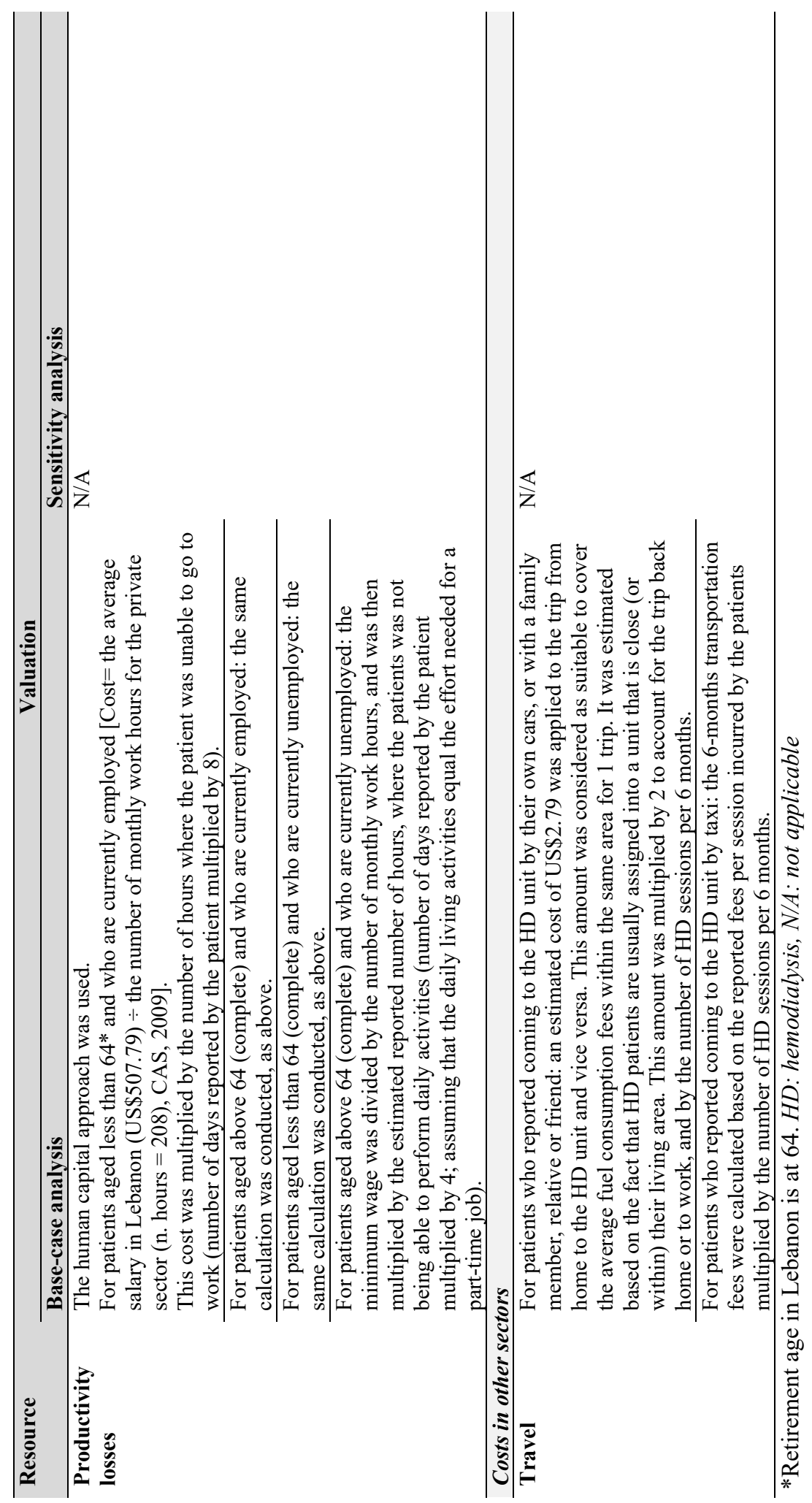



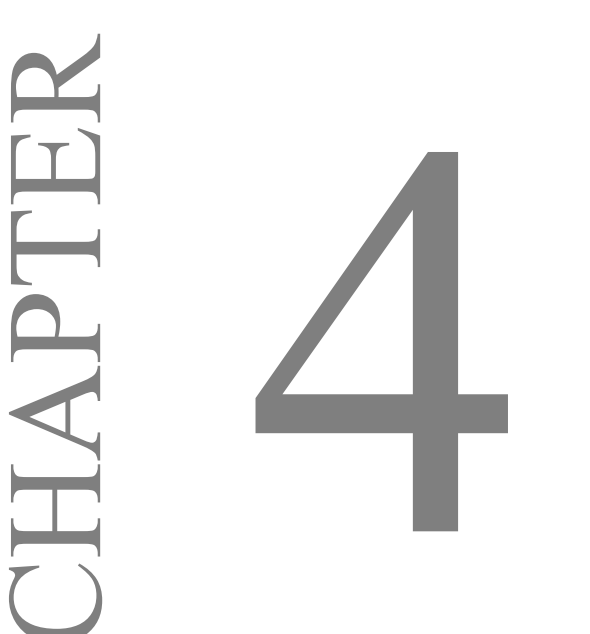

EFFECT OF STAGE-BASED EDUCATION PROVIDED BY DEDICATED DIETITIANS ON HYPERPHOSPHATAEMIC HAEMODIALYSIS PATIENTS: RESULTS FROM THE NUTRITION EDUCATION FOR MANAGEMENT OF OSTEODYSTROPHY RANDOMISED CONTROLLED TRIAL $\square$

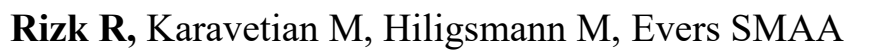




\section{Abstract}

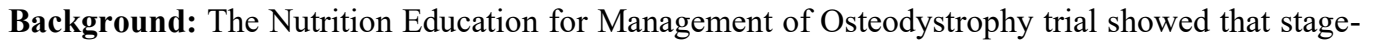

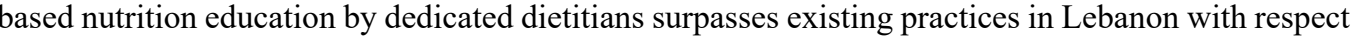
एயா

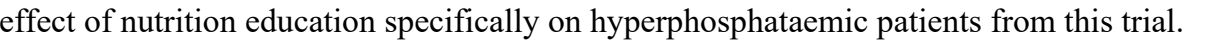

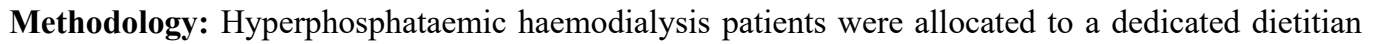

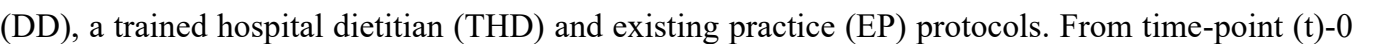

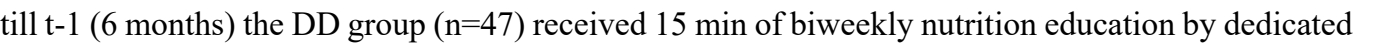

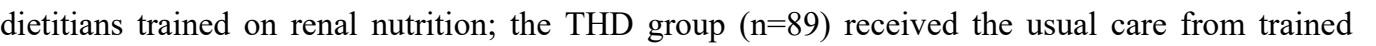

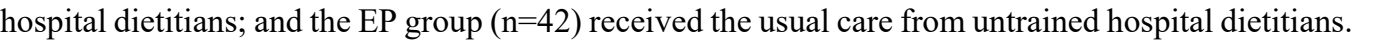

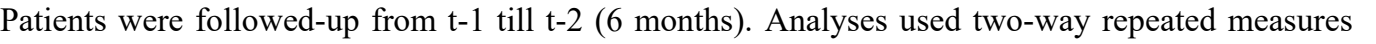

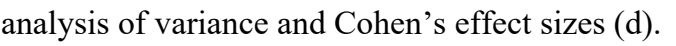

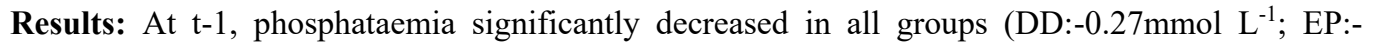

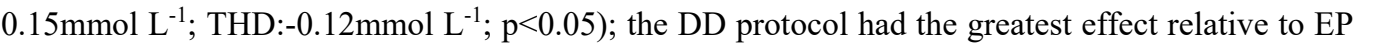

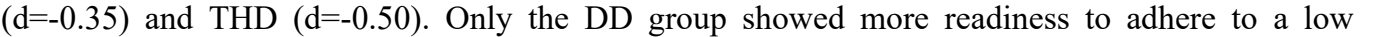

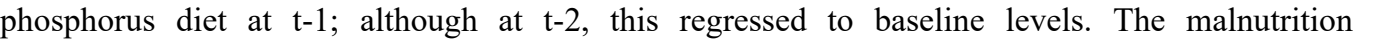
ए

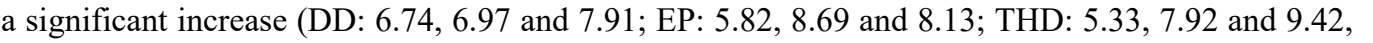

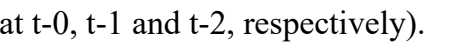

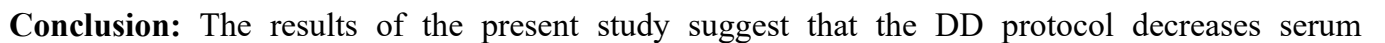

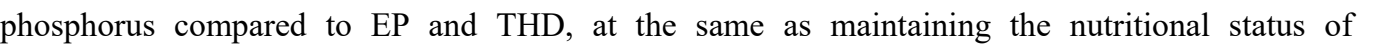

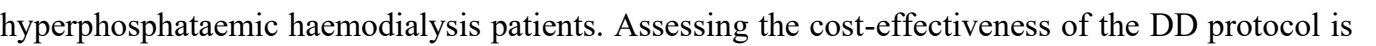
एणमिएाणा

\section{KEYWORDS}

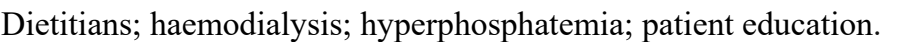




\section{INTRODUCTION}

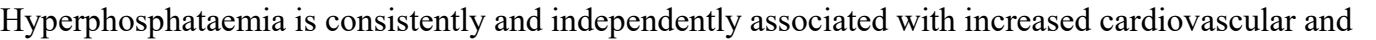

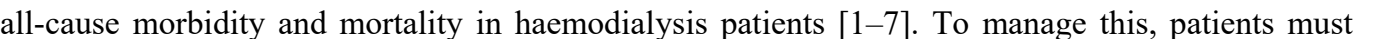

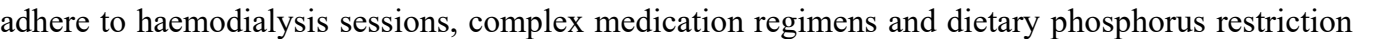

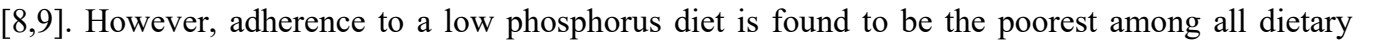

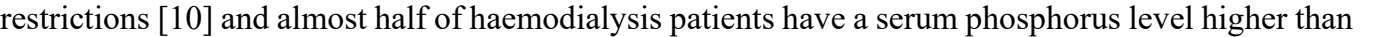

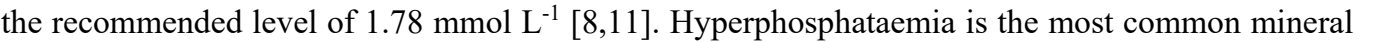

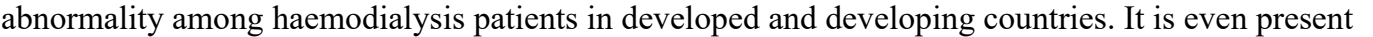

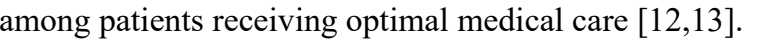

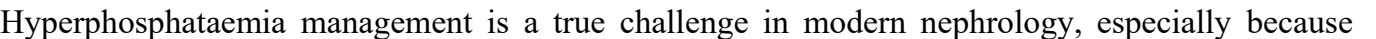

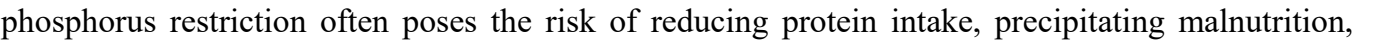

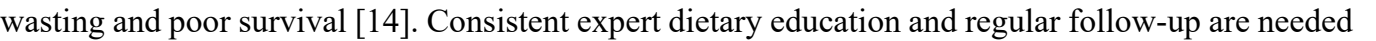

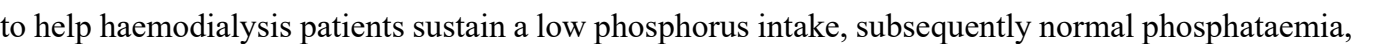

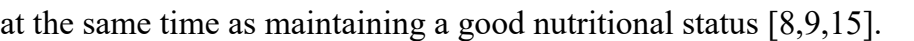

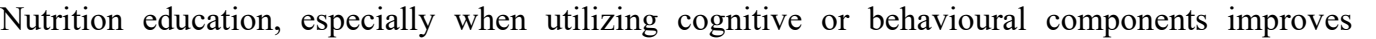

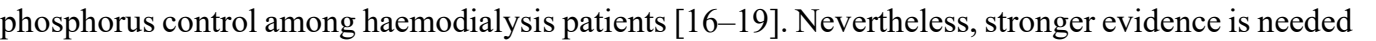

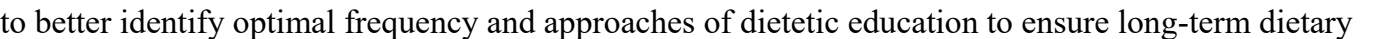

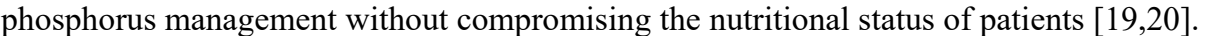

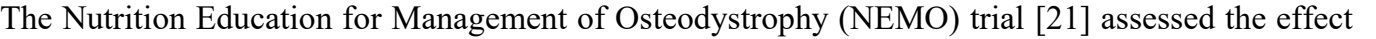

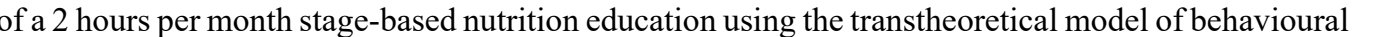

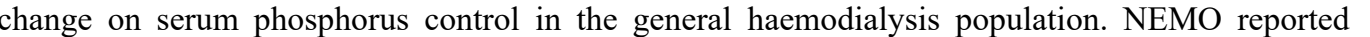

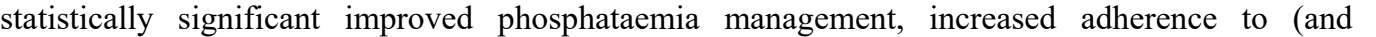

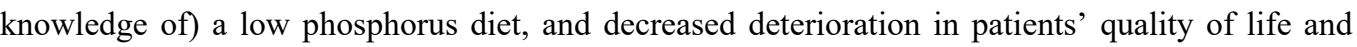

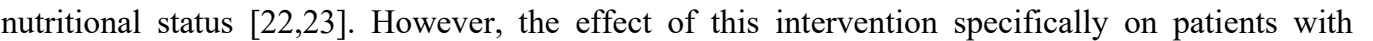

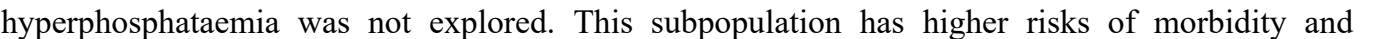

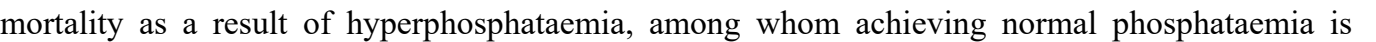

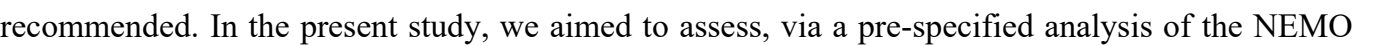
एव

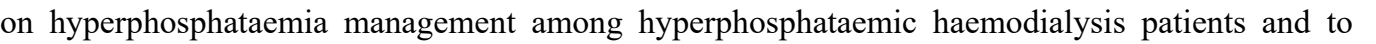

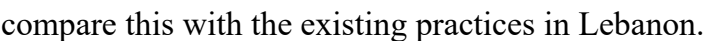

$\square$

\section{MATERIALS AND METHODS}

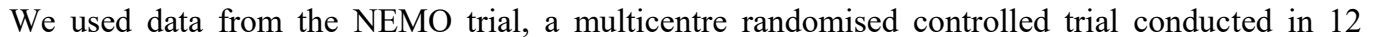

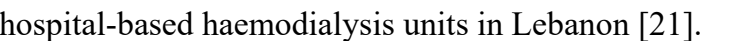




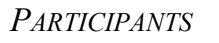

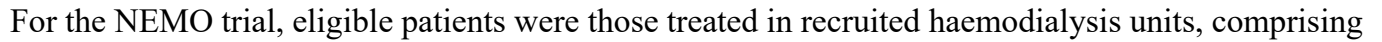

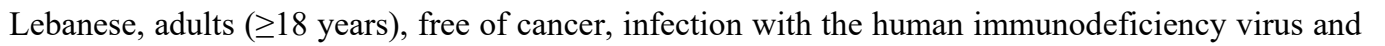

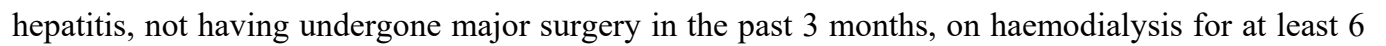

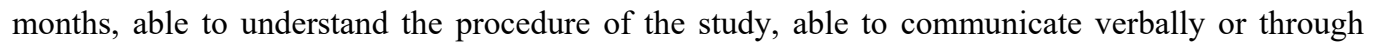

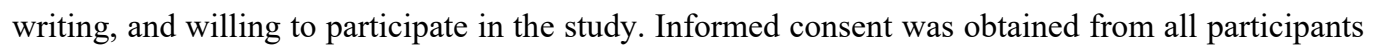

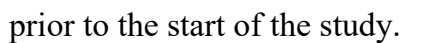

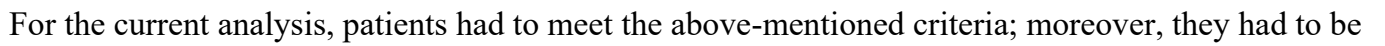

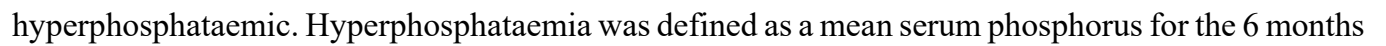

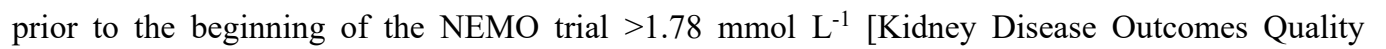

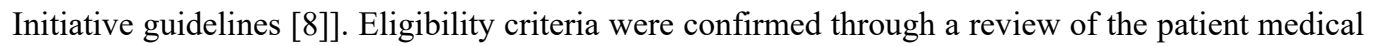

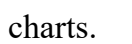

$\square$

$\square \square \square \square \square \square$

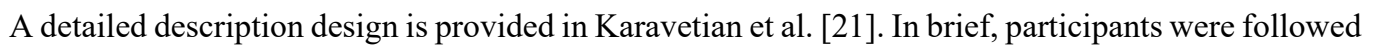

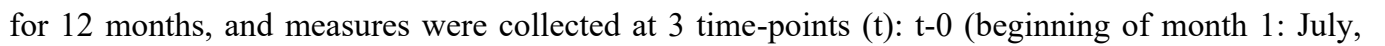

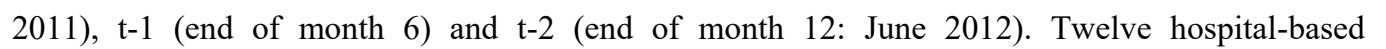

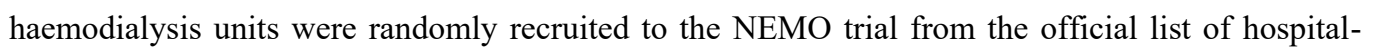

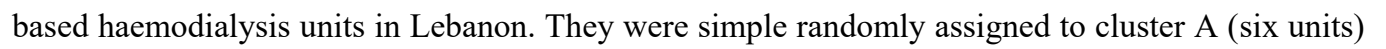

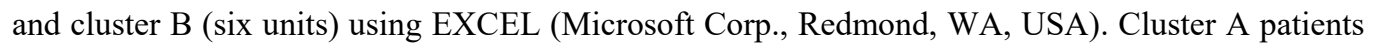

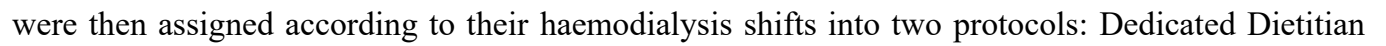

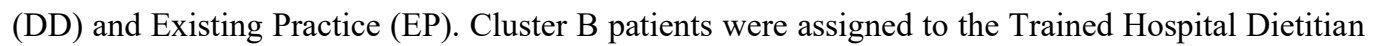

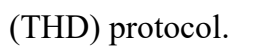

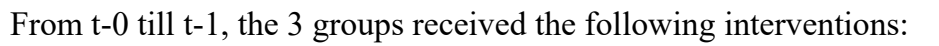

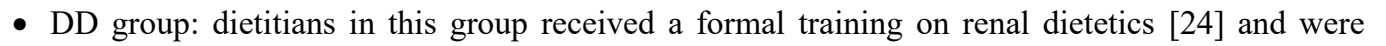

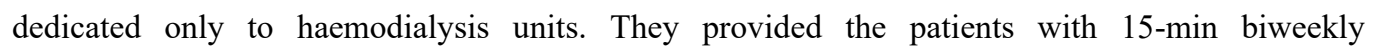

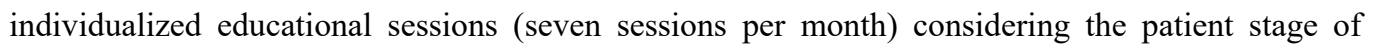

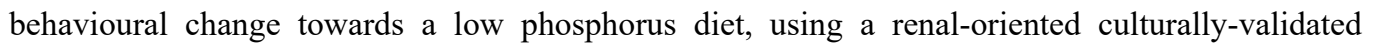

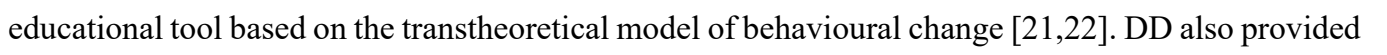
ए

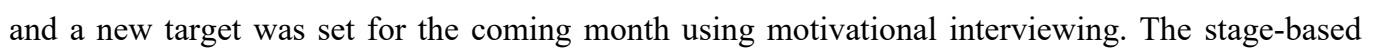

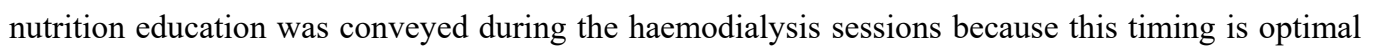

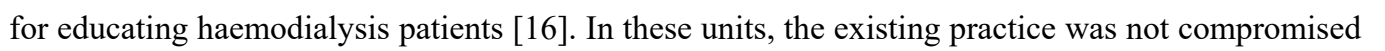

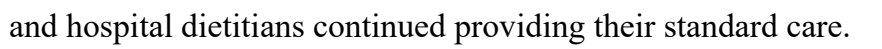




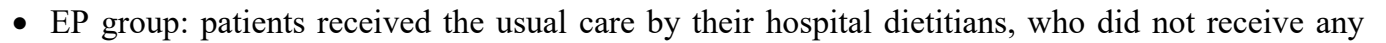

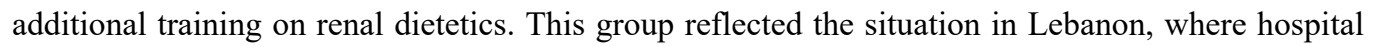

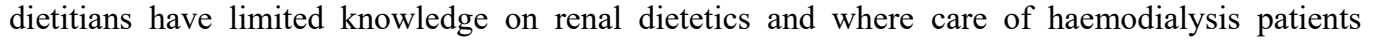

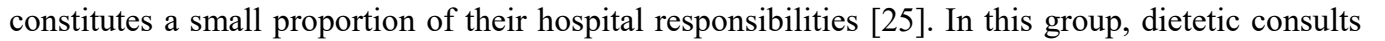

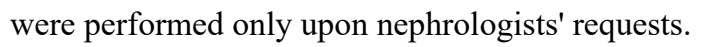

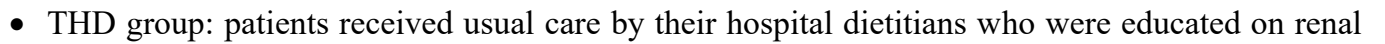

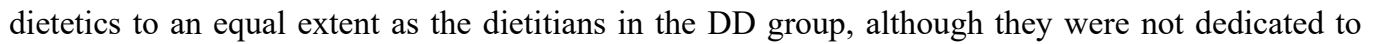

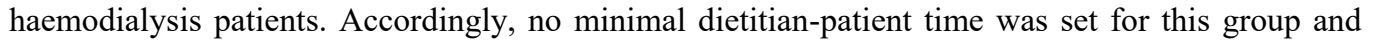

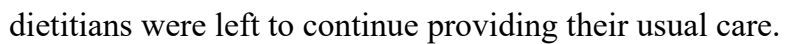

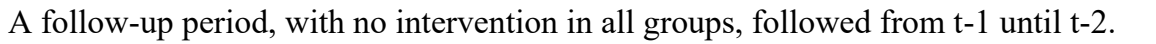

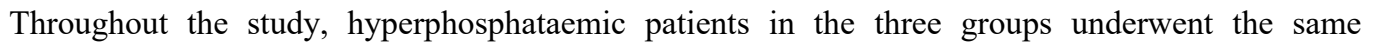

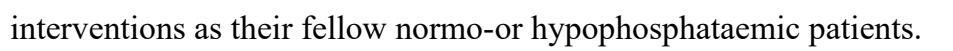

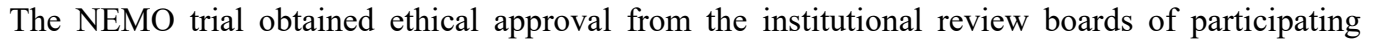

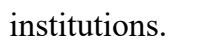

$\square$

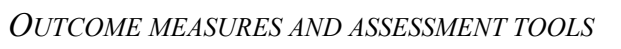

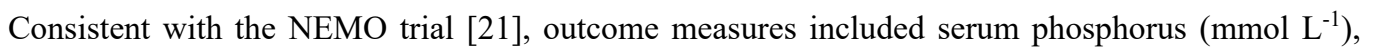

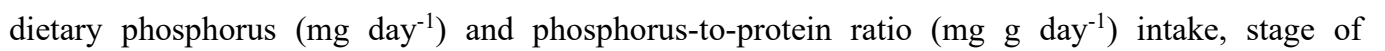

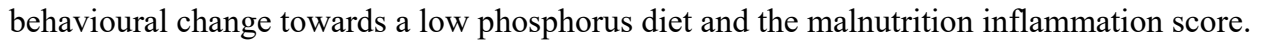

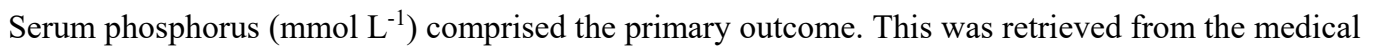

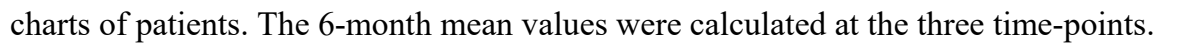

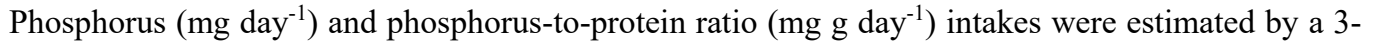

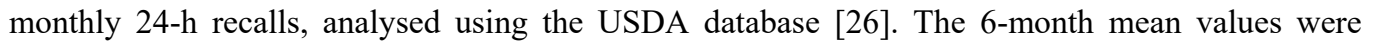

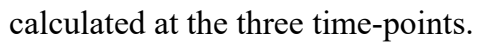

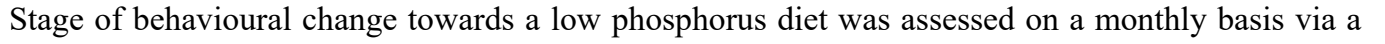

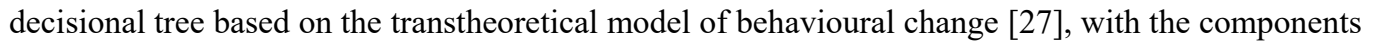

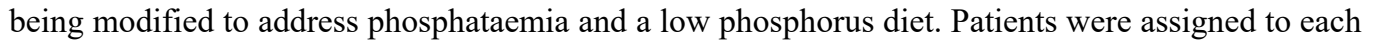

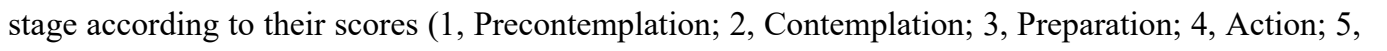

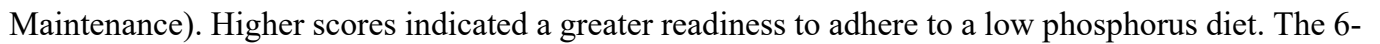

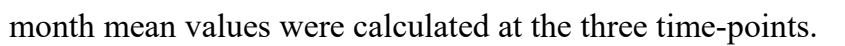

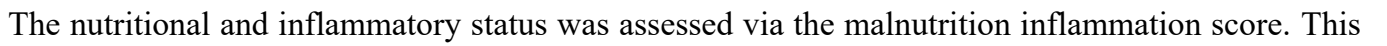

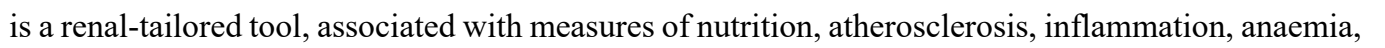

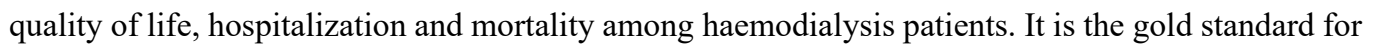

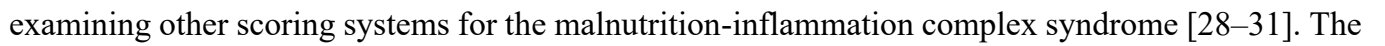




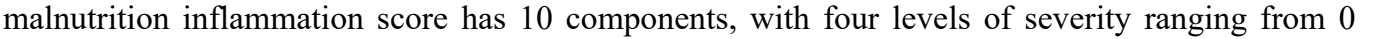

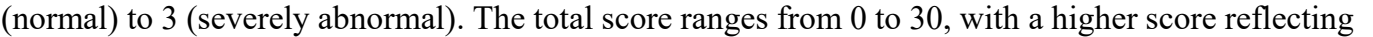

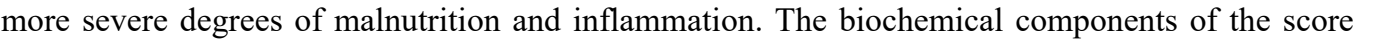

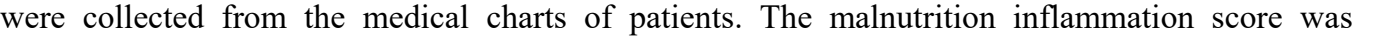

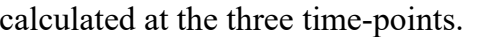

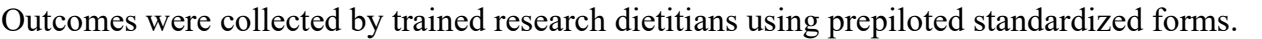

$\square$

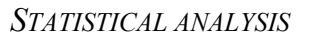

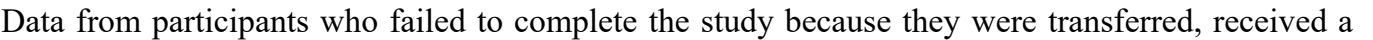

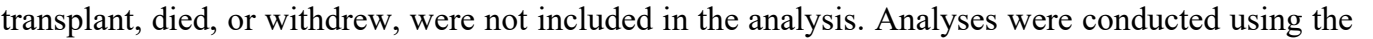

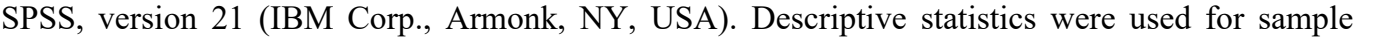

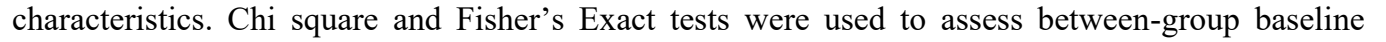

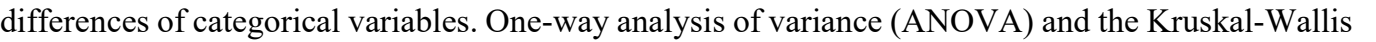

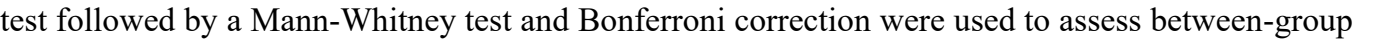

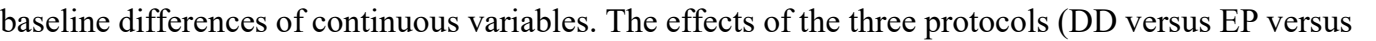

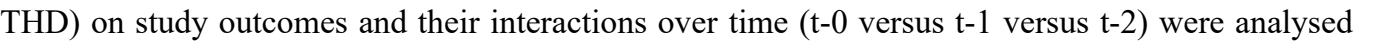

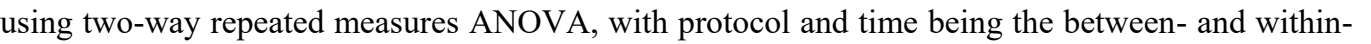

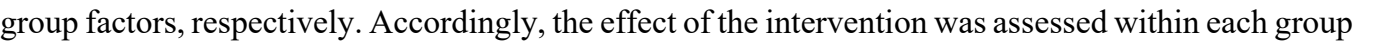

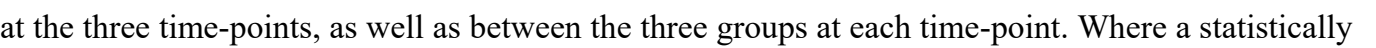

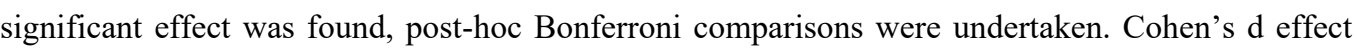

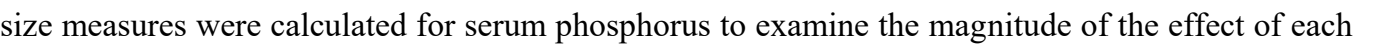

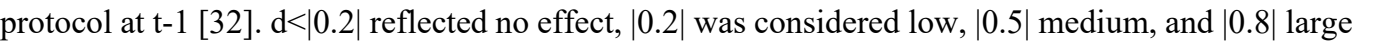

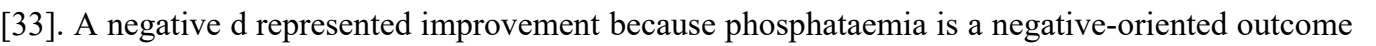

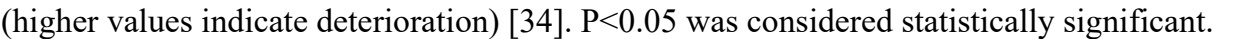

\section{$\square$}




\section{RESULTS}

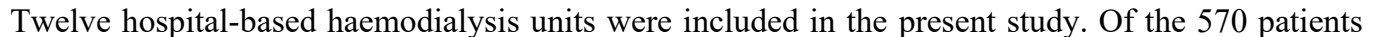

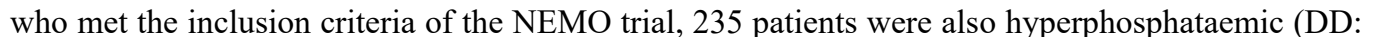

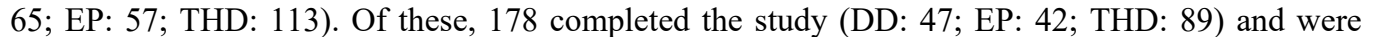

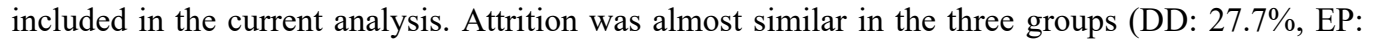

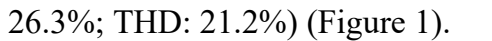

$\square$

\section{Enrollment}

\section{Allocation}

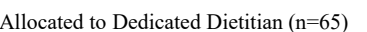

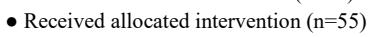

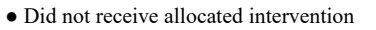

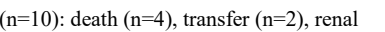

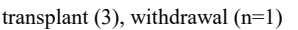

$\sqcap$

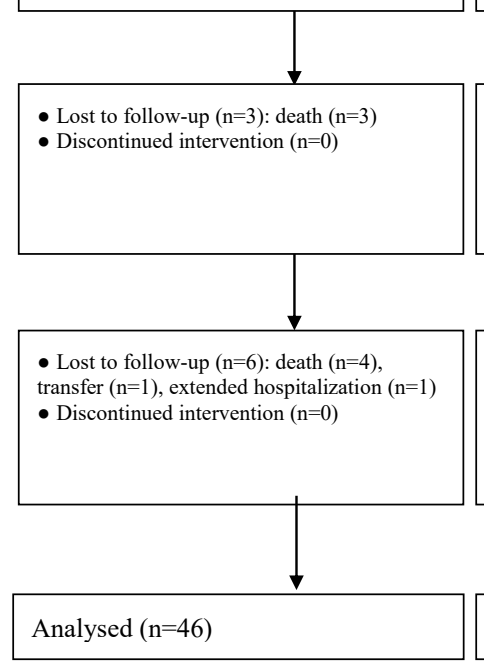

Time-point 0

•

•

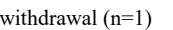

\section{Time-point 1}

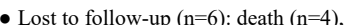

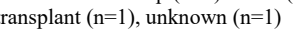

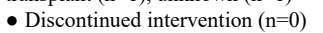

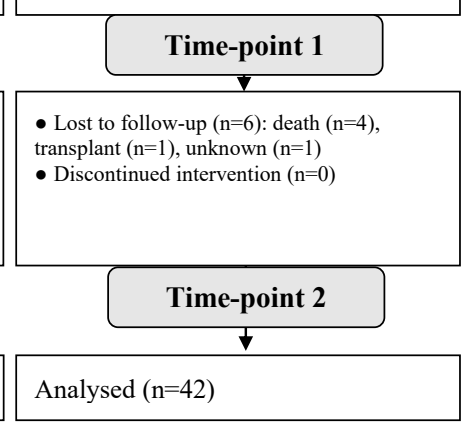

-

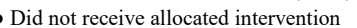

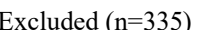

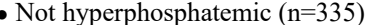

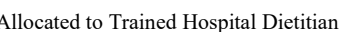

$\|\square\| \Pi \| \mathbb{1}$

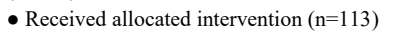

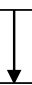

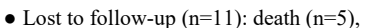

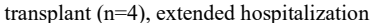

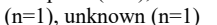

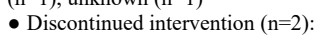

पणाणापणाणाप

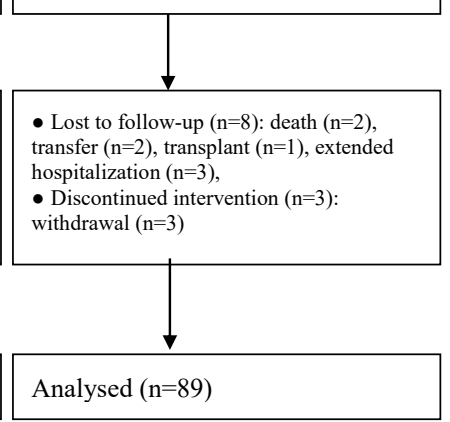

Figure 1. 


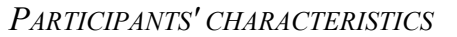

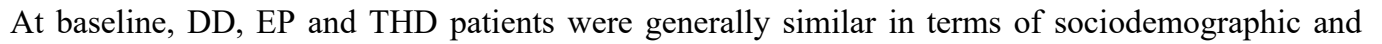

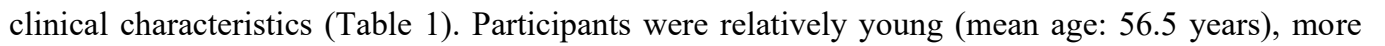
ए ए।

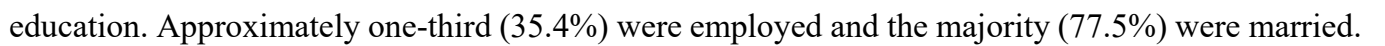

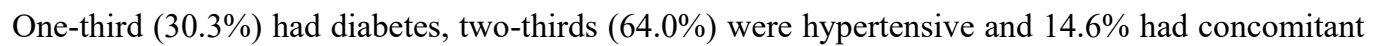

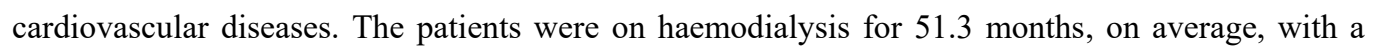

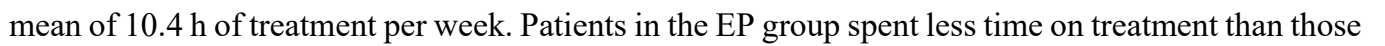

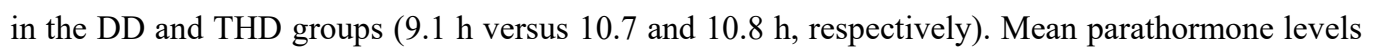

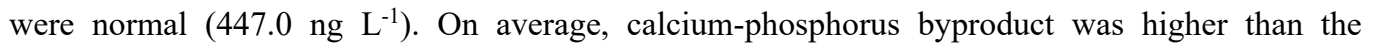

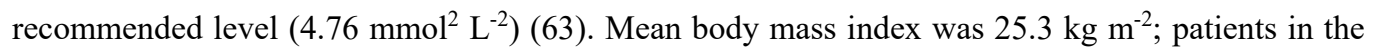

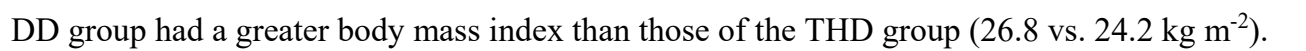

$\square$

Table 1. Participants' Characteristics [\% or mean $(S D)](n=178) \square$

\begin{tabular}{|c|c|c|c|}
\hline & $\begin{array}{l}\text { Dedicated } \\
\text { Dietitian }(n=47)\end{array}$ & $\begin{array}{l}\text { Existing } \\
\text { Practice }(n=42)\end{array}$ & $\begin{array}{l}\text { Trained Hospital } \\
\text { Dietitian }(n=89)\end{array}$ \\
\hline 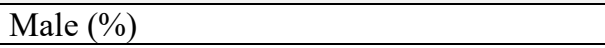 & $\square \| \square$ & 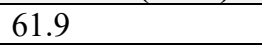 & 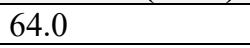 \\
\hline \multicolumn{4}{|l|}{ 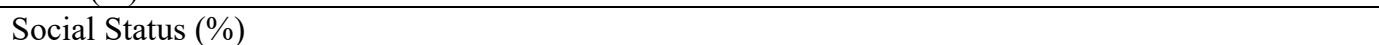 } \\
\hline \begin{tabular}{rl|l|l|} 
\\
\end{tabular} & \begin{tabular}{l|l|l|}
$\square \square \square$ \\
\end{tabular} & $\square \square$ & 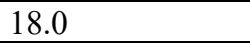 \\
\hline$\square \square\|\| \square$ & $\square \square \square$ & $\square \| \square$ & $\square \mid \square$ \\
\hline 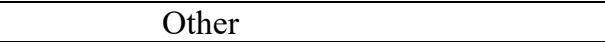 & $\mathrm{amb}$ & $\mathrm{QW \textrm {L }}$ & 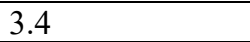 \\
\hline 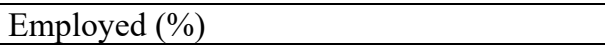 & $\square \square \square$ & $\square \| \square$ & $\square \square \square$ \\
\hline \multicolumn{4}{|l|}{ 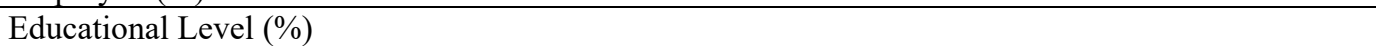 } \\
\hline 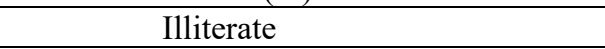 & $\square \square \square$ & $\square \| \square$ & 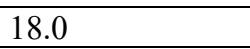 \\
\hline 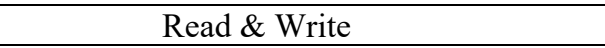 & 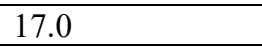 & 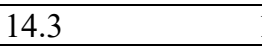 & 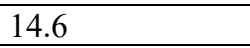 \\
\hline 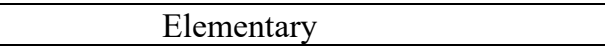 & \begin{tabular}{l|l|l|}
$\square \square \square$ \\
\end{tabular} & 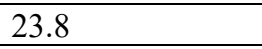 & 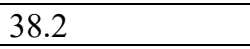 \\
\hline 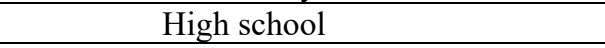 & 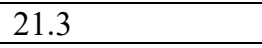 & 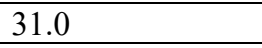 & $\square \square \square$ \\
\hline 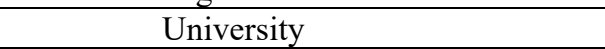 & $\square \square$ & $\square \mid \square \square$ & $\square|\square|$ \\
\hline \multicolumn{4}{|l|}{ 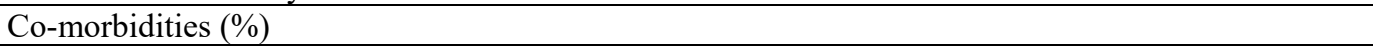 } \\
\hline 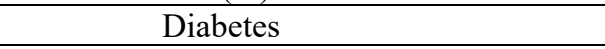 & $\square \square \square$ & $\square \square \square$ & 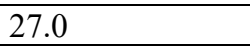 \\
\hline 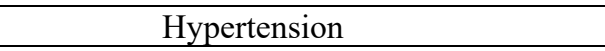 & $\square \square \square$ & $\square \square \square$ & $\square \square \square$ \\
\hline 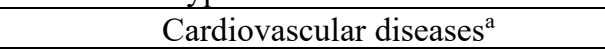 & $\square|\Pi|$ & $\square \square$ & $\square|\square|$ \\
\hline 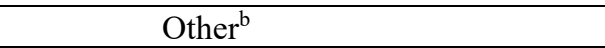 & 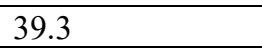 & 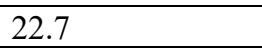 & 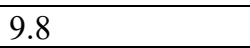 \\
\hline 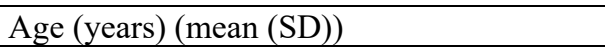 & 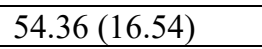 & 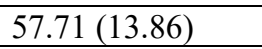 & 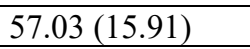 \\
\hline 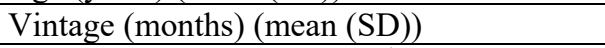 & 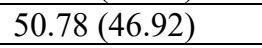 & 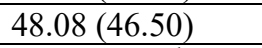 & 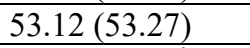 \\
\hline 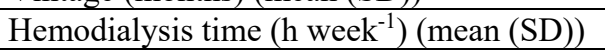 & 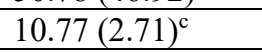 & 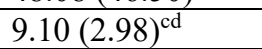 & 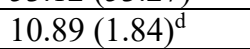 \\
\hline 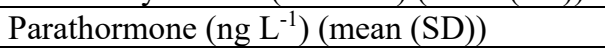 & 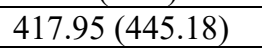 & 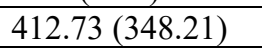 & 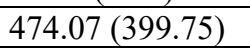 \\
\hline 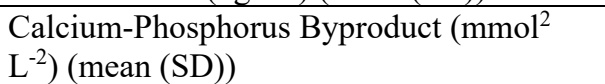 & 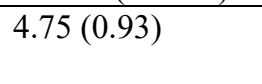 & 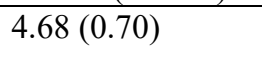 & 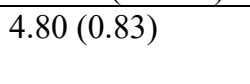 \\
\hline 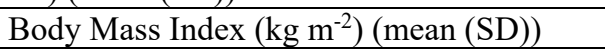 & 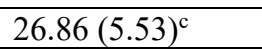 & 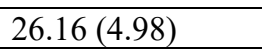 & 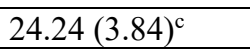 \\
\hline
\end{tabular}

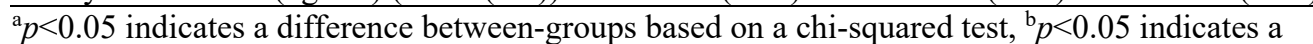

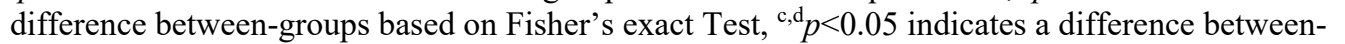

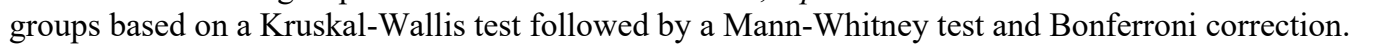

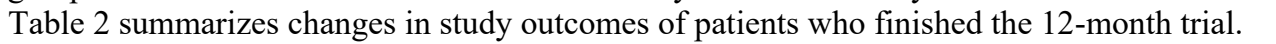




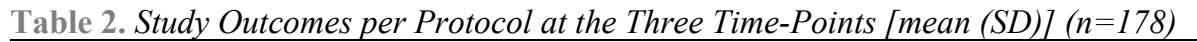

\begin{tabular}{|c|c|c|c|c|}
\hline$\square$ & & $\begin{array}{l}\text { Dedicated } \\
\text { Dietitian }(n=47)\end{array}$ & $\begin{array}{l}\text { Existing Practice } \\
(n=42)\end{array}$ & $\begin{array}{l}\text { Trained Hospital } \\
\text { Dietitian }(n=89)\end{array}$ \\
\hline \multirow{3}{*}{$\begin{array}{l}\text { Serum Phosphorus } \\
\left(\mathrm{mmol} \mathrm{L}^{-1}\right)\end{array}$} & $t-0$ & 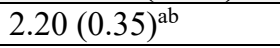 & 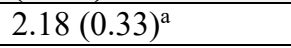 & 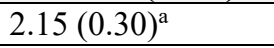 \\
\hline & $t-1$ & 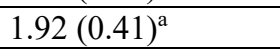 & 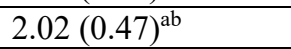 & 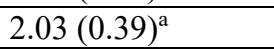 \\
\hline & $t-2$ & 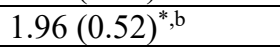 & 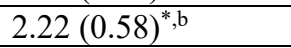 & 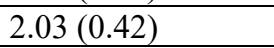 \\
\hline \multirow{3}{*}{$\begin{array}{l}\text { Malnutrition- } \\
\text { Inflammation Score }\end{array}$} & t-0 & 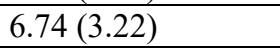 & 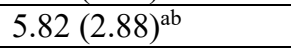 & 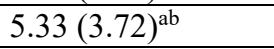 \\
\hline & $t-1$ & 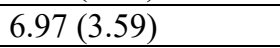 & 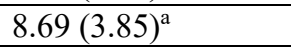 & 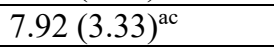 \\
\hline & $t-2$ & 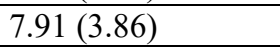 & \begin{tabular}{|l|l|l|l|l|l|} 
\\
\end{tabular} & 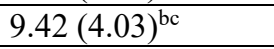 \\
\hline \multirow{3}{*}{$\begin{array}{l}\text { Stage of Behavioural } \\
\text { Change }\end{array}$} & $t-0$ & 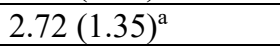 & 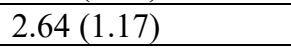 & 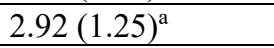 \\
\hline & $t-1$ & 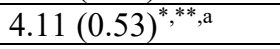 & 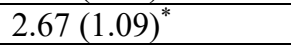 & 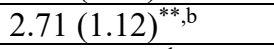 \\
\hline & $t-2$ & 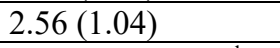 & 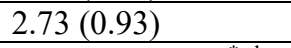 & 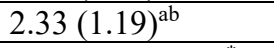 \\
\hline \multirow{3}{*}{$\begin{array}{l}\text { Phosphorus Intake (mg } \\
\text { day }^{-1} \text { ) }\end{array}$} & $t-0$ & 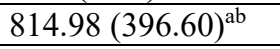 & 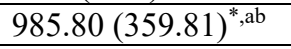 & 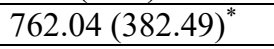 \\
\hline & $t-1$ & 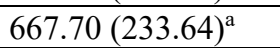 & 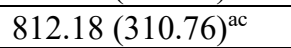 & 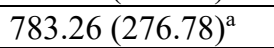 \\
\hline & $t-2$ & 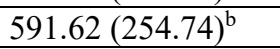 & 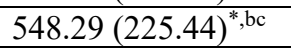 & 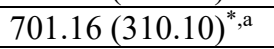 \\
\hline \multirow{3}{*}{$\begin{array}{l}\text { Phosphorus-to-Protein } \\
\text { Ratio (mg g day }{ }^{-1} \text { ) }\end{array}$} & $t-0$ & 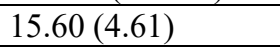 & 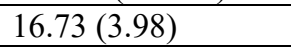 & 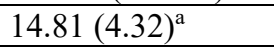 \\
\hline & $t-1$ & 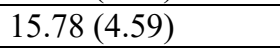 & 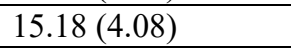 & 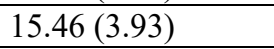 \\
\hline & $t-2$ & 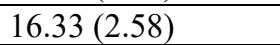 & 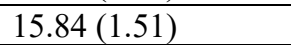 & 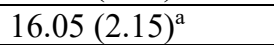 \\
\hline
\end{tabular}

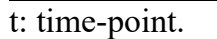

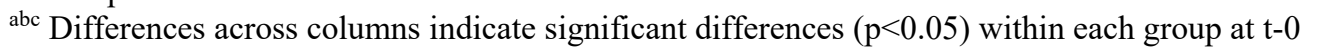

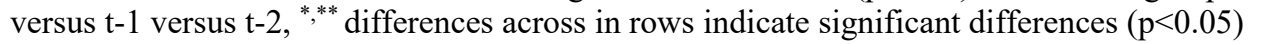

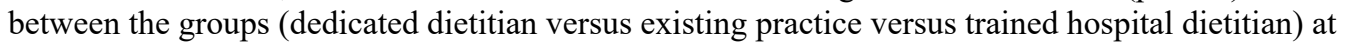

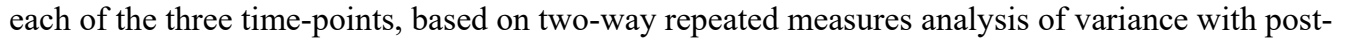

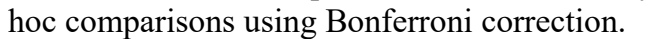

$\square$

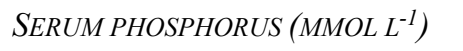

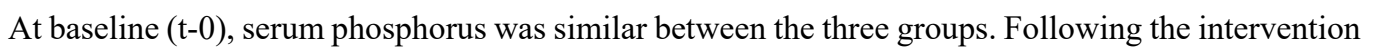

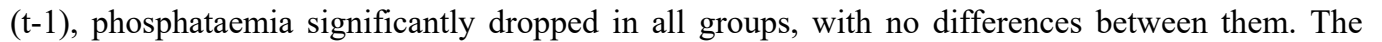

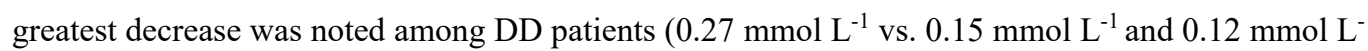

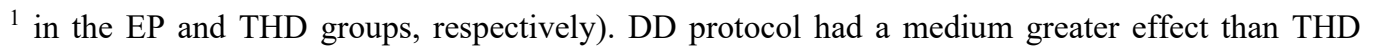

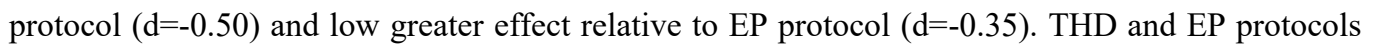

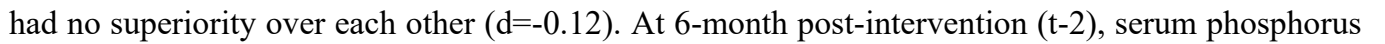

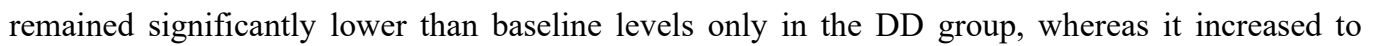

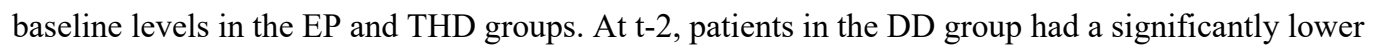

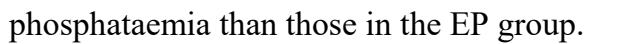

$\square$

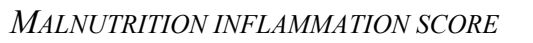

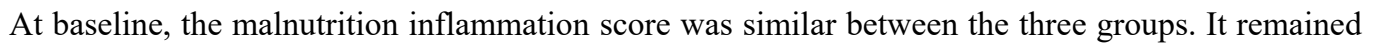

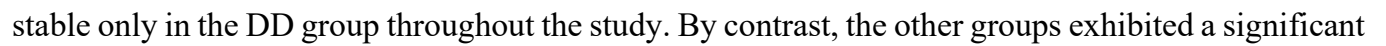

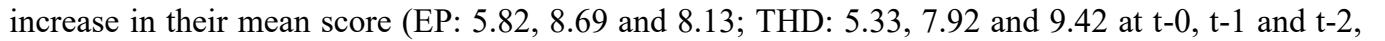

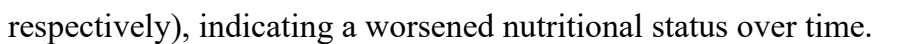




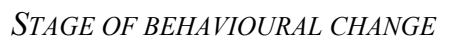

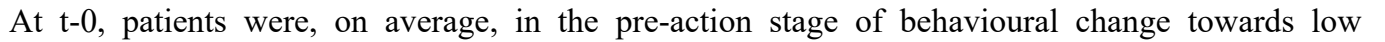

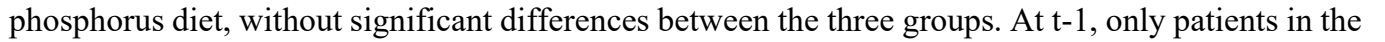

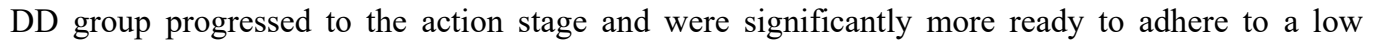

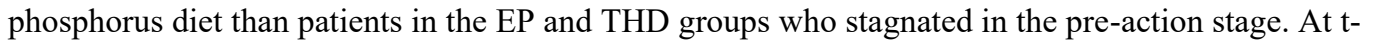

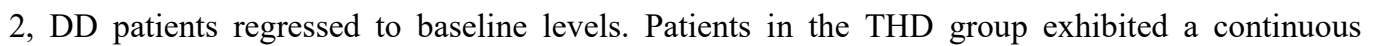

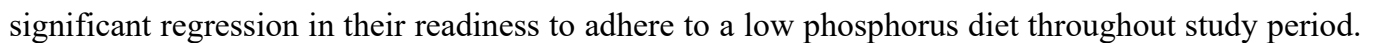

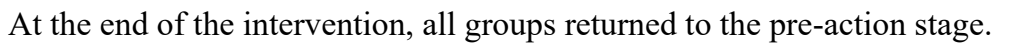

$\square$

ㅁำ

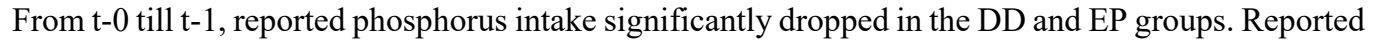

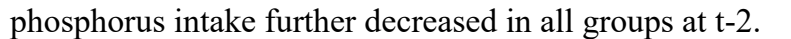

$\square$

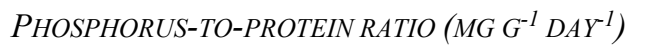

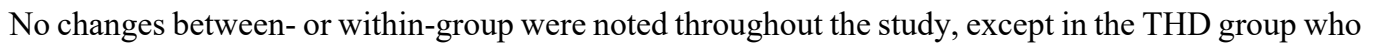

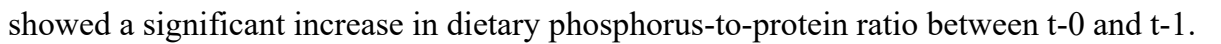

\section{DISCUSSION}

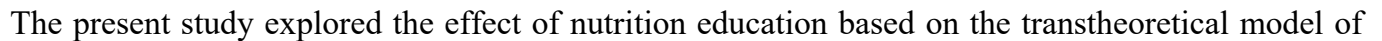

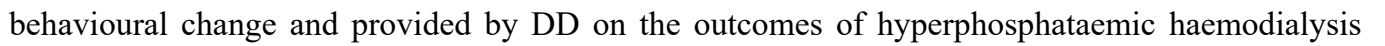

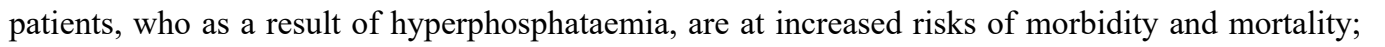

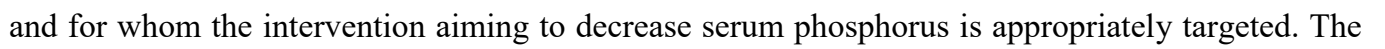

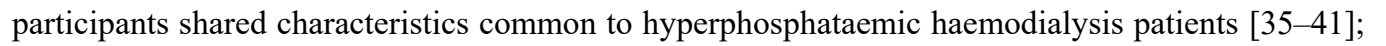

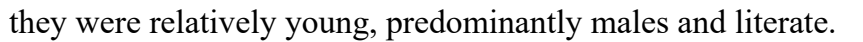

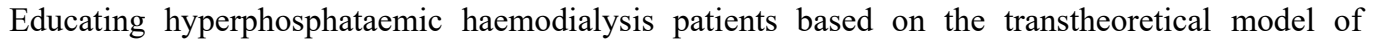

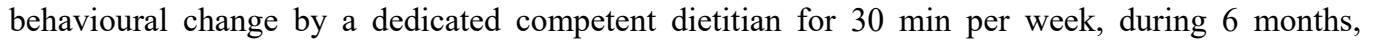

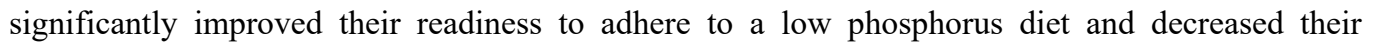

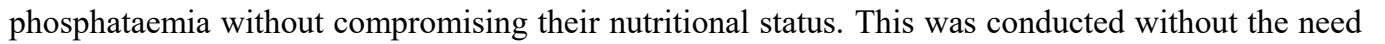
ए एव

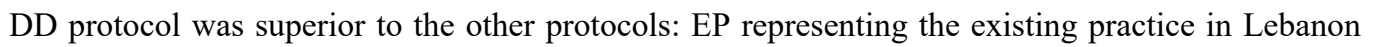

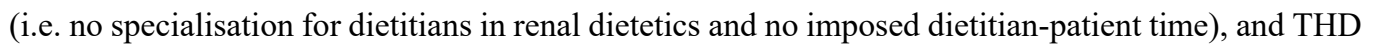

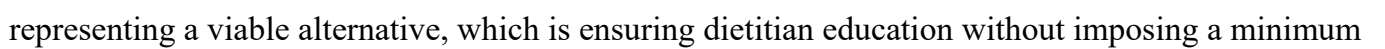

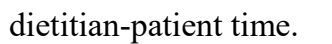




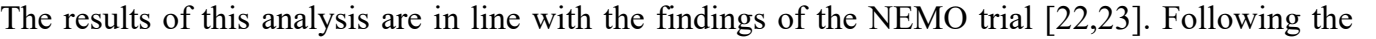

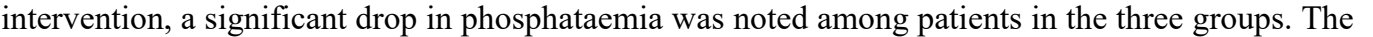

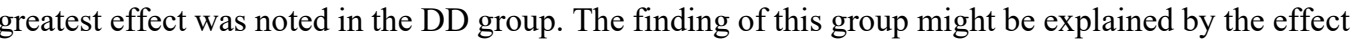

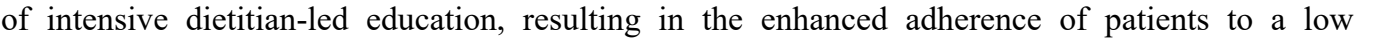

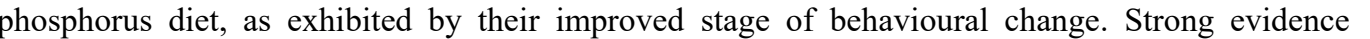

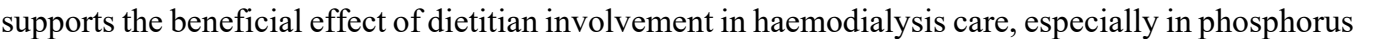

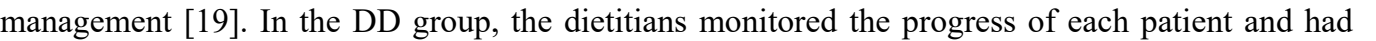

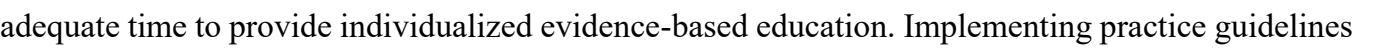

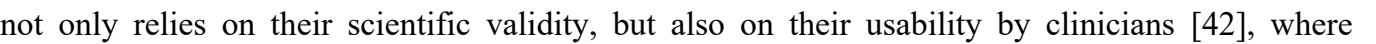

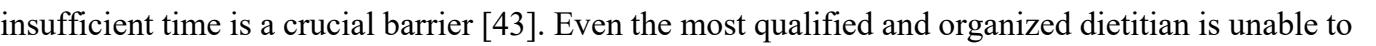

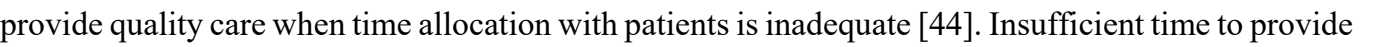

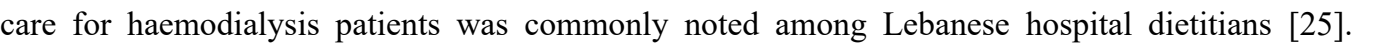

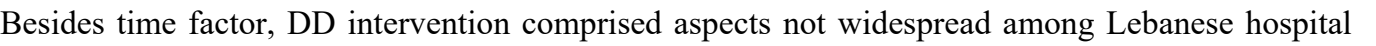

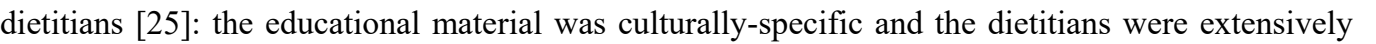

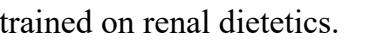

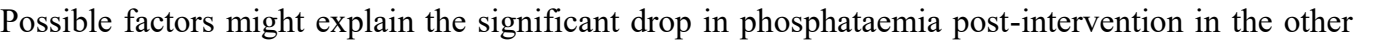

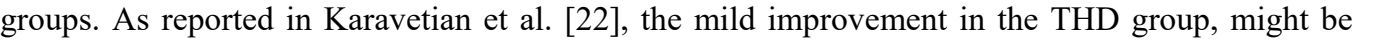

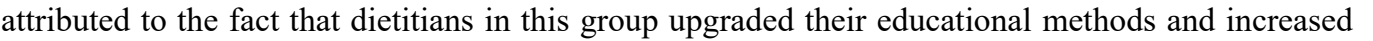

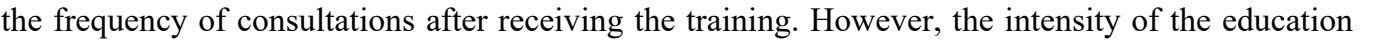

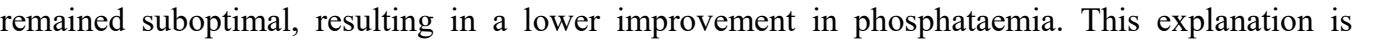

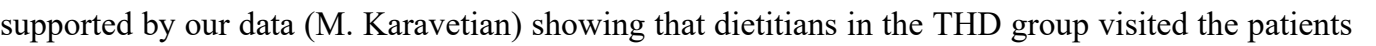

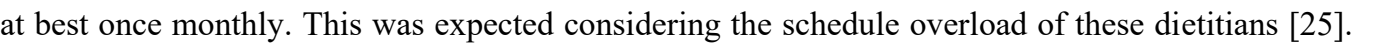

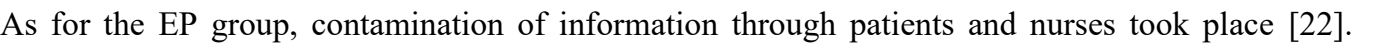

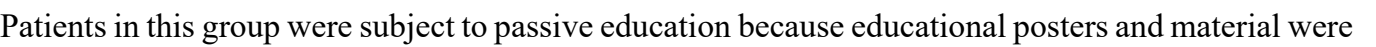
hung on the dialysis units' walls during th

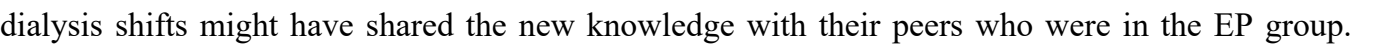

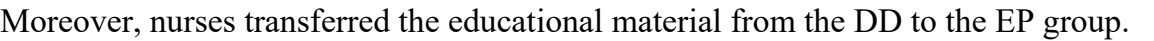

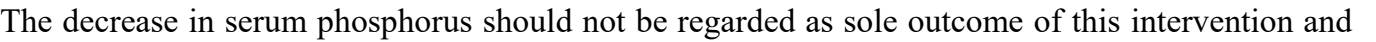

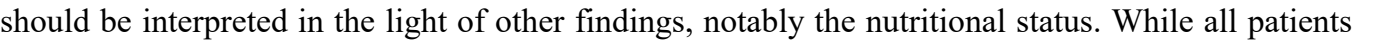

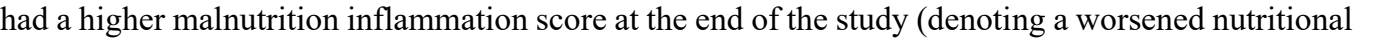

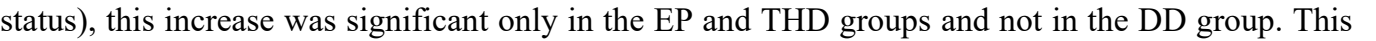

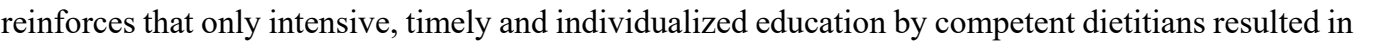

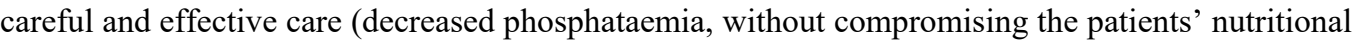

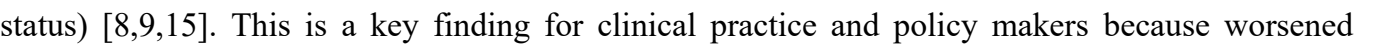

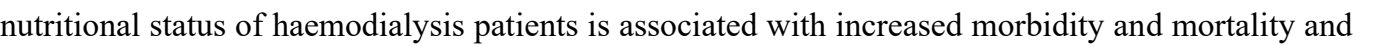

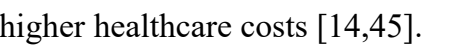




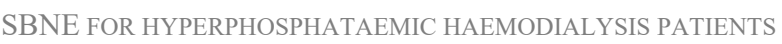

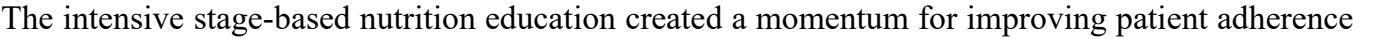

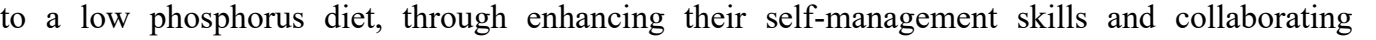

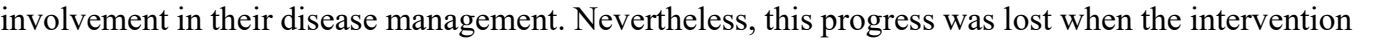

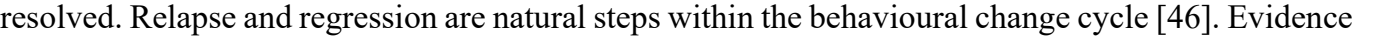

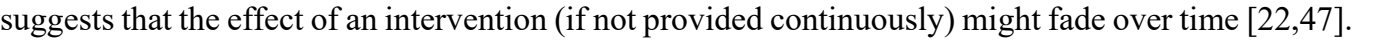

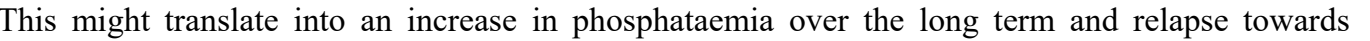

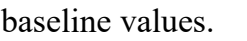

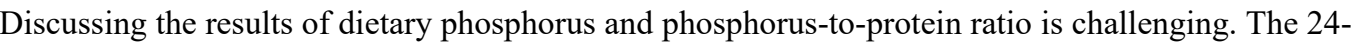

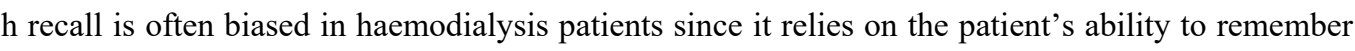

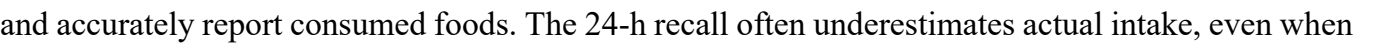

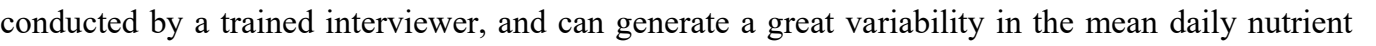

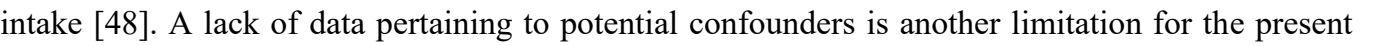

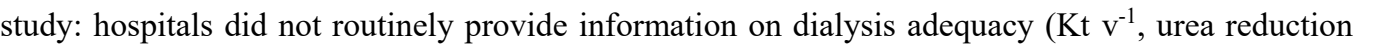

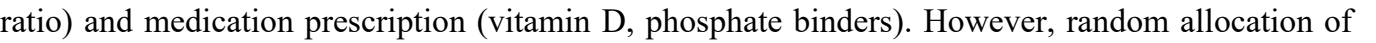

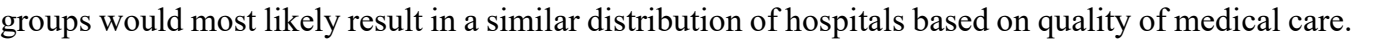

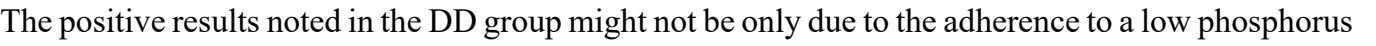

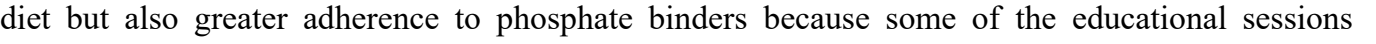

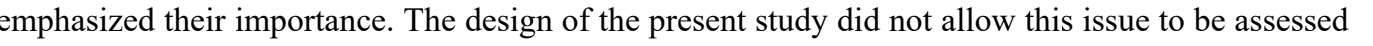

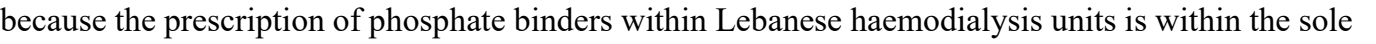

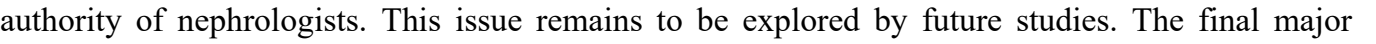

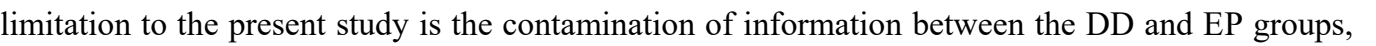

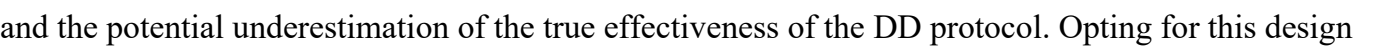

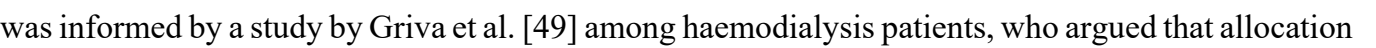

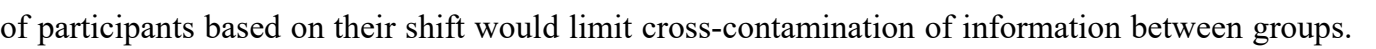

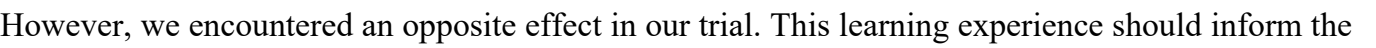

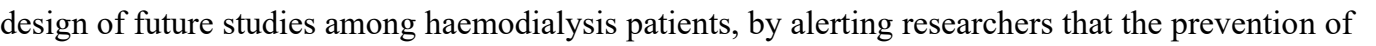

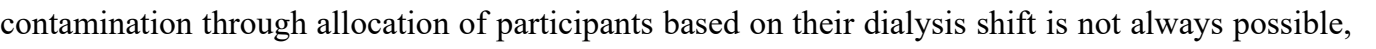

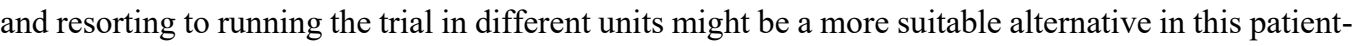

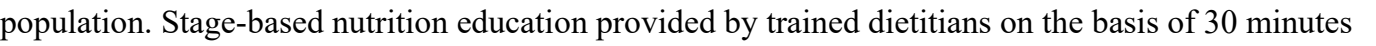

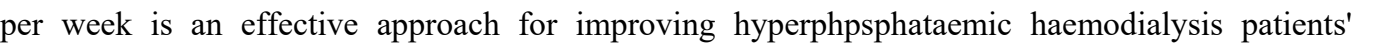

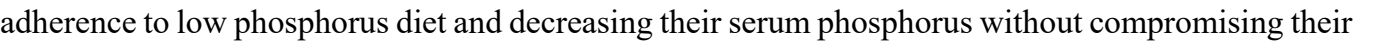

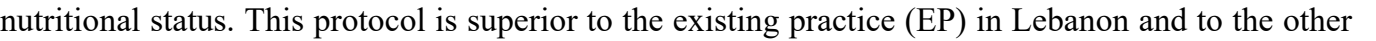

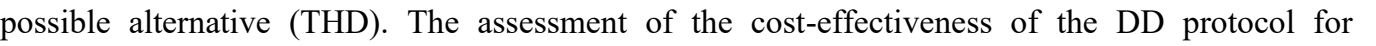

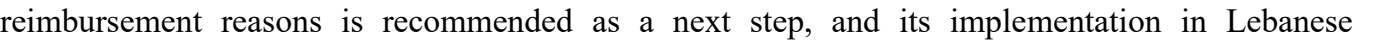

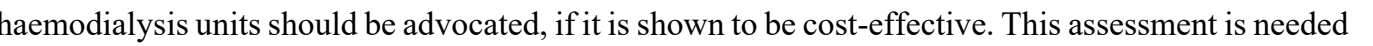

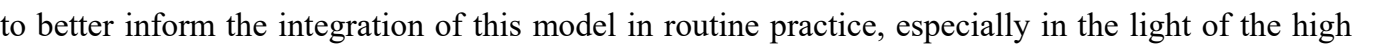




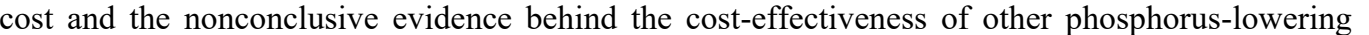

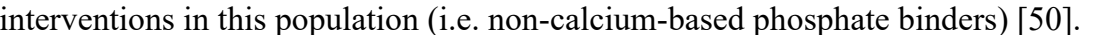

\section{Acknowledgments}

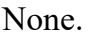




\section{REFERENCES}

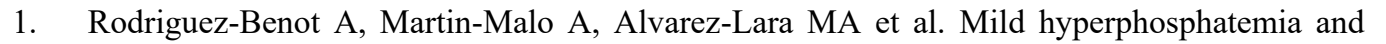

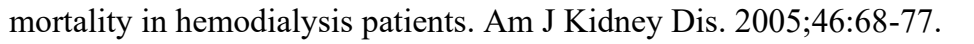

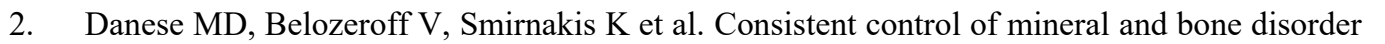

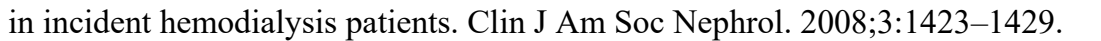

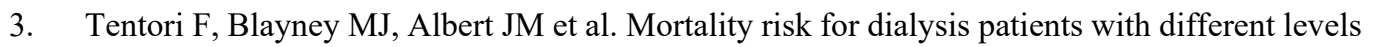

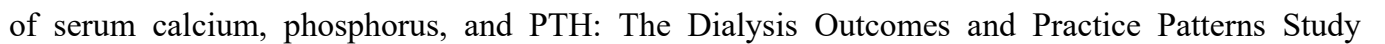

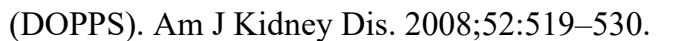

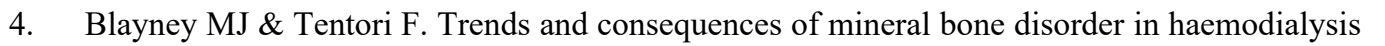

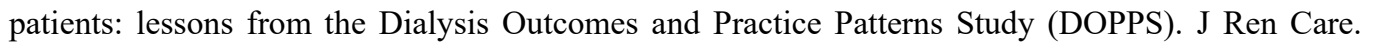

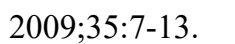

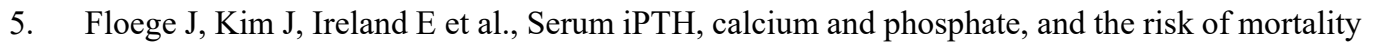

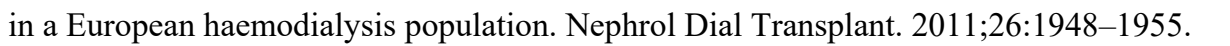

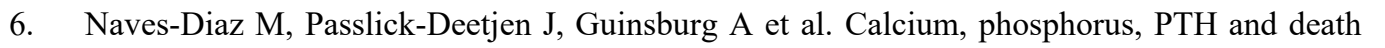

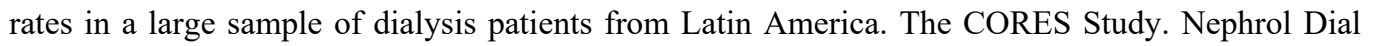

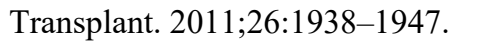

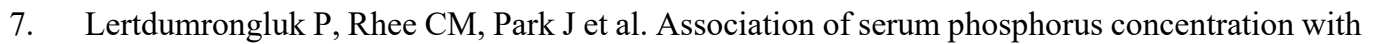

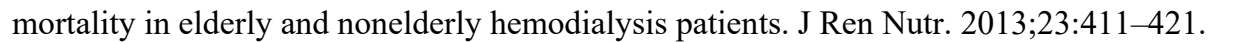

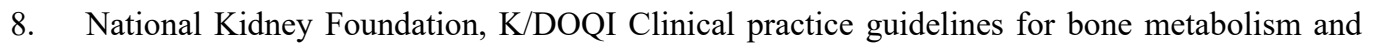

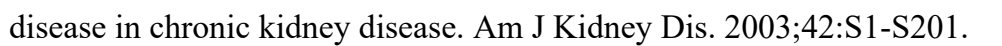

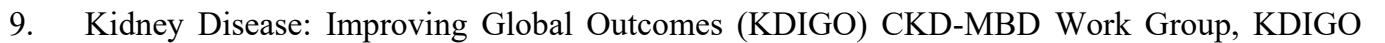

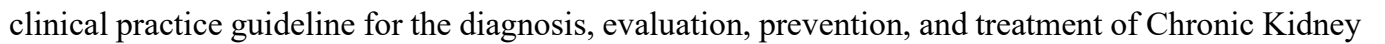

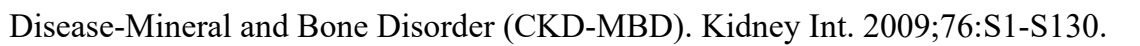

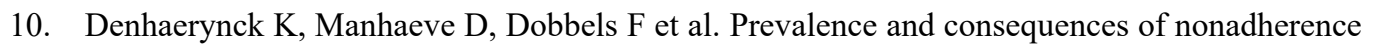

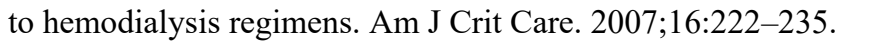

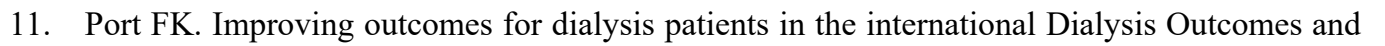

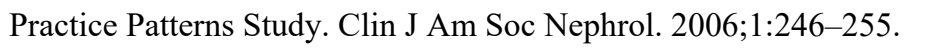

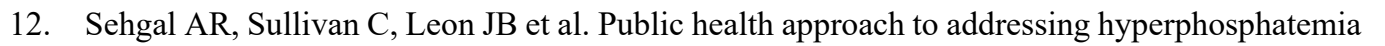

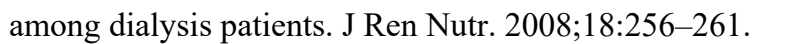

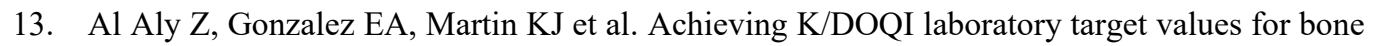

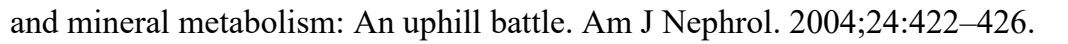

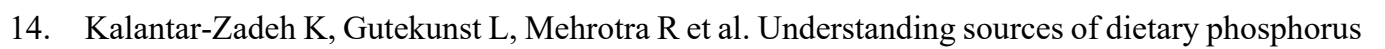

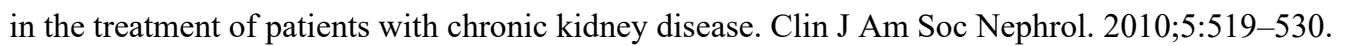




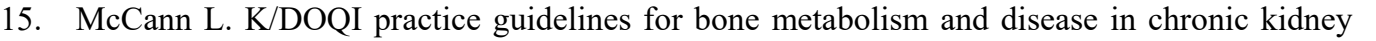

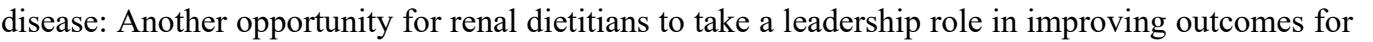

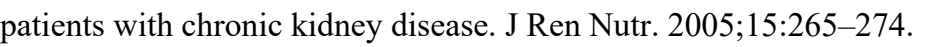

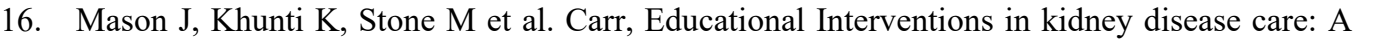

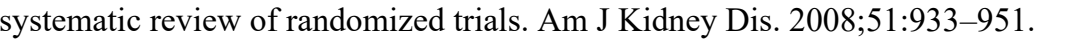

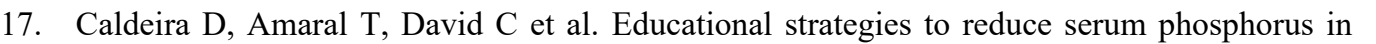

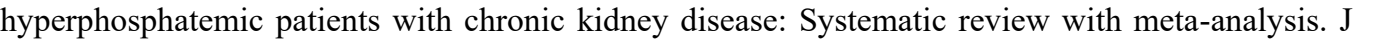

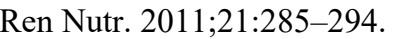

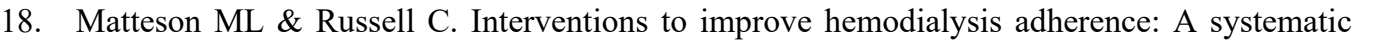

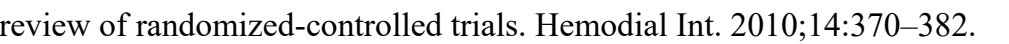

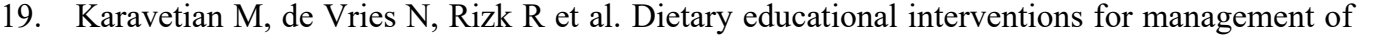

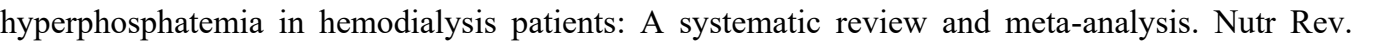

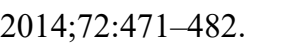

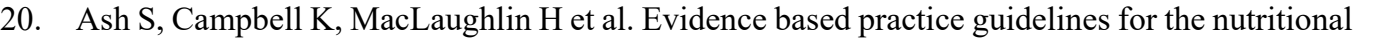

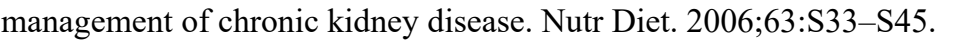

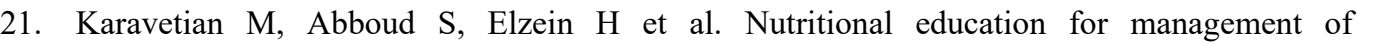

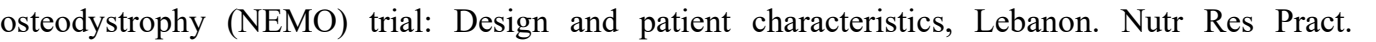
$\square|1| \Pi 1+\square \square \|$

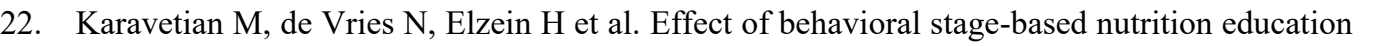

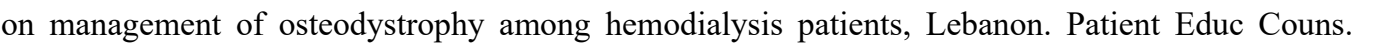

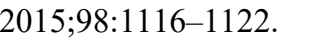

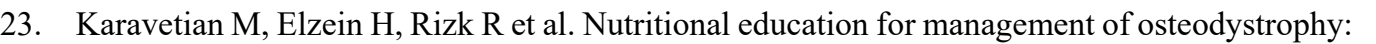

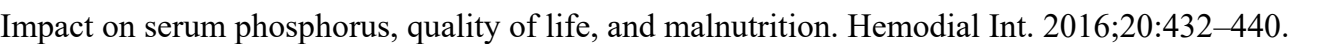

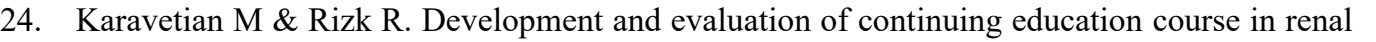

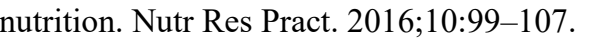

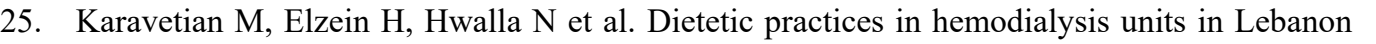

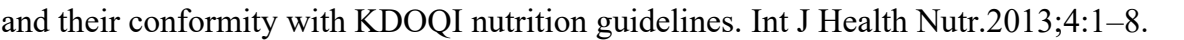

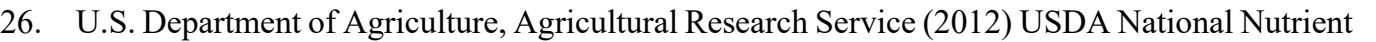

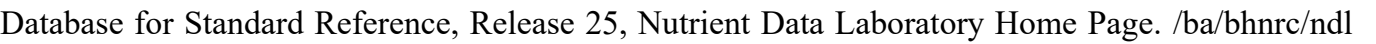

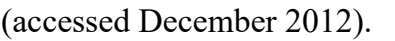

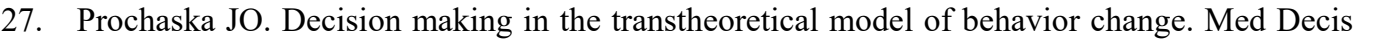

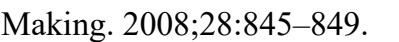

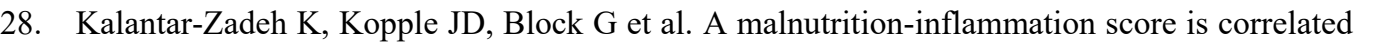

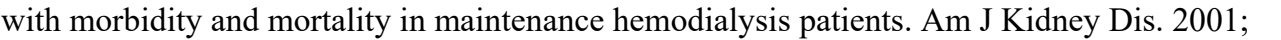




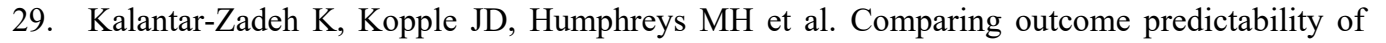

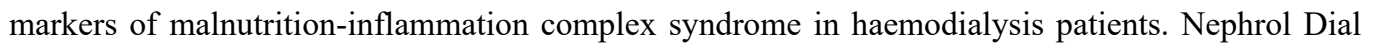

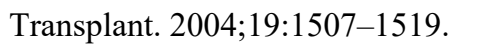

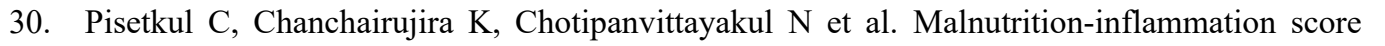

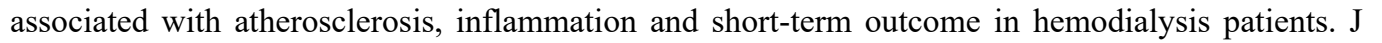

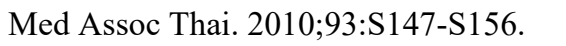

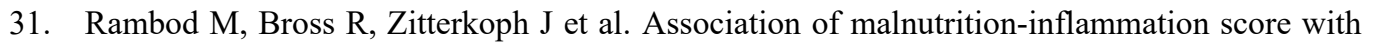

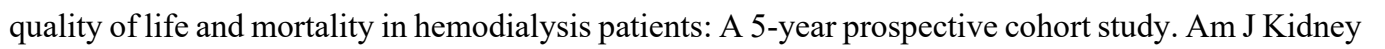

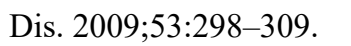

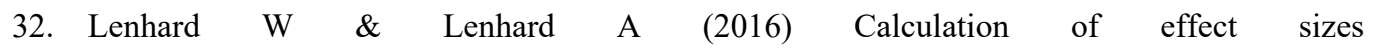

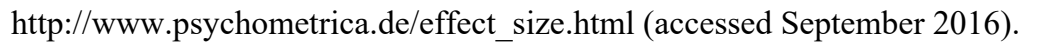

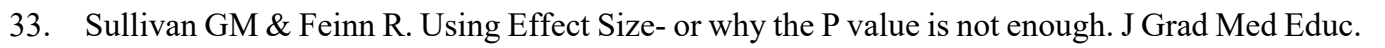

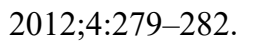

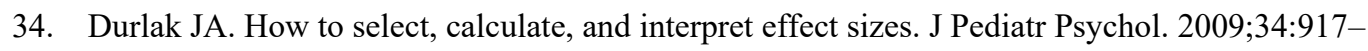
$\square|\Pi| \mathbb{1}$

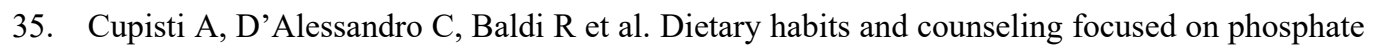

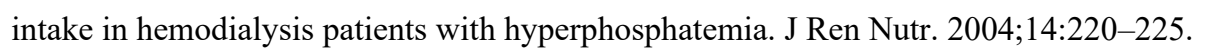

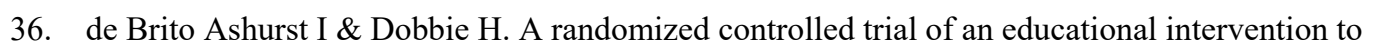

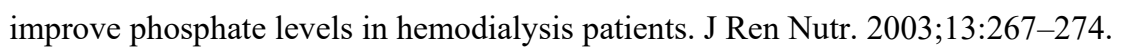

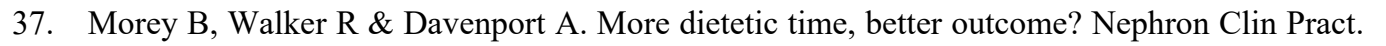
$\square|||||||-\square| \Pi$

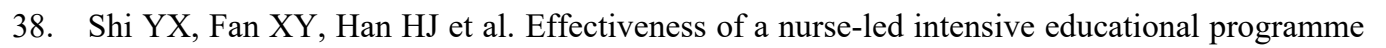

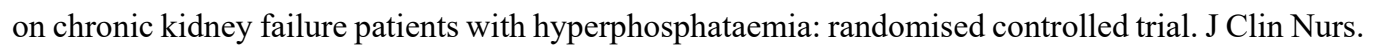
$\square|\Pi| \Pi \mid-\square \square \square \square$

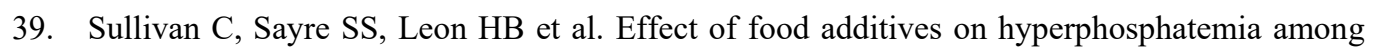

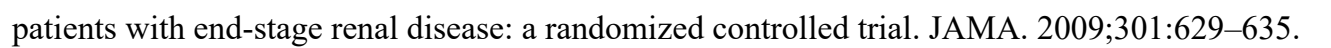

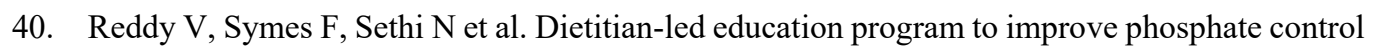

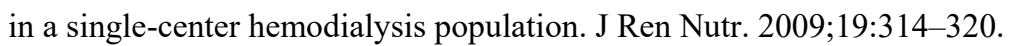

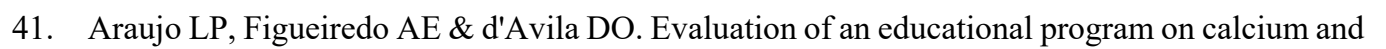

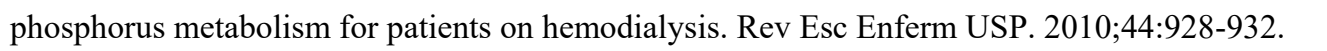

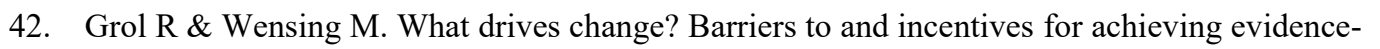

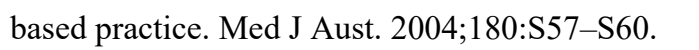

$\square$ Burrowes JD, Russell GB \& Rocco MV. Multiple factors affect renal dietitians' use of the NKF $\square$

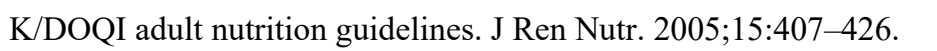




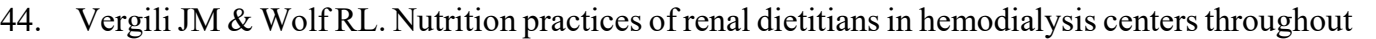

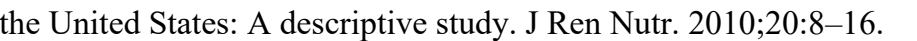

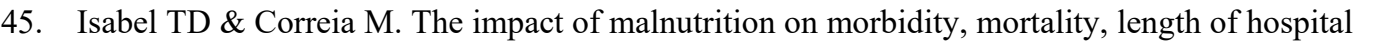

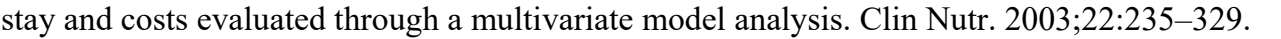

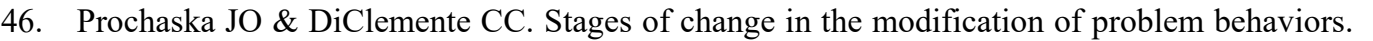

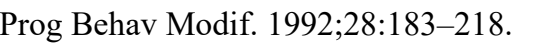

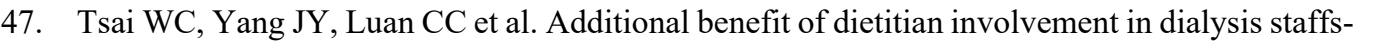

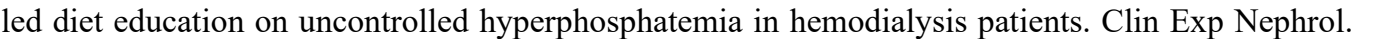

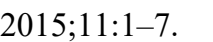

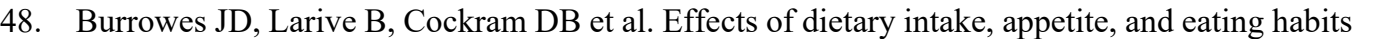

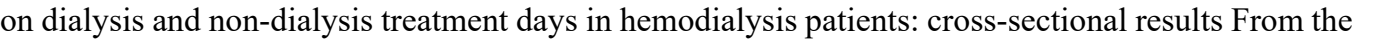

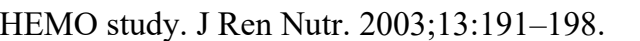

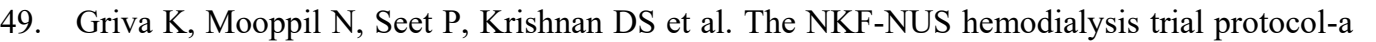

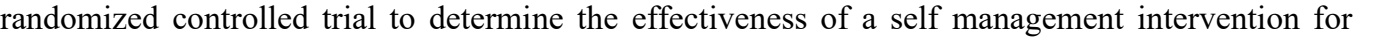

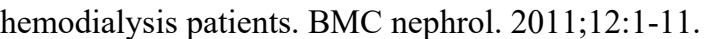

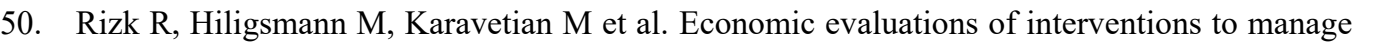

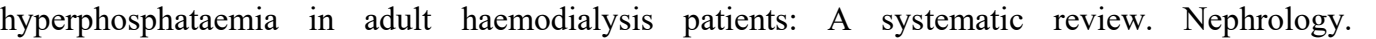

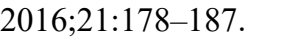



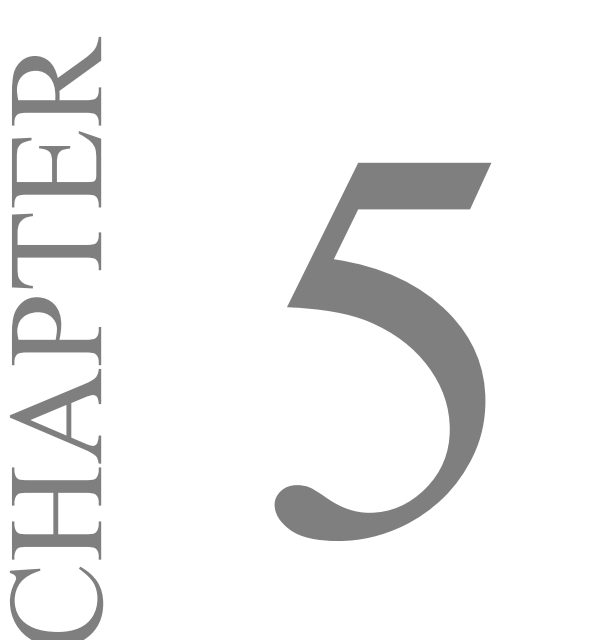

COST-EFFECTIVENESS OF DEDICATED DIETITIANS FOR HYPERPHOSPHATEMIA MANAGEMENT AMONG HEMODIALYSIS PATIENTS IN LEBANON: RESULTS FROM THE NUTRITION EDUCATION FOR MANAGEMENT OF OSTEODYSTROPHY TRIAL $\square$

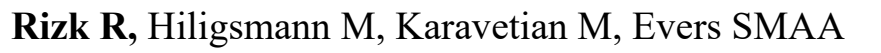




\section{Abstract}

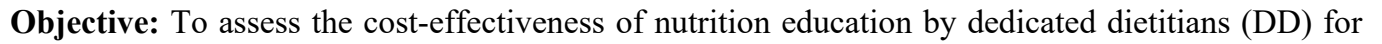

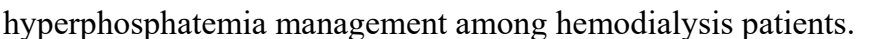

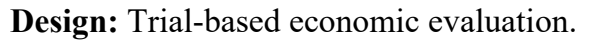

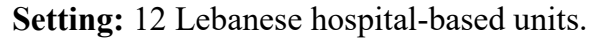

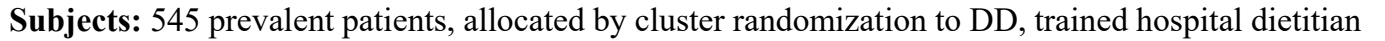

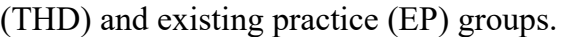

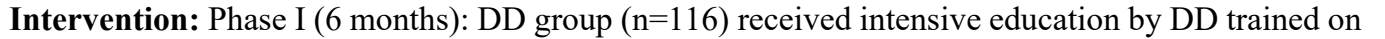

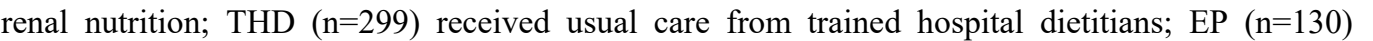

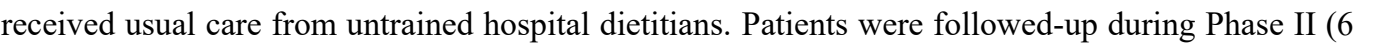

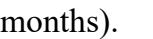

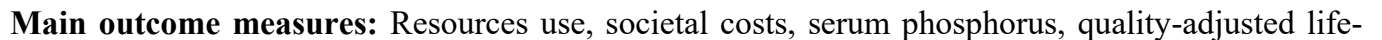

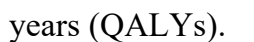

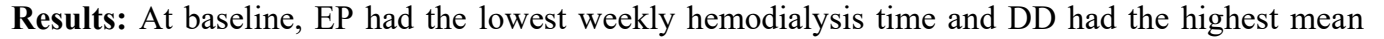

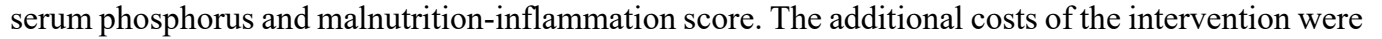

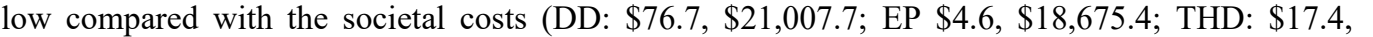

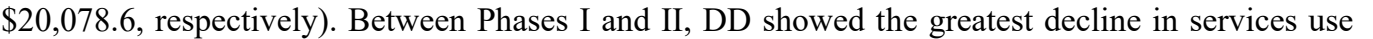

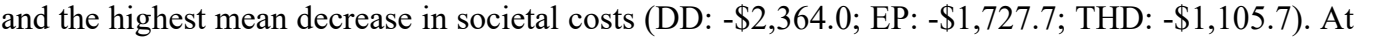

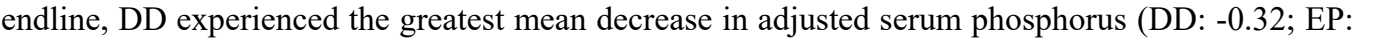

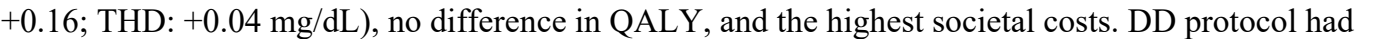

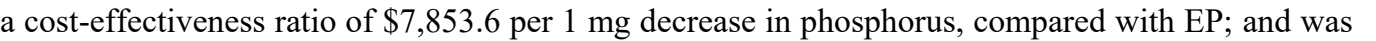

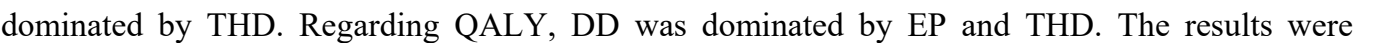

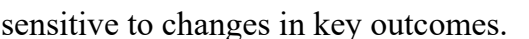

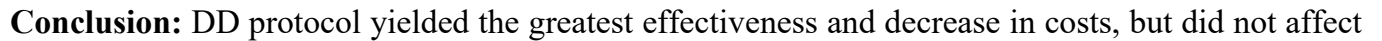

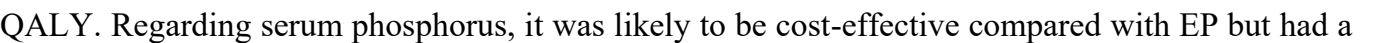

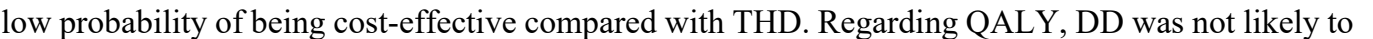

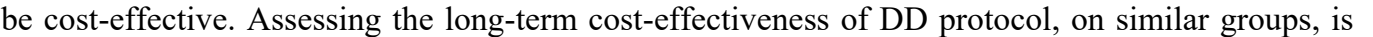
एणामाणाप

\section{KEYWORDS}

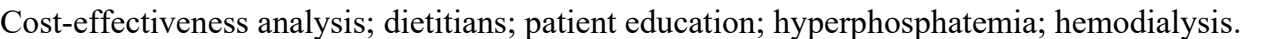




\section{INTRODUCTION}

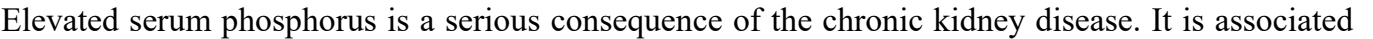

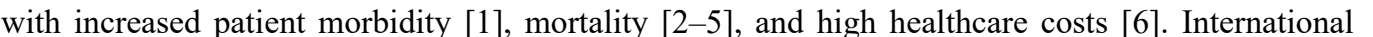

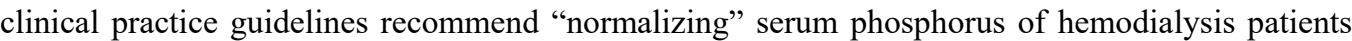

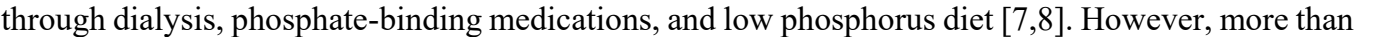

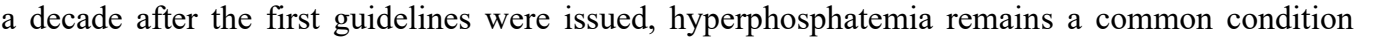

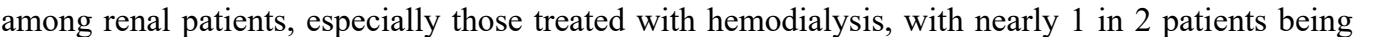

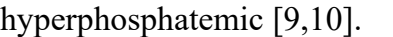

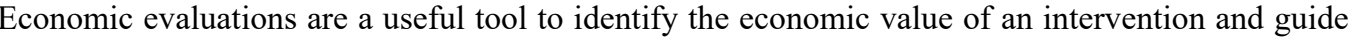

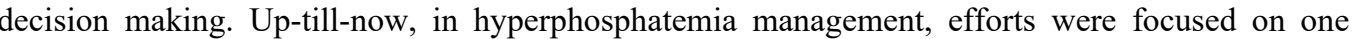

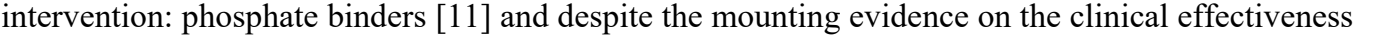

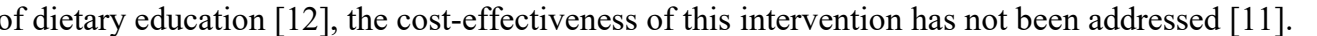

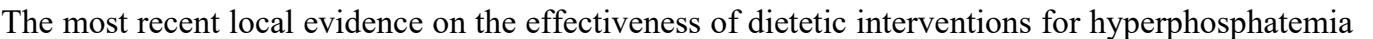

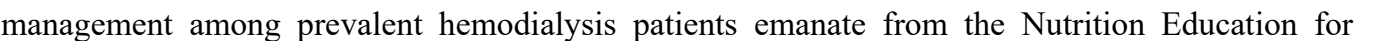

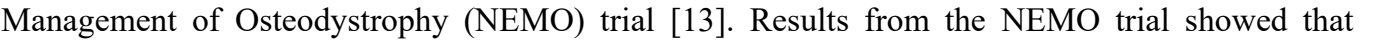

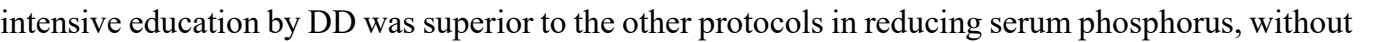

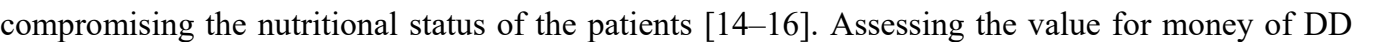

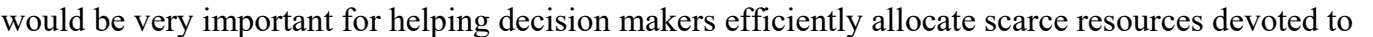

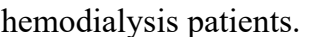

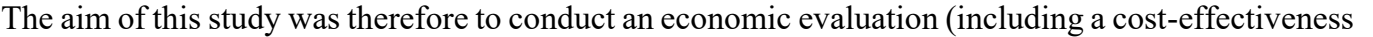

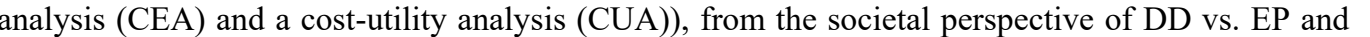

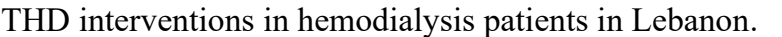

$\square$

\section{METHODS}

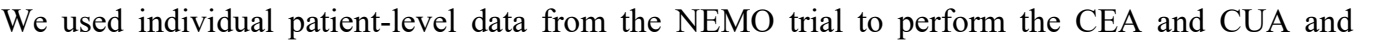

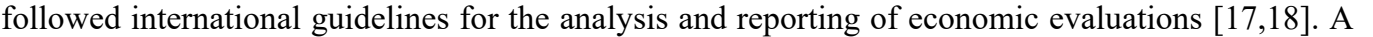

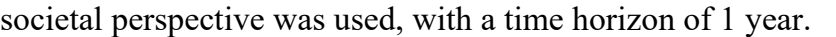

$\square$

$\square \square\left[\begin{array}{ll}\square \\ 0\end{array}\right.$

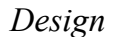

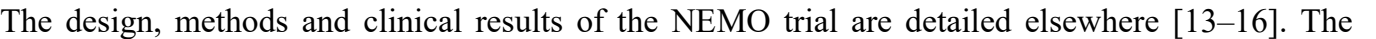

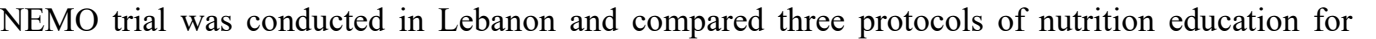

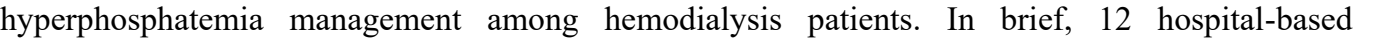

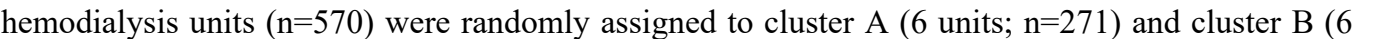




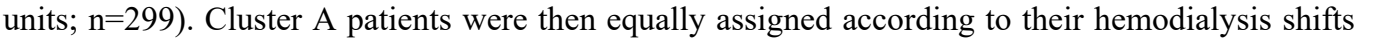

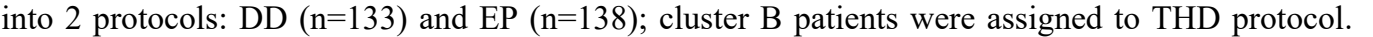

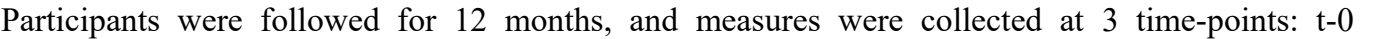
ए ए।

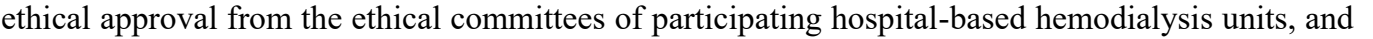

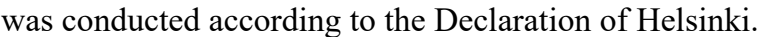

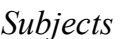

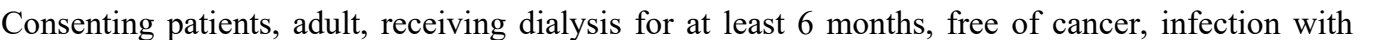

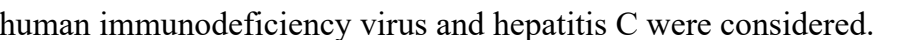

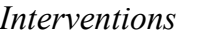

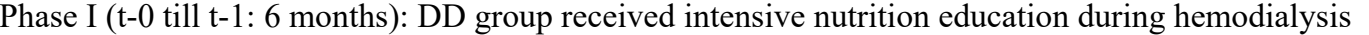

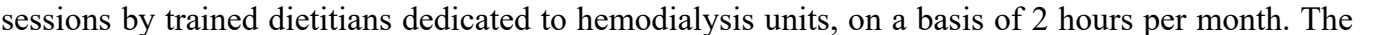

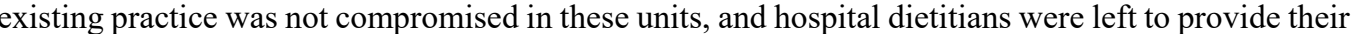
\sual care; EP group received usual care by their hospital dietitians, where patients' consults are upon

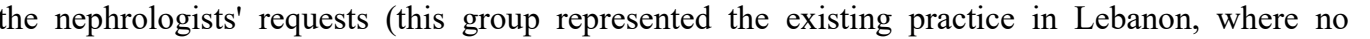

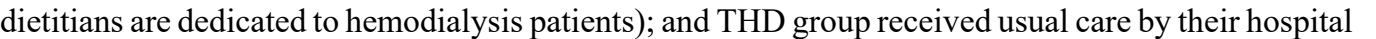

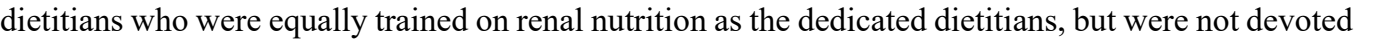

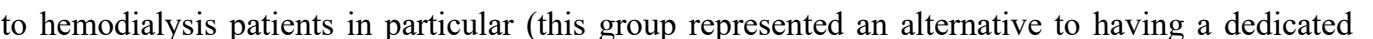
पाitian; whereby hospital dietitian's education is ensured, but dietitian

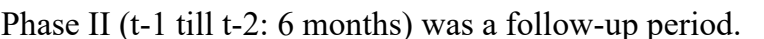

$\square$

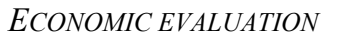

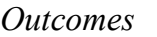

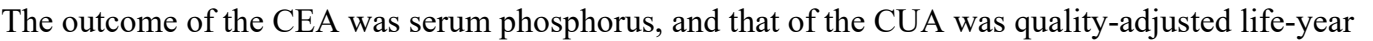

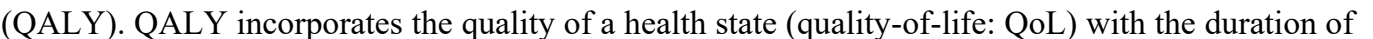

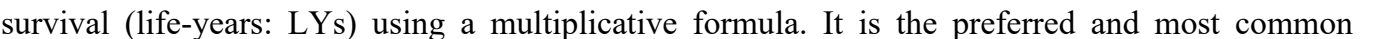

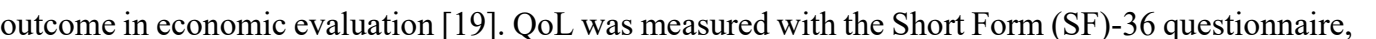

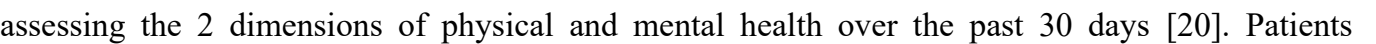

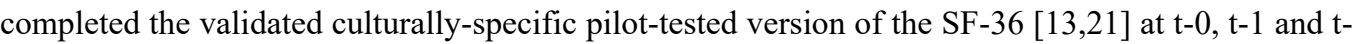

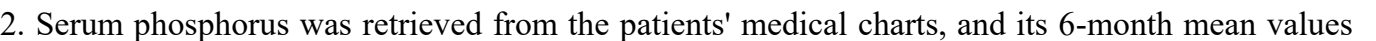

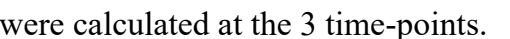

\section{$\square$ \\ $\square$}




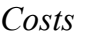

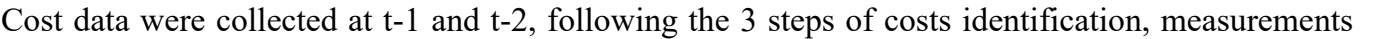

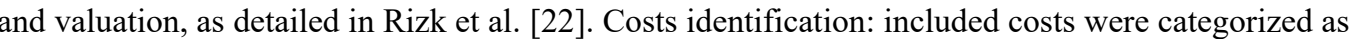

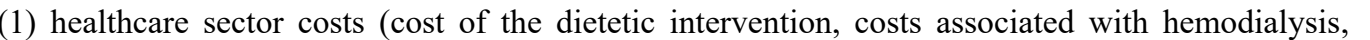

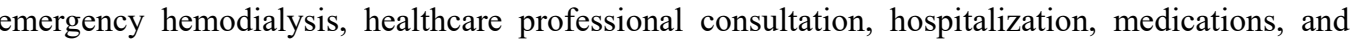
ए

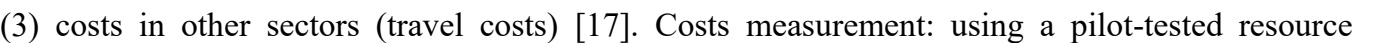

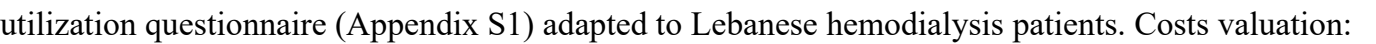

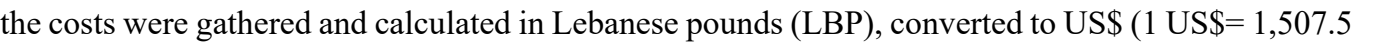

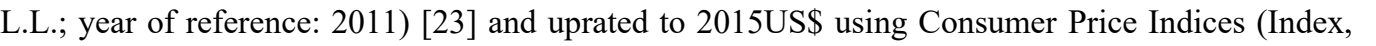

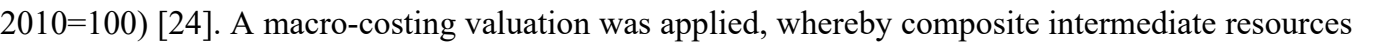

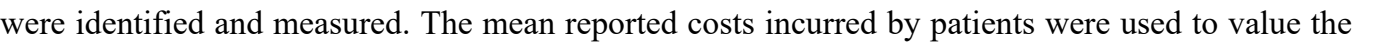

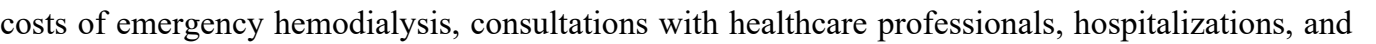

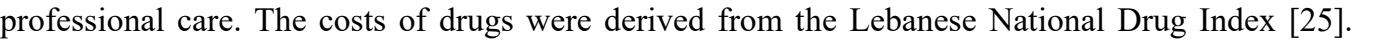

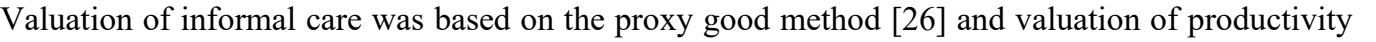

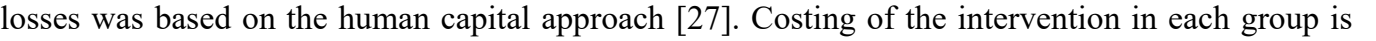

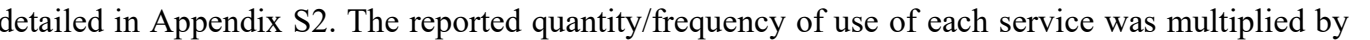

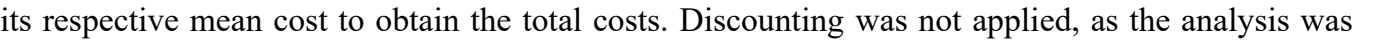

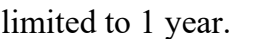

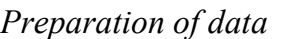

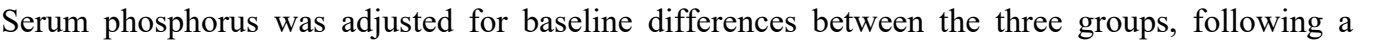

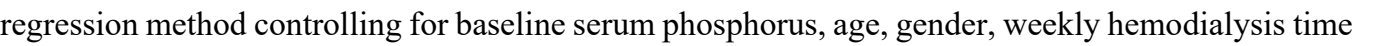

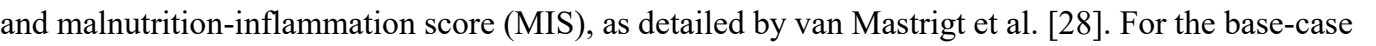

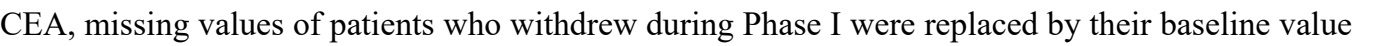

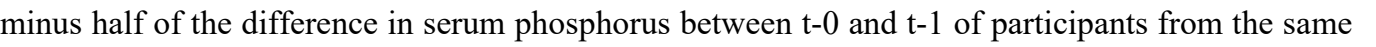

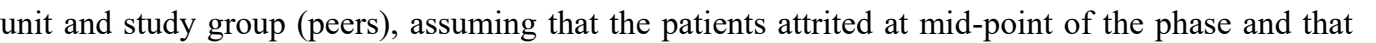

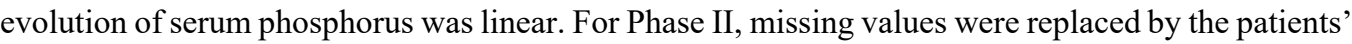

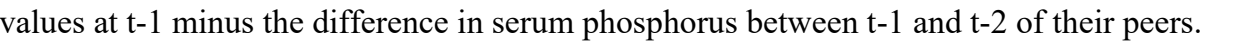

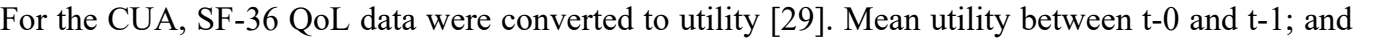

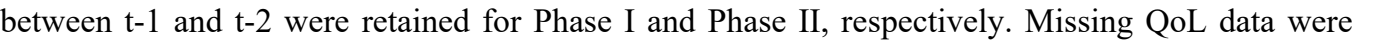

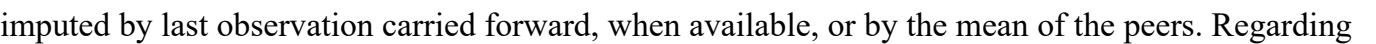

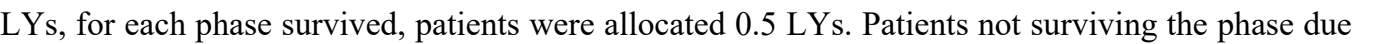

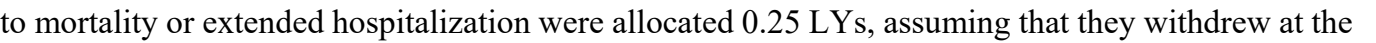

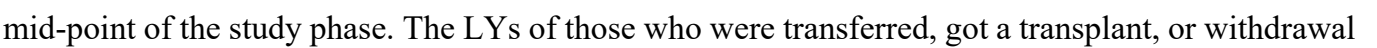

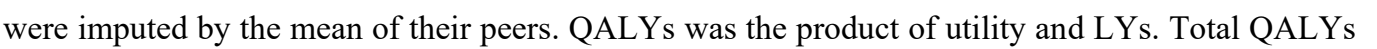

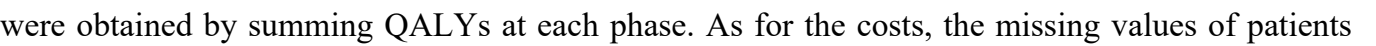




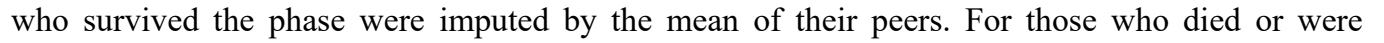

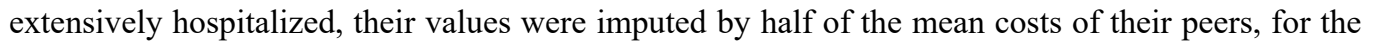

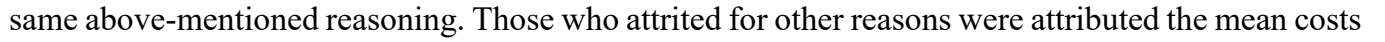

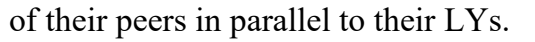

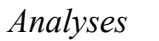

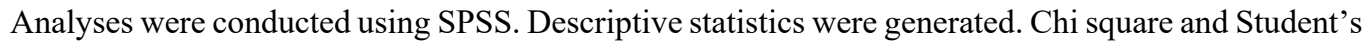

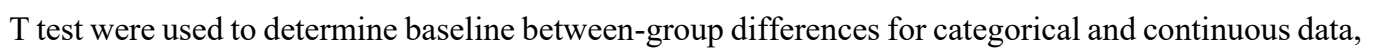

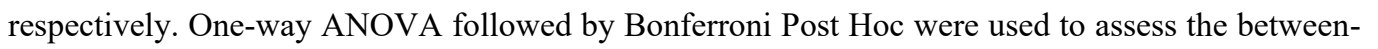

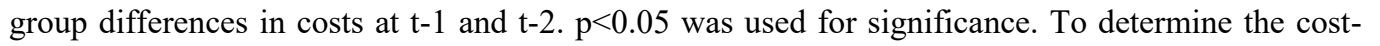

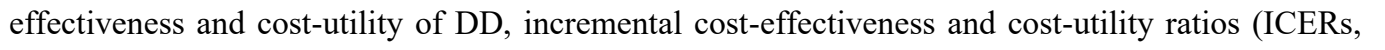

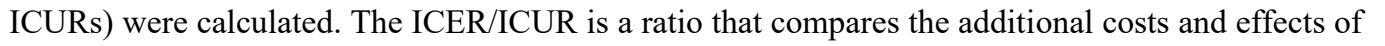

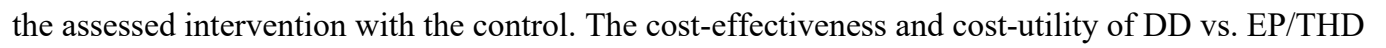

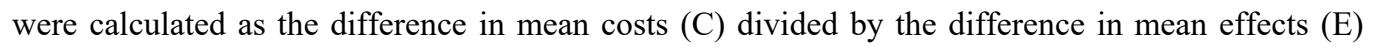

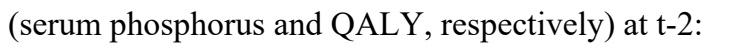

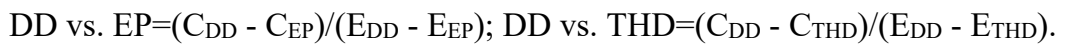

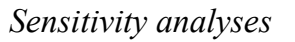

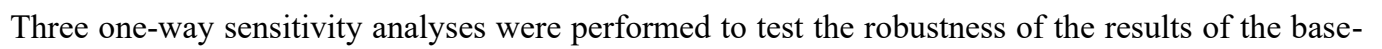

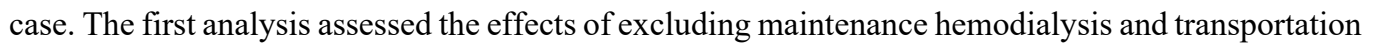

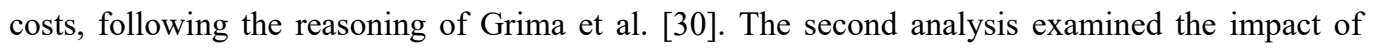

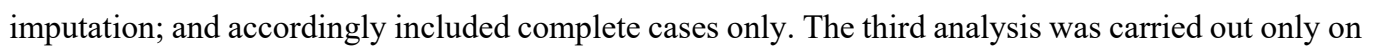

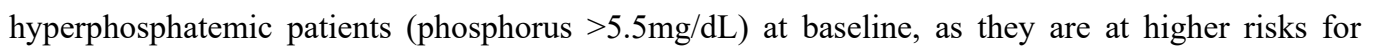

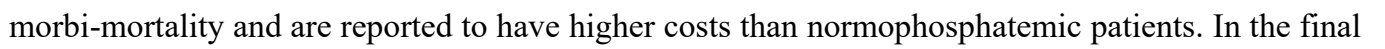

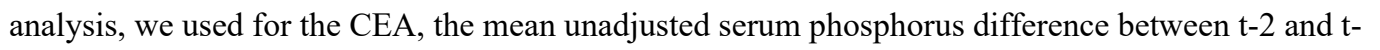

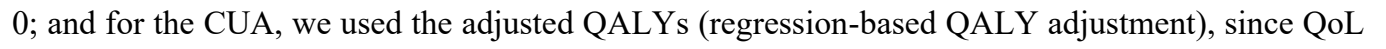

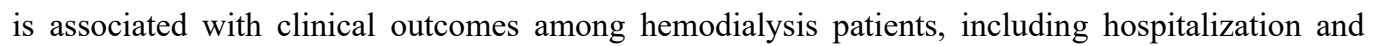

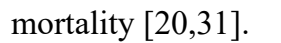

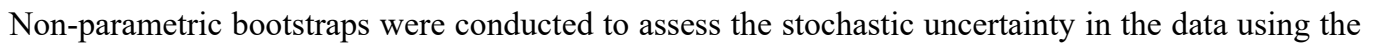

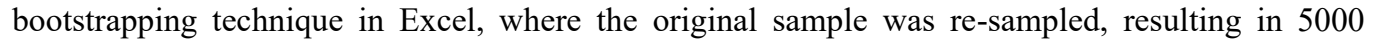

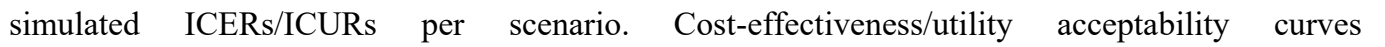

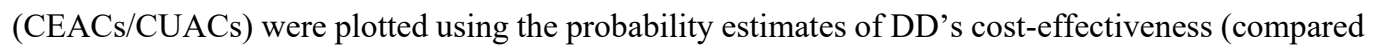

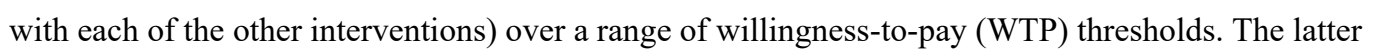

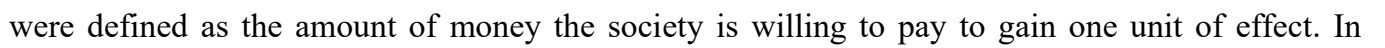

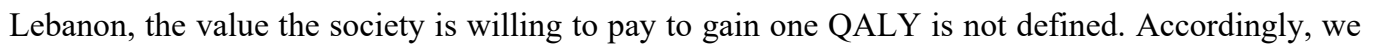

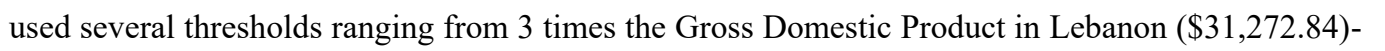

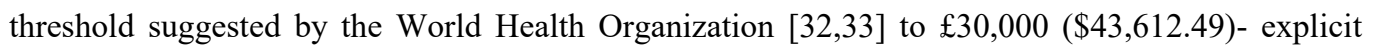




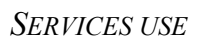

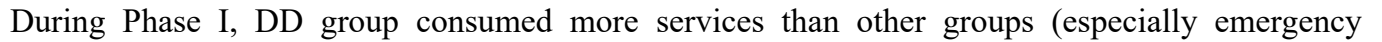

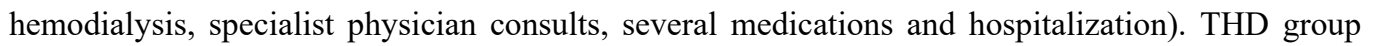

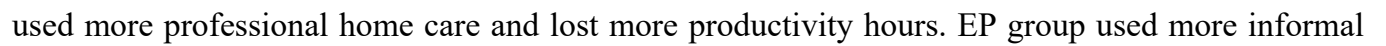

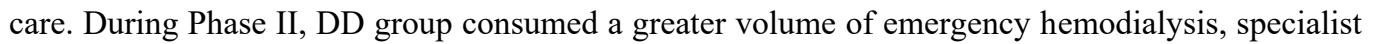

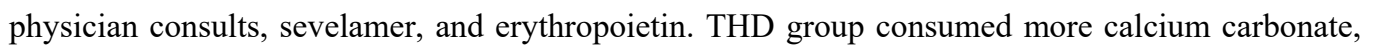

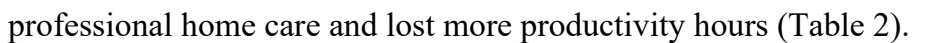

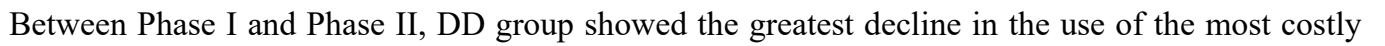

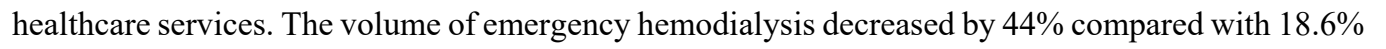

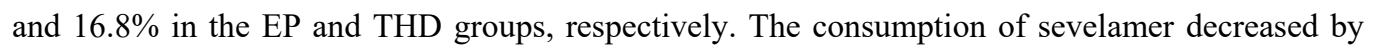

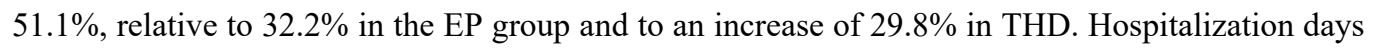

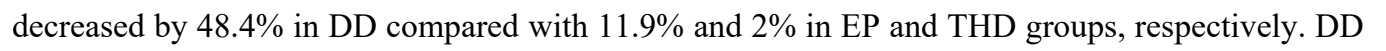

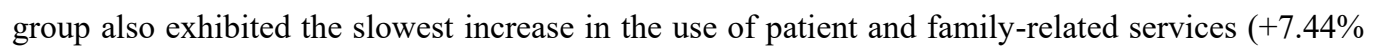

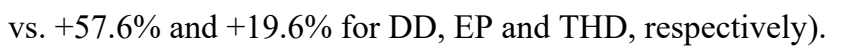

$\square$

$\square \square \square \square \square$

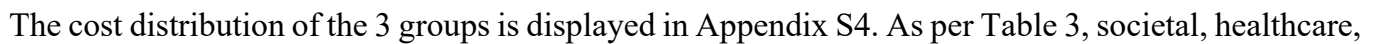

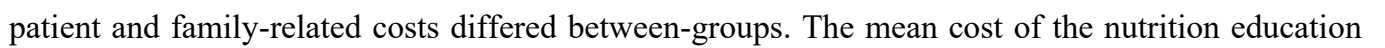

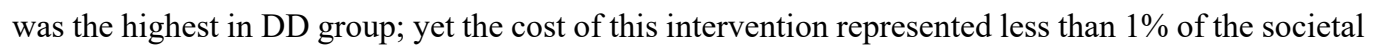

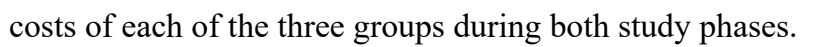

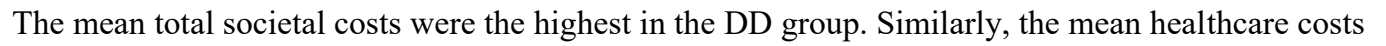

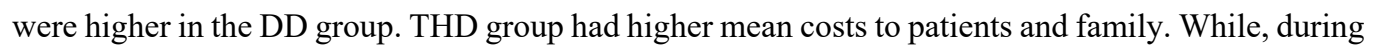

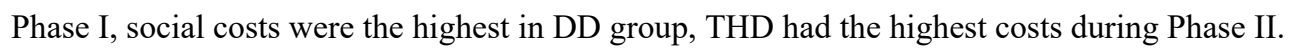

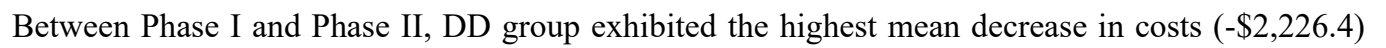

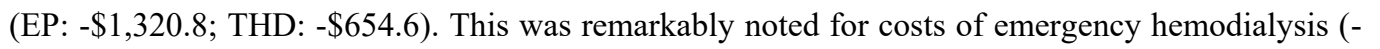

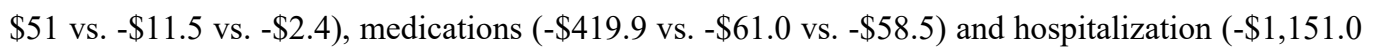

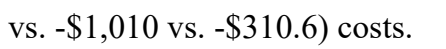

$\square$ 


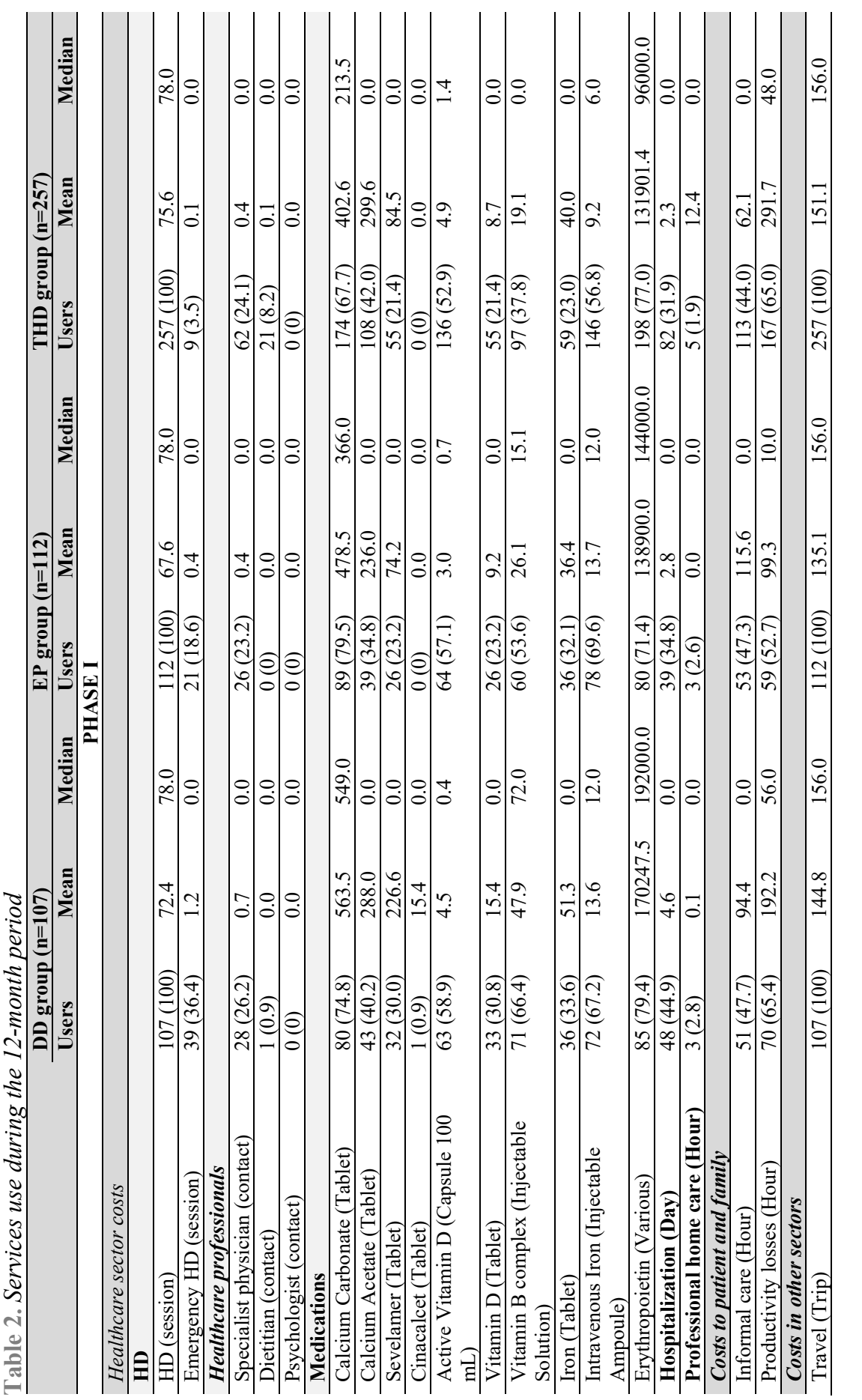




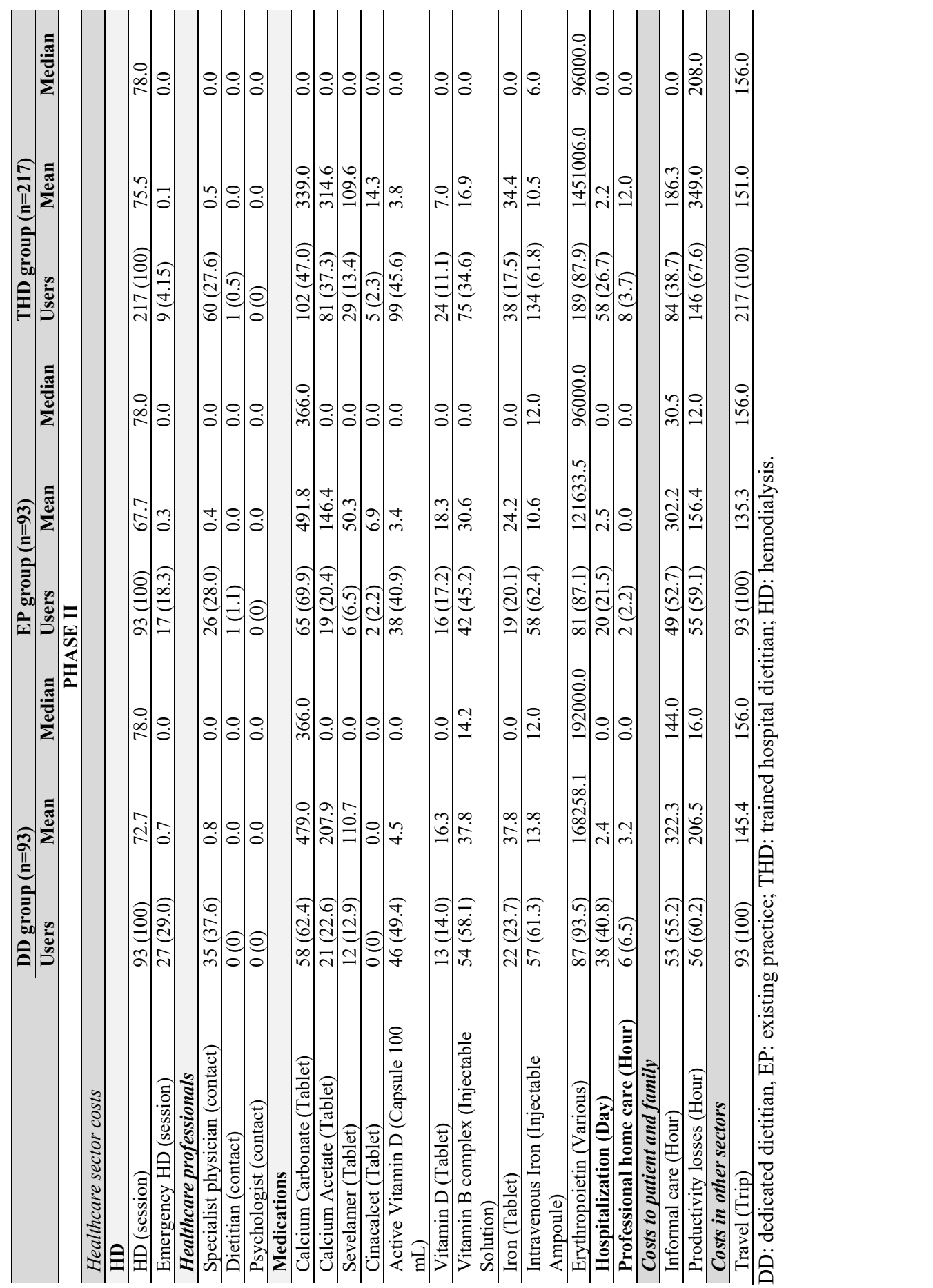




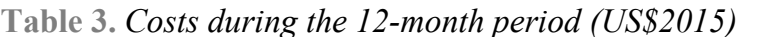

\begin{tabular}{|c|c|c|c|c|c|c|}
\hline \multirow[t]{2}{*}{$\square$} & \multicolumn{2}{|c|}{ DD group $(n=116)$} & \multicolumn{2}{|c|}{ EP group $(n=130)$} & \multicolumn{2}{|c|}{ THD group $(n=299)$} \\
\hline & Mean & Median & Mean & Median & Mean & Median \\
\hline \multicolumn{7}{|c|}{ PHASE I } \\
\hline \multicolumn{7}{|l|}{ Healthcare sector costs } \\
\hline HD & 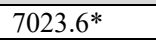 & 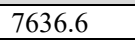 & 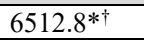 & 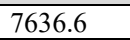 & $\square\left|\|\mid\|^{\circ} \square\right.$ & 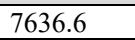 \\
\hline Maintenance HD & 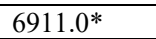 & 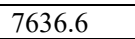 & 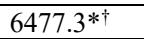 & 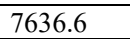 & $\square||||^{\prime} \square$ & 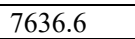 \\
\hline Emergency HD & 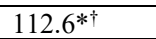 & $\square \square$ & $\square|\square| \square$ & $\square \square$ & $\square \square ं \square$ & $\square \square$ \\
\hline Healthcare professionals & 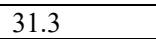 & $\square \square$ & $\square|\square|$ & $\square \square$ & $\square \| \square$ & $\square \square$ \\
\hline Medications & $\square|| \mid ण \dot{\square}$ & 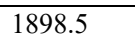 & $\square \square \| \square \square$ & 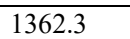 & $\square|\square| \square ं \square$ & 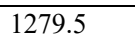 \\
\hline Hospitalization & $\square|||\|| \square$ & $\square \square$ & 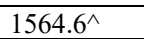 & $\square \square$ & $\square \mid\|\| \dagger$ & $\square \square$ \\
\hline Professional home care & $\square \square$ & 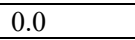 & 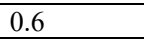 & $\mathrm{DII}$ & 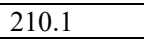 & 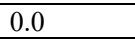 \\
\hline Total & 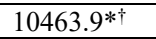 & 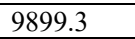 & 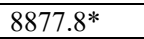 & 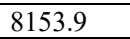 & 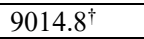 & पाप⿴囗十 \\
\hline \multicolumn{7}{|l|}{ Costs to patient and family } \\
\hline Informal care & \begin{tabular}{l|l|l|}
$\square$ \\
\end{tabular} & 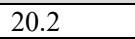 & \begin{tabular}{l|l|l|}
$\square$ \\
\end{tabular} & 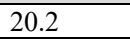 & \begin{tabular}{l|l|l|}
$\square$ & \\
\end{tabular} & 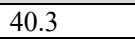 \\
\hline Productivity losses & 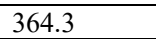 & $\square \square \square \square$ & $\square\|\| \square$ & $\square \square \square$ & $\square\|\| \|$ & $\square \square \square$ \\
\hline Total & \begin{tabular}{l|l|l|}
$\square$ \\
\end{tabular} & 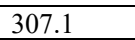 & 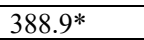 & 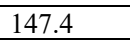 & $\square \square|\square| \square$ & 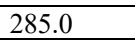 \\
\hline \multicolumn{7}{|l|}{ Costs in other sectors } \\
\hline Travel & $\square \square \square \square$ & $\square \square \square \square$ & $\square\|\| \| \square$ & 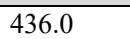 & $\square\|\| \| \square$ & $\square|\square| \square$ \\
\hline Intervention costs & 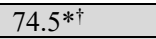 & $\square 111$ & 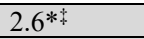 & पIII & $\square 1 \|^{\dagger *} \square$ & प1⿴囗十 \\
\hline Total societal costs & 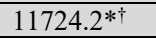 & 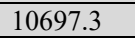 & \begin{tabular}{l|l|l|l|}
$\square\|\|\|\|$ \\
\end{tabular} & $+\square\|1\| 1 \|$ & 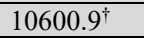 & 4\|\|$\|$ \\
\hline \multicolumn{7}{|c|}{ PHASE II } \\
\hline \multicolumn{7}{|l|}{ Healthcare sector costs } \\
\hline HD & 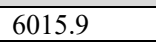 & $\square \Pi 110$ & 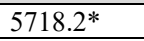 & 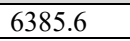 & 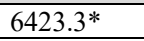 & 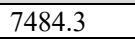 \\
\hline Maintenance HD & 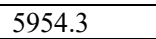 & 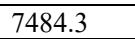 & 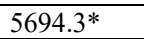 & 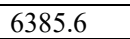 & $\square\|\| \square \square$ & 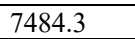 \\
\hline Emergency HD & $\square \square \square \vec{\square} \square$ & $\square \square$ & 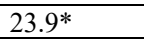 & $\square \square$ & $\square \square \dagger$ & $\square \square$ \\
\hline Healthcare professionals & 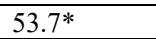 & $\square \square$ & 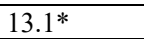 & $\square \square$ & $\square \square \square$ & $\square \square$ \\
\hline Medications & $\square\|1\|$ & 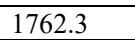 & 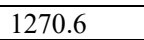 & $\square\|1\|$ & $\square \square|\square| \square$ & 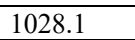 \\
\hline Hospitalization & 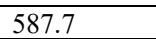 & 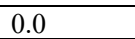 & 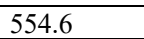 & $\square \square$ & $\square|||| \mid$ & $\square \square$ \\
\hline Professional home care & पापाप & 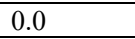 & 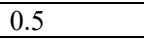 & 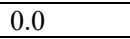 & 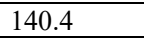 & 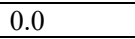 \\
\hline Total & 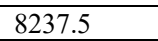 & 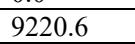 & $\square \| 10$ & 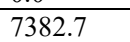 & $\square \square \square \square$ & $\square \square \square \square$ \\
\hline \multicolumn{7}{|l|}{ Costs to patient and family } \\
\hline Informal care & 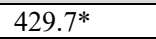 & 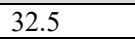 & 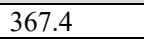 & $\square \square$ & 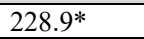 & $\square \square$ \\
\hline Productivity losses & $\square \square \square \square$ & 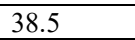 & 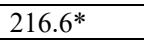 & 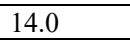 & $\square \||\square| \square$ & 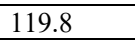 \\
\hline Total & 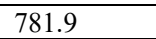 & 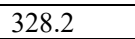 & 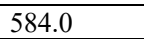 & \begin{tabular}{l|l|l|}
$\square$ \\
\end{tabular} & $\square|||| \mid$ & 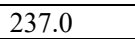 \\
\hline \multicolumn{7}{|l|}{ Costs in other sectors } \\
\hline Travel & 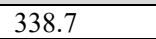 & $\square \square \square$ & $\square\|\| \square$ & 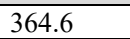 & $\square \square \| \square$ & 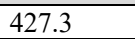 \\
\hline Intervention costs & \begin{tabular}{ll|}
$11 \square$ \\
\end{tabular} & पाII & पां & 10 & $\square \square+\square$ & पाII \\
\hline Total societal costs & 0111111 & 4111111 & $2 \square\|\| \square$ & $4 \square 1111$ & 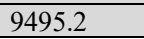 & $4\|1\| \square$ \\
\hline \multicolumn{7}{|c|}{ TOTAL COSTS } \\
\hline \multicolumn{7}{|l|}{ Healthcare sector costs } \\
\hline HD & 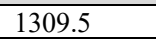 & 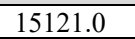 & 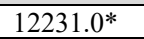 & 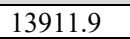 & 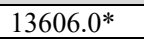 & 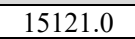 \\
\hline Maintenance HD & 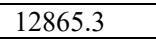 & 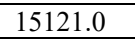 & 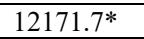 & 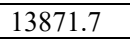 & 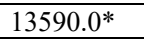 & 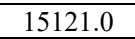 \\
\hline Emergency HD & $\square|| ⿴ 囗 十 丁$ & 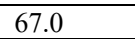 & 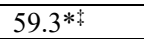 & 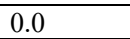 & $\square \mid \square^{*} \square$ & $\square \square$ \\
\hline Healthcare professionals & 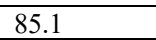 & $\square \square \square$ & $\square|\square|$ & $\square \square \square$ & 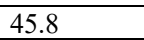 & $\square \square \square$ \\
\hline Medications & 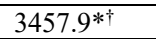 & 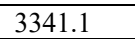 & 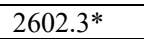 & 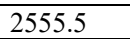 & $\left.\square \square||\right|^{\prime} \square$ & 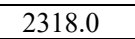 \\
\hline Hospitalization & 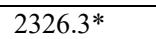 & 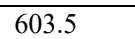 & $\square\|\| \| \square$ & 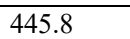 & $\square|||||| \mid$ & $\square|| \| \mid$ \\
\hline Professional home care & $\square \square$ & 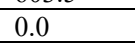 & पIII & पाण & 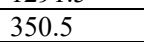 & $\square \square$ \\
\hline Total & $\square\|\|\|\|$ & $\square\|\| \|$ & 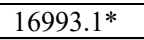 & 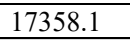 & $\square\|\|\|\|$ & $\square\|\| \| \square$ \\
\hline \multicolumn{7}{|l|}{ Costs to patient and family } \\
\hline Informal care & 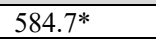 & 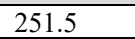 & 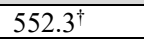 & 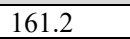 & 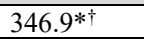 & 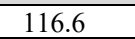 \\
\hline Productivity losses & $\square \| \Pi \square$ & 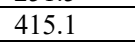 & $\square 1 \| \dagger \square$ & $\square 1 \Pi$ & $\square\|\| \| \dot{\square} \square$ & $\square \| \Pi$ \\
\hline Total & 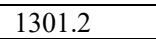 & \begin{tabular}{l|l|l|}
$\square$ \\
\end{tabular} & $\square\|\| \| \square$ & 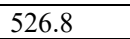 & 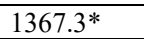 & \begin{tabular}{l|l|l|} 
\\
\end{tabular} \\
\hline \multicolumn{7}{|l|}{ Costs in other sectors } \\
\hline Travel & $\square\|\Pi\|$ & $\square \square \square$ & पापंप & 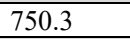 & 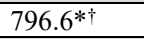 & 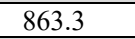 \\
\hline Intervention costs & पापांप & पाIII & $4111+\square$ & पIII & $\square 1 U^{* *} \square$ & प1111 \\
\hline Total societal costs & 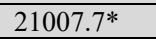 & $\square\|\|\|\|$ & 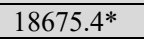 & 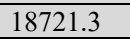 & 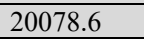 & $+\square||||||$ \\
\hline
\end{tabular}




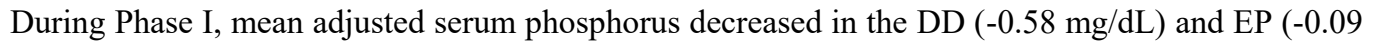

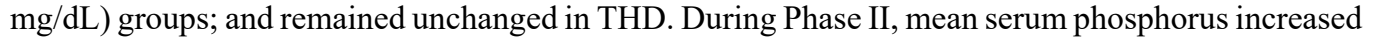

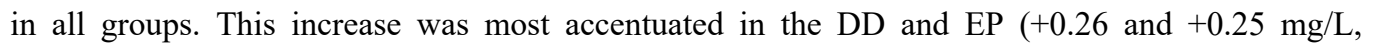

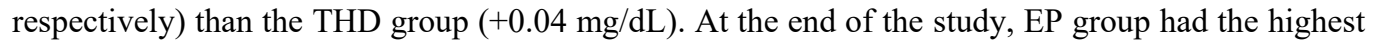

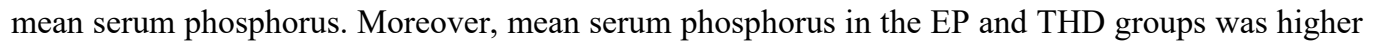

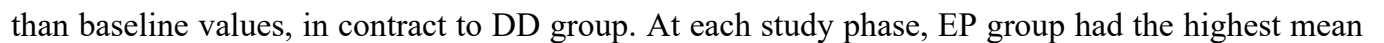

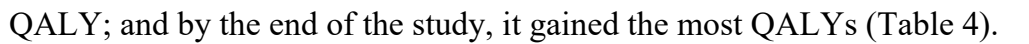

$\square$

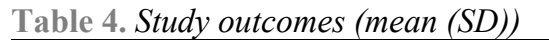

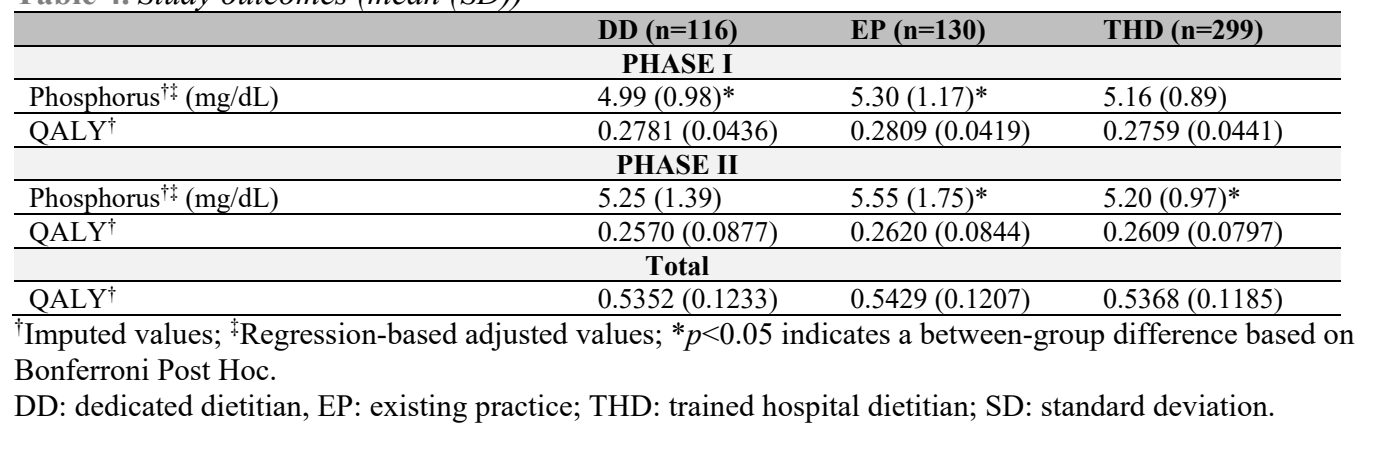

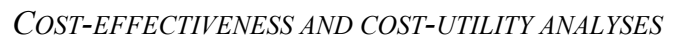

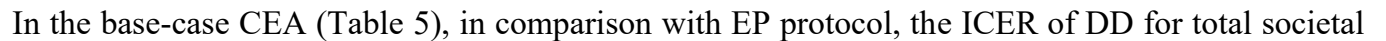

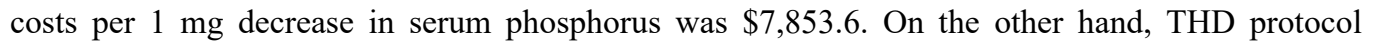

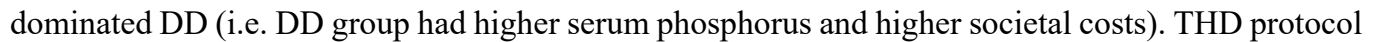

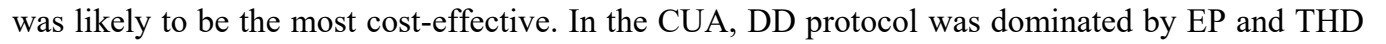

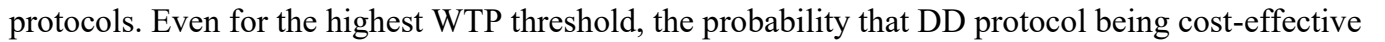
ए

$\square$

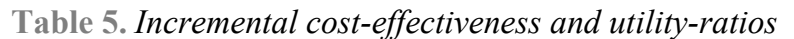

\begin{tabular}{|c|c|c|c|c|c|c|}
\hline \multirow[t]{2}{*}{$\square$} & \multicolumn{3}{|l|}{ DD vs. EP } & \multicolumn{3}{|l|}{ DD vs. THD } \\
\hline & $\begin{array}{l}\text { Incremental } \\
\text { effects }\end{array}$ & $\begin{array}{l}\text { Incremental } \\
\text { costs }\end{array}$ & ICER & $\begin{array}{l}\text { Incremental } \\
\text { effects }\end{array}$ & $\begin{array}{l}\text { Incremental } \\
\text { costs }\end{array}$ & ICER \\
\hline \multicolumn{7}{|c|}{ Serum phosphorus (mg/dL) } \\
\hline 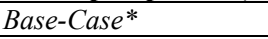 & 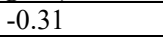 & 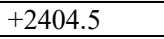 & $\square\|\| \|^{\circ} \square$ & \begin{tabular}{l|l|l|}
$\square \square$ \\
\end{tabular} & 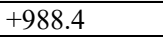 & 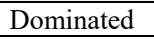 \\
\hline 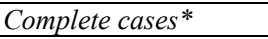 & \begin{tabular}{|l|l|l|l|l|}
$\square|l|$ \\
\end{tabular} & 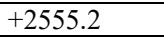 & \begin{tabular}{l|l|l|}
$\square$ \\
\end{tabular} & 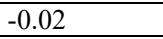 & 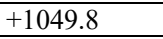 & 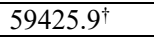 \\
\hline 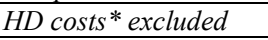 & 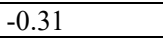 & 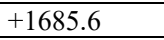 & 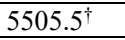 & \begin{tabular}{l|l|l|}
$\square \square$ \\
\end{tabular} & 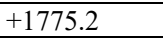 & 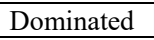 \\
\hline 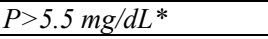 & 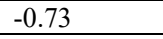 & \begin{tabular}{l|l|l|l|}
$\square \square$ & \\
\end{tabular} & 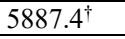 & 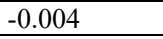 & \begin{tabular}{l|l|l|l|l|}
$\square$ & \\
\end{tabular} & 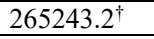 \\
\hline 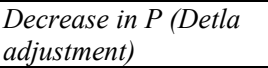 & \begin{tabular}{l|l|l|}
$\square|l|$ &
\end{tabular} & 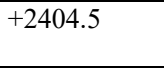 & 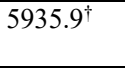 & 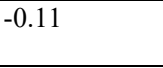 & 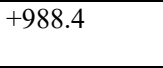 & 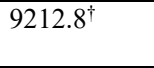 \\
\hline
\end{tabular}




\begin{tabular}{|c|c|c|c|c|c|c|}
\hline \multirow[t]{2}{*}{$\square$} & \multicolumn{3}{|l|}{ DD vs. EP } & \multicolumn{3}{|l|}{ DD vs. THD } \\
\hline & $\begin{array}{l}\text { Incremental } \\
\text { effects }\end{array}$ & $\begin{array}{l}\text { Incremental } \\
\text { costs }\end{array}$ & ICER & $\begin{array}{l}\text { Incremental } \\
\text { effects }\end{array}$ & $\begin{array}{l}\text { Incremental } \\
\text { costs }\end{array}$ & ICER \\
\hline \multicolumn{7}{|l|}{ QALY } \\
\hline 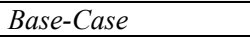 & 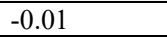 & 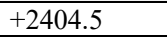 & 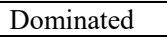 & 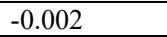 & 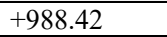 & 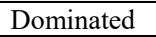 \\
\hline 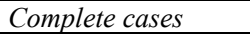 & $\begin{array}{lll}\mathrm{m} \\
\mathrm{n}\end{array}$ & $\square \square 1110$ & पमापाण & 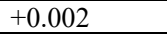 & 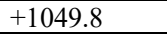 & 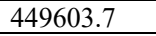 \\
\hline 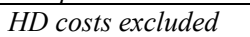 & एवाणा & 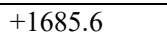 & प्मापाणा & एखायाए & 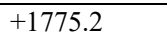 & 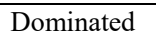 \\
\hline 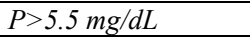 & 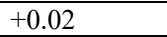 & 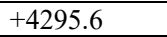 & $\square 11111010$ & 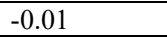 & 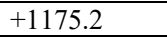 & 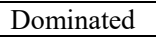 \\
\hline 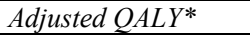 & $\begin{array}{lll}1010 \\
\end{array}$ & $\square \square \mid 110$ & पमापाण & 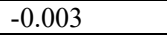 & $\square \square 110$ & 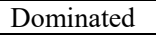 \\
\hline
\end{tabular}

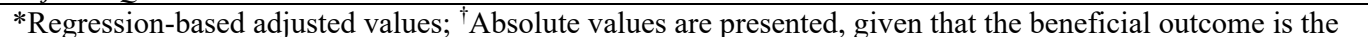

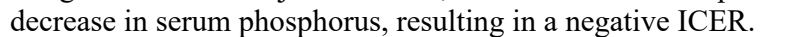

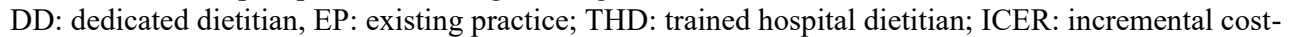

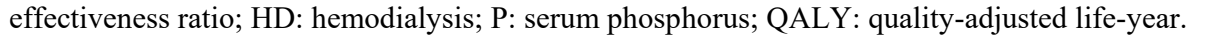

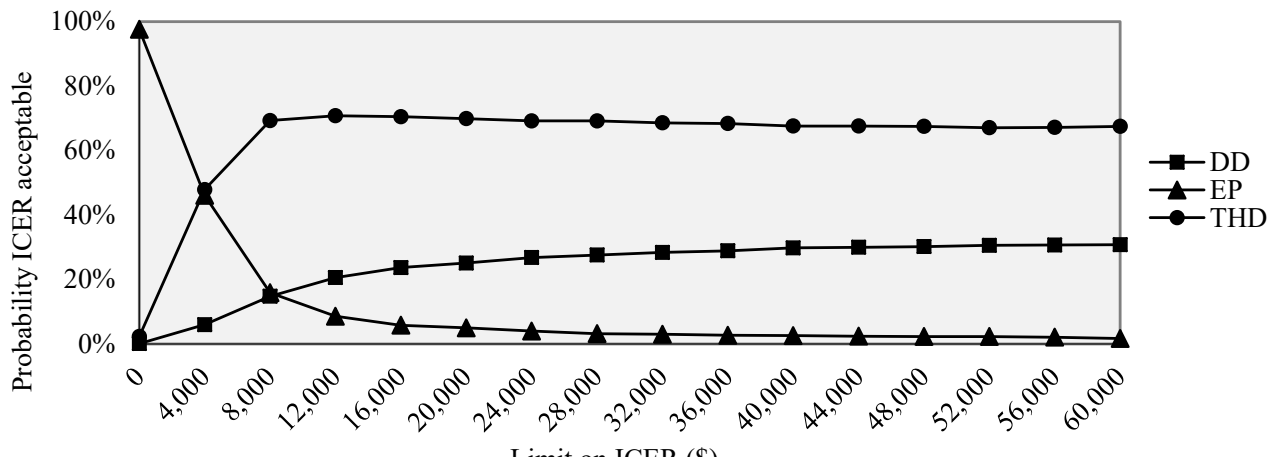

Figure 1a.

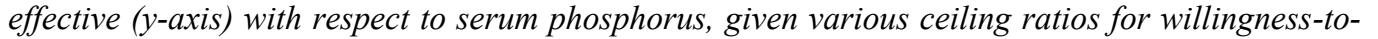

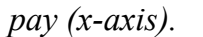

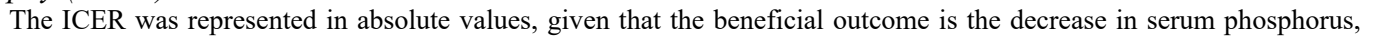
ए एण

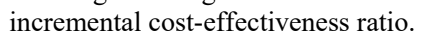

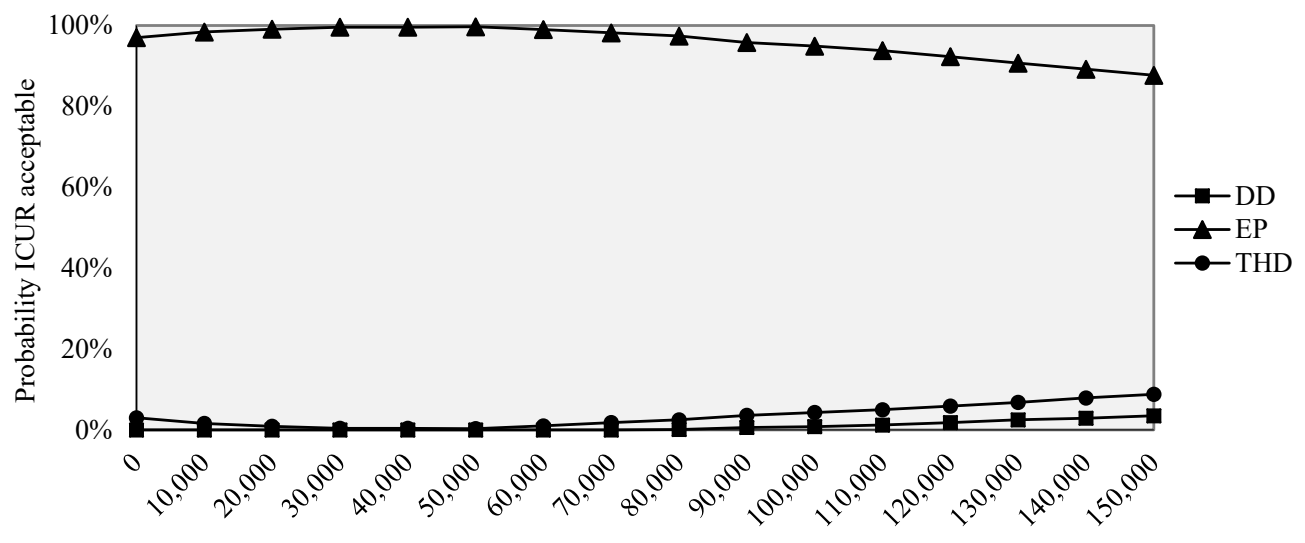

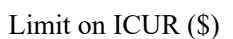

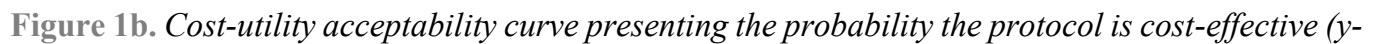

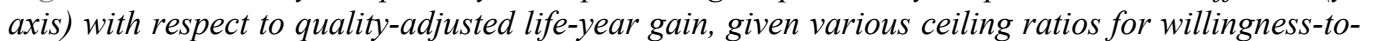

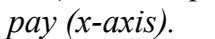

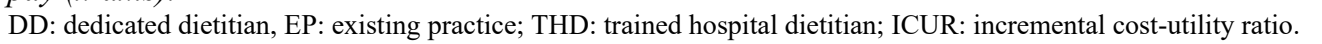




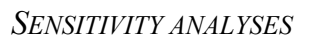

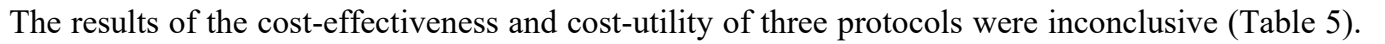

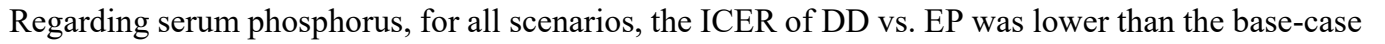

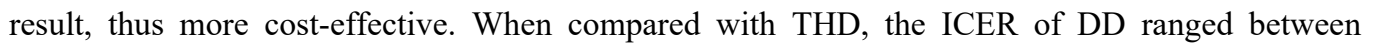

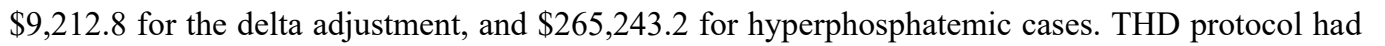

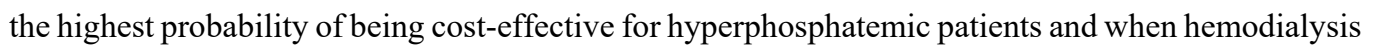

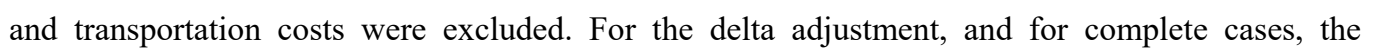

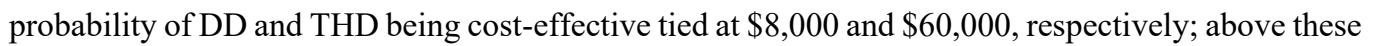

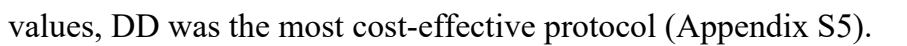

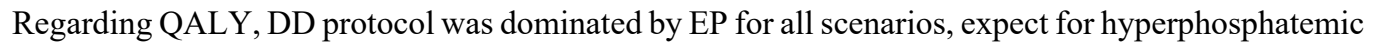

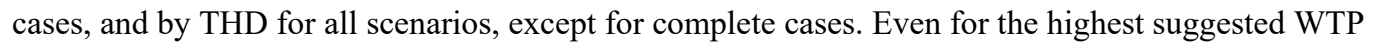
ए

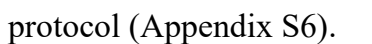

$\square$

\section{DISSCUSSION}

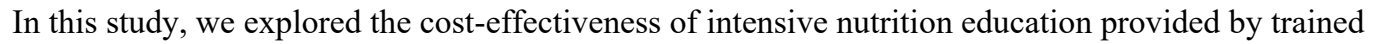

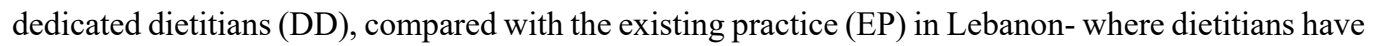

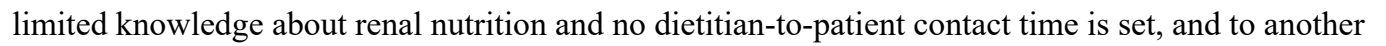

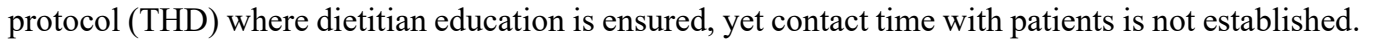

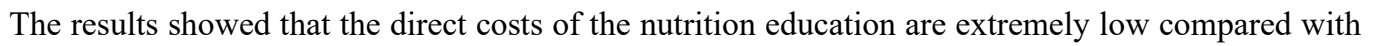

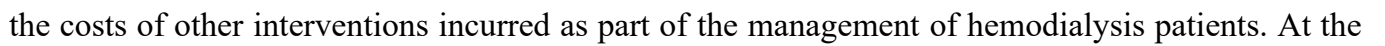

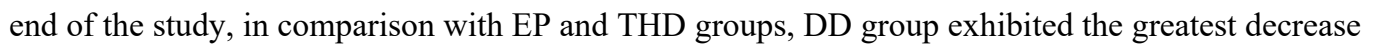

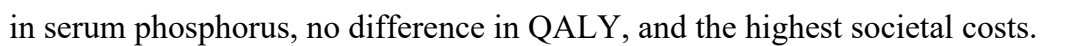

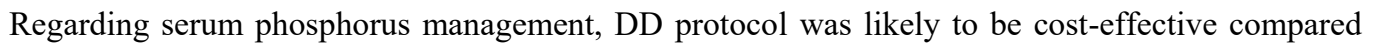

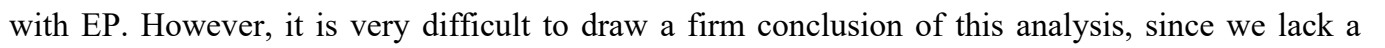

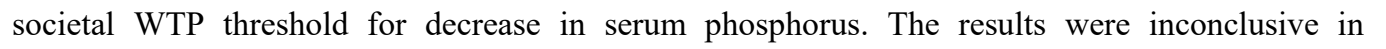

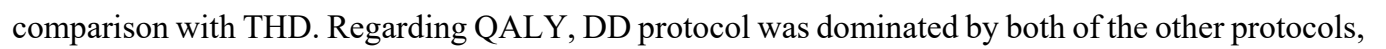

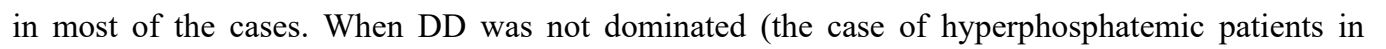

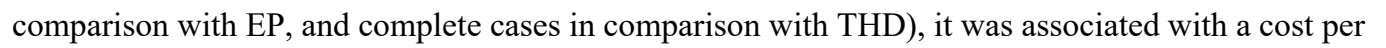

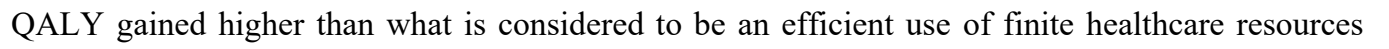

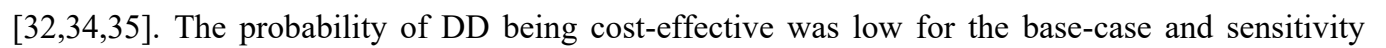
$\square\|\|\|\| \| \square$

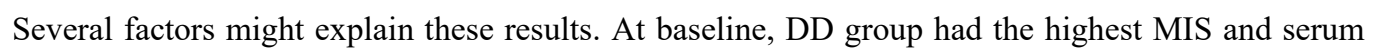

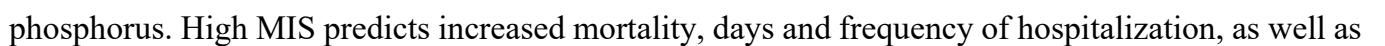


ए

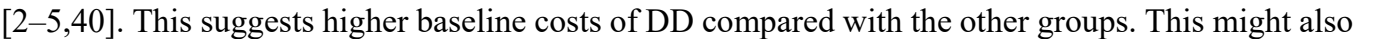

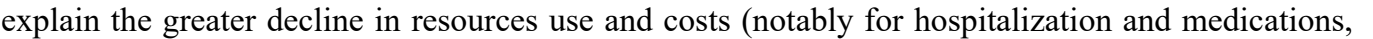

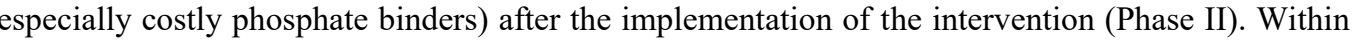

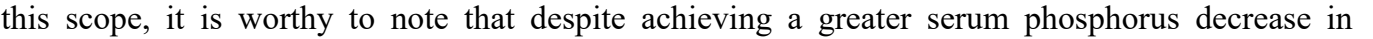

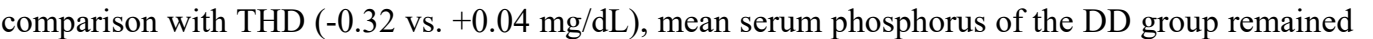

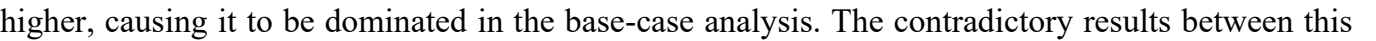

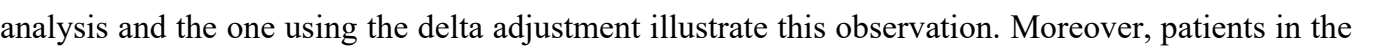

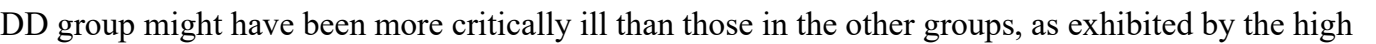

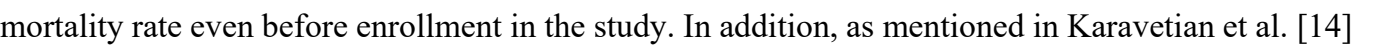

\section{the effectiveness of the DD protocol might have been partially "masked" by the improvement noted}

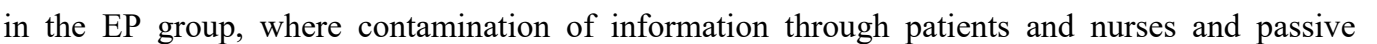

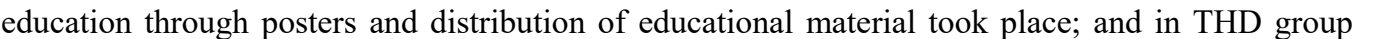

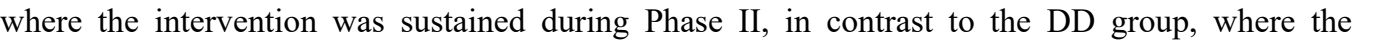

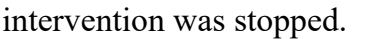

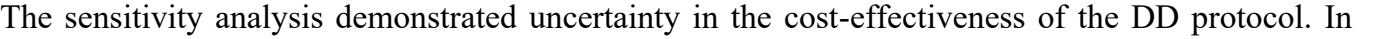

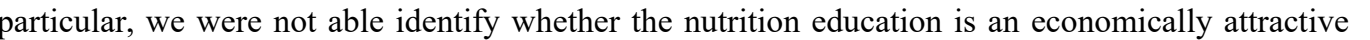

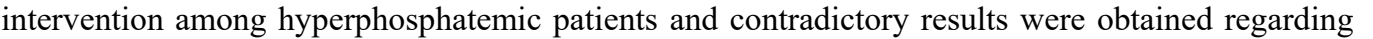

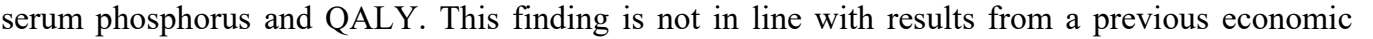

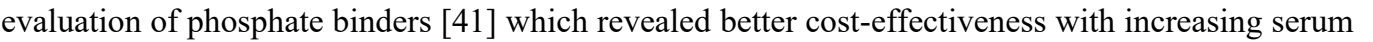

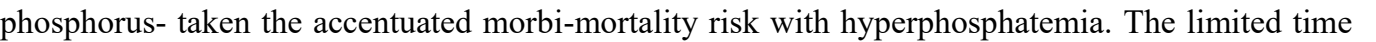

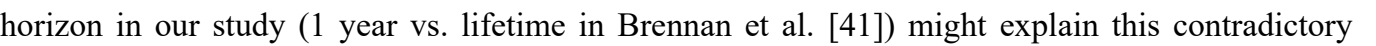

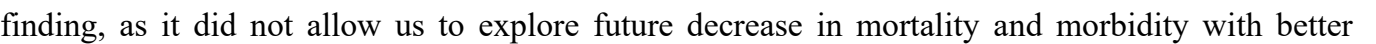

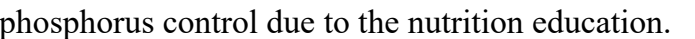

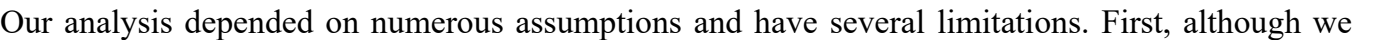

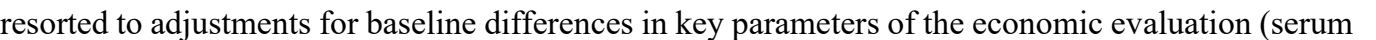

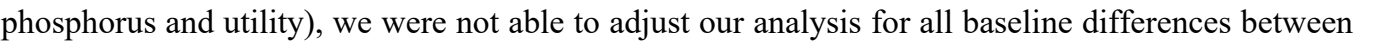
प

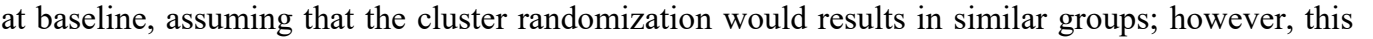

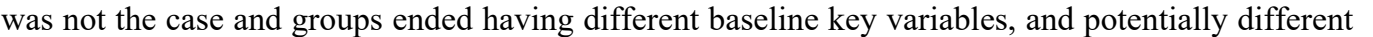

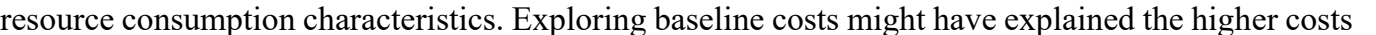

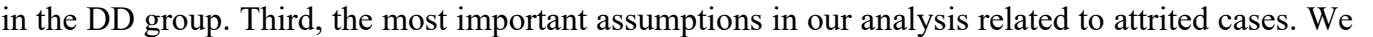

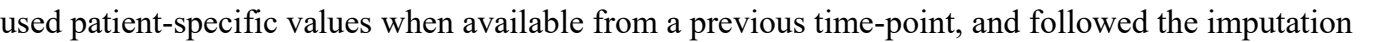

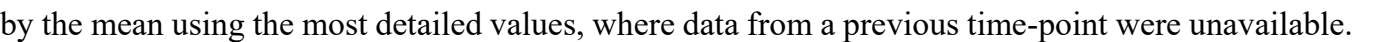

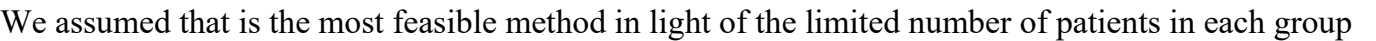

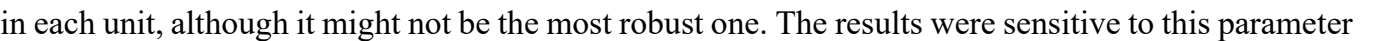




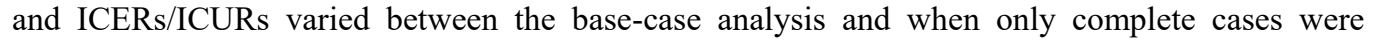

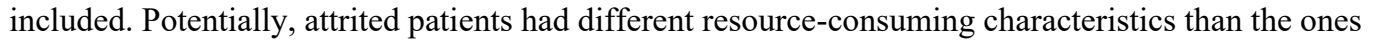

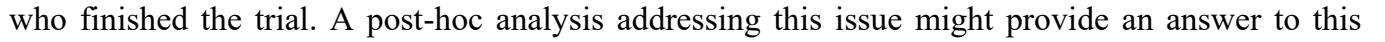

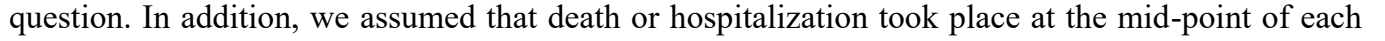

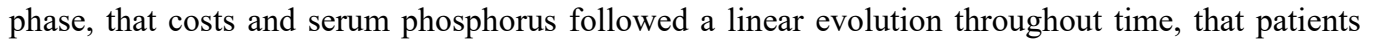

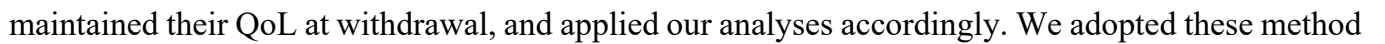

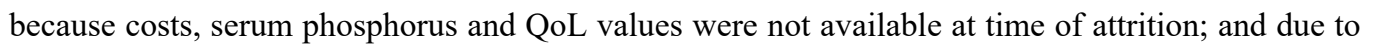

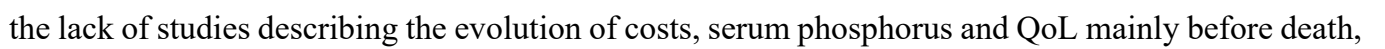

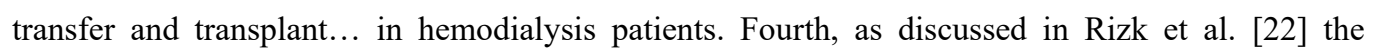

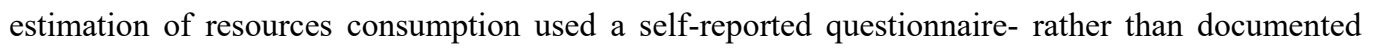

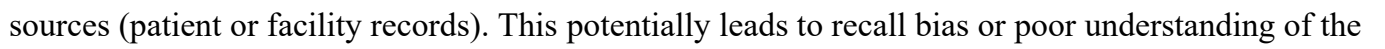

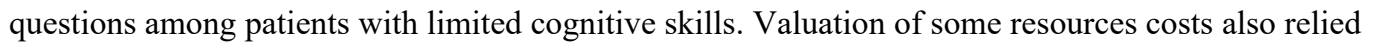

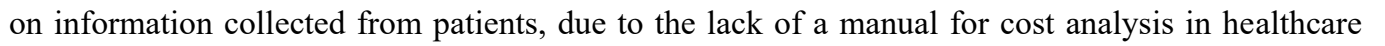

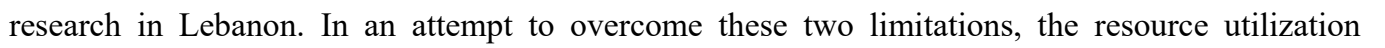

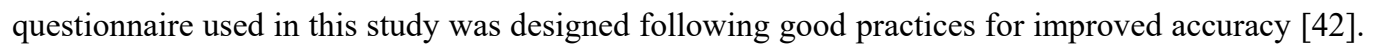

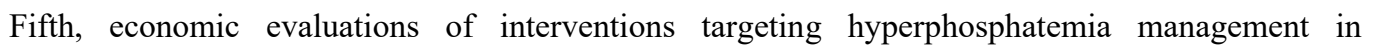

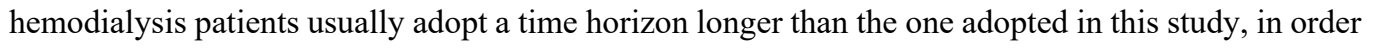

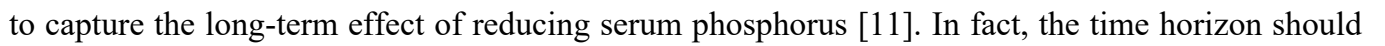

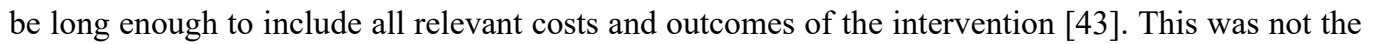

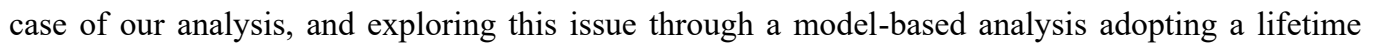

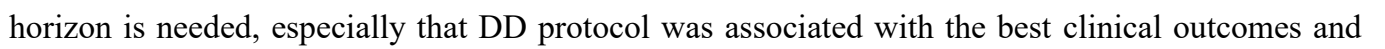

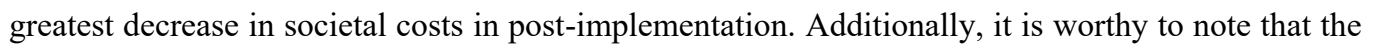

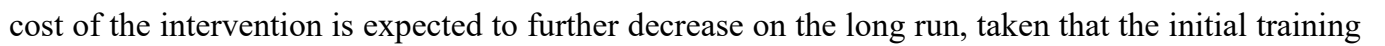

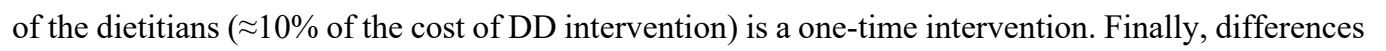

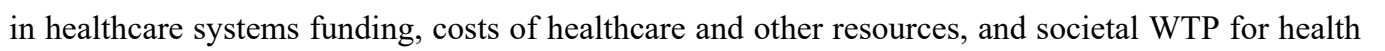

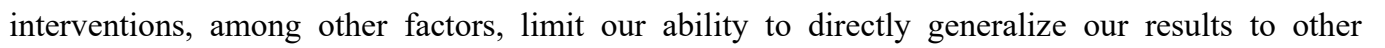

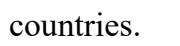

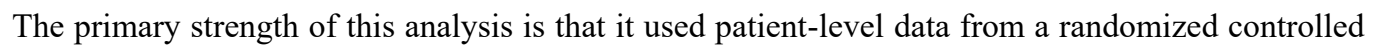

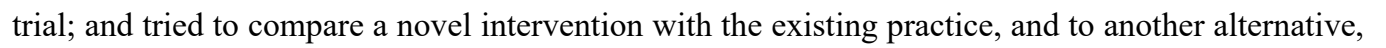

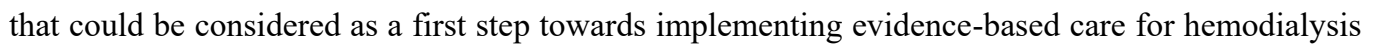

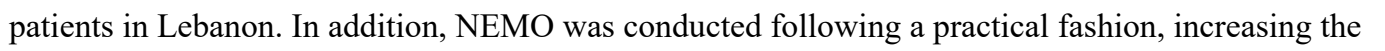

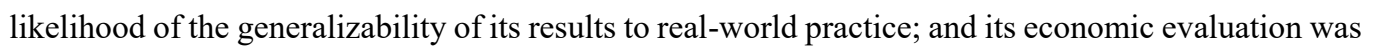

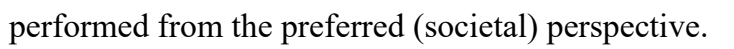

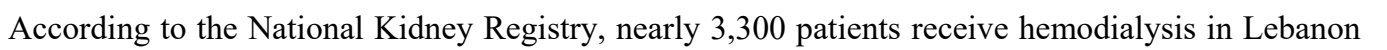

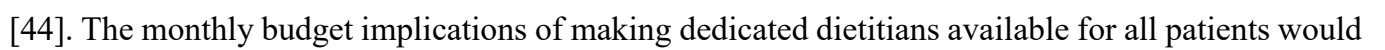
be substantial $(\approx \$ 40,000)$. Yet, a closer look at the cost savings of this intervention are also likely to 


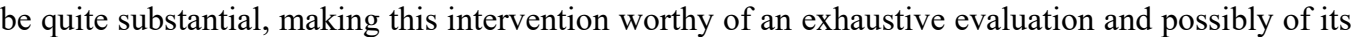

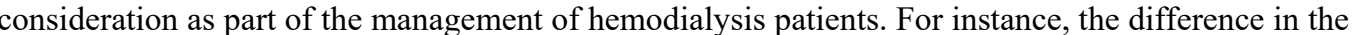

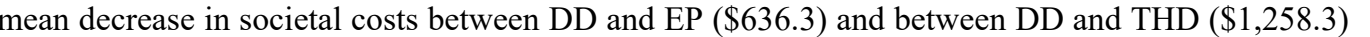

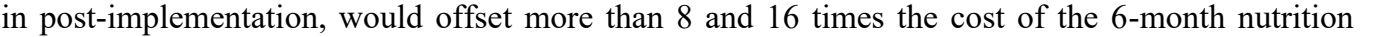

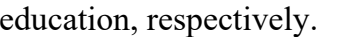

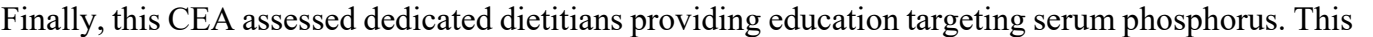

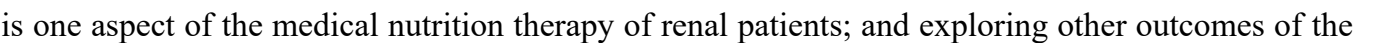

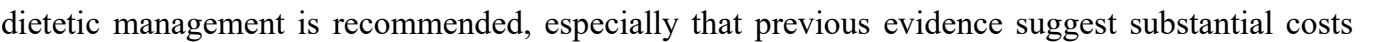

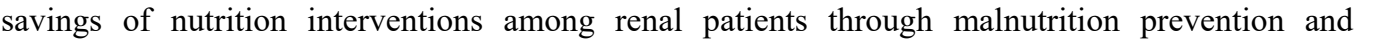

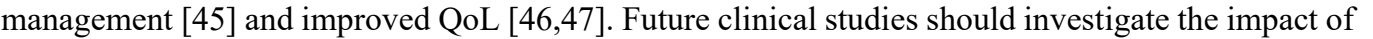

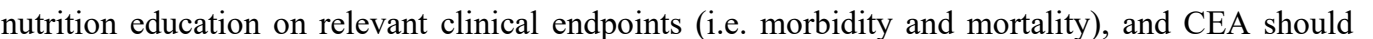

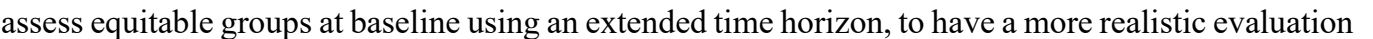

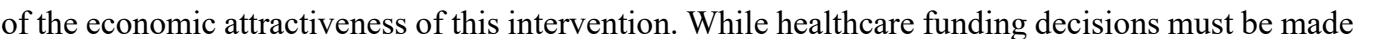

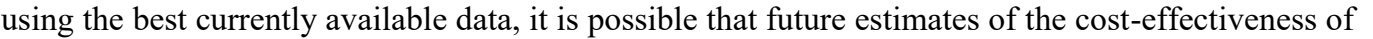

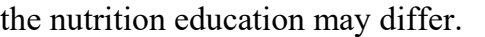

$\square$

\section{PraCticAl IMPLiCATIONS}

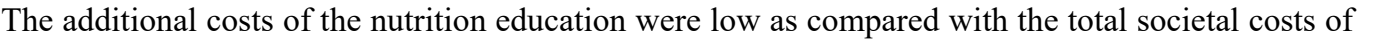

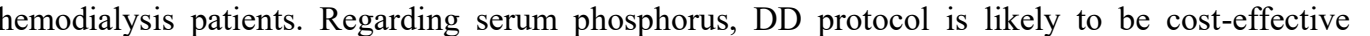

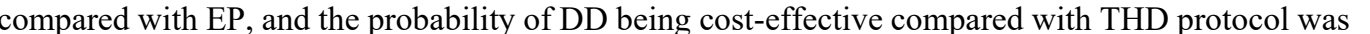

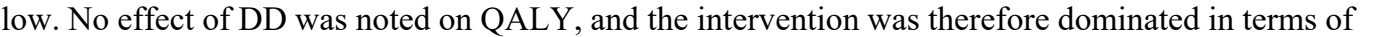

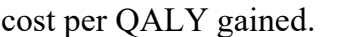

\section{Acknowledgments}

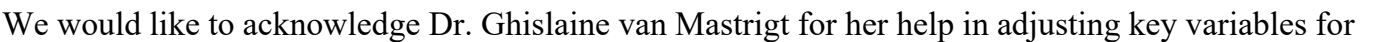

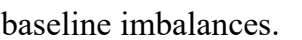




\section{REFERENCES}

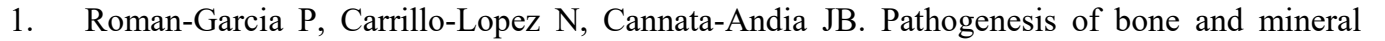

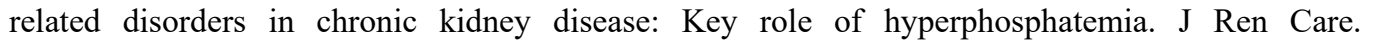

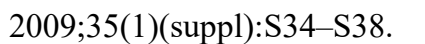

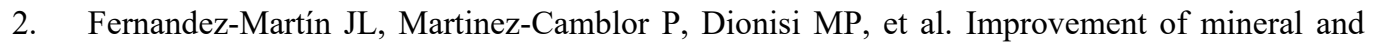

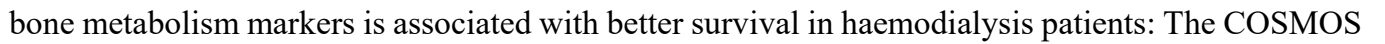

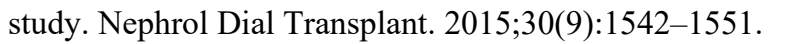

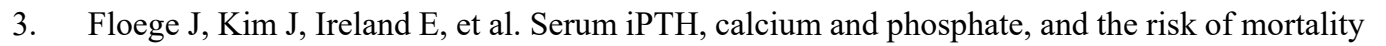

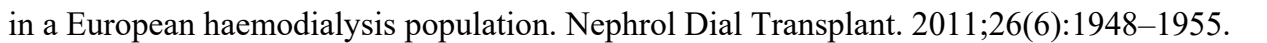

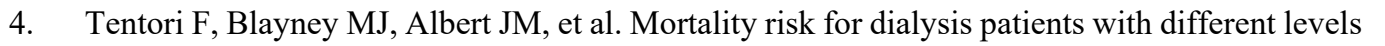

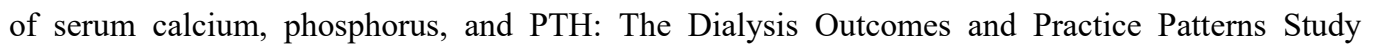

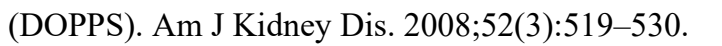

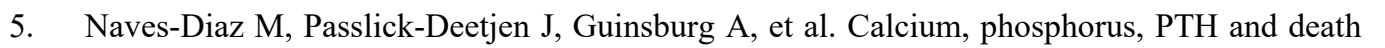

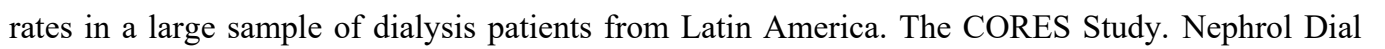

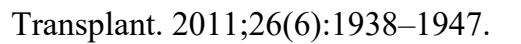

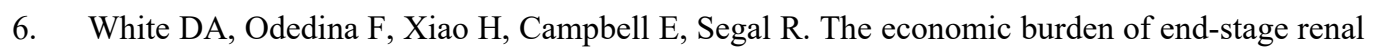

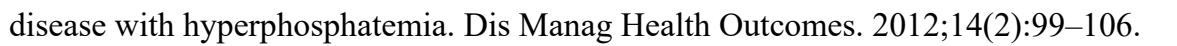

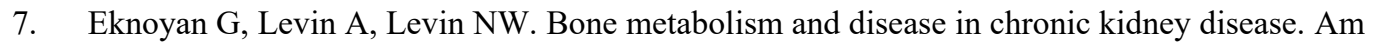

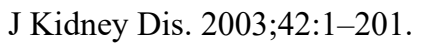

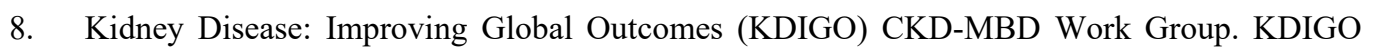

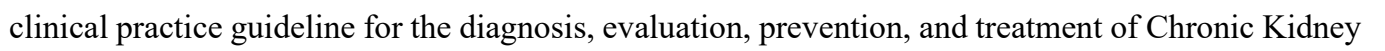

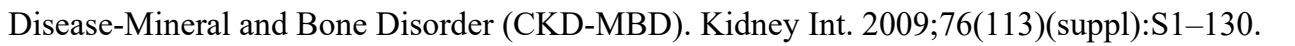

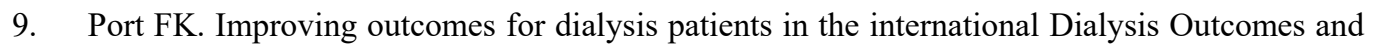

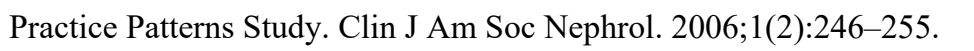

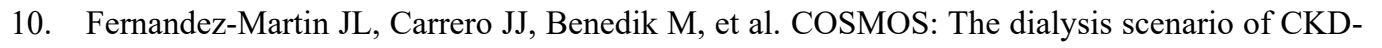

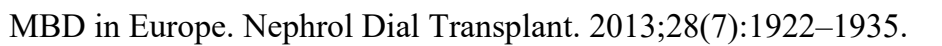

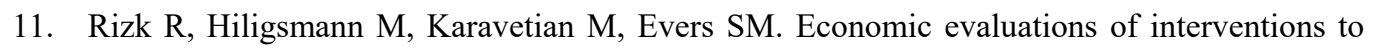

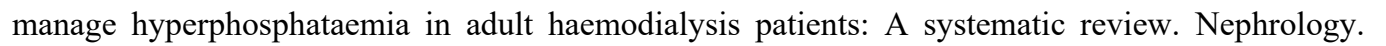

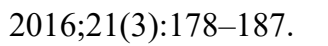

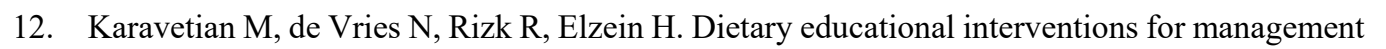

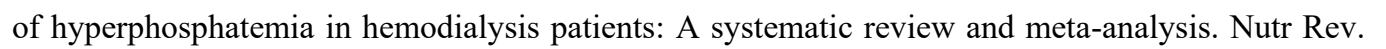

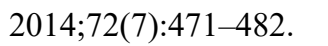

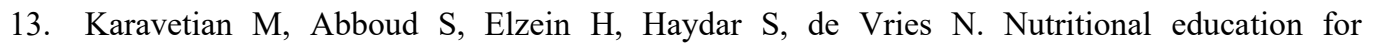

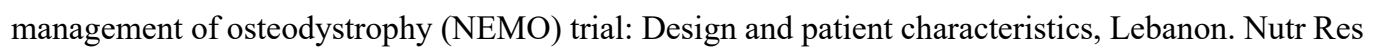

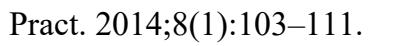




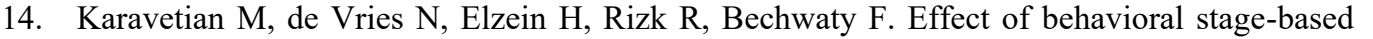

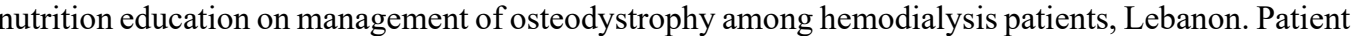

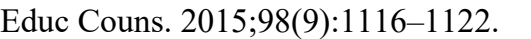

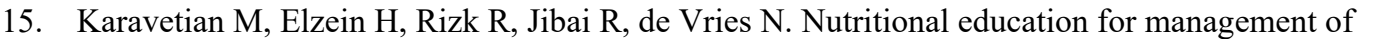

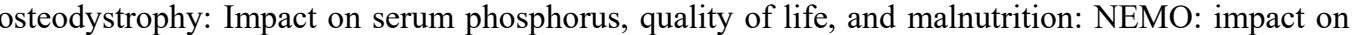

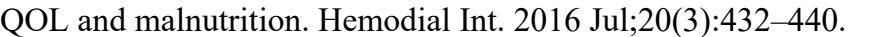

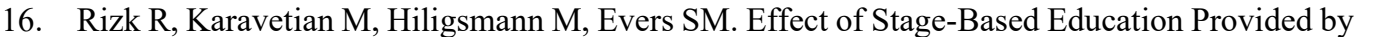

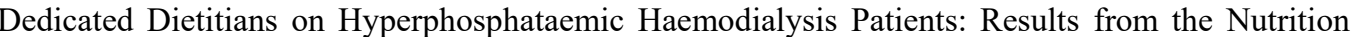

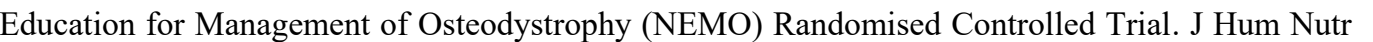

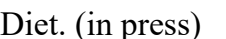

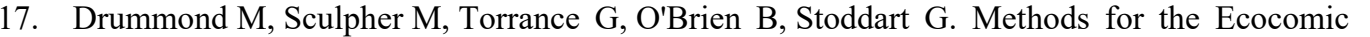

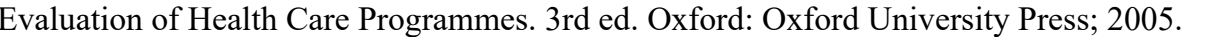

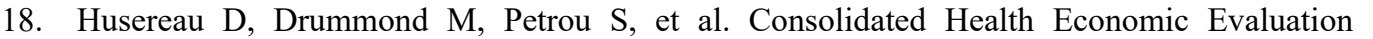

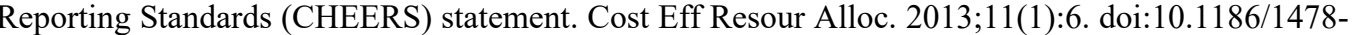

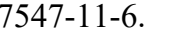

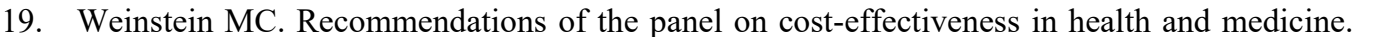

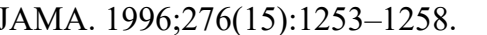

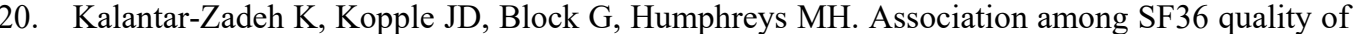

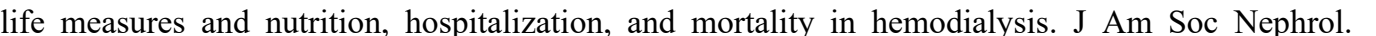

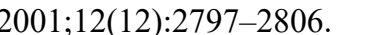

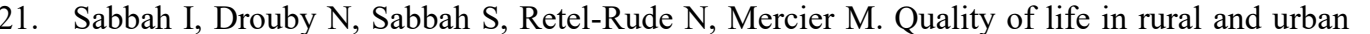

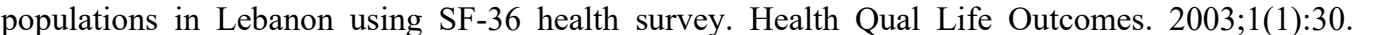

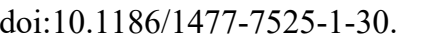

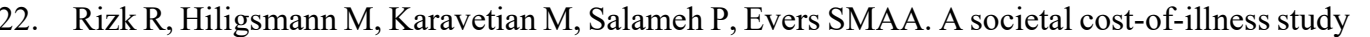

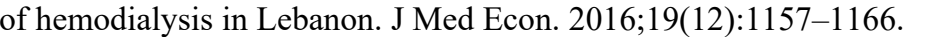

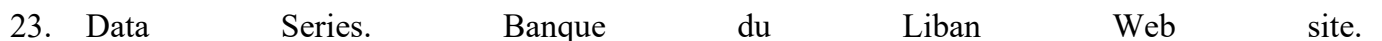

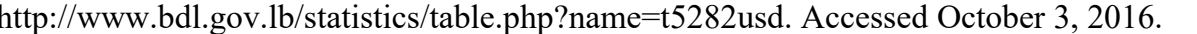

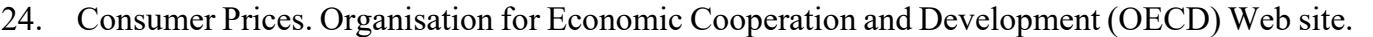

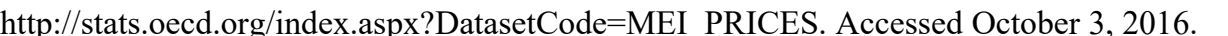

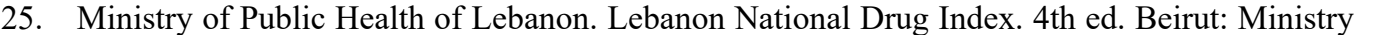

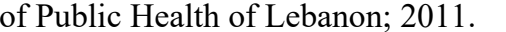

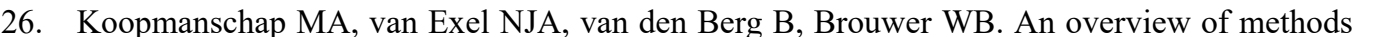

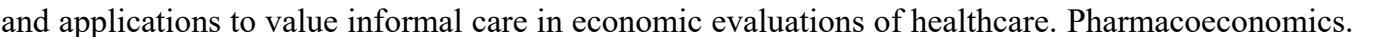

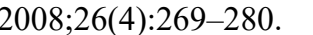




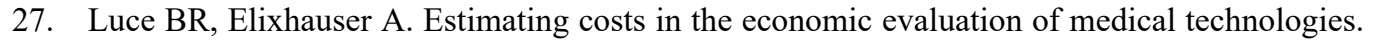

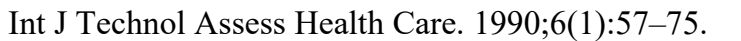

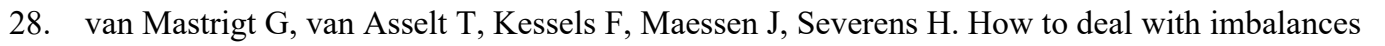

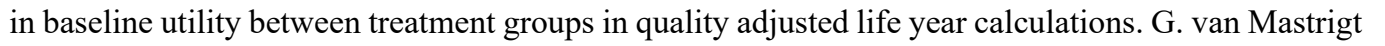

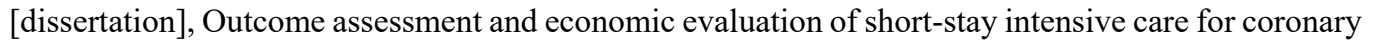

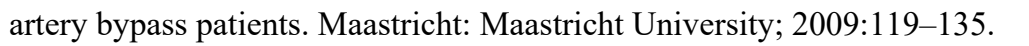

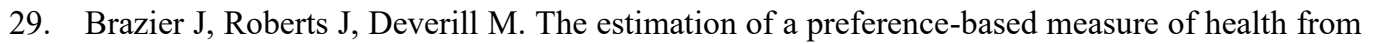

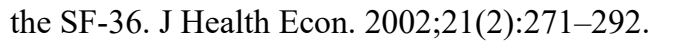

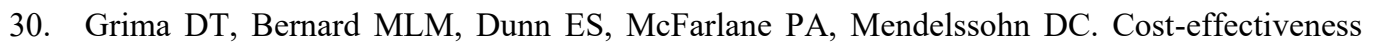

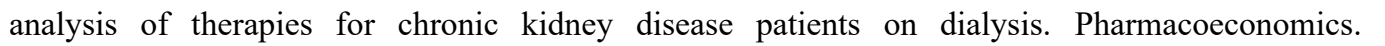

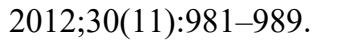

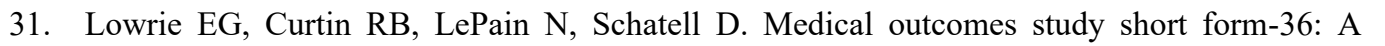

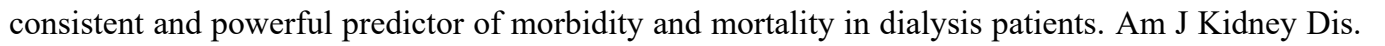

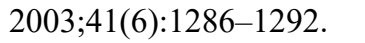

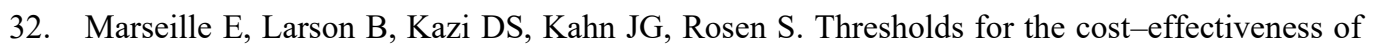

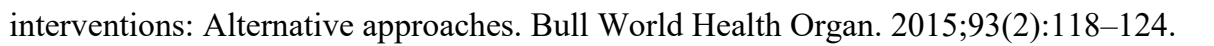

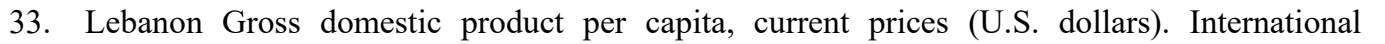

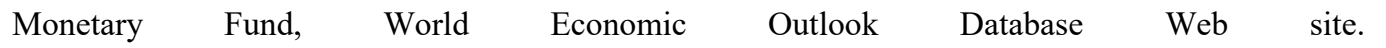

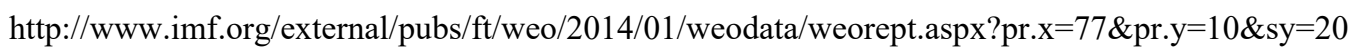

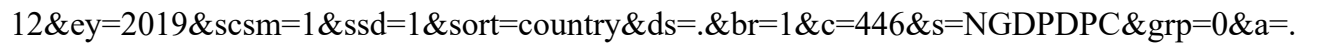

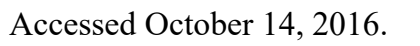

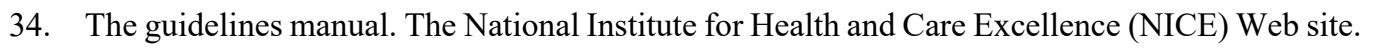

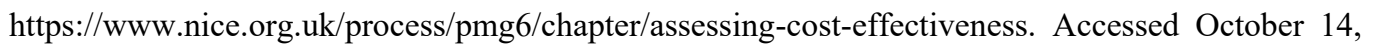

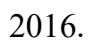

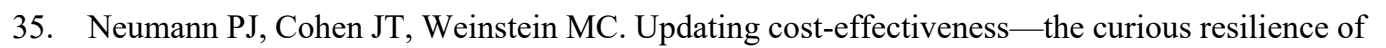

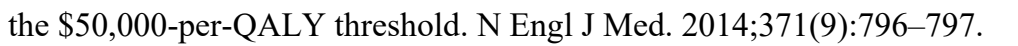

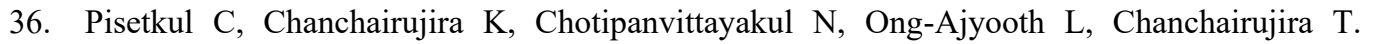

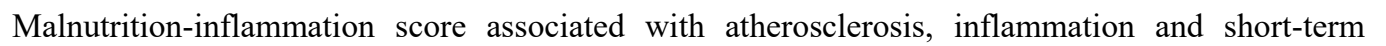

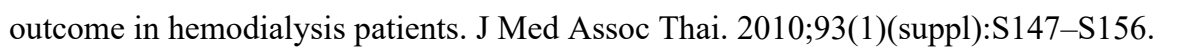

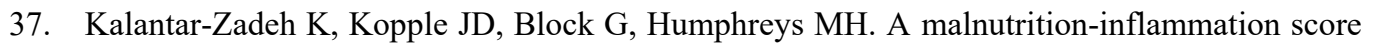

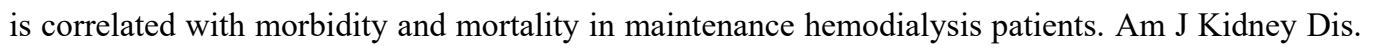
$\square|||||||||||-\square| \mid \Pi$

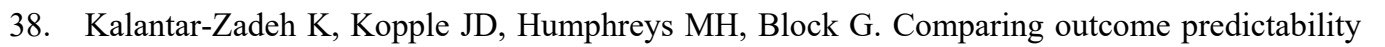

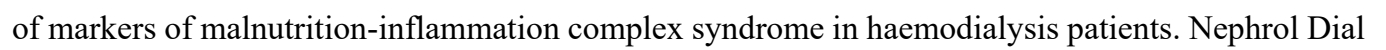

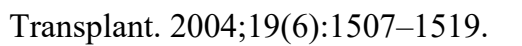




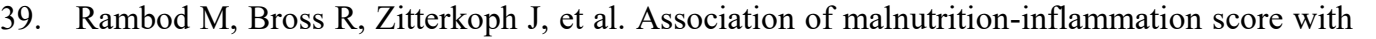

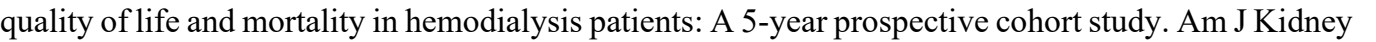

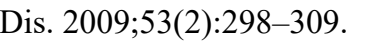

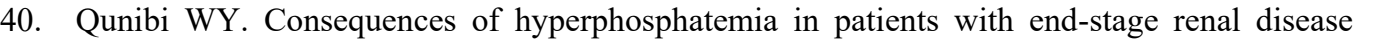

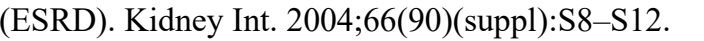

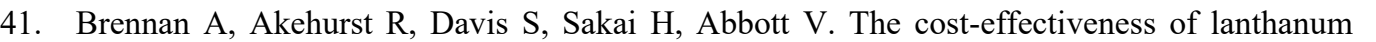

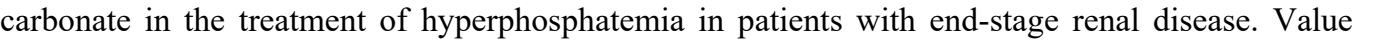

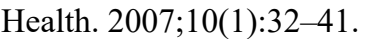

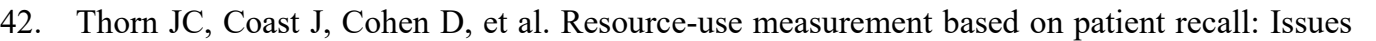

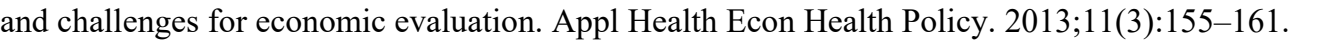

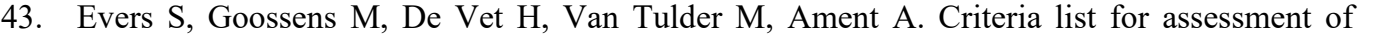

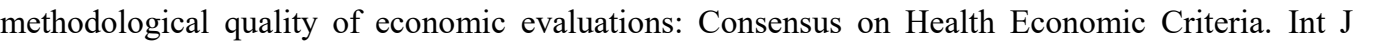

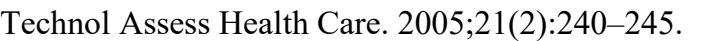

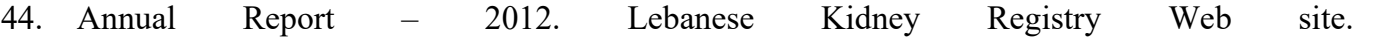

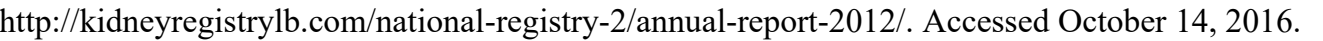

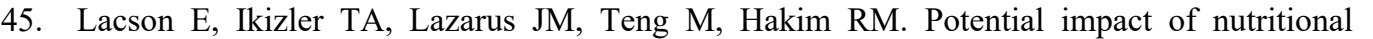

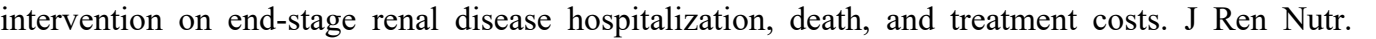

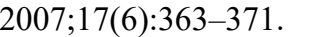

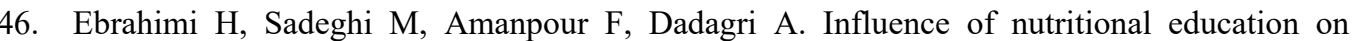

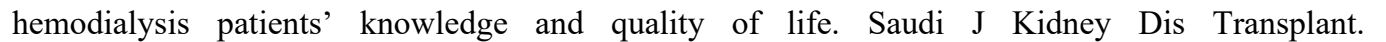

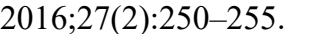

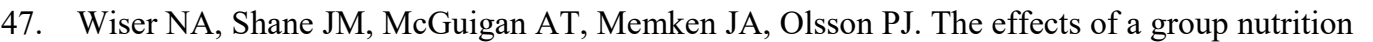

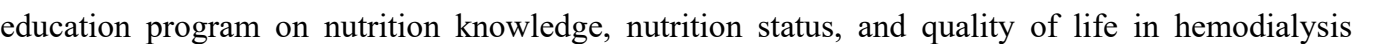

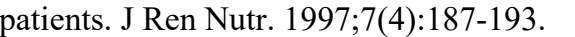


Appendix S1: Resource utilization questionnaire

\begin{tabular}{|c|c|c|c|c|c|c|}
\hline \multirow[t]{2}{*}{$\square$} & \\
\hline & \multicolumn{6}{|c|}{ 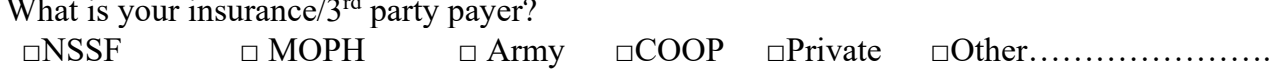 } \\
\hline \multirow[t]{4}{*}{$\square$} & \multicolumn{6}{|c|}{ 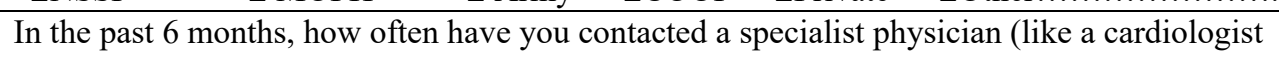 } \\
\hline & \multicolumn{6}{|c|}{ 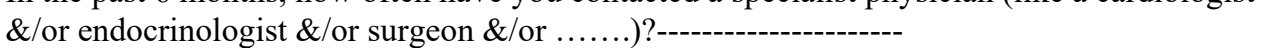 } \\
\hline & \multicolumn{6}{|c|}{ 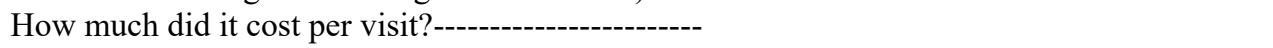 } \\
\hline & \multicolumn{6}{|c|}{ 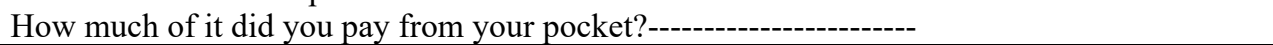 } \\
\hline$\square$ & \multicolumn{6}{|c|}{ 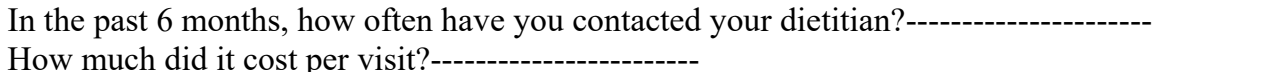 } \\
\hline \multirow[t]{2}{*}{$\square$} & \multirow{2}{*}{\multicolumn{6}{|c|}{ 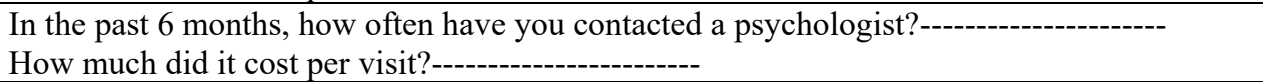 }} \\
\hline & & & & & & \\
\hline \multirow[t]{3}{*}{$\square \square$} & \multicolumn{6}{|c|}{ 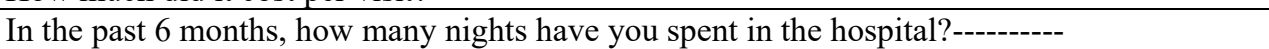 } \\
\hline & \multicolumn{6}{|c|}{ 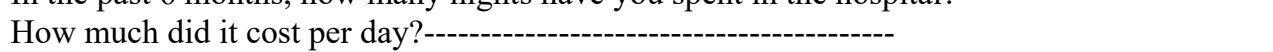 } \\
\hline & \multicolumn{6}{|c|}{ 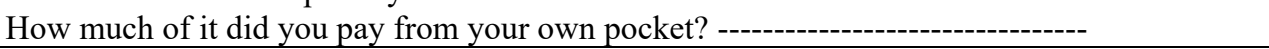 } \\
\hline \multirow[t]{2}{*}{$\square \square$} & \multicolumn{6}{|c|}{ 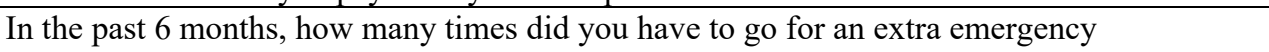 } \\
\hline & \multicolumn{6}{|c|}{ 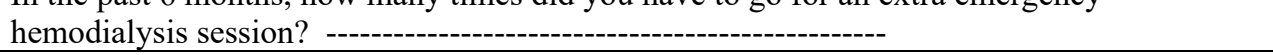 } \\
\hline \multirow[t]{10}{*}{$\square$} & \multicolumn{6}{|c|}{ 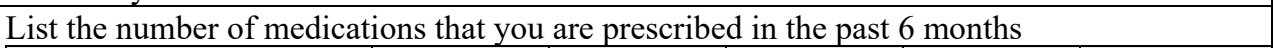 } \\
\hline & $\square$ & \begin{tabular}{l|l|l|l|l|l|}
$\square \square \square$ \\
\end{tabular} & 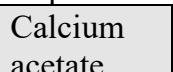 & $\square \square \mid \Pi \square \square \square$ & 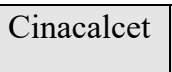 & 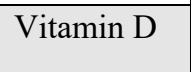 \\
\hline & 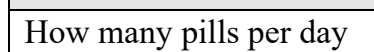 & $\square$ & $\square$ & $\square$ & $\square$ & $\square$ \\
\hline & & & & & & \\
\hline & $\square$ & 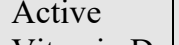 & 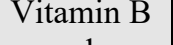 & 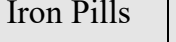 & 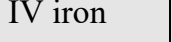 & \\
\hline & & & & & & \\
\hline & 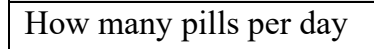 & $\square$ & $\square$ & $\square$ & $\square$ & $\square$ \\
\hline & \multicolumn{6}{|c|}{ 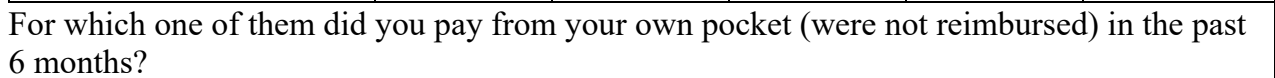 } \\
\hline & \multicolumn{6}{|l|}{$\square$} \\
\hline & \multicolumn{6}{|c|}{$\square$} \\
\hline \multirow[t]{3}{*}{$\square$} & \multirow{3}{*}{\multicolumn{6}{|c|}{ 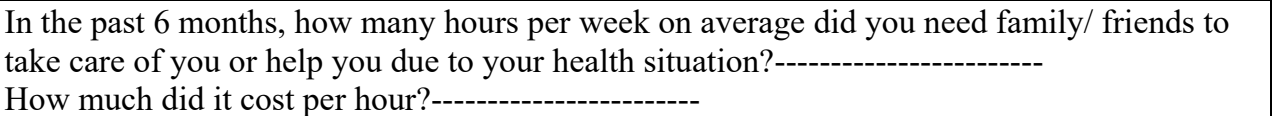 }} \\
\hline & & & & & & \\
\hline & & & & & & \\
\hline \multirow[t]{3}{*}{$\square$} & \multirow{2}{*}{\multicolumn{6}{|c|}{ 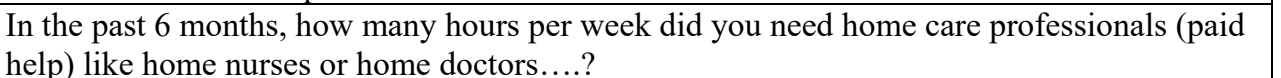 }} \\
\hline & & & & & & \\
\hline & 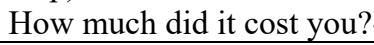 & 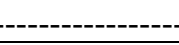 & 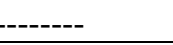 & & & \\
\hline$\square \square$ & 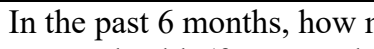 & 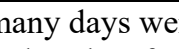 & 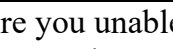 & & & 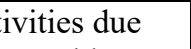 \\
\hline & & & 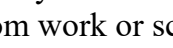 & & & \\
\hline & & & & & & \\
\hline
\end{tabular}


Appendix S2: Costing of the nutrition education

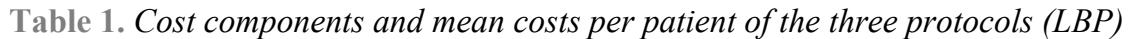

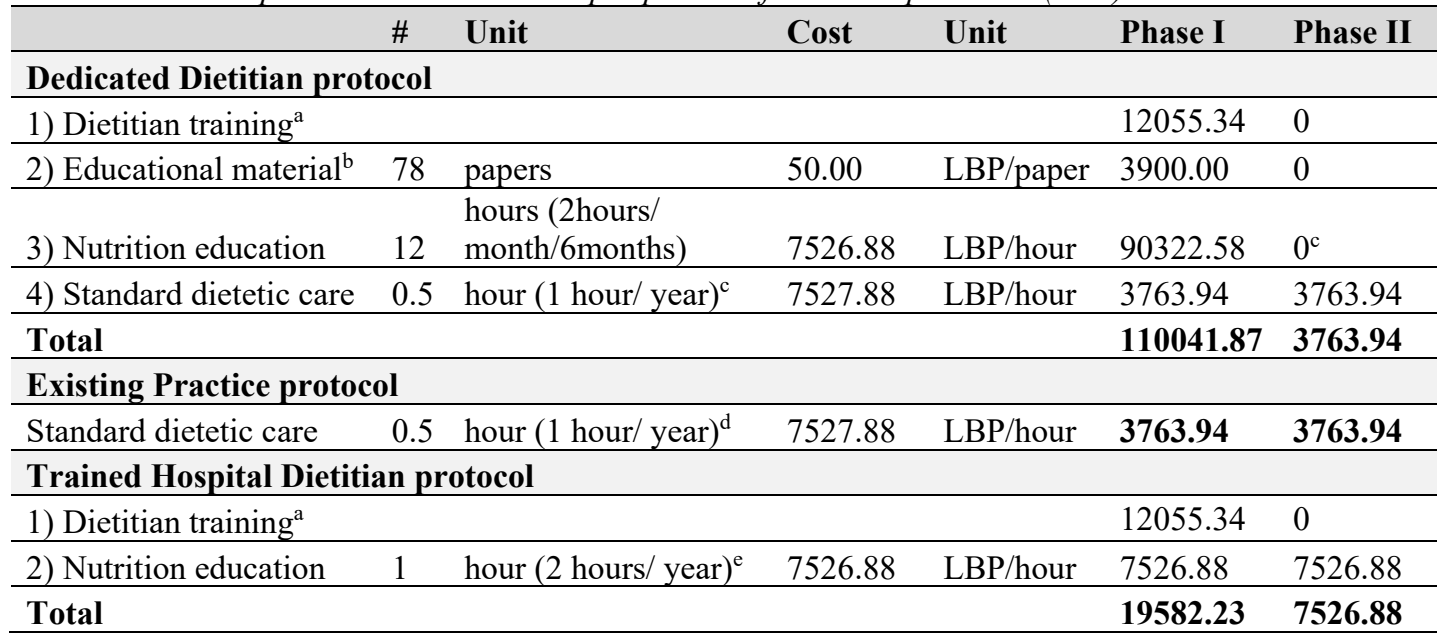

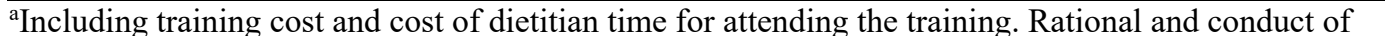

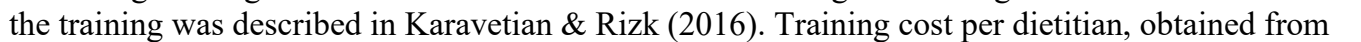

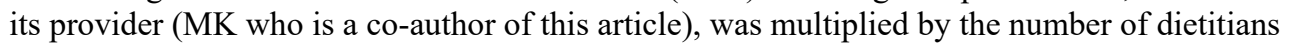

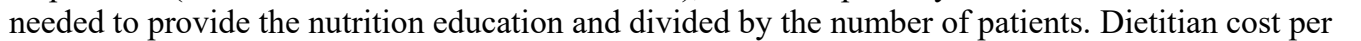

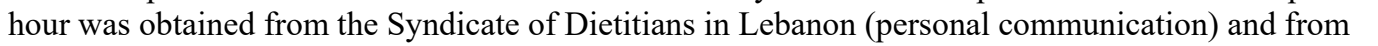

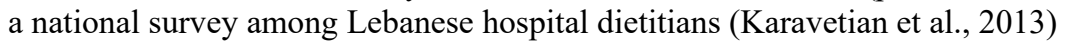

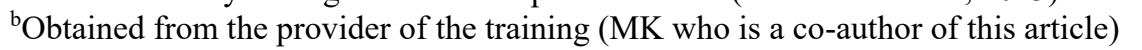

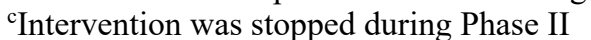

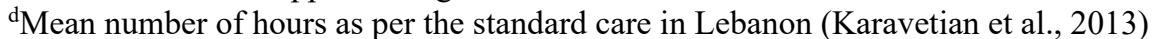

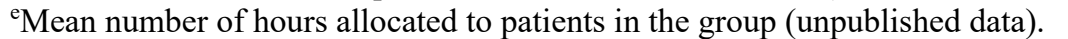

$\square$

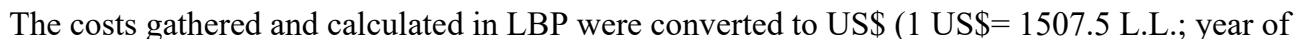

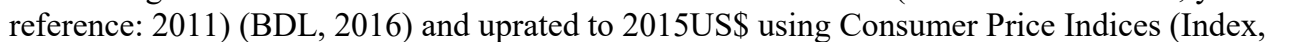

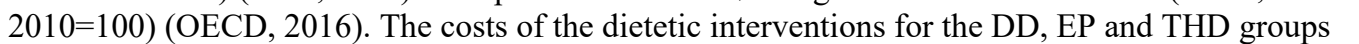

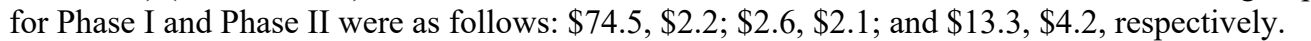
$\square$ 


\section{Appendix S3: Flow diagram of the trial}

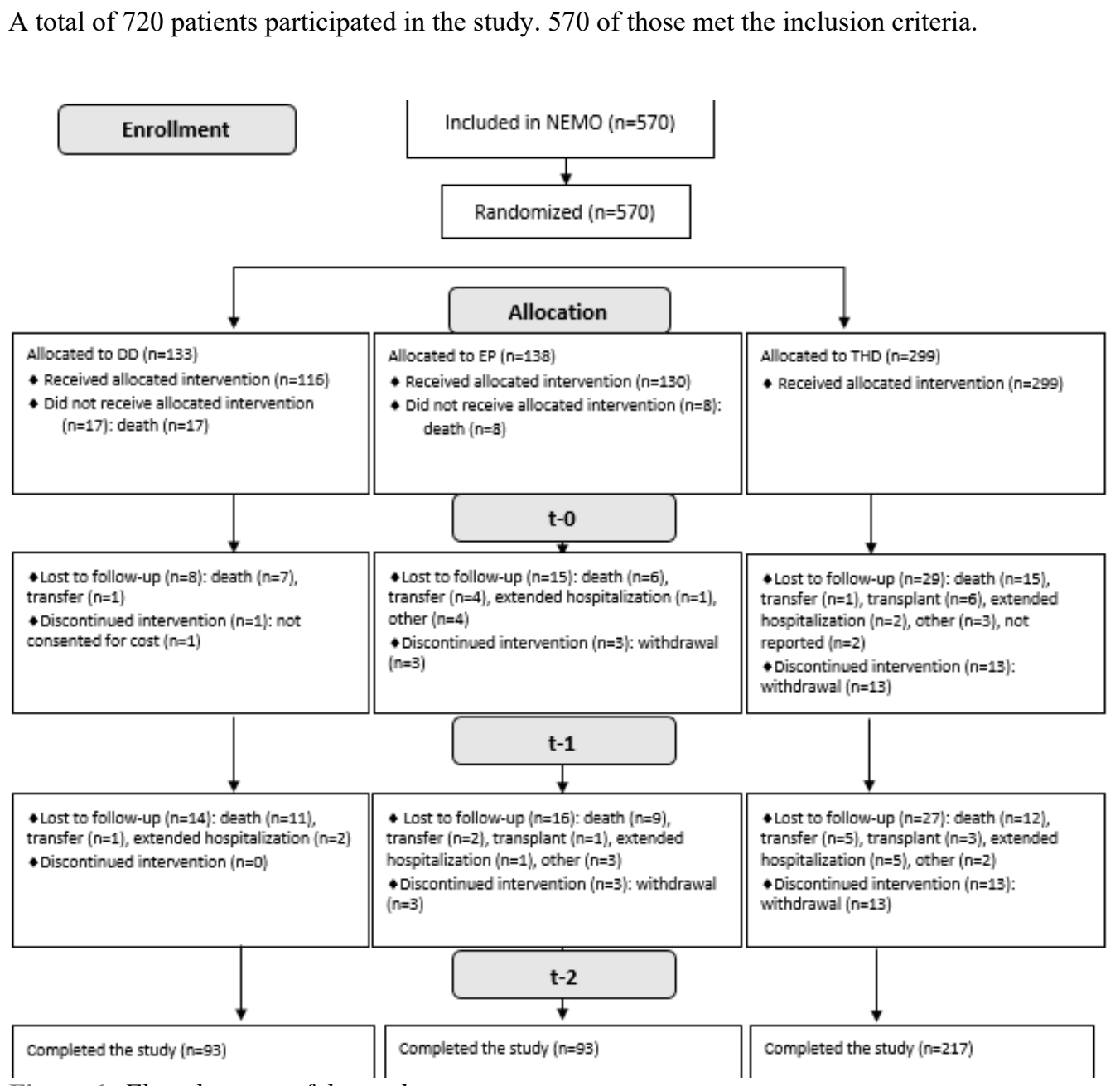

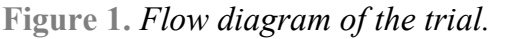




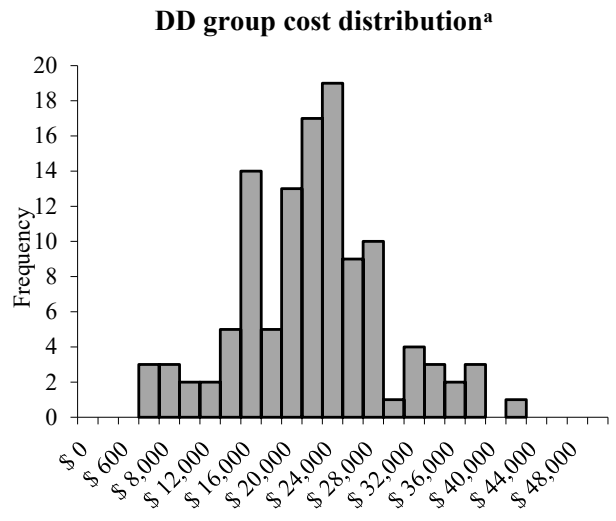

THD group cost distribution ${ }^{c}$

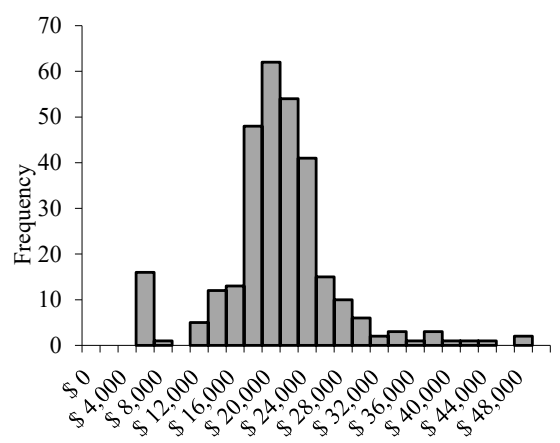

EP group cost distribution ${ }^{b}$

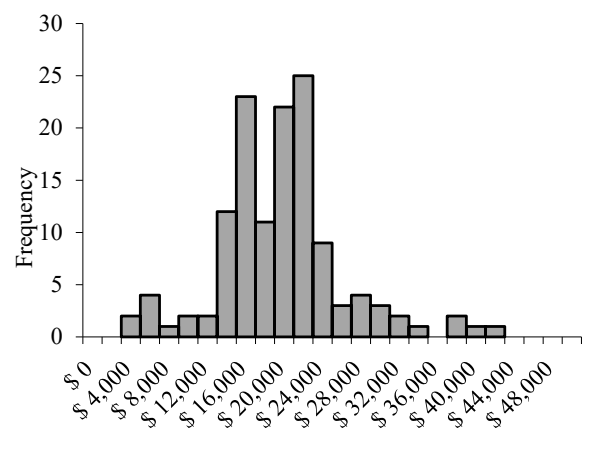

$\square \square \mathbb{1 0}$

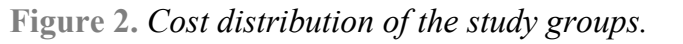

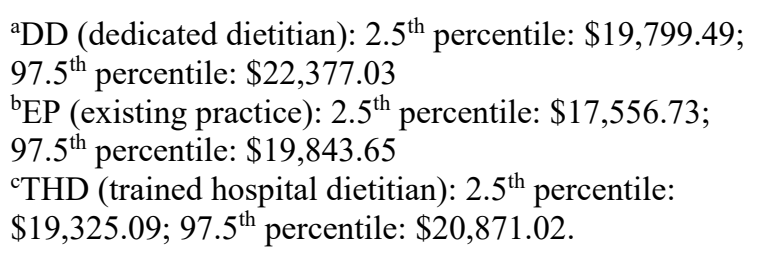

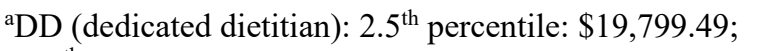

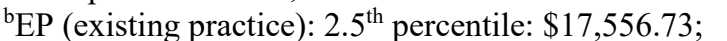

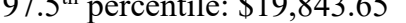

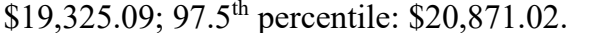

$\square \square \mathbb{1 1}$ 
Appendix S5: Cost-effectiveness acceptability curves for sensitivity analyses

Complete cases

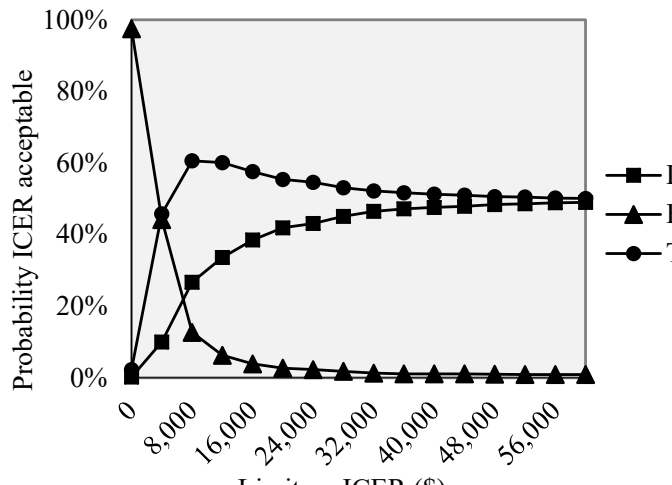

Hyperphosphatemia at baseline

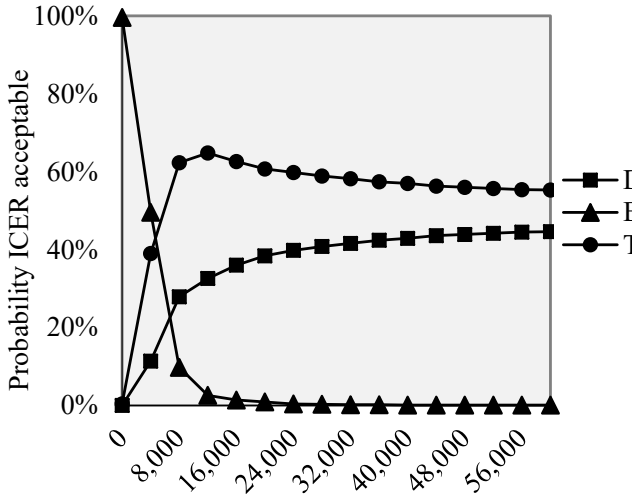

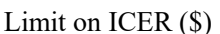

Hemodialysis costs excluded

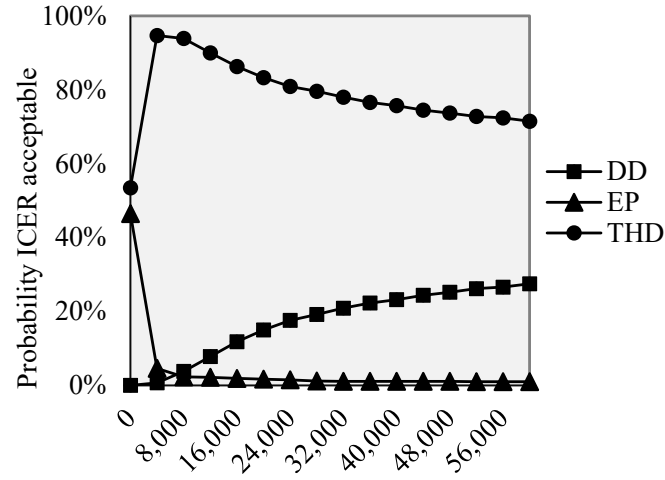

Difference in serum phosphorus

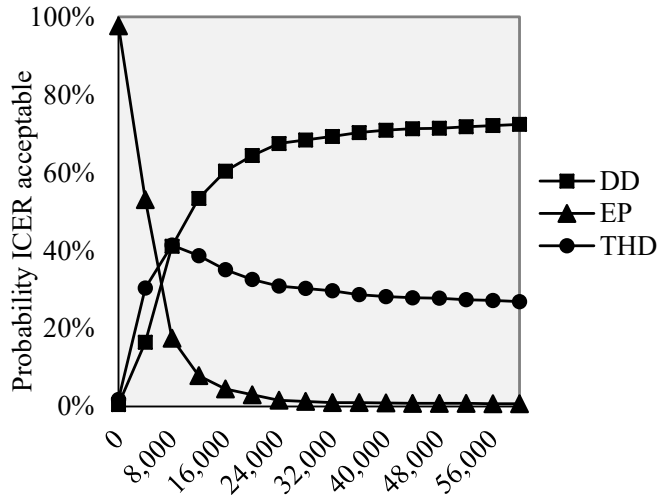

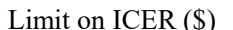

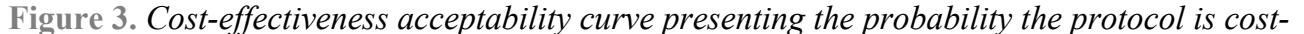

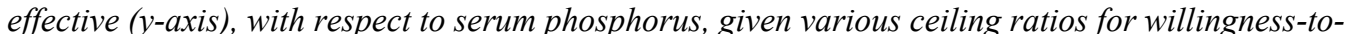

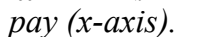

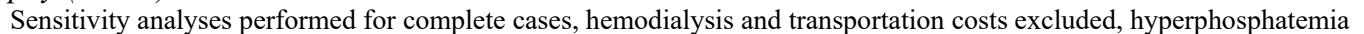

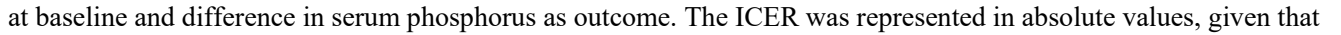
ए एव

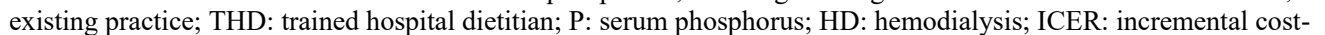

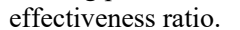


Appendix S6: Cost-utility acceptability curves for sensitivity analyses

Complete cases

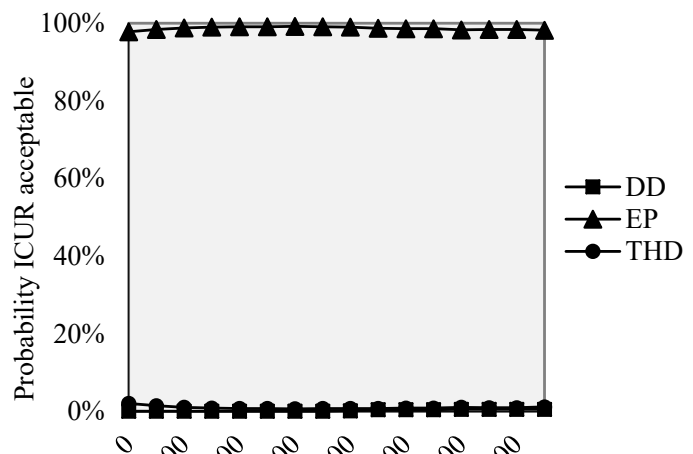

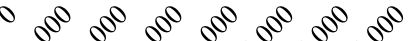

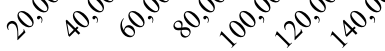

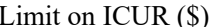

Hyperphosphatemia at baseline

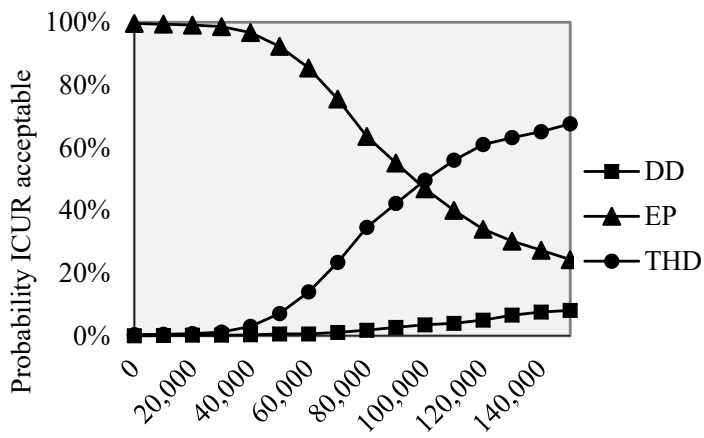

Hemodialysis costs excluded

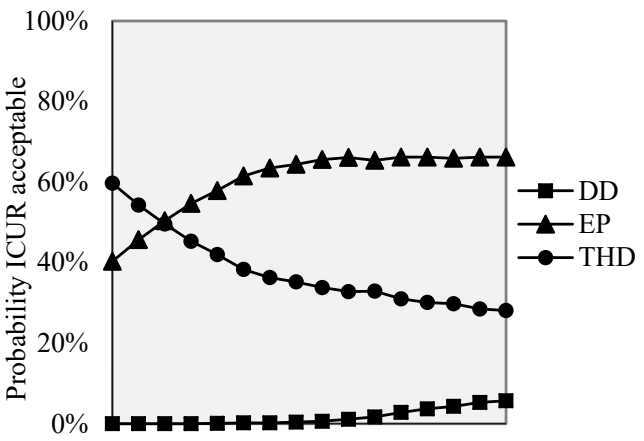

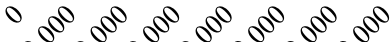

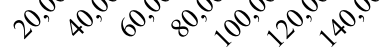

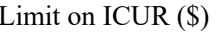

Adjusted QALY

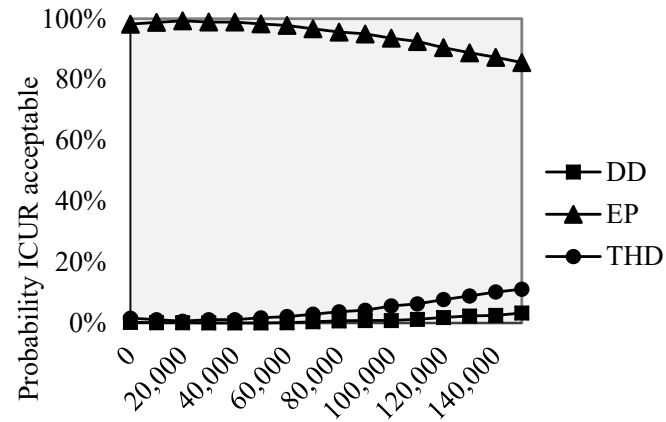

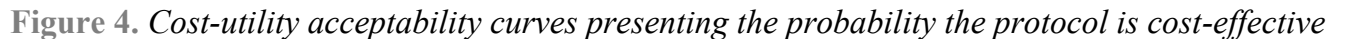
प

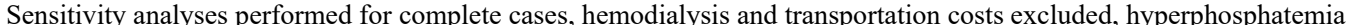

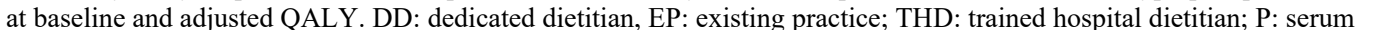

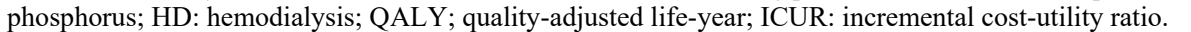




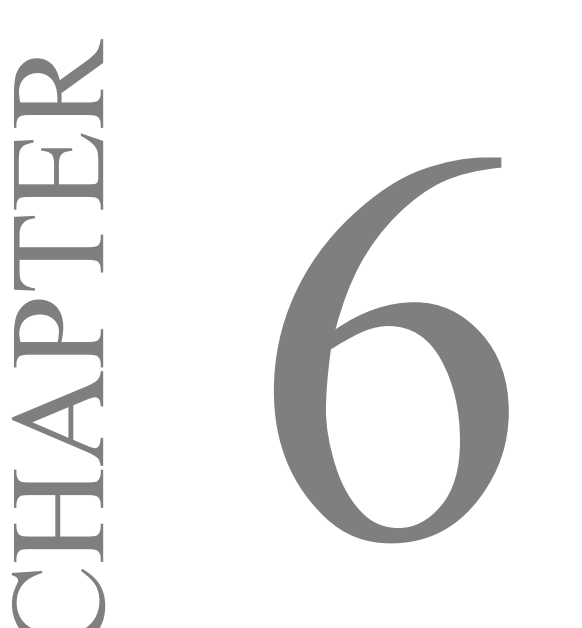


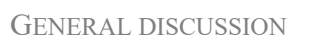

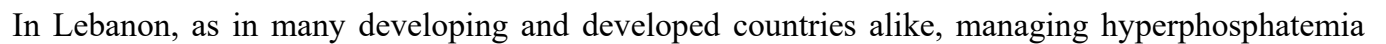

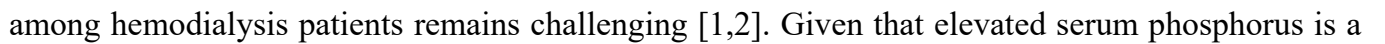

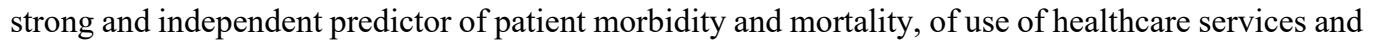

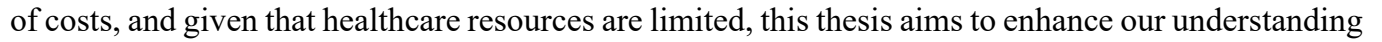

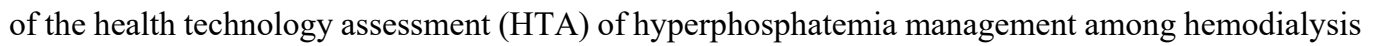
प Im

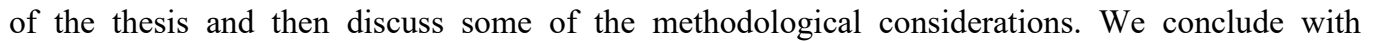

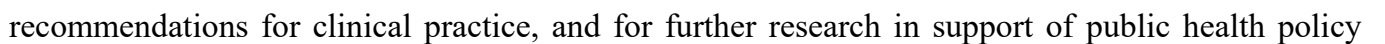

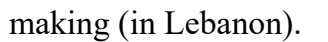

$\square$

\section{MAIN FINDINGS OF THE THESIS}

\section{Box 1: Main findings}

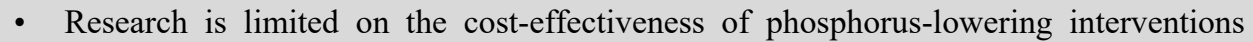

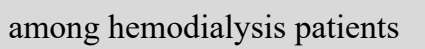

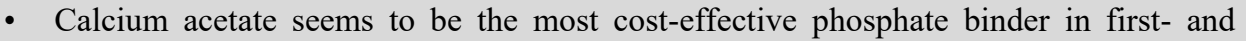

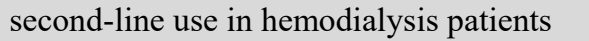

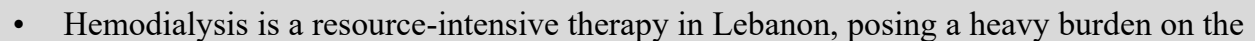

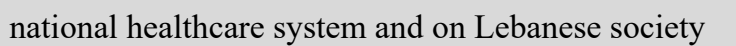

•

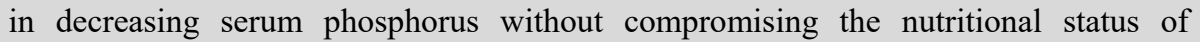

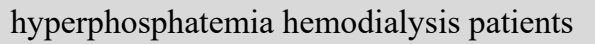

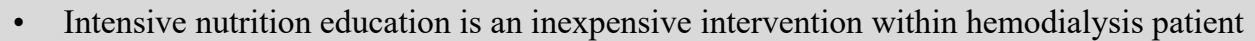

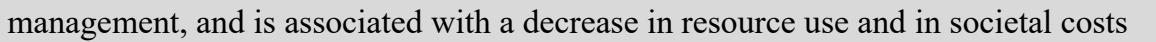

•

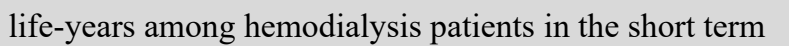

$\square$

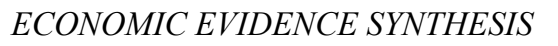

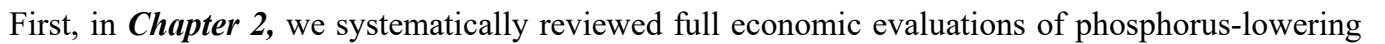

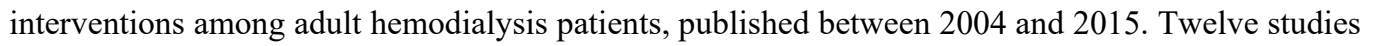

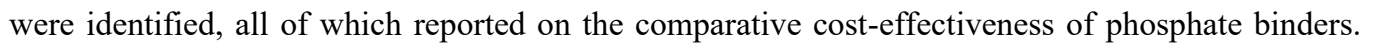

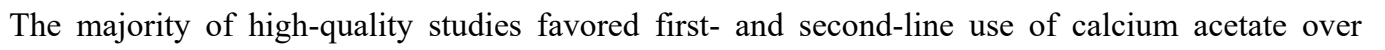

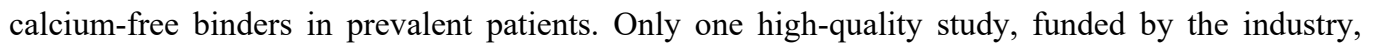

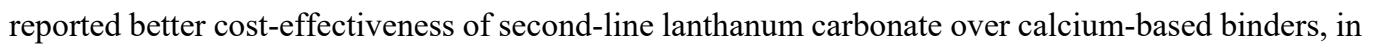

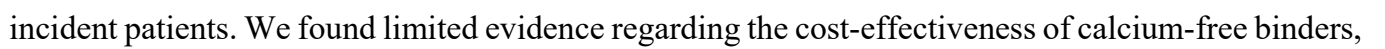

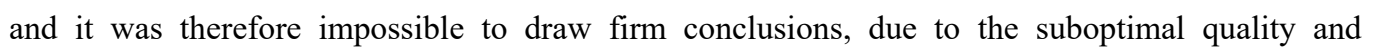

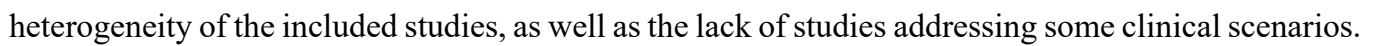




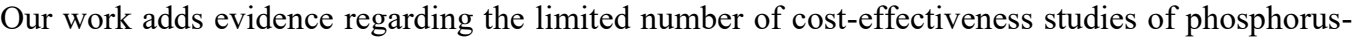

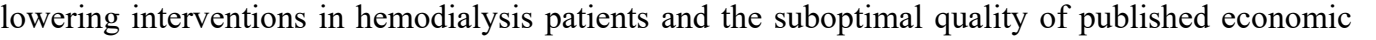

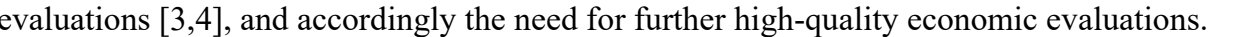

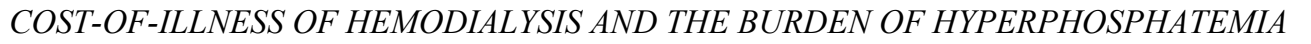

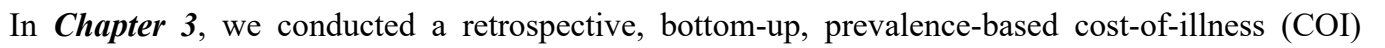

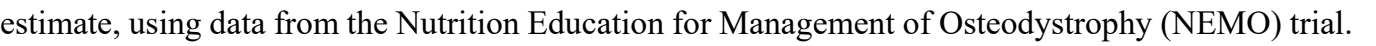

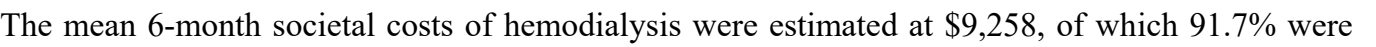

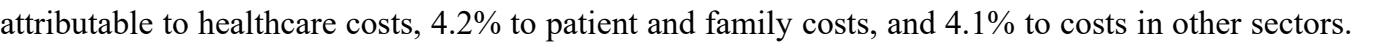

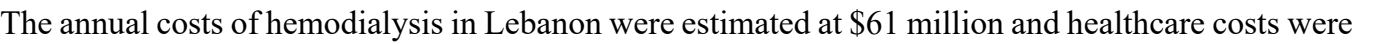

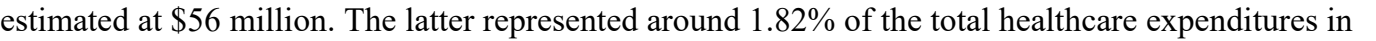

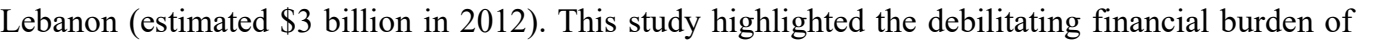

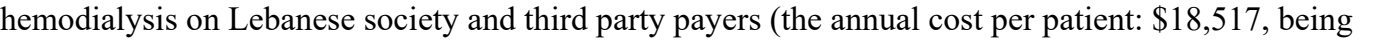

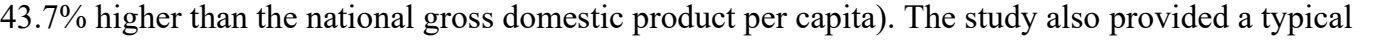

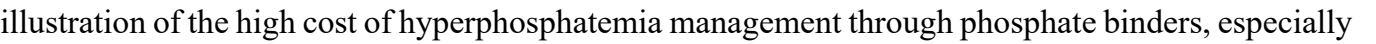

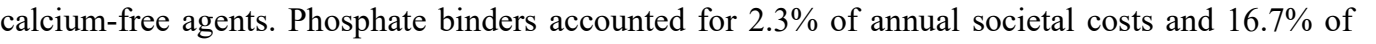

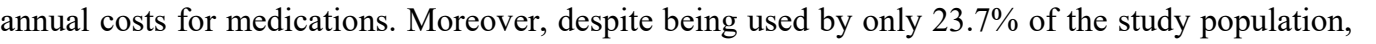

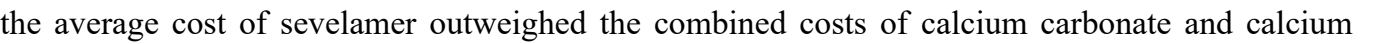

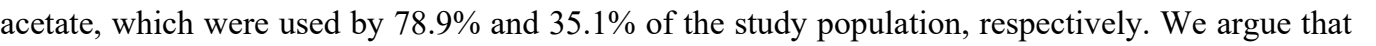

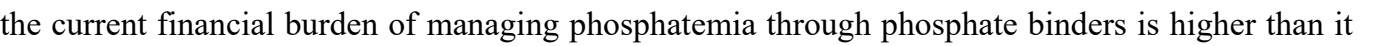

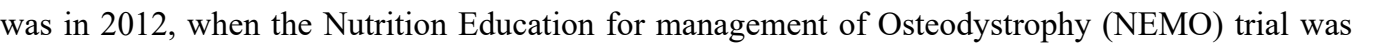

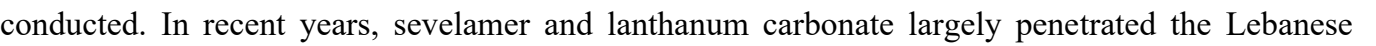

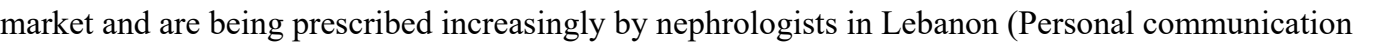

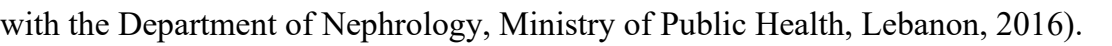

$\square$

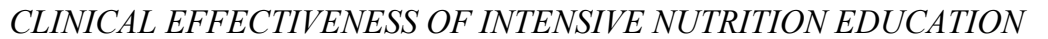

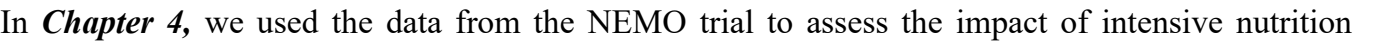

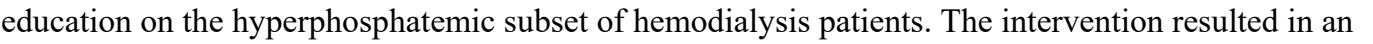

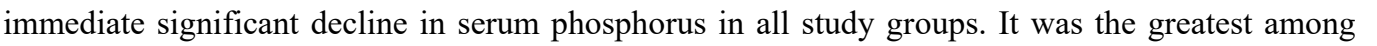

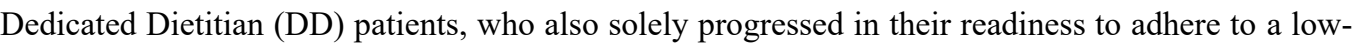

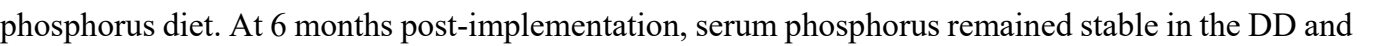

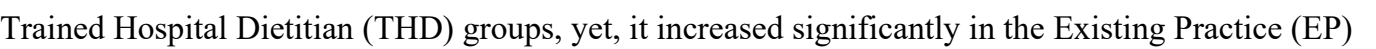

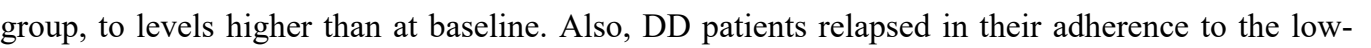

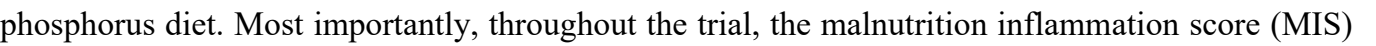

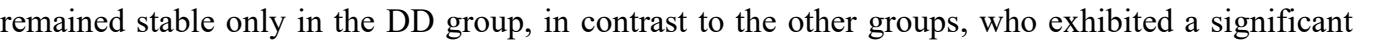




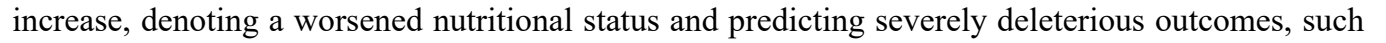

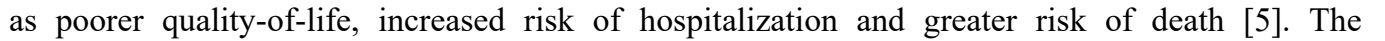

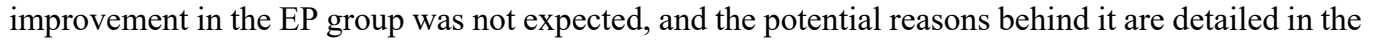

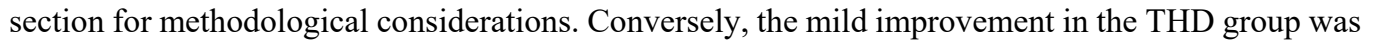

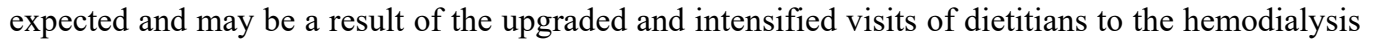

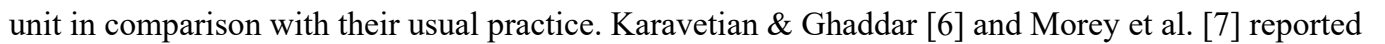

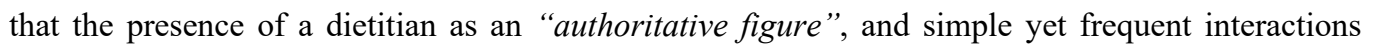

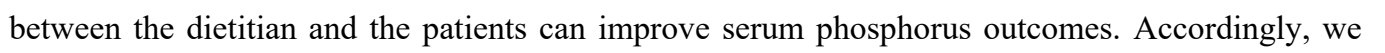

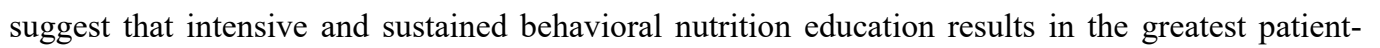

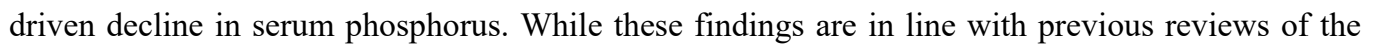

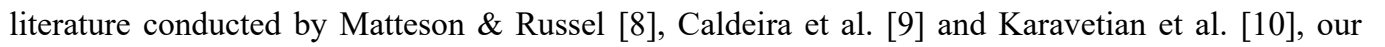
एण

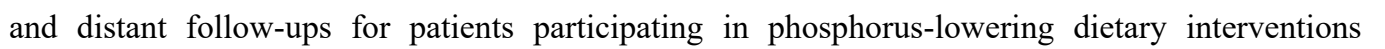

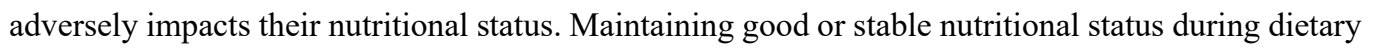

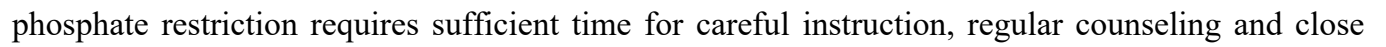

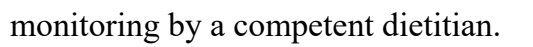

$\square$

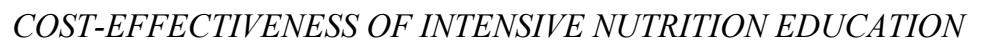

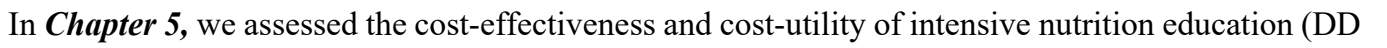

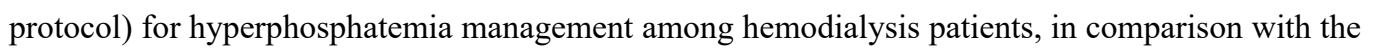

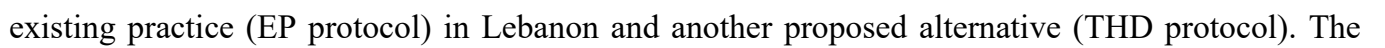

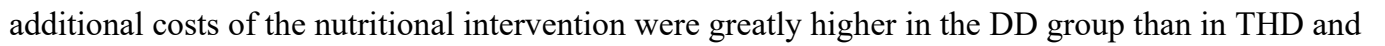

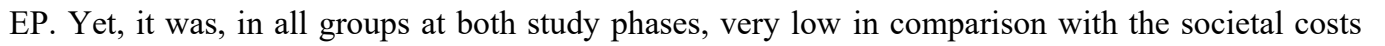

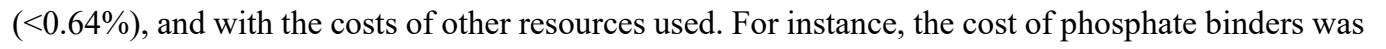

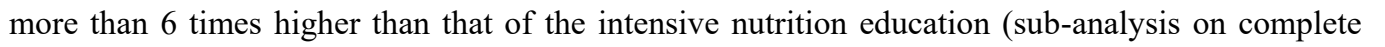

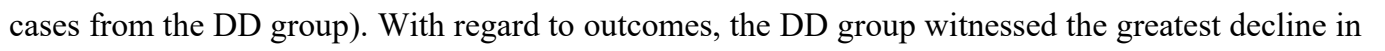

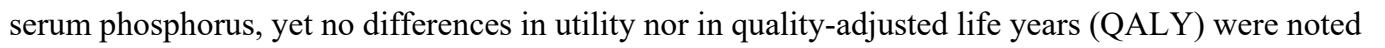

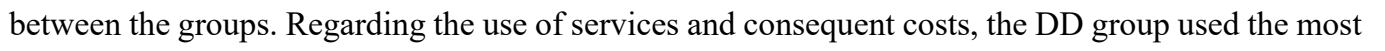

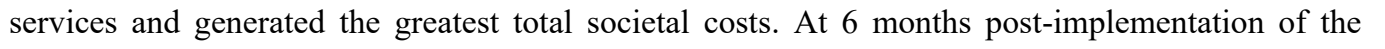

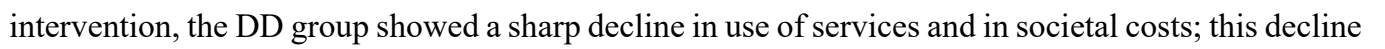

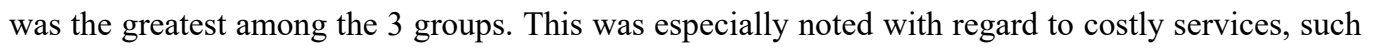

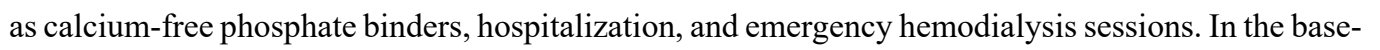

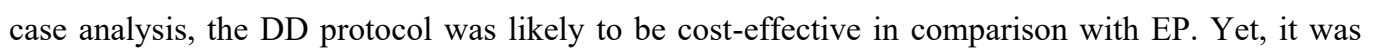

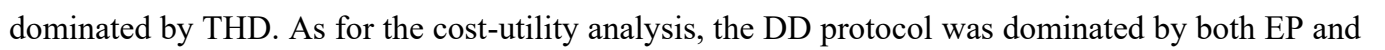

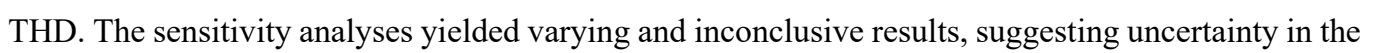

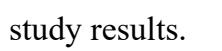




\section{METHODOLOGICAL CONSIDERATIONS}

\section{Box 2: Methodological considerations}

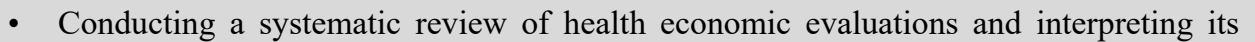

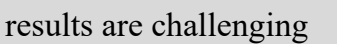

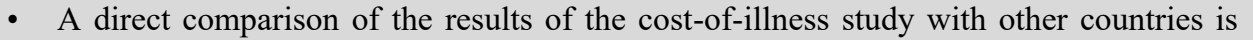

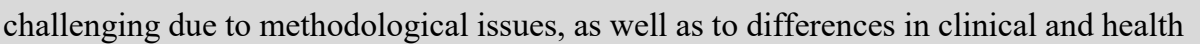

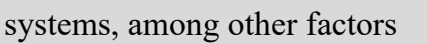

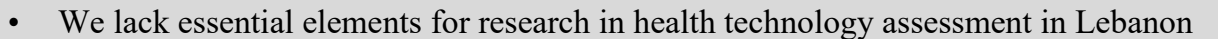

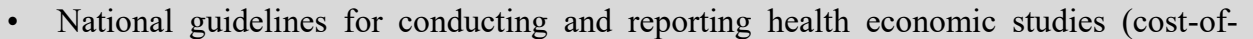

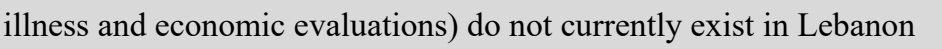

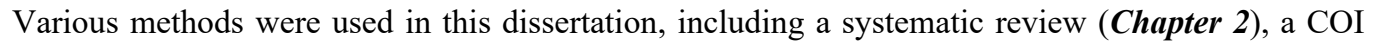

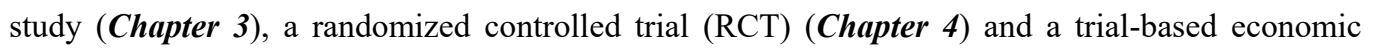

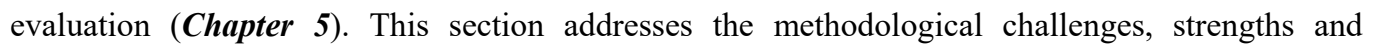

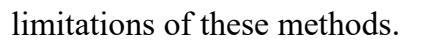

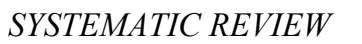

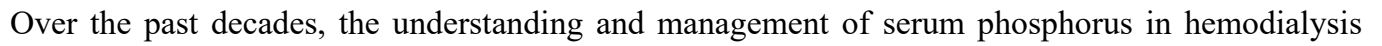

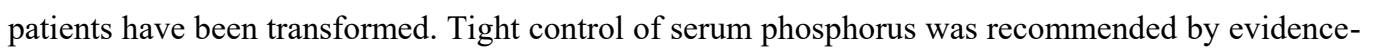

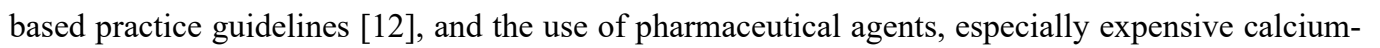

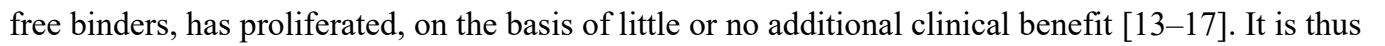

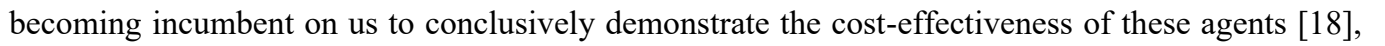

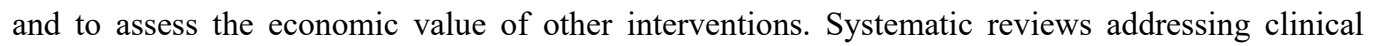

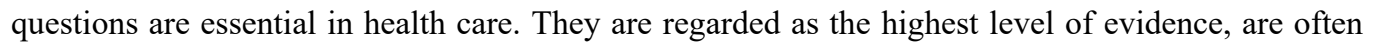

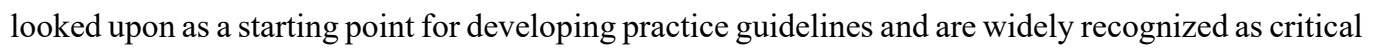

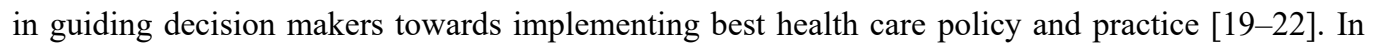

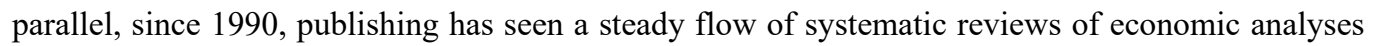

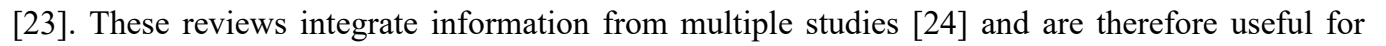

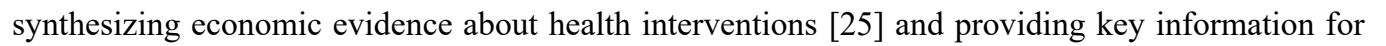

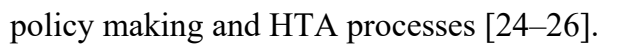

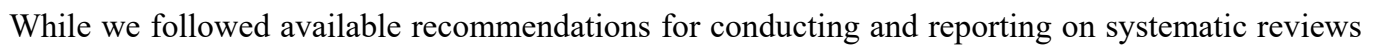

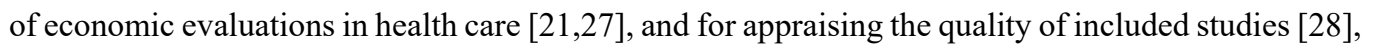

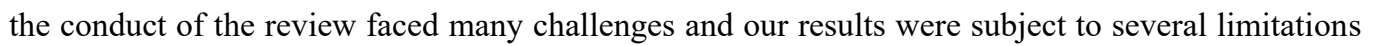

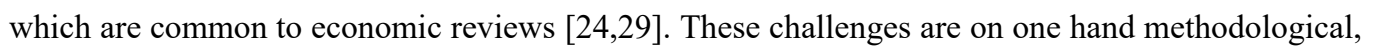

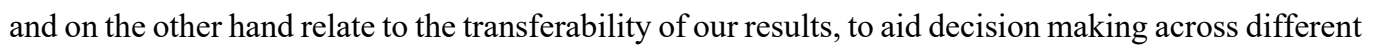

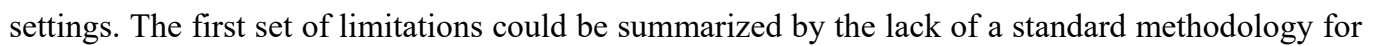




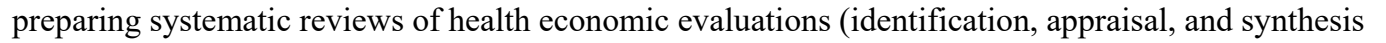

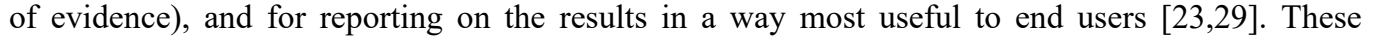

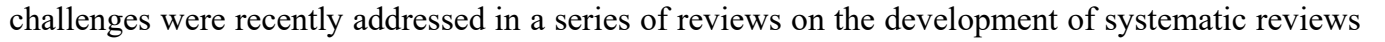

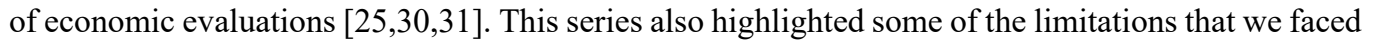

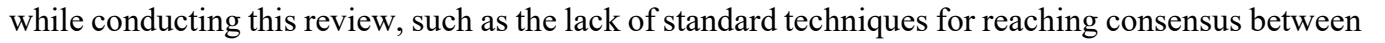

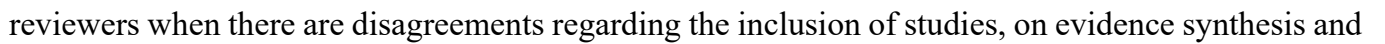

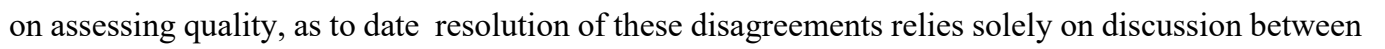

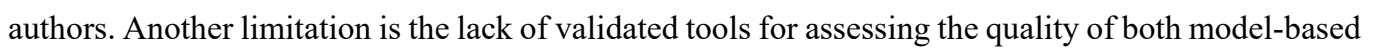

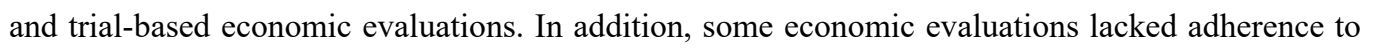

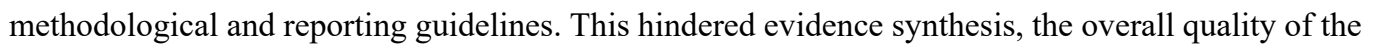

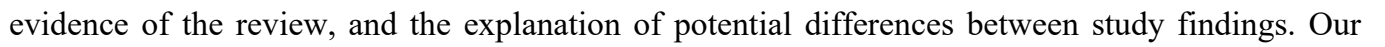

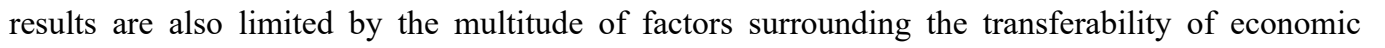

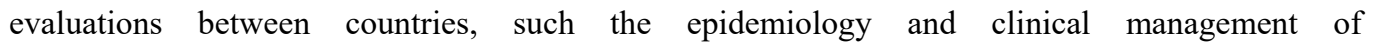

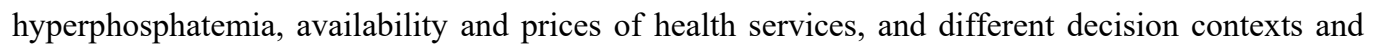

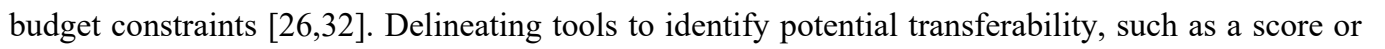

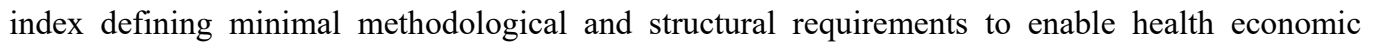

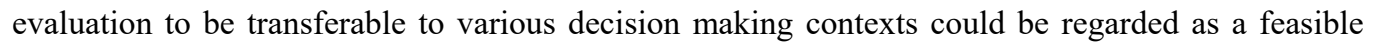

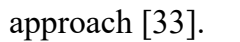

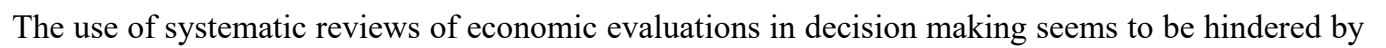

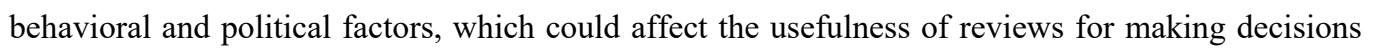
ए

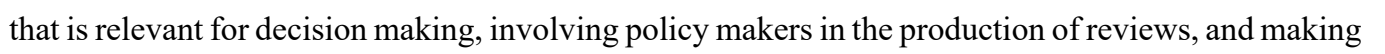

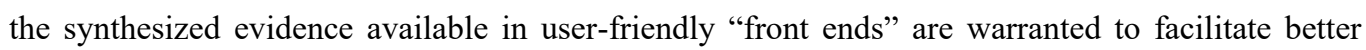

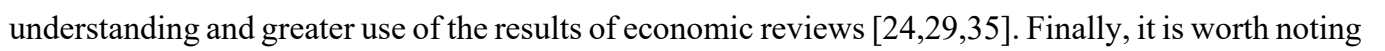

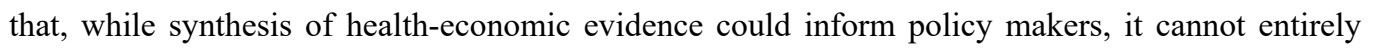

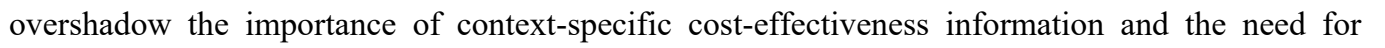

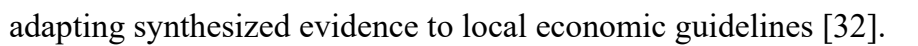

$\square$

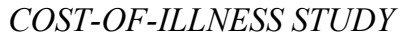

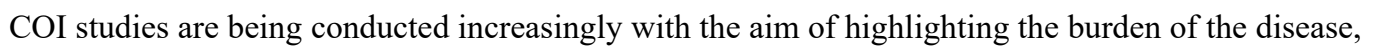

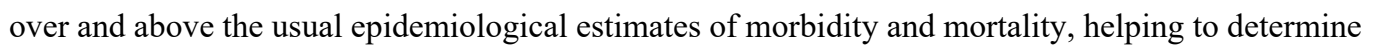

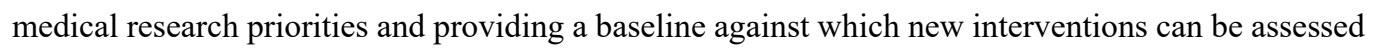
पाणाणाएण

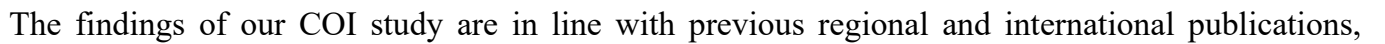

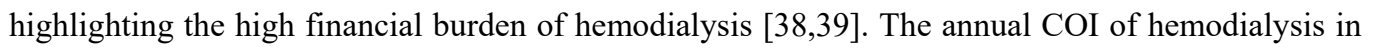




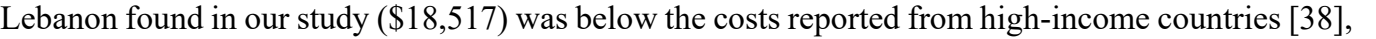

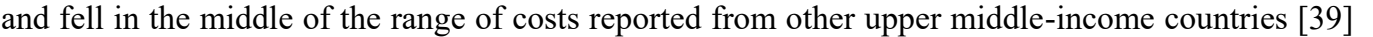
ए

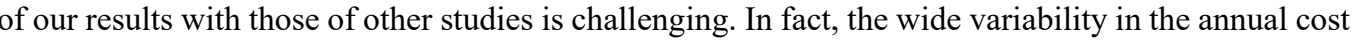

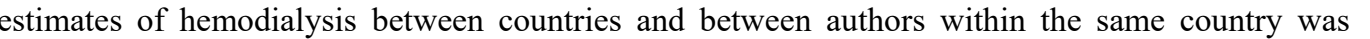

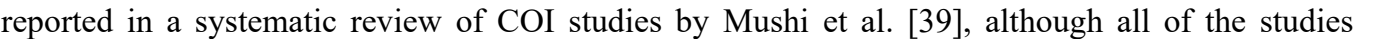

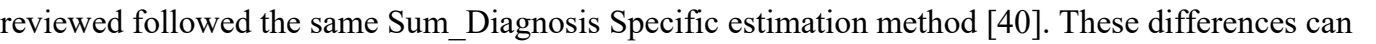

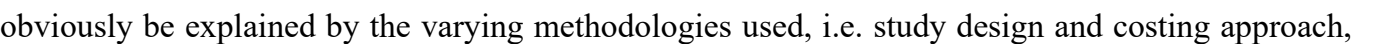

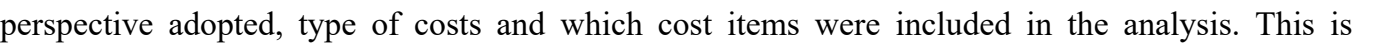

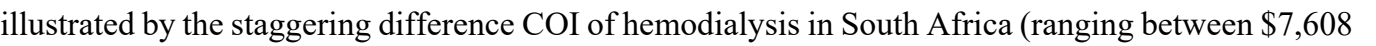

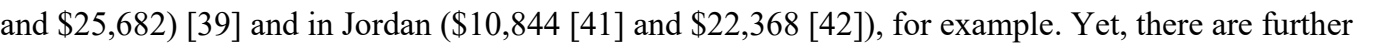

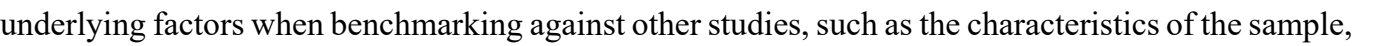

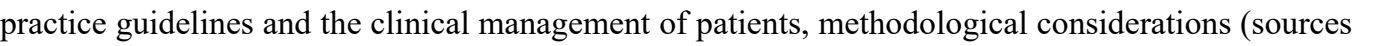

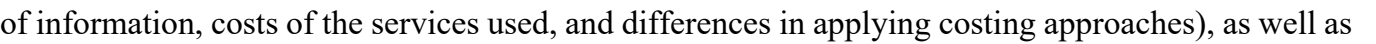

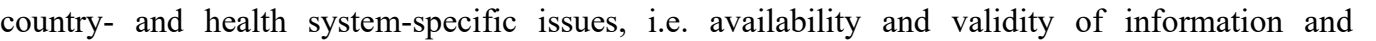

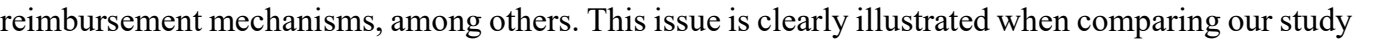

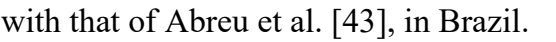

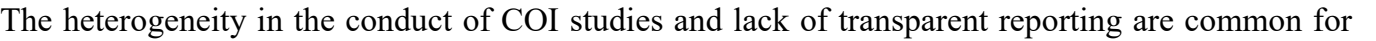

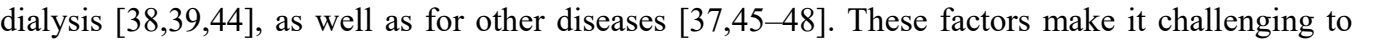

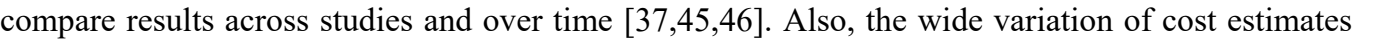

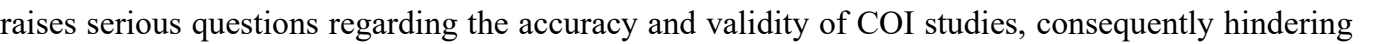

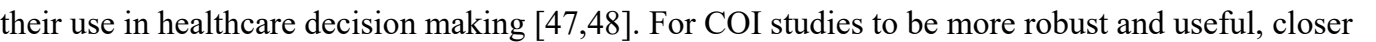
agreement among researchers and strict adherence to available recommendations around "best

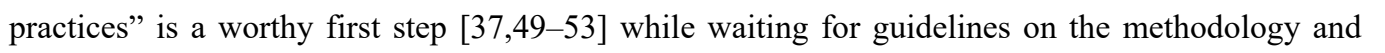

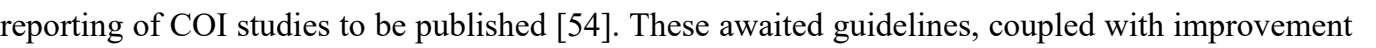

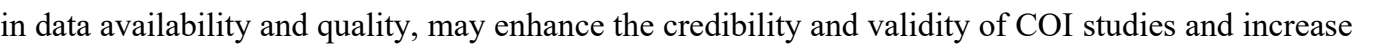

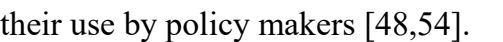

एव

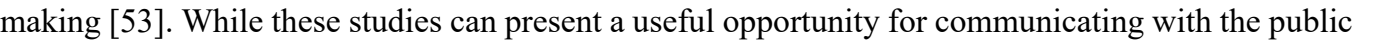

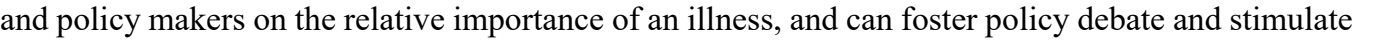

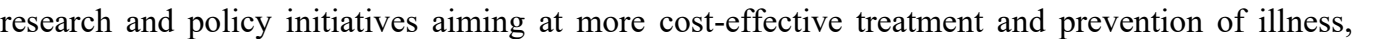

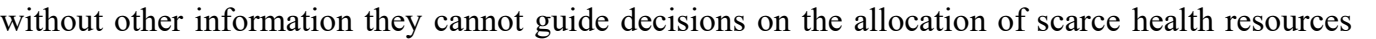

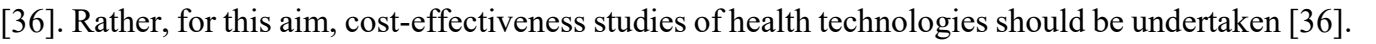

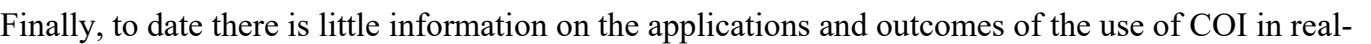

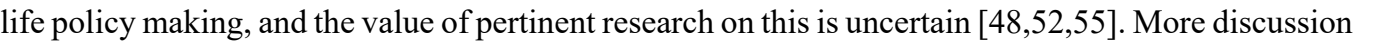

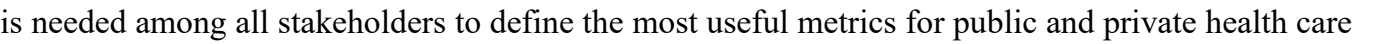




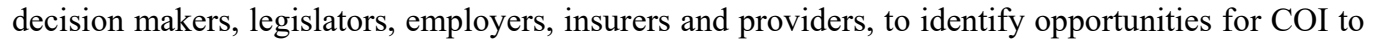

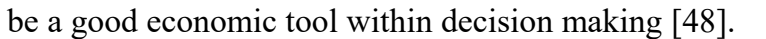

$\square$

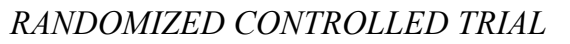

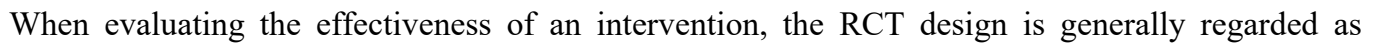

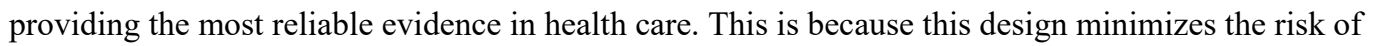

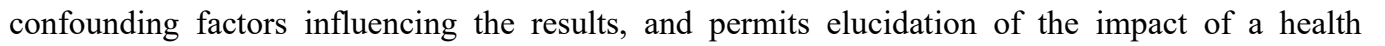

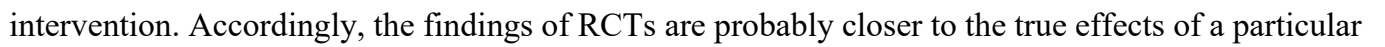

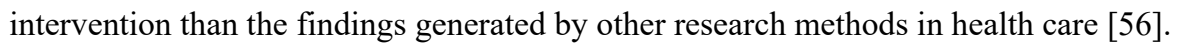

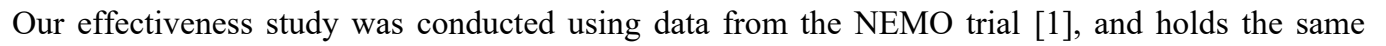

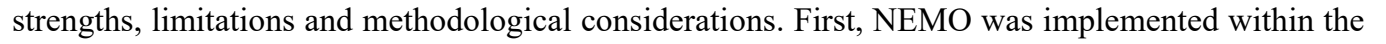

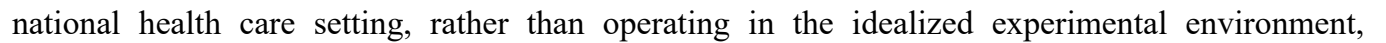

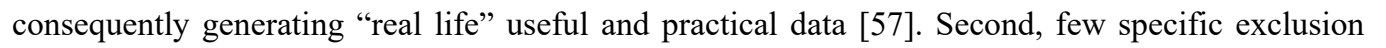

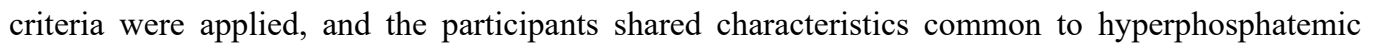

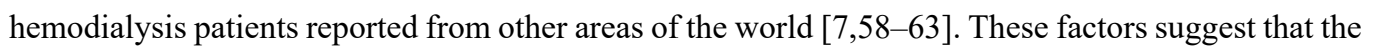
ए

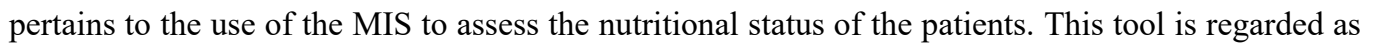

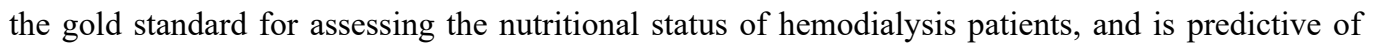

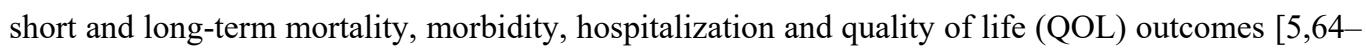

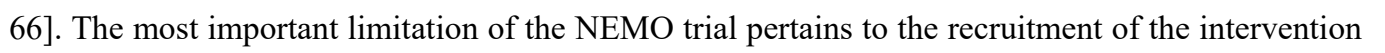

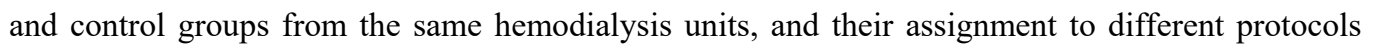

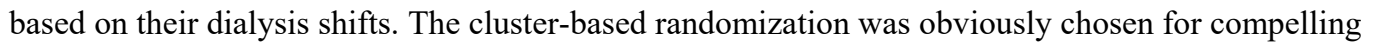

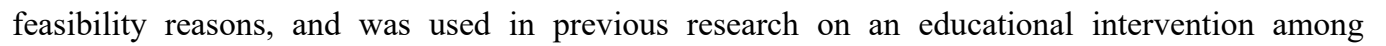

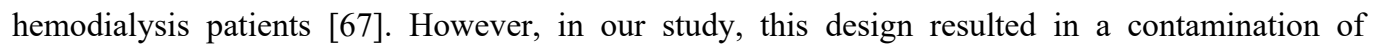

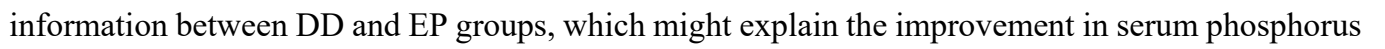

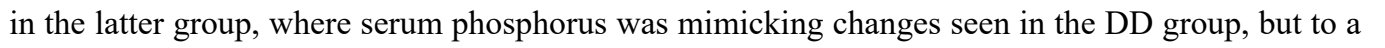

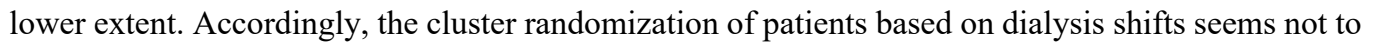

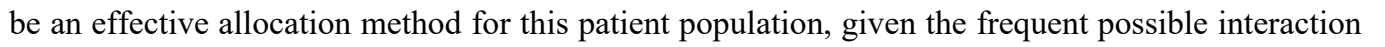

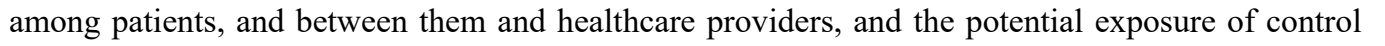

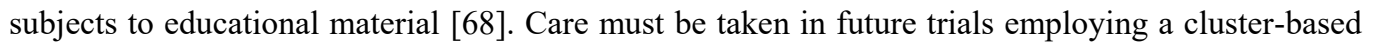

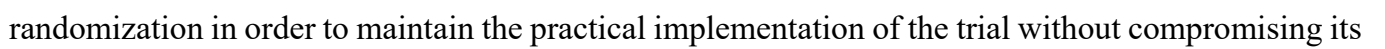

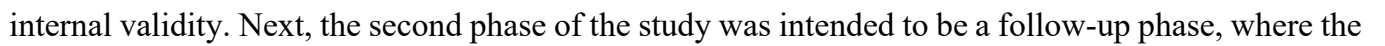

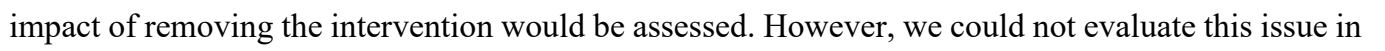

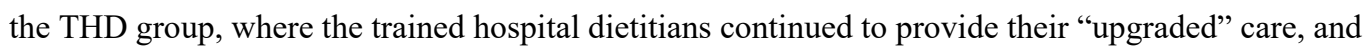

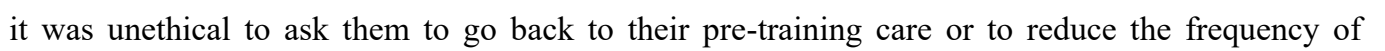

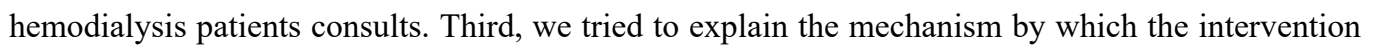




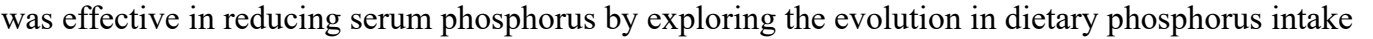

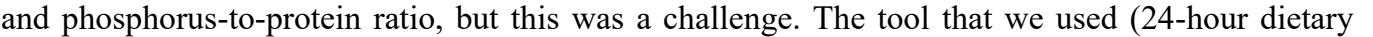

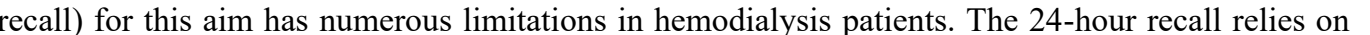

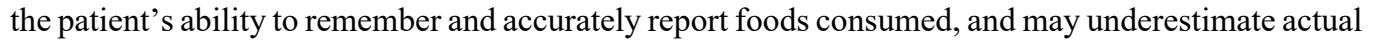

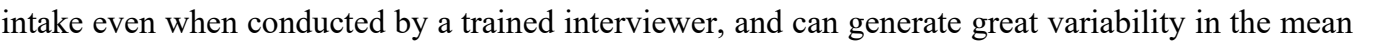

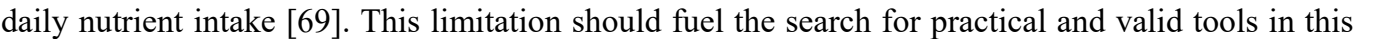

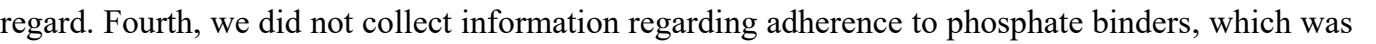

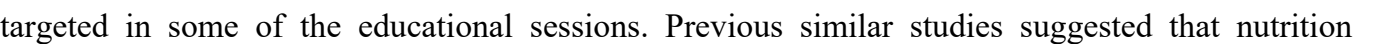

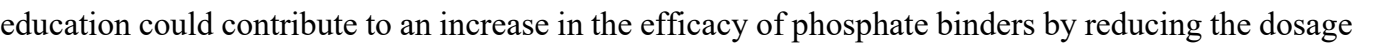

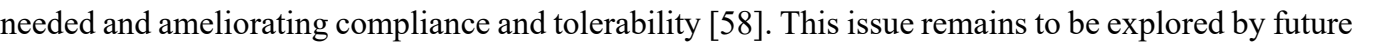
एणाणाणाए

$\square$

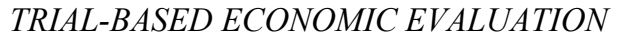

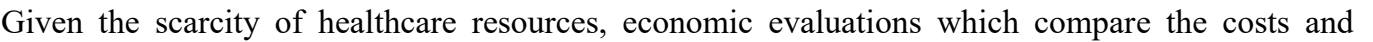

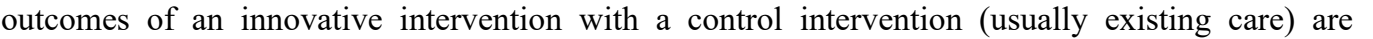

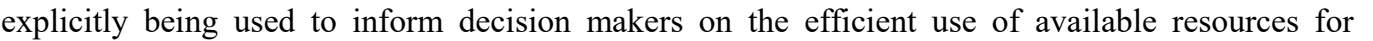

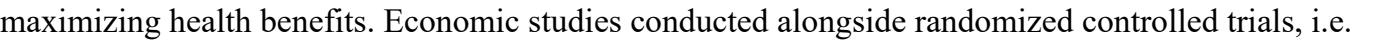

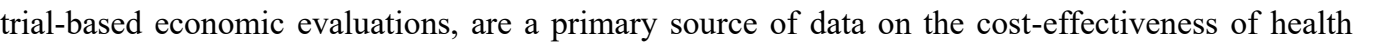

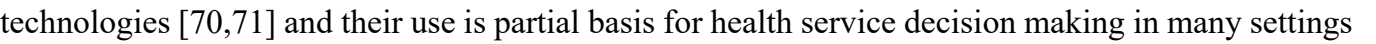
पाएणाए

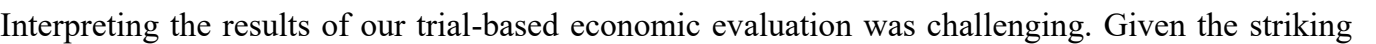

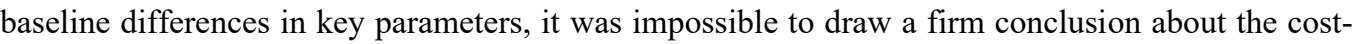

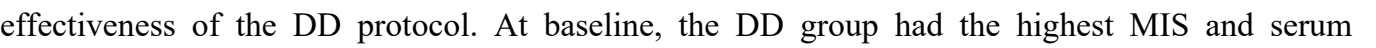

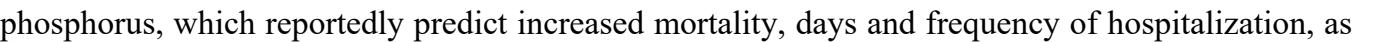

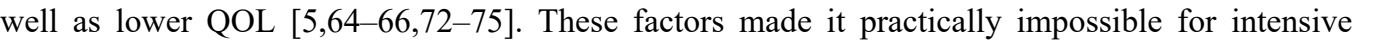

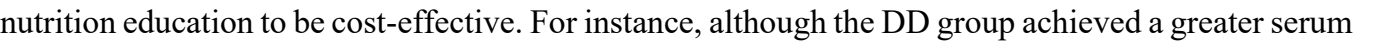

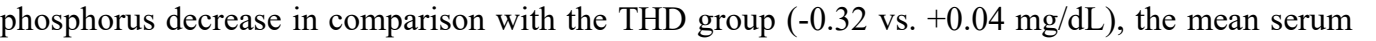

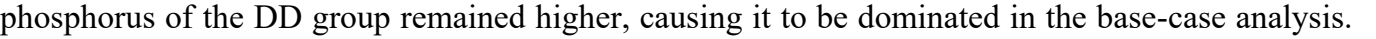

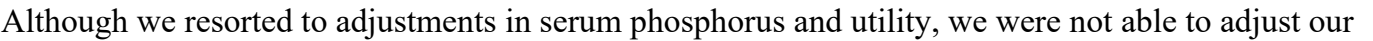

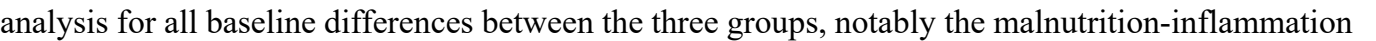

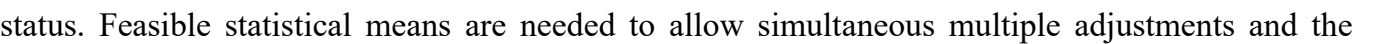

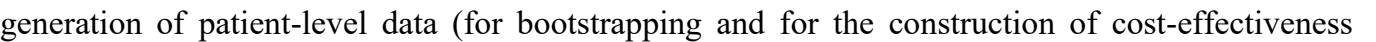

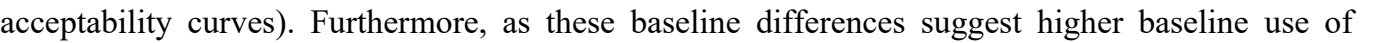

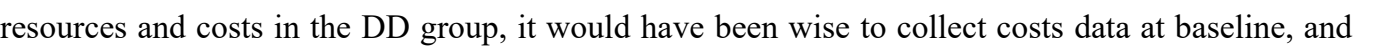

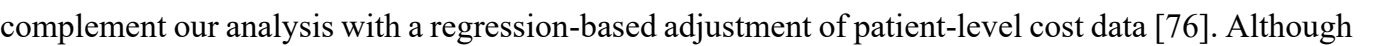

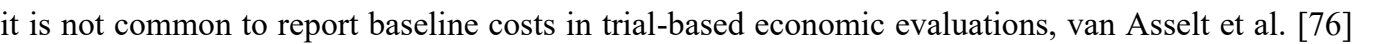




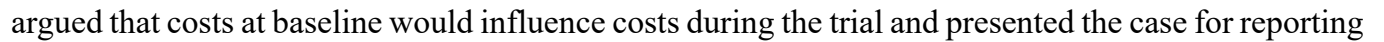

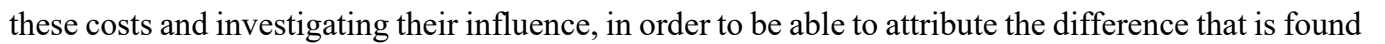

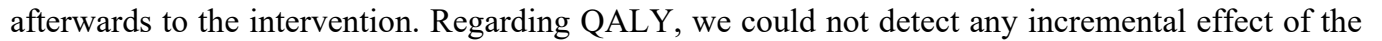

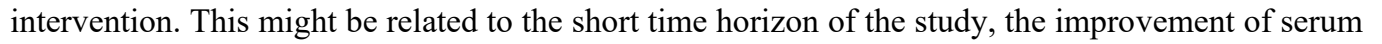

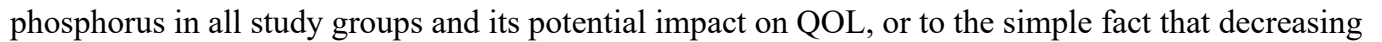

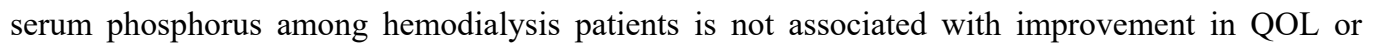

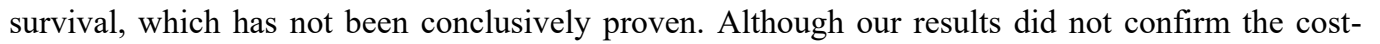

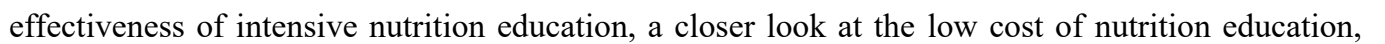

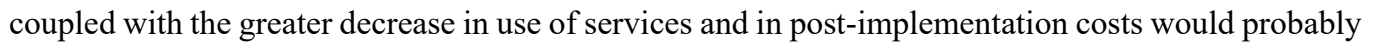

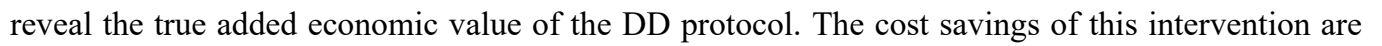

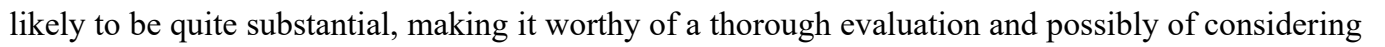

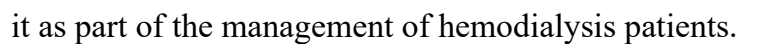

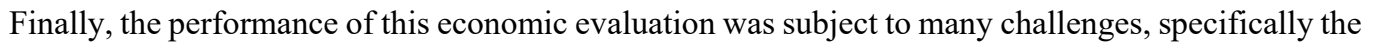

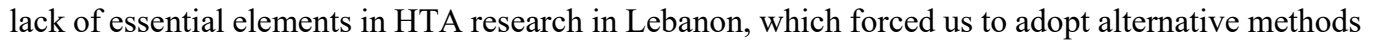

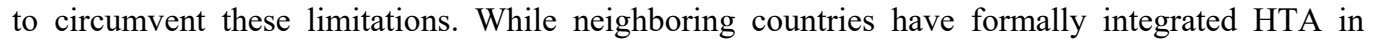

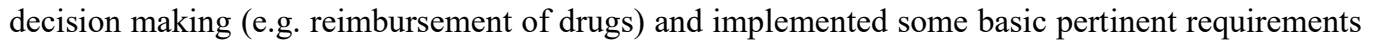

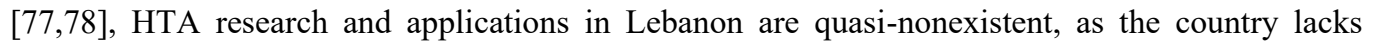

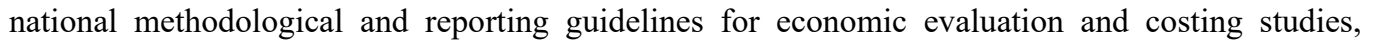

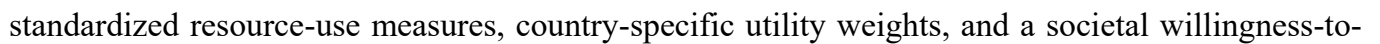

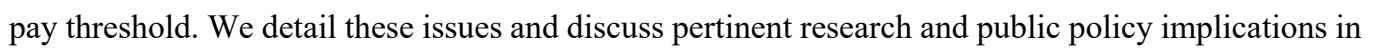

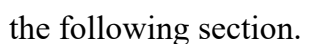

$\square$

\section{IMPLICATIONS}

\section{Box 3: Implications}

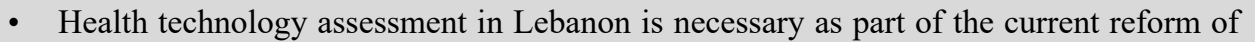

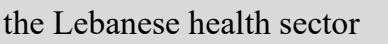

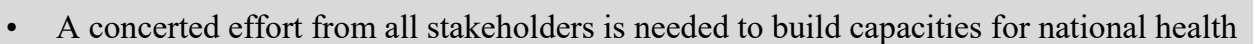

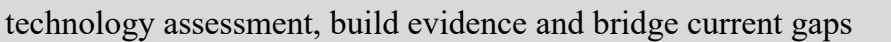

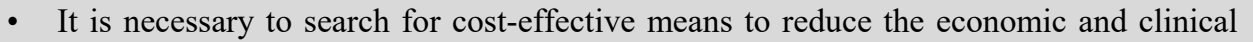

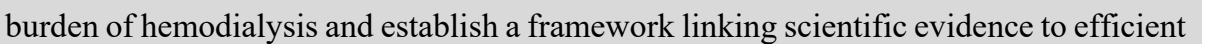

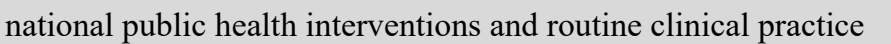

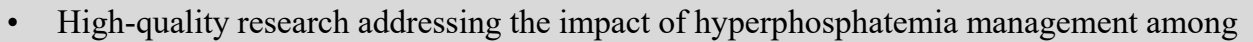

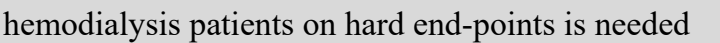

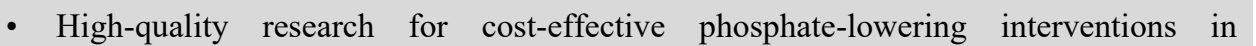

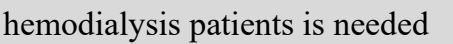

$\square$ 


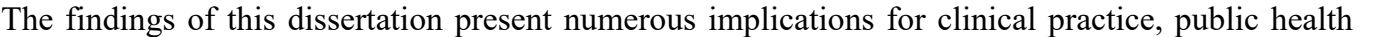

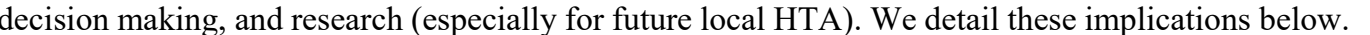

$\square$

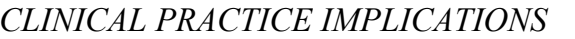

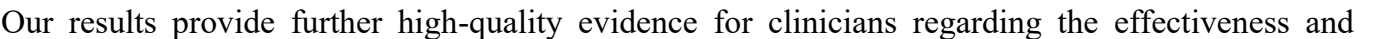

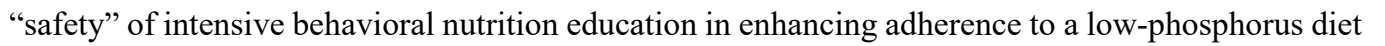

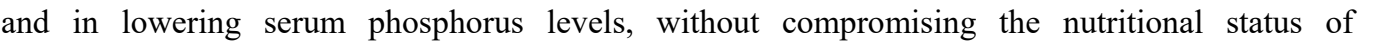

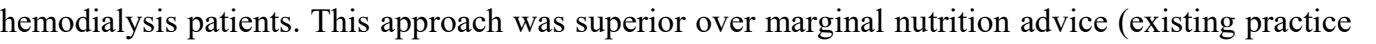

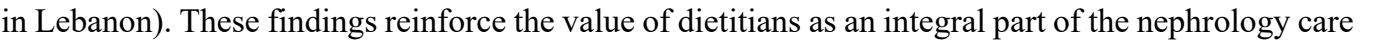

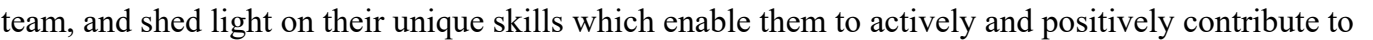

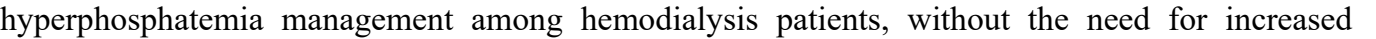

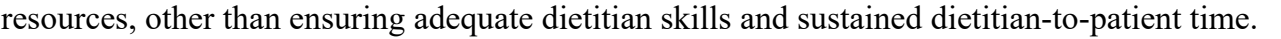

$\square$

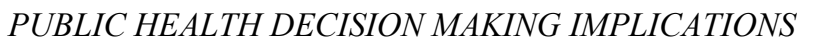

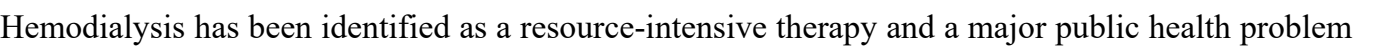

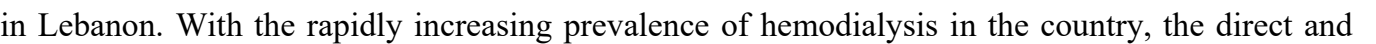

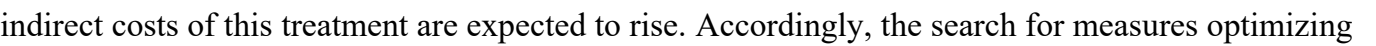

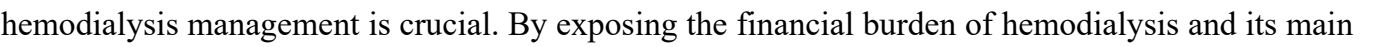

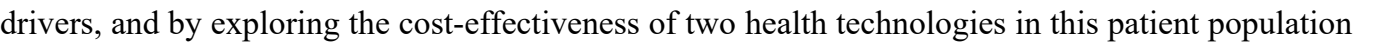

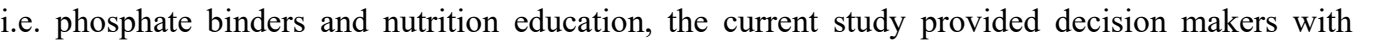

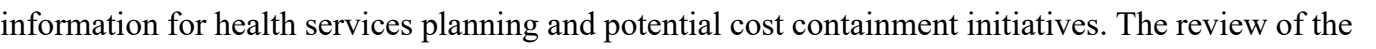

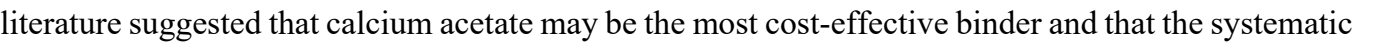

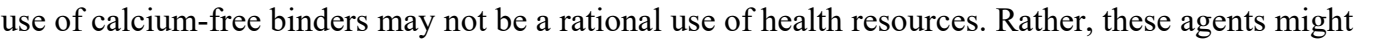

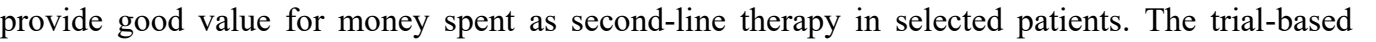

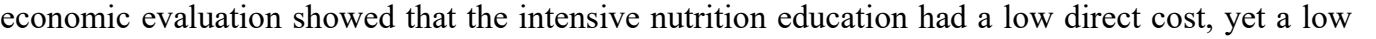

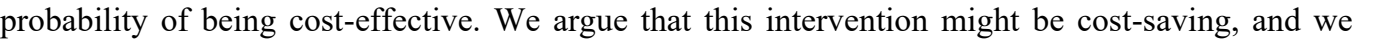
घण口

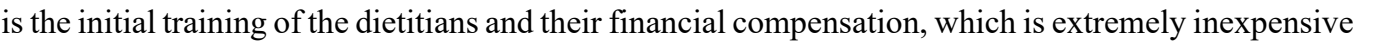

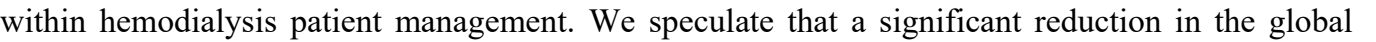

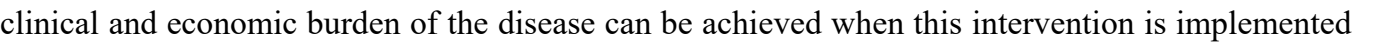
पापाणामाप

\section{Concrete implications for health technology assessment implementation in Lebanon}

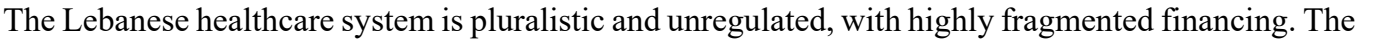

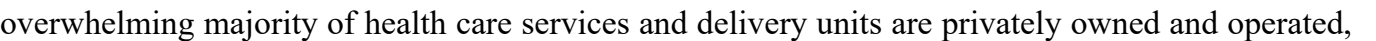




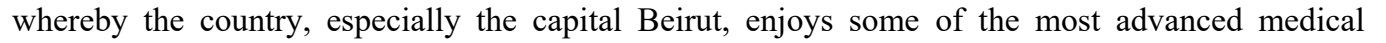

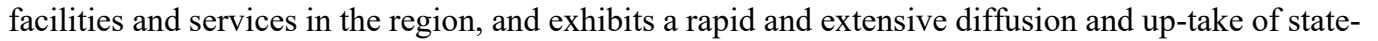

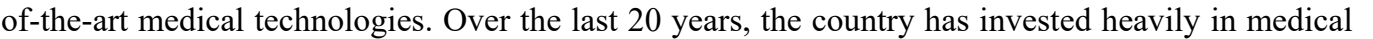

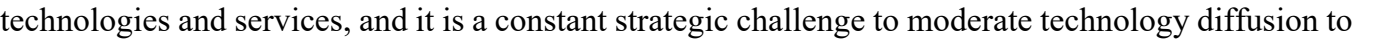
प

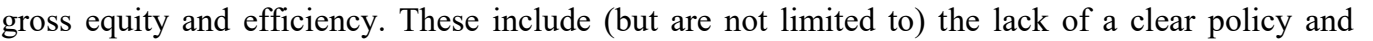

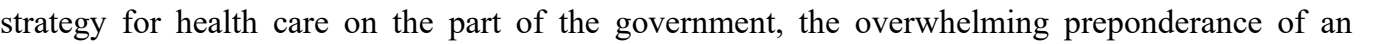

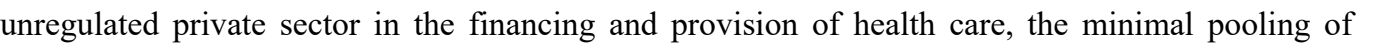

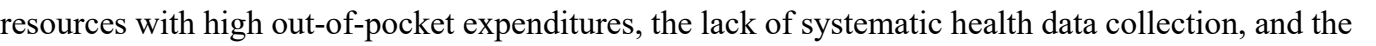

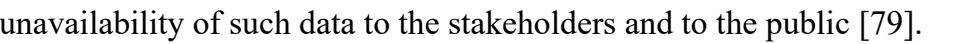

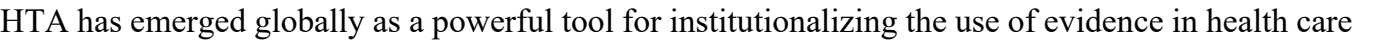

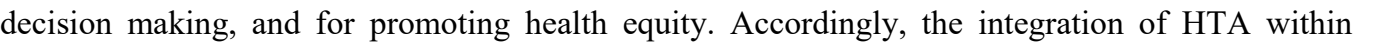

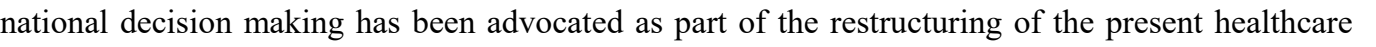

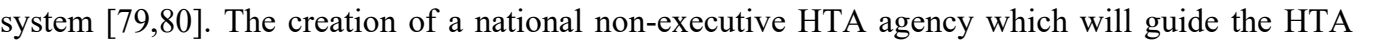

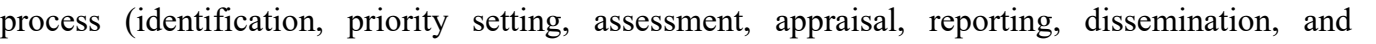

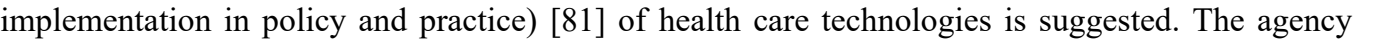

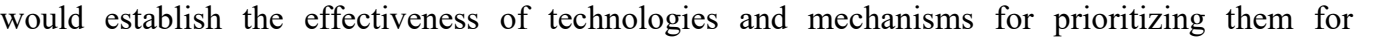

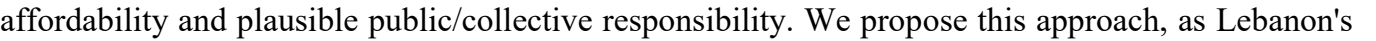

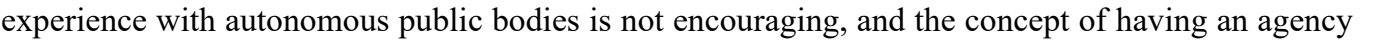

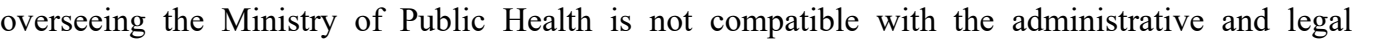

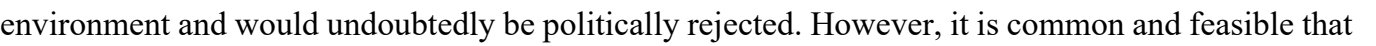

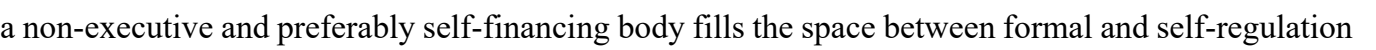

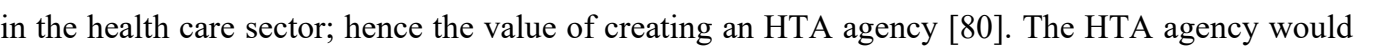

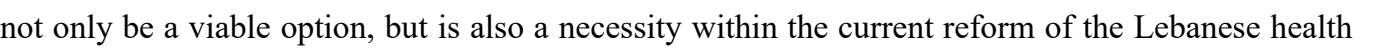

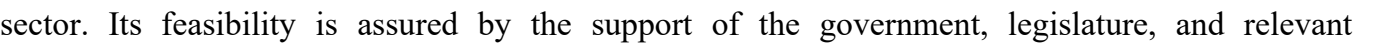

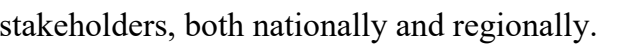

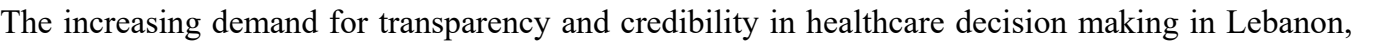

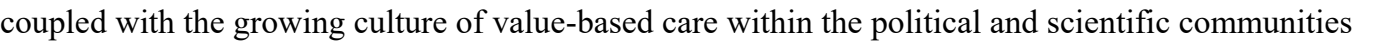

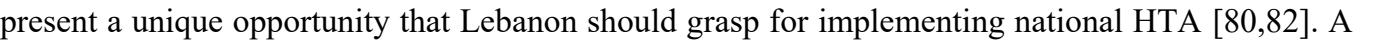

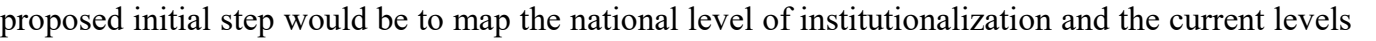

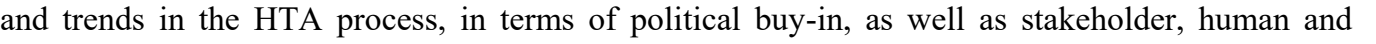

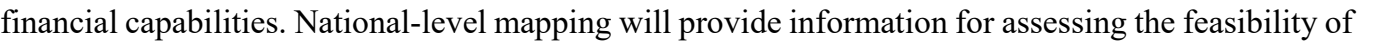

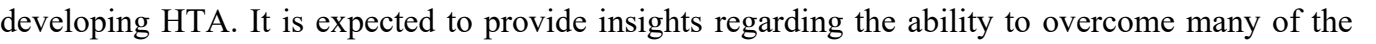

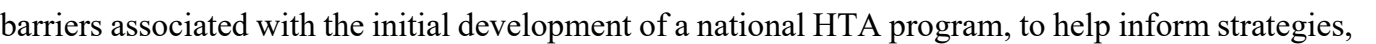

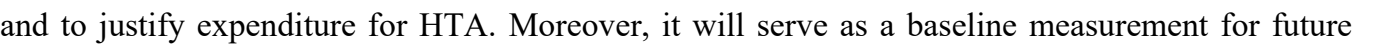

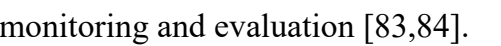




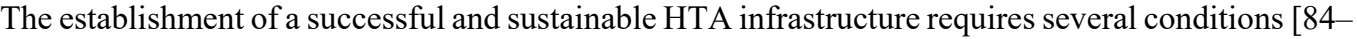

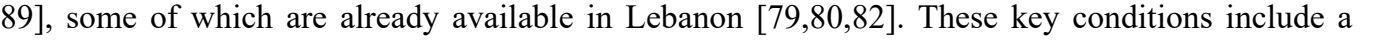

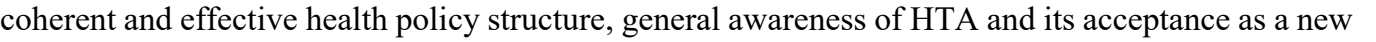

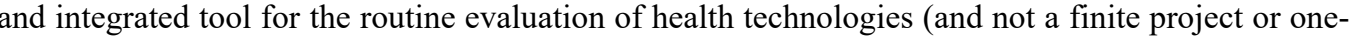

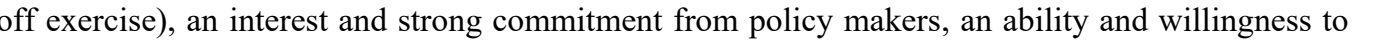

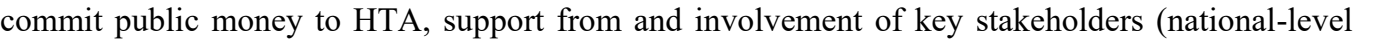

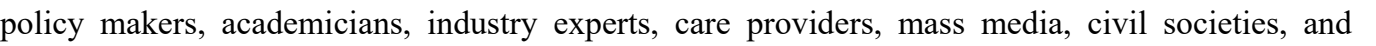

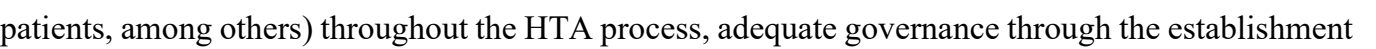

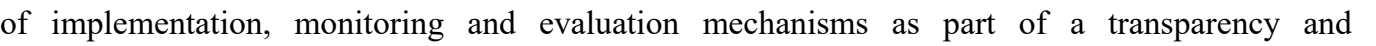

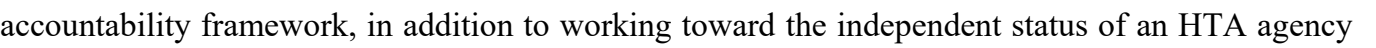

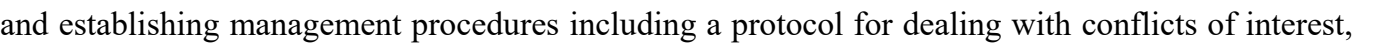

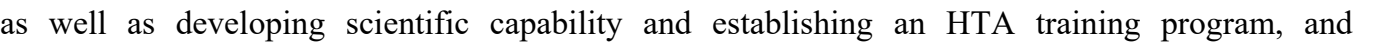

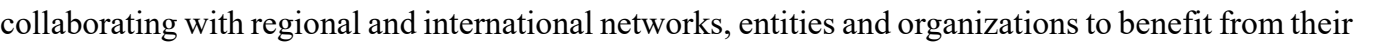

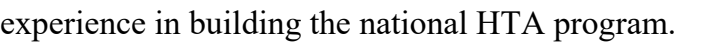

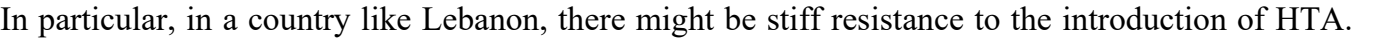

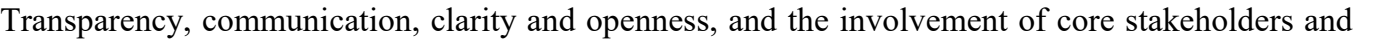

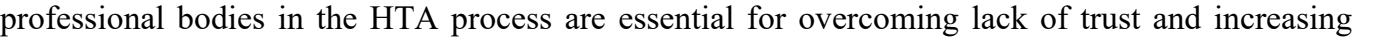

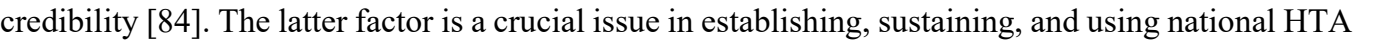

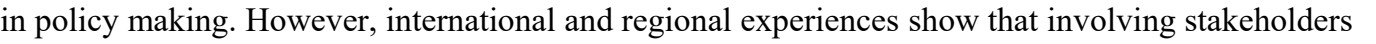

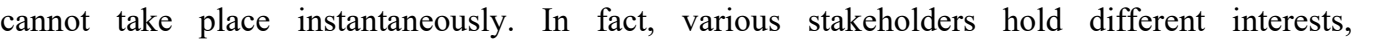

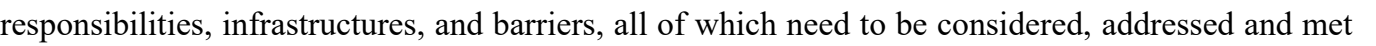

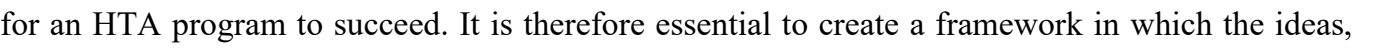

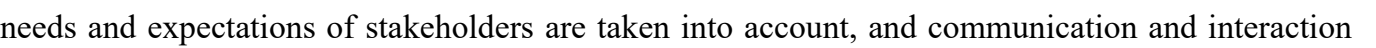

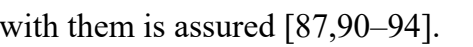

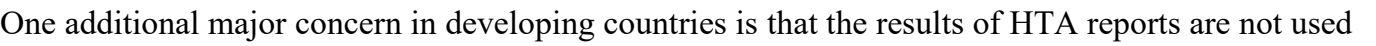

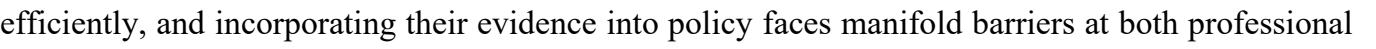

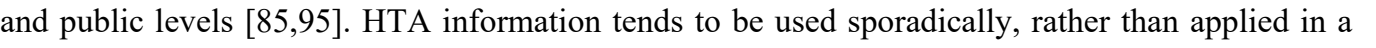

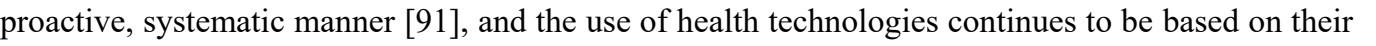

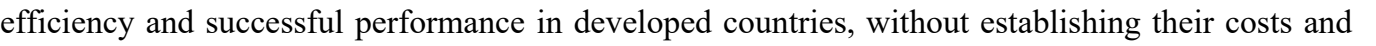

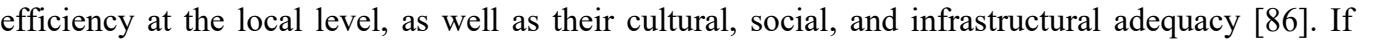

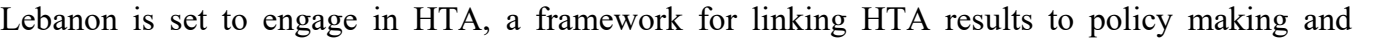

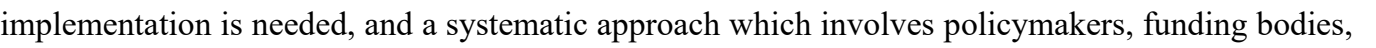

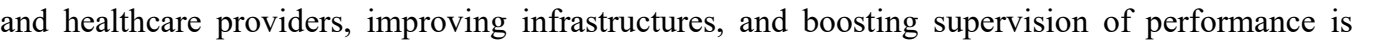

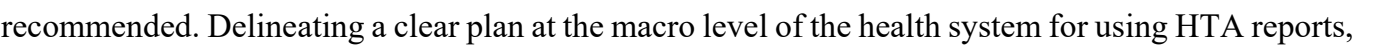

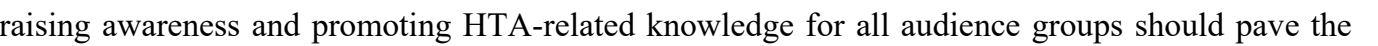

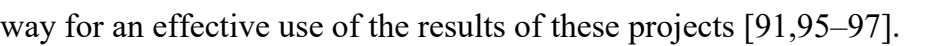




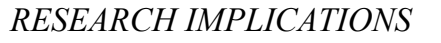

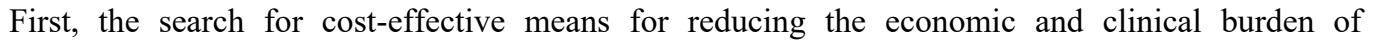

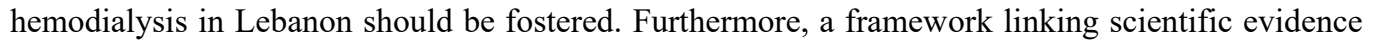

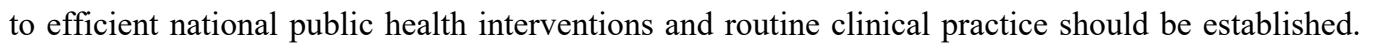

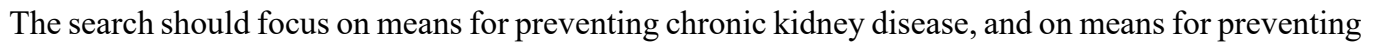

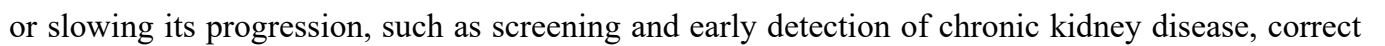

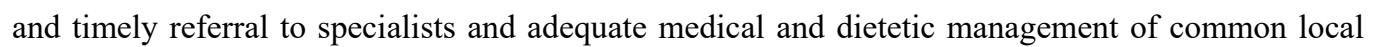

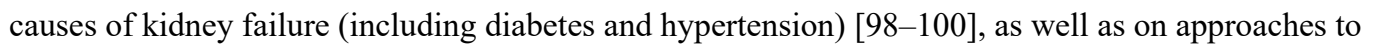

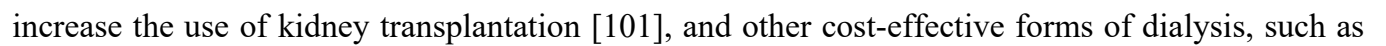

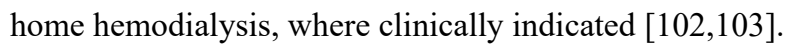

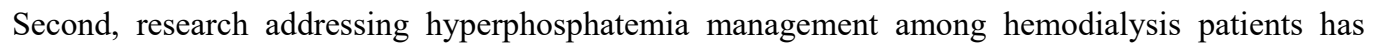

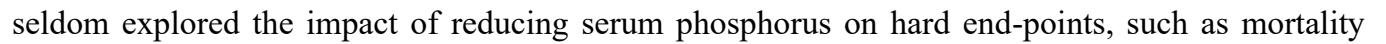

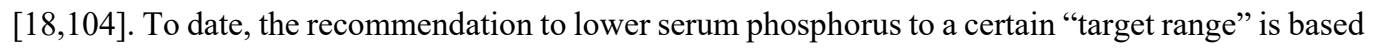

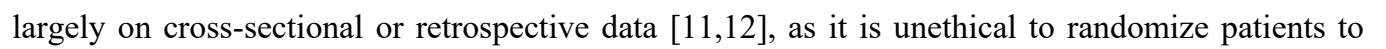

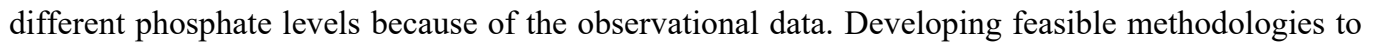

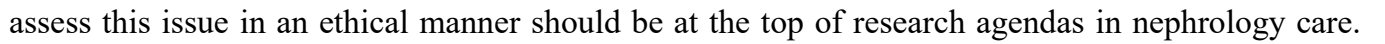

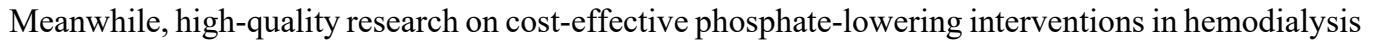

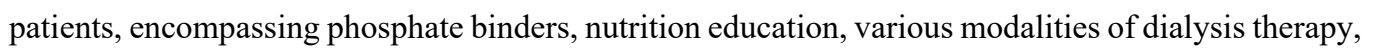

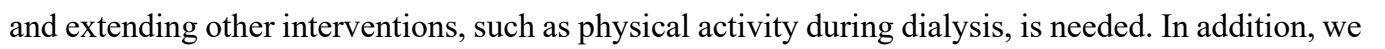

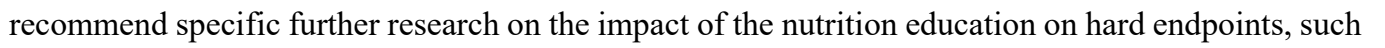

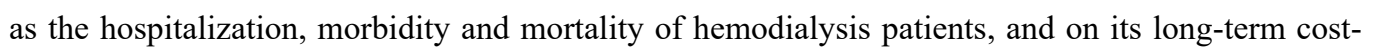

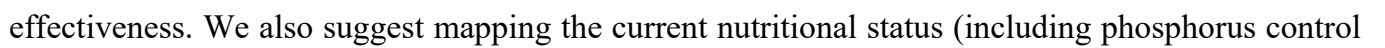

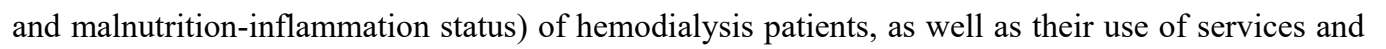

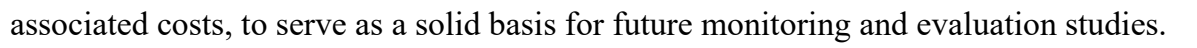

Third, the economic evaluation conducted as part of this dissertation exposed the "virgin" territory of

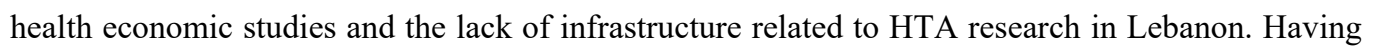

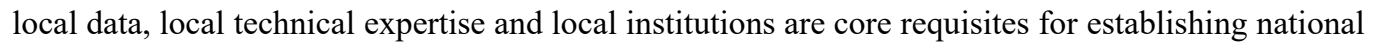

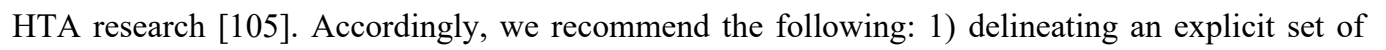

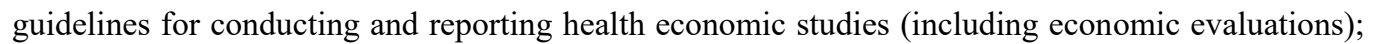

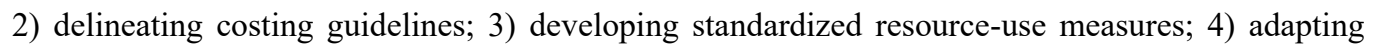

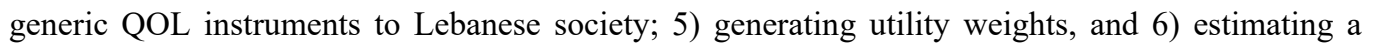

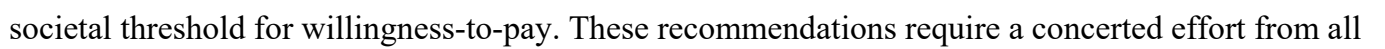

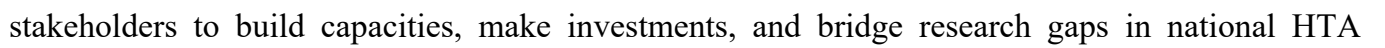

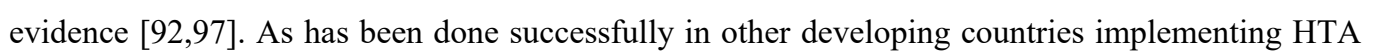

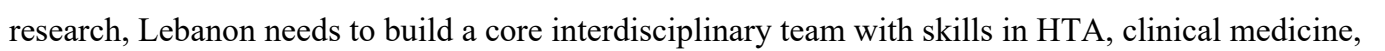

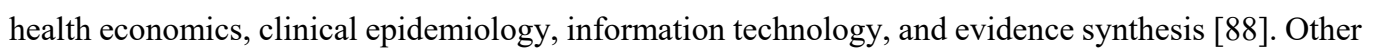




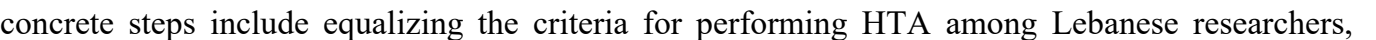

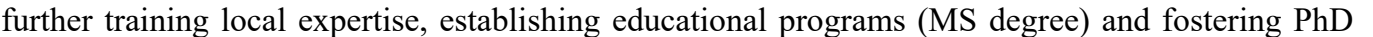

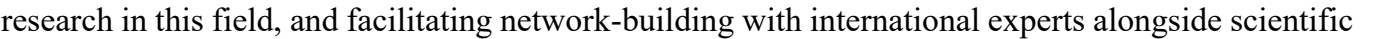

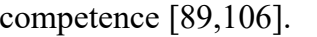




\section{REFERENCES}

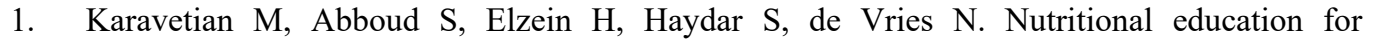

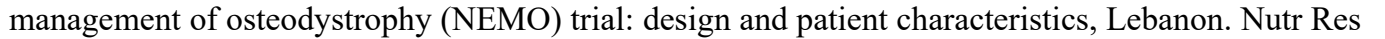

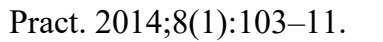

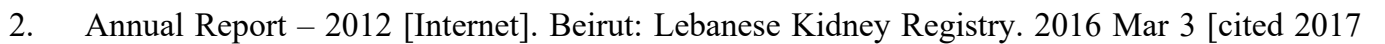

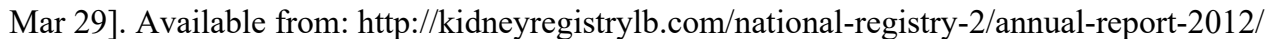

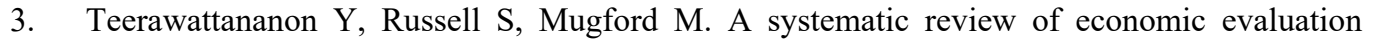

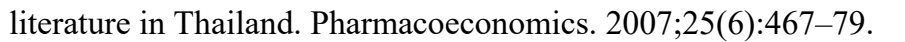

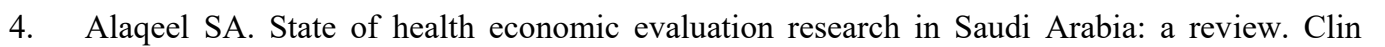

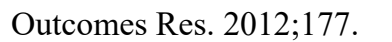

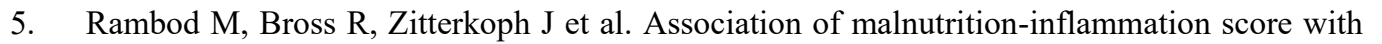

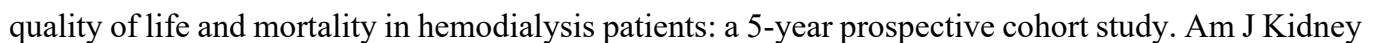

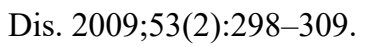

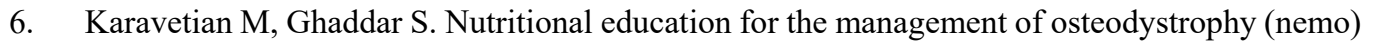

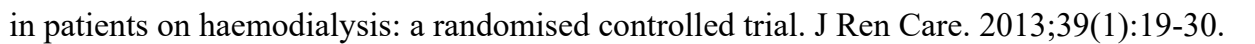

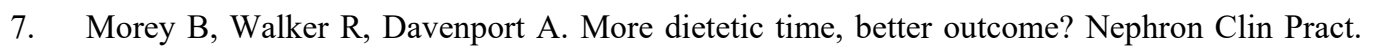

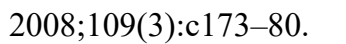

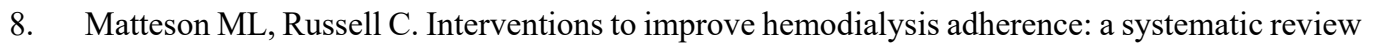

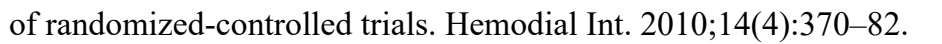

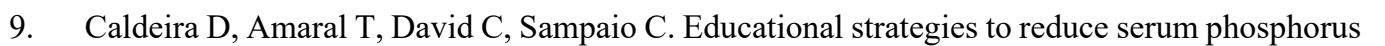

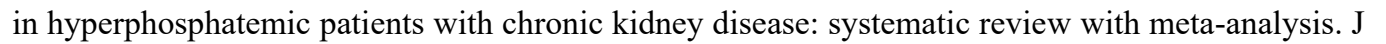

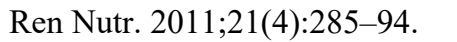

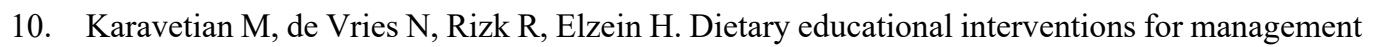

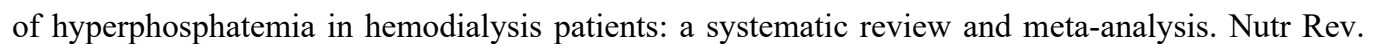

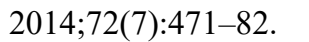

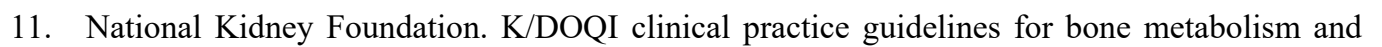

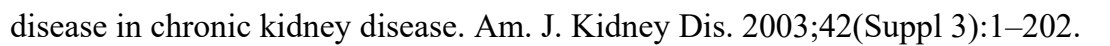

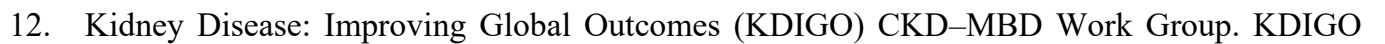

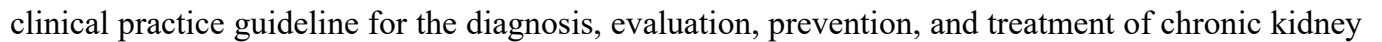

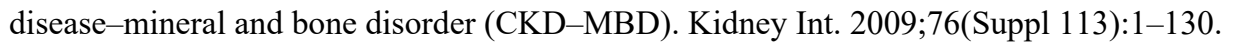

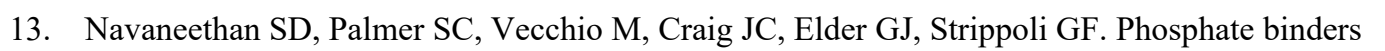

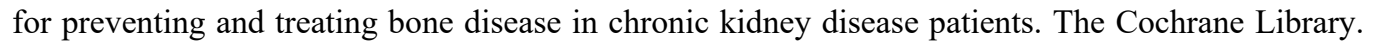
$\square 110$

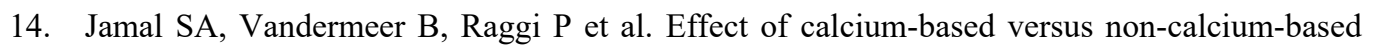

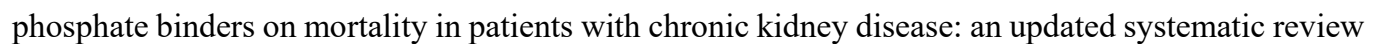

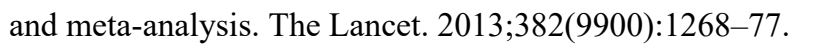

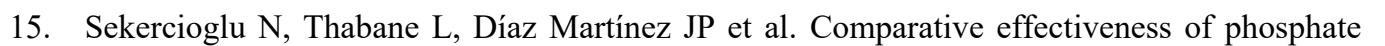

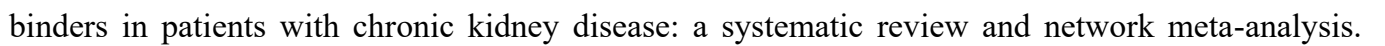

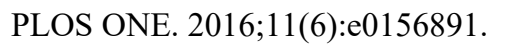




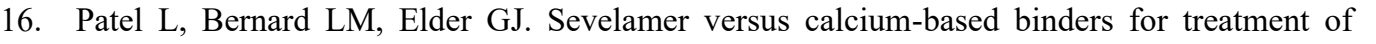

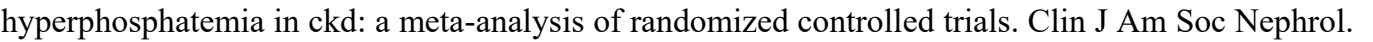

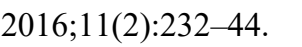

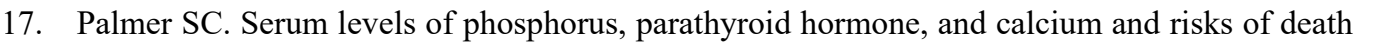

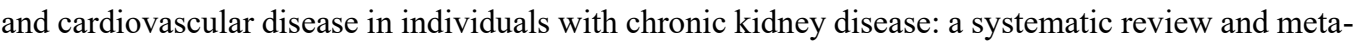

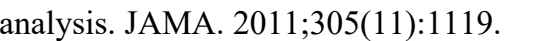

$\square \square$ Hutchison AJ. Novel phosphate binders: plus ça change, plus c'est la même chose. $\square \square \square \square \square \square \|$

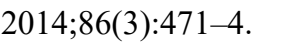

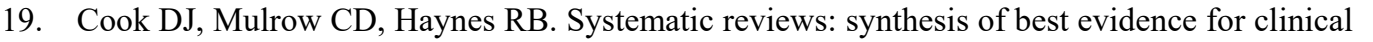

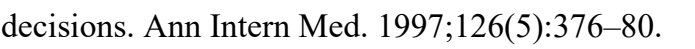

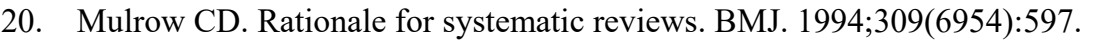

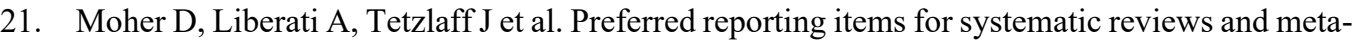

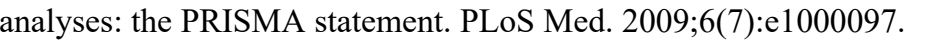

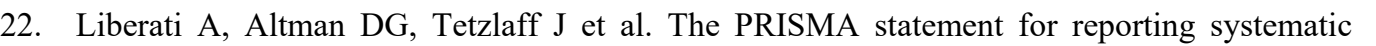

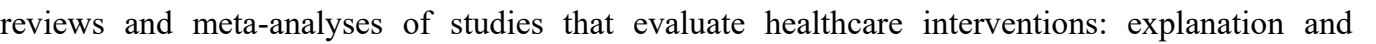

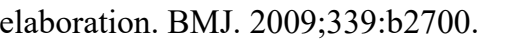

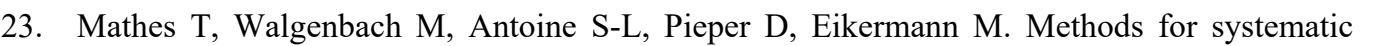

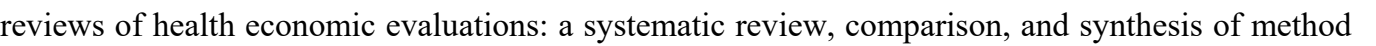

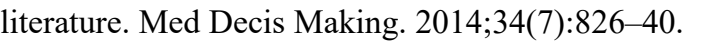

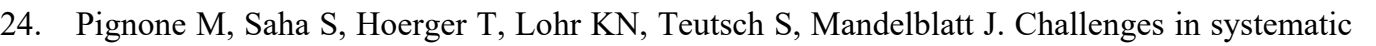

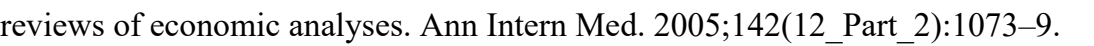

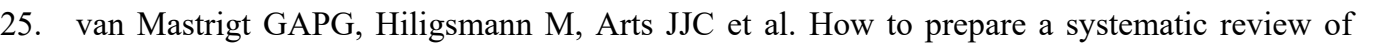

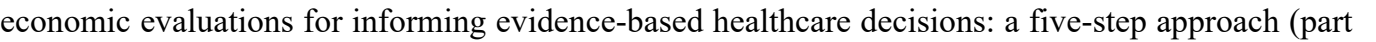

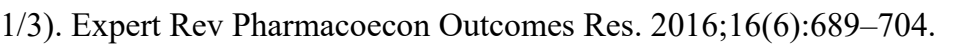

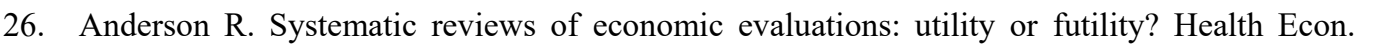

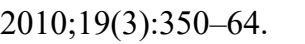

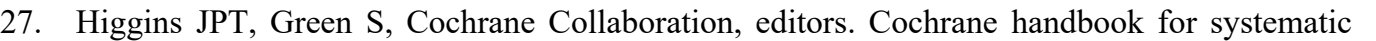

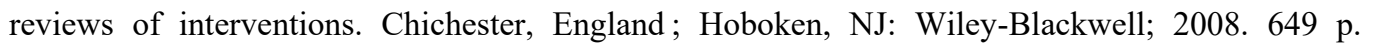

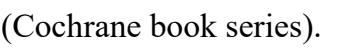

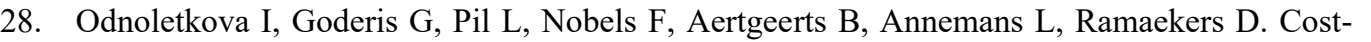

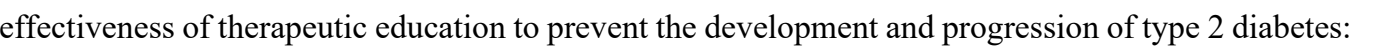

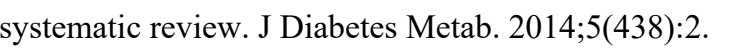

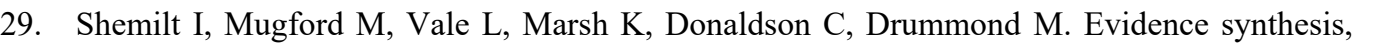

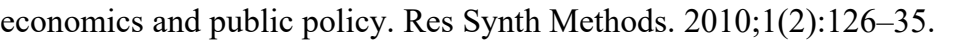

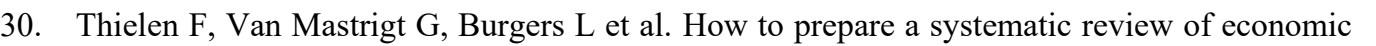

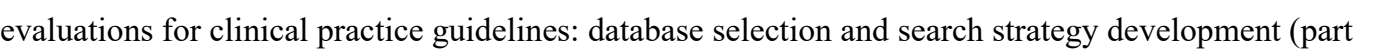

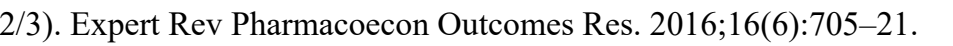

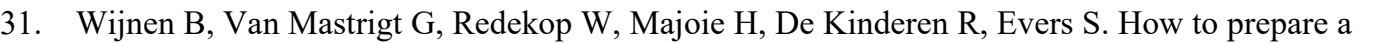

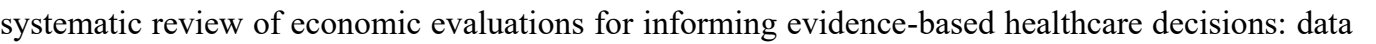




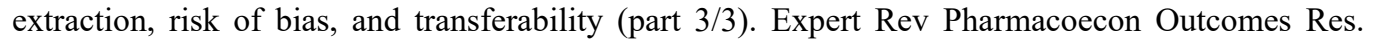

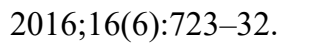

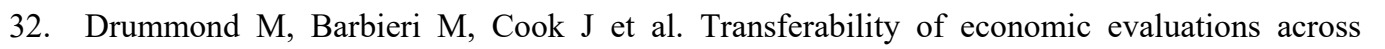

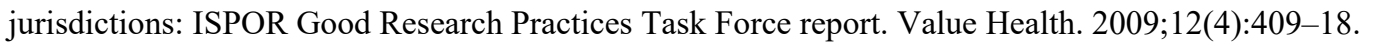

\section{$\square \mathrm{IU}$ Goeree R, He J, O'Reilly D et al. Transferability of health technology assessments and}

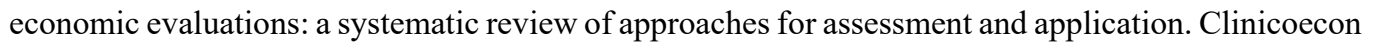

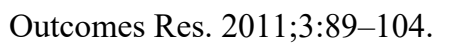

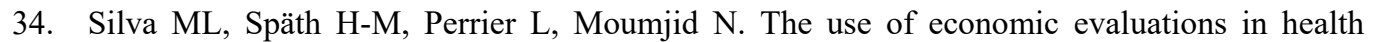

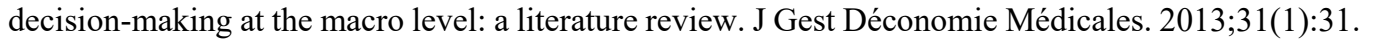

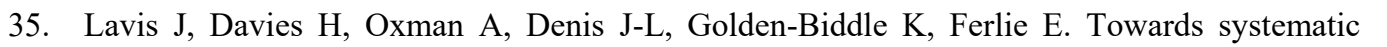

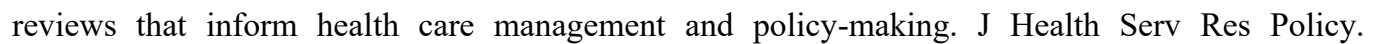

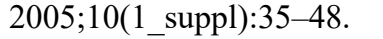

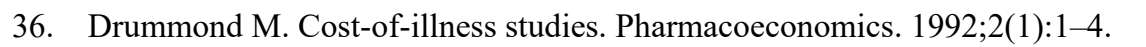

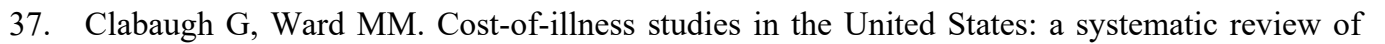

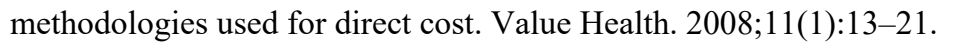

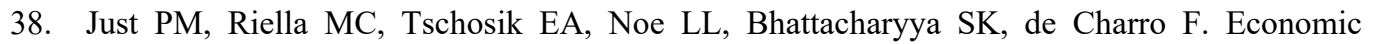

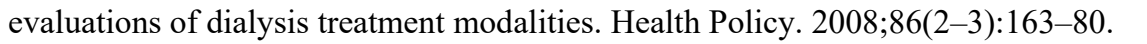

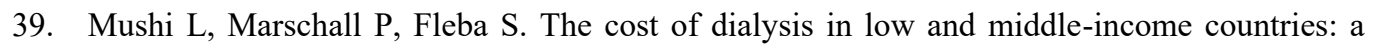

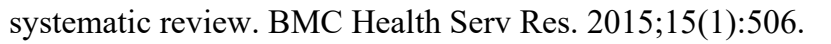

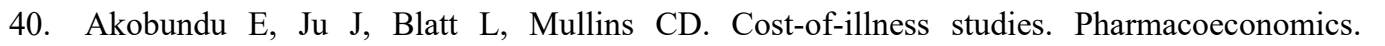

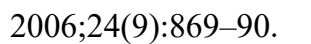

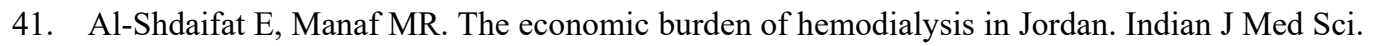

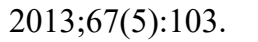

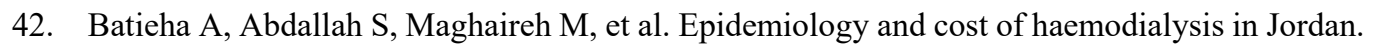

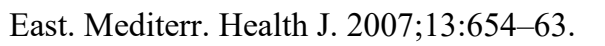

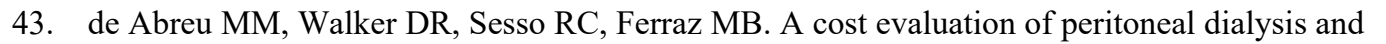

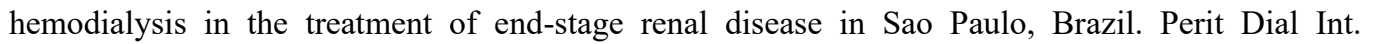

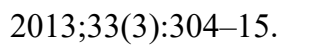

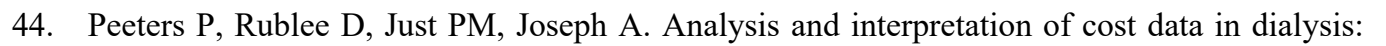

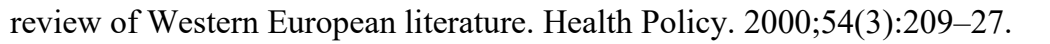

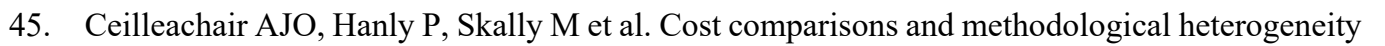

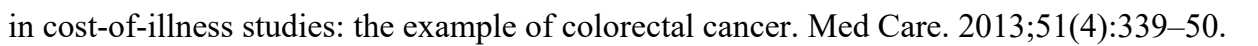

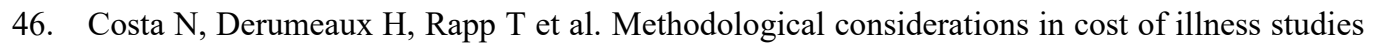

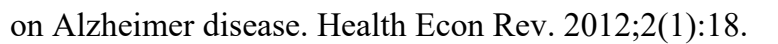

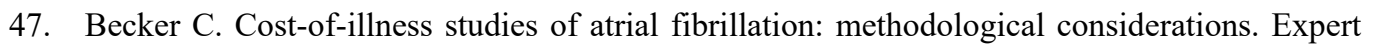

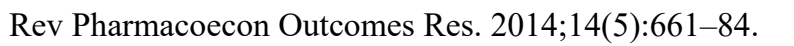

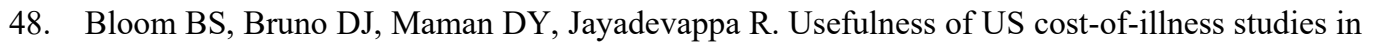

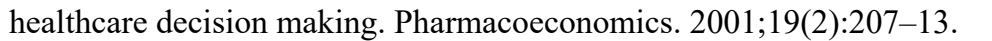

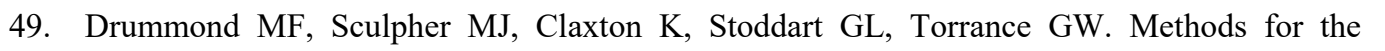

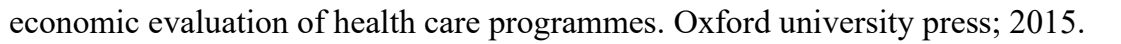




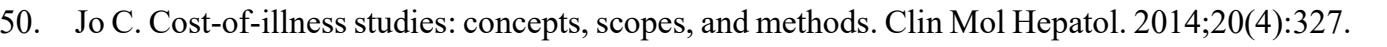

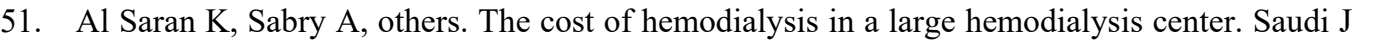

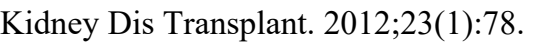

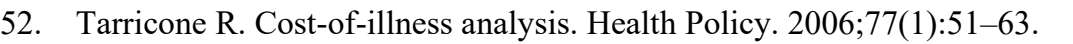

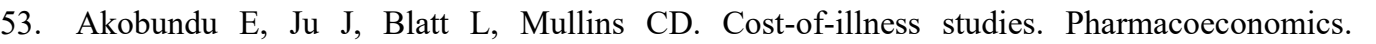

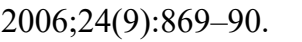

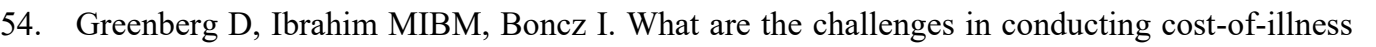

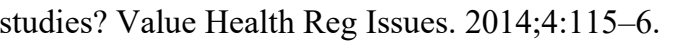

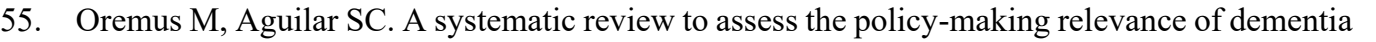

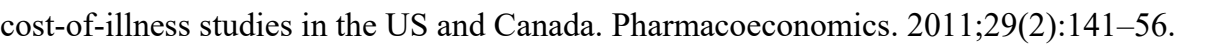

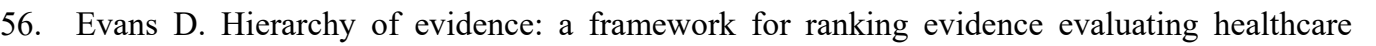

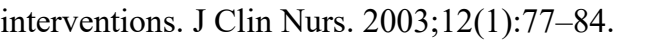

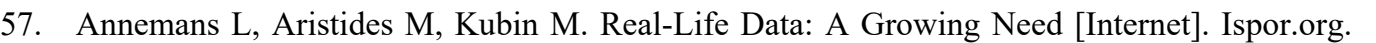

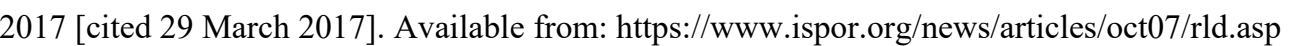

\section{$\square \square$ Cupisti A, D’Alessandro C, Baldi R, Barsotti G. Dietary habits and counseling focused on}

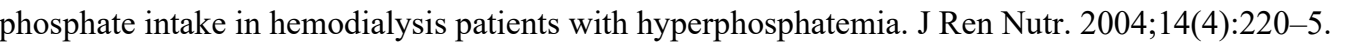

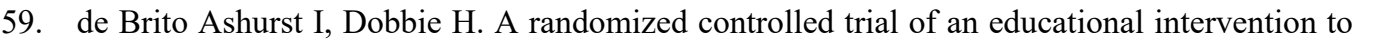

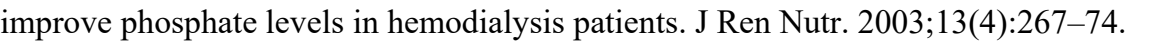

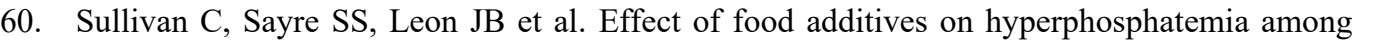

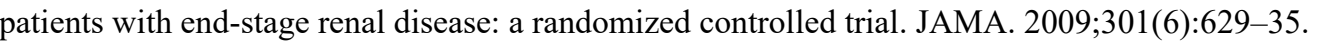

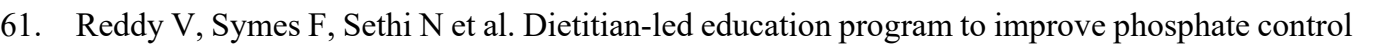

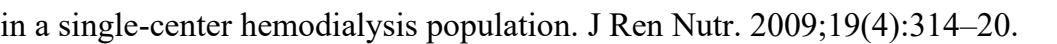

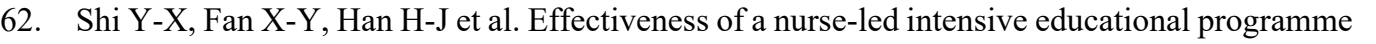

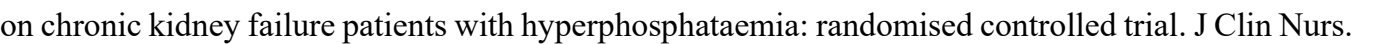

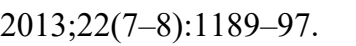

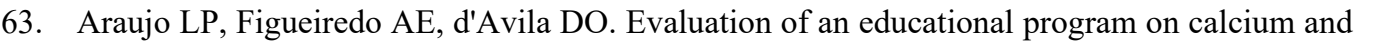

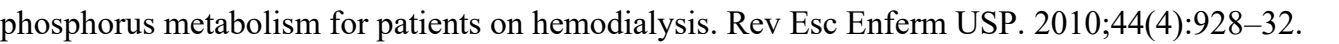

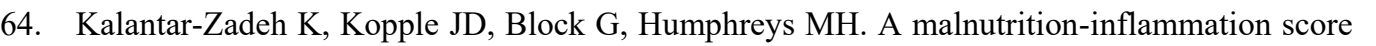

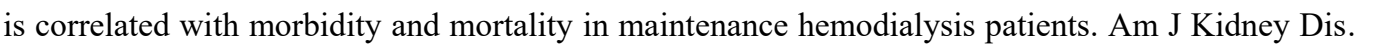
$\square|||||||||||-\square| \Pi$

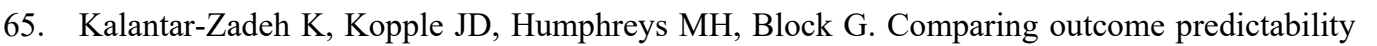

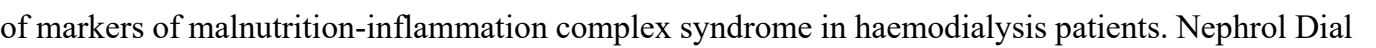

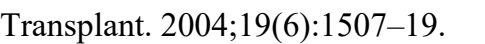

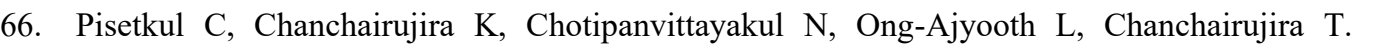

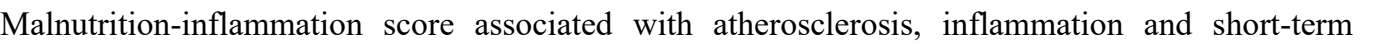

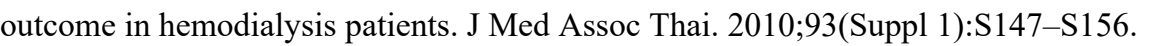

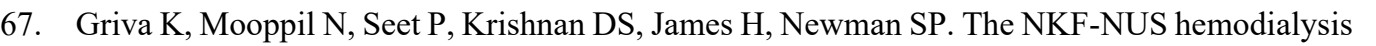

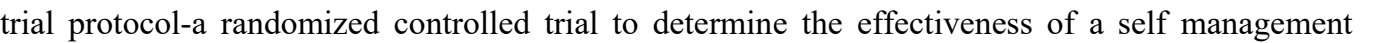

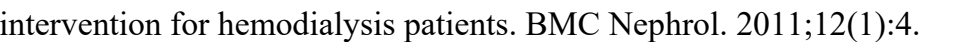




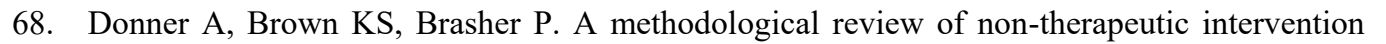

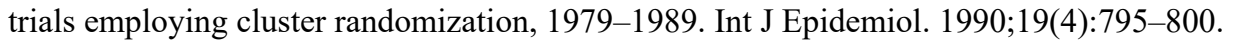

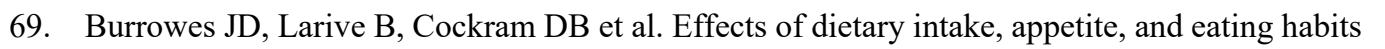

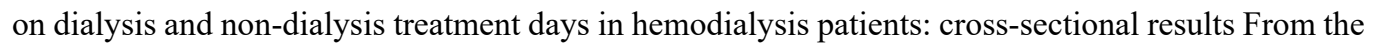

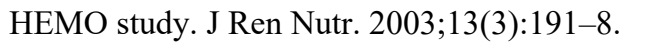

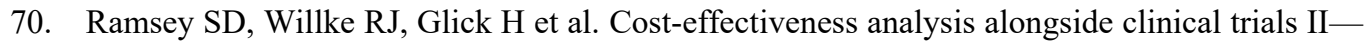

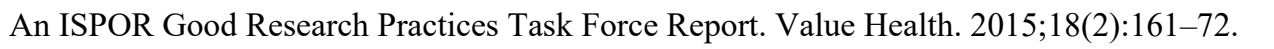

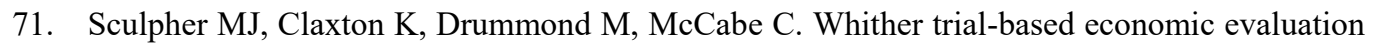

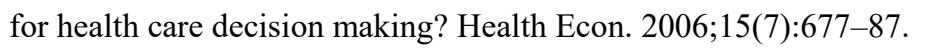

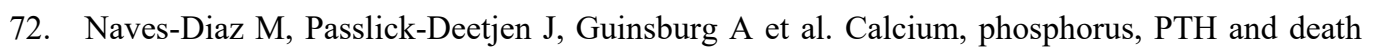

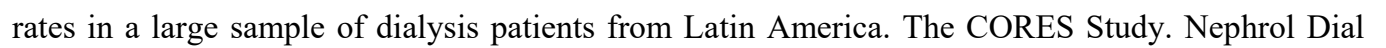

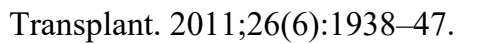

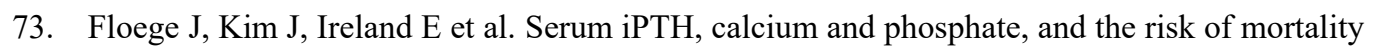

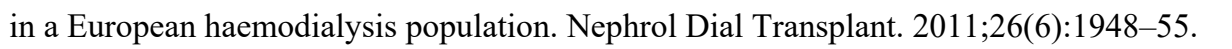

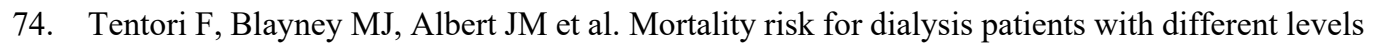

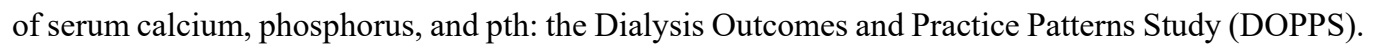

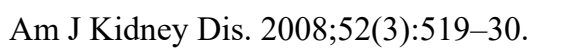

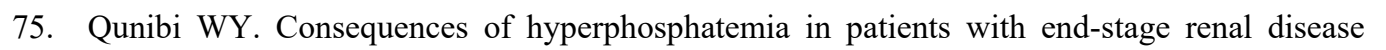

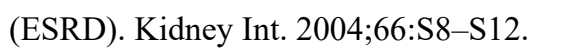

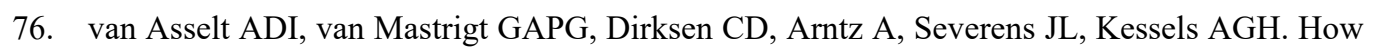

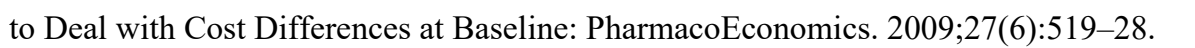

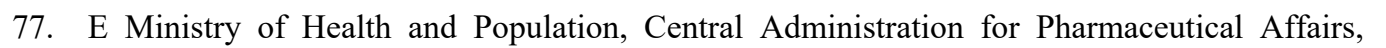

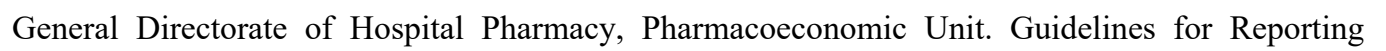

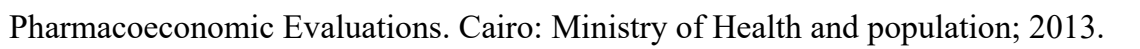

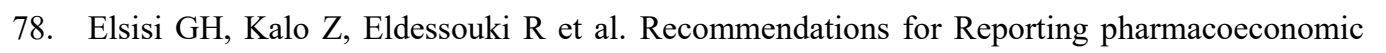

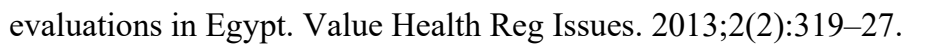

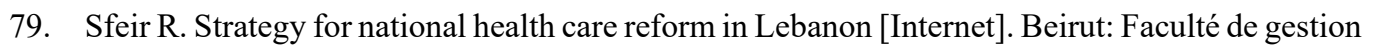

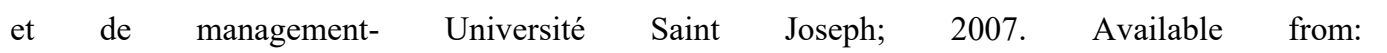

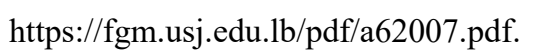

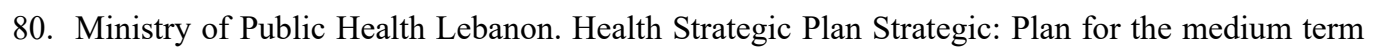

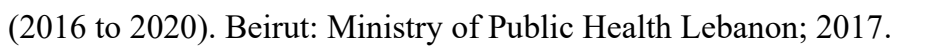

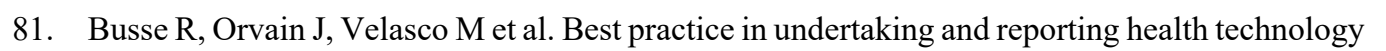

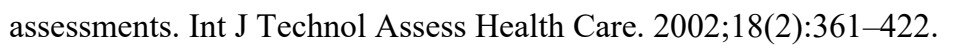

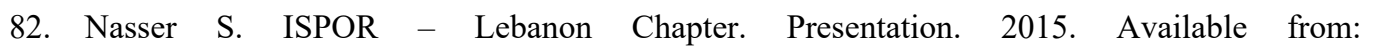

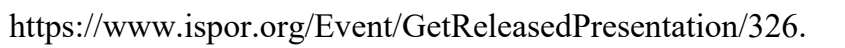

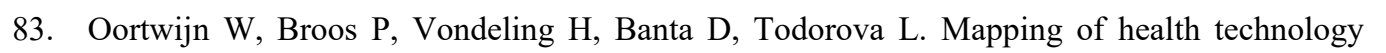

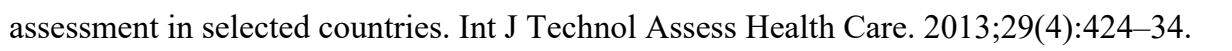

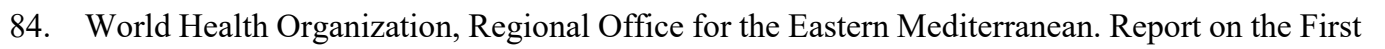

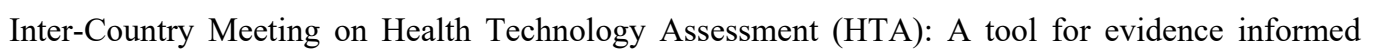




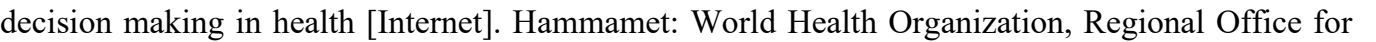

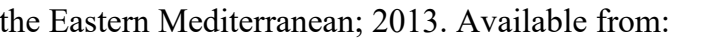

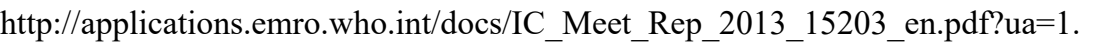

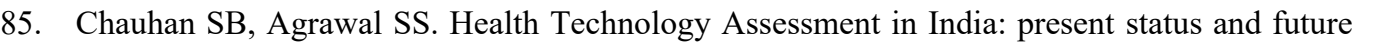

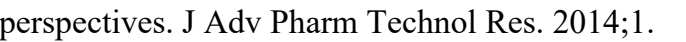

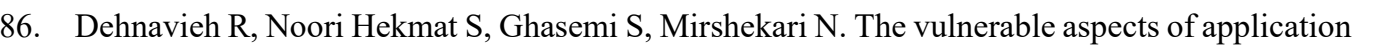

of "health technology assessment. Int J Technol Assess Health Care. 2015;31(3):197- $\square \square$

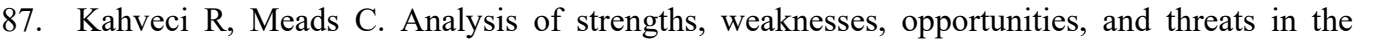

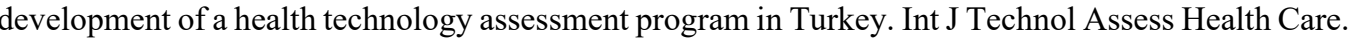

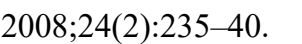

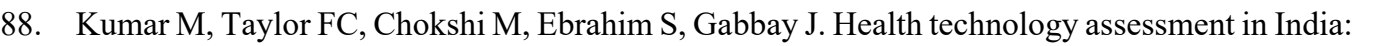

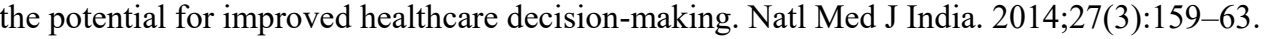

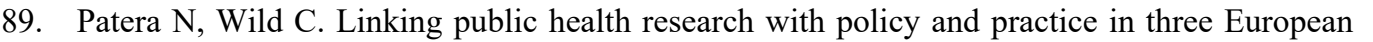

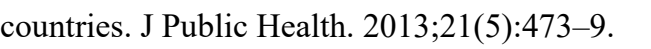

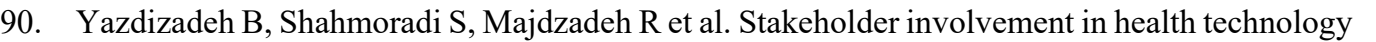

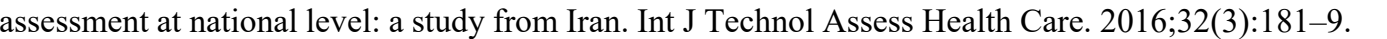

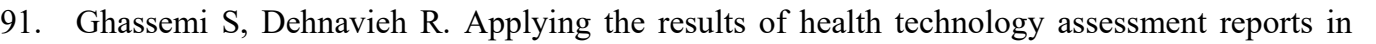

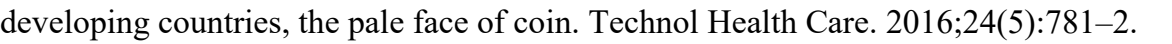

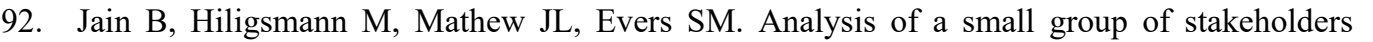

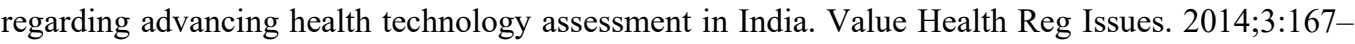
$\square \mathrm{III}$

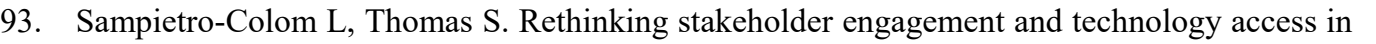

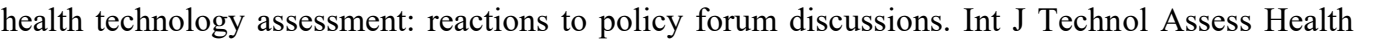

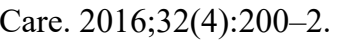

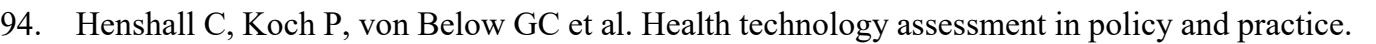

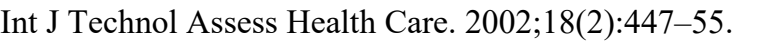

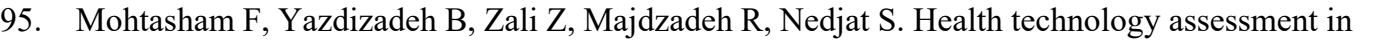

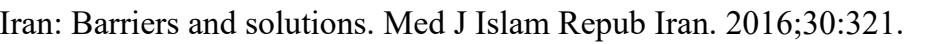

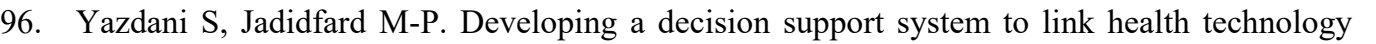

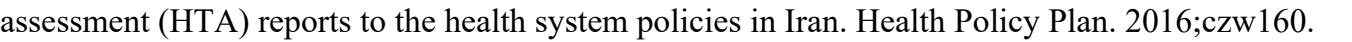

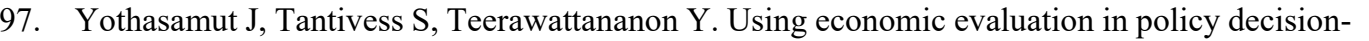

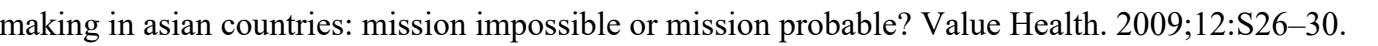

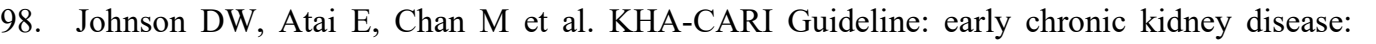

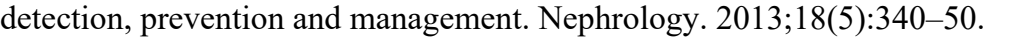

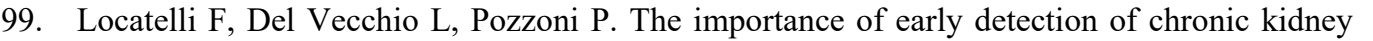

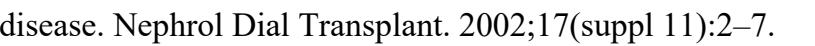

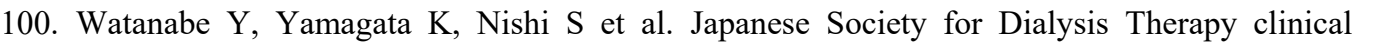

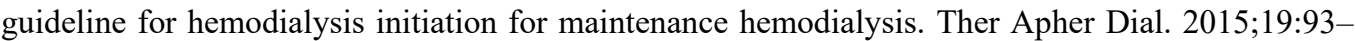
$\square \square \square$ 


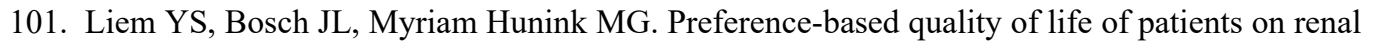

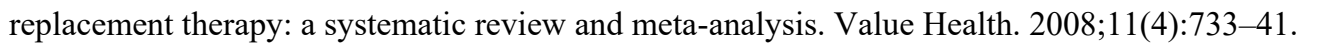

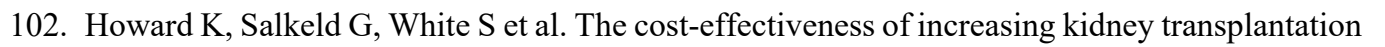

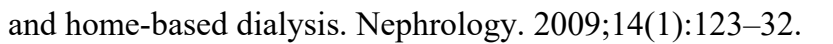

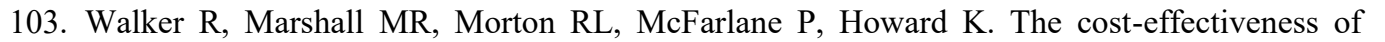

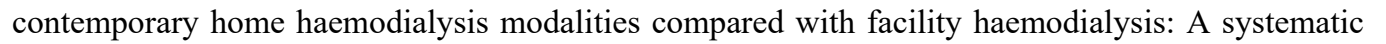

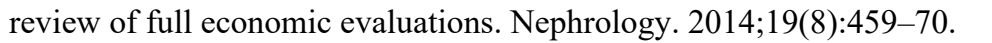

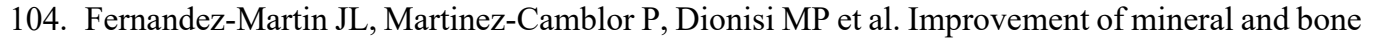

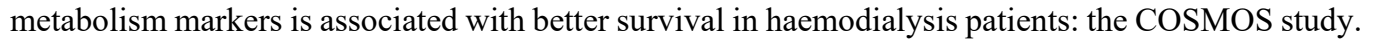

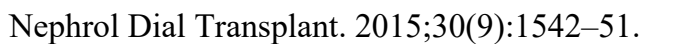

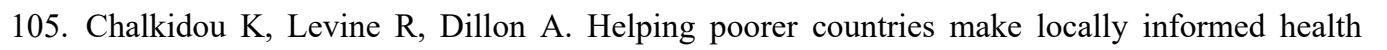

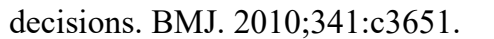

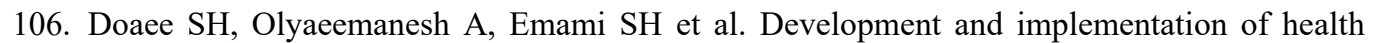

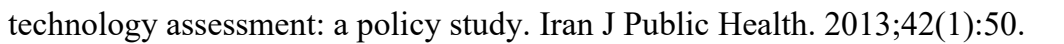

\section{$\square$}


LIST OF PUBLICATIONS OF THE THESIS $\square$ 


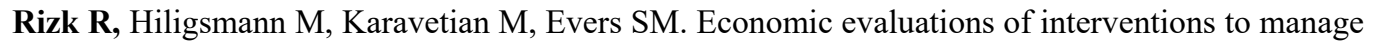

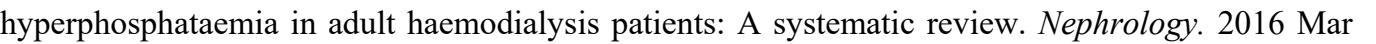

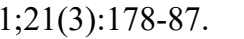

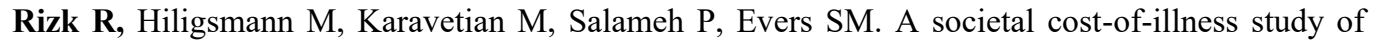

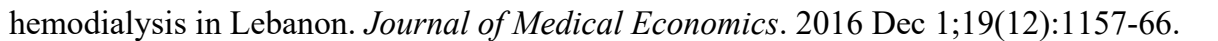

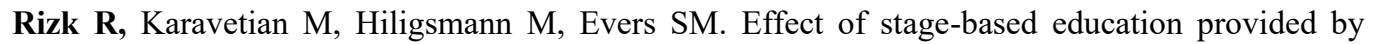

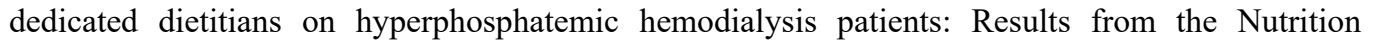

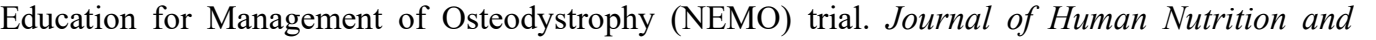

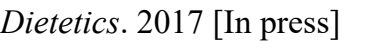

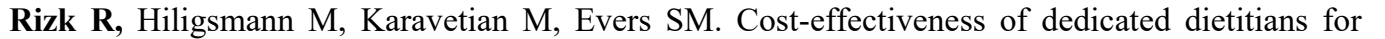

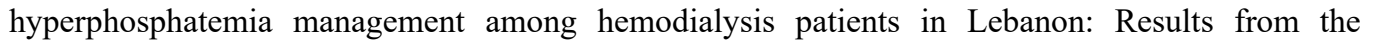

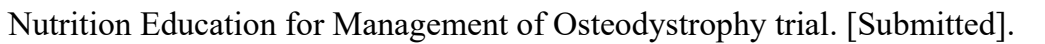


VALORIZATION $\square$ 


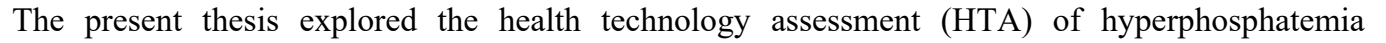

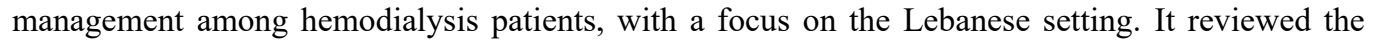

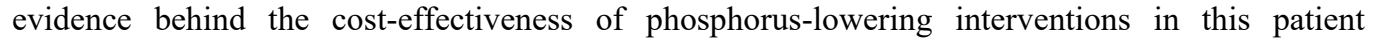

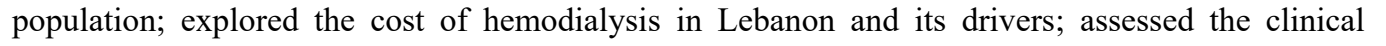

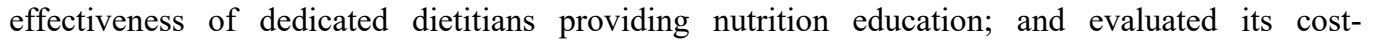

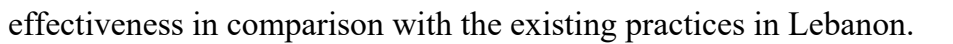

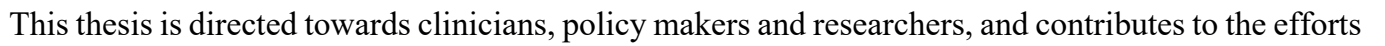

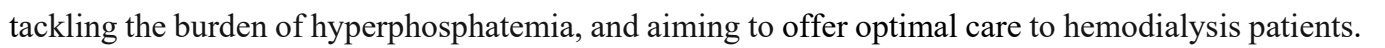

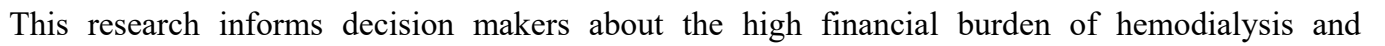

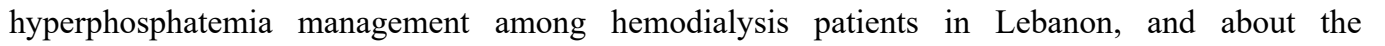

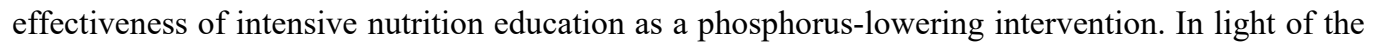

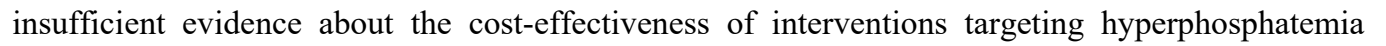

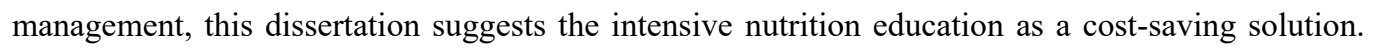

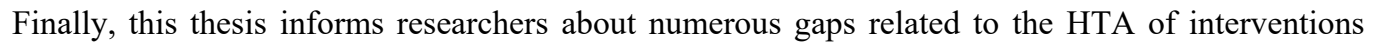

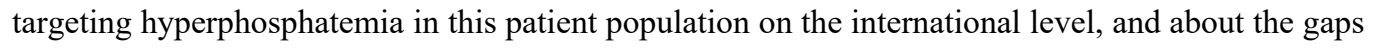

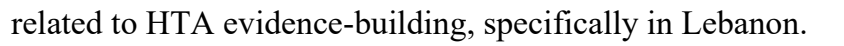

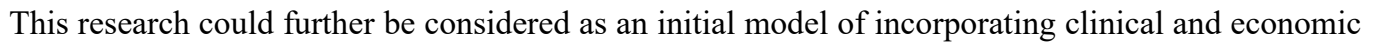

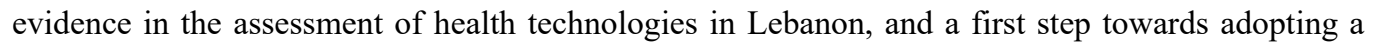

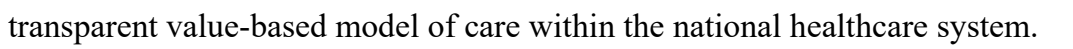

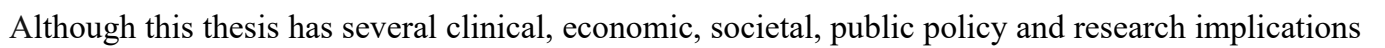

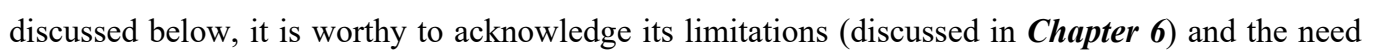

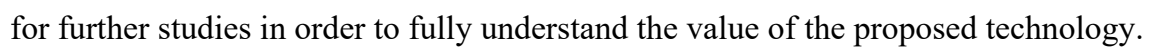

$\square$

\section{PROPOSED TECHNOLOGY AND IMPLEMENTATION ROADMAP}

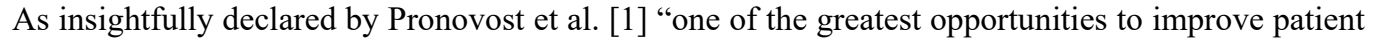

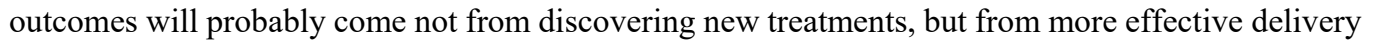
of existing therapies"

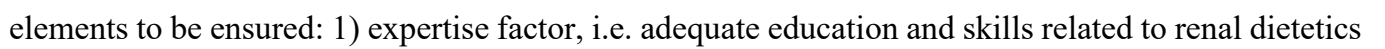

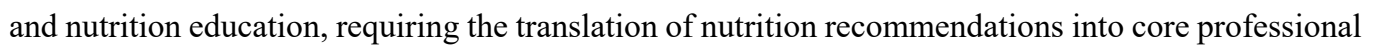

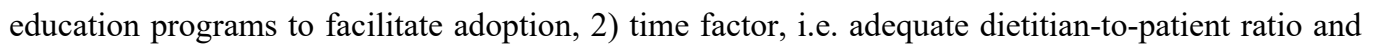

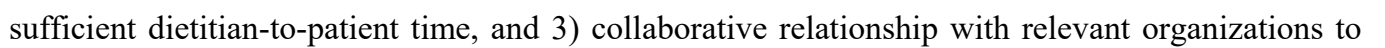

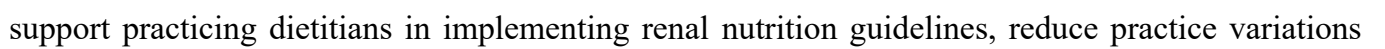

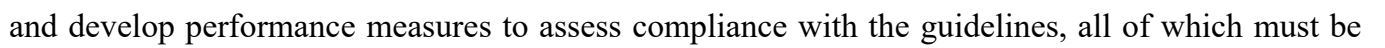

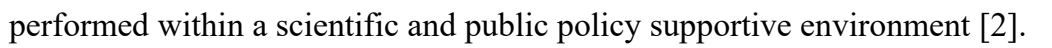




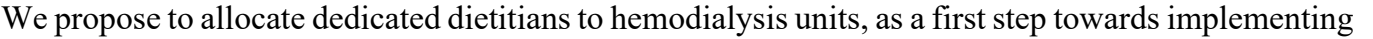

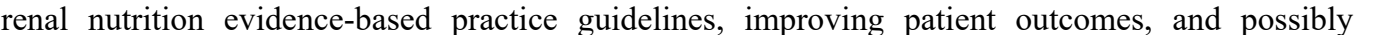

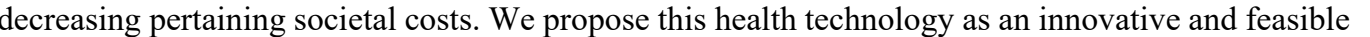

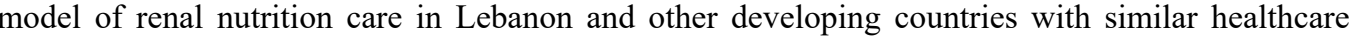

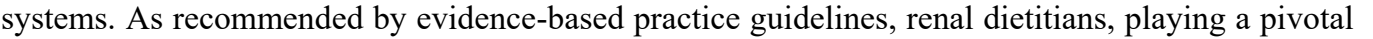

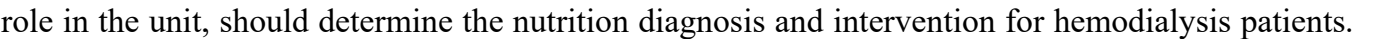

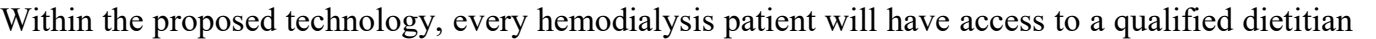

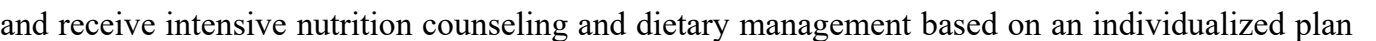

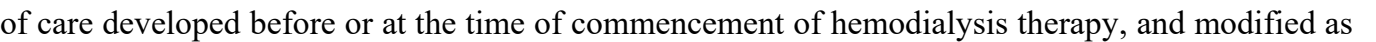
पाणाणाएा

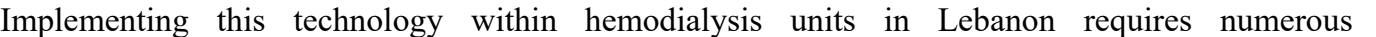

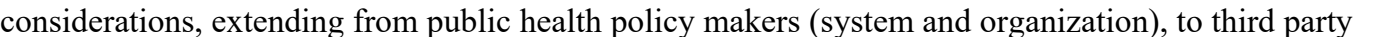

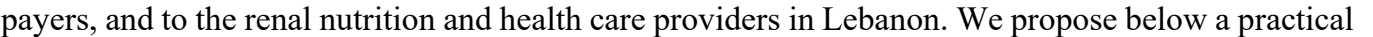

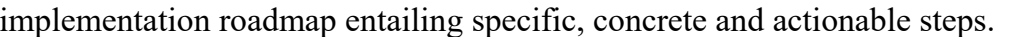

$\square$

\section{Governance considerations}

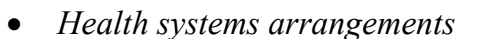

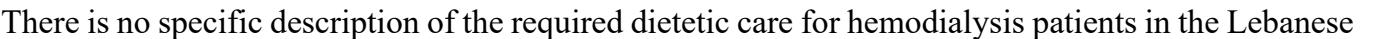

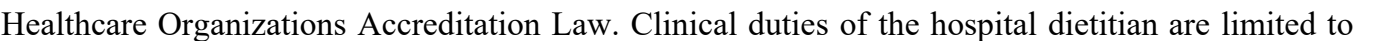

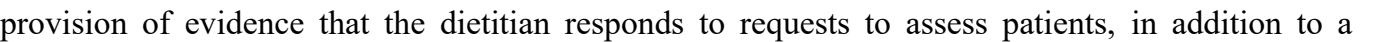

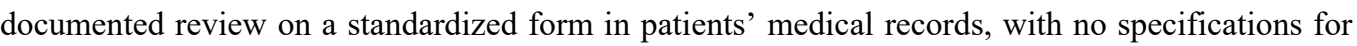

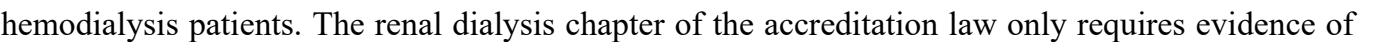

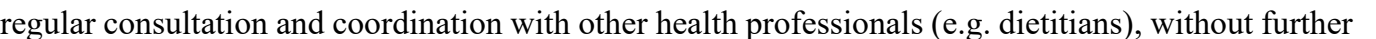

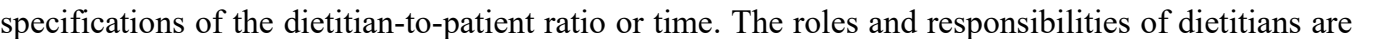
ए।

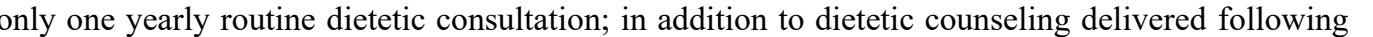
nephrologists' consult requests $\square$

$\rightarrow \square[||||||||||||||$

एवण

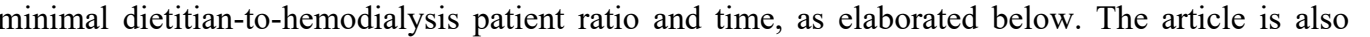

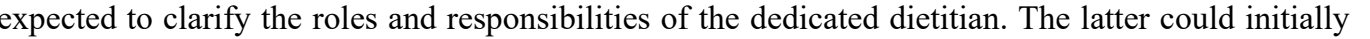

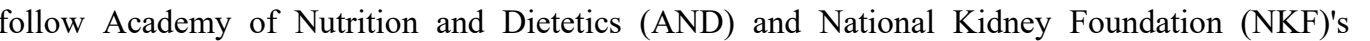

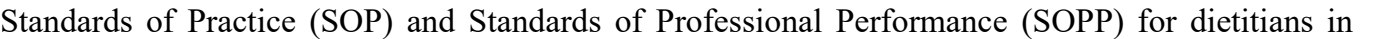

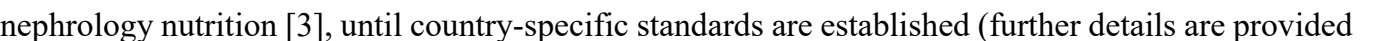
$\square[\square \square \square$ 


\section{$\underline{\text { Organizational considerations }}$}

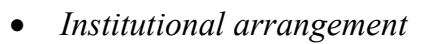

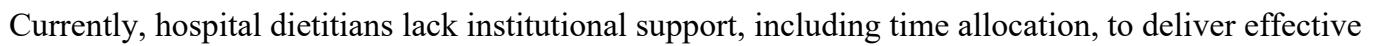

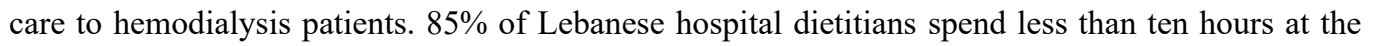

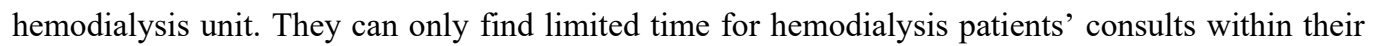

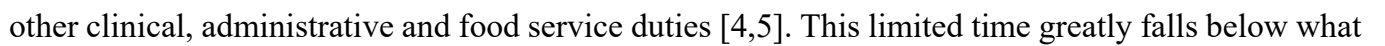

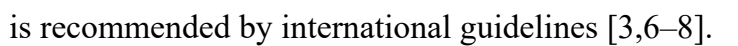

$\rightarrow \square \||||||||||||| \mid$

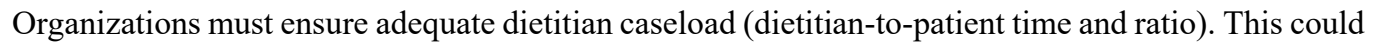

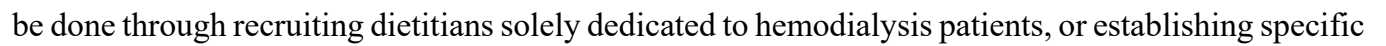

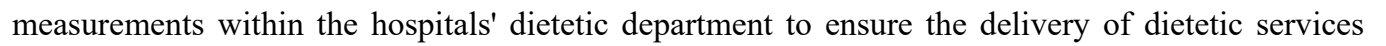

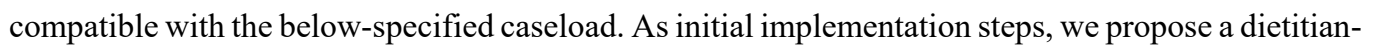

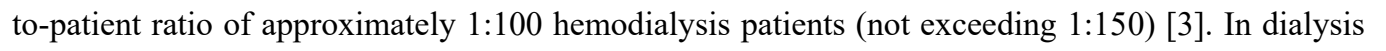

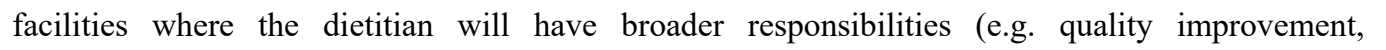

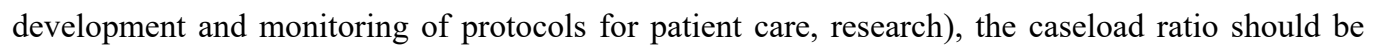

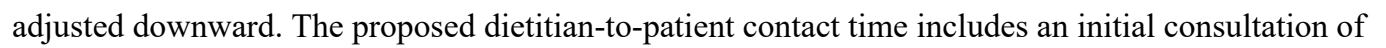

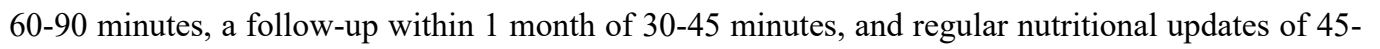

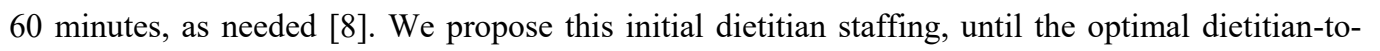

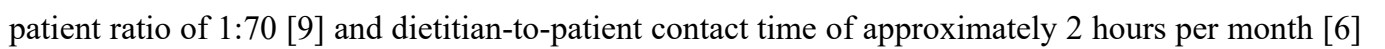

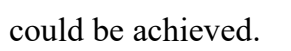

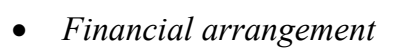

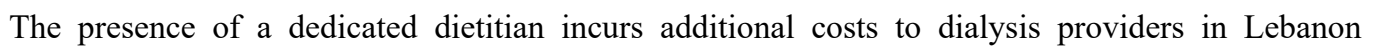

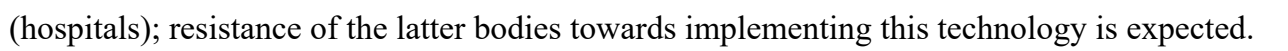

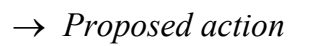

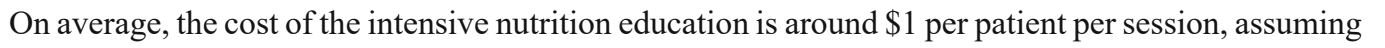

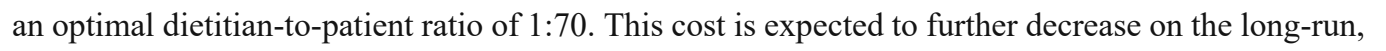

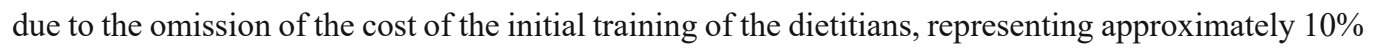

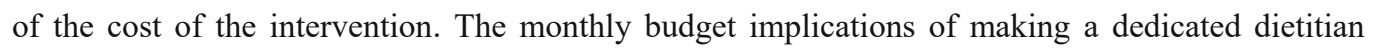

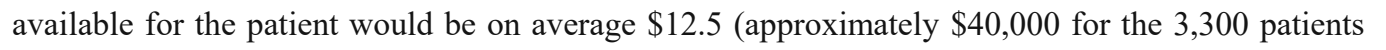

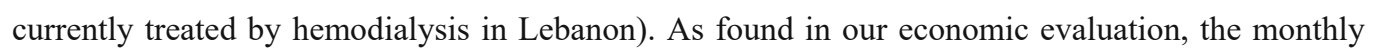

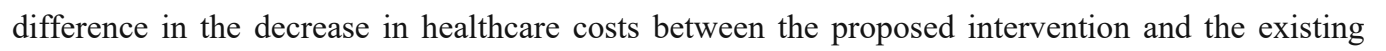

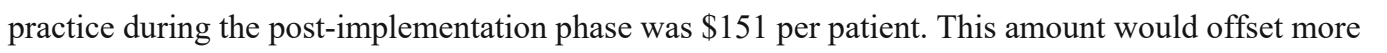

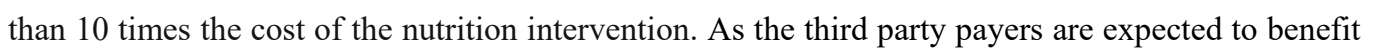

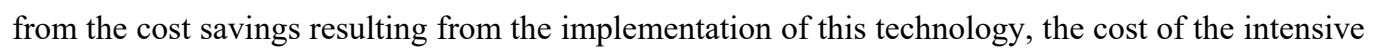




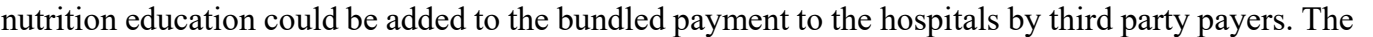

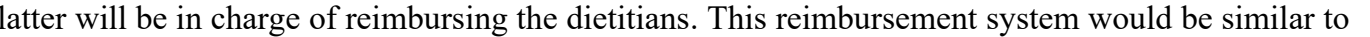

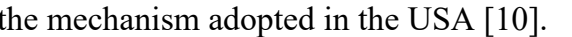

$\square$

\section{$\underline{\text { Provider considerations }}$}

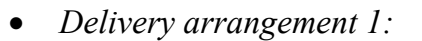

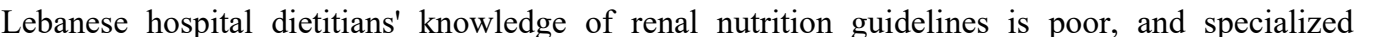

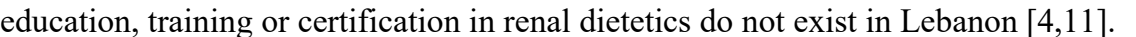

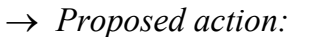

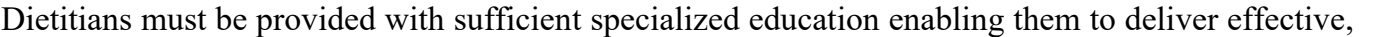

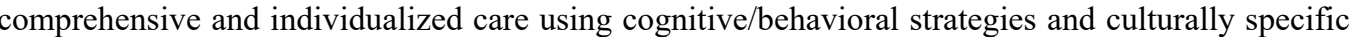

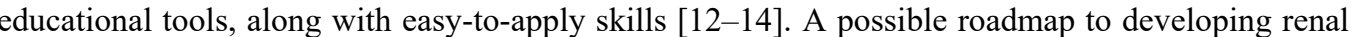

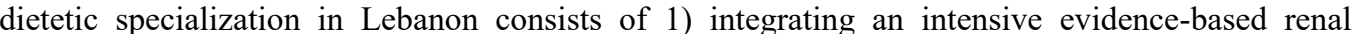

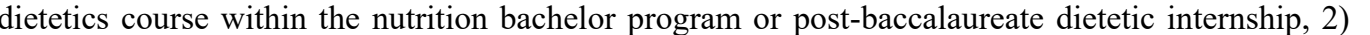

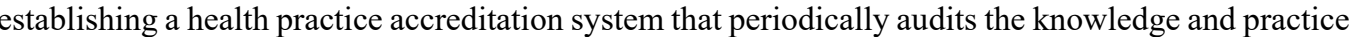

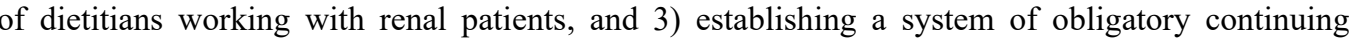

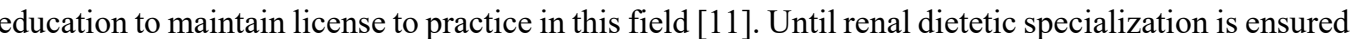

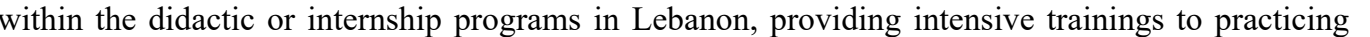

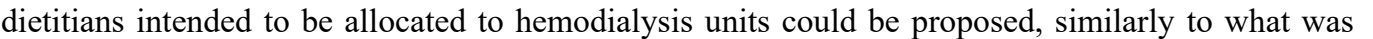

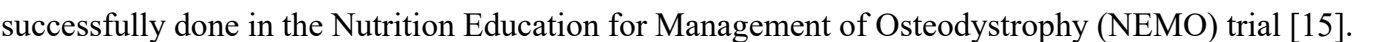

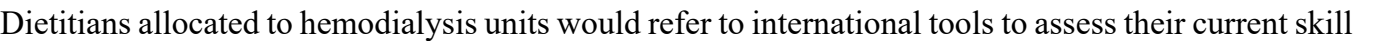

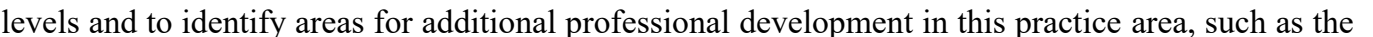

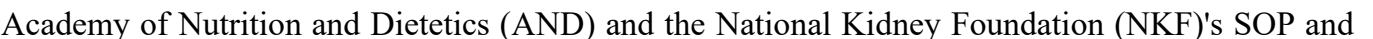

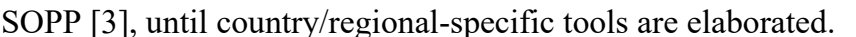

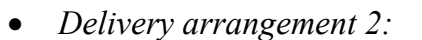

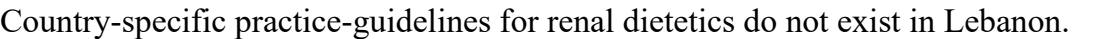

$\rightarrow \quad \square[||||||||||||||$

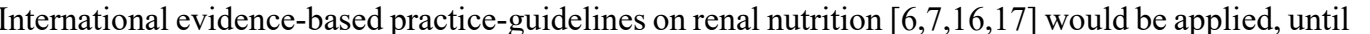

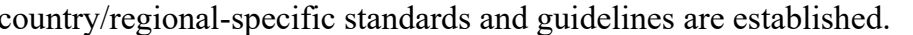

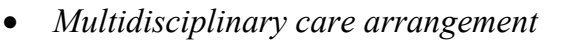

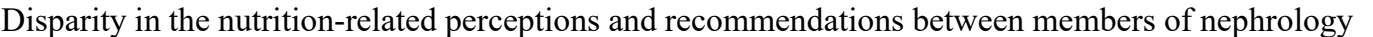

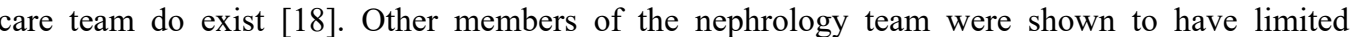

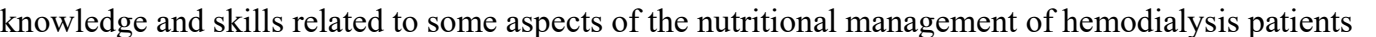




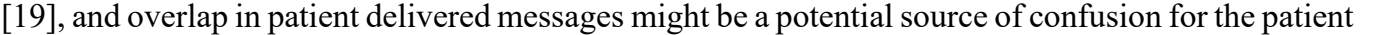
पामाणा

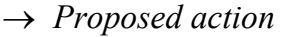

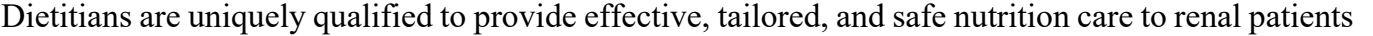

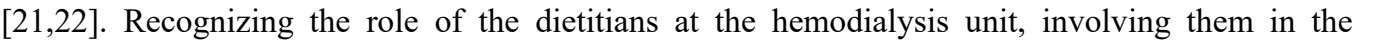

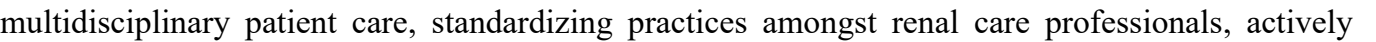

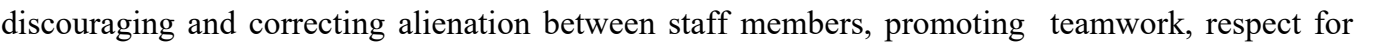

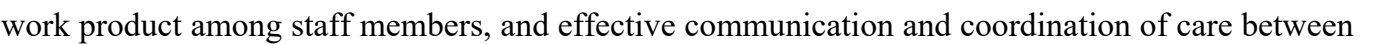

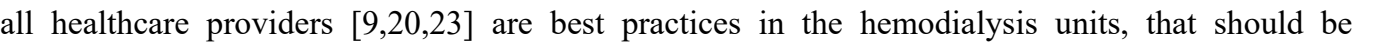

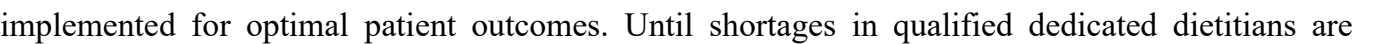

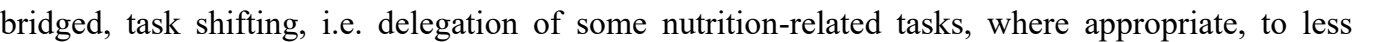

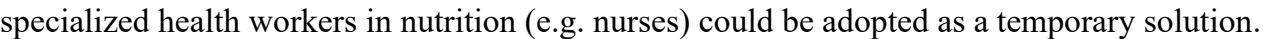




\section{REFERENCES}

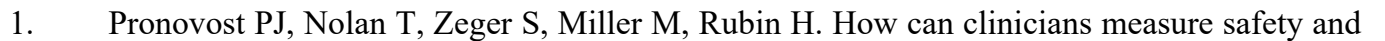

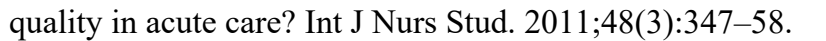

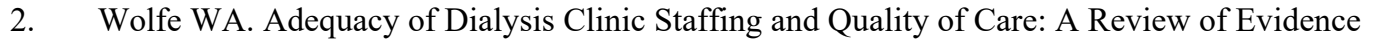

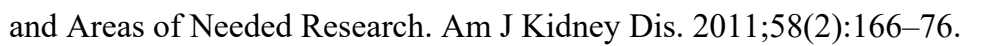

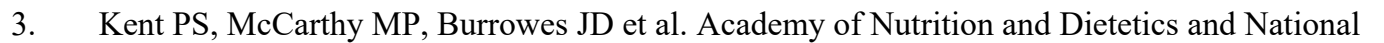

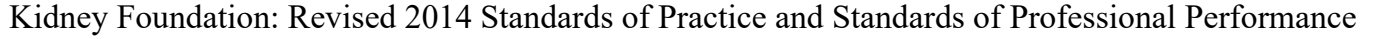

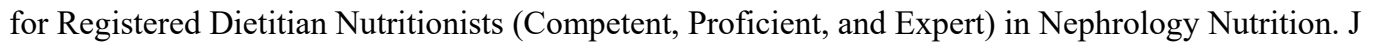

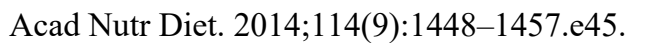

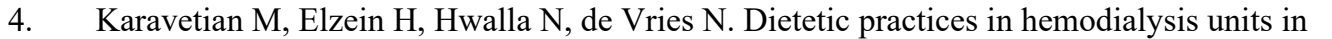
पा

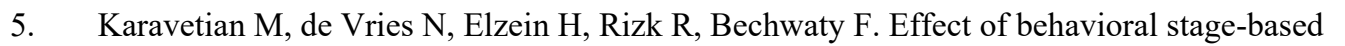

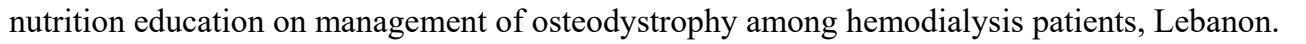

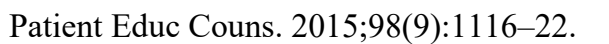

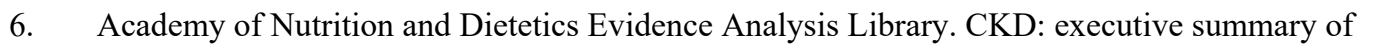

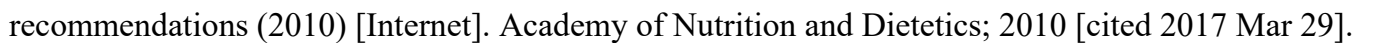

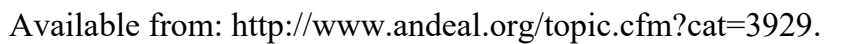

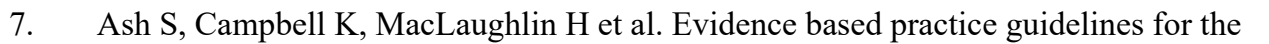

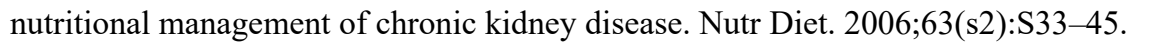

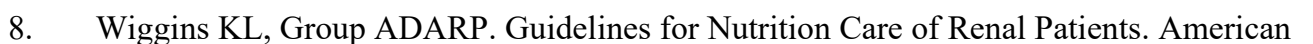

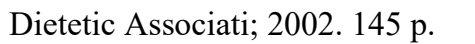

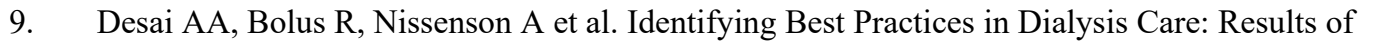

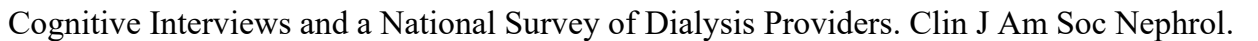

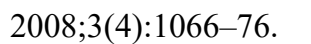

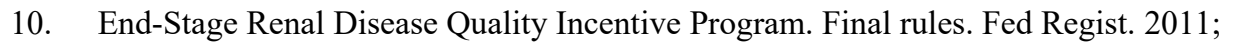

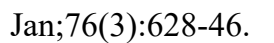

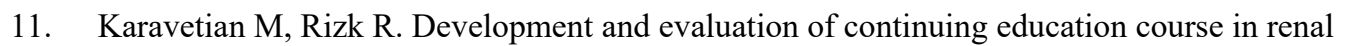

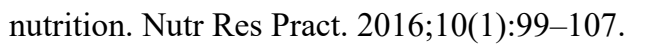

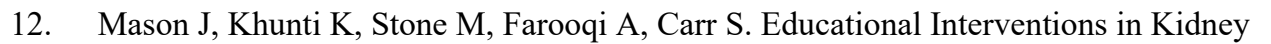

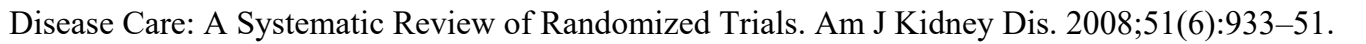

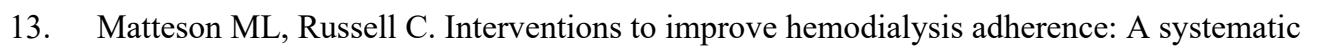

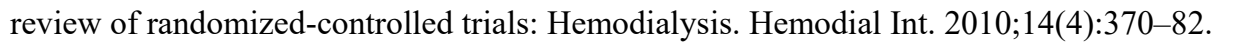

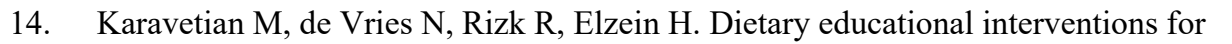

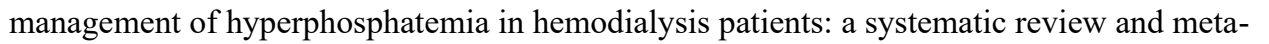

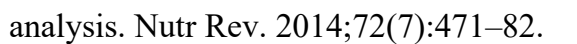

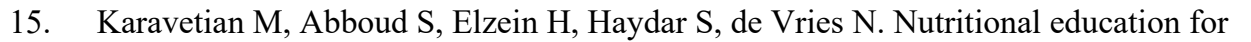

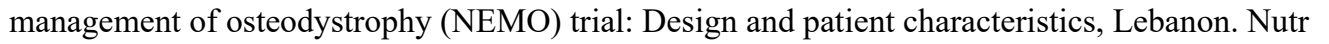

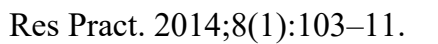




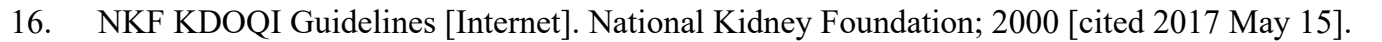

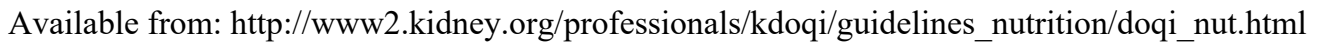

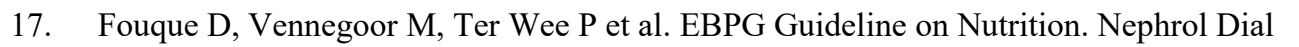

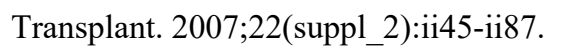

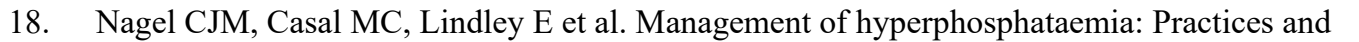

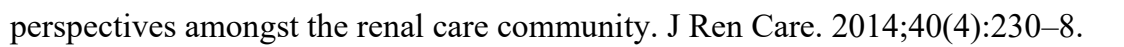

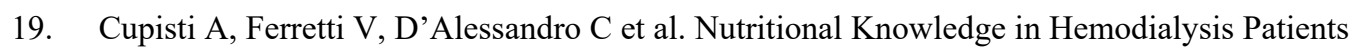

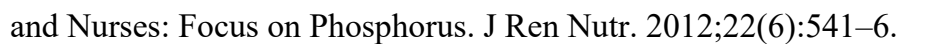

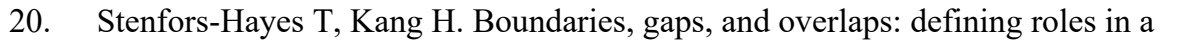

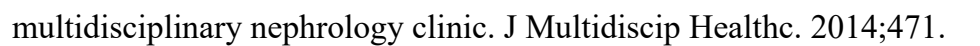

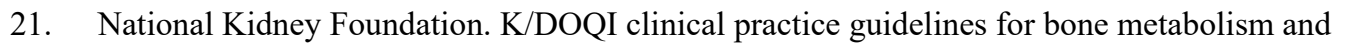

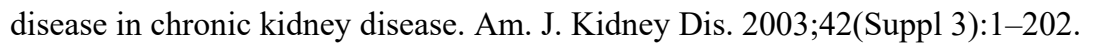

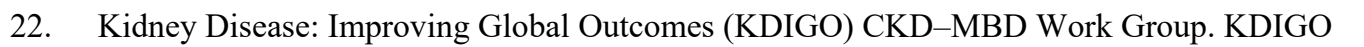

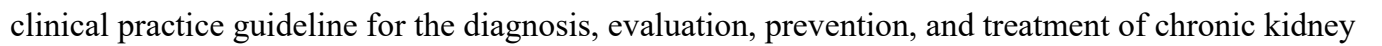

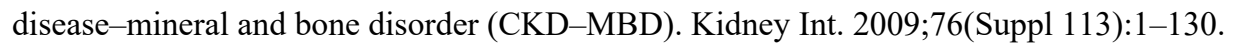

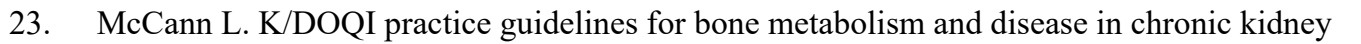

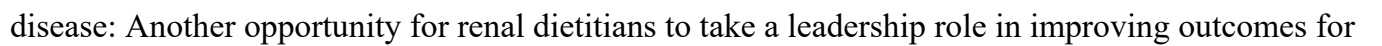

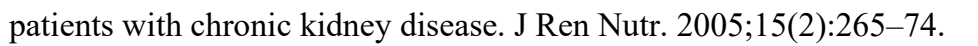

$\square$ 
SUMMARY $\square$ 


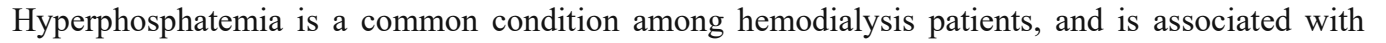

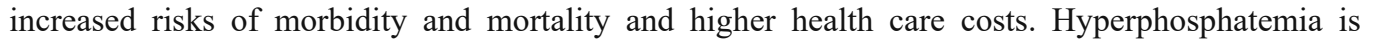

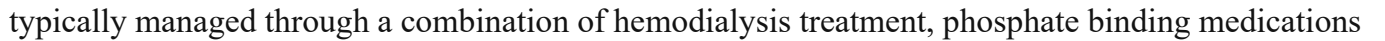

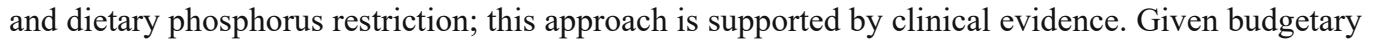

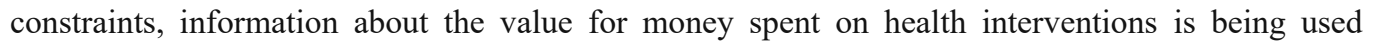

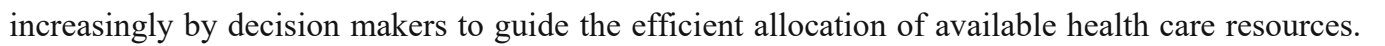

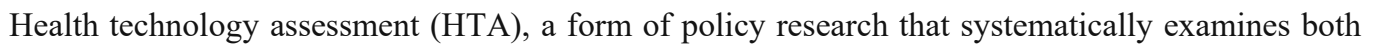

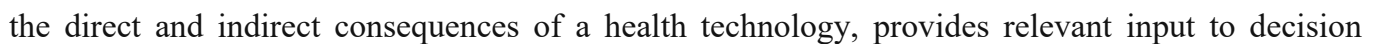

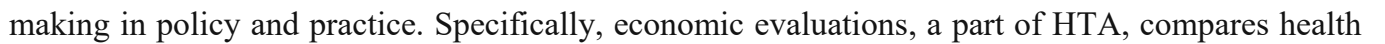

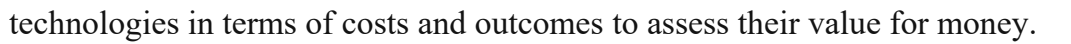

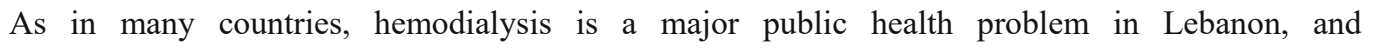

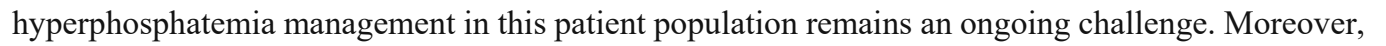

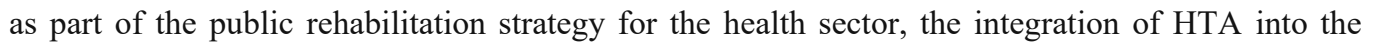

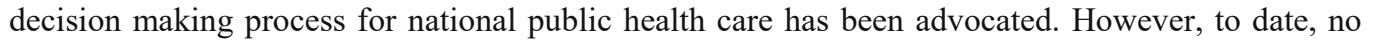

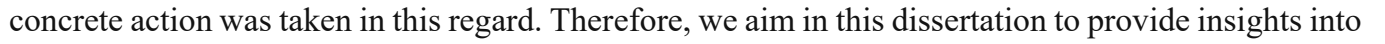

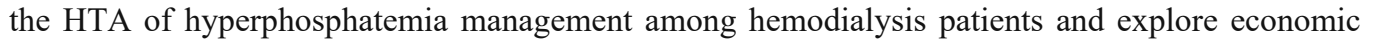

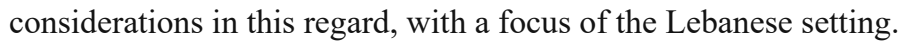

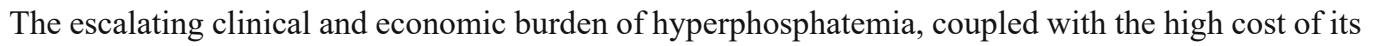

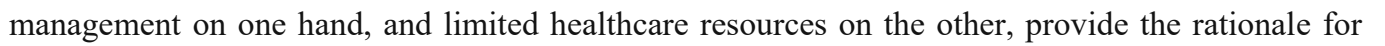

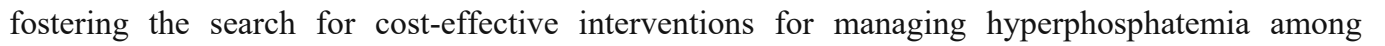

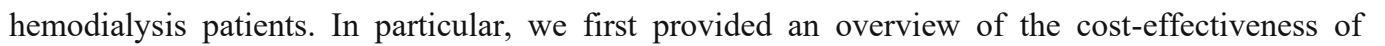

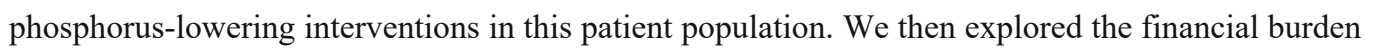

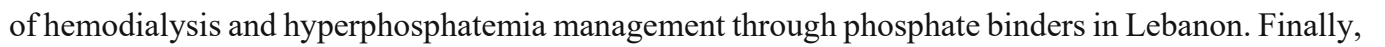

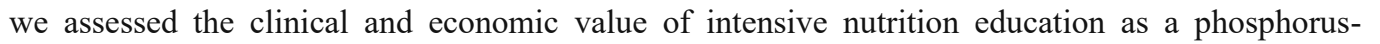

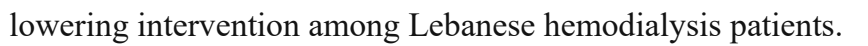

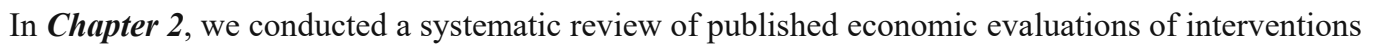

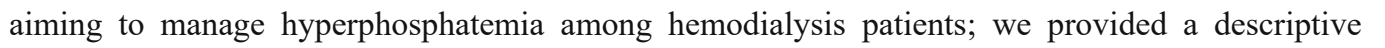

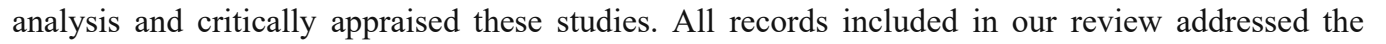

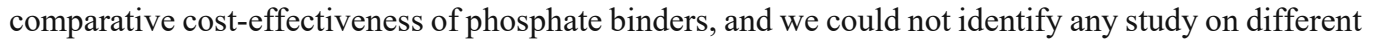

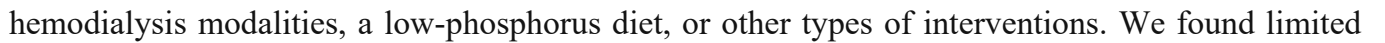

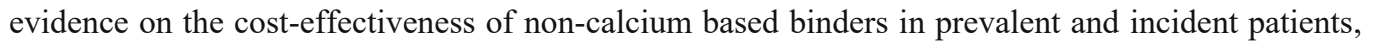

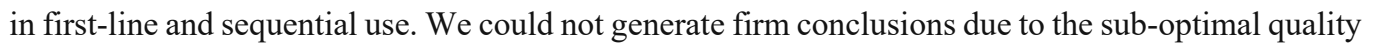

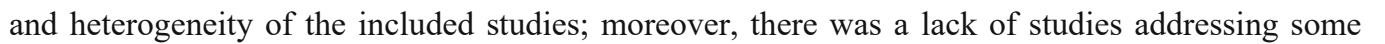

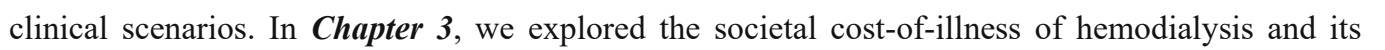

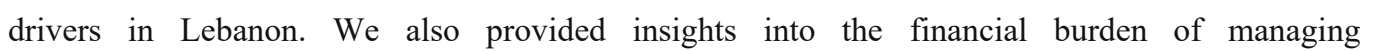

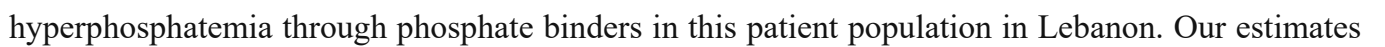


ए

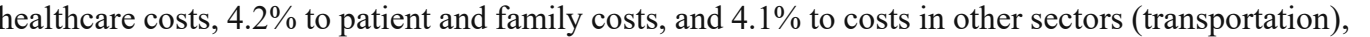

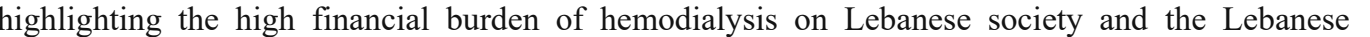

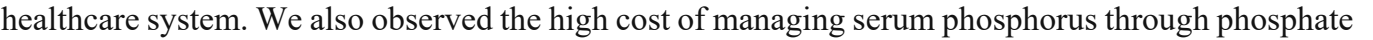

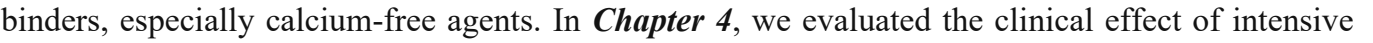

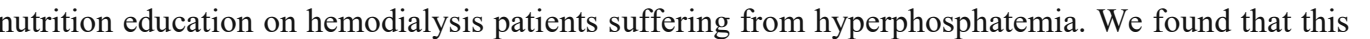

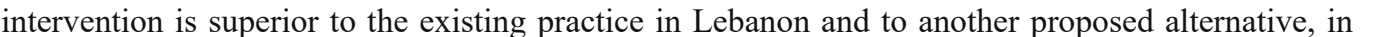

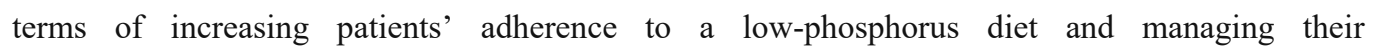

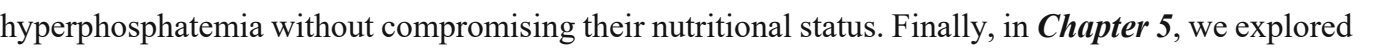

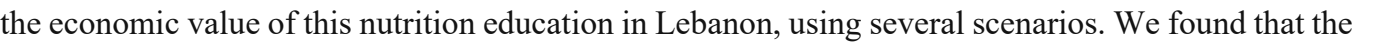

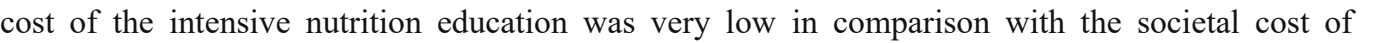

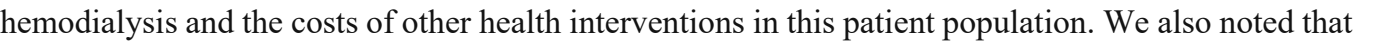

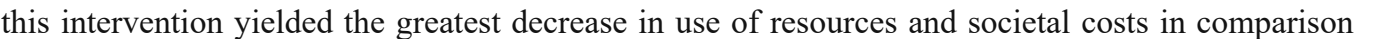

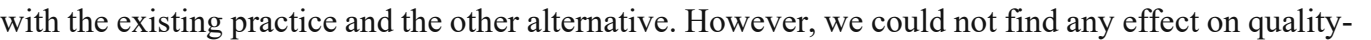

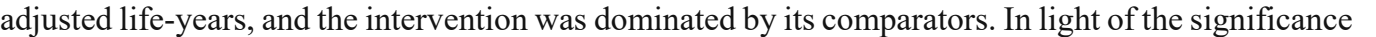

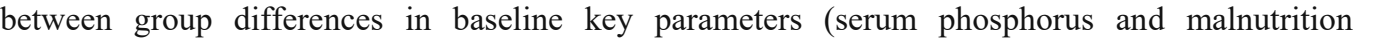

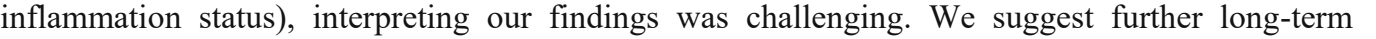

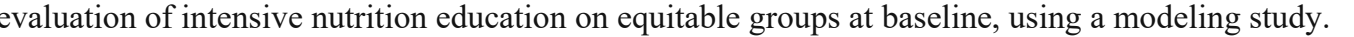

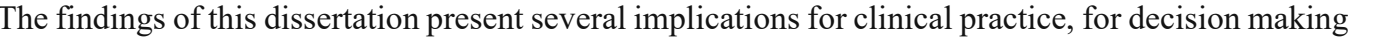

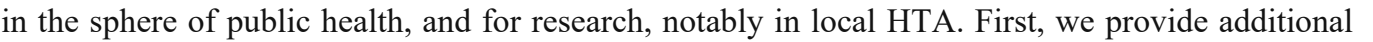

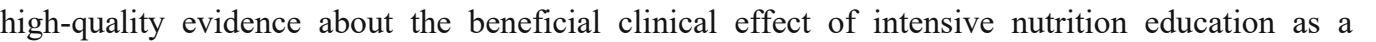

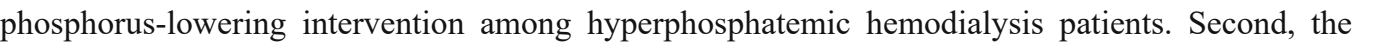

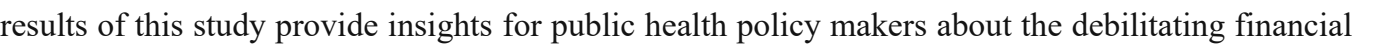

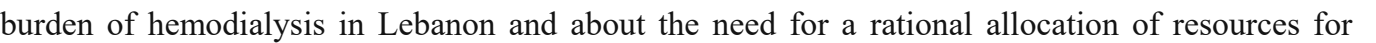

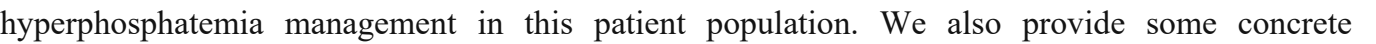

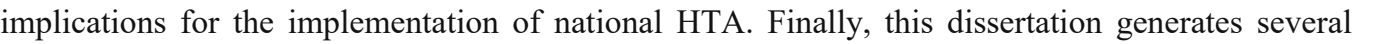

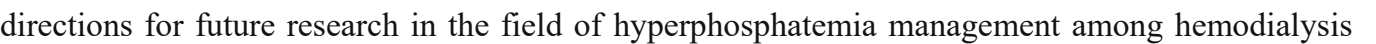

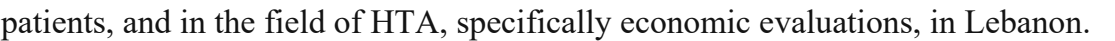

$\square$ 
WORDS OF THANKS $\square$ 


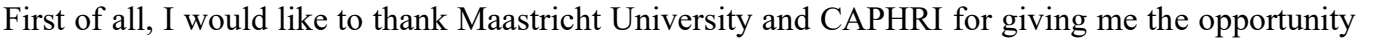

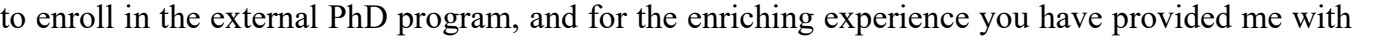

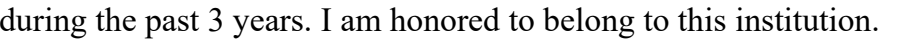

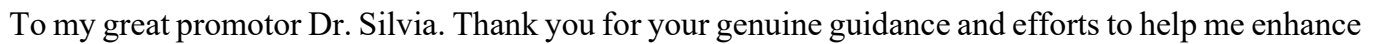

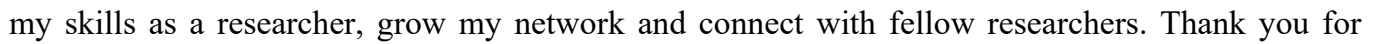

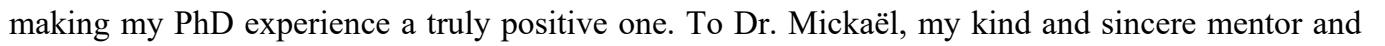

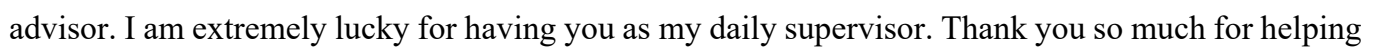

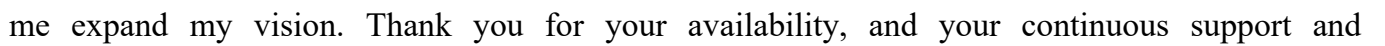

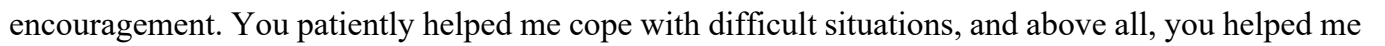

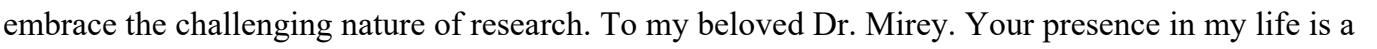

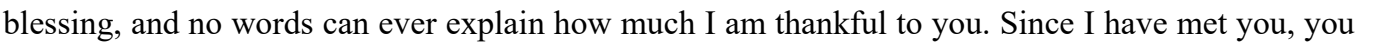

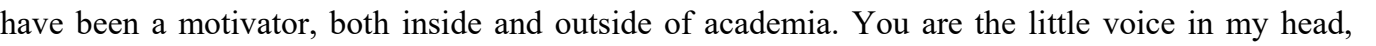

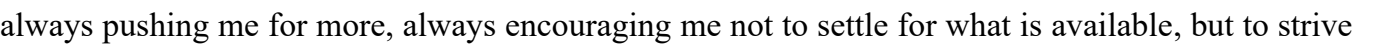

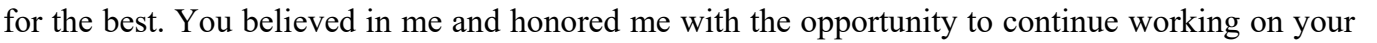

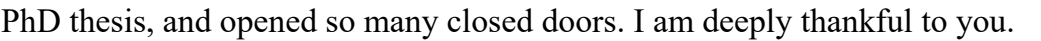

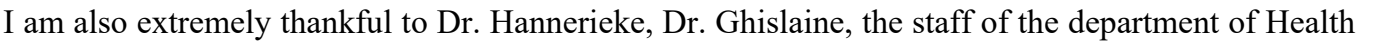

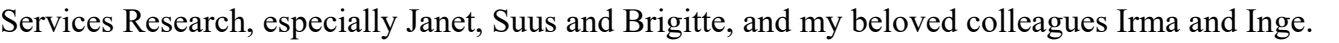

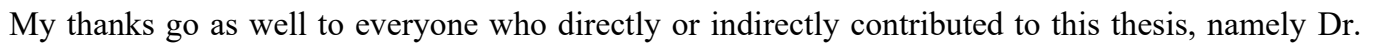

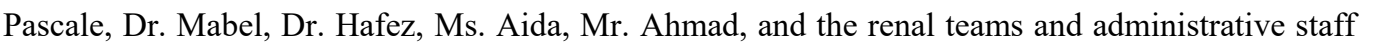

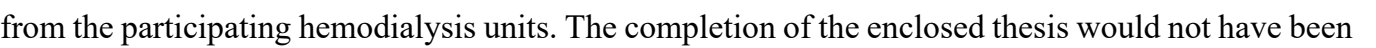

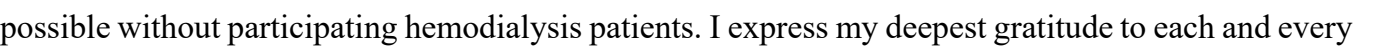

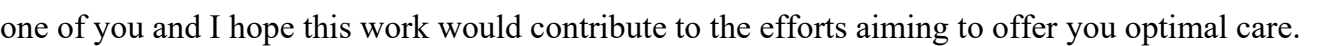

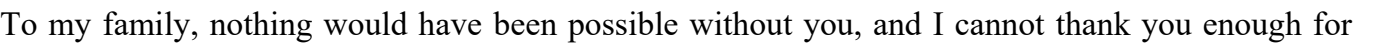

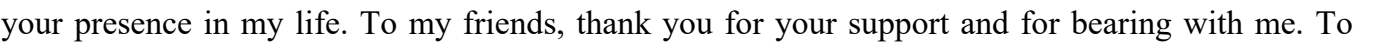
everyone who ever closed a door in my face, thank you... Without you, I would have never grown to

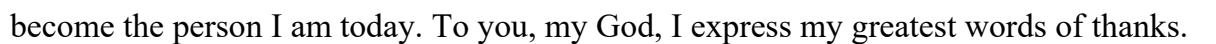

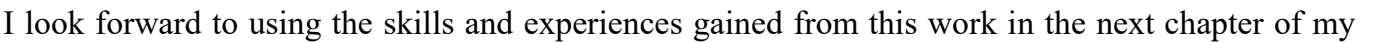

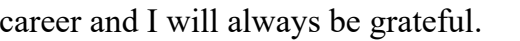

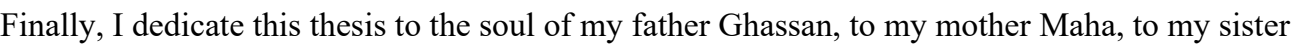

Rima, to my brother Tony... and to you my God, my all... $\square$ 
CURRICULUM VITAE $\square$ 


\section{About The AUthor}

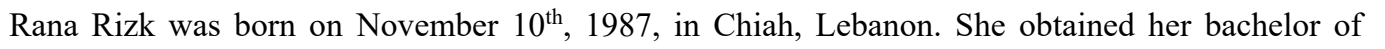

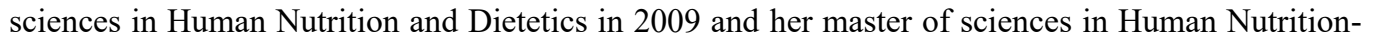

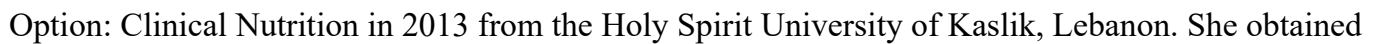

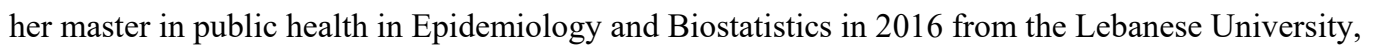
$\square[1111 \mathrm{~m}$

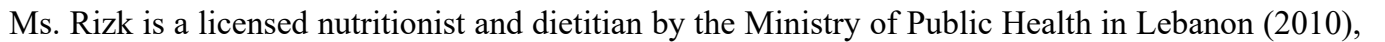

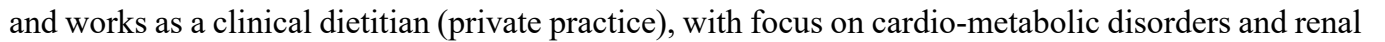

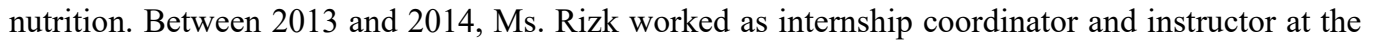

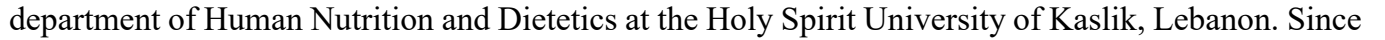

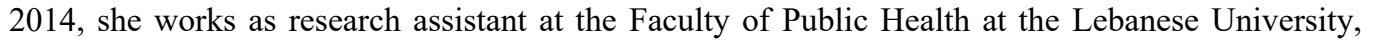

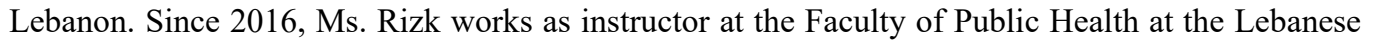

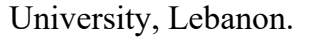

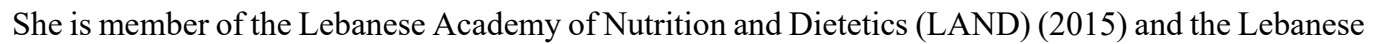

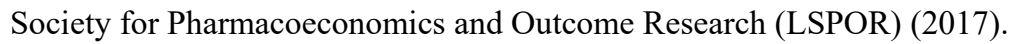




\section{PEER-REVIEWED ARTICLES}

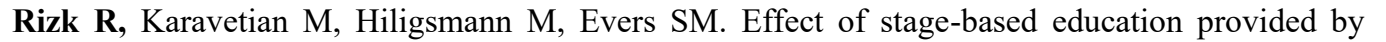

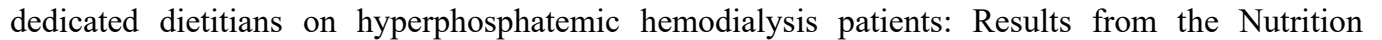

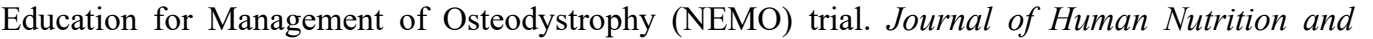

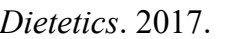

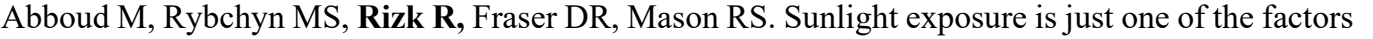

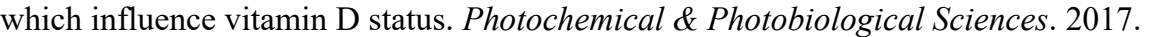

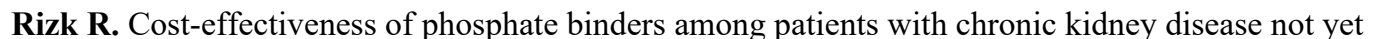

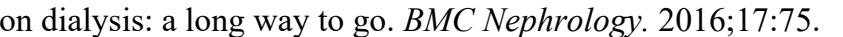

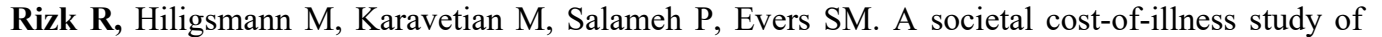

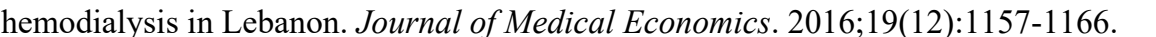

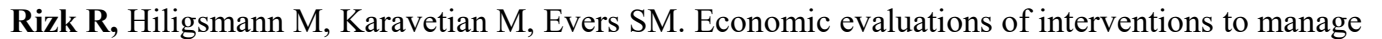

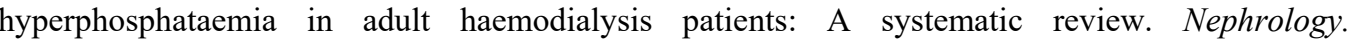

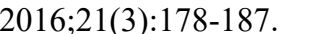

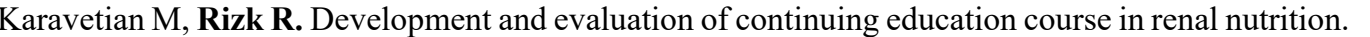

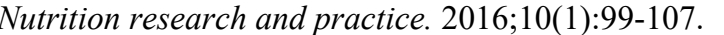

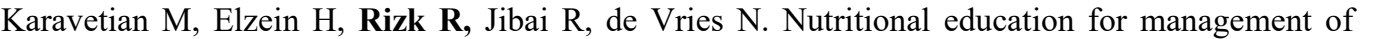

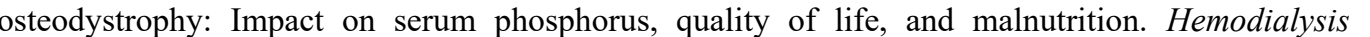

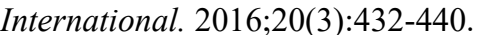

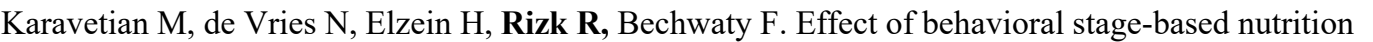

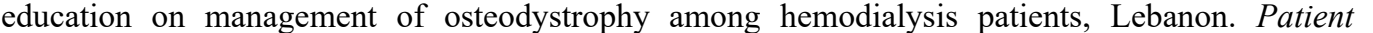

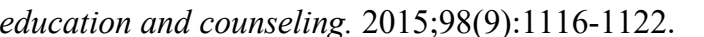

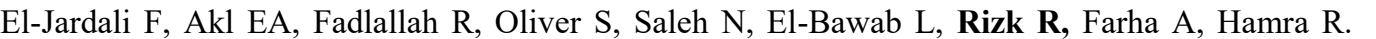

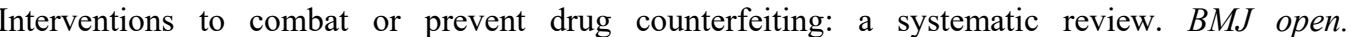
पामापाणामामाप

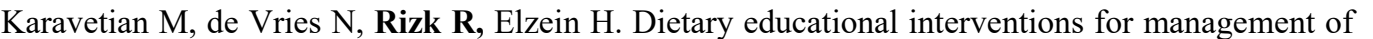

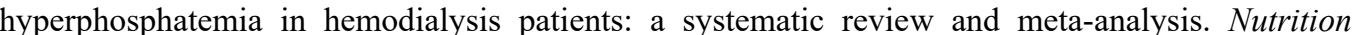

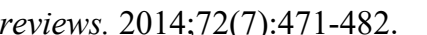

